\title{
Wireless Sensor Network for Advanced Energy Management Solutions
}

\section{Final Report}

\author{
DOE Award Number: DE-FC36-04G014000 \\ Reporting Period: January 2004 - June 2009 \\ Principal Investigator: Peter J. Theisen \\ (414) 449-6924 \\ PeterJTheisen@eaton.com \\ Bin Lu, Ph.D. \\ (414) 449-6036 \\ BinLu@eaton.com \\ Charles J. Luebke \\ (414) 449-7264 \\ CharlesJLuebke@eaton.com \\ Recipient: Eaton Corporation \\ Innovation Center \\ 4201 N. $27^{\text {th }}$ Street \\ Milwaukee, WI 53216
}




\section{DOCUMENT AVAILABILITY, ACKNOWLEDGEMENT, DISCLAIMER and PROPRIETARY DATA NOTICE}

Document Availability:

Reports are available free via the U.S. Department of Energy (DOE) Information Bridge:

http://www.osti.gov/bridge

Reports are available to DOE employees, DOE contractors, Energy Technology Data Exchange (ETDE) representatives, and Information Nuclear Information System (INIS) representatives from the following source:

Office of Scientific and Technical Information

P.O. Box 62

Oak Ridge, TN 37831

Tel: (865) 576-8401

Fax: (865) 576-5728

E-mail: reports@osti.gov

Web Site: http://www.osti.gov/contact.html

Acknowledgement:

This report is based upon work supported by the U.S. Department of Energy under Award No. DE-FC36-04G014000.

\section{Disclaimer:}

Any findings, opinions, and conclusions or recommendations expressed in this report are those of the author(s) and do not necessarily reflect the views of the Department of Energy. 
Table of Contents

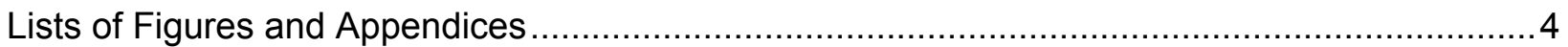

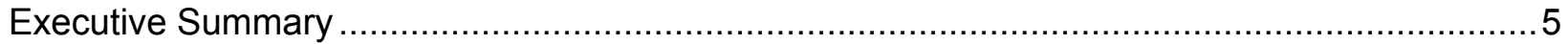

Introduction

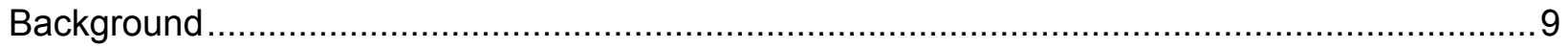

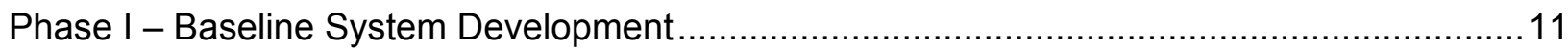

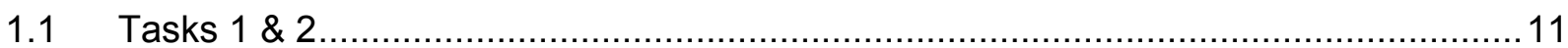

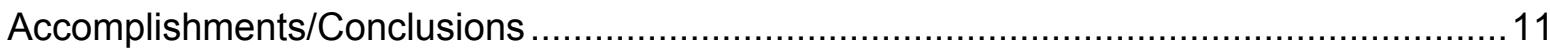

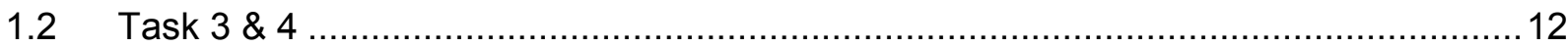

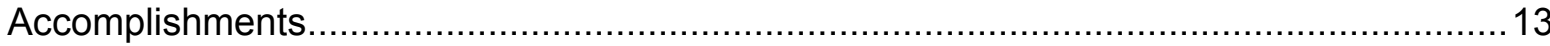

1.3 Task 5 w

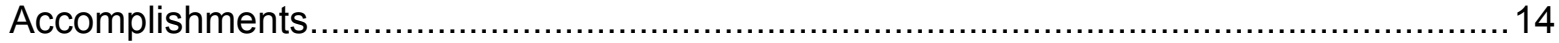

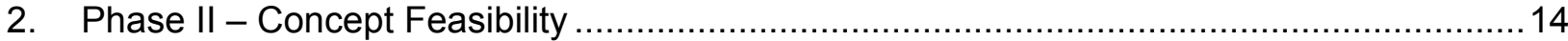

$2.1 \quad$ Task 6

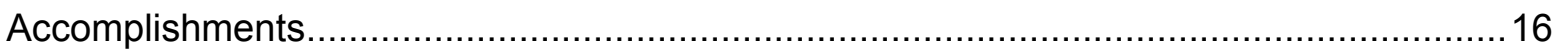

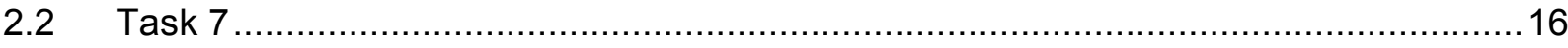

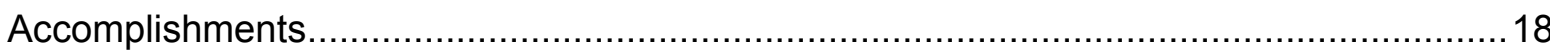

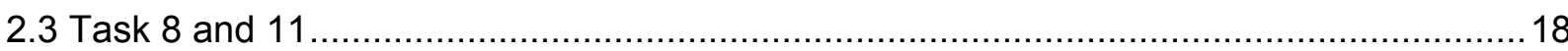

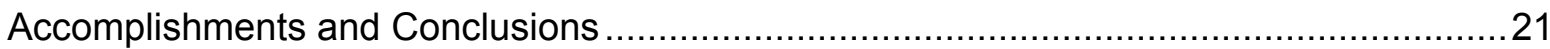

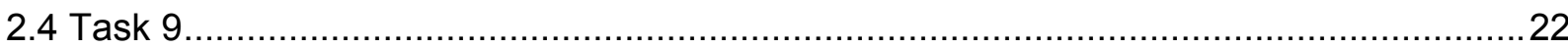

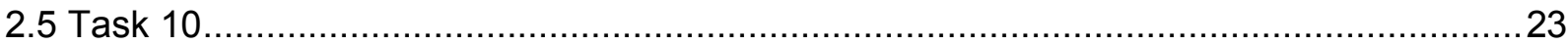

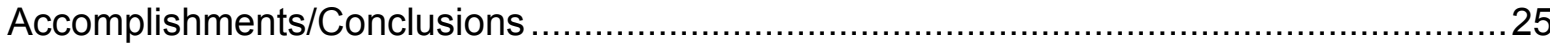

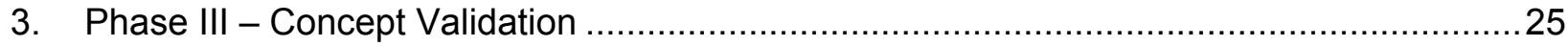

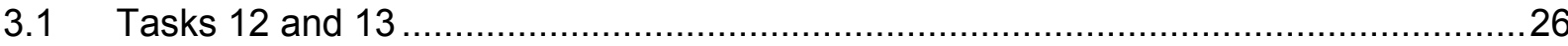

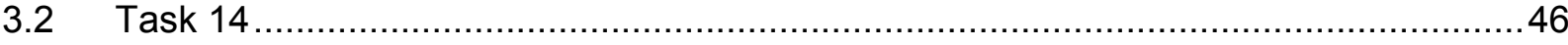

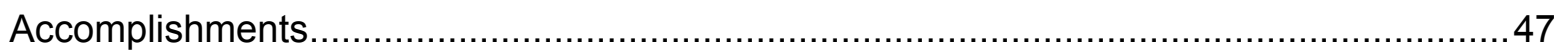

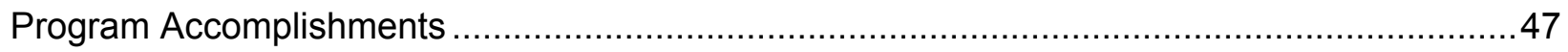

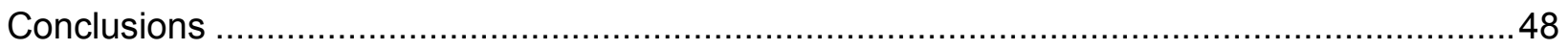

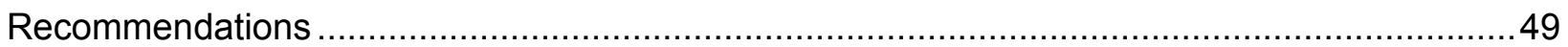




\section{Figures}

\section{Lists of Figures and Appendices}

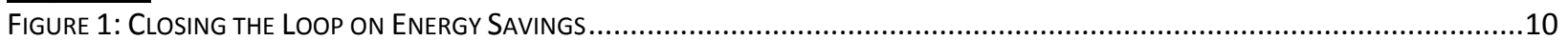

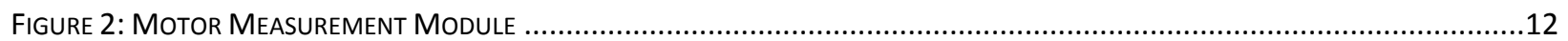

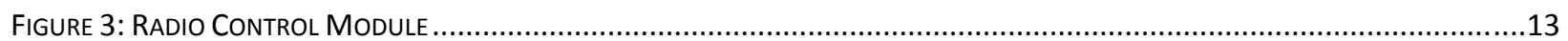

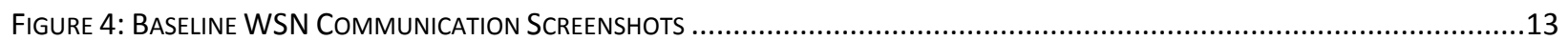

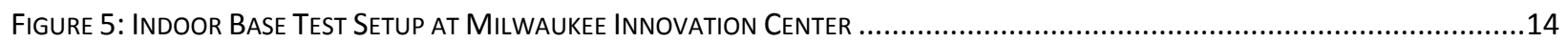

FIGURE 6: POWER CONSUMPTION OF WIRELESS SENSOR

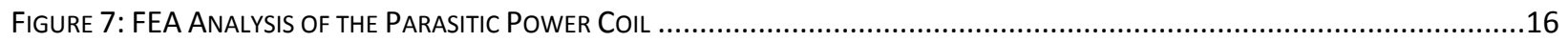

FIGURE 8: DUAL RADIO INDUSTRIAL WIRELESS TEST NOdE INSIDE MOTOR CENTER ............................................................17

Figure 9: Top VieW of GReNAdINE PART OF THE EATON WIRELESS LINK ASSESSMENT SYSTEM ................................................20

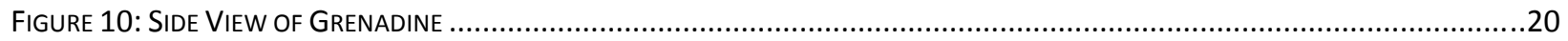

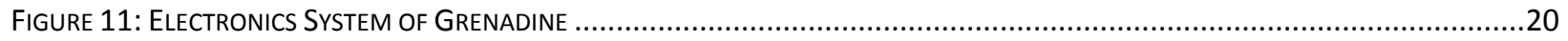

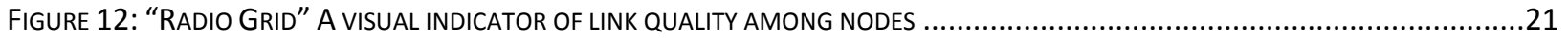

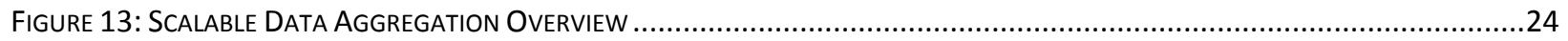

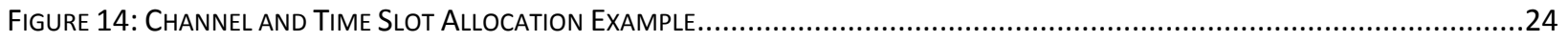

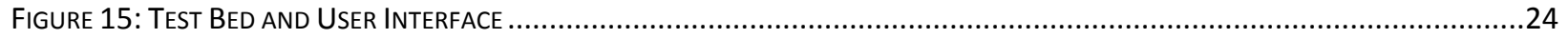

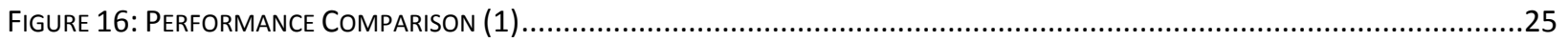

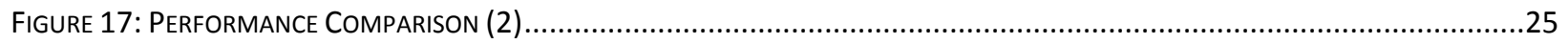

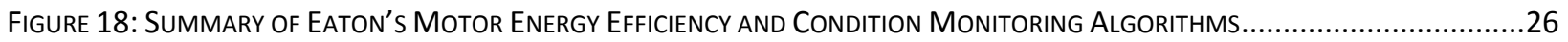

FIGURE 19: NONINTRUSIVE AND INFERENTIAL BASED ALGORITHM DEVELOPMENT METHODOLOGY ..............................................27

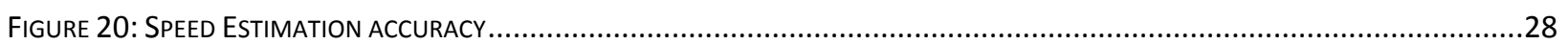

FIGURE 21: A GENERAL APPROACH FOR NONINTRUSIVE MOTOR EFFICIENCY ESTIMATION …......................................................29

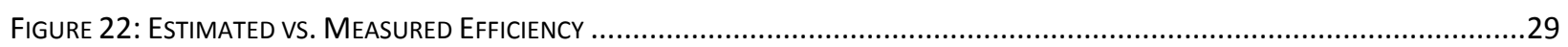

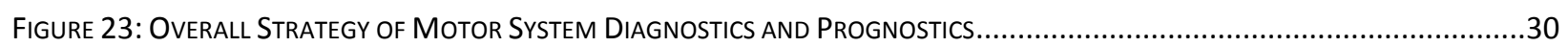

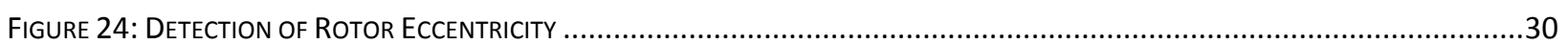

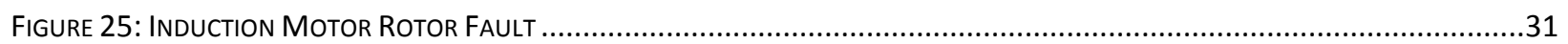

FigURE 26: EXPERIMENTAL RESULTS (MOtOR: LESSON 7.5 HP, 230 V, 20 A, NEMA-B, ODP, 1760 RPM) ..................................31

FIGURE 27 : TYPICAL MOTOR CURRENT SIGNATURE WITH AND WITHOUT PUMP CAVITATION...................................................32

FIGURE 28: DESCRIPTION OF THE INSULATION FAULT DETECTION METHOD USING NEURAL NETWORKS. ...........................................32

Figure 29: Lab Validation Setup at Georgia TeCH and Experimental Results of Bearing Fault Algorithm ........................33

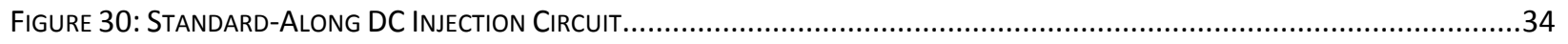

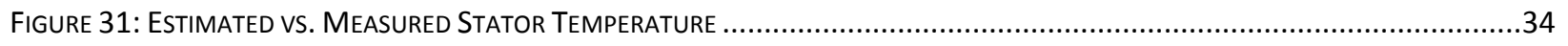

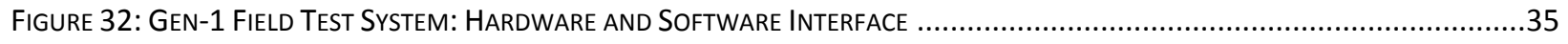

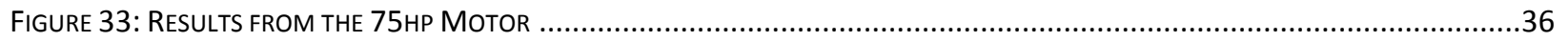

FIGURE 34: POWER, LOADING AND EFFICIENCY RESULTS OF THE 200HP COMPRESSOR MOTOR …............................................37

FIGURE 35: FoRMAL REPORT TO COMPANY A WITH OBSERVATIONS AND SUGGESTIONS .........................................................37

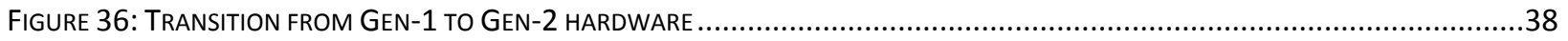

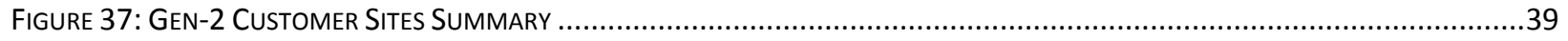

Figure 38: Gen-2 Customer Site Installations at Waste Water Treatment Plant- Company B.......................................40

Figure 39: Energy SaVIng Potential as WASte Water TReatment Plant of Company B ................................................42

Figure 40: Gen-2 Customer Site Installations at -Container Box Plant Company C ................................................42

Figure 41: Energy SaVing Potential At ContaIner Box Plant of Company C.............................................................4

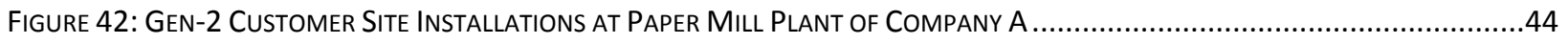

Figure 43: Energy SaVIng Potential at A Paper Mill Plant of Company A .................................................................46

\section{Appendices}

Appendix A - Supporting Documentation and Internal Reports: Tasks 1-14

Appendix B - External Publications

Appendix C - Patents 


\section{Executive Summary}

Eaton has developed an advanced energy management solution that has been deployed to several Industries of the Future (IoF) sites. This demonstrated energy savings and reduced unscheduled downtime through an improved means for performing predictive diagnostics and energy efficiency estimation. Eaton has developed a suite of online, continuous, and inferential algorithms that utilize motor current signature analysis (MCSA) and motor power signature analysis (MPSA) techniques to detect and predict the health condition and energy usage condition of motors and their connect loads. Eaton has also developed a hardware and software platform that provided a means to develop and test these advanced algorithms in the field. Results from lab validation and field trials have demonstrated that the developed advanced algorithms are able to detect motor and load inefficiency and performance degradation.

Eaton investigated the performance of Wireless Sensor Networks (WSN) within various industrial facilities to understand concerns about topology and environmental conditions that have precluded broad adoption by the industry to date. A Wireless Link Assessment System (WLAS), was used to validate wireless performance under a variety of conditions. Results demonstrated that wireless networks can provide adequate performance in most facilities when properly specified and deployed. Customers from various loF expressed interest in applying wireless more broadly for selected applications, but continue to prefer utilizing existing, wired field bus networks for most sensor based applications that will tie into their existing Computerized Motor Maintenance Systems (CMMS). As a result, wireless technology was deemphasized within the project, and a greater focus placed on energy efficiency/predictive diagnostics. Commercially available wireless networks were only utilized in field test sites to facilitate collection of motor wellness information, and no wireless sensor network products were developed under this project.

As an outgrowth of this program, Eaton developed a patented energy-optimizing drive control technology that is complementary to a traditional variable frequency drives (VFD) to enable significant energy savings for motors with variable torque applications, such as fans, pumps, and compressors. This technology provides an estimated energy saving of $2 \%-10 \%$ depending on the loading condition, in addition to the savings obtained from a traditional VFD. The combination of a VFD with the enhanced energy-optimizing controls will provide significant energy savings (10\% to $70 \%$ depending on the load and duty cycle) for motors that are presently connected with across the line starters. It will also provide a more favorable return on investment (ROI), thus encouraging industries to adopt VFDs for more motors within their facilities. The patented technology is based on nonintrusive algorithms that estimate the instantaneous operating efficiency and motor speed and provide active energy-optimizing control of a motor, using only existing voltage and current sensors. This technology is currently being commercialized by Eaton's Industrial Controls Division in their next generation motor control products. Due to the common nonintrusive and inferential nature of various algorithms, this same product can also include motor and equipment condition monitoring features, providing the facility owner additional information to improve process uptime and the associated energy savings.

Calculations estimated potential energy savings of 261,397GWh/Yr (\$15.7B/yr), through retrofitting energy-optimizing VFDs into existing facilities, and incorporating the solution into building equipment sold by original equipment manufacturers (OEMs) and installed by mechanical and electrical contractors. Utilizing MCSA and MPSA for predictive maintenance (PM) of motors and connected equipment reduces process downtime cost and the cost of wasted energy associated with shutting down and restarting the processes. Estimated savings 
vary depending on the industry segment and equipment criticality per facility/process. Average downtime for an industrial facility is $4-12$ hours with a cost $/ \mathrm{hr}$ of $\$ 7500 / \mathrm{hr}$, with large, critical processes reaching $\$ 50-100 \mathrm{k} / \mathrm{hr}$. Specific downtime costs are not included in this report because of customer confidentiality, but projected savings across the Industries of the Future (loF) are still expected to be comparable to the original program estimates.

Two generations of customer field deployments and evaluation have been completed during the course of this project. Customer sites included:

- a $200 \mathrm{hp}$ compressor motor at a container box plant,

- a $100 \mathrm{hp}$ pump and a $15 \mathrm{hp}$ spindle motor at an industrial hydraulic plant,

- a $400 \mathrm{hp}$ digester mixer machine at a paper pulp plant, and

- a $125 \mathrm{hp}$ remote lift station pump, a $50 \mathrm{hp}$ pump, and a $350 \mathrm{hp}$ blower at a waste water treatment plant.

Results from these customer sites have been used for identifying the scope and improving the developed energy and wellness algorithms. The field deployments have confirmed that the hardware for sensing and sampling motor currents and voltages are reliable and able to provide an adequate signal-to-noise ratio from the electrical noise present on the motor signals.

A series of customer review meetings have been held with the lead customer sites to review motor operating conditions estimated by the developed technologies to the site maintenance staff, and validate/ improve the value proposition of the technology. The monitoring results provided to the customers over the course of the project have generated very positive impact on their PM and production activities. Meanwhile, the feedback from the customers helped the development team to quantify the savings of the developed technologies provided to their facilities and project the overall energy saving opportunities when the technologies are widely used across the industries. The following is a list of a few specific examples from the customer field trials, showing how the Eaton motor wellness prototypes using the developed technologies provided energy savings and reduced unscheduled downtime through the application of predictive motor diagnostics:

- The shaft misalignment detection algorithm indicated a rising misalignment pattern for a $200 \mathrm{hp}$ compressor motor, allowing for planned maintenance and repair, resulting in the elimination of an estimated 12 hours of unscheduled down time and the estimated costs of $\$ 90,000$.

- The algorithms in the wellness unit identified a highly dynamic load cycling pattern and associated reduced efficiency of a $200 \mathrm{hp}$ compressor system. Improvements were made working with the equipment vendor resulting in \$2,172 annual energy saving and an undetermined uptime improvement by reduced ageing of the equipment. The site maintenance supervisor offered this quote "This is the closest thing to a crystal ball that I have seen yet".

- The algorithms in the wellness unit identified a highly under-loaded condition (10\% to $40 \%$ load variation) for a $125 \mathrm{hp} \mathrm{VFD}$ driven pump motor. The motor efficiency estimation algorithm estimated the average efficiency of only $55 \%$, which is greatly lower than the $95 \%$ rated motor efficiency. A VFD with an energy-optimizing control feature will provide an additional $\$ 2,500$ savings per year. The projected savings have been confirmed by two field tests using the customer's VFD with Eaton's energy-optimizing control algorithm.

- The pump cavitation detection algorithm in the wellness unit identified occasional early pump cavitation symptoms for a $125 \mathrm{hp} \mathrm{VFD} \mathrm{driven} \mathrm{pump} \mathrm{motor.} \mathrm{Remedy} \mathrm{of} \mathrm{this} \mathrm{situation}$ during a scheduled maintenance interval eliminated an estimated 10 hours of unscheduled down time and the estimated costs of $\$ 72,000$. 
- The broken rotor bar fault detection algorithm in the wellness unit identified an early stage induction motor rotor fault for a $400 \mathrm{Hp}$ VFD driven mixer motor. Remedy of this situation during a scheduled maintenance interval eliminated an estimated 15 hours of unscheduled down time and the estimated costs of $\$ 112,000$.

- The algorithms in the wellness unit identified a highly dynamic load variation of 58 to $71 \%$ for a $350 \mathrm{Hp}$ VFD driven blower motor. Modern drive technology has the potential for $8 \%$ energy savings resulting in $\$ 2,540$ in yearly cost improvement. 


\section{Introduction}

The early tasks within the program were to assess the application of wireless technologies to enable broad deployment of wellness and energy monitoring. It was recommended at the annual program review in 2007 that the emphasis be shifted more to energy savings and predictive diagnostic methods. The instantaneous efficiency estimation provides a means to assess how well energy is being utilized by individual motors and the connected equipment. As a result of this project, Eaton is now pursuing the commercialization of several products to provide the Industries of the Future with a cost effective solution of predicting motor and connected load health and instantaneous motor efficiency. These products will enable broad, continuous coverage for monitoring industrial equipment that is deployed today. The information provided by these products will drive a dramatic reduction in the amount of unscheduled downtime (lost production and lost energy) and provide a means to identify and improve the efficiency of motor driven equipment. This will reduce energy use, thus making businesses more competitive. This family of equipment health and efficiency monitoring products will be primarily applied as a retrofit solution into existing facility systems. In order to enable low cost installation and commissioning, integration with motor controls was employed.

The core technology behind the Eaton motor and equipment health and efficiency monitoring is inferring (or extracting) diagnostics and prognostics information from the sensed power (current and voltage) signature. Initially it was thought that the value of this diagnostic was to verify the performance of rewound or damaged motors. However, this technology provides a means for most facilities to easily and cost-effectively expand beyond the present coverage of preventative maintenance (PM). Typically only large, critical motors are monitored today by periodic vibration analysis, or with embedded sensors. Maintenance personnel are very interested in inferential diagnostics from power sensing as a means to get broader coverage of equipment, and obtain an early indication to identify motors that need a closer PM check. As a result, Eaton is pursuing the development and commercialization of a predictive motor health monitor that can be easily retrofit into motor control centers within existing facilities.

Providing instantaneous efficiency estimation as well as energy usage and other power quality parameters is of high interest to many loF. As a result of the field trials, Eaton determined that customers were particularly interested in having the motor controls (drives) directly optimize the energy use, versus just metering/monitoring efficiency and energy use. As a result, Eaton is developing and commercializing an energy optimizing drive that will minimize the energy required to maintain a variable torque load.

Targeted market segments for Predictive Diagnostics and Energy Savings include: forestry, wastewater treatment, Industrial Continuous Processes, and primary metals. These markets were identified because they want to save energy, have many medium size motors on critical equipment, already perform PM to minimize unscheduled downtime (vs. run to failure), and are optimized for throughput (lost production $=$ lost revenue). Targeted equipment in those facilities are variable torque loads (centrifugal pumps, fans, and compressors including air handling and HVAC applications) operating at varying loads with service factor of $70 \%$ or less. 


\section{Background}

DOE research indicates that motors below 200 horsepower make up $98 \%$ of those in service and consume $85 \%$ of the energy used. Widespread deployment of energy management systems is critical in realizing possible energy savings. Wireless sensor technology was recognized as a potential enabler for existing and new electrical distribution and power control systems to communicate and report information for diagnostics and prognostics purposes. Today, only a small percentage of this equipment is capable of communicating information due to cost of installation and the use of proprietary interfaces that are non-interoperable among different sensor manufacturers. A Wireless Sensor Network (WSN) can provide a low-cost path to deliver voltage, current, power, load, and other key process information to facility/enterprise systems or applications. The WSN enables these systems to save energy, provide diagnostics and prognostics, and improve uptime across the entire plant.

To illustrate how this broad monitoring and diagnostic information can be applied, consider the problem of properly sizing replacement electric motors. An early Xenergy study for the DOE in 1998 estimates that 6,786 GWh/year can be saved simply by properly sizing replacement motors (United States Industrial Electric Motor Systems Market Opportunities Assessment, prepared for Department of Energy, by Xenergy Inc., December 1998). The number of motors in industries have increased since 1998, therefore the expected energy saving opportunity became even more significant. This report identifies lack of detailed power usage information as the primary cause of incorrectly sizing replacement motors. WSN enables this information to be collected across the plant in a cost effective manner.

The objective is to research, develop, test, and deploy a Wireless Sensor Network (WSN) for the purpose of enabling significant energy savings and Advancing Energy Management Solutions (AEMS) in the loF. The WSN will support open wireless protocols and solve existing industrial application issues through self-configuring, robust and secure performance.

Figure 1 illustrates how Eaton envisions facilitating this energy savings. The focus of this program was centered on the first two models; the success of these will pave the way for the local and enterprise optimization models since they represent incremental changes to the overall system. 


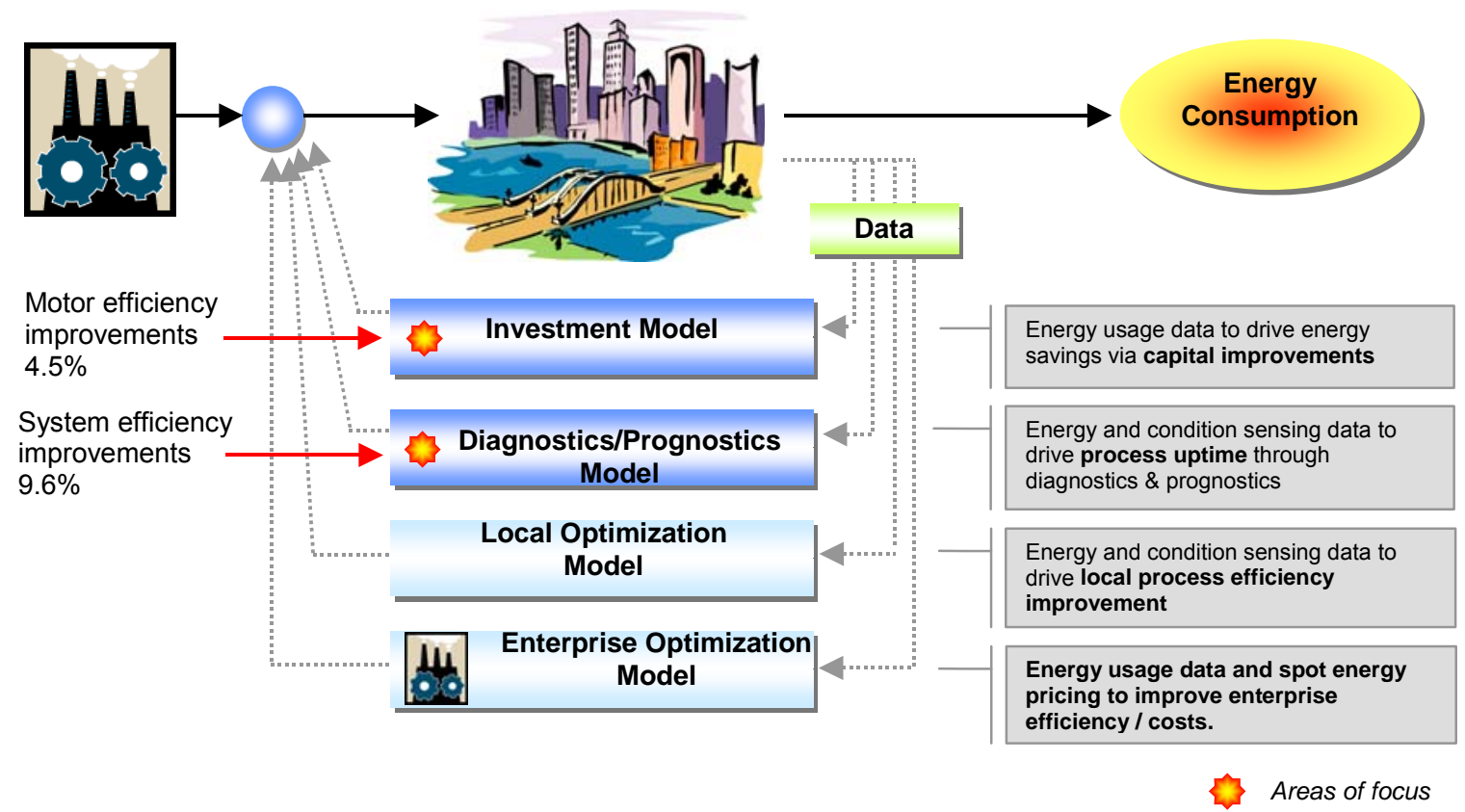

Figure 1: Closing the Loop on Energy Savings

The concept behind Eaton's energy management architecture is to primarily utilize voltage and current measurements to estimate motor energy usage, motor efficiency and diagnostics and prognostics. This lowest cost architecture will facilitate deploying the technology to all motors (even the smallest) utilized in the loF.

Eaton's Innovation Center in Milwaukee, WI leads the research effort for this project; it employs approximately 90 scientists, engineers and support staff with a mission to create growth and value through innovation and technology development. Eaton's Innovation Center focuses on breakthrough opportunities, emerging disruptive technologies, strategic technology capabilities, and closing capability gaps to provide Eaton with a distinctive and sustainable advantage. Technology Thrusts include: Communications, Arc Science for Electrical Power Protection and Control, Prognostics for Wellness enhancements to products, and Sensor/Transducer/Actuator design. Eaton has more than 1000 patents issued in the areas of sensing, monitoring and communications; 150 of these are focused in communications technology (mostly focused in sensors).

Several companies supported the project by helping to define requirements, field testing the technology, and participating in the evaluation of the field test results. Their involvement in the program was critical and ensured the system addresses the broadest range of loF requirements. 


\section{Phase I - Baseline System Development}

The objective of this phase was to evaluate a baseline Wireless Sensor Network (WSN) suitable for operation in an industrial environment. The baseline WSN demonstrated feasibility of the basic networking concepts. Demonstrations used wireless-enabled sensors that consisted of current, voltage, and other monitoring sensors and on/off actuators (mains powered devices).

\subsection{Tasks $1 \& 2$}

Objective: Work with team and end users to derive and document the WSN requirements, investigate, assess and document alternative solutions

Eaton worked with end users to derive and document the Wireless Sensor Network requirements for the targeted industrial applications based on assessments of Customer Needs, Technology, Competition, and Regulatory requirements. An end-user discussion guide was developed and used to effectively obtain needs during interviews with key individuals in each industry segment. A total of 36 interviews were completed. Eaton also conducted a search of published material to complete the technical and regulatory assessment of competing Energy Management (EM) and Condition Based Monitoring (CBM) solutions. Study objectives included: Identify best practices in motor management, identify measured parameters and their locations, understand deployment of current wired EM/CBM systems, trends, barriers, and value of EM/CBM systems, and testing of a WSN concept.

The findings have been used to help drive the direction of an overall energy management strategy. The required motor data and the necessary measurement parameters for the wireless sensor network have been identified. Investigations into suitable methods for on-line efficiency estimation were also performed.

An assessment of various wireless communications technologies resulted in the selection of IEEE 802.15.4 as the best fit to meet the WSN requirements including installed cost, reliability, and performance. Unlike Wireless LANs (WLANs), Low Rate-Wireless Personal Area Networks (IEEE 802.15.4 LR-WPAN) are designed to convey information without requiring preestablished network setup. Self-configuration and multi-hop capabilities are key attributes of LRWPAN that enable large scale, mesh type networks to be formed to cover long distances and provide redundant paths within large facilities. LR-WPAN is ideally suited to communicate sensing and monitoring information.

Non-LR-WPAN wireless technologies require higher installation costs due to required configuration (by specialized personnel) and they need frequent maintenance. On the other hand, LR-WPAN technology was specifically designed for operation in industrial environments with enough features to allow for the creation of self-configuring mesh networks with a minimal cost on a per node basis and focus on enabling WSNs. IEEE 802.15.4 LR-WPANs use direct sequence spread-spectrum techniques to mitigate the effect of jamming and improve wireless communications reliability. IEEE 802.15.4 and the higher layer functionality of Zigbee are an ideal fit for this application due to its scalability, low power consumption, low-cost radio hardware, self-configuration and self-organization, its meshing capabilities as well as the low data rates required by this application.

\section{Accomplishments/Conclusions}

- Verified energy management and condition-based monitoring systems are currently deployed on larger critical motors to save money 
- Voltage and current measurements at the motor controls can provide large part of value for EM system

- Wireless becoming better understood / more prevalent for monitoring applications

- $\quad-90 \%$ of motors are arranged in the plant in a fashion compatible with LR-WPAN (less than $300 \mathrm{ft}$ from an adjoining motor)

- Motor controls located within buckets of a Motor Control Center (MCC) are also compatible with the use of LR_WPAN (typically less than 30ft apart)

- IEEE 802.15.4 provides best tradeoff for range, power consumption, and as an open industry standard. Has received broad industry endorsement but has not yet been widely deployed and was not implemented in any products.

- Environments for first system components are familiar to Eaton

- WSN concept resonates with those in the Industries of the Future (IOF)

- Need to establish a clear end-user cost/benefit justification to help define WSN system requirements and user adoption

- $\quad$ Larger \& critical un-spared motors are next targets for both EM and CBM

- Facility Managers interested in enhanced EM/CBM algorithms and data, less concerned about how the data is obtained (i.e. wired vs. wireless) as long as it does not increase the demands on maintenance staff.

\subsection{Task 3 \& 4}

Objectives: Develop design specifications for the hardware, wireless devices, power supplies/coupling and software; build the wireless system, integrate hardware and software, and construct a test bed system with sensors, motors and loads, and document

The goal of these tasks was to create a baseline Wireless Sensor Network (WSN) suitable for operation in an industrial environment and demonstrate feasibility of the basic networking concepts. Demonstrations used wireless-enabled sensors that consisted of current, voltage, and other monitoring sensors and on/off actuators (mains powered devices).

Based on the previous findings we proceeded to design the hardware and wireless devices of the baseline WSN test bed software. This hardware design and implementation was completed on September 2004. The hardware includes 250 Radio Control Modules (RCMs, see Figure 3), approximately 60 daughter I/O boards and three Motor Measurement Modules (Figure 2). The core of the wireless nodes consisted of an Atmel mega128L controller and a CC2420/EM2420 as the radio controller.

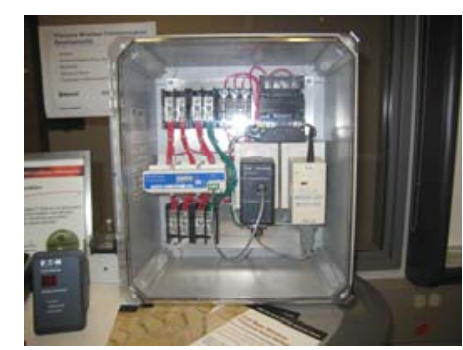

Figure 2: Motor Measurement Module 


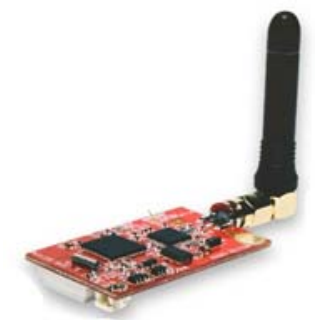

Figure 3: Radio Control Module

Two of the Motor Measurement Modules were connected to motors and were tested as part of the baseline network. Each Motor Measurement Module consisted of an I/Q Universal Power Sentinel (existing Eaton product capable of making 3 phase energy and power measurements), a protocol adapter, power supply and Radio Control Module.

The baseline WSN communications stack chosen for this baseline test was based on the IEEE Std 802.15.4, 2003 version. The stack including the Physical (PHY), Medium Access (MAC) and Network (NWK) layer were modeled using the Rhapsody UML modeling tool and simulated in both Rhapsody and OPNET (a network simulation environment) (Figure 4). Code for the embedded hardware was automatically generated from these high level models using Rhapsody in C++.
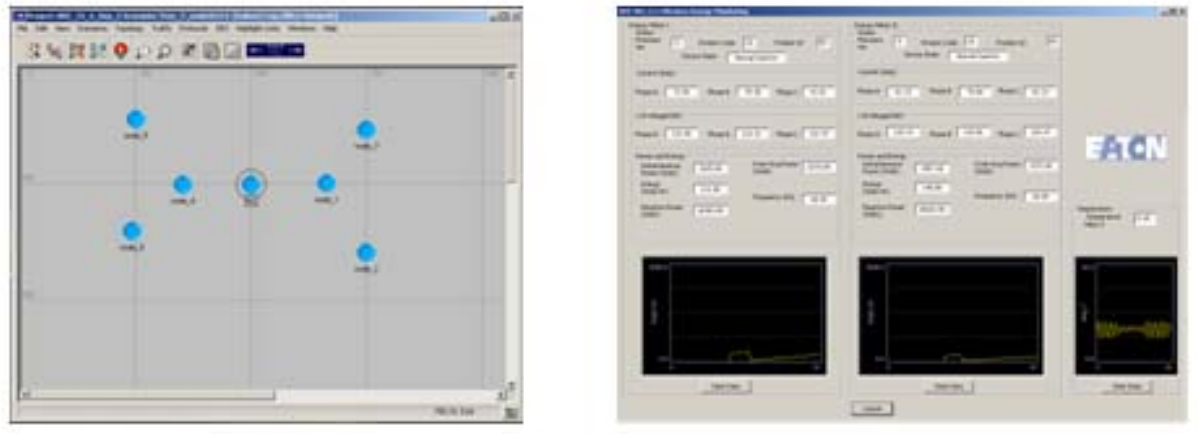

Figure 4: Baseline WSN Communication Screenshots Left to right: Simulation using the OPNET environment, data waveform capture from a lab test motor

\section{Accomplishments}

The development of this baseline system proved that it was possible with the existing technology to build WSN to transmit motor data to a central point for further analysis and was the stepping stone for the next piece of effort to validate the suitability of WSN in industrial environments, converging in two types of testing efforts, described in the Task 5 section of this report. The first was to perform scalability analysis with networks up to 50 nodes, where various data traffic patterns were systematically placed on the network to determine scalability and robustness. The second was to do performance testing measuring latency, packet error rate, throughput, network setup time, range, and reconfiguration delays (dropped nodes, rerouting etc. The results of these tests were compared against the requirements and used to define the required improvements to the baseline network. 


\section{$1.3 \quad$ Task 5}

Objective: Develop test plan, perform baseline tests to determine system performance and document

The objective of this task was to test the baseline Wireless Sensor Network (WSN) developed under Task 4 for operation in an industrial environment and demonstrate feasibility of the basic networking concepts. Demonstrations used wireless-enabled sensors that consisted of current, voltage, and other monitoring sensors and on/off actuators (mains powered devices).

The baseline WSN test bed demonstrated an initial metrics of robustness by deploying a network of 23 nodes (including 2 motors, 2 wall switches, and 1 temperature sensor), with all nodes sending and consuming periodic traffic. This baseline network operated continuously for two weeks (Figure 5).

The planned approach was to perform a scalability and performance testing to the network. The scalability analysis with networks up to 50 nodes, where various data traffic patterns were systematically placed on the network to determine scalability and robustness was not performed due to insufficient hardware, later compensated with the scalability work on Tasks 7 and 8 . The second was to do performance testing measuring latency, packet error rate, throughput, network setup time, range, and reconfiguration delays (dropped nodes, rerouting etc.).

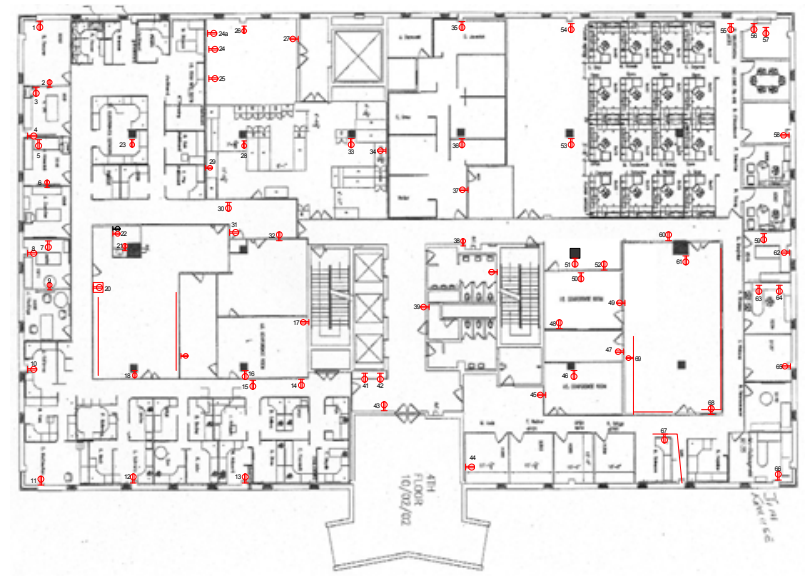

Figure 5: Indoor Base Test Setup at Milwaukee Innovation Center

\section{Accomplishments}

The results of the tests that were completed in this task were compared against the requirements and used to define the required improvements to the baseline network. This demonstrated the feasibility of the WSN approach in the environments of interest and gave us insight on the types of software and hardware modifications needed to deploy WSN in industrial environments.

\section{Phase II - Concept Feasibility}

The objective of this phase is to extend the baseline by addressing four issues critical to fielding a robust wireless industrial network: Ultra low-power sensors/Power harvesting, Security, Radiofrequency transmission in the industrial environment, and Power-Aware routing. 


\section{$2.1 \quad$ Task 6}

Objective: Develop and evaluate concepts to enable wireless nodes to be either ultra-low or zero-power for use beyond the electrical system

As can be seen in the Wireless Sensor Power consumption in Figure 6, a significant amount of power is used by the microprocessor to perform all of the local data sampling, signal analysis, and communications. A parasitic power coil was designed and sized to provide adequate power for a wireless device in a switchgear based application (Figure 7). However to extend this wireless device to other long life and battery less applications, further reductions in energy consumption will be required. Ultra low power (ULP) design is critical in remotely powered applications such as applications powered by batteries, fuel cells, or power harvesting sources. Power harvesting sources provide power in the 100s of micro-watts range whereas present-day sensors draw milli-watts of power. ULP is a technology that can bridge this gap.

Areas in analog processing, digital processing and the radio interface were researched in 20062007. Digital and analog designs were researched included sub-threshold operation of devices used to lower power consumption by an order of magnitude while operating at low supply voltages. In the digital domain, new low voltage circuit techniques based on dynamic voltage and frequency scaling and new leakage reduction techniques to maintain standby power consumption significantly smaller as compared to the harvested power were researched.

ULP could revolutionize wireless mesh networks with applications in industrial automation and controls, distributed process controls and smart sensors in hydraulics, aerospace and automotive markets. ULP products have started to enter the market in building automation and HVAC (EnOcean as one example). Increased market penetration will soon be seen and the first NanoPower Forum held June 2007 is indicative of the elevated interest from industry.

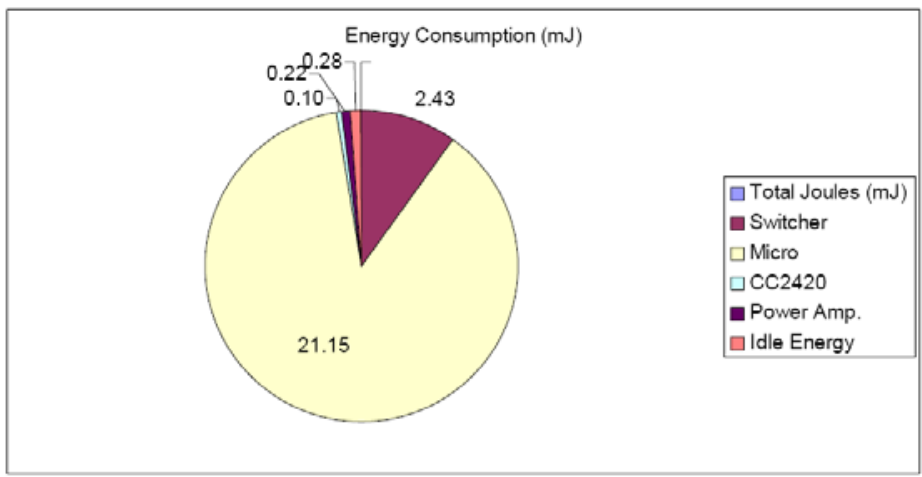

Figure 6: Power Consumption of Wireless Sensor 


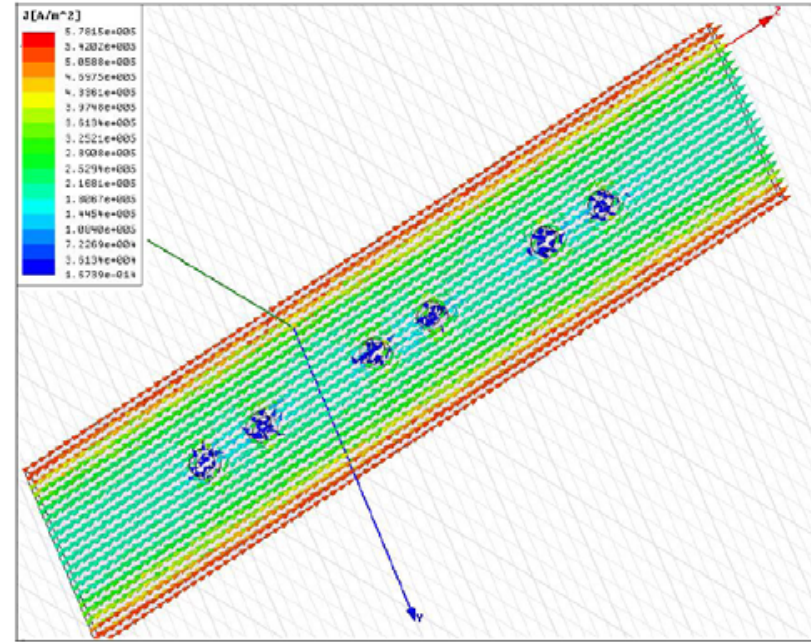

Figure 7: FEA Analysis of the Parasitic Power Coil

\section{Accomplishments}

A 3-D FinFET structure in Athena/Atlas with sharp geometry in the $32 \mathrm{~nm}$ node was created. This was created without any process models in Athena. The gate length for this node was 18 $\mathrm{nm}$. The 3-D structure was simulated to obtain drain current vs. gate voltage for different bias conditions and structural parameters. The correct models to use were explored and modeling of quantum effects was studied. The effect of variation in parameters (gate oxide thickness, fin width, fin height, gate length) on device and circuit characteristics such as threshold voltage, drive current and leakage was also studied. The research explored a promising technology of ultra-low power electronics, which can be used in wireless sensors to greatly reduce energy consumption and improve battery life.

\section{Conclusions}

While significant progress has been made in ULP devices, further work is still required to make practical, low power sensors that can be motor/machine mounted without requiring external (mains) power. Locating EM/CBM sensors within the MCC where electrical power is available provides a viable alternative.

\section{$2.2 \quad$ Task 7}

\section{Objective: Increase robustness of self-configuration and routing algorithms}

The baseline WSN test bed demonstrated an initial metrics of robustness by deploying a network of 23 nodes, as described in Task 5. Performance testing measured end-to-end latency, network setup times, and range. 


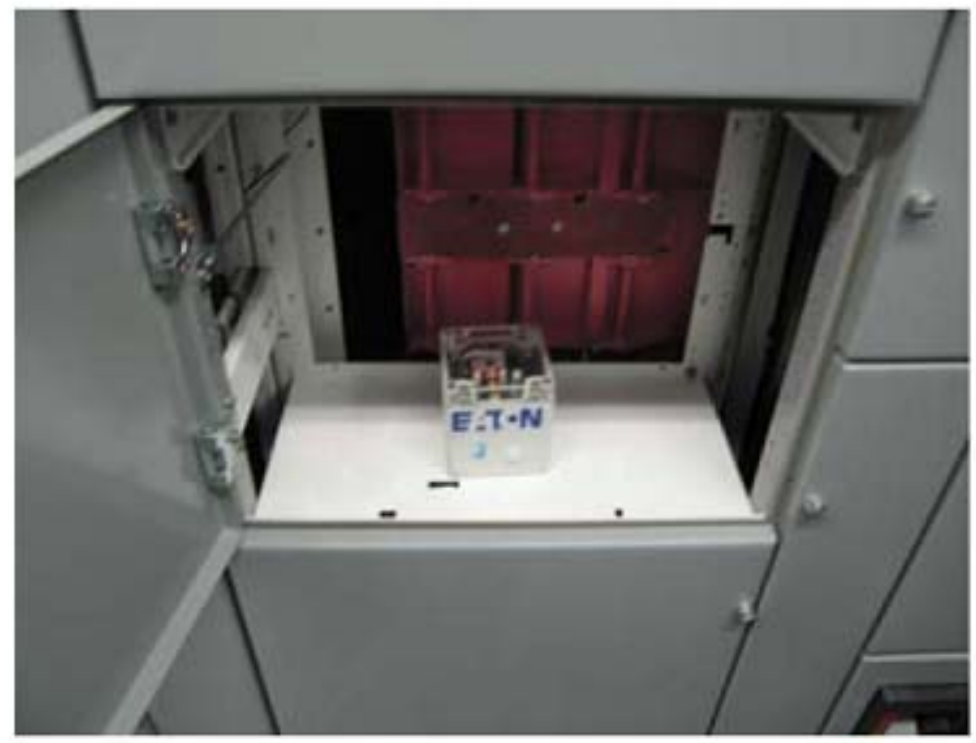

Figure 8: Dual Radio Industrial Wireless Test Node Inside Motor Center

We determined the performance of the WSN was consistent in the lab and proceeded to test again in an industrial environment. Results of those tests gave us insight on the enhancements needed to the stack and/or applications. The basic conclusions were:

- At $2.4 \mathrm{GHz}$, IEEE 802.15.4 wireless radios can transmit reliably from one end of the motor control center to the other (less than 30') without any repeater (Figure 8). However, multi-hop mesh networking can be utilized to increase robustness of the communication medium.

- The radios can communicate in and out of the metal switchgear enclosures. Metal enclosures do, however, reduce the range of the radio communication.

- These wireless networks will perform quite reliably in refinery industrial environment, with packet success rates measured between $94 \%$ and $100 \%$ without higher layer protocol retrial.

As part of these results, we performed changes to the WSN hardware and software, and initiated efforts to utilize the ZigBee network layer (Z-Stack purchased from Figure8Wireless, a company acquired by $\mathrm{TI}$ ) in order to take advantage of the growing standards in this area, and to improve interoperability in the future.

We performed hardware reliability analysis for the wireless modules, being reconciled with the MIL (Military) reliability standards. Eaton also developed a system reliability analysis approach using the AADL architecture modeling framework. These system models were able to combine the reliability models for hardware and wireless link assessment data from Eaton's Wireless Link Assessment System (WLAS). (See Appendix: A1: Grenada test plan v10)

Noise injection tests were conducted to assess the impact of a wide range of possible external RF sources on IEEE 802.15.4 wireless nodes. With these tests, industrial noise sources have been identified and classified according to the level of interference it will cause (e.g. WiFi and Microwave ovens are worst interference sources whereas Bluetooth is least interfering). These results have been incorporated in the design of a run-time RF environment sensing algorithm. 
With this knowledge, Eaton designed and tested a channel agile wireless network, boosting the reliability of a ZigBee network by avoiding channels being used by other external interfering systems.

\section{Accomplishments}

In conclusion, all of these efforts reached the goal of having a wireless network that improved the reliability of communication with the following characteristics:

- Unlike the standard implementation, the enhanced Eaton implementation is capable of using all the wireless channels available in the allocated band, ensuring that if one section of the band is interfered, another can be used immediately. This factor alone can improve the communication reliability by a factor of 16 times when compared with a standard ZigBee implementation.

- Poor communication performance is constantly monitored in each node of the network in a distributed fashion. This is done in order to make on-the-fly corrective decisions that will look for better portions of the spectrum to transfer data. This makes our network robust to intentional or unintentional attacks, external interference, and noise factors.

- Reports on bad wireless channel conditions and other critical data are sent over a subset of the channels with best performance. This ensures that both data and management/control information reaches their destination with high probability.

Additional to the points mentioned above, Eaton identified that these solutions should be backward compatible to standard Zigbee nodes. This means nodes that do not implement Eaton's channel agile approach can still be part of the network ensuring in this way interoperability of Eaton's solution.

\section{Conclusions}

This work demonstrated that it was technically feasible to construct a reliable and robust wireless communications network based on IEEE 802.15.4. However these features were not incorporated into any products as the backward compatibility mechanism was outside the scope of the project.

\subsection{Task 8 and 11}

\section{Objective: Task 8- Address packaging and other environmental issues for robust radio frequency (RF) transmission performance in industrial applications}

\section{Objective: Task 11- Conduct testing to evaluate the performance of the WSN}

The purpose of the channel agility effort, Task 8, was to investigate and, if necessary, enhance the reliability of the wireless sensor network used for energy monitoring as well as better understand the effects of industrial environments on wireless communications. With the knowledge gained from the tests in Task 7, we developed a wireless link assessment instrument tool that performs simple and automated mesh network communication reliability assessment for Eaton customers, measuring wireless network performance at customer premises (i.e., industrial, residential and commercial). 
The main problem in wireless communications is the interference caused by other networks or devices over the communication links in the network of interest. Therefore we focused on the effect of interference on the performance of the network. Our findings can be summarized as follows:

- The Zigbee Wireless Sensor Network performance is barely degraded even for large levels of interference. We had to increase the interference level substantially in order to see degradation in the transfer of application information packets. Typical interference sources were used, such as Microwave ovens, WiFi nodes, and other proprietary Wireless Network solutions. In all cases, the performance degradation was minimum to nonexistent at the application layer. In order to see degradation we co-transmitted a carrier at the center of the Zigbee channel at levels of $20 \mathrm{dBm}$ and higher. We were able to see degradation under these rare circumstances.

- We devised several strategies to change channels automatically and pro-actively in case interference was unusually high. We devised and implemented a method to detect interference, and also to change channels in case that measured interference was high. We performed tests with these methods, and the network was able to successfully change channels and continue performing satisfactorily.

The Eaton Wireless Link Assessment System (codenamed "Grenada") is a system composed of a set of devices that are connected in a wireless manner for measuring the qualities, including the delivery rate, the received signal strength, and many other wireless communication links. Each individual device is named "Grenadine". The Eaton Wireless Link Assessment System is capable of measuring wireless link qualities along six dimensions of factors that affect the link performance metrics:

- Time

- Transmitter location

- Receiver location

- Channel

- Transmitting power level

- Packet size

The Grenadines, or individual devices, are identical in terms of hardware and software. A Grenadine of the current version is packaged in a water-proof NEMA-4 enclosure to prevent hazards from the environment to the device, or from the device to critical deployment infrastructures (Figure 9). On one side of the NEMA-4 enclosure there is a power connector and a three-way switch for turning on/off the Grenadine, as shown in Figure 10. An AC-DC adapter (120V AC to $12 \mathrm{~V} \mathrm{DC}$ ), in addition to the NEMA-4 enclosed Grenadine device, is provided to utilize main power, or batteries if the application requires it. The electronics system inside the NEMA-4 enclosure for each Grenadine includes a voltage regulator, a Compact Flash (CF) card data logger Print Circuit (PC) board, a radio board (which can be either the RM2420 module from RAE Systems, or a board internally developed by Eaton Corporation) with an antenna, an interface PC board and a Compact Flash (CF) card, as shown in Figure 11. The CF data logger PC board and the development PC board for the radio board are connected through two sets of serial connectors, both of which are powered from the voltage regulator. During tests, the radio board and the CF card need to be plugged into the development board and CF data logger, respectively. A detailed description of the instrument, including the test plan associated with it can be found in (Appendix: A1: Grenada test plan v10). 


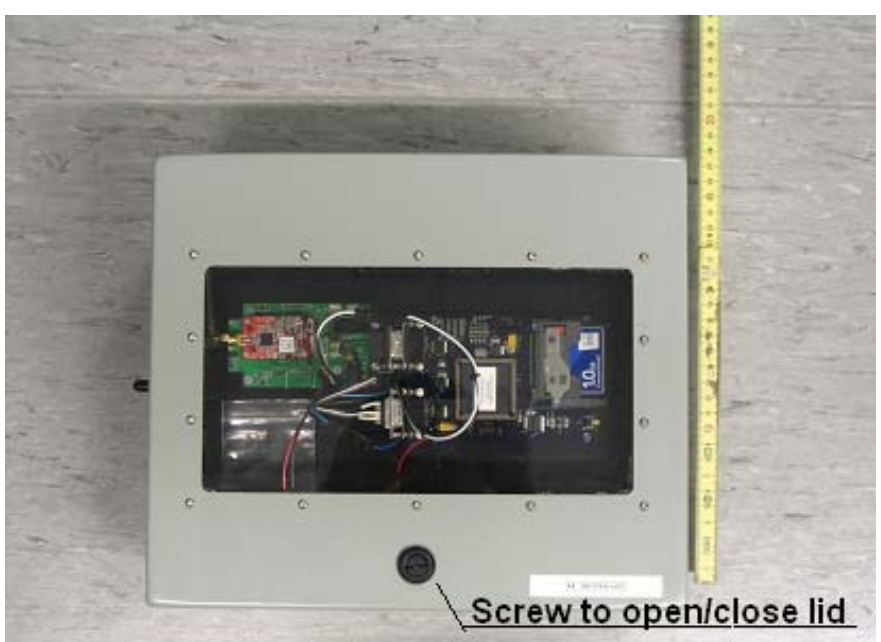

Figure 9: Top View of Grenadine Part of the Eaton Wireless Link Assessment System

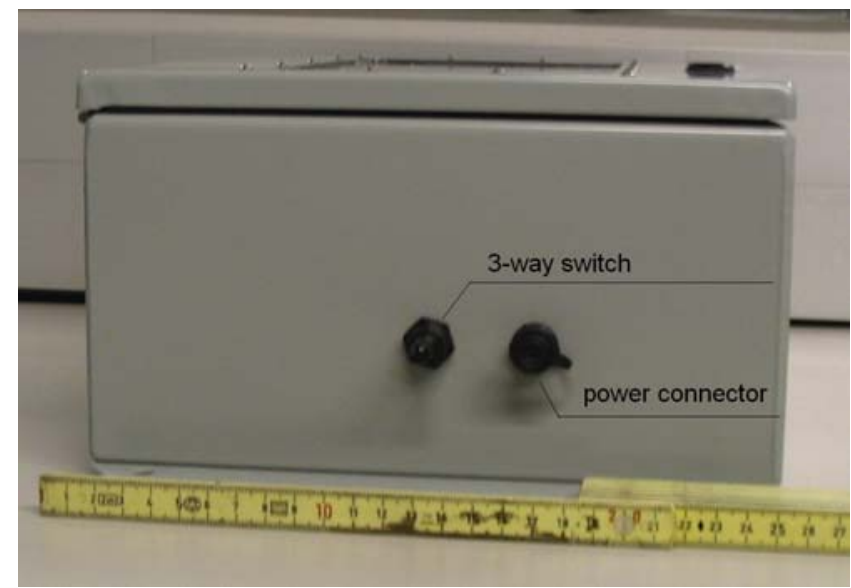

Figure 10: Side View of Grenadine

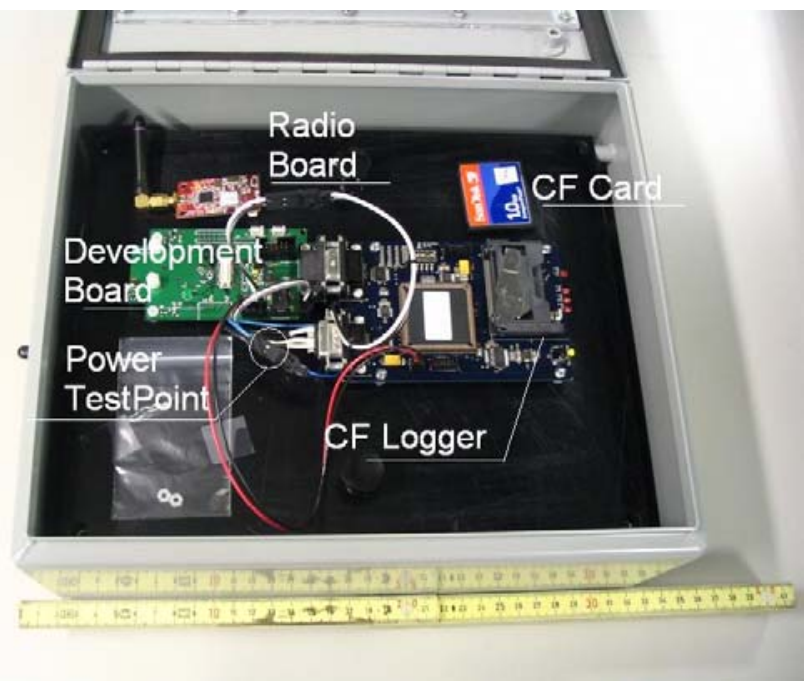

Figure 11: Electronics System of Grenadine 
This instrument allowed us to capture packets of data under a variety of conditions, where we could assess the effects of the environment on the data transmission. A typical plot of the data processed from the instrument is shown in Figure 12. Each point to point link between two nodes can behave completely different, but taken as an aggregate the information can be used for a routing algorithm to find paths that are more reliable over time.

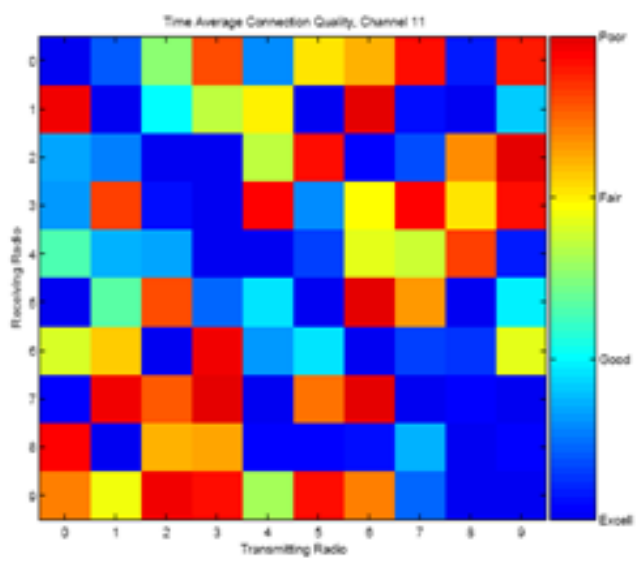

Figure 12: "Radio Grid" A visual indicator of link quality among nodes

In addition to the assessment system, Eaton developed a test bed of $100+$ nodes which included a graphic interface. Fast aggregation of status information from the network coordinator, a critical requirement for wireless operation has been proven; more than $95 \%$ of the nodes report within a 0.6 second or less. This significantly improves the existing technology where a success rate of only $40 \%$ data aggregation can be achieved. This technology was successfully demonstrated, and the results were published in the SODA paper (see Appendix A2: DOE Task 10 Appendix).

In conclusion we believe in most situations a Zigbee Wireless Sensor Network is reliable enough to perform the intended tasks of energy monitoring. Zigbee only needs extra reliability support in extreme cases of interference levels (e.g., intended or un-intended attacks). Zigbee is suitable for most industrial, commercial and residential applications. In case extra reliability is needed, an adaptive channel hopping strategy can be implemented in nodes with minimum processing power and spending small amounts of energy.

\section{Accomplishments and Conclusions}

- A channel agility method was developed, simulated and tested for robust communications using a LR-WPAN.

- A Wireless Link Assessment System was developed that can evaluate the RF environment at multiple frequencies within a customer's facility.

- Radios can be effectively packaged to protect the electronics from severe plant environments, yet still provide adequate RF transmission/reception. In our specific case, we found NEMA4 enclosing did not diminish the RF transmission and reception while providing protection for nearby industrial equipment, facilitating the implementation and adoption of wireless technologies in the plant floor.

- Investigations showed technical feasibility for channel agility and data aggregation algorithms to improve performance. More research is needed to incorporate these features in routing protocols to have a complete solution and therefore these features were not incorporated into any products. 


\subsection{Task 9}

\section{Objective: Implement enhanced security to meet the industrial application's needs}

This task presents the report for characterizing the performance of wireless sensor networks (WSN) using ZigBee security. The idea behind these tests is to provide a measure of the delay introduced in packet processing and packet transmission in nodes communicating using the ZigBee 1.0 standard, after security has been introduced. The tests also compare the performance of ZigBee security-enabled nodes running a particular application to that of the nodes when ZigBee security mechanisms are disabled under the same setting.

The goal of this task was to measure the time taken for the following when security is disabled and when it is enabled:

- Packet processing time: The time taken for the lower layers to ready a packet for transmission and send it, after the application has given a command to send a packet. When security is enabled, this would include all the time required for cryptographic processing of the packet.

- Packet transmission time: The time taken for the radio to send out the packet. This would depend on the number of transferred bits. When security is enabled, it shall be relative to the number of MIC bits used.

- Action time: This is the time taken for the command to be executed at the receiving node, indicating the transmission/reception times, the air time, and processing time at both sending and receiving ends.

- Confirmation Time: This is the time required for the application to receive a confirmation that the acknowledgement has been received.

Purpose and Rationale:

The test results shall enable application designers to decide whether the additional time incurred due to enabling security is acceptable for the given application. This additional time translates to extra response time, additional battery (power) requirements, etc.

Test Setup:

Two CC2420 boards programmed with a modified version of the Texas Instrument Demo Application in ZStack, one acting as the input (the router) and the other as the output (the coordinator). The router sends toggle LED messages to the coordinator. Each time it receives an acknowledgement from the coordinator, it transmits another toggle message. The tests are first carried out with security level 0 (no security), then at level 5 (Encryption with 32 bit Message Integrity Code or MIC). Finally, the test is repeated at security level 7 (Encryption with 128 bit MIC). This is to give an idea of the additional time requirement if a higher level of security is chosen. Various network configurations have been utilized to assess different security levels.

Inferences:

- The difference in processing times is nearly equal for levels 5 and 7 which is as expected because in both levels, the cryptographic processing required is equal. (Only 
more bits of the MIC are transmitted at level 7). Hence, levels with 128-bits of MIC must be preferred to 64 or 32 bits of MIC.

- Transmission times range from 1 to $2 \mathrm{~ms}$ for various levels of security and hence, need not be regarded with concern.

- Performing AES (Advanced Encryption Standard) in hardware is far more beneficial than in software and should be opted for. (The default mode is software).

Conclusions:

- For commercial applications, it is advisable to use level 7 of security in hardware.

- For applications where the time associated with level 7 may seem a concern, levels 4 or 3 may be used.

- Level 4 however uses no MIC. Integrity of the message would be of more concern than their secrecy in most applications.

\subsection{Task 10}

Objective: Update the demonstration to include advances from previous tasks in a test bed operating within an industrial environment

The objective of this task was to update the Wireless Sensor Network (WSN) demonstration in a test bed within an industrial environment including the advances from previous tasks. The improvements are focused on addressing one of the key challenges of real industrial applications: scalability.

For time-critical industrial WSN systems, it is a fundamental requirement that after the base node (the node in control of the whole network) sends a broadcast query message to the whole network, all nodes in the network shall respond with their status information in a timely fashion. Carrier Sense Multiple Access/Collision Avoidance (CSMA/CA) media access control protocols (including variations) are typically used in industrial WSN systems. Although they are capable of satisfying this requirement when the network contains about a dozen nodes, when the number of nodes in the network increases, these protocols can only deliver a small fraction of all the responses expected.

We designed and tested a novel algorithm for this critical requirement for industrial WSN systems. The algorithm uses a near-optimal channel assignment scheme that maximizes the utilization of multiple available channels to deliver all responses in a short amount of time (typically within 3 seconds for a 100-node network). The query response data are aggregated to minimize the usage of time and bandwidth. This solution is designed to reside on top of a widely used commercial off-the-shelf ZigBee protocol stack based on IEEE 802.15.4 with backward compatibility. Figure $\mathbf{1 3}$ gives an overview of the scalable data aggregation solution. 

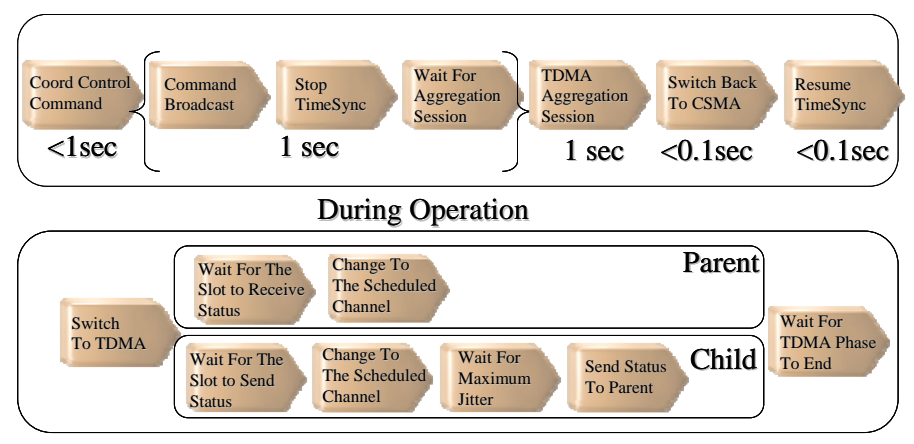

TDMA Aggregation Session

Figure 13: Scalable Data Aggregation Overview

An example of the constraint-based channel and time slot allocation is shown in Figure 14. Details of the scheme are described in Appendix: A2: DOE Task 10. The algorithm has been implemented in a test bed including 100 wireless test nodes ("MAUI" nodes developed by Eaton) as shown in Figure 15. The test bed includes a user interface that collects experimental data as well.

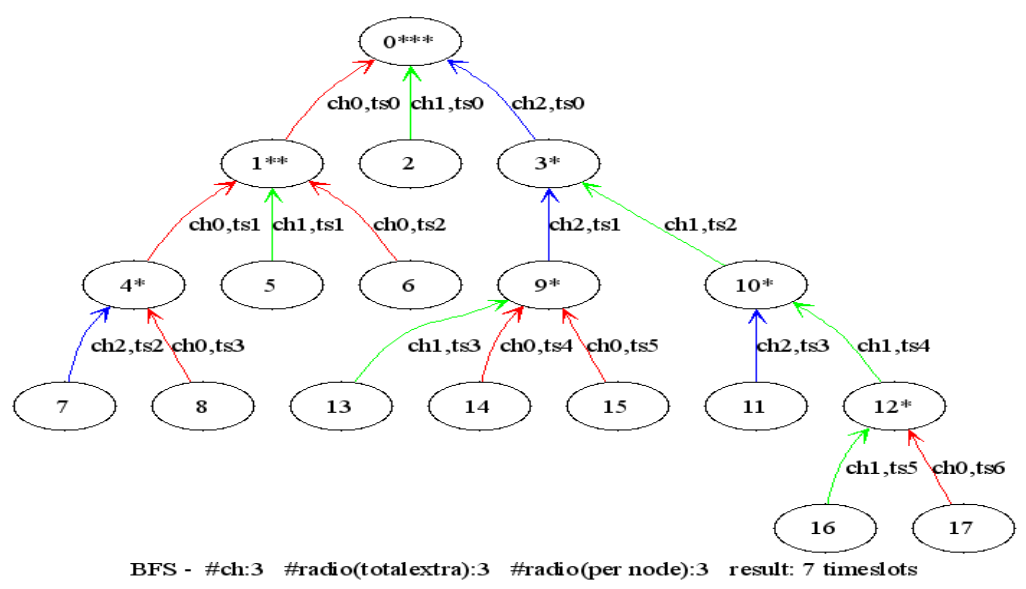

Figure 14: Channel and Time Slot Allocation Example

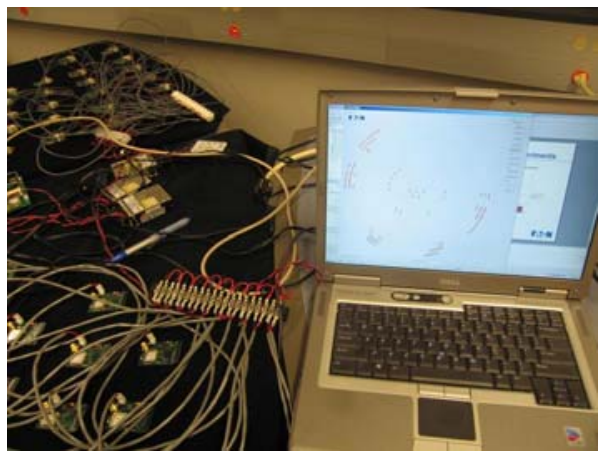

Figure 15: Test Bed and User Interface 


\section{Performance Comparis on Between Data Aggregation and Baseline Scheme}

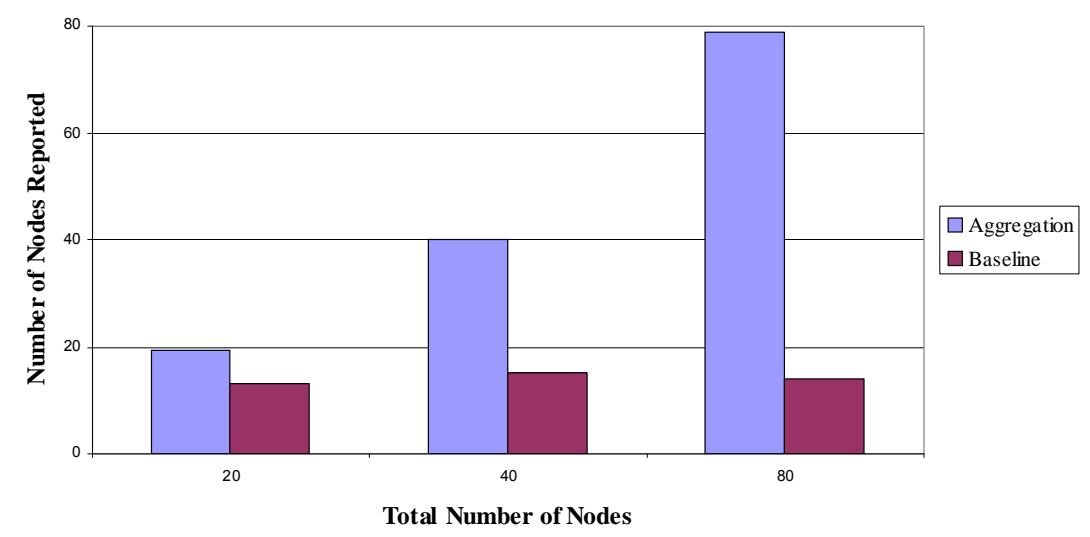

Figure 16: Performance Comparison (1)

Performance Comparison Between Data Aggregation and $B$ aseline Scheme

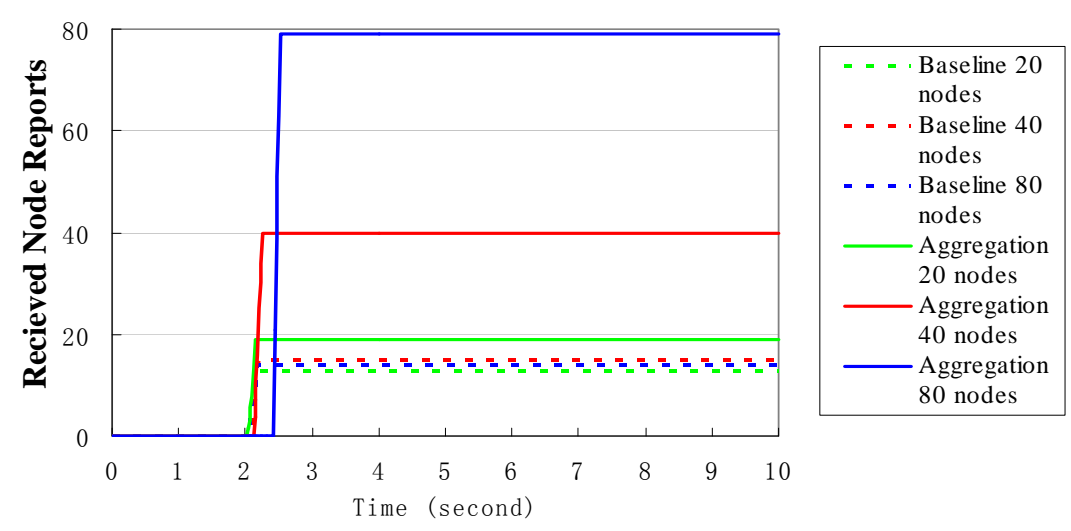

Figure 17: Performance Comparison (2)

\section{Accomplishments/Conclusions}

The test bed shows that the conventional CSMA/CA media access control protocol adopted in the ZigBee/802.15.4 standards can only deliver responses from less than 20 nodes (even when responses from all (100) nodes are expected), while our proposed solution is capable of delivering more than $95 \%$ of all the expected responses within 3 seconds (Figure 16 and Figure 17 above). Note that this investigation was to confirm technical feasibility.

\section{Phase III - Concept Validation}

The objective of this phase is to field an alpha system and conduct field tests of that system. Final plans to bring the new energy management products to market will also be developed. The initial product is envisioned to focus on optimization of motor systems within the Industries of the Future (IoF). 


\subsection{Tasks 12 and 13}

Task 12 and 13 Objective: Identify applications that, when combined with the energy management system, will provide benefits to the IOF. Create alpha site demonstrations that are instrumented and installed on-site in an end user's manufacturing plant. Support system during the entire alpha test, conduct experiments based on real life scenarios and document. Based on test results at the alpha sites, implement additional design improvements to enhance the performance of the system.

With the main scope on improving energy savings in the industries of the future, Eaton Innovation Center developed a complete set of motor energy efficiency and condition monitoring algorithms that can be incorporated into Eaton's industrial control products as advanced energy saving and condition monitoring features. The objective of this phase is to further validate these algorithms in various industrial facilities and demonstrate the energy saving capabilities to the industries. This is a collaborative effort consisting of experts from industrial manufacturers, universities, and customer industrial partners.

\section{Summary of Energy Efficiency and Condition Monitoring Technology Development}

Achievements in energy efficiency and condition monitoring technology development from Eaton Innovation Center and Georgia Institute of Technology during the period of the research program have been significant. The work has focused on both aspects of predictive maintenance (PdM): Nonintrusive motor system impending failure diagnostics as well as energy efficiency monitoring. A series of intelligent and inferential-based algorithms on motor/load fault diagnostics and energy efficiency estimation has been developed. Algorithms include diagnostics and prognostics of pump cavitation, shaft misalignment, rotor fault, stator winding fault, motor bearing failure, motor winding temperature estimation, motor speed estimation, motor efficiency/torque estimation, power quality and harmonic analysis, and energy-optimized drive controls, as in Figure 18.

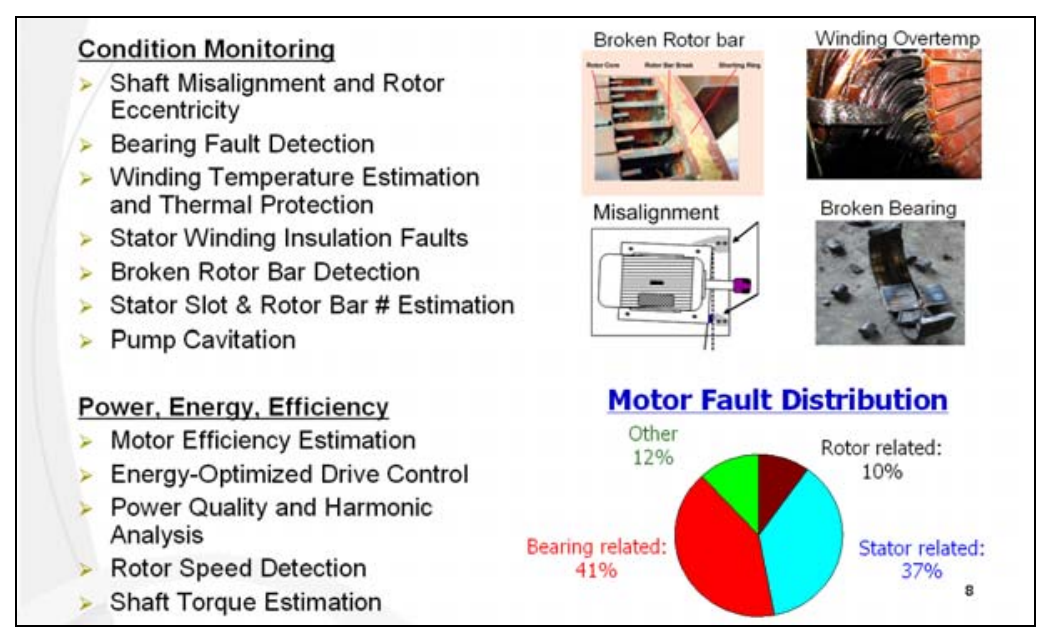

Figure 18: Summary of Eaton's Motor Energy Efficiency and Condition Monitoring Algorithms 


\section{A Nonintrusive Inferential Based Methodology}

As a major advancement in the PdM technology, Eaton Innovation Center and Georgia Institute of Technology developed a "nonintrusive" and "inferential" methodology of PdM modeling for induction motor drive systems, as illustrated in Figure 19. It has been observed that the motor input voltages and currents (or input power) contain certain harmonics that are caused by certain motor system failures and can be used as the raw data for various PdM algorithms. Using these data, inferential-based energy efficiency estimation and fault diagnostics/prognostic algorithms can be developed to estimate the energy usage and health conditions of the motor driven system.

As shown in Figure 19, these algorithms use only samples of motor input voltages and currents as well as the motor nameplate information as the input data. Each algorithm starts first with a study of the motor/load itself and unveils the fault mechanism from a physics standpoint. Then, advanced data processing techniques are applied to infer the fault signatures and patterns from the input data in an online fashion. To develop inferential algorithms that can predict impending failures at an early stage, the following advanced modeling and intelligent data processing techniques are commonly used: Motor modeling and analysis, system identification and optimization (e.g., Kalman filter and particle swarm optimization), pattern recognition (e.g., clustering and grouping), stochastic and statistical process (e.g., statistical process control), digital signal processing (e.g., adaptive filters), and other intelligent methods (e.g., artificial neural networks, fuzzy logic, etc.). The novelty this research lies in its nonintrusive, online, and inferential characteristics.

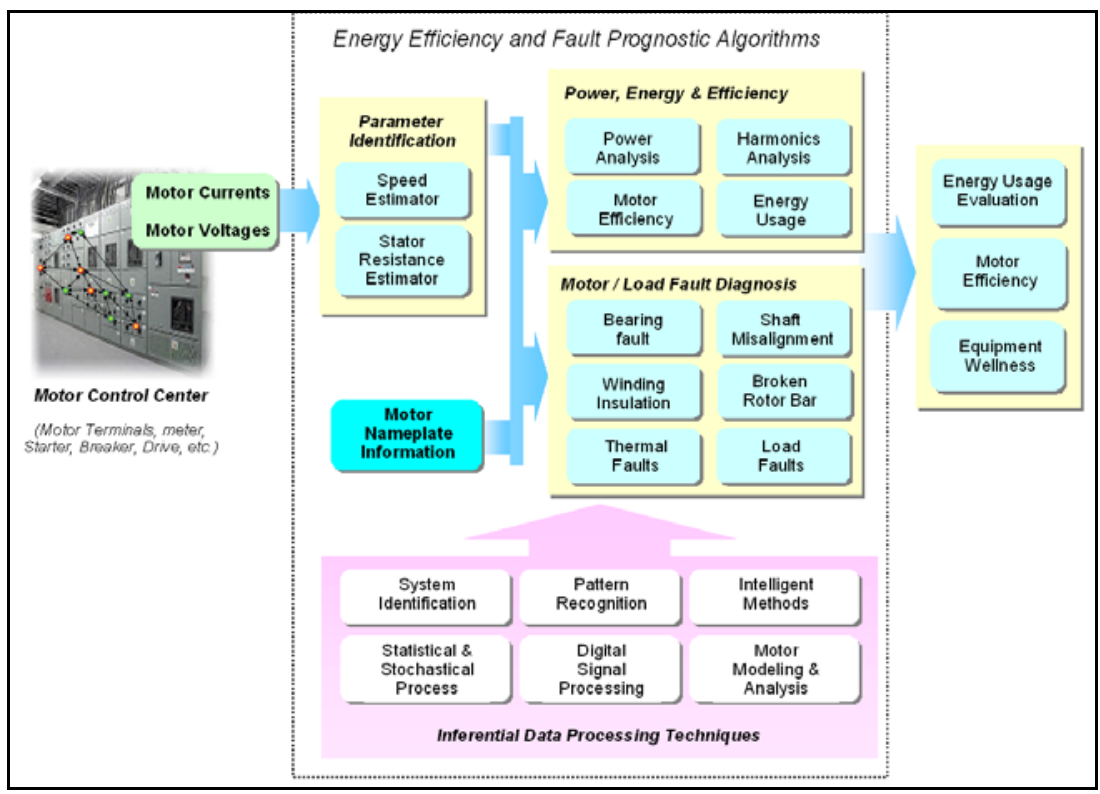

Figure 19: Nonintrusive and inferential based algorithm development methodology

\section{Summary of Key Algorithms}

PdM mainly focuses on two aspects: Energy efficiency improvement, and unscheduled downtime reduction. The algorithms are also developed accordingly and can be generally divided into these two categories: 
1) Power Metering, Power Quality, and Energy Efficiency:

One key of energy savings is the power quality and instantaneous motor efficiency evaluation. With accurate power quality information, the equipment can work under a more reliable environment and reduce the process downtime caused by utility power quality issues. With reliable efficiency information, low-efficiency motors can be identified and proper actions can be taken to improve the overall efficiency by adjusting the motor control settings, replacing lowefficiency motors, etc.

Efficiency estimation requires that the motor speed to be estimated first. Commonly, direct measurement of the rotor speed requires a shaft-mounted speed encoder or an optical tachometer to be installed. However, in many circumstances, installing additional sensors is not possible under field conditions. In this work, an online sensor less rotor speed estimation method is developed. This method provides robust speed estimate down to $1 \mathrm{~Hz}$ operation with a high accuracy. Figure $\mathbf{2 0}$ below shows experimental results of this algorithm from a motor under various speed conditions.

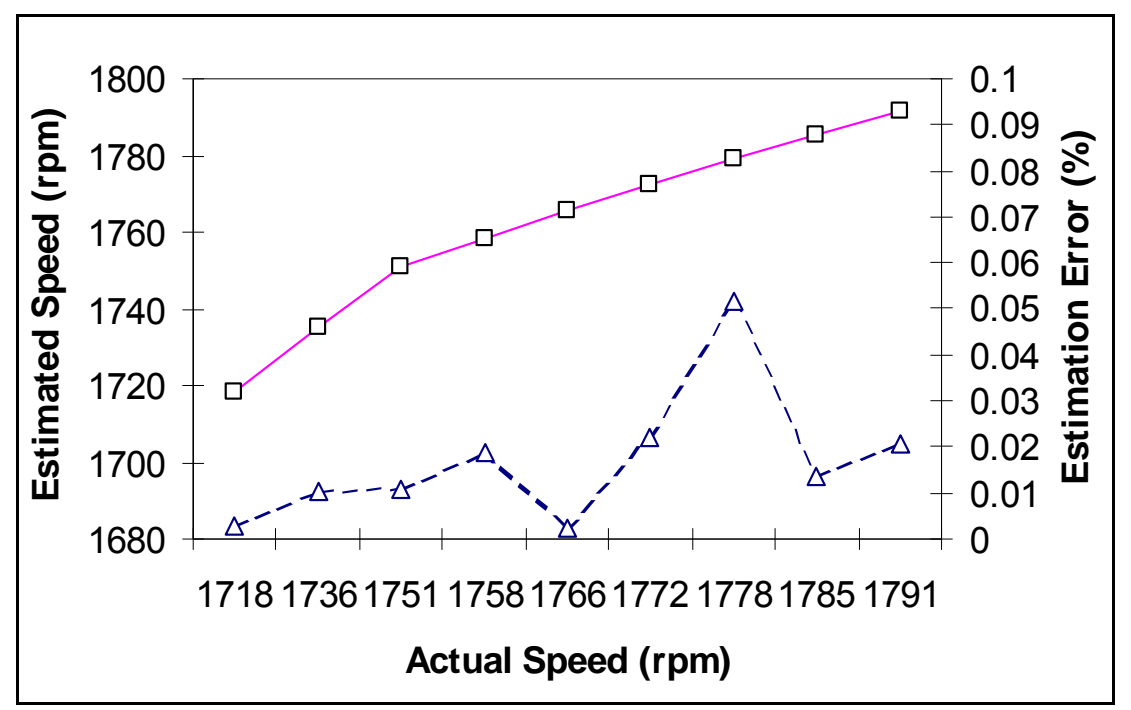

Figure 20: Speed Estimation accuracy

In this program, many motor efficiency evaluation methods and devices have been evaluated and potential methods are identified. Today, online and sensorless speed and stator resistance estimation methods have been developed using only motor voltages, currents, and nameplate information. Incorporating these recent advances, in this program, two novel nonintrusive methods are developed to estimate the motor efficiency. They estimate the motor efficiency using only terminal voltages and currents, and motor nameplate information. US patent applications for both methods were submitted in 2007. Details of the algorithms developed are included in Appendix A. 


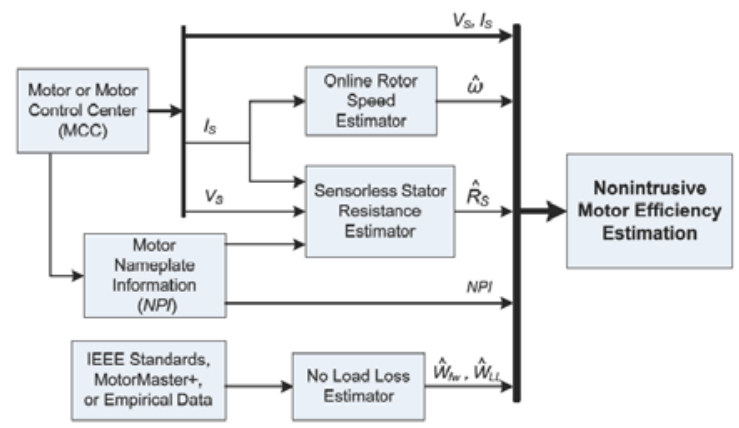

Figure 21: A general approach for nonintrusive motor efficiency estimation

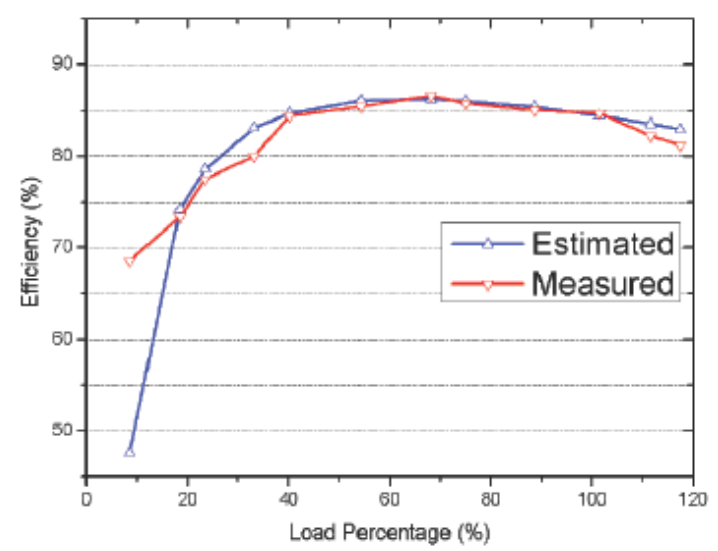

Figure 22: Estimated vs. Measured Efficiency

2) Motor/Load Fault Diagnosis:

The fault diagnostics and prognostics for induction motors and their connected loads include the detection of many impending faults including: Air-gap eccentricities, shaft misalignment, worn bearing, winding insulation faults, broken rotor bars, winding thermal overheating, load torque oscillations and pump cavitation. Various motor signature analysis based fault detection techniques have been extensively studied in this work. The focus is to develop algorithms that only need motor input voltages and currents. The overall strategy of motor system diagnostics and prognostics are shown in Figure 23. 


\section{Diagnostics and Prognostic Strategy}

- Motor Driven Systems (Motors, Drives, Loads).

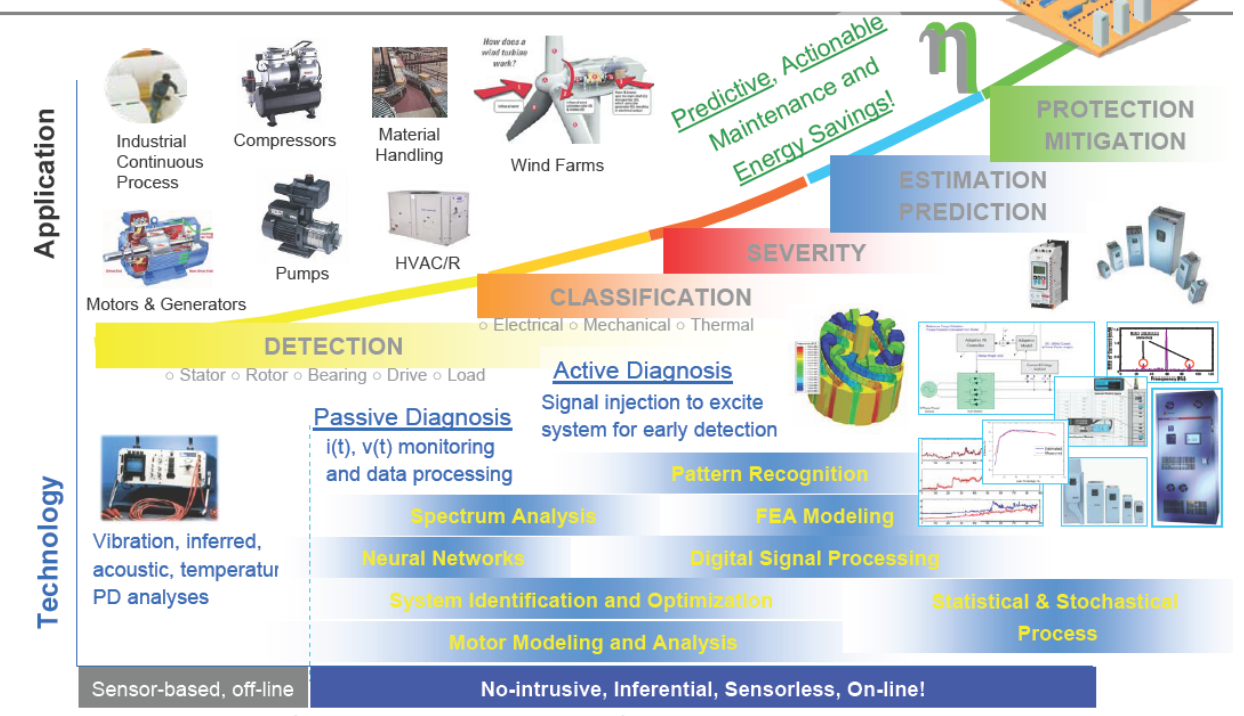

Figure 23: Overall Strategy of Motor System Diagnostics and Prognostics

Among all the motor and load faults, the algorithms for rotor mechanical faults (e.g., rotor bar and ring breakage), rotor eccentricity, and shaft misalignment have been extensively researched and "standard methods" have been developed. These methods are all based on motor (current or power) fault signatures that appear besides the fundamental frequency. A brief description of the mechanical fault algorithm is shown below in Figure 24.

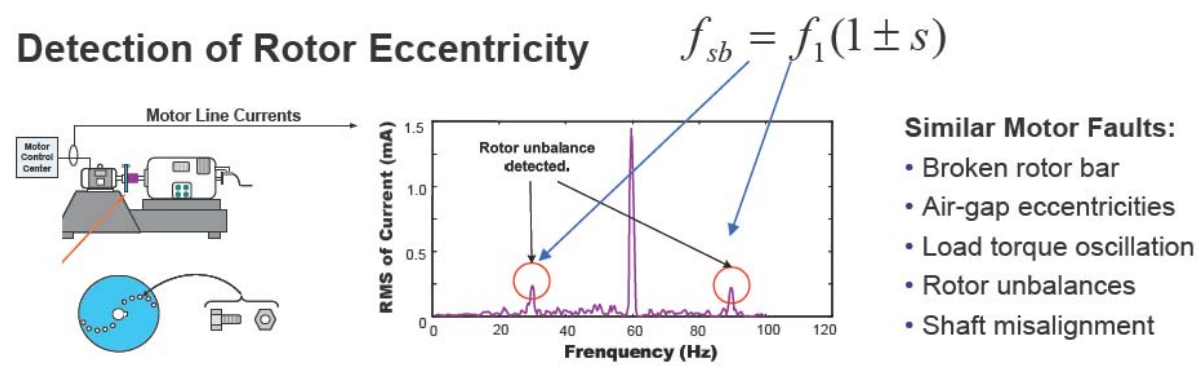

Figure 24: Detection of Rotor Eccentricity

In this work, a method for induction motor rotor fault diagnosis using wavelet analysis is developed to address the rotor fault with higher signal to noise ratio under varying load conditions. It also includes an interactive technique to detect broken rotor bar in varying load conditions. The fault severity is derived by wavelet analysis of single-phase active one-cycle average power. Wavelet allows analyzing non-stationary waveform and one-cycle average power allows detecting fault characteristic frequency component under low load conditions without removing the fundamental component. A photo showing a typical broken bar fault is shown in Figure 25. The results from the rotor fault detection algorithm for a motor with different number of broken rotor bars are shown in Figure 26. 


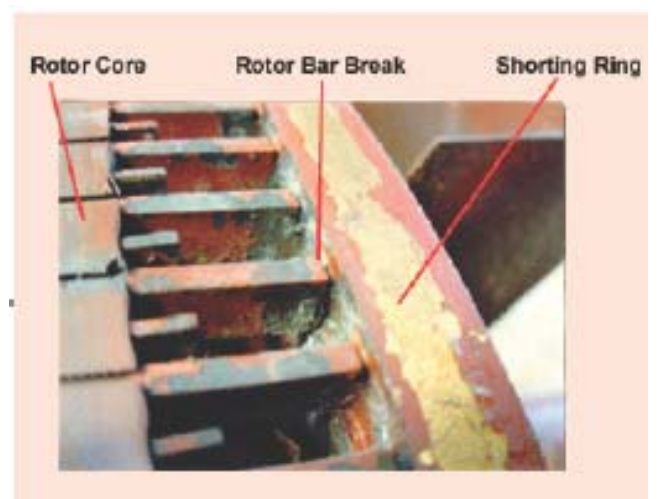

Figure 25: Induction Motor Rotor Fault

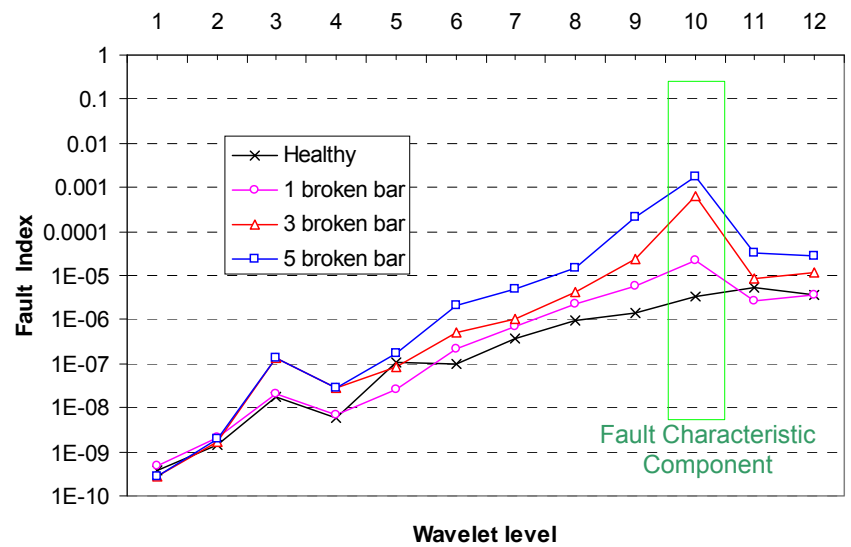

Figure 26: Experimental Results (Motor: Lesson 7.5 hp, 230 V, 20 A, NEMA-B, ODP, 1760 rpm)

Pump cavitation algorithms have also been developed, mostly based on the experience observed from experimental testing results. For example, Figure 27 below shows an example of a 4-pole, 10 horsepower motor current spectrum via Fast Fourier transform (FFT) along the sideband frequencies, with the $60 \mathrm{~Hz}$ fundamental frequency notched out using a hardware notch filter. The linear average magnitude of a lower sideband (LSB), e.g., 25 to $55 \mathrm{~Hz}$, and an upper sideband (USB), e.g., 65 to $85 \mathrm{~Hz}$, is used to monitoring the pump cavitation. However, the range of LSB and USB is based on the experimental results. More work needs to be continued to better model the fault mechanism under cavitation conditions. 


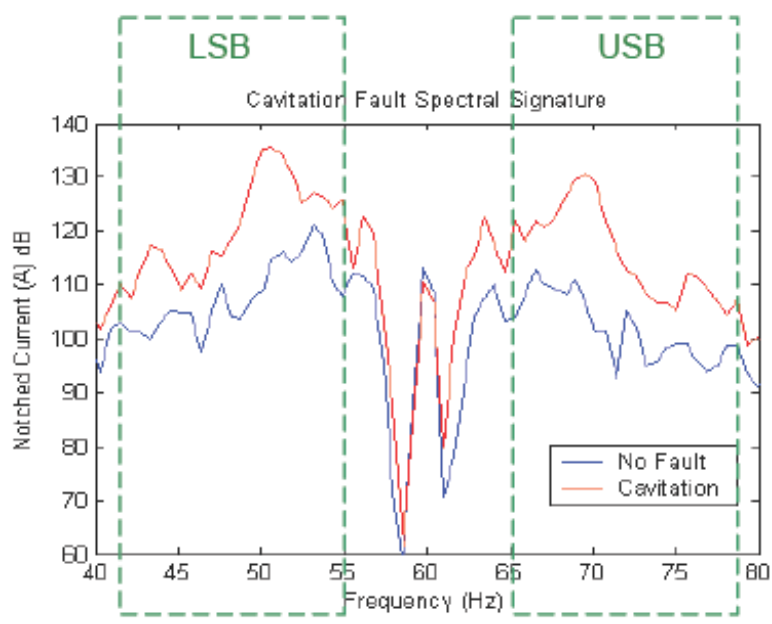

Figure 27 : Typical motor current signature with and without pump cavitation

The winding insulation faults, especially the inter-turn fault, still remains as one of the most interesting, while challenging, topics in today's motor diagnostics and prognostics research. Inter-turn winding insulation failure typically develops within 30 to 60 seconds before the iron core is melted. Such short time and devastating post outcome poses a large challenge on the time response and accuracy of the detection algorithm, making insulation failure more of a motor protection problem than a prognostic problem. An algorithm based on artificial neural networks is developed in this work that provides at least 10 second early indication of inter-turn winding insulation fault indication to avoid severe damage of the motor core. A brief description of this algorithm is shown in Figure 28 below.

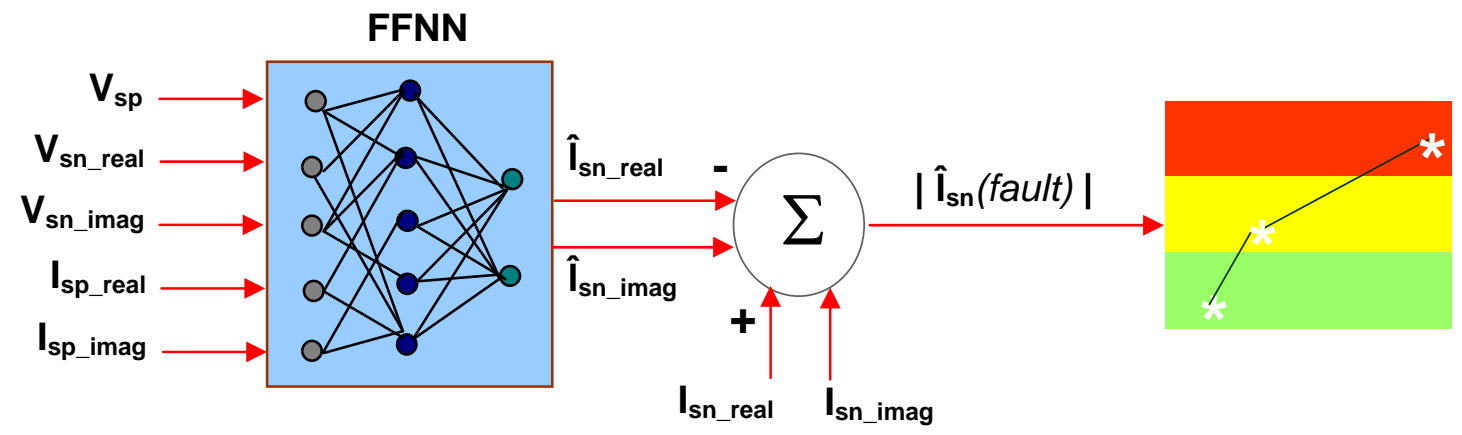

Figure 28: Description of the insulation fault detection method using neural networks.

Bearing fault is the most common maintenance problem in almost all industries. The bearing fault happens as a complicated multi-stage process. The majority of previous work in bearing fault detection was based on motor signature analysis to detect a "single-point defect," which means a fault happens at a specific part of the bearing, such as outer or inner races, and the balls. Single-point defect occurs during the late stage of the bearing life. However, in most cases, bearings start to fail as a "general roughness" on the surfaces of the races and balls, which may not have a unique fault characteristic frequency, but rather cause broadband changes in the motor current noise floor. A stator current noise-cancellation bearing algorithm has been developed to address this fault without using motor signature analysis. A brief description of this algorithm is shown below in Figure 29. 


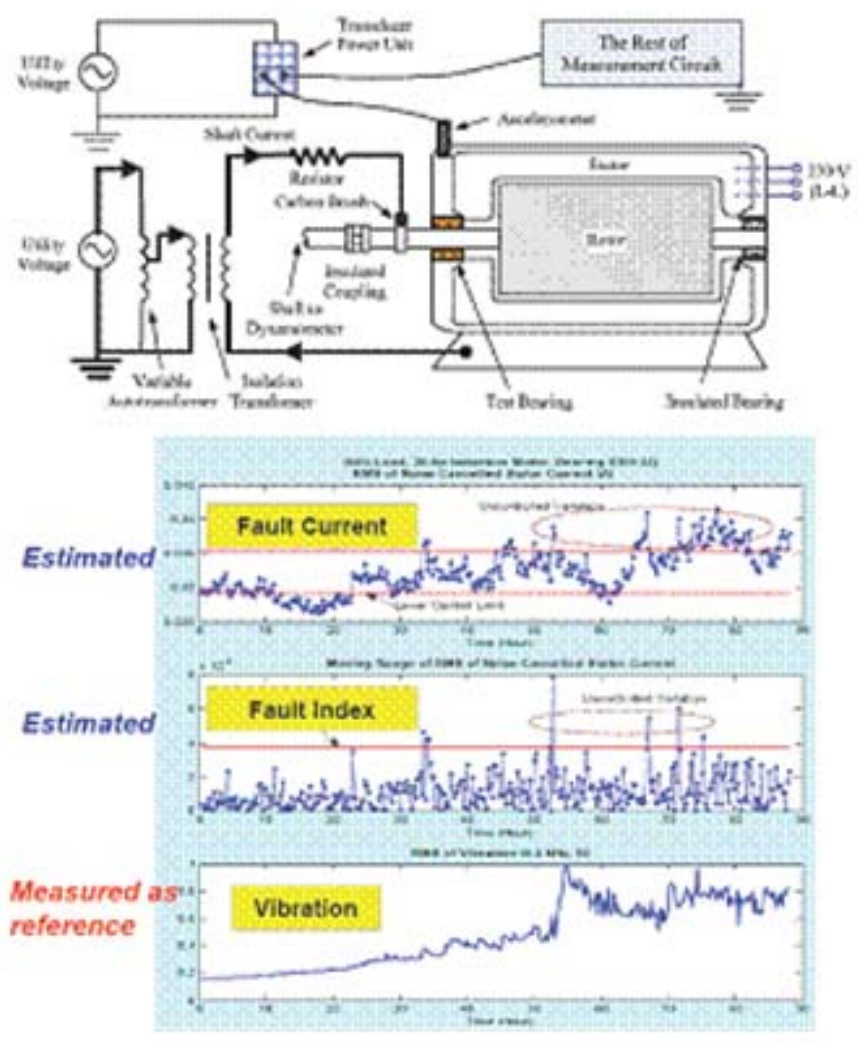

Figure 29: Lab Validation Setup at Georgia Tech and Experimental Results of Bearing Fault Algorithm

Reliable thermal protection of ac motors is crucial for reducing the motor failure rate and prolonging a motor's lifetime. In this work, conventional thermal protection devices and state-ofthe-art thermal protection techniques are reviewed. The research team has developed a series of active motor thermal protection methods, as a low-cost alternative approach to the traditional passive and sensor-based methods. These active thermal protection methods monitor the average stator temperature via stator resistance estimation based on the dc equivalent model of ac motors, using only motor stator voltage and current measurements. In particular, the active thermal protection techniques for line-started, soft-starter-connected and inverter-fed ac motors are developed. The active thermal protection techniques are capable of providing accurate, and non-invasive thermal protection of ac motors. A prototype of an external standalone dc injection module is shown in Figure $\mathbf{3 0}$ and Figure $\mathbf{3 1}$ shows the typical experimental results of motor temperature estimation using dc injection compared with directly measured winding temperature from thermal couples. 


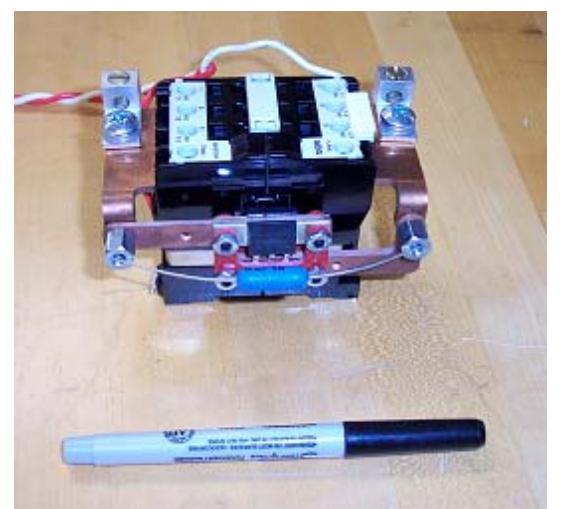

Figure 30: Standard-Along DC Injection Circuit

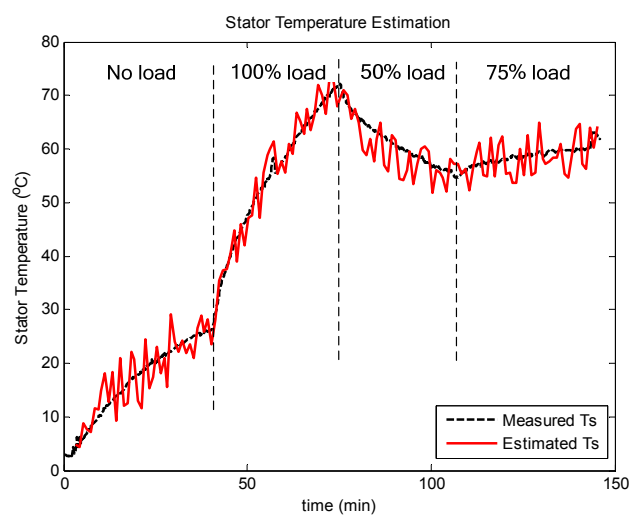

Figure 31: Estimated vs. Measured Stator Temperature

The main benefits of the developed PdM modeling methodology come from its nonintrusive, online and remote nature. As discussed above, only motor input voltages and currents need to be measured in the proposed scheme. This eliminates the needs of all other types of transducers and sensors in the motors, such as torque, temperature, speed, vibration, etc. From a product perspective, it makes it possible that the developed algorithms can be implemented in a "sensor less" fashion. This is because most PdM algorithms tend to be added as intelligent functionalities in the motor control devices. These devices directly feed electric power to the motors. For protection or control purposes, all these devices already measure motor currents and most of them also measure voltages or input power.

Therefore, the PdM algorithms can be implemented using existing measurement already in these devices without the need for additional sensors. The independence of other physical measurements also means that the energy monitoring and fault prognostics can be done "remotely" and "non-intrusively", without interfering with the motor's normal operation. Compared with the periodic checks at certain intervals used in the PM activities today, this scheme enables continuous monitoring and prognosis of the plant system, greatly reducing the risk of severe process breakdowns.

\section{Gen-1 Field Experience at a Container Box Plant of Company}

Beginning in November 2006, the research group began validating and consolidating selected algorithms through field evaluations by installing first generation (Gen-1) prototype systems in the industrial partners' manufacturing plants. As one of the initiators of this DOE program, a pulp and paper company A actively supported the Gen-1 field evaluation. This section presents 
the field experience from their packaging plant in a mid-west state, USA. This plant has been acquired by another paper company C in 2008.

In an effort to bring the emerging science forward, it was important to gain experience from the field evaluation of the nonintrusive PdM based diagnostics systems. A corrugated containerboard plant of Company A was selected as the site for prototype testing. A containerboard plant was selected instead of a pulp \& paper mill because the motor applications in this facility are generally aligned with material handling and somewhat less complex than systems found in a process environment. The development team and company A believed that deployment and troubleshooting would present less risk in this type of manufacturing environment.

Since the offering is yet to be commercialized, during the field verification phase of the project, this technology was implemented in a "motor wellness unit (MWU)," which is a research prototype including hardware and software that support the various algorithms discussed above. A prototype field testing system was installed and commissioned in January 2007 . The system was monitored and evaluated over the course of a six-month period.

A web interface displaying critical parameters including motor power, temperature, mechanical fault, cavitation, speed, efficiency and network communications was used to interrogate the selected motor driven systems. This allowed interrogation and troubleshooting by the project team from a remote site in Milwaukee, Wisconsin. Three wellness prototype systems were installed in the plant monitoring three critical motors including a 75hp blower motor, a 50hp hydraulic pump motor, and a $200 \mathrm{hp}$ compressor motor. Based on the algorithm results, service suggestions and energy saving suggestions are made to the plant manager on the 75hp and 200hp motors. Figure $\mathbf{3 2}$ and Figure $\mathbf{3 3}$ show the installed systems and sample results obtained.

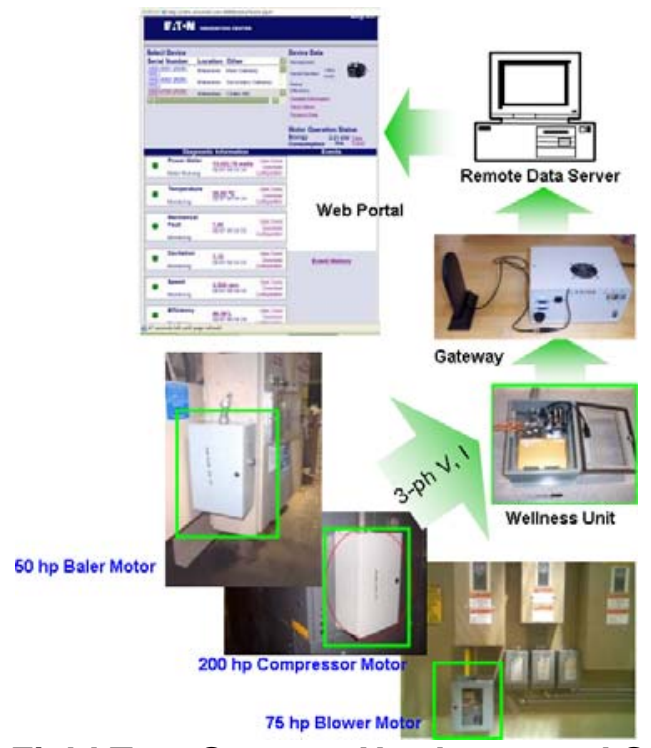

Figure 32: Gen-1 Field Test System: Hardware and Software Interface 


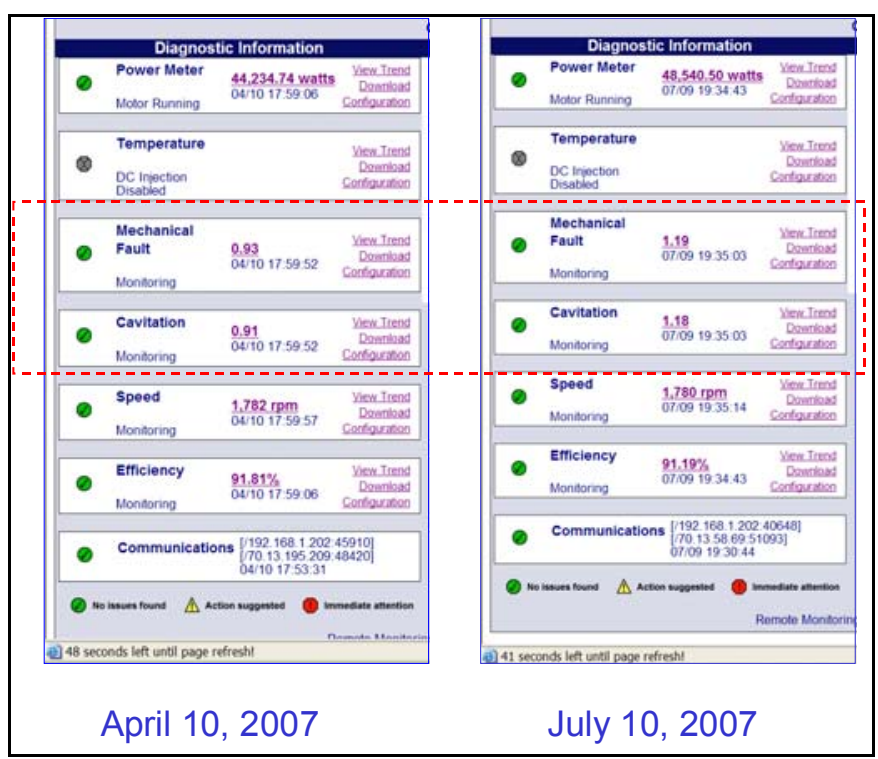

Figure 33: Results from the 75hp Motor

\section{Horsepower Blower Motor Installation}

The wellness unit for the $75 \mathrm{hp}$ blower motor was installed in a wall-mounted enclose near the existing NEMA enclosed motor starter. The planned commercialized version of this product technology would have a form factor that would enable a retrofit into the existing starter enclosure. However, the prototype was substantially larger, requiring a separate enclosure.

During the monitoring period of April 2007 through July 2007, the wellness unit algorithms indicated a rising misalignment pattern. Figure 33 shows the web-based data recorded on April 10th and July 10th. The wellness unit misalignment index was 0.94 on April 10, 2007, which meant the motor was well aligned with the driven load. The misalignment index jumped up to 1.12 on July 10, 2007. Because of the alignment change, company A maintenance scheduled an equipment outage for this plant load in order to perform an alignment check for the 75hp blower motor. The equipment alignment problem was identified and corrected during the outage. After the correction, the wellness algorithm verified a normal level for alignment. The misalignment situation was also identified during the survey period by the monthly motor vibration analysis service that company A employs at this site. The plant is currently spending \$2000-3000 per month for regular motor vibration measurement and analysis.

\section{Horsepower Compressor Installation}

Another wellness unit for the $200 \mathrm{hp}$ compressor motor was installed as a unit-mount NEMA type 1 enclosure directly on the compressor assembly. Since the compressor was a larger motor in this plant, the starter was a Wye-Delta type, designed to reduce the inrush current during the starting cycle. The wellness unit was able to track the actual line input starting current for the 200hp motor. The starting current profile shows that the Wye-delta starter is well designed and effectively reduces the starting current and torque stress. During the motor start, the maximum starting current was measured at about 2.5 times of rated motor current (as opposed to 6 times using a direct across the line start). The input voltages and currents of the motor look normal at around 180 amperes. The input 3 phase currents are well balanced, meaning the torque ripple or the vibration caused by the current unbalance is very negligible. Power factor is about 0.83 at full load, which is also normal. Motor speed at full load is $1786 \mathrm{rpm}$, compared with $1785 \mathrm{rpm}$ rated speed. 
The system suggested a possible issue with the 200hp compressor load cycling as shown in Figure 34 below. The data show that the compressor has an oscillating load profile, which is normal. However, the load profile shows that the load is varying from about $50 \%$ of rated load to around $110 \%$ overloaded condition. The load oscillation varied from 1 minute to 5 minutes. In full operation, the period was measured at about 2 minutes. The motor can work under $110 \%$ overloaded condition for a short period of time. However, constantly overloaded situation will cause overheat and increased vibration, leading to eventual premature failure. Also reduced efficiency is observed from this motor using the efficiency estimation algorithm.
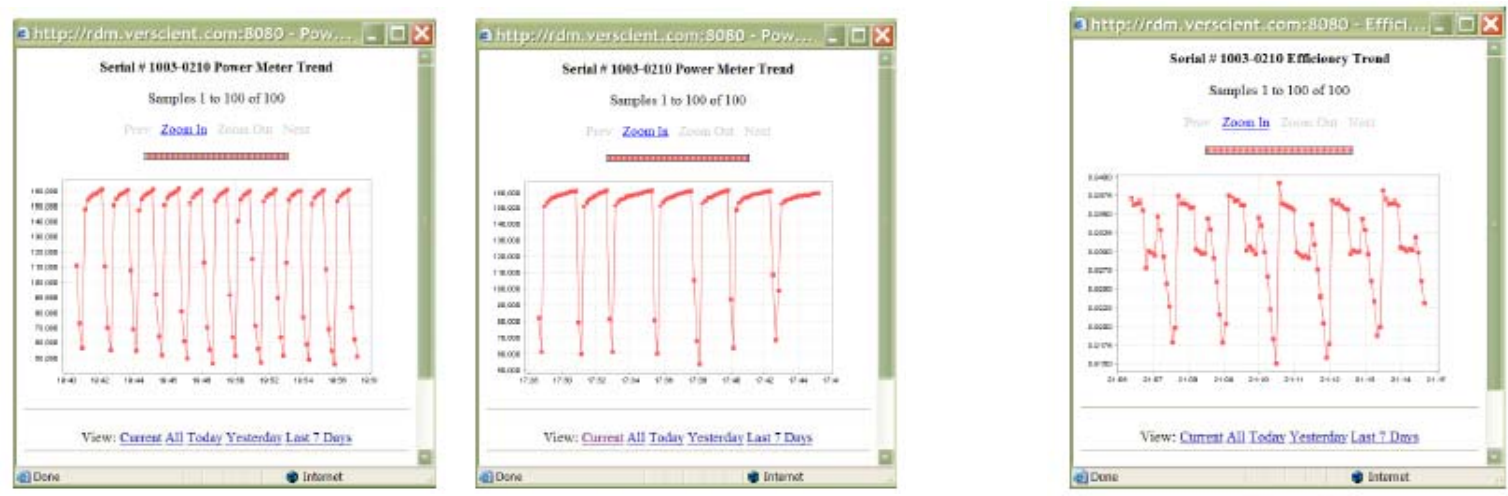

Figure 34: Power, Loading and Efficiency Results of the 200hp Compressor Motor

A formal report was provided to the plant staff with all observations and supporting data, as shown in Figure 35 below. The recommendation was to find a way to unload the compressor, or upgrade the system to a larger size, at least $250 \mathrm{hp}$. Company A elected to show the diagnostic results to the compressor manufacturer and work to resolve this issue.

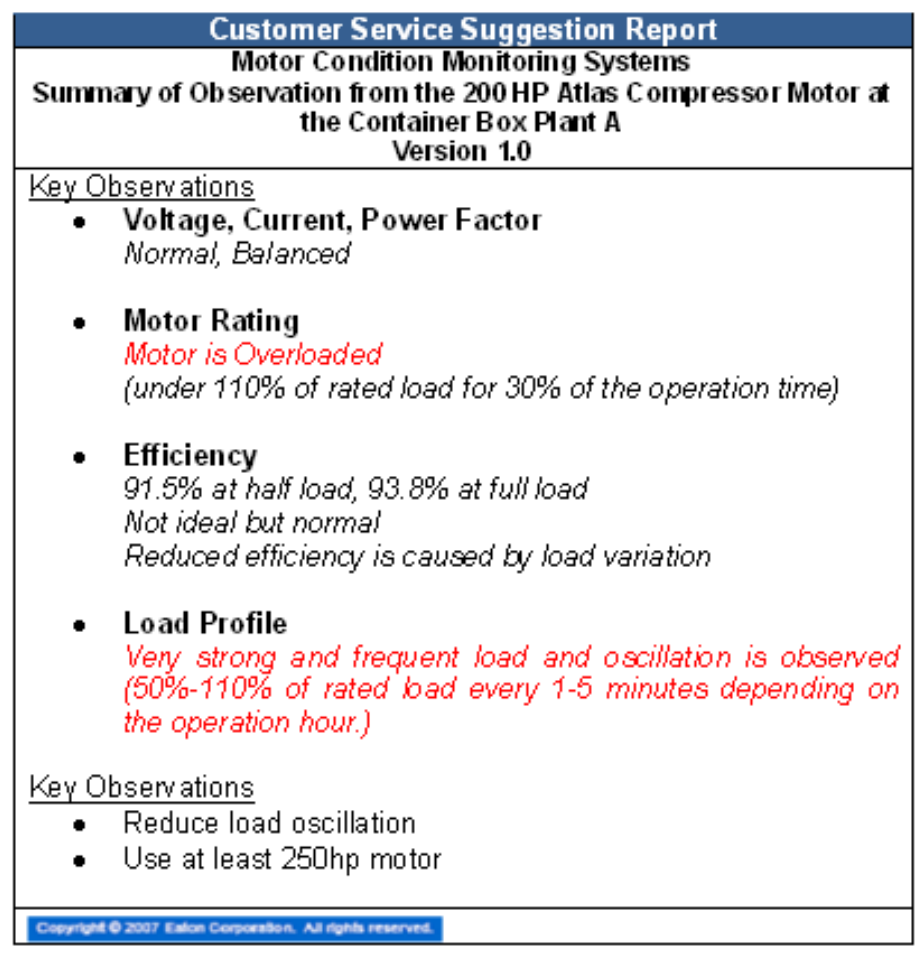

Figure 35: Formal Report to Company A with Observations and Suggestions 
Company A site maintenance responded favorably to the addition of the wellness system as a method to implement $\mathrm{PdM}$, offering a new window of information to assist maintenance personnel. The site maintenance supervisor offered the quote "This is the closest thing to a crystal ball that I have seen yet". The maintenance leadership agreed to share the results of the wellness installed system in their monthly internal motor maintenance meetings. A suggestion was made that development was needed for wellness monitoring of motors connected to variable frequency drives. This is finally realized in the Gen-2 field testing.

\section{Gen-2 Field Experience at Multiple Customer Sites}

Starting from mid 2007, the research team began to launch a major effort in improving the functionality and reliability of the field testing hardware and software. The goal was to design a more flexible field testing system that would enable us to validate the complete set of developed algorithms in more customer sites. The second generation (Gen-2) field testing system was completed with the basic functions with selected algorithms in April 2008 and later enhanced with full capabilities by July 2008.

The Gen-2 system was designed based on the same hardware platform in Gen-1. The enclosure and power supply were redesigned to fit better in industrial environments. Software operating system was changed from Microsoft Windows to Linux to provide better support for shutdown recovery and wireless communications. Figure $\mathbf{3 6}$ below shows the pictures of a few key hardware components in the Gen-2 system.
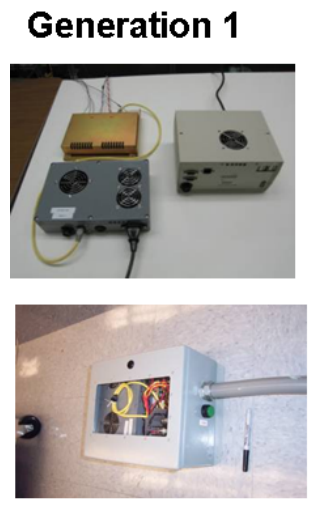

- Closer to commercialization

- More algorithms

- More communication options

- Less footprint, easy retrofit

- Better reliability, flexibility

- Remote and dynamic algorithm management

- Enable more customer engagement - Provide more values to the customer for more energy savings.

\section{Generation 2}

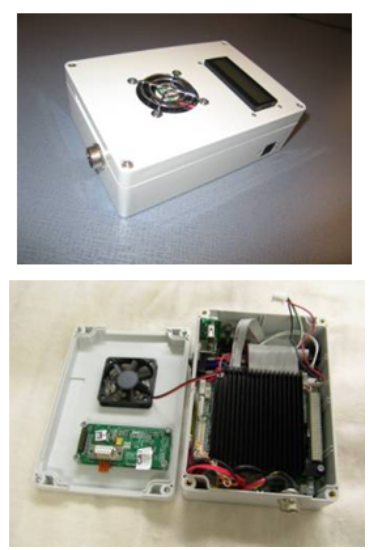

Figure 36: Transition from Gen-1 to Gen-2 hardware

The system architecture was redesigned to provide more options for remote access by the research team depending on the preference by the facility manager of the test site, and the diverse set of conditions at potential test sites.

The software information architecture was also redesigned to enable more flexible data acquisition, data analysis, and data storage capabilities. In the Gen-2 motor wellness unit, three phase motor terminal voltages and currents are sampled in the data acquisition board and digitalized signals are passed to the data processing unit. A complete set of 10 algorithms (as discussed in previous sections) are implemented as executable applications and run in a parallel architecture in a Linux operating system. The raw voltage and current samples are stored for two days and processed results are stored permanently in the hard disk in the MWU. The research team can remotely access the MWU through Secure Shell (SSH) protocol connections via Wi-Fi access point, repeaters, and cell net modems. This gives the research 
team full access to all raw data acquired by the MWU and all the processed results from the algorithms in a remote fashion.

In the Gen-2 field tests, the team initially contacted a large group of customers from a variety of industries. After initial site assessment and customer interviews, the team decided to focus on the following industries: Pulp and paper, water and waste water, petro/chem, material handling. The targeted applications include pumps, fans (blowers), and compressors (see Figure 37). Nine customer sites have been visited and accessed. Ten Gen-2 MWU were finally installed in four customers who actively supported the field testing efforts. The following chart shows the detailed site and motor application information of all the applications identified in Gen-2 and their status. Due to the location convenience, the Gen-2 algorithm analysis and customer reviews were focused on the sites that are located relatively close to Milwaukee, Wisconsin. This includes a waste water treatment plant of Company B and the Gen-1 container box plant from company $\mathrm{C}$ (previously owned by company A). A pulp and paper mill plant of Company $A$ at a northwest state also produced results for algorithm validation and review meetings were held as well. Eaton Eden Prairie hydraulic manufacturing plant (D) also produced valid results for algorithm validation. However, the $15 \mathrm{hp}$ spindle drive motor operates at almost no load in the majority of time and many algorithms were designed to operate at higher load conditions. Therefore, the $15 \mathrm{hp}$ spindle drive motor results were not extensively studied after the initial algorithm analysis in September 2007, and the site disabled.

\begin{tabular}{|c|c|c|c|c|}
\hline CUSTOMER & INDUSTRY & APPLICATION \#1 & APPLICATION \#2 & APPLICATION \#3 \\
\hline B & Wastewater & $\begin{array}{l}350 \text { Hp Blower } \\
\text { Oxygenization of influent } \\
\text { Soft Start }\end{array}$ & \begin{tabular}{|l}
50 Hp Pump \\
Final effluent \\
Drive
\end{tabular} & $\begin{array}{l}125 \mathrm{Hp} \text { Pump (Kentucky) } \\
\text { Primary influent } \\
\text { Drive }\end{array}$ \\
\hline \multirow[t]{3}{*}{ C } & Box Plant & $\begin{array}{l}3 \mathrm{Hp} \text { Boiler Pump } \\
\text { Across the line starter }\end{array}$ & $\begin{array}{l}200 \mathrm{Hp} \text { Compressor } \\
\text { Across the line starter }\end{array}$ & \\
\hline & Refinery & $\begin{array}{l}20 \text { Hp sump pump } \\
\text { Across the line starter }\end{array}$ & $\begin{array}{l}200 \mathrm{Hp} \text { Cooling Tower Fan } \\
\text { Across the line starter }\end{array}$ & \\
\hline & Steel & $\begin{array}{l}30 \mathrm{Hp} \text { Fan } \\
\text { Annealing process circulation } \\
\text { Across the line starter }\end{array}$ & & \\
\hline \multirow[t]{2}{*}{ D } & Manufacturing & $\begin{array}{l}100 \text { Hp Pump } \\
\text { Test stand - hydraulic pump } \\
\text { Across the line starter }\end{array}$ & $\begin{array}{l}15 \mathrm{Hp} \text { Spindle Drive } \\
\text { Multitool machining center } \\
\text { Drive }\end{array}$ & \\
\hline & Process & $\begin{array}{l}10 \mathrm{Hp} \text { Dryer \#1 Motor } \\
\text { Drive }\end{array}$ & $\begin{array}{l}50 \text { Hp Dryer \#2 motor } \\
\text { Drive }\end{array}$ & $\begin{array}{l}10 \text { Hp Dryer \#1 New Motor } \\
\text { Drive }\end{array}$ \\
\hline \multirow[t]{3}{*}{ A } & Paper/Pulp & $\begin{array}{l}400 \mathrm{Hp} 600 \mathrm{~V} \\
\text { Digester Mixer } \\
\text { Drive }\end{array}$ & $\begin{array}{l}125 \mathrm{Hp} 575 \mathrm{~V} \\
\text { Centrifugal Sump Pump } \\
\text { Across the line starter }\end{array}$ & $\begin{array}{l}75 \mathrm{Hp} 600 \mathrm{~V} \\
\text { Mixing System Agitator } \\
\text { Across the line starter }\end{array}$ \\
\hline & $\begin{array}{c}\text { Automotive } \\
\text { Manufacturer }\end{array}$ & $\begin{array}{l}200 \mathrm{Hp} 480 \mathrm{~V} \\
\text { Ventilation Fan } \\
\text { Across the line Starter }\end{array}$ & & \\
\hline & Building Mgmt/HVAC & \begin{tabular}{|l}
\multicolumn{1}{|c|}{$\mathrm{Hp} 480 \mathrm{~V}$} \\
Chiller \\
Drive
\end{tabular} & & \\
\hline \multirow{4}{*}{$\begin{array}{l}\text { Last updated: } \\
\qquad \text { 12-Jan-09 }\end{array}$} & & & Commissioned/Operational & $\frac{8}{2}$ \\
\hline & & & Planned & 3 \\
\hline & & & Identified & 5 \\
\hline & & & Total & 18 \\
\hline
\end{tabular}

Figure 37: Gen-2 Customer Sites Summary 
These Gen-2 customer sites provided great opportunities for the research team to obtain real motor data under actual industrial loading conditions. With the data obtained from the customer sites, the developed algorithms are validated in actual industrial environment. Besides, the energy efficiency and motor health information produced by the algorithms provide great value to the customers to improve their process. A series of customer interview/review meetings were held with the Gen-2 customers. The discussions in these review meetings helped the research team to determine the value proposition of the developed technology in this program, and also provided insightful information to quantify the potential energy saving opportunities when the developed technology is implemented in industrial control products and deployed in a large basis. The details of these business development related information will be discussed in more details in Section 3.2 Task 14.

The following sections summarize the conclusions and observations made in these customer review meetings. All the conclusions are made based on the continuously monitored algorithm results for at least three months. Formal reports with detailed algorithm results and recommendations were also provided to the customers in these meetings.

\section{A Waste Water Treatment Plant of Company $B$}

Three MWU's were installed at this site monitoring three critical motors: A variable frequency drive (VFD) driven $125 \mathrm{hp}$ lift pump motor, a $340 \mathrm{hp}$ blower motor, and a VFD-driven $50 \mathrm{hp}$ pump motor. Figure $\mathbf{3 8}$ show the pictures of the motors and MWU's taken from the field. Two review meetings were held on December $5^{\text {th }} 2008$ and January $20^{\text {th }} 2009$. Figure 39 and Figure 40 summarize the algorithm validation status and key observations/conclusions from this site, respectively.

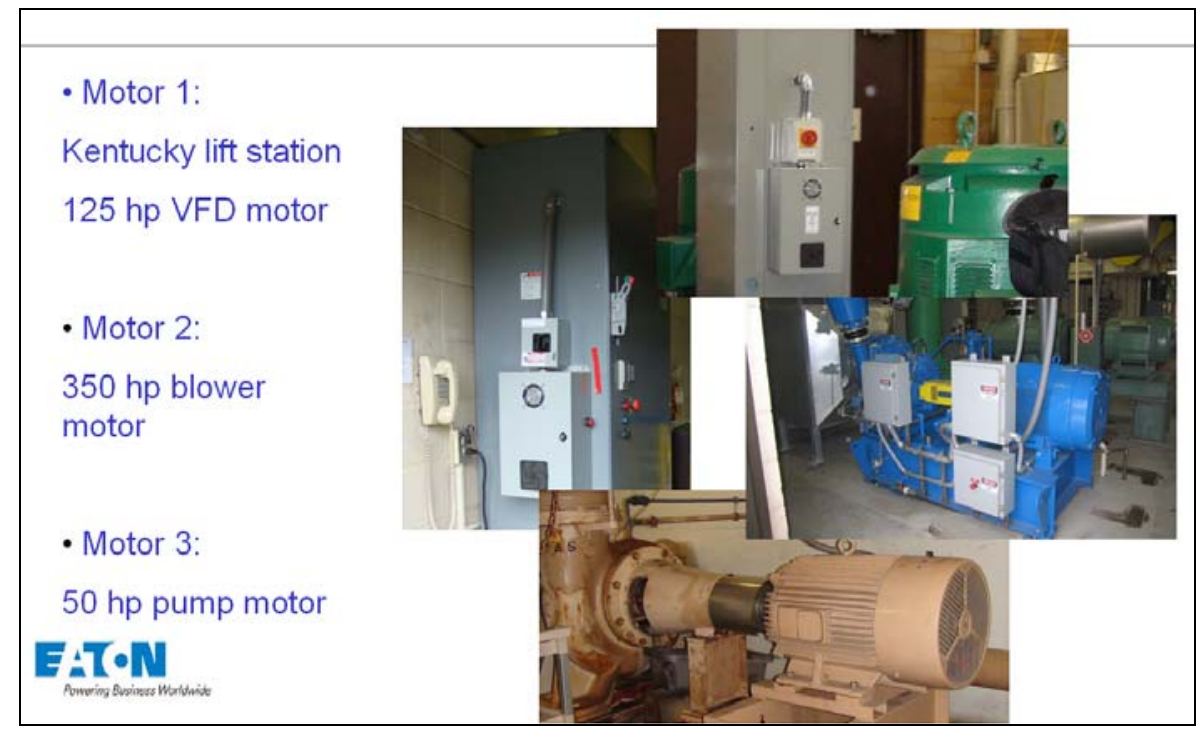

Figure 38: Gen-2 Customer Site Installations at Waste Water Treatment Plant- Company B 
Key Algorithm Validation Status at Waste Water Treatment Plant of Company B

Algorithms successfully validated from Alpha side results:

- Power metering

- Power quality

- Harmonic analysis

- Speed detection

- Efficiency estimation

- Torque estimation

- Shaft misalignment/Rotor eccentricity (non VFD)

- Pump cavitation

Algorithm under improvement and further testing

- Bearing fault for VFD (follow-up testing on 125hp pump in Q3 2009)

- Shaft misalignment/Rotor eccentricity for VFD

\section{Summary of Alpha Site Algorithm Results}

Motor 1: 125 hp VFD pump motor

- $10 \%$ to $40 \%$ load variation- highly under loaded

- Average efficiency is $55 \%$ (v.s. $94.5 \%$ rated efficiency)

- Opportunity for Eaton energy saving drive (8-9\% additional energy savings or $\$ 2500$ per year Validated from actual date)

- Observed very infrequent pump cavitations when load is above $25 \%$

- No motor shaft eccentricity fault is observed

Motor 2: $50 \mathrm{hp} \mathrm{VFD} \mathrm{pump} \mathrm{motor}$

- $30 \%$ to $70 \%$ load variation- under loaded most of time (e.g. $30 \%$ load in week of $12 / 25 / 08)$

- Average efficiency under average load (50-70\%) is 70-85\% (v.s 93.6\% rated efficiency)

- Opportunity for Eaton energy saving drive (5-10\% of energy savings- expected from load profile.)

- No motor shaft eccentricity and pump cavitation faults were observed

- All results (voltage, current, load, power quality, power factor, VFD performance) before and after the VFD failure on 11/30/2008 are Consistent and Normal. > VFD failure was not caused by motor or pump problems. Could be caused by VFD internal faults or upstream $P Q$ issues (VFD input).

Motor 3: 350 hp blower motor- non VFD

- $50 \%$ to $70 \%$ load variation- motor is well loaded

- Using VFD expects to get $15 \%$ of energy savings under light load

- No motor shaft eccentricity and pump cavitation are observed

Based on the actual dynamic load profile and power quality information observed from the power metering algorithm, harmonic analysis algorithm, efficiency estimation algorithm, a recommendation was made to the customer to use a variable frequency drive for the $350 \mathrm{hp}$ blower motor. By using a standard VFD, it is estimated that a $15 \%$ energy savings is achievable. The team also performed field tests twice on the $125 \mathrm{hp} \mathrm{lift}$ station pump motor at this customer to verify the energy saving potential for the Eaton energy saving drive control algorithm, which was developed in Eaton based a few algorithms originated from this DOE research program. 
The measured energy savings (input power reductions) with actual load from this $125 \mathrm{hp}$ motor is $8-9 \%$ incremental to the energy savings obtained from a standard VFD. The detailed results are shown in Figure 39.

\section{Energy Savings Drive Validation}

- Eaton has performed onsite tests twice to validate Optimized Drive Configuration for Energy Saving Drive on this motor on 12/11/08 and 01/07/09.

- Test results confirmed $8-9 \%$ additional energy savings on top of energy savings already obtained from using a VFD.

\begin{tabular}{|c|c|c|c|c|c|c|c|c|c|}
\hline & $\begin{array}{l}\text { Existing VFD } \\
\text { Configuration }\end{array}$ & $\begin{array}{c}\text { Quadratic } \\
\text { Confguration }\end{array}$ & \multicolumn{3}{|c|}{$\begin{array}{l}\text { Quadratic Confy. } \\
\text { Energy Savings }\end{array}$} & $\begin{array}{c}\text { Eaton Optimized } \\
\text { Configurmion }\end{array}$ & \multicolumn{3}{|c|}{$\begin{array}{c}\text { Eaton Optimized } \\
\text { Configuration Savings }\end{array}$} \\
\hline Frequency & $\mathrm{kW}$ & $\mathrm{kW}$ & $\mathrm{kW}$ & $\%$ & \$lyear & $\mathrm{kW}$ & $\mathrm{kW}$ & $\%$ & S/ year \\
\hline 60 & 88 & 88 & 0 & $0 \%$ & $\$ 0$ & 828 & 0.5 & $0.6 \%$ & $\$ 438$ \\
\hline 45 & 37.9 & 36.4 & 1.5 & $4 \%$ & $\$ 788$ & 35 & 29 & $8 \%$ & $\$ 2,540$ \\
\hline 40 & 26.3 & 24.8 & 1.5 & $6 \%$ & $\$ 788$ & 23.9 & 2.4 & $9 \%$ & $\$ 2,102$ \\
\hline
\end{tabular}

Figure 39: Energy Saving Potential as Wäste Water Treatment Plant of Company B

\section{A Container Box Plant of Company C (previously owned by Company A)}

This plant was formerly owned by company A before 2008. The site supported the Gen-1 field test. Gen-2 MWU systems were installed in this site. Customer review meeting was held on April $21^{\text {st }}$ 2009. A MWU was installed to monitor a critical $200 \mathrm{hp}$ compressor motor, as shown in Figure 40. Below is a summary of the algorithm validation status and key observations/conclusions from this site, respectively.

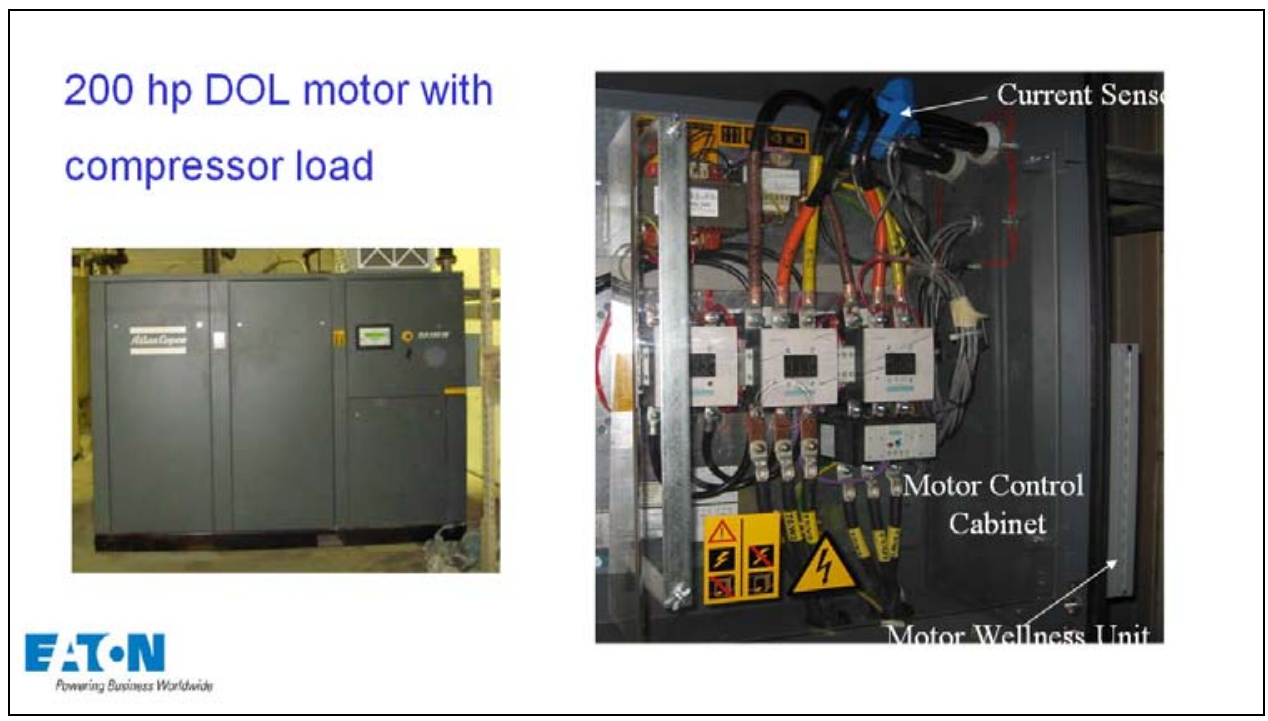

Figure 40: Gen-2 Customer Site Installations at -Container Box Plant Company C 
Key Algorithm Confirmation Status at Container Box Plant of Company $C$

The motor was closely monitored between November 08 and April 09. Four weeks of continuous algorithm results are provided in this report from November 08 to April 09.

Algorithms successfully confirmed from Alpha site results:

- Power metering

- Power quality

- Harmonic analysis

- Speed detection

- Efficiency estimation

- Torque estimation

- Shaft misalignment/Rotor eccentricity (DOL)

- Pump cavitations (for False Positives)

- Bearing fault detection (DOL)

Algorithm under improvement and further testing:

- Bearing fault for VFD

- Shaft misalignment/ Rotor eccentricity for VFD

Summary of Results at Container Box Plant of Company C

Key observations and conclusions:

- Voltage supply is clean with THD $<4 \%$

- Highly dynamic load variation and consistently overloaded condition:

a) $20 \%$ to $110 \%$ rated load at $1.5-2$ minute cycle

b) Service factor is 1.15 hence motor can be continuously loaded up to $115 \%$ of rated load

- Voltage THD $<5 \%$ but overall current THD $>10 \%$ - excessive power losses in motor

- Voltage and current unbalances are low (<4\%) indicating balanced cable wiring and motor windings

- Power factor is 0.85 at rated load (vs rated PF 0.86 ) and 0.5 at low load.

- Average motor efficiency varies from $84-94 \%$ (vs. $96 \%$ rated efficiency)

- Potential for energy savings

a) Replacing starter by VFD expects to get $10-15 \%$ of energy savings under light load

b) Eaton energy savings drive is expected to provide $\sim 5 \%$ incremental energy savings estimated from actual load profile

- Mechanical fault index is consistent around 1. Indicating healthy alignment

- Bearing fault index is consistent below threshold- as of now bearing is healthy

- Pump cavitations fault index is consistently close to 1 . Indicating no false alarms.

Based on the actual dynamic load profile and power quality information observed from the power metering algorithm, harmonic analysis algorithm, efficiency estimation algorithm, a recommendation was made to the customer to replace the wye-delta starter with a VFD for the $200 \mathrm{hp} \mathrm{pump} \mathrm{motor.} \mathrm{By} \mathrm{using} \mathrm{a} \mathrm{standard} \mathrm{VFD,} \mathrm{it} \mathrm{is} \mathrm{estimated} \mathrm{that} \mathrm{a} 15 \%$ energy savings is achievable.

The team also estimated the energy saving potential if a VFD with Eaton energy saving drive control algorithm is used, based on the field test results from the $125 \mathrm{hp}$ lift station pump motor at plant B. It is estimated that for this $200 \mathrm{hp}$ motor $8-9 \%$ energy saving (input power reduction) 
incremental to the energy savings obtained from a standard VFD could be obtained using the Eaton energy saving drive control method. The detailed results are shown in Figure 41.

\section{Energy Savings Potential}

- Using VFD is expected to get $10-15 \%$ of energy savings under light load

- Eaton has performed onsite tests twice to validate Optimized Drive Configuration for Energy Savings Drive on a $125 \mathrm{hp}$ motor at a waste water facility in Wisconsin on 12/11/08 and 01/07/09

- Based on the validation data and the field data collected from the $200 \mathrm{hp}$ motor at Manitowoc, a potential $\sim 5 \%$ incremental energy savings per year could be achieved using Eaton energy savings drive technology.

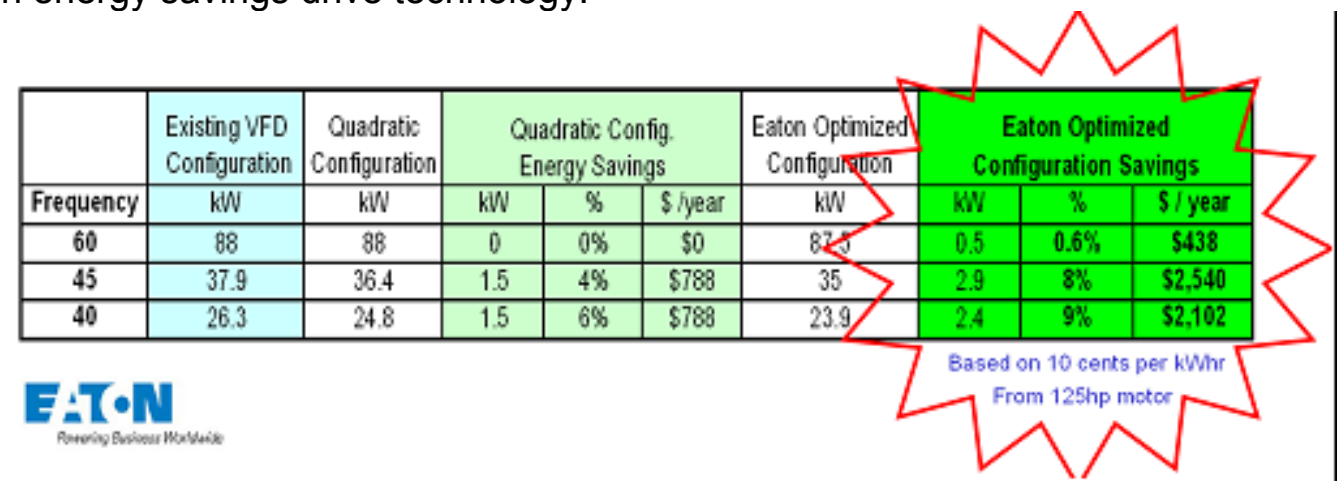

Figure 41: Energy Saving Potential at Container Box Plant of Company C

\section{A Paper Mill Plant of Company A}

Customer review meeting was held on February $19^{\text {th }}$ 2009. A MWU was installed to monitor a critical $400 \mathrm{hp}$ blower motor, as shown in Figure 42. The next sections below summarize the algorithm validation status and key observations/conclusions from this site, respectively.

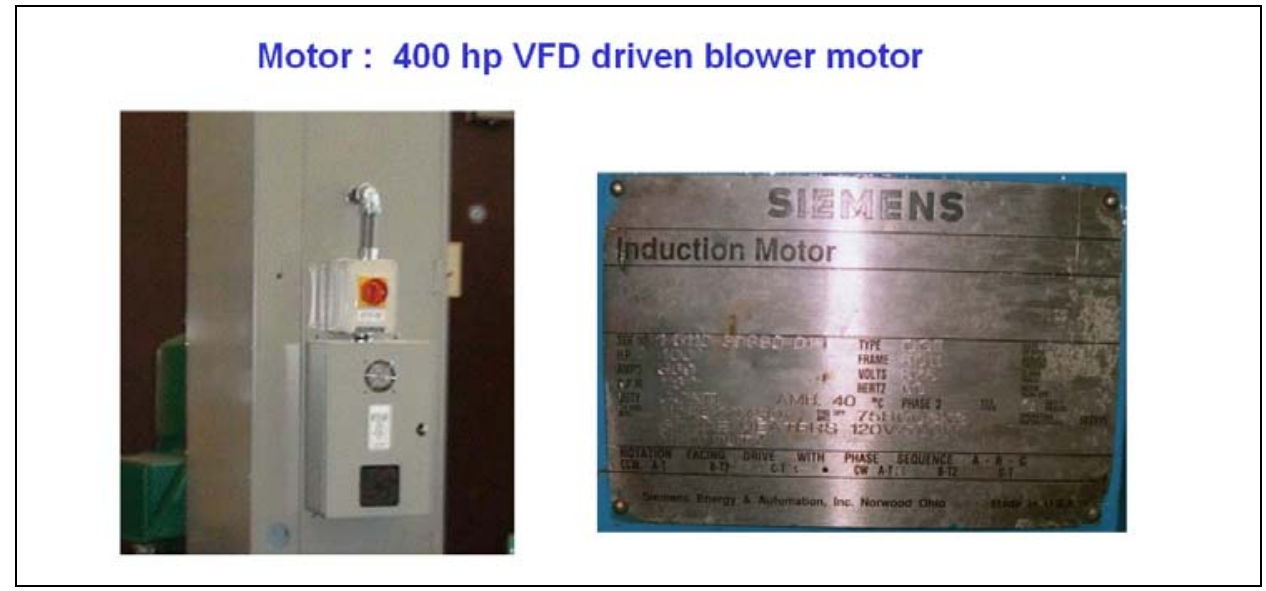

Figure 42: Gen-2 Customer Site Installations at Paper Mill Plant of Company A 
Key Algorithm Validation Status at a Paper Mill Plant of Company A

Algorithms successfully validated from Alpha site results:

- Power metering

- Power quality

- Harmonic analysis

- Speed detection

- Efficiency estimation

- Torque estimation

- Rotor Fault Detection (initial results)

Algorithm under improvement and further testing:

- Bearing Fault for VFD

- Shaft misalignment/ Rotor exxentricity for VFD

Summary of Alpha Site Algorithm Results

24 hour period 12/24/08-12/25/08

Motor: 400 hp VFD driven blower motor:

- Load variation: $58-71 \%$

- VFD voltage variation: $408 \mathrm{~V}$ to $489 \mathrm{~V}$

- Speed variation: 853 RPM to 940 RPM (rated speed 1200 RPM)

- Power factor: 0.805 to 0.837 (rated PF missing from nameplate)

- Average efficiency $87 \%$ is about 5 points below the rated efficiency

- Supply power quality of the VFD is normal, which verifies normal VFD operation

- Harmonic analysis results are normal (voltage THD < 2.4\%, current THD < 1.6\%)

- Motor current and power frequency spectrum suggest early stage for rotor fault (broken bar or broken end ring.)

- Potential opportunity for Eaton energy saving drive (about 5-8\& incremental energy savings per year- estimated based on acutal field validation data from a waste water plant $125 \mathrm{hp}$ pump motor)

- Existing mechanical fault, pump cavitation, and bearing fault detection algorithms are developed for $60 \mathrm{~Hz}$ ALS applications. We are currently in the process of modifying and improving these algorithms for VFD applications.

The team also estimated the energy saving potential if a VFD with Eaton energy saving drive control algorithm is used, based on the field test results from the $125 \mathrm{hp}$ lift station pump motor from site B. It is estimated that for this 400hp motor a 5-8\% energy saving (input power reduction) incremental to the energy savings obtained from the existing VFD could be obtained using the Eaton energy saving drive control method. The detailed results are shown in Figure 43.

\section{Energy Savings Drive}

- Eaton has performed onsite tests twice to validate Optimized Drive Configuration for Energy Saving Drive on a $125 \mathrm{hp}$ motor at a waste water facility in Wisconsin on 12/11/08 and 01/07/09.

- Based on the validation data and the field data collected from the 400hp motor, a potential $5-8 \%$ incremental energy saving per year can be achieved using Eaton energy saving drive technology. 


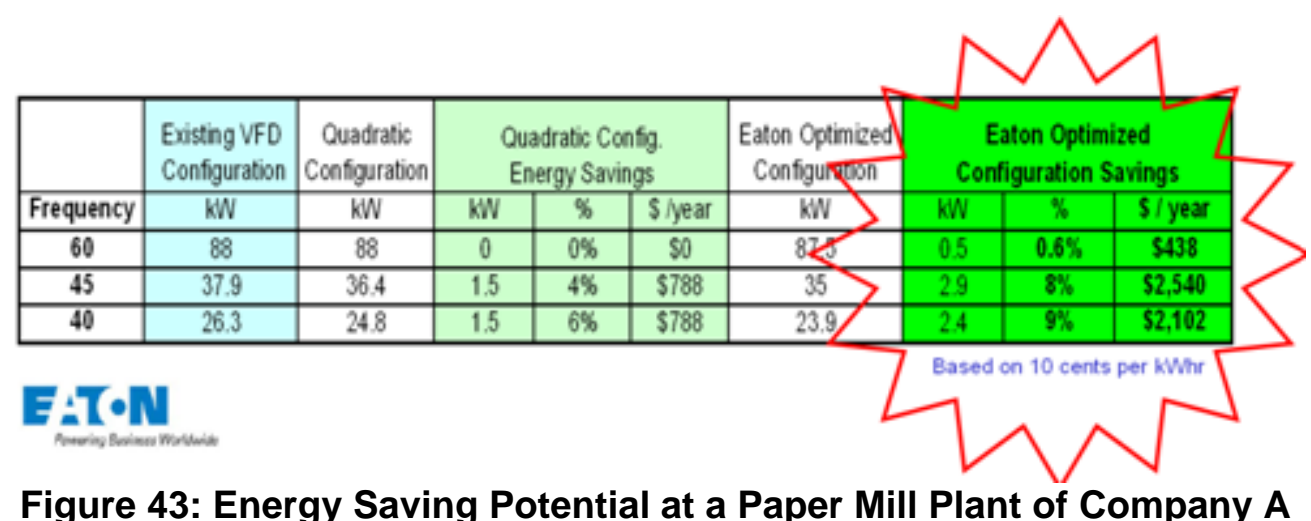

\subsection{Task 14}

Objective: Develop and implement product introduction plan covering the manufacturing, marketing, and introduction of the WSN into an energy management system optimization product

Eaton Innovation Center (IC) and Industrial Controls Division (ICD) gathered Voice of Customer across multiple industry segments during Alpha site customer visits and interviews. Customers identified needs and requirements for initial product offerings, confirmed the value algorithms and products provide in order to construct and validate business cases. The needs statements from VOC interviews have been captured in a searchable database. Several customers indicated that the timing is perfect - they have been looking for a motor prognostic tool that could automate their current preventative maintenance procedures and enable them to cost effectively monitor more points (motors) within their facilities to reduce unscheduled downtime. Eaton also initiated discussions with key Building Equipment OEMs regarding the benefits of incorporating this technology into new installations as well as retrofits.

The Alpha site field deployments also confirmed that the hardware for sensing and sampling motor currents and voltages was reliable and able to provide an adequate signal-to-noise ratio with the electrical noise present on the power line within various facilities. To facilitate product industrialization, the Innovation Center (IC) performed a Technology Transfer to the Industrial Controls Division (ICD) of the algorithms, software, hardware, test results, and associated documentation.

Two additional product development projects are targeted for commercialization. The first is an enhanced motor control device that incorporates selected wellness and efficiency algorithms to provide early warnings and alarms of impending or changing motor and connected load conditions. This product will be targeted towards industrial processes that have motor driven equipment in critical applications where downtime costs are significant. Average downtime for an industrial facility is $4-12$ hours with a cost of $\$ 7500 / \mathrm{hr}$, with large, critical processes reaching $\$ 50-100 \mathrm{k} / \mathrm{hr}$. The enhanced motor control device would support various wired industrial field bus protocols to enable communications to other existing PLC, DCS, and SCADA type systems to report alarms, warnings, and trend data. While wireless communications was incorporated and evaluated in the field prototypes, it was not targeted for the final product as Customers typically wanted any new/additional predictive diagnostic products to tie into their existing wired network infrastructure and computerized maintenance systems. 
Alpha site field trial units also contained energy efficiency and power quality algorithms. Monitoring energy usage for individual pieces of equipment is informative to facility and energy managers for targeting energy saving initiatives, but what they really want is to have the motor controls (drives) automatically optimize the energy used, especially when motors are running at less than full load, which constitutes almost $50 \%$ of the motors in US industry today. Although many facilities recognize that a drive saves energy on motors that they chose to run at less than full speed, they do not always identify energy saving opportunities on other applications, much less to optimize and quantify the actual energy use. Drives often are applied for speed or torque control. Eaton is now targeting applications where an Eaton drive can provide for energy optimization, even beyond that of existing "conventional" drives. The second product development project is for an "automatic" energy optimizing control product which can provide "outer loop" control commands for a Variable Frequency Drive. Simulation, implementation, and validation testing of the Energy Optimized Drive Control are underway. Laboratory and field data analysis confirm the potential for $2-10 \%$ incremental energy savings for applications that run continuously or intermittently at less than full load. The product would also calculate and displays the energy savings. An independent PC based software tool would also be available to help sales personnel and customers calculate projected energy savings for different equipment and load profiles prior to purchase and installation of the product.

\section{Accomplishments}

As a result of this program, Eaton is now incorporating motor wellness and efficiency technology into its strategic plans, product roadmaps, and product development. Eaton IC and ICD held a business implementation summit in December 2008 to select and plan for the commercialization of specific products based on the motor wellness and energy saving technologies developed within this program, and based on the Customer validation received through Alpha site visits, detailed interviews, surveys, and product concept reviews. The Industrial Controls Division is a market leader in motor controls and protective devices. In 2007 ICD hired a product manager to develop the business plan and product introduction for an enhanced overload called Motor Insight, which was released for sale in 2008. This ICD product was developed independently of this program, but it was identified as a potential platform for incorporation of additional wellness and efficiency algorithms that resulted from this program.

The product roadmap expands the family of motor control products that will incorporate motor wellness and energy saving/efficiency algorithms. Follow on R\&D is also being conducted in 2009 to modify and validate selected wellness algorithms for motors driven at variable frequencies, and to modify and validate the wellness algorithms for Medium Voltage motor applications. Broad deployment across Industrial facilities will enable achieving significant energy savings, and reduce unscheduled downtime.

\section{Program Accomplishments}

Confirmed that deployment across Industrial facilities will enable achieving significant energy savings, and reduce unscheduled downtime.

Overall project accomplishments are highlighted in each individual Task section throughout the main body of the report. 
A total of six motor diagnostics and energy saving related patents have been filed during this program. Eighteen additional patent disclosures are still pending review by Eaton management for patent filing.

Nineteen papers were published, with numerous presentations made at Industry Conferences.

Please see Appendix B for a complete listing of external publications.

\section{Conclusions}

- The combination of inferential motor and connected load monitoring, built into conventional motor protective relays, motor starters and motor drives provides a low cost customer solution to monitor overall energy efficiency and system degradation / impending motor and load failures.

- Low cost motor and connected load condition monitoring provides the industrial customer with a long sought solution to identify and improve efficient operation of facility equipment and provide a dramatic reduction in unscheduled downtime.

o The following motor wellness algorithms have been proven through simulation lanalysis, lab testing and field demonstrations

- Power metering, power quality and energy efficiency

- Motor/Load fault analysis

- Rotor eccentricity

- Rotor bar fault

- Pump cavitation

- Winding insulation fault

- Bearing fault

- The broader adoption of Energy-Optimizing Control Technology will enable many applications to "automatically" save energy when the motor is not at full load.

Field trials and customer interviews confirmed that nonintrusive, motor current signature analysis (MCSA) and motor power signature analysis (MPSA) provide an effective means to diagnose and predict changes in motor health without requiring individual sensors to be mounted to a motor, or subjected to the environment at the motor. This enables broader deployment to monitor medium size (20-200hp) motors and loads on critical equipment within a facility. Primary benefits to the customer are early indication of impending failures to provide more proactive/effective PM coverage, and to reduce unscheduled downtime and ensure equipment productivity. Continuous process users want alerts/results sent to their existing SCADA/DCS/PLC system and do not want to implement remote monitoring. Target customers are those who value downtime, and have some form of condition based monitoring alreadyeasier adoption and integration.

Analysis of power quality, energy use, and instantaneous efficiency estimation on a per motor basis provides an effective means to determine the operating load profile of a motor, and can be used to identify methods to reduce energy use and improve motor life. This includes the broader identification adoption/installation of Drives for motors that do not run continuously at full load.

The field trials also confirmed that the performance of algorithms, current sensors, and data acquisition circuitry was not compromised by the industrial environment. Eaton was also able to enhance the robustness of the algorithms based on real world signal inputs. 
Other Lessons Learned:

- Customers desire additional condition monitoring for Medium-Voltage motors (which are almost always critical)

- Difficult to retrofit Predictive Maintenance enhancements at lean or union based facilities that run to failure (reactive maintenance)

- Estimating Remaining Useful Life (RUL) desired but not required at many sites

\section{Recommendations}

- Passive, monitoring algorithms have provided great value to increase the "awareness" of energy efficiency and condition monitoring conditions. Active and automatic control related algorithms/devices are desired to actively correct and improve the conditions based on the monitoring results.

o On the energy efficiency side, funding support for Energy-Optimizing Controls to extend energy savings to the maximum level possible is recommended. Through large scale field tests and demonstrations, an additional $2-10 \%$ energy savings is possible with breakthrough optimization and active control algorithms. It is also recommended that DOE consider follow on work to demonstrate new technologies in the area of active energy savings through improved motor drive controls.

o On the motor condition monitoring side, the majority of research work in this project and other reported publications has focused on passive monitoring of motor and equipment failures. In order to be able to predict failures earlier and more accurately, active motor diagnostics such as online surge test techniques show great potentials. Another area worth further exploration is "actively" injecting specific signals into the motor and observing the response. This method can provide additional insights into the condition of the motor. Funding support for additional research in these areas is recommended.

- Large scale demonstration under different applications to remove risks associated with the inferential motor and connected load monitoring with communications capabilities, built into conventional motor protective relays, motor starters and motor drives, will dramatically improve the acceptance of this technology and solution in the marketplace defined by the Industries of the Future. 


\section{Appendices}

Appendix A - Supporting Documentation and Internal Reports: Tasks 1-14

List of Documents in Appendix A

1. Grenada_testplan_v10.pdf

2. DOE Task 10 Appendix.pdf

3. A Survey of Efficiency-Estimation Methods for In Service Induction Motors.pdf

4. 2006_TIA_A Nonintrusive and In-Service Motor Efficiency Estimation Method.pdf

5. 2008_TIE_A Remote and Sensorless Stator Winding Resistance Estimation Method.pdf

6. EffEstMethod.pdf

7. EffEstMethod_updated.pdf

8. EMC-08-T010_fullpaper.pdf

9. EnergyUsageProduct.pdf

10. ETN_WhitePaper_BrokenBar.pdf

11. ETN_WhitePaper_NCBearing.pdf

12. ETN_WhitePaper_PowerQuality.pdf

13. Induction motor rotor fault detection via wave let analysis of one-cycle average power.pdf

14. IPEMC06_NPB.pdf

15. NAGT_paper.pdf

16. PESC06_NPB_full.pdf

17. WSNonMediumMotors.pdf

Appendix B - External Publications

Appendix C -Patents 


\title{
Wireless Sensor Network for Advanced Energy Management Solutions
}

\section{Appendix A-Supporting Documentation and Internal Reports}

\author{
DOE Award Number: DE-FC36-04G014000 \\ Reporting Period: January 2004 - June 2009 \\ Principal Investigator: Peter J. Theisen \\ (414) 449-6924 \\ PeterJTheisen@eaton.com \\ Bin Lu, Ph.D. \\ (414) 449-6036 \\ BinLu@eaton.com \\ Charles J. Luebke \\ (414) 449-7264 \\ CharlesJLuebke@eaton.com \\ Recipient: Eaton Corporation \\ Innovation Center \\ 4201 N. $27^{\text {th }}$ Street \\ Milwaukee, WI 53216
}




\section{Appendices}

Appendix A - Supporting Documentation and Internal Reports

\section{List of Documents in Appendix A}

1. Grenada_testplan_v10.pdf

2. DOE Task 10 Appendix.pdf

3. A Survey of Efficiency-Estimation Methods for In Service Induction Motors.pdf

4. 2006_TIA_A Nonintrusive and In-Service Motor Efficiency Estimation Method.pdf

5. 2008_TIE_A Remote and Sensorless Stator Winding Resistance Estimation Method.pdf

6. EffEstMethod.pdf

7. EffEstMethod_updated.pdf

8. EMC-08-T010_fullpaper.pdf

9. EnergyUsageProduct.pdf

10.ETN_WhitePaper_BrokenBar.pdf

11.ETN_WhitePaper_NCBearing.pdf

12.ETN_WhitePaper_PowerQuality.pdf

13. Induction motor rotor fault detection via wave let analysis of one-cycle average power.pdf

14.IPEMC06_NPB.pdf

15. NAGT_paper.pdf

16. PESC06_NPB_full.pdf

17. WSNonMediumMotors.pdf 


\section{Test Plan of the Eaton Wireless Link Assessment System}

\section{Scope}

This document describes the detailed operational steps that need to be carried out for testing the Eaton Wireless Link Assessment System.

\section{System, Hardware and Software Description}

The Eaton Wireless Link Assessment System is a system composed of a set of devices that are connected in a wireless manner for measuring the qualities, including the delivery rate, the received signal strength among many others, of wireless communication links. Each individual device is named "Grenadine". The Eaton Wireless Link Assessment System is capable of measuring wireless link qualities along six dimensions of factors that affect the link performance metrics:

1. Time,

2. Transmitter location,

3. Receiver location,

4. Channel,

5. Transmitting power level,

6. Packet size. 


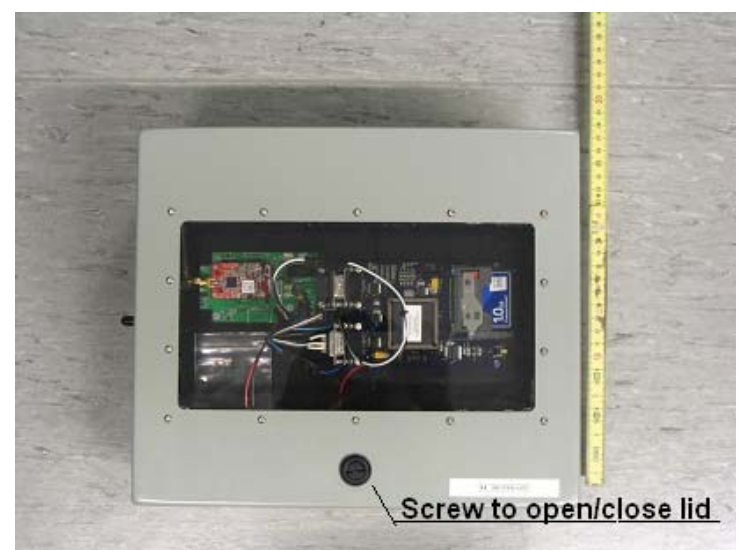

Figure 1. Top View of Grenadine

The Grenadines, or individual devices, are identical in terms of hardware and software. A Grenadine of the current version is packaged in a water-proof NEMA-4 enclosure, as shown in Figure 1, to prevent hazards from the environment to the device, or from the device to critical deployment infrastructures. On one side of the NEMA-4 enclosure there are a power connector and a three-way switch for turning on/off the Grenadine, as shown in Figure 2. An AC-DC adapter (120V AC to 12V DC), in addition to the NEMA-4 enclosed Grenadine device, is provided to utilize mains power. If mains power is not available, 1 bank of 3 Energizer 528 Alkaline 6V batteries, or 6 Energizer 528 batteries in 2 banks are equipped in the NEMA-4 enclosure for each Grenadine device. Each bank includes 3 batteries connected in serial, and banks are connected in parallel to provide even longer lifetime for the device.

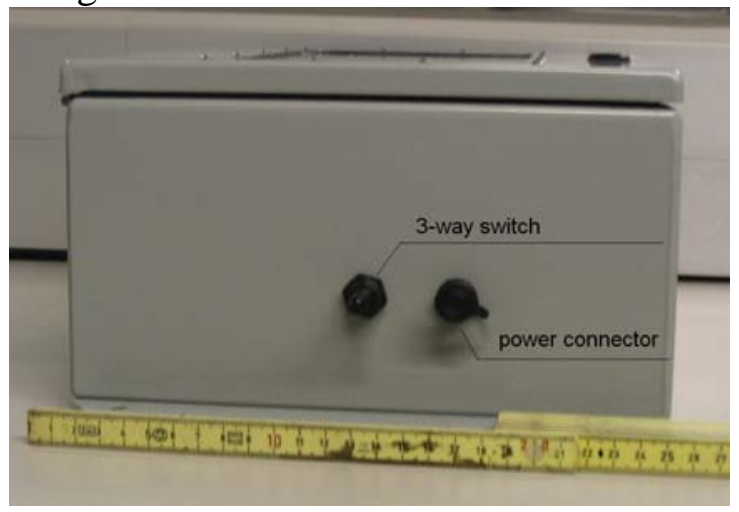

Figure 2: Side View of Grenadine

The electronics system inside the NEMA-4 enclosure for each Grenadine includes a voltage regulator, which converts the input $12 \sim 18 \mathrm{~V}$ DC power to $5 \mathrm{~V}$ DC power, a Compact Flash (CF) card data logger Print Circuit (PC) board, a radio board (currently the RM2420 module from RAE Systems, or the "red board", to be replace by the MAUI board developed by Eaton Corporation) with an antenna, a development/interface PC board for the radio board, and a Compact Flash (CF) card, as shown in Figure 3. The CF data logger PC board and the development PC board for the radio board are connected through two sets of serial connectors, and both are powered from the voltage regulator. During tests, the radio board and the CF card need to be plugged into the development board and CF data logger, respectively. 


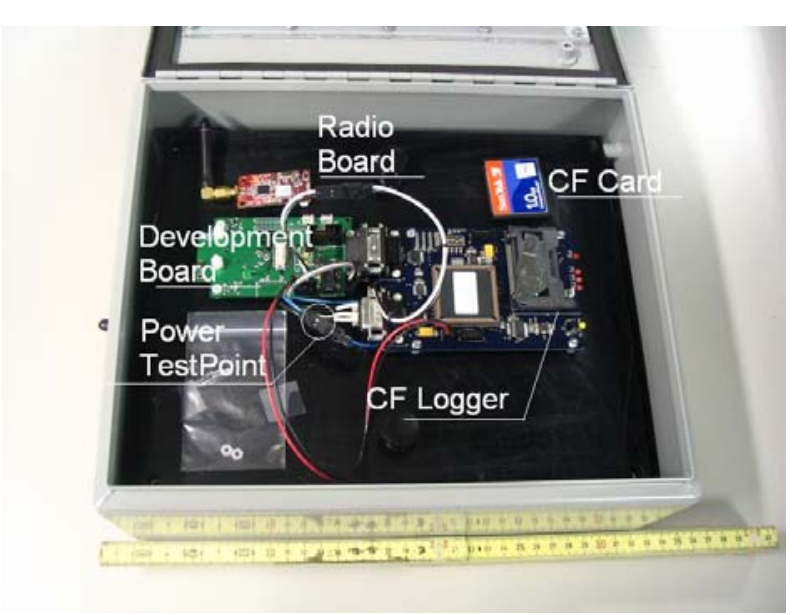

Figure 3. Electronics System of Grenadine

\section{Device Preparation for A Test Campaign} campaign.

A test sheet is attached as a template for recording the information for each test

\section{Power Source Check}

The first preparing step is to check power source for each individual Grenadine device. The three-way switch is used to turn on/off the device and select the power source. If it is put in the middle position, the Grenadine is powered off.

If mains power is available, the steps for the power supply check are listed below:

a) Check the AC-DC adapter as follows.

b) Plug the adapter into the mains power outlet. Make sure it is NOT connected to any Grenadine.

c) Measure the output voltage with a multimeter from the two small metal tubes in the round connector at the other end of the cable. If it is not within the range of $11 \mathrm{~V} \sim 13 \mathrm{~V}$, replace the adapter. Run the check again from step a).

d) After the AC-DC adapter passes the check, remove it from mains power.

e) Make sure the three-way switch on the NEMA-4 enclosure is in the middle position (power off).

f) Make sure the CF card and the radio board are NOT plugged in the NEMA-4 enclosure.

g) Plug the AC-DC adapter cable to the power connector on one side of the NEMA-4 enclosure, rotate the cable connector till it snaps in the enclosure connector, and rotate the "ring" surrounding it to lock it.

h) Connect the adapter to the mains power outlet.

i) Turn the three-way switch TOWARDS the power connector (AC power). 
j) In the NEMA-4 enclosure, there are a blue wire and a black wire connected by a black plastic connector. On one side of the connector, two very small pieces of metal are exposed. Measure the voltage cross the two metal pieces, as shown in Figure 3. If the difference between the voltage and the voltage obtained from the AC-DC adapter is greater than $0.2 \mathrm{~V}$, the Grenada needs to be replaced and examined later for power connection.

k) After the Grenadine passed the AC power supply test, turn off the power by switching the three-way to the middle position (power off). Record the input voltage from the last step onto the test sheet.

If mains power is not available, batteries have to be used as the power source. Check the power source following these steps:

a) Make sure the three-way switch on the NEMA-4 enclosure is in the middle position (power off).

b) Make sure the CF card and the radio board are NOT plugged in the NEMA-4 enclosure.

c) Turn the three-way switch AWAY FROM the power connector (battery power).

d) Measure the voltage cross the two metal pieces on the blue-black wire connector. If it is below $11 \mathrm{~V}$ or above $21 \mathrm{~V}$, replace the batteries and run the check from step a) again.

e) After the Grenadine passed the battery power supply test, turn off the power by switching the three-way to the middle position (power off). Record the input voltage from the last step onto the test sheet.

\section{Preparing Devices}

Both the radio board and the CF card needs to be properly prepared for the tests. For each radio board, the correct version of the Grenadine software needs to be downloaded using the IAR software tool and a JTAG In Circuit Emulator (ICE). The software downloaded is identical for all Grenadine radio boards. The only difference among the Grenadines lie in the configuration files in the CF cards.

A CF card is marked with a label reading a name for the card, such as "C0", "B5", and "A14". The first character is a capital letter denoting the set of CF cards. The rest are a number, from 0 to 7 for an 8-Grenadine test, or from 0 to 15 for a 16 -Grenadine test. The Grenadines are also marked with numbers from 0 to 7 for an 8-Grenadine test, or 0 to 15 for a 16-Grenadine test. A test must use the same set of CF cards, so the capital letter must be identical for a single test. The number on a Grenadine and the CF card plugged in it must match. For example, for a single test, C0, C1, .., C7 can be used for Grenadine $\# 0, \# 1, \ldots, \# 7$, respectively. Grenadine \#0 is a special device and has to be used in all tests, since it is the root for synchronization and triggers the transmission of all other Grenadines. For tests with less than 8 Grenadines, any subset of Grenadine \#1 to 
Grenadine \#7 can be used, as long as the numbers of CF cards in them match their numbers.

Two sets of CF cards need to be used for a complete set of tests. The first set is used to validate the functionalities of the Grenadines for a short period of time. The second set is used for the real test. Here are the steps to prepare the CF cards for an 8Grenadine test:

a) Prepare the configuration file directory. A template directory is provided for the users as an example for tests. Create an empty directory. Without loss of generality, we name it test/ Copy the subdirectories CF0, CF1, ..., CF7 from the template directory to the empty directory test/. Each subdirectory (CF0 CF7) consists of three files: CONFIG.TXT, NODEID.TXT, Schedule.txt. The CONFIG.TXT files in all subdirectories are identical. So are the Schedule.txt files. The only difference among the Grenadines is that the NODEID.TXT is different. Each NODEID.TXT file includes a line that denotes the CF card's ID. For example, for CF5, or the Compact Flash card that is plugged in Grenadine \#5, it includes a line:

\section{NodeID $=5$}

The files in test/CF0 needs to be copied into CF card 0 (marked as A0, B0, $\mathrm{C} 0, \ldots$, depending on the set of CF cards used). Similarly, files in test/CF1 needs to be copied into CF card 1, etc. Without loss of generality, we choose $\mathrm{A} 0, \mathrm{~A} 1, \ldots, \mathrm{A} 7$ as the cards for the short-term validation test, and $\mathrm{B} 0, \mathrm{~B} 1, \ldots$, B7 as the cards for the real, long test. The user can select any set for either purpose. The first set of CF cards can be as small as 128Mbytes each. The sizes of the second set of CF cards need to be decided based on the test settings. When all the debugging switches are on (LoggingDebugMode1 $=1$, LoggingDebugMode2 $=1$, LoggingDebugMode3 $=1$ in CONFIG.TXT), and the uTicksPerTick parameter is 2048, a 2-day test can be accommodated in a 1 Gbyte CF card on each Grenadine. A 4-day test can be accommodated in a 2Gbyte CF.

b) Format all CF cards. Backup the files in CF cards if necessary.

c) Launch the software tool "GrenadaCFManager.exe" by double clicking the program icon from the PC. A dialog window will appear similar to the Figure 4: 


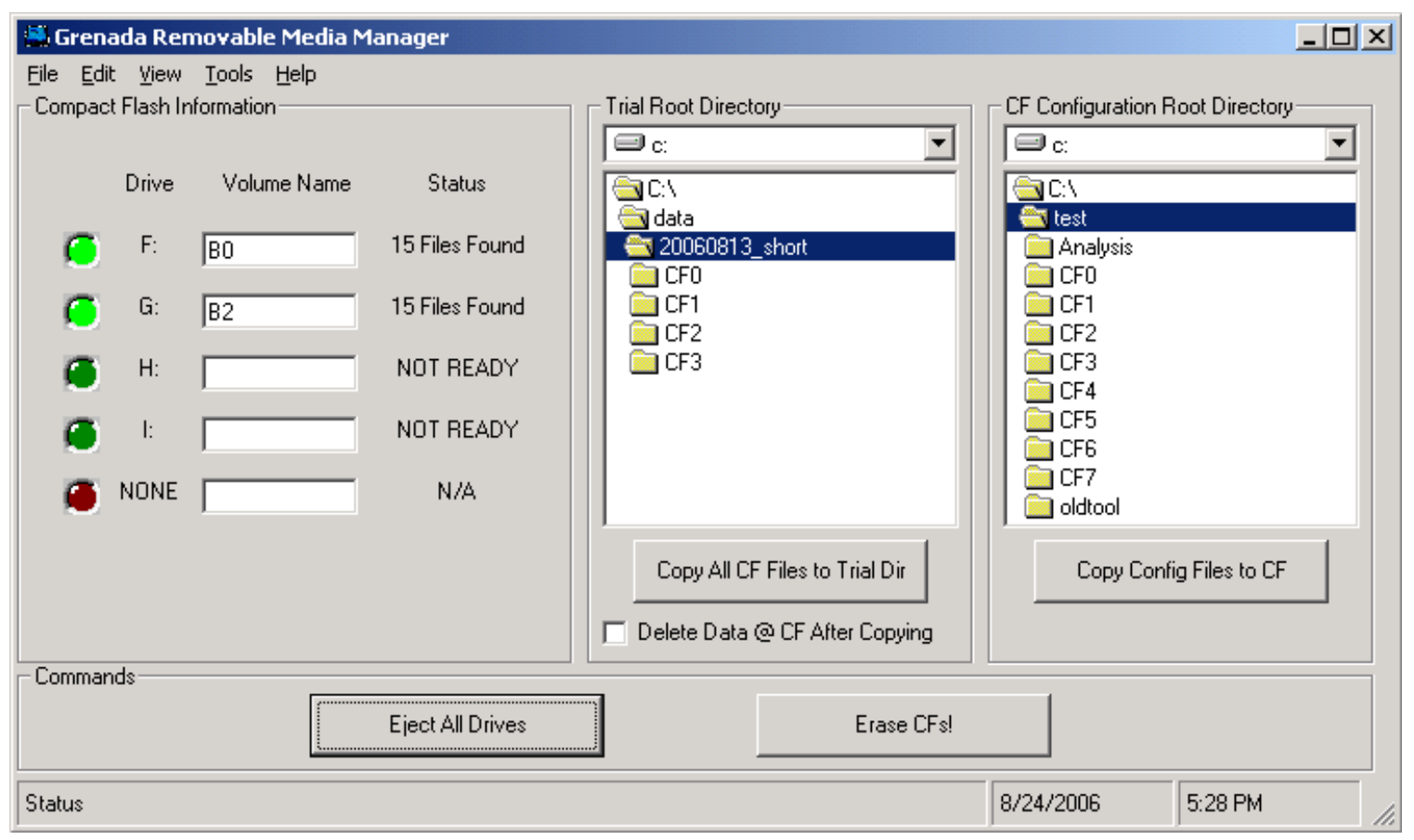

Figure 4. GrenadaCFManager

d) Select the test/ directory in the file tree under "CF Configuration Root Directory”.

e) Plug CF cards A0 to A3 to the CF card readers attached to the PC. The dark green lights to the left of Drive symbols on the Dialog window will turn to bright green. If the CF cards are properly formatted, the Status for each of them should show "0 Files Found".

f) Click on the button "Copy Config Files to CF". The LEDs on the CF card readers will blink. Wait till the blinking stops and all Status field change to "3 Files Found".

g) Click on the "Eject All Drives" button, wait till all Status fields become "NOT READY" and the four LEDS on the CF card readers are off. Push the buttons on the CF card readers to eject the CF cards.

h) Repeat from step e) for CF cards A4 A7, B0 B3, B4 B7.

\section{$\underline{\text { In-Lab Functional Validation }}$}

Before the actual deployment to the test site, the Grenadines need to be verified for proper functionalities. Put the Grenadines in a small area (maximum range $<5$ meters) and take the following steps:

a) First make sure that all the three-way power switches on all Grenadines are in the middle (power-off) position.

b) For each Grenadine, attach the properly programmed radio board on to the development board; push the CF card for short-term validation test into the CF slot till stably attached. 
c) Turn the three-way power switch away from the power connector (battery mode).

d) After one minute, check the LEDs on the radio board, development board and the CF data logger for each Grenadine.

e) For each Grenadine, the yellow LED on the radio board and the green LED on the development board should be blinking constantly. The red LED on the radio board may or may not blink. On the CF data logger, the Power Indicator and the Record Indicator LEDs should be on stably, the Fault Indicator LED should be off stably, the data indicator should be blinking constantly. If any of the above mentioned LEDs do not work as specified, the Grenadine node should be taken away from the test for diagnosis. As long as Grenadine \#0 and a few other Grenadines are functioning properly, the test can still be conducted.

f) For each Grenadine, push the record button, turn off the power by putting the three-way switch to the middle.

\section{$\underline{\text { Recording Test Site Information }}$}

It is of vital importance to record the test site information in as much details as possible. The level of details depends on the availability of information. Ideally, a floor plan for the test site is very helpful and fundamental for recording test site information. If a copy of floor plan is available, the locations of the deployed Grenadines can be marked on the floor plan for documentation. If it is not available, a rough sketch needs to be drawn for documentation purposes. The following are the items that need to be documented:

a) Location of the test site: the address, or longitude/latitude of the test location; the floor plan if available; if available, a satellite picture of the test site is also very useful to align with the floor plan.

b) The architectural information of the test site. For example, how much metal is used to build the walls and roof and whether the doors are metal.

c) The wireless environment of the test site. For example, the locations of the closest cell phone base stations; the types $(\mathrm{a} / \mathrm{b} / \mathrm{g})$ and locations of 802.11 access points; the estimated number and locations of Wi-Fi enabled laptops/desktops; the locations of microwave ovens; nearby Bluetooth devices; nearby ZigBee devices; and so on.

d) The electromagnetic radiation sources. For example: locations and capacities of transformers; locations and capacities of motors; locations and capacities of electrically powered machines.

A digital camera or camcorder may be used to take pictures of the test site if allowed. The pictures should cover all areas of the test site and there should be overlapping areas among pictures for continuity. For any area in the test site, pictures from at least three angles need to be taken. If a precise floor plan is not available, the dimensions of the test site needs to be measured manually. All the pictures, documentations and files need to be attached to the test report. 


\section{Deploy Devices}

There are two types of deployments of Grenadines for a test: predefined and ad hoc. For predefined deployments, the location of each Grenadine is set a priori. For ad hoc deployments, the user has the freedom to choose the locations of the Grenadines. The following steps need to be taken for ad hoc deployments to provide connectivity.

a) Make sure the radio board and the CF card for short-term validation test on each Grenadine are properly installed.

b) Choose a location for Grenadine \#0. A good choice is a location close to the middle of the whole deployment area. Switch the power of Grenadine \#0, to either mains power or batteries.

c) Choose a location for the next Grenadine. Switch the power on the Grenadine to either mains power or batteries. After two minutes, check the LEDs on this Grenadine and Grenadine \#0. On the CF data logger, the Power Indicator and the Record Indicator LEDs should be on stably, the Fault Indicator LED should be off stably, the data indicator should be blinking constantly. If not, the CF data logger needs to be diagnosed and the corresponding Grenadine needs to be taken away from the test. If the green LED on the development board or the yellow LED on the radio board of either Grenadine is not blinking, try to bring the second Grenadine closer to Grenadine \#0 until the two LEDs on both Grenadines blink.

d) Choose a location for another Grenadine. Switch the power on the Grenadine to either mains power or batteries. After two minutes, check the LEDs on this Grenadine. The check for LEDs on the CF data logger is the same as step c). If the green LED on the development board or the yellow LED on the radio board does not blink, try to bring it closer to other already deployed Grenadines until the two LEDs blink.

e) Repeat step d) for all the rest Grenadines.

Note that if there are some areas or points in the test site that have to be covered and there are not enough Grenadines for the test, some nodes may be deployed unconnected, and the test can be conducted anyway. In the deployments, it is good practice to deploy the Grenadines with smaller numbers first.

After the above steps, record the locations of the deployed Grenadines. Manual measurements between the Grenadines and the reference points in the floor plans are suggested, so that the locations of the Grenadines can be marked on the floor plan copy. The reference points also needs to be marked on the floor plan. It is important to also record the heights of the nodes from the floor. The orientation of each Grenadine also needs to be recorded. For each deployed Grenadine, pictured from at least three angles need to be taken, and each needs to include a reference point from the floor plan. The 
bottom line is, from the information from the documentation and pictures, the deployment of nodes can be exactly reconstructed.

For the long time test, take the following steps:

a) For each Grenadine, push the record button on the CF data logger, and turn off the power by put the three-way switch to the middle position. Eject the CF card.

b) For each Grenadine, push the CF data logger for long duration test into the CF slot till stably installed. Turn on the power by put the three-way switch to the proper power source (mains power if available, or batteries).

c) Close the lid on each NEMA-4 enclosure and fasten the screw on it.

d) Record the starting time of the test.

e) Record events such as the operations of machines, opening/closing doors, moving vehicles, and so on. The rule of thumb is any operation involving electricity or any movement of metal in the close neighborhood of the running Grenadines. Keep record of temperature and humidity. For outdoor testing, also record the weather conditions during the test.

\section{End A Test}

At the end of each test, take the following steps:

a) For each Grenadine (following the opposite order of deployment), loosen the screw on top of the NEMA-4 lid, open the lid, and record the status of all LEDs (on the radio board, on the development board and on the CF data logger).

b) For each Grenadine, push the record button on the CF data logger, and eject the CF card.

c) Turn off the power of each Grenadine by put the three-way switch to the middle position.

d) Collect all CF cards for post processing.

\section{Data Post Processing}

a) Create an empty directory for data, such as “C:Idata\20060809_1200” on the PC.

b) Launch GrenadaCFManager.exe by clicking on the icon. A dialog window similar to Figure 4 will appear.

c) Select the data directory in the directory tree under "trial root directory".

d) Push CF cards B0 B3 into CF card readers connected to the PC.

e) Click on the button "Copy All CF Files to Trial Dir”.

f) After the status bar stops changing, click on the button "Eject All Drives". Wait till all Status fields become "NOT READY" and the four LEDS on the 
CF card readers are off. Push the buttons on the CF card readers to eject the CF cards.

g) Push CF cards B4 B7 into CF card readers connected to the PC and repeat steps e) and $\mathrm{f}$ ).

h) Lauch MATLAB, and cd to the Analysis directory. Open file "GrenadaProc_ReadDataFiles.m”

i) Change the directory to the directory in step a), in the line LogFilePath =

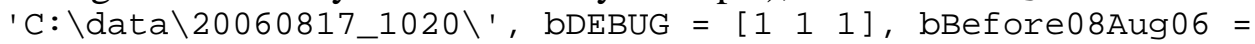
$\odot$;

j) Run the following MATLAB programs in sequence:

“GrenadaProc_ReadDataFiles.m”, “GrenadaProc_BuildEventArrays.m”, “GP_AnalyzePlotPSR.m”, “GP_AnalyzePlotEvents.m”, and “GP_AnalyzePlotSync.m”. 
Eaton Wireless Link Assessment System Test Sheet

Operator Name:

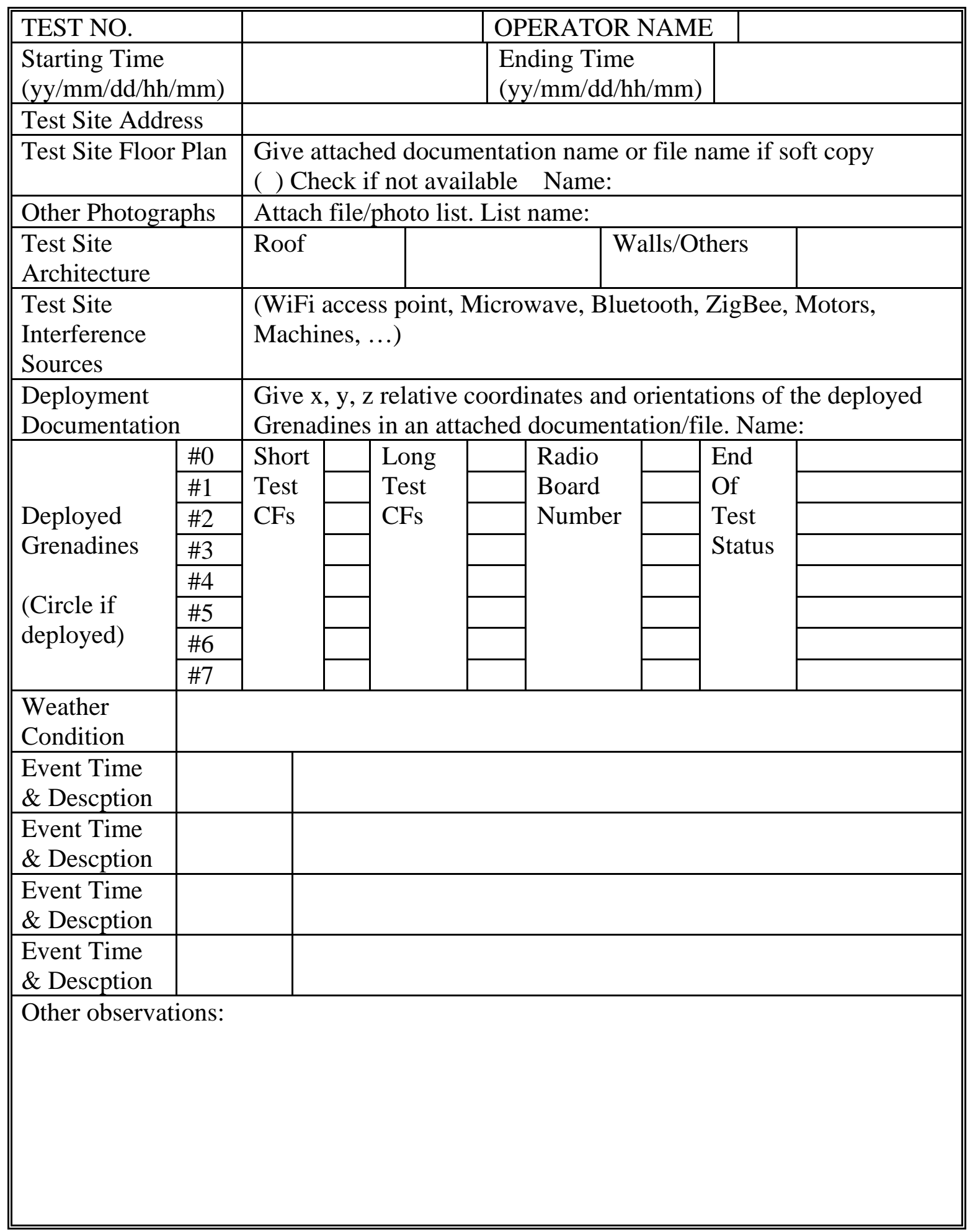




\title{
SODA: Scalable On-Demand Aggregation for Wireless Sensor Networks
}

\author{
Ting Yan, Member, IEEE, Sujit R. Das, Member, IEEE, and Luis R. Pereira, Member, IEEE
}

\begin{abstract}
This paper proposes a novel approach to gather responses from a wireless sensor network in a fast and scalable fashion in response to a broadcast command/query. This is a wellknown difficult problem in real-world networks requiring timebounded responses for time-critical applications. The approach proposed is based on a near-optimal constraint based scheduling algorithm and tight time synchronization. We validated the efficiency and feasibility of our approach based on simulation studies and real system implementation, which is built upon and coexisting with a CSMA-based commercial off-the-shelf wireless protocol stack.
\end{abstract}

Index Terms-aggregation, channel assignment, scalability, wireless sensor networks.

\section{INTRODUCTION}

W ITHIN the last ten years, wireless sensor networks (WSNs) have attracted many important military, industrial and commercial applications because of their unprecedented capability of sensing and actuating the physical world with very low cost. It has been observed that the scale of WSNs has increased from tens to thousands of nodes and beyond during a very short period of time. Therefore, scalability has become a requirement of vital importance for WSN communication protocols.

Many communication protocols, from the application layer all the way down to the Media Access Control (MAC) layer, have been proposed from both academia and industry. Although facing challenges, among quite a few proposed MAC protocols, Carrier Sense Multiple Access (CSMA), including variations, is one of the most dominant MAC schemes used in WSNs due to its efficiency and flexibility. For example, IEEE 802.15.4 [1], one of the most widely used Wireless Personal Area Network (WPAN) standards, adopts CSMA with Collision Avoidance (CSMA/CA) on its MAC.

In sensor networks, it is a common scenario that the WSN base node, defined here as the node in control of the whole network and acting as the gateway between the network and the user or other subsystems, sends a broadcast message to the whole network and expects each node within it to acknowledge it. A very similar scenario is that after the WSN base node broadcasts a query message to the whole network, it expects all

Manuscript received May 15, 2008. This work was financially supported in part by the United States Department of Energy (DOE) under Grant DEFC36-04GO14000 and such support does not constitute an endorsement by DOE of the views expressed in the article.

T. Yan and L. R. Pereira are with the Innovation Center of Eaton Corporation, $4201 \mathrm{~N}$ 27th Street, Milwaukee, WI, 53216 USA (e-mail: \{Ting Yan, LuisRPereira\}@eaton.com).

S. R. Das is with QuantSoft LLC, 13310 Crestwood Ct, New Berlin, WI, 53151 USA (e-mail: SujitRDas@quantsoft.biz). other nodes to respond with their status information in a timely fashion. In both scenarios, the fundamental requirement is to deliver all responses timely assuming there are no physical layer errors. This requirement is very important for timecritical systems. It is also important for good usability to provide prompt feedback to user requests. CSMA/CA and similar MAC protocols are capable of handling such scenarios efficiently when the network contains about a dozen nodes. However, when the number of nodes in the network increases, these protocols can only deliver a small fraction of all the responses expected. The cause of this phenomenon will be explained later in this paper.

One key novelty of the proposed algorithm is a near-optimal channel assignment scheme that maximizes the utilization of multiple available channels to deliver all responses in a short amount of time given the above two scenarios based on tight time synchronization. Another novelty is that our solution is designed to work on top of a commercial off-the-shelf (COTS) ZigBee protocol stack based on IEEE 802.15.4 with backward compatibility. We validated our claims by both simulation and real system implementation.

The rest of the paper is organized as follows: section II analyzes the problem to solve and gives detailed description of our design; section III describes the evaluation of the proposed algorithm based on both simulations and real-system implementation; section IV introduces related work and concludes the paper.

\section{Problem Analysis And Algorithm Design}

\section{A. Problem Analysis}

Here we examine the reason why CSMA/CA MAC cannot meet the requirement of quickly delivering a large amount of acknowledges or status messages in response to the broadcast command/query. After each node receives the broadcast message, it tries to send its response/acknowledge to the base node based on the CSMA/CA algorithm. As an example let us assume that we use the (unslotted) CSMA/CA algorithm as specified in the IEEE 802.15.4 standard.

The CSMA/CA algorithm as depicted in Fig. 1 employs two variables, $N B$ (number of backoffs) and $B E$ (backoff exponent), limited by macMinBE, aMaxBE (the minimum and maximum values of $B E$ ) and macMaxCSMABackoffs (the maximum number of backoffs the algorithm attempts before declaring a channel access failure). In the the $2.4 \mathrm{GHz}$ PHY, the unit backoff period is 0.32 milliseconds.

Before a new transmission attempt (step $<1>$ in Fig. 1), $N B$ and $B E$ are initialized, then (step $<2>$ ) the node waits 


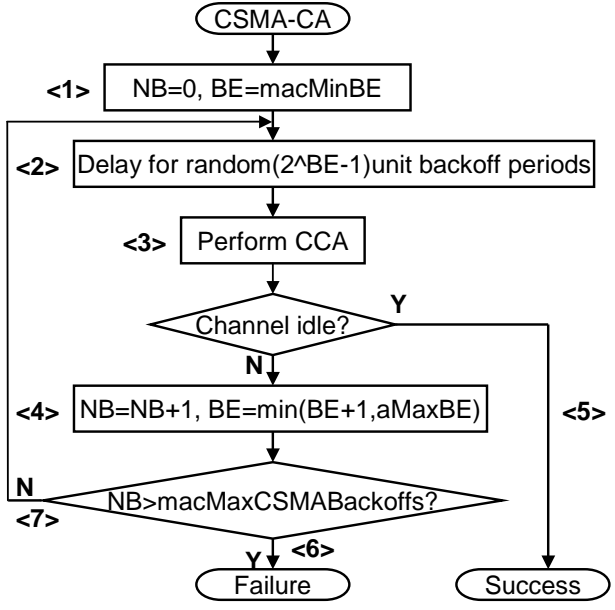

Fig. 1. The CSMA-CA Algorithm (from [1])

for a random number (selected from 0 to $2^{B E}-1$ ) of unit backoff periods. After that (step $<3>$ ) it performs the channel assessment and if the channel is idle (step $<5>$ ), it sends the message. Otherwise $($ step $<4>$ ) the node will update $N B$ and $B E$ and checks if the maximum number of backoffs is reached and reports unsuccessful attempt if that is true (step $<6>$ ), or otherwise starts another iteration of random delay (step $<7>$ ).

This leads to an important issue when there are a large amount of nodes trying to send messages starting very close in time. The maximum time window from when any node starts the first backoff to when it gives up retrying is less than 50 milliseconds. Since an IEEE 802.15.4 packet takes from 1 to 4 milliseconds to be transmitted, it limits the maximum possible number of packets that may be delivered to the base node to 50, not considering other factors like multiple hop transmissions. Based on our experiments (see Fig. 7 in section III), the average number of packets delivered in this scheme is smaller than 20: only a small fraction of the responses can be delivered successfully. Other transmissions either fail due to collisions caused by random number selection, or give up retry attempts after the maximum number of retries (macMaxCSMABackoffs) is reached, an issue common for all CSMA based MAC protocols, although the exact time window duration may vary based on the implementation mechanisms and parameters selected.

One natural alternative solution is to enforce random waiting after receiving the broadcast message in the application layer instead of in the MAC layer, where parameters like $a M a x B E$ and macMaxCSMABackoffs can be set larger. However, this approach has several issues. First, without tight time synchronization, the nodes have to use longer backoff periods to tolerate a quite large jitter among them, therefore causing longer response time than expected. Second, the random selection of backoff time may cause unnecessary collisions and negatively affect the total number of responses delivered. The problem can be modeled like a "birthday paradox" with formula (1) (from [2]). Even with 200 (T) timeslots to select from, $p(n)$, the probability of the same number selected by at least two nodes is greater than $50 \%$ only when there are 18
( $n$ ) nodes in the network. The probability increases to greater than $99 \%$ when there are 44 nodes in the system.

$$
p(n)=1-\prod_{k=1}^{n-1}\left(1-\frac{k}{T}\right) \geq 1-e^{-\frac{n(n-1)}{2 T}}
$$

Another alternative solution is to use Time Division Multiple Access (TDMA) MAC with predefined transmission sequence. This approach may deliver the responses in a short amount of time without collisions. However, it will cause interoperability issues for network designers and users who have already adopted network stacks conforming to CSMA-based standards because these two MAC schemes are drastically different and incompatible.

\section{B. Design Overview: The Big Picture}

Based on the above observations we propose a Scalable OnDemand Aggregation scheme (SODA) to deliver all responses in a very short period of time. This solution is designed to work on top of CSMA-based MAC layer protocols instead of replacing them. The base node first schedules the transmission sequence and sends it to all other nodes, and after a broadcast command/query is sent to the network, they transmit their responses based on the schedule received earlier. The expected responses often only contain a limited amount of information (for example, success or failure, operation mode, power level), which can be represented by few bytes, or even several bits. Our proposed solution also takes advantage of the fact that for large-scale networks, many nodes need to send the responses through multiple hops, so the relaying nodes may aggregate the responses from further nodes into fewer packets to reduce transmission overhead.

In our proposed approach, we apply a tight synchronization mechanism and make nodes transmit information following a precomputed schedule based on a tree topology to avoid transmission collisions upon the broadcast command/query.

We design four initialization phases for the network to accomplish this goal. 1) After the network is started, a spanning tree is generated so that every non-base node identifies its parent, the base node being the common ancestor, and each child can send messages to its parent in a single hop. The base node gathers information of the tree topology into a local data structure. 2) Based on the tree structure, the base node computes a collision free schedule defining when and through which channel each node will send its response to its parent after the broadcast is received. 3) The base node sends this schedule to all non-base nodes. 4) The base node starts a time synchronization process.

After the whole network achieves synchronization, the base node is allowed to send on-demand broadcast query/command to the whole network. After a node receives this broadcast message, it waits until its own transmitting time (specified by the schedule sent from the base node) and sends its response. If a node is a parent node, the response is aggregated from all its descendents and its own. The node's parent needs to switch to the same channel to receive the response. The schedule is designed in a way so that a parent node always sends its aggregated response after it receives all the responses from 
its descendents. Please note that the parent is able to obtain all responses directly or indirectly from all of its descendents. After this short time window, the network switches back to normal CSMA mode for compatibility.

The most important part of the SODA design is the channel scheduling algorithm. For some MAC/PHY standards, there are multiple non-overlapping channels defined. For example, IEEE 802.15.4 defines 16 non-overlapping channels for the $2.4 \mathrm{GHz}$ physical layer. Typically only a few of them are utilized in a deployment environment. Therefore, we assume that it is possible to use those available channels for very short time windows. However, SODA also works for a single channel, and the total time needed for the responses' transmissions is roughly inversely proportional to the number of channels used (see section III-A).

In our design, we divide the time for the transmissions of the responses into timeslots with equal intervals. Each nonbase node is assigned a timeslot and a channel, and when the assigned timeslot becomes current after the broadcast query/command, the node transmits its own response (if it is not a parent) or the aggregated response of all its descendents and its own to its parent in one hop. We assume that simultaneous transmissions from different channels do not interfere with each other.

\section{SODA Design: Details}

The algorithm design now reduces to the following problem. Given an input of the set of node indices $N=\{1,2, \ldots, n\}$, where $n$ is the total number of non-base nodes (the index of the base node is 0 ), the tree topology of the network $G$, and the set of channel indices $M=\{1,2, \ldots, m\}$, where $m$ is the number of available channels, the algorithm needs to produce for each node $i \in N$ a pair $(T S(i), C I(i))$ of timeslot $(T S)$ and channel index $(C I)$ that satisfies the following three constraints.

Constraint 1 (C1). $\forall i, j \in N$ and $i \neq j$, if $T S(i)=T S(j)$, $C I(i) \neq C I(j)$. This constraint guarantees that for any given timeslot, there is only one node transmitting at each channel to avoid collision.

Constraint 2 (C2). $\forall i \in N, T S(\operatorname{parent}(i))>T S(i)$, where parent $(i)$ is defined as the node index of node $i$ 's parent. This constraint guarantees that a parent obtains all responses from its descendents before it is allowed to send the aggregated response to its own parent.

Constraint 3 (C3). $\forall i, j \in N$, if $i \neq j$ and parent $(i)=$ $\operatorname{parent}(j), T S(i) \neq T S(j)$. If parent $(i)=\operatorname{parent}(j)$, we define that node $i$ is node $j$ 's sibling, and vice versa. This constraint is to ensure that a parent does not receive response from multiple children at the same timeslot.

It can be derived that the lower bound of the number of timeslots needed is $\lceil n / m\rceil$ based on $\mathbf{C 1}$ and the pigeonhole principle.

The SODA allocation algorithm is shown in Listing 1 . The algorithm visits all nodes in the network following a traversing order of either Breadth First $(B F)$ or Depth First $(D F)$. The algorithm allocates $T S$ and $C I$ for each node when it is visited. However, since both $B F$ and $D F$ visit a node after its parent, the straightforward assignments of $T S \mathrm{~s}$ will violate C2, Therefore, we have to define an auxiliary array, "reverse order index" $(R O I)$, which reverses the $T S$ order, so that we can allocate $R O I$ s while traversing the tree topology with $B F$ or $D F$. The algorithm maintains a 2dimensional allocation table pair $[0 . . n][1 . . m]$ in which each element represents a $(R O I, C I)$ pair, whose row index is the $R O I$ value and column index, $C I$. The algorithm also maintains arrays $R O I[0 . . n]$ and $C I[0 . . n]$ for all nodes. When node $i$ is assigned an $R O I$ value of $j$ and a $C I$ value of $k$, we set $\operatorname{pair}[j][k]=i, R O I[i]=j$, and $C I[i]=k$. Before the assignment, the corresponding pair, $R O I$, and $C I$ values are set to -1 to indicate that no valid values have been assigned (line 3). The algorithm then traverses the network following the order of $B F$ or $D F$ (line 6). When node $i$ is visited, the algorithm searches for unassigned cells in the allocation table starting from row $R O I($ parent $(i))+1$ to satisfy C2 (line 7). It checks each cell in the row pair $[n x t R O I][1 . . m]$ to see whether there is an unassigned cell (line 11) and record $C I$ of the first unassigned cell (line 13). It also checks whether a sibling is assigned with the same $R O I$ (line 15). If there is an unassigned cell $(\mathbf{C} 1)$ and no sibling is assigned the same $R O I(\mathbf{C 3})$, it fills the first unassigned cell with $i$ (line 20), and assigns the $(R O I, C I)$ pair to node $i$ (line 21). Otherwise, it searches the next $R O I$ row (line 23). After the traversing is completed, the formula in line 28 reverses $R O I$ to get $T S$ for each node.

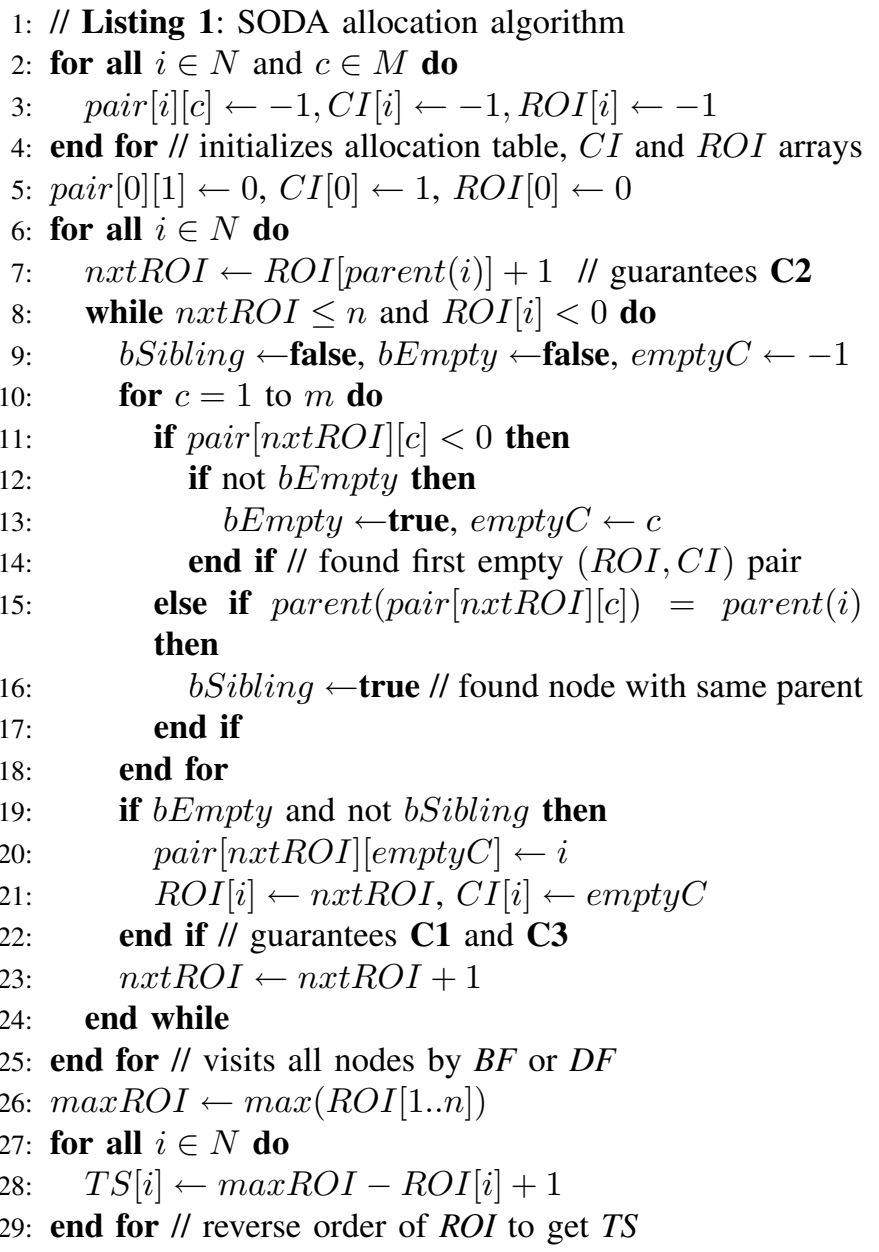




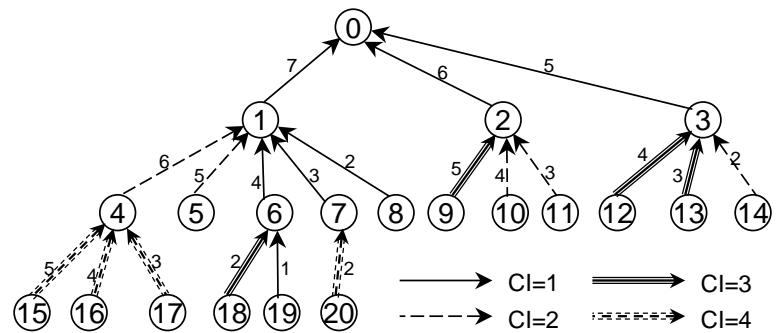

Fig. 2. Tree Topology and Algorithm Example and Results - Timeslots (edge labels) and Channel Index Numbers (edge styles)

TABLE I

ROI/CI ALLOCATION TABLE

\begin{tabular}{l||c|c|c|c} 
& CI=1 & CI=2 & CI=3 & CI=4 \\
\hline \hline ROI=0 & Node 0 & & & \\
\hline ROI=1 & Node 1 & & & \\
\hline ROI=2 & Node 2 & Node 4 & & \\
\hline ROI=3 & Node 3 & Node 5 & Node 9 & Node 15 \\
\hline ROI=4 & Node 6 & Node 10 & Node 12 & Node 16 \\
\hline ROI=5 & Node 7 & Node 11 & Node 13 & Node 17 \\
\hline ROI=6 & Node 8 & Node 14 & Node 18 & Node 20 \\
\hline ROI=7 & Node 19 & & &
\end{tabular}

Note that it can be proved by contradiction that the maximum number of $T S \mathrm{~s}$ (or equivalently $R O I \mathrm{~s}$ ) needed will not exceed $n$, the number of the non-base nodes, independent of the traversing order, and $m$, the number of available channels. The algorithm uses memory space for arrays pair $[0 . . n][1 . . m]$, $R O I[0 . . n], C I[0 . . n]$, and few other auxiliary variables. Therefore, the space complexity of the algorithm is $O(n \cdot m)$. It can be derived that the time complexity of the algorithm is $O\left(n^{2} \cdot m\right)$.

\section{Example of Algorithm Execution}

Fig. 2 shows a tree topology of a 21 node network, where node 0 is the base node. For convenience we label the nodes based on a breadth first (BF) traversing order, although the labeling order does not affect how the algorithm performs. We assume that there are $m=4$ available channels.

Table I shows how $(R O I, C I)$ pairs are allocated. Assuming nodes 0 to 18 have already been allocated, we illustrate how the allocations for nodes 19 and 20 are conducted. Node 19 has to select an $R O I$ value greater than ROI(parent(19)), which is $4(\mathbf{C 2})$. However, all four pairs for $R O I=5$ have been allocated to other nodes earlier $(\mathbf{C 1})$. Since parent $(18)=$ parent(19) (nodes 18 and 19 are siblings) and $R O I(18)=6$, node 19 cannot take 6 as its $R O I$ value either $(\mathbf{C 3})$. The next candidate $R O I$ is 7 , and among 4 possible $C I$ numbers, it chooses the smallest one, 1 . For node 20, it needs to select an $R O I$ value greater than $R O I($ parent $(20))$, which is 5 (C2). Since it has no siblings, and there is one unused pair for $R O I=6(\mathbf{C 3}$ and $\mathbf{C 1})$, this value is selected as its $R O I$ and the only unused $C I$ number, 4.

From the table we can see that $\max R O I=7$, and we can obtain the allocation of $T S$ s for all nodes based on formula $T S(i)=\max R O I-R O I(i)+1$ (line 28 in Listing 1). Fig. 2 also shows in the tree topology when and through which channel a node transmits the response to its parent.

\section{EVALUATION AND IMPLEMENTATION}

In this section we first evaluate the algorithm efficiency based on simulations. To prove the feasibility of the algorithm on top of a CSMA based network stack, we implemented the algorithm within the framework of a COTS ZigBee stack and measured the performance compared with a straightforward approach.

\section{A. Evaluation Through Simulation}

The key questions we want to get answers from simulations are: 1) how efficiently the algorithm makes use of all the available timeslot/channel pairs compared with the lower bound; 2 ) how scalable the algorithm is, in terms of the number of $T S \mathrm{~s}$ used, when the number of nodes increases.

We developed a simple Monte Carlo simulator using the $\mathrm{C}$ programming language to obtain the number of $T S \mathrm{~s}$ that the allocated schedule uses under various configurations. Our simulator generates tree topologies with random numbers of children per parent and various numbers of nodes in the network. Then the simulator applies the allocation algorithm (Listing 1) to assign $(T S, C I)$ pairs for all non-base nodes and gives results.

We compare the simulation results with the lower bound shown in section II-C: $\lceil n / m\rceil$ and examine how many extra time slots are needed above this bound.

The simulations cover the combination of the following parameters: $n \in\{50,100,200,500,1000\}, m \in\{2,4,8\}$, and the traversing order (BF or DF). For each configuration the simulations were run 100 rounds. The average numbers of timeslots needed are plotted in Fig. 3. The 90\% confidence interval of each TS number plotted is within $10 \%$ of the corresponding average value. We observed that $95 \%$ of the numbers of timeslots needed are smaller than the lower bound plus 8 for all configurations we tested. In real-world implementations, the duration of timeslots is typically between 4 milliseconds and 50 milliseconds, therefore the extra time caused by the suboptimality of the algorithm is below 0.4 seconds. Based on this observation, we can see that the total number of timeslots needed is roughly proportional to the total number of nodes in the network, and roughly inversely proportional to the number of channels available. Another interesting observation is that in general, typically $B F$ takes 1 or 2 less timeslots than $D F$. Therefore, our real system implementation uses $B F$ for traversing.

It is worth mentioning two important results from other simulations. First, the number of average children in the tree structure has little effect on the total number of timeslots needed. Second, if $B F$ is used and the tree structure is very unbalanced in the sense that some node has many more children than others at the same depth, the total number of timeslots needed can be reduced quite significantly if the nodes with more children are visited earlier than those with less children.

\section{B. System Implementation and Evaluation}

We implemented SODA on top a commercial off-the-shelf (COTS) ZigBee stack. The purpose is to show that SODA 


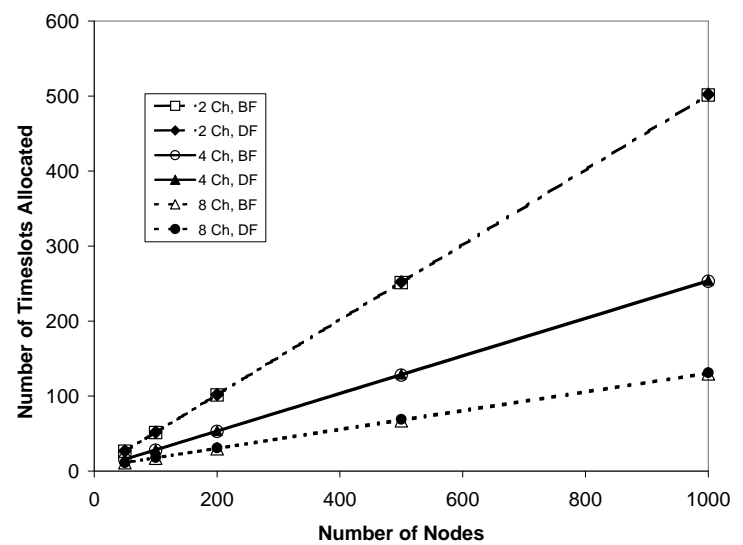

Fig. 3. Node Number vs. Number of Timeslots Allocated (from simulation)

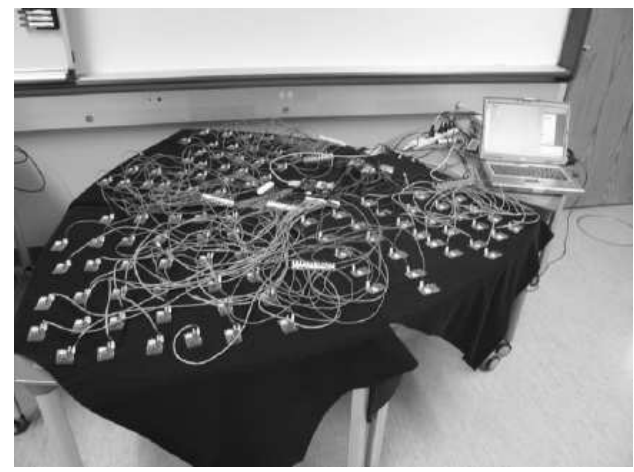

Fig. 4. Experimental 100 Node Test Setup

coexists with CSMA based MAC protocols that are rather prevalent in wireless personal area networks, requires minimum modification of the original stack, and is backward compatible with the original protocol. Fig. 4 shows the 100 node test setup with the SODA algorithm implemented.

Time synchronization is one of the key implementation issues. Each node needs to switch to its allocated channel during its allocated timeslot for transmission of response to its parent. During this timeslot, its parent needs to switch to the same channel to receive the response. It is very important for the switching time to be aligned with minimum jitter, otherwise packet loss may happen because the transmitter (child) and the receiver (parent) may be at different channels due to timeslot misalignment. Therefore, all nodes in the whole network need to maintain a global time with minimum jitter. Embedding a relative time delay in the broadcast and having each node waiting for this amount of time and switch work poorly. The reason is that in a multiple hop network the broadcast message is typically retransmitted for robust coverage of the whole network, and the retransmissions are not synchronized, therefore the time when the retransmission arrives plus the relative time delay may generate unaligned switching time. Thus specifying an absolute time based on the synchronized global time is the prefered solution. Our time synchronization implementation is based on the flooding time synchronization protocol (FTSP) described in [3]. It does add some communication overhead. However, based on our experi-

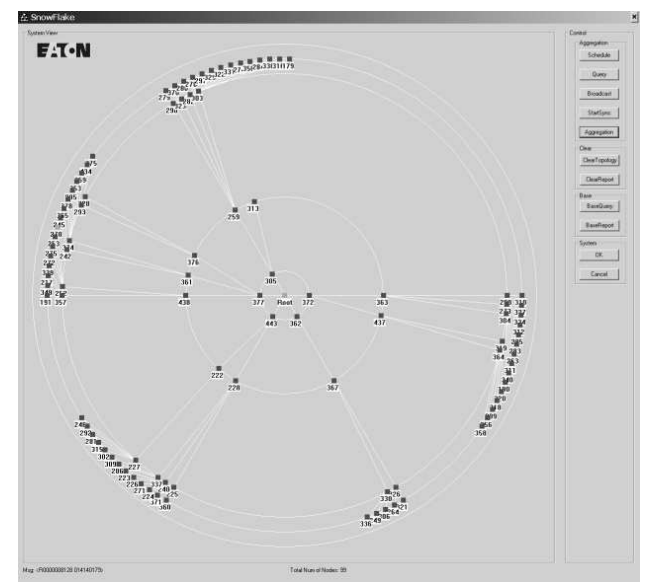

Fig. 5. 100 Node Responses as Shown in GUI

ments, a 1 minute time synchronization update rate results in a one hop time error smaller than 100 microseconds. We need to increase the timeslot duration due to this 100 microsecond per hop maximum timing error, but compared with a typical packet duration of several milliseconds, this overhead is small. FTSP requires an accurate time capture when a packet is transmitted and received. This is typically supported by the synergy of the radio transceiver, the microcontroller and the MAC layer software.

In our implementation, we gather the tree topology from the network upon deployment. We use the child-parent relation in the existing ZigBee association tree structure for that purpose. We run the algorithm on the base node (or the PAN coordinator in ZigBee's terminology), and broadcast the schedule containing all $(T S, C I)$ pairs to the whole network. Please note that all the above are just conducted once during the initialization phase after the network is deployed. Each broadcast command/query includes an absolute global starting time for all nodes in the network, and each node computes when it should switch based on this absolute global starting time, and the $(T S, C I)$ pair it receives during initialization. We set the global starting time 2 seconds after the broadcast command/query from the base node to avoid collisions with broadcast retransmissions. In our testing each timeslot is 10 milliseconds. Each node sends a 3 bit status information to the base node in response to the broadcast command/query. Under this setup the aggregated response can be contained in exactly one IEEE 802.15.4 packet for a 100 node network.

Fig. 5 shows the graphical user interface (GUI) of our system that displaying the result of one test. In this test, 99 nodes out of 100 delivered their status information within 0.5 seconds after the global starting time.

Fig. 6 shows more detailed response delivery timing from a set of tests with 20, 40 and 80 nodes measured at the base node. For each node number, the tests were conducted 50 times. Note that since responses are aggregated at the parents, and the children of the base node sends their aggregated responses at the latest timeslots generated, the base waits longer for the responses for 80 nodes to arrive. More than $95 \%$ of the responses in all cases are delivered within 0.5 


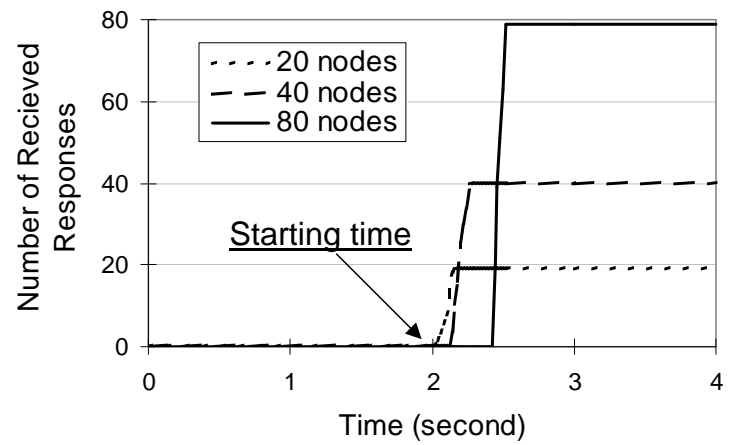

Fig. 6. Number of Node Responses Over Time (experimental results)

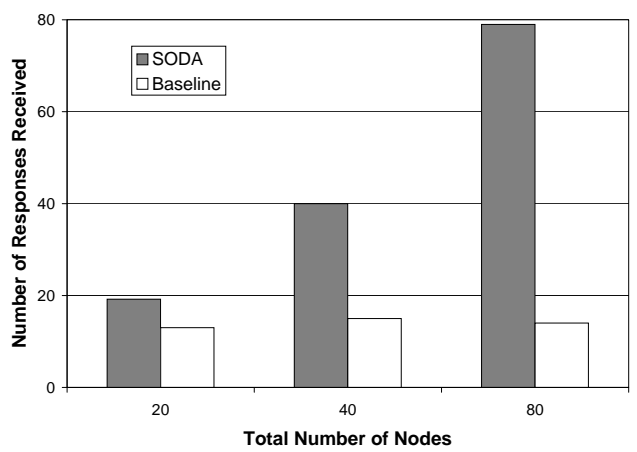

Fig. 7. Comparison of SODA and Baseline (experimental results)

seconds after the global starting time.

Fig. 7 shows the comparison of the SODA algorithm proposed in this paper and the straightforward method (as the baseline). The straightforward method requires all nodes in the network to send their responses after a short delay upon reception of the broadcast command/query in an ad hoc, besteffort fashion. Each value is based on the average of the results from 50 tests, and the $90 \%$ confidence interval is within $10 \%$ of the average value. Our results show that with the total number of nodes increasing from 20 to 80 , the straightforward method always receives responses from only about 15 nodes in the network, while SODA receives responses from 19.2, $40,79.0$ nodes in average, for $20,40,80$ node networks respectively. In our tests SODA always achieves a delivery rate higher than $95 \%$. For the 80 node network, SODA achieves more than $400 \%$ improvement over the baseline. This set of simulations highlights the scalability of SODA.

\section{RELATED WORK AND CONCLUSION}

\section{A. Related Work}

Data aggregation techniques have been an active area in wireless sensor network research. A comprehensive review of various protocols and techniques was reported in survey paper [4]. Among these techniques, improvement of communication efficiency, reduction of energy consumption, and real-time features have attracted a lot of research interests. Among them $\mathrm{He}$ et al. proposed AIDA [5], an application independent aggregation approach that balances the end-to-end transmission delay and energy consumption. Heuristic algorithms presented in [6][7] built aggregation trees that minimize energy consumption under latency bounds for wireless sensor networks. None of the above publications adopts a time-division scheme based on scheduling as proposed in this paper.

Other techniques related to the approach proposed in this paper include MAC protocols for optimizing performance of data aggregation, scalable broadcast protocols, multi-channel MAC protocols, and channel allocation schemes. Lu et al. developed DMAC [8], a MAC protocol that optimizes nodes' active/sleep schedules to save energy consumption and reduce latency for data gathering applications for wireless sensor networks. A scalable broadcast protocol, BPS [9], was proposed to minimize the number of retransmissions and therefore the energy consumption is reduced as a result. MMSN [10] is a MAC protocol that applies a sophisticated scheduling algorithm to efficiently use multiple channels for communications in wireless sensor networks, but it was not aimed at the problem we solved in this paper. DCA [11] is a dynamic channel allocation scheme that uses distributed 2-hop coloring techniques to avoid both primary and secondary interferences.

\section{B. Conclusion}

In this paper, we propose a fast and scalable approach to gather responses from a wireless sensor network triggered by a broadcast command or query. The near-optimal efficiency of our algorithm is validated by simulation studies. We also showed that SODA is incorporated into and coexsits with a commercial off-the-shelf CSMA-based wireless protocol stack. Based on testing on the real-system implementation, we validated that SODA delivers within 0.5 seconds up to four times more responses compared with the straightforward approach for the same number of nodes.

\section{REFERENCES}

[1] “IEEE Std 802.15.4-2006 (Revision of IEEE Std 802.15.4-2003)," IEEE Computer Society, 2006.

[2] T. H. Cormen, C. E. Leiserson, R. L. Rivest, and C. Stein, Introduction to Algorithms. MIT Press, Cambridge, MA, 2001.

[3] M. Maróti, B. Kusy, G. Simon, and A. Lédeczi, "The Flooding Time Synchronization Protocol," in ACM SenSys, 2004, pp. 39-49.

[4] E. Fasolo, M. Rossi, J. Widmer, and M. Zorzi, " In-Network Aggregation Techniques for Wireless Sensor Networks: A Survey," in IEEE Wireless Communications, April 2007.

[5] T. He, B. M. Blum, J. A. Stankovic, and T. Abdelzaher, "AIDA: Adaptive Application-Independent Data Aggregation in Wireless Sensor Networks," in ACM TECS, May 2003.

[6] H. Cheng, Q. Liu, and X. Jia, "Heuristic Algorithms for Real-time Data Aggregation in Wireless Sensor Networks ," in ACM IWCMC, 2006.

[7] Y. Hu, N. Yu, and X. Jia, "Energy Efficient Real-Time Data Aggregation in Wireless Sensor Networks," in ACM IWCMC, 2006.

[8] G. Lu, B. Krishnamachari, and C. S. Raghavendra, "An Adaptive Energy-Efficient and Low-Latency MAC for Data Gathering in Sensor Networks," in IEEE IPDPS, 2004.

[9] A. Durresi, V. K. Paruchuri, S. S. Iyengar, and R. Kannan, "Optimized Broadcast Protocol for Sensor Networks," in IEEE Transactions on Computers, August 2005.

[10] G. Zhou, C. Huang, T. Yan, T. He, J. A. Stankovic, and T. F. Abdelzaher, "MMSN: Multi-Frequency Media Access Control for Wireless Sensor Networks," in IEEE INFOCOM, 2006.

[11] K. R. Chowdhury, P. Chanda, D. P. Agrawal, and Q.-A. Zeng, "DCA - A Distributed Channel Allocation Scheme for Wireless Sensor Networks," in IEEE PIMRC, 2005. 


\title{
A Survey of Efficiency-Estimation Methods for In-Service Induction Motors
}

\author{
Bin Lu, Student Member, IEEE, Thomas G. Habetler, Fellow, IEEE, and Ronald G. Harley, Fellow, IEEE
}

\begin{abstract}
Condition monitoring of electric motors avoids severe economical losses resulting from unexpected motor failures and greatly improves the system reliability and maintainability. Efficiency estimation, which shares many common requirements with condition monitoring in terms of data collections, is expected to be implemented in an integrated product. This brings more considerations into the selection of the efficiency-estimation methods. This paper presents the results of an up-to-date literature survey on efficiency-estimation methods of in-service motors, particularly with considerations of the motor-condition-monitoring requirements. More than 20 of the most commonly used methods are briefly described and classified into nine categories according to their physical properties. Six categories of these methods are more related to in-service testing and are compared in a table summarizing the required tests and measurements, intrusion level, and average accuracy. Estimation of the rotor speed and the stator resistance, the two stumbling blocks of various efficiencyestimation methods, is also carefully studied; commonly used methods are summarized. Based on the survey results, four efficiency-estimation methods are suggested as candidates for nonintrusive in-service motor-efficiency estimation and conditionmonitoring applications. Another contribution of this paper is that a general approach for developing nonintrusive motorefficiency-estimation methods is proposed, incorporating rotor speed, stator resistance, and no-load loss estimations.
\end{abstract}

Index Terms-Condition monitoring, efficiency estimation, IEEE Standard 112, induction motor, in-service testing, rotorspeed estimation, stator-resistance estimation.

\section{INTRODUCTION}

$\mathbf{I}$ $\mathrm{T}$ IS estimated that over two-thirds of the total electric energy generated in the United States is consumed by motordriven systems. On average, the motors in industrial plants operate at $60 \%$ of their rated load because of oversized installations or underloaded conditions and, as a result, have fairly low efficiencies. As the global energy shortage and the green house effect have worsened, the improvement of the motor energy usage in industry is drawing more attention. This could be done through many ways, such as replacing oversized motors or applying more efficient control techniques. Obviously, all of

Paper IPCSD-06-027, presented at the 2005 IEEE International Electric Machines and Drives Conference, San Antonio, TX, May 15-18, and approved for publication in the IEEE TRANSACTIONS ON INDUSTRY APPLICATIONS by the Electric Machines Committee of the IEEE Industry Applications Society. Manuscript submitted for review July 30, 2005 and released for publication April 5, 2006. This work was supported in part by the Department of Energy (DOE) and in part by Eaton Corporation.

The authors are with the School of Electrical and Computer Engineering, Georgia Institute of Technology, Atlanta, GA 30332 USA (e-mail: binlu@ece.gatech.edu; thabetler@ece.gatech.edu; rharley@ece.gatech.edu).

Digital Object Identifier 10.1109/TIA.2006.876065 these ways are based on the precise estimation of the energyusage condition of the motors [1].

Condition monitoring and diagnostics are very important issues in motor-driven and power-electronics systems since they can greatly improve the reliability, availability, and maintainability of the system. An industry expects the energyusage estimation and condition-monitoring functions to be implemented in an integrated product, since they share many common needs in terms of data collection. This introduces further considerations into the selection of motor-efficiencyestimation methods in these applications. For example, in the detection of the stator-winding turn faults, broken rotor bars, and worn bearings, the current-based spectral-analysis techniques are used, which require the stator-current waveforms to be sampled and collected. Having the recorded current data, we would naturally seek an efficiency-estimation method that uses the stator current [2].

Over the years, many motor-efficiency-estimation methods for general purposes have been proposed. A series of laboratory assessments have been conducted back in 1990s and form an important resource of this survey. In 1996, a team at the Oak Ridge National Laboratory (ORNL) reviewed 28 of the previously proposed methods and evaluated them according to their intrusion levels and cost of equipment sponsored by the U.S. Department of Energy (DOE) [3]. Shortly after the first report, the same team selected six candidate methods for a more detailed estimation [4]. These six methods particularly target the estimation of motor efficiency and load under field conditions. This report finally suggested three best candidate methods ranging from low to high intrusion level for the efficiency testing under field conditions. These two reports did give a relatively comprehensive assessment and suggest an important new method "air-gap torque method," however, they did not consider any condition-monitoring requirements. Besides this, because of the development of the advanced techniques of sensorless speed estimation [21]-[31] and in-service statorresistance estimation [33]-[43] during the last decade, some of the testing procedures and measurements described in these reports are now obsolete.

Based on these reports, in 1999, the Washington State University Co-Operative Extension Energy Program tested 12 motor-efficiency testing methods [5]. A more detailed test of four methods was then performed, and the authors claimed these methods as "nonintrusive." However, these methods should be called "low-intrusive" or even "medium-intrusive" methods more precisely, since either speed measurement with a transducer or a stator-resistance measurement with an unpowered test is required. Again, the methods in this test can be 
simplified greatly using the speed and stator-resistance estimation now available.

Generally, the measurements needed for each method are different, but most of them require the following common data: input line voltages and line currents. Some methods require the nameplate data (rated voltage, current, horsepower, speed, etc.), stator resistance $R_{\mathrm{s}}$, or rotor speed $\omega_{\mathrm{r}}$. Among these, the measurements or estimates of stator resistance and speed have been regarded as the stumbling blocks of various efficiencyestimation methods for years. However, recent research has made a great progress in the area of stator resistance and speed estimation. Most of these estimators utilize the terminal voltage and current, which are already available in conditionmonitoring systems.

\section{Definition of Motor Losses And EfFiciency}

The total losses in the motor are of interest here, since finding the efficiency is equivalent to finding the total losses. IEEE Std-112 part 5 defines five types of losses in induction motors as (1) [13]

$$
\begin{aligned}
W_{\text {Losses }} & =P_{\text {input }}-P_{\text {output }} \\
& =W_{\mathrm{s}}+W_{\mathrm{r}}+W_{\text {core }}+W_{\mathrm{fw}}+W_{\mathrm{LL}}
\end{aligned}
$$

where $P_{\text {input }}$ and $P_{\text {output }}$ are the input and output powers of the motor, respectively, and $W_{\text {Losses }}$ is the total loss in the motor.

Stator copper loss $W_{\mathrm{s}}$ is the loss in the stator windings. It equals to $1.5 I^{2} R$ for a three-phase motor, where $I$ is the measured or calculated rms current per line terminal at specified load; and $R$ is the average dc resistance between any two line terminals corrected to the specified temperature. For a perfectly balanced motor, $R$ is twice of $R_{\mathrm{s}}$.

Rotor copper loss $W_{\mathrm{r}}$ is the loss in the rotor windings including the brush contact losses for wound-rotor motors. It should be determined from the per-unit slip using (2)

$$
W_{\mathrm{r}}=\left(P_{\text {input }}-W_{\mathrm{s}}-W_{\text {core }}\right) \cdot s
$$

where $s$ is the slip.

Core loss $W_{\text {core }}$ is due to magnetizing hysteresis and eddy currents in the iron. It varies approximately with the square of the input voltage, but for fixed input voltage, it remains approximately constant from no load to full load. It is usually measured from the no-load test.

Windage and friction loss $W_{\mathrm{fw}}$ is the mechanical rotational loss due to the friction and windage. It is also nearly constant from no load to full load and usually measured from the no-load test.

Stray load loss $W_{\mathrm{LL}}$ is the loss in a motor not accounted for by the sum of $W_{\mathrm{s}}, W_{\mathrm{r}}, W_{\text {core }}$, and $W_{\mathrm{fw}}$. It can be divided into two parts: stator-stray load loss $W_{\mathrm{LLs}}$ and rotor-stray load loss $W_{\mathrm{LLr}}$.

The motor efficiency $\eta$ is then defined as

$$
\eta=\frac{P_{\text {output }}}{P_{\text {input }}} \times 100 \%=100 \%-\frac{W_{\text {Losses }}}{P_{\text {input }}} \times 100 \% .
$$

\section{EfFiciency-Estimation Methods}

An in-service motor-efficiency-estimation method can be a single basic algorithm or a combination of different basic algorithms listed in this section. The methods are commonly categorized in the literature by their intrusion levels and accuracies. The intrusion level is determined by the type of data required for each method, the cost associated with the equipment installation and data collection process, and the operation planning of the motor. The accuracy is evaluated via comparison with a direct efficiency measurement, e.g., shaft torque and rotor speed.

This section lists more than 20 of the most important motorefficiency methods, which are categorized according to their physical properties [3]-[20]. As indicated before, some of these methods are combinations of several basic methods and can be classified into more than one category. These methods are finally classified into the most related category. For an instance, the ORMEL96 method is classified into equivalentcircuit methods, other than nameplate methods, statistical methods, or dedicated instrument methods.

\section{A. Nameplate Methods}

The least intrusive field evaluation method is to obtain motor information from the nameplate [3]-[5].

1) Standard Nameplate Method: The efficiency is assumed to be constant and equal to the nameplate value [3]. However, this method has poor accuracy because: 1) the nameplate data may not be given according to IEEE Std-112 Method B; 2) the motor may have been rewound; and 3) the field environment may be different from that where the nameplate data are derived from.

2) Volgelsang and Benning $(V \& B)$ Method I, II: The V\&B method I and II are variations of the standard nameplate method and used in a dedicated commercial instrument: the "Motor Analyzer." Option I ( $\pm 1 \%$ error) requires testing under three conditions: no load, normal load, and unpowered. Option II ( $\pm 3 \%$ error) does not require the no-load test, but, instead, uses motor nameplate data. Consequently, the accuracy is reduced. The principles of this instrument are based on the theory detailed in [7]. This method might also be classified as a segregated loss method.

\section{B. Slip Methods}

The slip methods rely on the motor speed measurement [3]-[6], [8]-[11]. The main advantage is their simplicity.

1) Standard Slip Method: The percentage of the load is presumed to be proportional to the ratio of the measured slip to the full-load slip. The motor efficiency is, thus, approximated using (4). However, the error comes from the fact that the slip ratio represents the percentage of load, and the efficiency is not equal to the percentage of load [8]

$$
\eta=\frac{\text { slip }}{\text { slip }_{\text {rated }}} \cdot \frac{P_{\text {output,rated }}}{P_{\text {input }}} .
$$


2) Ontario Hydro Modified Slip Method: As given by (5), the standard slip method is improved by correcting the rated nameplate speed for voltage variations, especially when the efficiency-load curve is not flat [9]. However, the estimate could still have a relatively large error, since the nameplate speed is allowed to deviate as much as $20 \%$ from the actual rated speed according to National Electric Manufacturing Association (NEMA) MG1 standard [12]

$$
\eta=\frac{\text { slip }}{\operatorname{slip}_{\text {rated }}} \cdot \frac{P_{\text {output,rated }}}{P_{\text {input }}} \cdot\left(\frac{V}{V_{\text {rated }}}\right)^{2} .
$$

3) Upper Bound Slip Method: As the simplest efficiencyestimation method, the original upper bound slip method assumes $W_{\mathrm{s}}$ to be zero as (6) [10]

$$
\eta \leq(1-\text { slip }) .
$$

It has been observed that $W_{\mathrm{s}}$ counts about $40 \%$ of the total losses in a typical motor. Therefore, this method can be improved by including $W_{\mathrm{s}}$, as shown in (7) [5]

$$
\eta \leq(1-\operatorname{slip})\left(1-\frac{3 I^{2} R_{\mathrm{s}}}{P_{\text {input }}}\right) .
$$

The resulting efficiency estimate is always higher than the actual efficiency since it neglects $W_{\mathrm{r}}, W_{\text {core }}, W_{\mathrm{fw}}$, and $W_{\mathrm{LL}}$.

\section{Current Methods}

Like the slip methods, the current methods use minimum measurements and manufacturer's data to estimate the efficiency [3]-[6], [8]. Their main advantage is simplicity.

1) Standard Current Method: In this case, the motor efficiency is approximated using (8), assuming that the percentage of load is closely proportional to the ratio of the measured current to full-load current. In reality, the current-load curve is slightly nonlinear. This is considered in an improved current method as (9) [8]. It is suggested in [3] that the average of these two approaches may give a more accurate efficiency estimate

$$
\begin{aligned}
& \eta=\frac{I}{I_{\text {rated }}} \cdot \frac{P_{\text {output,rated }}}{P_{\text {input }}} \\
& \eta=\frac{2 I-I_{\text {no load }}}{2 I_{\text {rated }}-I_{\text {no load }}} \cdot \frac{P_{\text {output,rated }}}{P_{\text {input }}} .
\end{aligned}
$$

\section{Equivalent-Circuit Methods}

The efficiency of an induction motor can be calculated from its equivalent electric circuit. These methods can provide an efficiency estimate for a motor operating under load conditions other than those at which measurements are made.

1) Standard Equivalent-Circuit Method (IEEE Std-112 Method F/F1): The IEEE Std-112 method F/F1 is the standard equivalent-circuit method [12], [13]. It requires an impedance measurement, no-load, variable-voltage, removed-rotor, and reverse-rotation tests. These tests are too intrusive and impractical for in-service monitoring, although the method can be quite accurate. This method is mentioned here mainly as the basis for other modified methods.
2) Ontario Hydro Modified Method F (OHMF): A modified version of the IEEE Std-112 method F1 is proposed by Ontario Hydro [6], [9]. It uses a slightly different equivalent circuit: The magnetizing impedance is taken to be a series combination of resistance and inductance instead of the standard parallel combination. A variable voltage test is not required, but both no-load and full-load tests at rated voltage must be run. Line voltage, input power, line current, power factor, and stator resistance are measured while operating at no load and at full load. The slip is also measured at full load.

3) Nameplate Equivalent-Circuit (ORMEL96) Method: This method derives the motor equivalent circuit from the nameplate data and the value of the stator resistance [3]-[6]. A parasitic resistance is inserted in the rotor circuit to account for stray load loss. Only the rotor speed is measured online. The stator resistance can be estimated from nameplate data [6], although the accuracy of efficiency estimate could be increased substantially if it can be accurately determined [5]. The ORMEL96 method also has an advanced mode, which allows the user to adjust certain "tunable parameters."

It is a low-intrusion method, however, the parameters of the equivalent circuit are solved from imaginary rated load condition and locked rotor condition, which completely rely on motor nameplate information and may have up to $20 \%$ inaccuracies according to NEMA MG1 [12].

Because of its inherent low intrusion level and good accuracy (within $\pm 4 \%$ error [3]), the ORMEL96 method is proposed as one of the four candidate methods for in-service motorefficiency estimation and condition-monitoring applications.

4) Rockwell Motor-Efficiency Wizard (RMEW) Method: Another interesting equivalent-circuit-based method is proposed by Rockwell Reliance and used as the efficiency estimator in their product "Motor Efficiency Wizard" [14]. This method calculates the parameters of the standard inductionmotor equivalent circuit using data from two different load operating points. It requires measurements of stator resistance and stator-winding temperature. Besides, the solution of motor parameters requires the actual value of stator leakage reactance, which is not available for in-service testing. Furthermore, the motor parameters are calculated using stator voltage and current phasors, and the harmonics in the power supply are not considered.

The experimental results in [14] show a high accuracy of within $\pm 1 \%$ errors. Although this accuracy seems to be overoptimistic according to its physical nature, this method is still considered as a candidate for in-service motor-efficiency estimation.

5) Locked Rotor Method: This method uses an equivalent circuit with two rotor loops [6], [15]. The parameters of the circuit are obtained from locked rotor tests. A no-load test must also be run. These tests make this a highly intrusive method.

6) Standstill Frequency Response Method: The equivalent circuit of this method also has two rotor loops [6], [16]. The parameters of the circuit are derived by measuring the impedance of the motor over a frequency range of $0.01 \mathrm{~Hz}$ to $500 \mathrm{~Hz}$, with its rotor stationary. The major advantage of this method is that the low voltage, no-load test is not required. However, it is still inherently a high-intrusion method. 
TABLE I

ASSUMEd VALUES For STRAY-LOAD LOSS IN IEEE STD-112

\begin{tabular}{|c|c|c|}
\hline \multicolumn{2}{|c|}{ Machine Rating } & $\begin{array}{c}\text { Stray load loss percent of } \\
\text { rated output }\end{array}$ \\
\hline $1-125 \mathrm{hp}$ & $1-90 \mathrm{~kW}$ & $1.8 \%$ \\
\hline $126-500 \mathrm{hp}$ & $91-375 \mathrm{~kW}$ & $1.5 \%$ \\
\hline $501-2499 \mathrm{hp}$ & $376-1850 \mathrm{~kW}$ & $1.2 \%$ \\
\hline $2500 \mathrm{hp}$ and up & $1851 \mathrm{~kW}$ and up & $0.9 \%$ \\
\hline
\end{tabular}

\section{E. Segregated Loss Methods}

These methods are most straightforward because they simply estimate (segregate) each loss component $\left(W_{\mathrm{s}}, W_{\mathrm{r}}, W_{\text {core }}\right.$, $W_{\mathrm{fw}}$, and $\left.W_{\mathrm{LL}}\right)$. These methods are generally very accurate (within $\pm 2 \%$ error [9]), although some of them are also quite complex and intrusive, while others rely on empirical values to estimate some of the losses.

1) Std. Segregated Loss Method (IEEE Std-112 Method E1): The IEEE Std-112 method E1 is the standard segregated loss method [12], [13]. Method E1 specifies an assumed value for the stray load loss at rated load for different size motors, as shown in Table I [13]. As with IEEE Std-112 method F1, it is not intended for in-service testing, since no-load, variablevoltage, removed-rotor, and reverse-rotation tests are required. It is listed here mainly as the basis for other modified methods.

2) Ontario Hydro Modified Method E (OHME): The IEEE Std-112 method E1 is simplified in [9] by assuming the combined windage, friction, and core losses $\left(W_{\mathrm{fw}}+W_{\text {core }}\right)$ to be $3.5 \%-4.2 \%$ of rated input power [3]-[6], [9]. The specific percentage could be optimized by testing samples of motors at different horsepower levels. The stray-load loss is also estimated from Table I [13]. This method can be simplified even further by using an assumed value of 0.8 for rated power factor. The stator resistance is estimated based on a simple approximation using the motor current to estimate the temperature rise. The only other measurements required are the input power and the rotor speed. Because of its low intrusion level and good accuracy (within $\pm 2 \%-3 \%$ error [3]), the OHME method is suggested as another candidate for in-service efficiency estimation.

\section{F. Torque Methods}

The motor efficiency can be defined in (10) in terms of the shaft torque $T_{\text {shaft }}$, the air-gap torque (AGT) $T_{\text {air gap }}$, and the rotor speed $\omega_{\mathrm{r}}$

$$
\eta=\frac{T_{\text {shaft }} \cdot \omega_{\mathrm{r}}}{P_{\text {input }}}=\frac{T_{\text {air gap }} \cdot \omega_{\mathrm{r}}-W_{\mathrm{fw}}-W_{\mathrm{LLr}}}{P_{\text {input }}} .
$$

With $P_{\text {input }}$ and $\omega_{\mathrm{r}}$ measured or estimated, the efficiency can be easily determined if $T_{\text {shaft }}$ is known. This is the basic principle of all the torque methods.

1) AGT Method: The AGT method proposed in [18] calculates the AGT using (11) from the motor instantaneous input line voltages and line currents [17]. The losses $W_{\mathrm{fw}}$ and $W_{\mathrm{LLr}}$ are obtained from the no-load test. Finally, the motor efficiency is calculated using (10)

$$
\begin{aligned}
T_{\text {air gap }}= & \frac{\text { poles }}{2 \sqrt{3}}\left\{\left(i_{A}-i_{B}\right) \cdot \int\left[\nu_{C A}-R_{\mathrm{s}}\left(i_{C}-i_{A}\right)\right] d t\right. \\
& \left.-\left(i_{C}-i_{A}\right) \cdot \int\left[\nu_{A B}-R_{\mathrm{s}}\left(i_{A}-i_{B}\right)\right] d t\right\}
\end{aligned}
$$

where poles is the number of poles, $i_{A}, i_{B}$, and $i_{C}$ are the three line currents, and $\nu_{C A}$ and $\nu_{A B}$ are the two line voltages.

A significant advantage of this method is that it considers the losses associated with the unbalances in the voltages and currents, which reflects the reality in an industrial plant. However, it requires a no-load test. Because of its extremely high accuracy (within $\pm 0.5 \%$ error [3]), this method is good for online monitoring. The high intrusion level is the main drawback of the original AGT method, but this can be overcome, as proposed in Section VI-B.

2) Shaft Torque Method: As the most straightforward of these approaches, this method measures the shaft torque and the rotor speed (and, thus, the output power) directly from the shaft, without the need to calculate the losses [3]. It offers the most accurate field-efficiency evaluation, but is also highly intrusive. Additionally, the high cost of the torque transducers makes this method not acceptable for most industrial applications.

The details of the rest three categories of efficiencyestimation methods are omitted here for simplicity, since they are either combinations of the previously discussed methods or product-specific methods that are out of interest here. The details of these methods are in [1].

\section{G. Empirical Methods}

\section{1) Stanford Wilke Method [3], [19]}

\section{H. Statistical Methods [3], [12]}

\section{Dedicated Instrument Methods}

1) Instantaneous Current Method [5], [20]

2) MotorMaster+ Methods [5]

3) Vectron (ECNZ) Method [5]

4) MAS-1000 Method [5]

5) Esterline Angus Method [5]

\section{RotoR-SPEED ESTIMATION}

Direct measurement of the rotor speed requires a shaftmounted speed encoder or optical tachometer to be installed. This reduces the reliability and increases the cost. During the past decade, numerous sensorless rotor-speed-estimation schemes have been proposed.

A large family of sensorless-speed-estimation schemes relies on an estimation of the back electromotive force (EMF) from stator voltages and currents, but these methods are inherently dependent on motor parameters and fail under a low-speed operation due to the degraded stator-voltage measurement [21], [22]. Digital-signal-processing (DSP) techniques have been effectively used to incorporate machine saliency harmonics into sensorless speed-estimation algorithms [23]-[31]. 


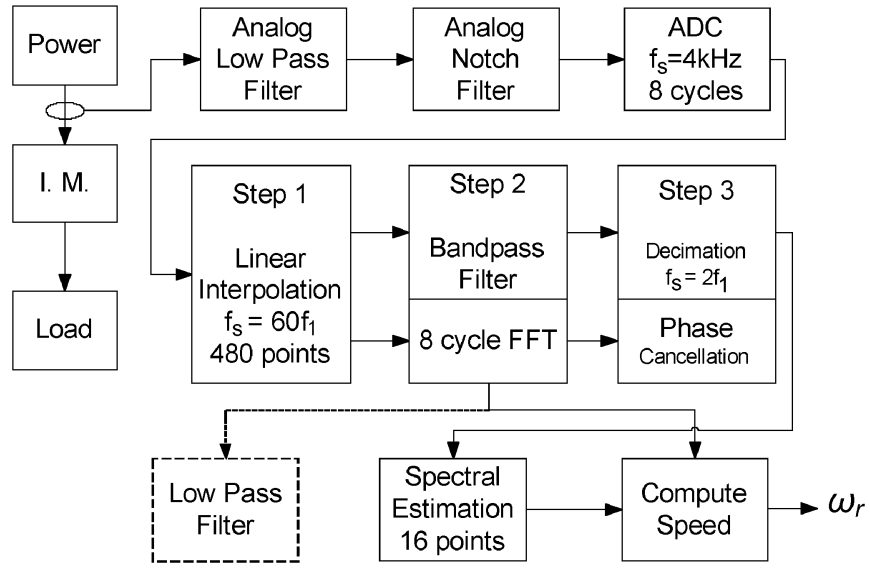

Fig. 1. Speed-detection algorithm using magnetic saliency harmonics.

In [23]-[25], the speed information from stator-voltage measurement is derived. In [23], the rotor-slot harmonics are isolated using the analog filtering, sampling, and zero-crossing detectors. This method fails below $10-\mathrm{Hz}$ operation. In [24], an external signal is injected to overcome the low-speed limit in [23]. In [25], a speed observer is developed based on a detailed motor magnetic model, which needs an extensive offline test to initialize. Again, a common problem of these methods is that the voltage is degraded at a low speed.

To overcome this problem, speed estimation schemes using current harmonics due to rotor unbalances and eccentricities are introduced in [26]-[30]. In [26], a scheme using an ana$\log$ filtering is described, but it cannot provide an accurate information from these low-bandwidth harmonics. In [27], DSP and a fast Fourier transform (FFT) are used to extract the slot harmonics over a much wider range of inverter frequencies than using an analog filtering. In [28], the same authors considered DSP spectral estimation methods as an alternative to FFT. However, a rather complex model is required to locate the desired harmonics. A common problem of these methods is that they are machine specific, since unknown machine parameters, e.g., rotor-slot number, are required.

In [29], a speed estimation method based on the current harmonics is developed requiring only the number of poles from the machine parameters. The speed-detection algorithm is shown in Fig. 1. This method can provide a robust and parameter-independent speed estimate down to $1-\mathrm{Hz}$ operation with a very high accuracy of within $5 \mathrm{r} / \mathrm{min}$ at high speeds and 0.005 p.u. slip at low speeds. In [30], the same authors further compare the accuracy, robustness, and computation time of two major classes of spectral estimation techniques: FFTbased methods and non-FFT parametric methods.

Other speed-estimation schemes require balanced polyphase high-frequency sense signals superimposed on the command voltage (or current) or modifications of machine designs [31]. These schemes are too intrusive for in-service testing and usually are not acceptable for line-connected motors.

\section{Stator-Resistance Estimation}

In many motor-efficiency-estimation methods, the stator resistance $R_{\mathrm{s}}$ is required. Traditionally, the dc resistance of the stator winding is measured through an unpowered test. The measurement procedure is given in IEEE Std-118 [32]. The obvious advantage of the direct resistance measurement is the high accuracy. However, direct measurement is not acceptable for inservice motor testing because of the following disadvantages.

1) It requires the motor to be disconnected from service to perform an unpowered test. Therefore, the intrusion level is very high.

2) It only records $R_{\mathrm{S}}$ at a certain temperature; for example, at the rated load temperature. The actual resistance, however, is linear with the winding temperature.

To overcome these problems, an online $R_{\mathrm{s}}$ estimate is necessary. Over the years, much work has been done on $R_{\mathrm{s}}$ estimation, especially as it relates to field control [33]-[35], speed estimation [36]-[38], and winding thermal monitoring and protection [39]-[43]. In [33]-[38], various $R_{\mathrm{S}}$ estimation methods have been proposed in the low-speed range for inverterfed machines using the stator-flux observers, state estimation, dc-voltage injection, or adaptive approaches. Stator-resistance estimation is also widely used for thermal-protection purposes [39]-[43]. Generally, there are two major families of methods in $R_{\mathrm{S}}$ estimation, as given below.

\section{A. Induction-Machine Model-Based $R_{\mathrm{S}}$ Estimation Methods}

The resistance estimate can be obtained by constructing an equivalent model of the induction motor [33]-[40]. In [33], $R_{\mathrm{S}}$ is estimated from the error between the measured stator current and the stator-current command. In [34], the stator-flux linkage $\lambda_{\text {qds }}$ is estimated from the rotor-flux-linkage estimate obtained from the rotor equations when the motor is operated at low speed. The value of $R_{\mathrm{S}}$ is then estimated from $\lambda_{\mathrm{qds}}$ using the stator-flux equation. In [36] and [39], $R_{\mathrm{S}}$ is calculated using the estimate of the rotor resistance $R_{\mathrm{r}}$ by assuming that $R_{\mathrm{S}} / R_{\mathrm{r}}$ is constant, which suffers accuracy because of skin effect and varying winding temperature. In [37], $R_{\mathrm{s}}$ is estimated using Lyapunov's direct method based on the error between the measured and estimated stator current. In [38], a mutual model reference adaptive system (MRAS) approach for estimating $R_{\mathrm{S}}$ and $R_{\mathrm{r}}$ is developed and improved. The MRAS is capable of estimating $R_{\mathrm{S}}$ under transient conditions. The structure of the MRAS is shown in Fig. 2.

In [40], a cascade $R_{\mathrm{S}}$ and $R_{\mathrm{r}}$ estimation scheme in the steady state is proposed. The overall structure of the estimator is shown in Fig. 3. This method minimizes the error in $R_{\mathrm{S}}$ caused by uncertainties in the measurements and motor parameters, and shows a good accuracy under normal-load conditions. This method only applies to steady state. The equations in Figs. 2 and 3 are listed in the Appendix.

The main advantage of the model-based $R_{\mathrm{s}}$ estimation methods is their nonintrusive nature, since the motor does not need to be removed from service. This is particularly important in the condition-monitoring applications where in-service testing is required. However, these methods suffer from the following disadvantages.

1) Accurate $R_{\mathrm{S}}$ estimate is usually not available under highspeed (light load) conditions. 


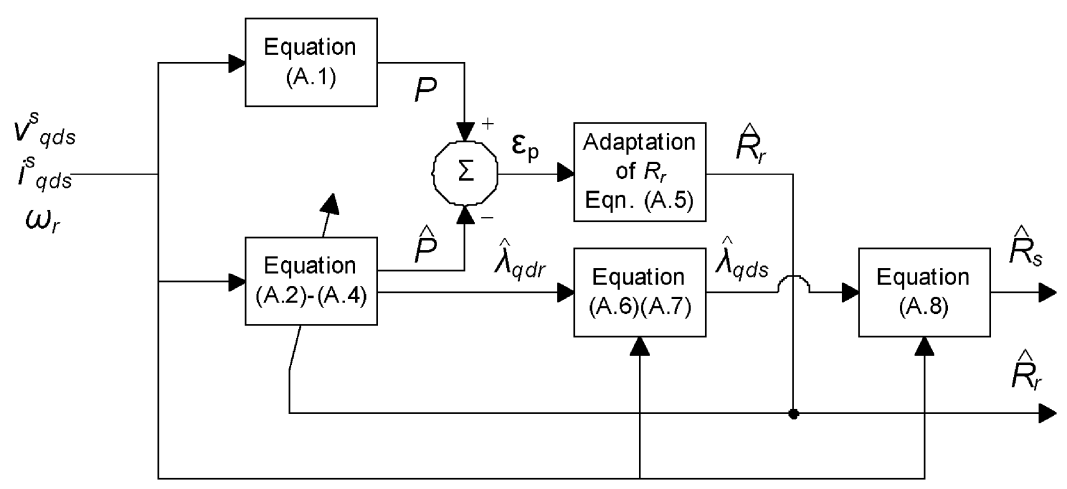

Fig. 2. Structure of the MRAS $R_{\mathrm{S}}$ and $R_{\mathrm{r}}$ estimator.

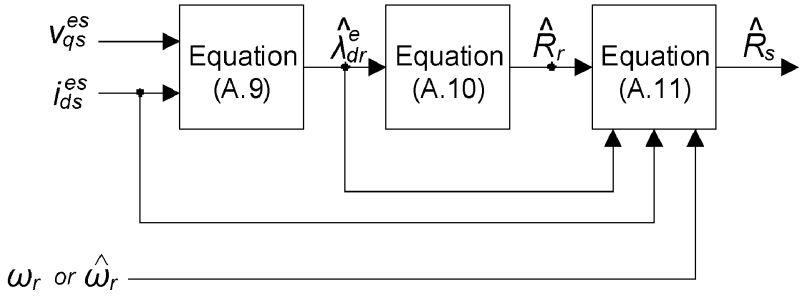

Fig. 3. Structure of the cascade $R_{\mathrm{S}}$ and $R_{\mathrm{r}}$ estimator.

2) $R_{\mathrm{S}}$ estimate is sensitive to errors in machine parameters, variables, and measurements, especially under light-load conditions.

3) Some methods require a complex computational effort, e.g., integration.

Because the average load factor of motors operating in industrial plant is around $60 \%$, the stator-resistance estimate using model-based methods is still optimistic.

\section{B. Signal-Injection-Based $R_{\mathrm{S}}$ Estimation Methods}

DC signal-injection methods have been proved to be very practical $R_{\mathrm{S}}$ estimation methods. In [41]-[43], an additional electric circuit is added between the source and the motor stator to inject a dc-voltage offset online. By measuring the associated dc-current offset, $R_{\mathrm{s}}$ is estimated. Particularly, [41] is for soft stared, and [42] is for line-connected induction machines using continuous dc injection. The problem of continuous dc injection is the additional power dissipation in the circuit and continuous torque pulsation, which are not accepted for some applications. In [43], a simple MOSFET controlled circuit is developed to intermittently inject a controllable dc bias into the motor. The circuit structure is shown in Fig. 4, and typical waveforms of voltage $\nu_{\mathrm{sw}}$ and current $i_{\mathrm{sa}}$ under dc injection mode are shown in Fig. 5. This method has very low power dissipation and torque distortion, and is capable of providing an accurate $R_{\mathrm{S}}$ estimate under motor startup, load variation, and abnormal cooling conditions.

The main advantages of the dc injection methods are the following.

1) The stator-resistance estimate has a very high accuracy.

2) They can be applied to a motor transient state.

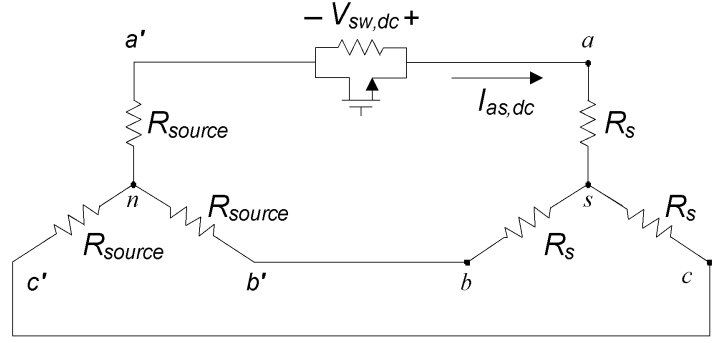

Fig. 4. Equivalent circuit of source, motor, and dc injection device.

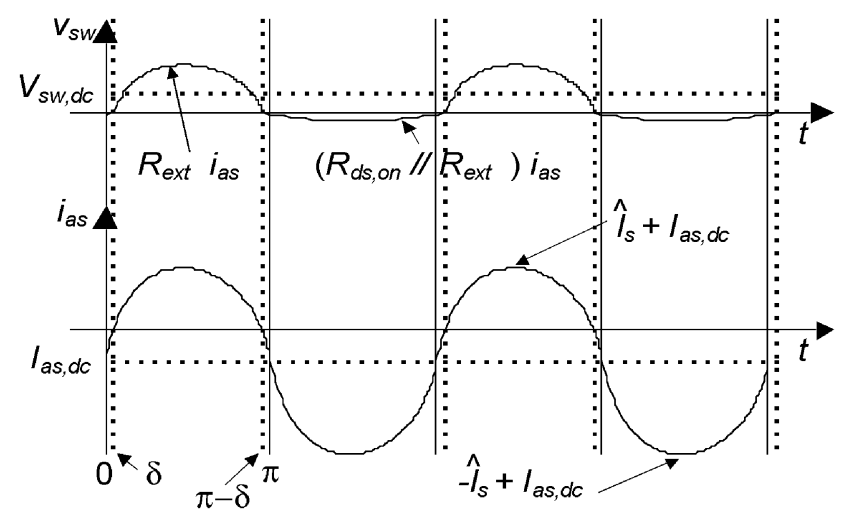

Fig. 5. Waveform of $\nu_{s w}$ and $i_{a s}$ under dc injection mode.

3) The stator-resistance-estimation process can be regarded as nonintrusive after the injection circuit is developed and included.

However, they also have the following disadvantages.

1) Additional circuit is required, and the cost is increased.

2) The installation of injection circuit is highly intrusive.

3) The injected dc signal produces unbalance in the stator voltages and currents and causes an additional power dissipation and torque distortion.

\section{Suggestions For Nonintrusive MOTOR-EFFICIENCY ESTIMATION}

\section{A. General Approach of Developing Nonintrusive Methods}

Nonintrusive in-service motor-efficiency-estimation methods usually use techniques for estimating the motor shaft torque without actually measuring it, or depend on the use of a motor equivalent circuit or some other mathematical model of the 


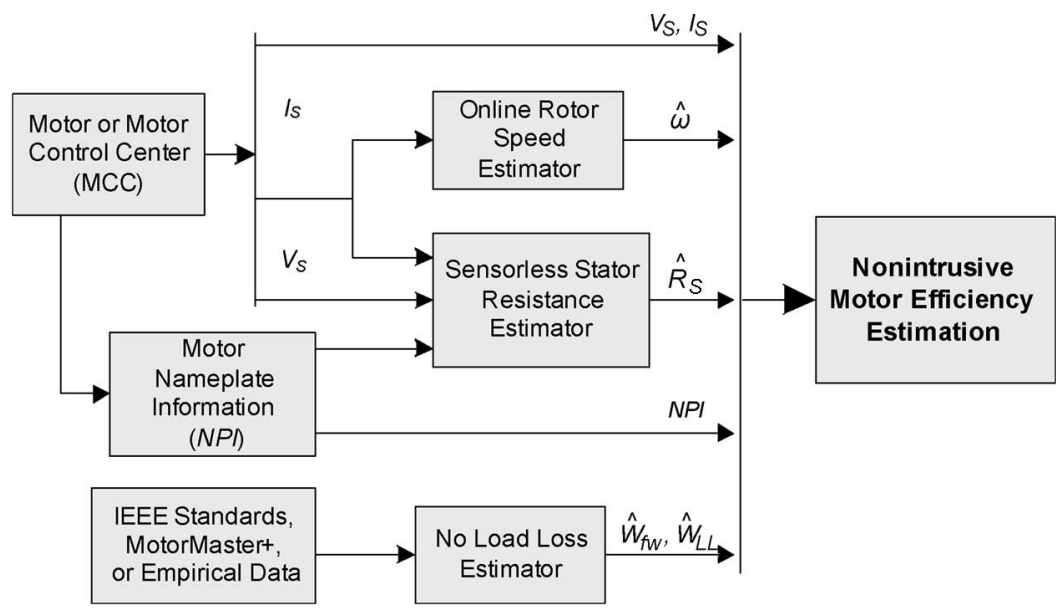

Fig. 6. General approach of developing nonintrusive methods.

motor's electromechanical power-transfer mechanism. Also, some of the losses, such as the friction and windage loss and stray-load loss, need to be estimated from the motor parameters. The degree of the accuracy of these methods is directly related to the accuracies of these estimations and motor parameters.

The development of a high-accuracy equivalent circuit and estimation of losses are normally done with information obtained from no-load testing and direct resistance measurement of the disconnected motor. Equivalent-circuit development is described in length in IEEE Std-112 [13]. In typical industrial applications, however, the user generally will not permit the motor to be disconnected to measure the stator resistance or allow uncoupled operation of the motor to measure the no-load power input. For this reason, alternate, less accurate, but less intrusive methods are often used.

An equivalent circuit may be developed, and losses may be estimated by using motor nameplate data and data from "MotorMaster+" or IEEE standards. MotorMaster+ is a motor selection and management tool developed by DOE, which includes a database of motor catalogue and performance information of more than 20000 ac motors [44]. The problem with this approach is that the nameplate and MotorMaster+ data are generic design data and may not be accurate in reflecting such parameters as the speed and power factor when the motor is delivering rated horsepower.

The high intrusiveness of many traditional methods also comes from the speed and resistance measurements. As summarized in Sections IV and V, online stator-resistance estimation and sensorless speed-estimation techniques can be used to obtaining the stator resistance and rotor speed.

\section{B. Candidate Methods}

Based on the survey results, the ORMEL96, REMW, OHME, and AGT methods are clearly the best candidates for nonintrusive motor-efficiency estimation, which only relies on line voltages, line currents, and motor-nameplate information. All four methods give more accurate efficiency estimates when the motor load is above $50 \%$. This is acceptable because, in cases where the load is low, it is less important to know the actual efficiency.
To minimize the intrusion levels of these methods, speed should be estimated from the current harmonics as in Section IV; stator resistance should be estimated using the methods in Section V. Specifically, the methods proposed in [29] and [43] are suggested for their proven high accuracy and reliability.

After the speed and the stator resistance are correctly estimated, the ORMEL96, REMW, and OHME methods do not need further modifications. The AGT method is regarded as the best in terms of accuracy (within $\pm 0.5 \%$ error) and ease of implementation. Besides, it mainly utilizes the shaft-torqueestimation techniques and, as a result, has least dependence on the motor parameters. Therefore, it is prone to provide a more accurate efficiency estimate compared with the other three methods even after nonintrusive modifications. However, it requires the no-load test, which is not accepted in many cases. Some additional modifications/assumptions must be added to estimate the no-load losses. This can be considered from the following perspectives.

1) If the nameplate information of the motor being tested is available, the no-load data could be estimated from the nameplate data.

2) The no-load losses could be estimated from the MotorMaster+ or empirical values.

A general approach of developing nonintrusive motorefficiency-estimation methods based on the candidate methods is illustrated in Fig. 6. The nonintrusiveness of this general approach comes from the fact that motor efficiencies are estimated using only the motor terminal quantities and motor nameplate data.

\section{CONCLUSION}

This paper has presented an up-to-date literature survey on in-service motor-efficiency estimation. More than 20 efficiency-estimation methods are studied and classified into nine categories according to their physical nature. Among them, the following six are of interest for in-service motor testing: 1) nameplate methods; 2) slip methods; 3 ) current methods; 4) equivalent-circuit methods; 5) segregated loss methods; and 6) torque methods. These six categories of methods are 
TABLE II

COMPARISON OF THE MOTOR-EFFICIENCY ESTIMATION METHODS

\begin{tabular}{|c|c|c|c|c|c|c|c|c|c|c|}
\hline \multirow{2}{*}{$\begin{array}{l}\text { Motor Efficiency } \\
\text { Estimation Methods }\end{array}$} & \multicolumn{8}{|c|}{ Tests and Measurements/Estimations Required } & \multicolumn{2}{|c|}{ Performance } \\
\hline & $\begin{array}{c}\text { No } \\
\text { Load }\end{array}$ & $\begin{array}{l}\text { Full } \\
\text { Load }\end{array}$ & $\begin{array}{c}\text { Un- } \\
\text { power }\end{array}$ & \begin{tabular}{|l} 
Variable \\
Volt/Freq
\end{tabular} & Speed & Torque & $\begin{array}{c}\text { Nameplate } \\
\text { Info }\end{array}$ & \begin{tabular}{|c|} 
Stator \\
Resistance
\end{tabular} & $\begin{array}{c}\text { Intrusion } \\
\text { Level }\end{array}$ & $\begin{array}{c}\text { Anticipated } \\
\text { Error }\end{array}$ \\
\hline Standard Nameplate Method & No & No & No & No & No & No & Yes & No & Low & $10 \%[3]$ \\
\hline V\&B Method I & Yes & No & Yes & No & Measure & No & No & Measure & High & N/A \\
\hline V\&B Method II & No & No & Yes & No & Measure & No & Yes & Measure & Medium & $2.5 \%[5]$ \\
\hline Standard Slip Method & No & No & Yes & No & Measure & No & Yes & Measure & Low & $7 \%[3]$ \\
\hline Upper Bound Slip Method & Yes & No & Yes & No & Measure & No & Yes & Measure & Medium & $8 \%[5]$ \\
\hline Standard Current Method & Yes & No & No & No & No & No & Yes & No & Medium & $6 \%[3]$ \\
\hline IEEE Std-112 Method F1 & Yes & No & Yes & Yes & Measure & No & Yes & Measure & High & $5 \%[3]$ \\
\hline ORMEL96 Method & No & No & Optional & No & Measure & No & Yes & Optional & Low & $4 \%[4]$ \\
\hline Rockwell Eff. Wizard Method & Optional & No & No & No & Measure & No & Yes & Measure & Medium & $1 \%[14]$ \\
\hline Locked Rotor Method & Yes & No & No & Yes & Measure & No & Yes & No & High & N/A \\
\hline Standstill Freq. Resp. Method & Yes & No & Yes & Yes & Measure & No & No & Measure & High & N/A \\
\hline IEEE Std-112 Method E1 & Yes & Yes & Yes & Yes & Measure & No & Yes & Measure & High & $3 \%[3]$ \\
\hline OHME Method & No & No & No & No & Measure & No & Yes & Measure & Medium & $2-3 \%[4]$ \\
\hline Air-gap Torque Method & Yes & No & No & No & Measure & Estimate & Yes & Measure & High & $0.5 \%[4]$ \\
\hline Shaft Torque Method & No & No & No & No & Measure & Measure & No & No & High & $<0.5 \%[3]$ \\
\hline
\end{tabular}

finally summarized and compared in Table II. In general, the more intrusive the method is, the more accurate the efficiency estimate is.

This paper also summarizes the recent progress in the online rotor speed and stator-resistance-estimation techniques. Appropriate adoption of these techniques can greatly reduce the intrusion levels of various efficiency-estimation methods, especially the four suggested candidate methods, in which direct resistance and speed measurements are the main source of the intrusiveness.

Another major contributions of this paper are: 1) a general approach for developing nonintrusive motor-efficiencyestimation methods is proposed, incorporating rotor-speed, stator-resistance, and no-load-loss estimations, and 2) four candidates are suggested out of more than 20 traditional methods for further modifications to become nonintrusive.

\section{APPENDIX}

Equations in the MRAS method [38] are

$$
\begin{gathered}
P=\left(\nu_{q s}^{s} i_{d s}^{s}-\nu_{d s}^{s} i_{q s}^{s}\right)+\sigma L_{s}\left(i_{d s}^{s} p i_{q s}^{s}-i_{q s}^{s} p i_{d s}^{s}\right) \\
\hat{P}=\frac{L_{m}}{L_{r}}\left\{\frac{\hat{R}_{\mathrm{r}}}{L_{r}}\left(\hat{\lambda}_{d r}^{s} i_{q s}^{s}-\hat{\lambda}_{q r}^{s} i_{d s}^{s}\right)\right. \\
\left.\quad+\omega_{\mathrm{r}}\left(\hat{\lambda}_{d r}^{s} i_{d s}^{s}-\hat{\lambda}_{q r}^{s} i_{q s}^{s}\right)\right\} \\
p \hat{\lambda}_{q r}^{s}=-\frac{\hat{R}_{\mathrm{r}}}{L_{r}} \hat{\lambda}_{q r}^{s}+\omega_{\mathrm{r}} \hat{\lambda}_{d r}^{s}+L_{m} \frac{\hat{R}_{\mathrm{r}}}{L_{r}} i_{q s}^{s} \\
p \hat{\lambda}_{d r}^{s}=-\frac{\hat{R}_{\mathrm{r}}}{L_{r}} \hat{\lambda}_{d r}^{s}-\omega_{\mathrm{r}} \hat{\lambda}_{q r}^{s}+L_{m} \frac{\hat{R}_{\mathrm{r}}}{L_{r}} i_{d s}^{s} \\
\Delta R_{\mathrm{r}}=k_{P} \varepsilon_{P}+k_{I} \int \varepsilon_{P} d t \\
\hat{R}_{\mathrm{r}}(n)=\hat{R}_{\mathrm{r}}(n-1)+\Delta R
\end{gathered}
$$

$$
\begin{aligned}
\hat{\lambda}_{q s}^{s} & =\frac{L_{m}}{L_{r}} \hat{\lambda}_{q r}^{s}+\sigma L_{s} i_{q s}^{s} \\
\hat{\lambda}_{d s}^{s} & =\frac{L_{m}}{L_{r}} \hat{\lambda}_{d r}^{s}+\sigma L_{s} i_{d s}^{s} \\
\hat{R}_{s} & =\frac{\nu_{q s}^{e s}-\omega_{s} \hat{\lambda}_{q s}^{e s}}{i_{q s}^{e s}} .
\end{aligned}
$$

Equations in Lee's methods [43] are

$$
\begin{aligned}
\hat{\lambda}_{d r}^{e} & =\left(\frac{L_{r}}{\omega_{e} L_{m}}\right) \nu_{q s}^{e}+\left(\frac{\sigma L_{s} L_{r}}{L_{m}}\right) i_{d s}^{e} \\
\hat{R}_{\mathrm{r}} & =\frac{\left(s \omega_{e}\right) L_{r}}{\sqrt{\left(\frac{L_{m} i_{d s}^{e}}{\hat{\lambda}_{d r}^{e}}\right)-1}} \\
\hat{R}_{s} & =\frac{\left(\frac{\nu_{d s}^{e}-s \omega_{e}^{2} L_{m} \hat{\lambda}_{d r}^{e}}{\hat{R}_{\mathrm{r}}}\right)}{i_{d s}^{e}} .
\end{aligned}
$$

\section{ACKNOWLEDGMENT}

The authors would like to thank Dr. Y. El-Ibiary, Dr. J. S. Hsu, Dr. X. Huang, and Dr. L. M. Tolbert for their assistance in collecting references.

\section{REFERENCES}

[1] B. Lu, T. G. Habetler, and R. G. Harley, "A survey of available methods for evaluating in-service motor efficiency," Georgia Inst. Technol., Atlanta, EATON WSN Res. Rep., Oct. 2004.

[2] T. G. Habetler, R. G. Harley, R. M. Tallam, S. B. Lee, R. Obaid, and J. Stack, "Complete current-based induction motor condition monitoring: Stator, rotor, bearings, and load," in Proc. CIEP VIII IEEE Int. Power Electron. Congr., Oct. 2002, pp. 3-8.

[3] J. Hsu, J. Kueck, M. Olszewski, D. Casada, P. Otaduy, and L. Tolbert, "Comparison of induction motor field efficiency estimation methods," IEEE Trans. Ind. Appl., vol. 34, no. 1, pp. 117-125, Jan./Feb. 1998.

[4] J. D. Kueck, M. Olszewski, D. A. Casada, J. Hsu, P. J. Otaduy, and L. M. Tolbert, "Assessment of methods for estimating motor efficiency 
and load under field conditions," Oak Ridge Nat. Lab., Oak Ridge, TN, Oak Ridge National Laboratory Rep. ORNL/ TM-13165, 1996.

[5] A. Wallance, A. Von Jouanne, E. Wiedenbrug, E. Matheson, and J. Douglass, "A laboratory assessment of in-service and non-intrusive motor efficiency testing methods," Electr. Power Compon. Syst., vol. 29, no. 6, pp. 517-529, Jun. 2001.

[6] J. D. Kueck, "Development of a method for estimating motor efficiency and analyzing motor condition," in Proc. IEEE Pulp and Paper Ind. Tech. Conf., Jun. 1998, pp. 67-72.

[7] W. Benning, "Method of determining the efficiency of asynchronous motors and apparatus for carrying out the method," U.S. Patent 5659232 , Aug. 19, 1997.

[8] R. L. Nailen, "Finding true power output isn't easy," Electr. App., Feb. 1994.

[9] "In-plant electric motor loading and efficiency techniques," Ontario Hydro, Toronto, ON, Canada, Market Literature.

[10] B. S. Guru and H. R. Hiziroglu, Electric Machinery and Transformers, 3rd ed. New York: Oxford Univ. Press, 2001, ch. 8.

[11] J. R. Holmquist, J. A. Rooks, and M. E. Richter, "Practical approach for determining motor efficiency in the field using calculated and measured values," IEEE Trans. Ind. Appl., vol. 40, no. 1, pp. 242-248, Jan./Feb. 2004.

[12] Motors and Generators, NEMA MG1-2003 Standard, 2003.

[13] IEEE Standard Test Procedure for Polyphase Induction Motors and Generators, IEEE Standard 112-2004, Nov. 2004.

[14] Y. El-Ibiary, "An accurate low-cost method for determining electric motors's efficiency for the purpose of plant energy management," IEEE Trans. Ind. Appl., vol. 39, no. 4, pp. 1205-1210, Jul/Aug. 2003.

[15] A. Dell'Aquila, L. Salvatore, and M. Savino, "A new test method for determination of induction motor efficiency," IEEE Trans. Power Appl. Syst., vol. PAS-103, no. 10, pp. 2961-2973, Oct. 1984.

[16] J. R. Willis, G. J. Brock, J. S. Edmonds, "Derivation of induction motor models from standstill frequency response tests," Electric Power Research Institute, Palo Alto, CA, Rep. GS-6250, Jul. 1991.

[17] J. O. Ojo, V. Ostovic, T. A. Lipo, and J. C. White, "Measurement and computation of starting torque pulsations of salient pole synchronous motors," IEEE Trans. Energy Convers., vol. 5, no. 1, pp. 176-182, Mar. 1990.

[18] J. Hsu and B. P. Scoggins, "Field test of motor efficiency and load changes through air-gap torque," IEEE Trans. Energy Convers., vol. 10, no. 3, pp. 477-483, Sep. 1995.

[19] K. Ikuanobe and T. Wilke, "Guildlines for implementing an energyefficient motor retrofit program," unpublished.

[20] E. J. Wiedenbrüg and A. K. Wallace, "Induction machine speed extraction by analysis of stator current signatures," in Proc. Electric Manuf. Conf., Oct. 1998.

[21] L. Ben-Brahim and A. Kawamura, "A fully digitized field-oriented controlled induction motor drive using only current sensors," IEEE Trans. Ind. Electron., vol. 39, no. 3, pp. 241-249, Jun. 1992.

[22] P. L. Jansen and R. D. Lorenz, "Accuracy limitations of velocity and flux estimation in direct field oriented induction machines," in Proc. Eur. Power Electron. Conf., Sep. 1993, vol. 4, pp. 312-318.

[23] M. Ishida and K. Iwata, "Steady-state characteristics of a torque and speed control system of an induction motor utilizing rotor slot harmonics for slip frequency sensing," IEEE Trans. Power Electron., vol. PE-2, no. 3, pp. 257-263, Jul. 1987.

[24] B. Hämmerli, R. Tanner, and R. Zwicky, "A rotor speed detector for induction machines utilizing rotor slot harmonics and an active threephase injection," in Proc. 2nd Eur. Power Electron. Appl. Conf., 1987, vol. 2, pp. 599-604.

[25] R. Cuzner, R. Lorenz, and D. Novotny, "Application of nonlinear observers for rotor position detection on an induction motor using machine voltages and currents," in Proc. IEEE-IAS Annu. Meeting, Oct. 1990, vol. 1, pp. 416-421.

[26] B. Williams, J. Goodfellow, and T. Green, "Sensorless speed measurement of inverter driven squirrel cage induction motors," in Proc. 4th IEEE Int. Conf. Power Electron. Variable-Speed Drives Conf., Jul. 1990, pp. 297-300.

[27] A. Ferrah, K. Bradley, and G. Asher, "Sensorless speed detection of inverter fed induction motors using rotor slot harmonics and fast Fourier transform," in Proc. IEEE Power Electron. Spec. Conf., Jun/Jul. 1992, vol. 1, pp. 279-286.

[28] _ _ "An investigation into speed measurement of induction motor drives using rotor slot harmonics and spectral estimation techniques," in Proc. Int. Elect. Mach. and Drive Conf., Sep. 1993, pp. 185-189.

[29] K. D. Hurst and T. G. Habetler, "Sensorless speed measurement using current harmonic spectral estimation in induction machine drives," IEEE Trans. Power Electron., vol. 11, no. 1, pp. 66-73, Jan. 1996.
[30] _ - "A comparison of spectrum estimation techniques for sensorless speed detection in induction machines," IEEE Trans. Ind. Appl., vol. 33, no. 4, pp. 898-905, Jul./Aug. 1997.

[31] P. L. Jansen and R. D. Lorenz, "Transducerless position and velocity estimation in induction and salient ac machines," IEEE Trans. Ind. Appl., vol. 31, no. 2, pp. 240-247, Mar./Apr. 1995.

[32] IEEE Standard Test Code for Resistance Measurement, IEEE Standard 118-1978, Mar. 1992

[33] S. Mir, M. E. Elbuluk, and D. S. Zinger, "PI and fuzzy estimators for tuning the stator resistance in direct torque control of induction machines," IEEE Trans. Power Electron., vol. 13, no. 2, pp. 279-287, Mar. 1998.

[34] T. G. Habetler, F. Profumo, G. Griva, M. Pastorelli, and A. Bettini, "Stator resistance tuning in a stator-flux field-oriented drive using an instantaneous hybrid flux estimator," IEEE Trans. Power Electron., vol. 13, no. 1, pp. 125-133, Jan. 1998.

[35] C. B. Jacobina, J. E. C. Filho, and A. M. N. Lima, "On-line estimation of the stator resistance of induction machines based on zero sequence model," IEEE Trans. Power Electron., vol. 15, no. 2, pp. 346-353, Mar. 2000.

[36] K. Minami, M. Velez-Reyes, D. Elten, G. C. Verghese, and D. Filbert, "Multi-stage speed and parameter estimation for induction machines," in Proc. IEEE Power Electron. Spec. Conf., Jun. 1991, pp. 596-604.

[37] H. Kubota and K. Matsuse, "Speed sensorless field-oriented control of induction motor with rotor resistance adaptation," IEEE Trans. Ind. Appl., vol. 30, no. 5, pp. 1219-1224, Sep./Oct. 1994.

[38] L. Zhen and L. Xu, "Sensorless field orientation control of induction machines based on a mutual MRAS scheme," IEEE Trans. Ind. Electron., vol. 45, no. 5, pp. 824-831, Oct. 1998.

[39] R. Beguanne and M. E. H. Benbouzid, "Induction motors thermal monitoring by means of rotor resistance identification," IEEE Trans. Energy Convers., vol. 14, no. 3, pp. 566-570, Sep. 1999.

[40] S. B. Lee, T. G. Habetler, R. G. Harley, and D. J. Gritter, "An evaluation of model-based stator resistance estimation for induction motor stator winding temperature monitoring," IEEE Trans. Energy Convers., vol. 17, no. 1 , pp. 7-15, Mar. 2002.

[41] A. D. Inuwa, "Smart motor protection," Ph.D. dissertation, Univ. Sussex, Brighton, U.K., 1992.

[42] D. A. Paice, "Motor thermal protection by continuous monitoring of winding resistance," IEEE Trans. Ind. Electron. Instrum., vol. IECI-27, no. 3, pp. 137-141, Aug. 1980.

[43] S. B. Lee and T. G. Habetler, "An online stator winding resistance estimation technique for temperature monitoring of line-connected induction machines," IEEE Trans. Ind. Appl., vol. 39, pp. 685-694, May/Jun. 2003.

[44] U. S. DOE Energy Efficiency and Renewable Energy (EERE), DOE Industry Tools. DOE MotorMaster+ Website. [Online]. Available: http://www1.eere.energy.gov/industry/bestpractices/software.html

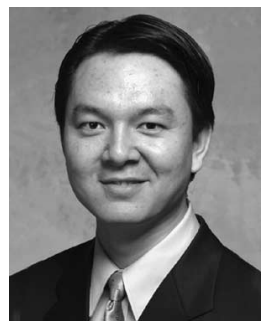

Bin Lu (S'00) received the B.Eng. degree in automation from Tsinghua University, Beijing, China, in 2001 and the M.S. degree in electrical engineering from the University of South Carolina, Columbia, in 2003. He is currently working toward the Ph.D. degree at the Georgia Institute of Technology, Atlanta.

From 2001 to 2003, he was with the virtue-testbed (VTB) group of the University of South Carolina, as a graduate Research Assistant. While there, he was involved in the development of a real-time VTB (VTB-RT) for the hardware-in-the-loop testing of multitechnical dynamic systems. Since July 2004, he has been working on the application of wireless sensor networks in energy evaluation and condition monitoring of electric machines in collaboration with Eaton Corporation, as a graduate Research Assistant in the electric power group of the Georgia Institute of Technology. His research interests include electric-machine efficiency estimation, condition monitoring and drives, control of power electronics, application of wireless sensor networks in electric power areas, and modeling and simulation. He has authored or coauthored over 20 papers and has one patent pending. 


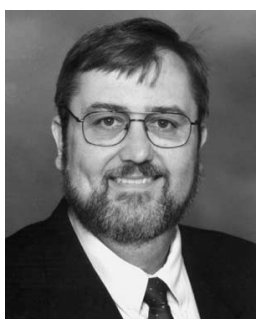

Thomas G. Habetler (S'83-M'83-SM'92-F'02) received the B.S. and M.S. degrees from Marquette University, Milwaukee, WI, in 1981 and 1984, respectively, and the Ph.D. degree from the University of Wisconsin, Madison, in 1989, all in electrical engineering.

From 1983 to 1985 , he was with the ElectroMotive Division of General Motors as a Project Engineer. Since 1989, he has been with the Georgia Institute of Technology, Atlanta, where he is currently a Professor of electrical engineering. His research interests are in electric-machine protection and condition monitoring, switching converter technology, and drives. He has published over 100 papers in the field. He is a regular consultant to industry in the field of condition-based diagnostics for electrical systems.

Dr. Habetler received four conference prize paper awards from the IEEE Industry Applications Society. He currently serves as IEEE Division II DirectorElect. He is the Past President of the IEEE Power Electronics Society and Past Chair of the Industrial Power Converter Committee of the IEEE Industry Applications Society.

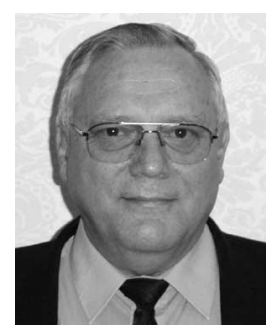

Ronald G. Harley (M'77-SM'86-F'92) received the B.Sc.Eng. (cum laude) and M.Sc.Eng. (cum laude) degrees from the University of Pretoria, Pretoria, South Africa, in 1960 and 1965, respectively, and the Ph.D. degree from London University, London, U.K., in 1969, all in electrical engineering.

In 1971, he was appointed as the Chair of Electrical Machines and Power Systems at the University of Natal, Durban, South Africa. At the University of Natal, he was a Professor of electrical engineering for many years, including the Department Head and Deputy Dean of Engineering. He is currently the Duke Power Company Distinguished Professor at the Georgia Institute of Technology, Atlanta. His research interests include the dynamic behavior and condition monitoring of electric machines, motor drives, power systems and their components, and controlling them by the use of power electronics and intelligent control algorithms. He has coauthored some 380 papers in refereed journals and international conference proceedings and is the holder of three patents.

Dr. Harley is a Fellow of the Institution of Electrical Engineers, U.K. $\mathrm{He}$ is also a Fellow of the Royal Society in South Africa, and a Founder Member of the Academy of Science in South Africa formed in 1994. During 2000 and 2001, he was one of the IEEE Industry Applications Society's six Distinguished Lecturers. He was the Vice-President of Operations of the IEEE Power Electronics Society (2003-2004) and Chair of the Atlanta Chapter of the IEEE Power Engineering Society. He is currently Chair of the Distinguished Lecturers and Regional Speakers program of the IEEE Industry Applications Society. He received the Cyrill Veinott Award in 2005 from the IEEE Power Engineering Society for "Outstanding contributions to the field of electromechanical energy conversion." He has received ten prize paper awards. 


\title{
A Nonintrusive and In-Service Motor-Efficiency Estimation Method Using Air-Gap Torque With Considerations of Condition Monitoring
}

\author{
Bin Lu, Member, IEEE, Thomas G. Habetler, Fellow, IEEE, and Ronald G. Harley, Fellow, IEEE
}

\begin{abstract}
Energy usage evaluation and condition monitoring for electric machines are important in industry for overall energy savings. They are often expected to be implemented in an integrated product because of many common requirements such as data collection. Because of the uninterrupted characteristic of industrial processes, traditional methods defined in IEEE Standard 112 cannot be used for these in-service motors. This paper proposes a truly nonintrusive method for in-service motor-efficiency estimation based on air-gap torque using only motor terminal quantities and nameplate information, with special considerations of motor condition monitoring requirements. Rotor speed and stator resistance, the stumbling blocks of most in-service testing methods, are extracted from motor input currents instead of being measured. The no-load test, which is required for calculating the rotational loss and core loss, is eliminated by using empirical values. Stray-load loss is assumed according to the motor horsepower as suggested in IEEE Standard 112. Finally, the proposed method is validated by testing three induction motors with different configurations. Experimental results show that the proposed method can estimate motor efficiencies with less than $2 \%$ errors under normal load conditions.
\end{abstract}

Index Terms-Air-gap torque (AGT), condition monitoring, efficiency estimation, electric machines, IEEE Standard 112, inservice testing.

\section{NOMENCLATURE}

$V_{s} \quad$ Magnitude of stator phase voltage phasor.

$I_{s} \quad$ Magnitude of stator phase current phasor.

$I_{r} \quad$ Magnitude of rotor phase current phasor.

$v_{a b}, v_{b c}, v_{c a} \quad$ Stator line voltages.

$i_{a}, i_{b}, i_{c} \quad$ Stator phase currents.

$\cos \varphi \quad$ Power factor.

$p \quad$ Number of poles.

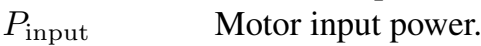

$P_{\text {output }} \quad$ Motor output power or shaft power.

$P_{\text {ag }} \quad$ Air-gap power or electromagnetic power.

Paper IPCSD-08-012, presented at the 2006 Industry Applications Society Annual Meeting, Tampa, FL, October 8-12, and approved for publication in the IEEE TRANSACTIONS ON INDUSTRY APPLICATIONS by the Electric Machines Committee of the IEEE Industry Applications Society. Manuscript submitted for review October 1, 2006 and released for publication February 19, 2008. Current version published November 19, 2008. This work was supported by the United States Department of Energy (DOE) under Grant DE-FC3604GO14000.

B. Lu is with the Innovation Center, Eaton Corporation, Milwaukee, WI 53216 USA (e-mail: binlu@ieee.org).

T. G. Habetler and R. G. Harley are with the School of Electrical and Computer Engineering, Georgia Institute of Technology, Atlanta, GA 30332 USA (e-mail: thabetler@ece.gatech.edu; rharley@ece.gatech.edu).

Color versions of one or more of the figures in this paper are available online at http://ieeexplore.ieee.org.

Digital Object Identifier 10.1109/TIA.2008.2006297

$\begin{array}{ll}P_{m} & \text { Developed mechanical power. } \\ R_{s} & \text { Stator resistance. } \\ R_{r} & \text { Rotor resistance. } \\ s & \text { Motor slip. } \\ T_{\mathrm{ag}} & \text { Air-gap torque or electromagnetic torque. } \\ T_{\mathrm{shaft}} & \text { Shaft torque or output torque. } \\ W_{\mathrm{LLs}} & \text { Stator stray-load loss. } \\ W_{\mathrm{LLr}} & \text { Rotor stray-load loss. } \\ W_{s} & \text { Stator copper loss. } \\ W_{\mathrm{core}} & \text { Core loss. } \\ W_{r} & \text { Rotor copper loss. } \\ W_{\mathrm{fw}} & \text { Friction and windage loss or mechanical loss. } \\ \omega_{\text {syn }} & \text { Synchronous speed. } \\ \omega_{r} & \text { Rotor mechanical speed. } \\ \eta & \text { Motor efficiency. } \\ \theta_{d a} & \text { Angle between } d \text {-axis and stator } a \text {-axis. } \\ v_{d q s} & \text { Stator voltage space vector. } \\ i_{d q s} & \text { Stator current space vector. } \\ \lambda_{d q s} & \text { Total flux linkage space vector. }\end{array}$

\section{INTRODUCTION}

$\mathbf{M}$ OTOR-DRIVEN systems use nearly $70 \%$ of the total electric energy consumed by industry in the United States. In industry, only motors above $500 \mathrm{hp}$ are usually monitored because of their high costs. However, motors below 500 hp make up $99.7 \%$ of the motors in service and consume $71 \%$ of the energy used. On average, these motors operate at no more than $60 \%$ of their rated load because of oversized installations or underloaded conditions, and thus at reduced efficiency which results in wasted energy [1]. Therefore, low-cost methods are needed in monitoring the motor energy usage and health conditions, particularly for medium- and small-size motors.

Energy usage evaluation and condition monitoring for electric machines are important in industry for overall energy savings. They are often expected to be implemented in an integrated product because of many common requirements such as data collections. In industrial plants, the motor terminal voltages and currents are readily available from the motor control centers, since there are already preinstalled potential transformers (PTs) and current transformers (CTs) for protection purpose. The terminal voltage and current measurements bring no additional costs in terms of data collection. Most traditional efficiency evaluation methods also require the rotor speed and shaft torque to be measured to calculate the output power [2]. The speed and torque transducers are very costly, and their installations are highly intrusive. In most cases, it is even 


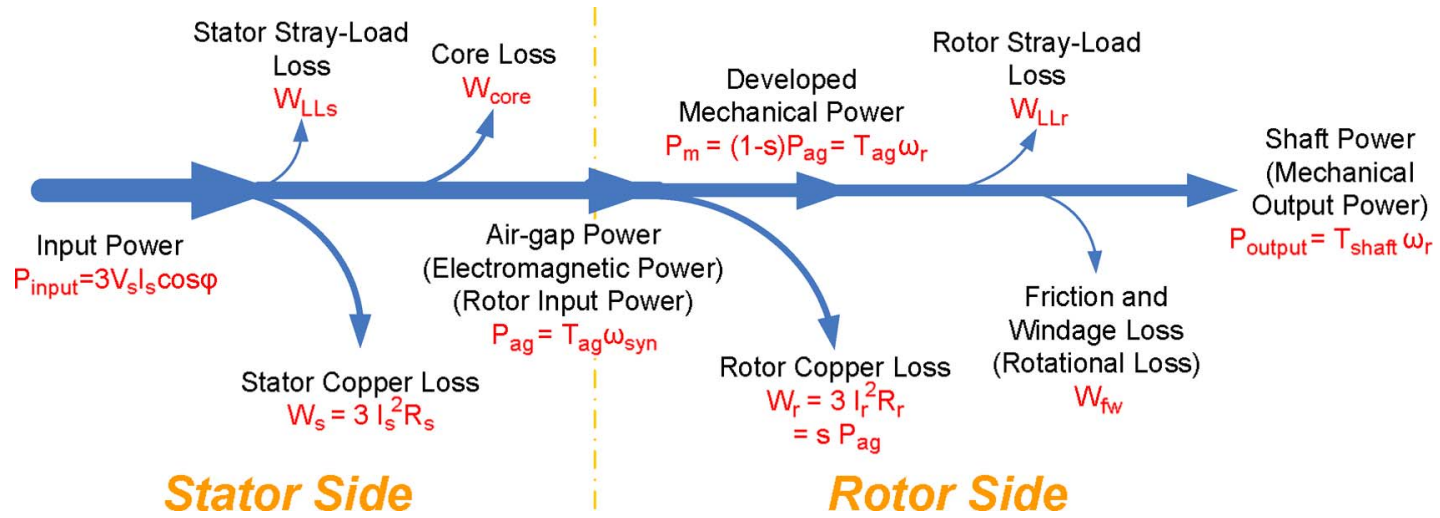

Fig. 1. Power flow and loss definition of induction motors.

not possible to install this equipment because the motors may be buried inside a machine or there is no space to attach such transducers between the motor and the load. Because of the uninterrupted characteristic of continuous industrial processes, traditional methods defined in IEEE Standard 112 cannot be used for these in-service motors. Nonintrusive methods, which only rely on terminal voltages and currents while a motor is running, have brought increasing attention during the recent years for these continuous applications [3], [4].

A possible approach of evaluating efficiency is to use the premeasured motor characteristic efficiencies under representative load conditions during motor development to predict the actual efficiencies in operation. This approach is nonintrusive in nature. However, its usage, in practice, is greatly limited by the following facts: 1) The characteristic efficiencies under representative load conditions are not always available from motor data sheets and 2) the characteristic efficiencies are generic data which could differ greatly from actual efficiencies for a specific motor due to many factors, such as inaccurate nameplate information and different working environments. Therefore, the authors only focus on online methods in this paper.

This paper proposes a nonintrusive air-gap torque (NAGT) method for in-service motor-efficiency estimation using only motor terminal quantities and nameplate information, with special considerations of motor condition monitoring requirements. Rotor speed and stator resistance, the stumbling blocks of most in-service testing methods, are extracted from motor input currents instead of being measured. No-load test, which is required for calculating the rotational loss and core loss, is eliminated by using empirical values. Stray-load loss is assumed according to the motor horsepower as suggested in IEEE Standard 112. As a continuation of the authors' previous work in [3], where a general approach is proposed for nonintrusive efficiency estimation based on a survey study, this paper specifically focuses on the experimental validation of the proposed NAGT method. Three induction motors with different configurations are tested, and the results are presented and analyzed.

\section{ORIGIN AND MOTIVATION}

The key to the electric machine energy usage evaluation is nonintrusive motor-efficiency estimation. The same authors conducted a survey on motor-efficiency estimation methods, specifically considering the advances in sensorless speed estimation and in-service stator resistance estimation techniques during the last decade [3]. Three candidate methods, ORMEL96, OHME, and AGT methods, are suggested in the survey to be improved for nonintrusive and in-service motor testing.

Among these methods, the air-gap torque (AGT) method is regarded as the best in terms of accuracy and ease of implementation. It was developed based on the "AGT" [5] or "sensorless torque" equations [6], developed in 1990s. This method has been verified by several experiments to be an effective motorefficiency estimation method [7]-[9]. The input power to the motor is calculated from input line voltages and phase currents, and the output power is calculated from rotor speed and shaft torque. Air-gap flux is calculated from the integral of currents and voltages subtracting the stator $I R$ drop. Shaft torque is obtained by subtracting the torque losses associated with the friction and windage loss $W_{\mathrm{fw}}$ and rotor stray-load loss $W_{\mathrm{LLr}}$ from the calculated AGT. The power flow and definition of each loss term in an induction motor are shown in Fig. 1.

The AGT method requires the following data to be measured: line voltages, phase currents, rotor speed, and stator resistance. In addition, to measure $W_{\text {core }}$ and $W_{\mathrm{fw}}$, a no-load test must be run. The requirement of speed, stator resistance measurements, and the no-load test are the main drawbacks of this method, which makes it a "high-intrusive" method and prevents its use in in-service motor testing.

To overcome these problems, a "nonintrusive" method based on AGT equations can be developed by making the following improvements to the original AGT method. It still keeps satisfactory accuracy but greatly reduces the intrusion levels.

1) The rotor speed does not come from direct measurement. It is estimated at high accuracy ( 0.005 p.u.) from motor current spectrum analysis extracting slot harmonics from stator currents [10].

2) The stator resistance is estimated using online dc signal injection methods using only the input line voltages and phase currents [11], [12], instead of direct measurement from unpowered testing or rough approximation from the nameplate data.

3) A no-load test should be avoided due to its high intrusiveness. Instead, no-load data are obtained by three options at different levels of accuracy/intrusiveness.

a) If the motor being tested is to be newly installed or has a regular scheduled maintenance, the no-load 
TABLE I

ASSUMEd VALUES FOR STRAY-LOAD LOSS IN IEEE STANDARD 112

\begin{tabular}{|c|c|c|}
\hline \multicolumn{2}{|c|}{ Machine Rating } & $\begin{array}{c}\text { Stray-Load Loss Percent of } \\
\text { Rated Output Power }\end{array}$ \\
\hline $1-125 \mathrm{hp}$ & $1-90 \mathrm{~kW}$ & $1.8 \%$ \\
\hline $126-500 \mathrm{hp}$ & $91-375 \mathrm{~kW}$ & $1.5 \%$ \\
\hline $501-2499 \mathrm{hp}$ & $376-1850 \mathrm{~kW}$ & $1.2 \%$ \\
\hline $2500 \mathrm{hp}$ and up & $1851 \mathrm{~kW}$ and up & $0.9 \%$ \\
\hline
\end{tabular}

test could be done during the scheduled operation downtime to give best accuracy.

b) If the motor under test is available in the motor master database, the no-load data are estimated from the generic design data [13].

c) If neither of the above is available, the no-load data can be estimated from empirical values using only motor nameplate data, similar to the OHME and ORMEL96 methods [8]. More specifically, the combined no-load losses are assumed to be $3.5 \%$ of rated output power, the friction and windage loss is $1.2 \%$ rated output power, and the stray-load loss is estimated from Table I as in IEEE Standard 112 [2].

\section{SENSORLESS Rotor SpeEd Estimation}

Direct measurement of the rotor speed requires a shaftmounted speed encoder or optical tachometer to be installed. This reduces the reliability and increases the cost. During the past decade, numerous sensorless rotor speed estimation schemes have been proposed [3]. A sensorless speed estimation method based on current harmonics has been developed by the same research group in [10]. It is based on the motor magnetic saliency harmonics, which arise from rotor slotting and eccentricity.

The frequency of these rotor and dynamic eccentricity harmonics is related to the rotor speed by

$$
f_{\mathrm{seh}}=f_{1}\left[\left(k R+n_{d}\right) \frac{1-s}{p / 2}+n_{w}\right]
$$

where $f_{\text {seh }}$ is the frequency of rotor-related harmonic components; $f_{1}$ is the supply frequency; $k=0,1,2, \ldots ; R$ is the number of rotor slots; $p$ is the number of poles; $s$ is the motor slip; $n_{d}=0, \pm 1, \pm 2, \ldots$ is the order of rotor eccentricity; and $n_{w}= \pm 1, \pm 3, \ldots$ is the air-gap MMF harmonics order.

The speed estimation process consists of two algorithms: an initialization algorithm and an online speed-detection algorithm. The initialization algorithm employs the eccentricity harmonics to determine the value of $R$ and an optimal set of numbers for $k, n_{d}$, and $n_{w}$, which are usually unknown quantities. After that, the subsequent online speed-detection algorithm estimates the rotor speed by

$$
s=1-\frac{p}{2} \cdot \frac{\frac{f_{\mathrm{seh}}}{f_{1}}-n_{w}}{k R+n_{d}} .
$$

The slip estimate is independent of motor parameters, only requiring the number of poles. The determination of slot harmonic frequency requires the motor to be in steady state so

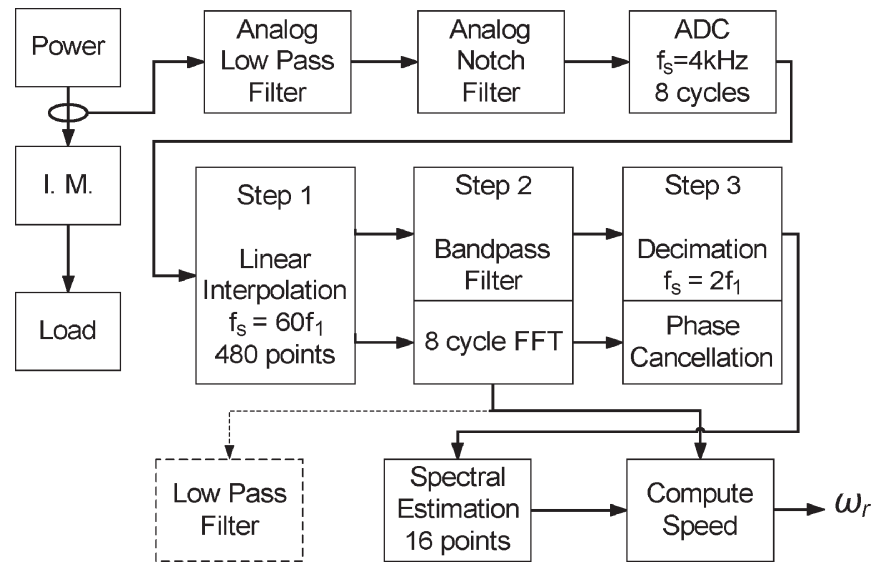

Fig. 2. Speed-detection algorithm using magnetic saliency harmonics.

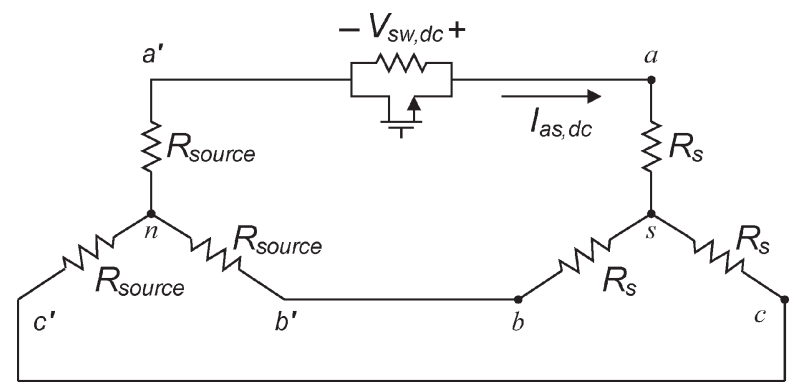

Fig. 3. DC equivalent circuit of source, motor, and dc injection.

that $f_{1}$ and $s$ are nearly constant; thus, this speed-detection method is ideal for online motor-efficiency estimation, where only steady states are concerned. This method provides robust speed estimate down to $1-\mathrm{Hz}$ operation with very high accuracy of within $5 \mathrm{r} / \mathrm{min}$ at high speeds and 0.005 -p.u. slip at low speeds. Fig. 2 shows the overall signal processing algorithm. For simplicity, the details are omitted here and available in [10].

\section{Online Stator Resistance Estimation}

Traditionally, the dc resistance of the stator winding is measured through an unpowered test. The measurement procedure is given in IEEE Standard 118. The advantage of the direct resistance measurement is the high accuracy. However, direct measurement is not acceptable for in-service motor testing because of the following disadvantages.

1) It requires the motor to be disconnected from service to perform an unpowered test. Therefore, the intrusion level is very high.

2) It only records the resistance value at a certain temperature. The actual resistance, however, is a linear function of the winding temperature.

To overcome these problems, many online estimation methods have been developed over the years [3]. Among them, the dc signal injection methods have proven to be very practical, in particular the use of a simple MOSFET-controlled circuit to intermittently inject a controllable dc bias into the motor [11]. The circuit structure is shown in Fig. 3. This method has low power dissipation and torque distortion and is capable of providing accurate stator resistance estimate under motor startup, load variation, and abnormal cooling conditions. 
The installation of the injection circuit can be easily done in the motor control center for mains-fed machines. The stator resistance estimation process can be regarded as nonintrusive after the injection circuit is developed and included. However, the injected dc signal produces unbalances in the stator voltages and currents and causes additional power dissipation and torque distortion. For soft-starter-connected and inverter-fed machines, this simple circuit is not required, since dc signal injection can be easily implemented in a software mode by controlling the excitation signals [12].

\section{NAGT METHOD}

The novelty and importance of this NAGT method come from the fact that only motor terminal quantities and nameplate information are needed for efficiency estimation while the motor is operating in service. The details of this method are given below.

\section{A. Data Measurements: Only Voltages and Currents}

For many induction motors, the neutral points are not accessible from the motor terminals. Therefore, only the line-to-line voltages are available. For a three-phase wye-connected motor, the line voltages and phase currents are assumed to add to zero as in

$$
\begin{aligned}
v_{a b}+v_{b c}+v_{c a} & =0 \\
i_{a}+i_{b}+i_{c} & =0
\end{aligned}
$$

where, $v_{a b}, v_{b c}$, and $v_{c a}$ are the line voltages, and $i_{a}, i_{b}$, and $i_{c}$ are the phase currents.

Let $\theta_{d a}(t)$ be the angle between the $d$-axis and stator $a$-axis at time $t$, and the transformation matrix $T$ is defined as in (4), shown at the bottom of the page.

Assuming that the zero-sequence components in the stator voltages and currents are negligible, the stator voltage and current space vectors, $v_{d q s}$ and $i_{d q s}$, can be derived as in (5), shown at the bottom of the page (time index $t$ omitted from here on for simplicity)

Note that

$$
\begin{aligned}
& v_{a} \cos \theta_{d a}+v_{a} \cos \left(\theta_{d a}-\frac{2 \pi}{3}\right)+v_{a} \cos \left(\theta_{d a}-\frac{4 \pi}{3}\right)=0 \\
& v_{a} \sin \theta_{d a}+v_{a} \sin \left(\theta_{d a}-\frac{2 \pi}{3}\right)+v_{a} \sin \left(\theta_{d a}-\frac{4 \pi}{3}\right)=0 .
\end{aligned}
$$

From (3)-(6), the stator voltage and current space vectors can be obtained using only two voltage sensors $\left(v_{a b}\right.$ and $v_{c a}$ ) and two current sensors $\left(i_{a}\right.$ and $\left.i_{b}\right)$ as in (7), shown at the bottom of the page.

In industrial plants, the motors are connected to motor control centers, where PTs and CTs are already installed for protection purpose. The proposed efficiency estimation method relies completely on motor terminal voltages and currents, requiring no torque and speed measurements. The entire data collection process can take place at the motor control center with almost no additional cost. Another benefit of this data

$$
T\left(\theta_{d a}(t)\right)=\sqrt{\frac{2}{3}}\left[\begin{array}{ccc}
\cos \theta_{d a}(t) & \cos \left(\theta_{d a}(t)-\frac{2 \pi}{3}\right) & \cos \left(\theta_{d a}(t)-\frac{4 \pi}{3}\right) \\
-\sin \theta_{d a}(t) & -\sin \left(\theta_{d a}(t)-\frac{2 \pi}{3}\right) & -\sin \left(\theta_{d a}(t)-\frac{4 \pi}{3}\right)
\end{array}\right]
$$

$$
\begin{aligned}
v_{d q s}= & {\left[\begin{array}{l}
v_{d s} \\
v_{q s}
\end{array}\right]=T\left(\theta_{d a}\right)\left[\begin{array}{l}
v_{a} \\
v_{b} \\
v_{c}
\end{array}\right] \quad i_{d q s}=\left[\begin{array}{l}
i_{d s} \\
i_{q s}
\end{array}\right]=T\left(\theta_{d a}\right)\left[\begin{array}{c}
i_{a} \\
i_{b} \\
i_{c}
\end{array}\right] } \\
v_{d q s}= & {\left[\begin{array}{c}
\sqrt{\frac{2}{3}}\left[v_{a} \cos \theta_{d a}+v_{b} \cos \left(\theta_{d a}-\frac{2 \pi}{3}\right)+v_{c} \cos \left(\theta_{d a}-\frac{4 \pi}{3}\right)\right] \\
-\sqrt{\frac{2}{3}}\left[v_{a} \sin \theta_{d a}+v_{b} \sin \left(\theta_{d a}-\frac{2 \pi}{3}\right)+v_{c} \sin \left(\theta_{d a}-\frac{4 \pi}{3}\right)\right]
\end{array}\right] } \\
i_{d q s}= & {\left[\begin{array}{c}
\sqrt{\frac{2}{3}}\left[i_{a} \cos \theta_{d a}+i_{b} \cos \left(\theta_{d a}-\frac{2 \pi}{3}\right)+i_{c} \cos \left(\theta_{d a}-\frac{4 \pi}{3}\right)\right] \\
-\sqrt{\frac{2}{3}}\left[i_{a} \sin \theta_{d a}+i_{b} \sin \left(\theta_{d a}-\frac{2 \pi}{3}\right)+i_{c} \sin \left(\theta_{d a}-\frac{4 \pi}{3}\right)\right]
\end{array}\right] }
\end{aligned}
$$

$$
\begin{aligned}
& v_{d q s}=\left[\begin{array}{l}
\sqrt{\frac{2}{3}}\left[v_{a b} \cos \left(\theta_{d a}-\frac{2 \pi}{3}\right)-v_{c a} \cos \left(\theta_{d a}-\frac{4 \pi}{3}\right)\right] \\
\sqrt{\frac{2}{3}}\left[v_{a b} \sin \left(\theta_{d a}-\frac{2 \pi}{3}\right)-v_{c a} \sin \left(\theta_{d a}-\frac{4 \pi}{3}\right)\right]
\end{array}\right] \\
& i_{d q s}=\left[\begin{array}{c}
\sqrt{\frac{2}{3}}\left[i_{a} \cos \theta_{d a}+i_{b} \cos \left(\theta_{d a}-\frac{2 \pi}{3}\right)-\left(i_{a}+i_{b}\right) \cos \left(\theta_{d a}-\frac{4 \pi}{3}\right)\right] \\
-\sqrt{\frac{2}{3}}\left[i_{a} \sin \theta_{d a}+i_{b} \sin \left(\theta_{d a}-\frac{2 \pi}{3}\right)-\left(i_{a}+i_{b}\right) \sin \left(\theta_{d a}-\frac{4 \pi}{3}\right)\right]
\end{array}\right]
\end{aligned}
$$


measurement scheme is that the motor control centers are more easily accessible compared with the motors themselves, which further reduces the deployment cost.

\section{B. AGT Estimation}

The stator voltage equation in the $d-q$ system is

$$
v_{d q s}=R_{s} i_{d q s}+\frac{d \lambda_{d q s}}{d t}+\frac{d \theta_{d a}}{d t}\left[\begin{array}{cc}
0 & -1 \\
1 & 0
\end{array}\right] \lambda_{d q s}
$$

where $\lambda_{d q s}$ is the total flux linkage vector and $R_{s}$ is the average value of the three phase stator resistances.

Choose the stationary reference frame (denoted as the upper script "S"), i.e., $d \theta_{d a} / d t=0$. Thus, the flux linkage is given by the integral of input voltage minus the stator $I R$ drop

$$
\lambda_{d q s}^{\mathrm{S}}=\int\left(v_{d q s}^{\mathrm{S}}-R_{s} i_{d q s}^{\mathrm{S}}\right) d t .
$$

Then, the AGT $T_{\mathrm{ag}}$ can be derived as

$$
\begin{aligned}
T_{\mathrm{ag}}= & \frac{p}{2}\left|\lambda_{d q s}^{\mathrm{S}} \times i_{d q s}^{\mathrm{S}}\right| \\
= & \frac{\sqrt{3} p}{6}\left\{\left(i_{a}-i_{b}\right) \cdot \int\left[v_{c a}+R_{s}\left(2 i_{a}+i_{b}\right)\right] d t\right. \\
& \left.\quad+\left(2 i_{a}+i_{b}\right) \cdot \int\left[v_{a b}-R_{s}\left(i_{a}-i_{b}\right)\right] d t\right\} .
\end{aligned}
$$

The integrals in (10) represent the corresponding flux linkages. Since the sampling frequency of motor voltages and currents is usually large (greater than $2 \mathrm{kHz}$ ), a simple trapezoidal integration method can be used. Other numerical integration methods, e.g., Simpson's rules, can also be applied or better accuracy.

\section{Efficiency Estimation}

The instantaneous input power $P_{\text {input }}$ of a three-phase induction motor can be calculated from instantaneous voltages and currents as

$$
P_{\text {input }}=v_{a} i_{a}+v_{b} i_{b}+v_{c} i_{c}=-v_{c a}\left(i_{a}+i_{b}\right)-v_{a b} i_{b} .
$$

To reduce the ripples caused by the energy stored in the windings, the average value of instantaneous power is used to calculate efficiency.

The shaft output power $P_{\text {output }}$ is the product of the rotor speed $\omega_{r}$ and shaft torque $T_{\text {shaft }}$. The shaft torque is the difference between the AGT and torque losses corresponding to mechanical loss and rotor stray-load loss produced by rotor current and is given by

$$
T_{\text {shaft }}=T_{\mathrm{ag}}-\frac{W_{\mathrm{fw}}}{\omega_{r}}-\frac{W_{\mathrm{LLr}}}{\omega_{r}} .
$$

Since the majority of the stray-load loss is produced by rotor current, the rotor stray-load loss at the rated load is assumed according to Table I.
Finally, the efficiency $\eta$ is

$\eta=\frac{P_{\text {output }}}{P_{\text {input }}}=\frac{T_{\text {shaft }} \cdot \omega_{r}}{P_{\text {input }}}=\frac{T_{\mathrm{ag}} \cdot \omega_{r}-W_{\mathrm{fw}}-W_{\mathrm{LLr}}}{P_{\text {input }}}$.

\section{Discussions}

A significant contribution of this method is that the efficiency estimate is completely based on motor terminal quantities and motor nameplate information, eliminating the use of costly torque and speed transducers. This guarantees the nonintrusive nature of this method.

It can be easily implemented together with motor condition monitoring functions. Almost all condition monitoring algorithms require stator voltages and currents, while some also need stator resistance and rotor speed. When these algorithms are integrated together with the proposed efficiency estimation method, a common application interface can be built using the same collected motor data.

In this method, the air-gap flux (torque) is estimated directly using instantaneous voltage and current data. It does not need phasor computations and, thus, does not limit the analysis within a certain frequency frame. Therefore, the usage of this method can be extended from mains-fed motors to inverter-fed motors with any form of supply, such as space vector PWM, square wave, etc. Moreover, this method also considers the additional losses associated with unbalances and harmonics in the power supply, as well as the stator copper loss and stator stray-load loss.

However, the nonintrusiveness is obtained by sacrificing some accuracy, since the estimates of rotor speed, stator resistance, and no-load loss produce additional errors.

\section{EXPERIMENTAL VALIDATION}

The proposed method has been verified by both computer simulations and real experiments. The computer simulation results are omitted here in order to conserve space.

\section{A. Experimental Setup}

To evaluate the generality of the proposed method, three new induction motors are tested. These motors are intentionally selected with various physical configurations, such as different sizes (7.5 and $10 \mathrm{hp}$ ), designs (NEMA A and B), and enclosures (TEFC and ODP). The motor parameters are listed in Table II.

In the experimental setup, these motors are line connected to a 230-V mains supply. The voltages and currents are slightly unbalanced $\left(I_{-} / I_{+}<10 \%\right)$ and reflect the actual motor working condition. A dc generator feeding resistor loads serves as the dynamometer. Two line voltages $\left(v_{a b}\right.$ and $v_{c a}$ ) and two phase currents $\left(i_{a}\right.$ and $\left.i_{b}\right)$ are sampled using an NI PCI-6250 $\mathrm{M}$-series multifunction data acquisition system with 16-b resolution and 1.25-MS/s single-channel sampling rate and stored in a personal computer using NI LabVIEW. 
TABLE II

PARAMETERS OF THREE INDUCTION MOTORS IN EXPERIMENT

\begin{tabular}{|l|c|c|c|}
\hline & Motor I & Motor II & Motor III \\
\hline Brand & Leeson & GE & Marathon \\
\hline Rated Output Power (hp) & 7.5 & 7.5 & 10 \\
\hline Catalogue NO. & G140417 & S231 & U639 \\
\hline Phase & 3 -phase & 3 -phase & 3 -phase \\
\hline Rated Speed (rpm) & 1760 & 1755 & 1750 \\
\hline Rated Line Voltage (volts) & $230 / 460$ & $230 / 460$ & $230 / 460$ \\
\hline Full Load Current (amps) & $20.0 / 10.0$ & $18.2 / 9.1$ & $25.6 / 12.8$ \\
\hline Nominal Power Factor & 0.72 & 0.865 & 0.86 \\
\hline Nominal Efficiency & 0.885 & 0.895 & 0.895 \\
\hline Stator Resistance (ohms) & 0.326 & 1.044 & 0.223 \\
\hline NEMA Design & $\mathrm{B}$ & $\mathrm{A}$ & $\mathrm{B}$ \\
\hline Enclosure Type & ODP & TEFC & TEFC \\
\hline
\end{tabular}

\section{B. Error Analysis}

To evaluate and validate the accuracy of the estimated efficiencies, the actual motor efficiencies must be measured and their accuracies must be guaranteed. This is a key step in the entire testing process. In the experiment, the actual efficiencies are calculated as the ratio between the mechanical output power and the electrical input power.

The input power is directly calculated from two line-toline terminal voltages and two line currents. The two voltage transducers are LEM LV 25-P with 2500: 1000 conversion ratio, $10-500-\mathrm{V}$ rms voltage range, $\pm 0.8 \%$ accuracy, and $<0.2 \%$ linearity. The two current transducers are LEM LA 55-P with $1: 1000$ conversion ratio, 50-A rms current range, $\pm 0.65 \%$ accuracy, and $<0.15 \%$ linearity. Both of these transducers use Hall effect devices.

The output power is directly calculated from the output shaft torque and the rotor speed. An accurate in-line rotary torque transducer is installed between the motor and the load to measure the shaft torque. The torque transducer is a TQ501$1 \mathrm{~K}$ made by Omega with the following specifications: $10-\mathrm{V}$ dc excitation, $0-1000$-in $\cdot \mathrm{lb}$ torque range, $\pm 0.18 \%$ accuracy, $\pm 0.10 \%$ linearity, $\pm 0.10 \%$ hysteresis, $\pm 0.10 \%$ repeatability, $\pm 1.0 \%$ zero balance, and $6000 \mathrm{r} / \mathrm{min}$ (maximum). The rotor speed is measured by an Extech-461501 noncontact digital tachometer with $\pm 0.10 \%$ accuracy.

The accuracy of the measured efficiencies is highly dependent on the accuracies of the voltage, current, speed, and torque measurements. The accuracy of these measured values depends on the inherent accuracy of the instruments, on the precision of the readings, and the noise present in the measuring system. By using the worst case estimation method [14]

$$
\begin{aligned}
\eta \pm \Delta \eta & =\frac{P_{\text {output }} \pm \Delta P_{\text {output }}}{P_{\text {input }} \pm \Delta P_{\text {input }}} \\
& =\frac{\left(T_{\text {shaft }} \pm \Delta T_{\text {shaft }}\right) \cdot\left(\omega_{r} \pm \Delta \omega_{r}\right)}{\left(V_{s} \pm \Delta V_{s}\right) \cdot\left(I_{s} \pm \Delta I_{s}\right)}
\end{aligned}
$$

TABLE III

EXPERIMENTAL RESUlTS OF MOTOR I (LEESON 7.5 hp ODP)

\begin{tabular}{|c|c|c|c|}
\hline $\begin{array}{c}\text { Load } \\
\text { Percentage (\%) }\end{array}$ & $\begin{array}{c}\text { Rotor Speed } \\
(\mathbf{r p m})\end{array}$ & $\begin{array}{c}\text { Estimated } \\
\text { Efficiency } \mathbf{( \% )}\end{array}$ & $\begin{array}{c}\text { Measured } \\
\text { Efficiency }(\%)\end{array}$ \\
\hline 8.54 & 1797.0 & 47.59 & 68.56 \\
\hline 18.54 & 1794.6 & 74.24 & 73.37 \\
\hline 23.50 & 1793.4 & 78.55 & 77.50 \\
\hline 33.14 & 1788.6 & 83.06 & 79.99 \\
\hline 40.23 & 1785.8 & 84.67 & 84.39 \\
\hline 54.37 & 1780.7 & 86.08 & 85.42 \\
\hline 68.22 & 1776.0 & 86.25 & 86.59 \\
\hline 75.07 & 1774.1 & 86.02 & 85.75 \\
\hline 88.75 & 1768.6 & 85.37 & 85.01 \\
\hline 101.51 & 1762.6 & 84.40 & 84.70 \\
\hline 111.66 & 1757.1 & 83.52 & 82.20 \\
\hline 117.53 & 1754.7 & 82.89 & 81.19 \\
\hline
\end{tabular}

the maximum error in the measured efficiencies $\Delta \eta / \eta$ is calculated as

$$
\begin{aligned}
1 \pm \frac{\Delta \eta}{\eta} & =\frac{\left(1 \pm \frac{\Delta T_{\text {shaft }}}{T_{\text {shaft }}}\right) \cdot\left(1 \pm \frac{\Delta \omega_{r}}{\omega_{r}}\right)}{\left(1 \pm \frac{\Delta V_{s}}{V_{s}}\right) \cdot\left(1 \pm \frac{\Delta I_{s}}{I_{s}}\right)} \\
& =\frac{(1 \pm 0.18 \%) \times(1 \pm 0.10 \%)}{(1 \pm 0.80 \%) \times(1 \pm 0.65 \%)} \approx 1 \pm 1.7 \% .
\end{aligned}
$$

Considering that the realistic error is normally much lower than the maximum error $\pm 1.7 \%$, conclusion can be drawn that the measured efficiencies are accurate enough to validate the estimates.

\section{Experiment Results}

The estimated motor efficiencies are calculated using the proposed NAGT method. The line voltages and phase currents are sampled at high frequency $(5 \mathrm{kHz}$ for Motors I and III, $2 \mathrm{kHz}$ for Motor II). Simpson's $1 / 3$ rule is applied for the numerical integration in the air-gap flux (torque) computation for its simplicity and accuracy [15]. The dc offset in the air-gap flux is filtered by a three-cycle $(50 \mathrm{~ms})$ moving average window.

Since the majority of motors in industrial plants operate at about $60 \%$ of their rated load, special attention is paid to $40 \%-90 \%$ load levels in this paper. For extremely low and high load conditions, an accurate motor-efficiency estimate is no longer necessary for making planning decisions. It can be predicted from a typical motor performance curve (efficiency versus load) that under these conditions, the motor has low efficiencies.

In the experiment, all three motors are tested under various load levels, ranging from almost no-load to overload conditions. Since the no-load test could be invasive in a range of circumstances in practice, during the experimental validation, the estimated no-load data are used for efficiency calculation, as suggested in Section II. The total combined no-load losses are estimated to be $3.5 \%$ of rated output power. The friction and windage loss is assumed as $1.2 \%$ rated output power, and the stray-load loss is estimated from Table I. 
TABLE IV

EXPERIMENTAL RESULTS OF MOTOR II (GE 7.5 hp TEFC)

\begin{tabular}{|c|c|c|c|}
\hline $\begin{array}{c}\text { Load } \\
\text { Percentage (\%) }\end{array}$ & $\begin{array}{c}\text { Rotor Speed } \\
\text { (rpm) }\end{array}$ & $\begin{array}{c}\text { Estimated } \\
\text { Efficiency }(\%)\end{array}$ & $\begin{array}{c}\text { Measured } \\
\text { Efficiency }(\%)\end{array}$ \\
\hline 19.09 & 1775.0 & 90.68 & 92.45 \\
\hline 21.77 & 1771.5 & 91.26 & 91.63 \\
\hline 24.45 & 1768.0 & 91.65 & 92.38 \\
\hline 27.17 & 1764.5 & 91.89 & 92.75 \\
\hline 29.77 & 1760.8 & 91.94 & 92.28 \\
\hline 35.03 & 1753.5 & 91.71 & 91.24 \\
\hline 40.37 & 1746.0 & 91.24 & 91.09 \\
\hline 50.78 & 1730.0 & 90.00 & 88.47 \\
\hline 61.14 & 1713.0 & 88.46 & 87.08 \\
\hline 71.26 & 1694.0 & 86.65 & 85.48 \\
\hline 81.56 & 1672.0 & 84.49 & 83.39 \\
\hline 91.27 & 1647.0 & 82.10 & 80.21 \\
\hline
\end{tabular}

TABLE V

EXPERIMENTAL RESULTS OF MOTOR III (MARATHON 10 hp TEFC)

\begin{tabular}{|c|c|c|c|}
\hline $\begin{array}{c}\text { Load } \\
\text { Percentage (\%) }\end{array}$ & $\begin{array}{c}\text { Rotor Speed } \\
\text { (rpm) }\end{array}$ & $\begin{array}{c}\text { Estimated } \\
\text { Efficiency (\%) }\end{array}$ & $\begin{array}{c}\text { Measured } \\
\text { Efficiency (\%) }\end{array}$ \\
\hline 4.04 & 1799.2 & 55.62 & 66.23 \\
\hline 12.85 & 1796.0 & 84.80 & 84.50 \\
\hline 26.10 & 1789.2 & 91.00 & 89.70 \\
\hline 32.70 & 1787.4 & 91.66 & 91.52 \\
\hline 39.69 & 1785.0 & 91.82 & 91.15 \\
\hline 48.49 & 1782.6 & 91.76 & 90.25 \\
\hline 56.44 & 1779.0 & 91.39 & 90.86 \\
\hline 65.52 & 1776.0 & 91.03 & 90.49 \\
\hline 74.11 & 1772.6 & 90.41 & 90.42 \\
\hline 82.27 & 1768.9 & 89.80 & 89.51 \\
\hline 96.42 & 1761.8 & 88.56 & 88.81 \\
\hline 106.17 & 1752.8 & 86.86 & 86.87 \\
\hline
\end{tabular}

Tables III-V list the experimental results from Motors I-III, respectively, including load percentage, rotor speed, and estimated and measured motor efficiencies. Figs. 4-6 graphically illustrate the estimated and actual measured efficiencies versus the load percentage and rotor speed.

For all three motors, the estimated efficiencies closely agree with the measured efficiencies (within $2 \%$ errors) during the normal motor operations (load ranges from $40 \%$ to $90 \%$ of rated load). Moreover, this method also gives relatively accurate efficiency estimates at very low load (less than 30\% rated load) and high load (greater than $90 \%$ rated load) conditions.

The experimental results also confirm the fact that the generic motor nameplate data could contain a large amount of inaccuracies. The experimental results from Motors I and III agree well with the nameplate data. When the motor operates at the rated load condition, the speed is close to its rated value. The maximum efficiency occurs at normal load levels, which is typically how these motors are designed to operate.

However, the results from Motor II show obvious inconsistencies with the nameplate data. At rated load, the rotor speed drops to around $1640 \mathrm{r} / \mathrm{min}$, which is well below the rated speed of $1755 \mathrm{r} / \mathrm{min}$. Moreover, the maximum efficiency occurs at around 30\% rated load, which is lower than the desired design value. Repetitive tests on two identical GE S231 motors give similar results. It can be concluded that the generic motor template data contain significant inaccuracies for Motor II.

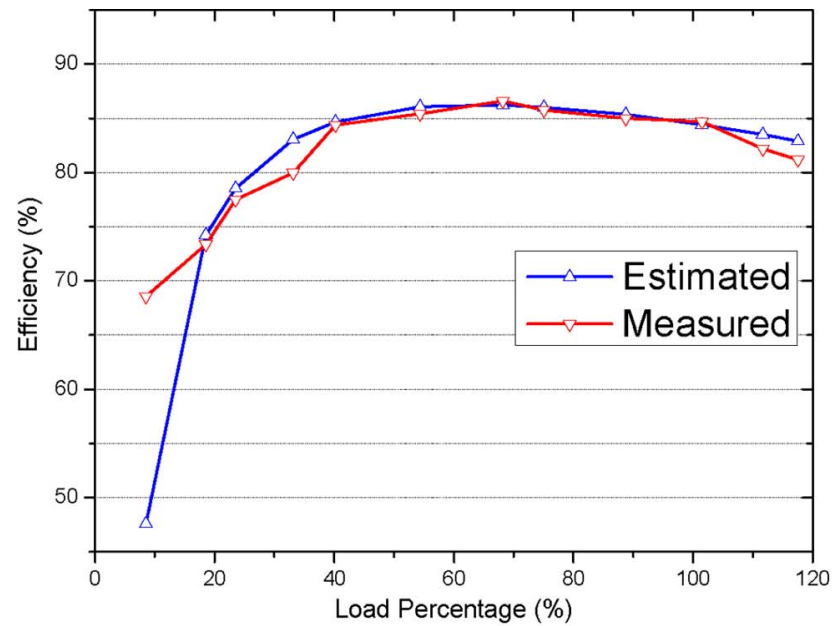

(a)

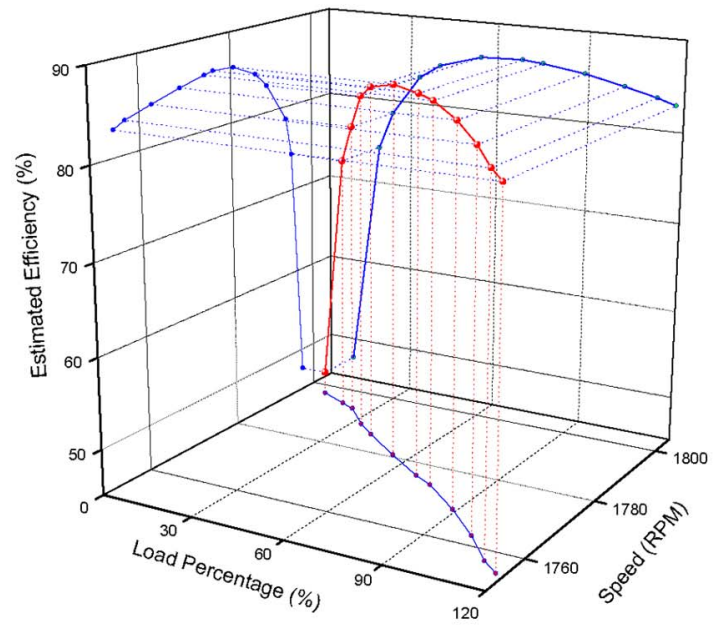

(b)

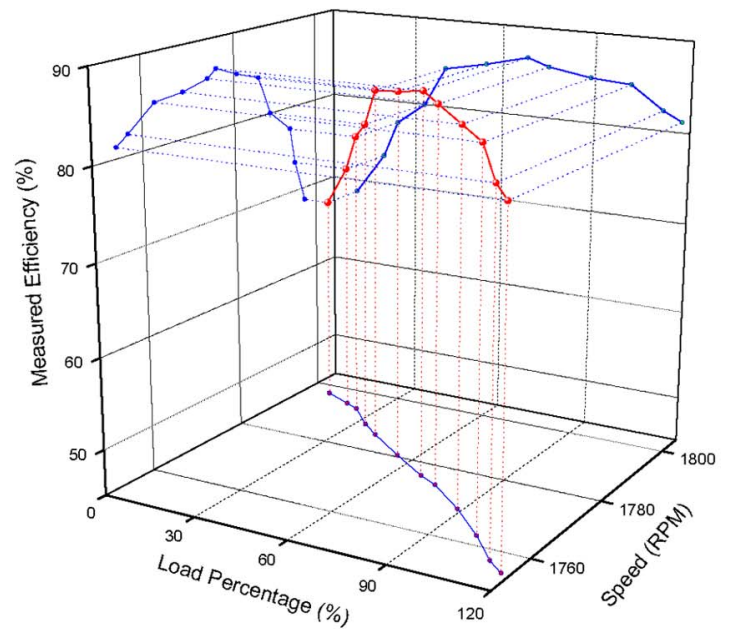

(c)

Fig. 4. Estimated and measured efficiencies of Motor I (Leeson, $7.5 \mathrm{hp}$, NEMA-B, ODP, $1760 \mathrm{r} / \mathrm{min}$ ). (a) Comparison of estimated and measured efficiencies. (b) Estimated efficiencies versus load percentage and speed. (c) Measured efficiencies versus load percentage and speed.

However, since the proposed NAGT method relies mostly on terminal voltages and currents, the inaccuracies in the nameplate data do not generate obvious errors in efficiency estimates, as shown in Fig. 5. 


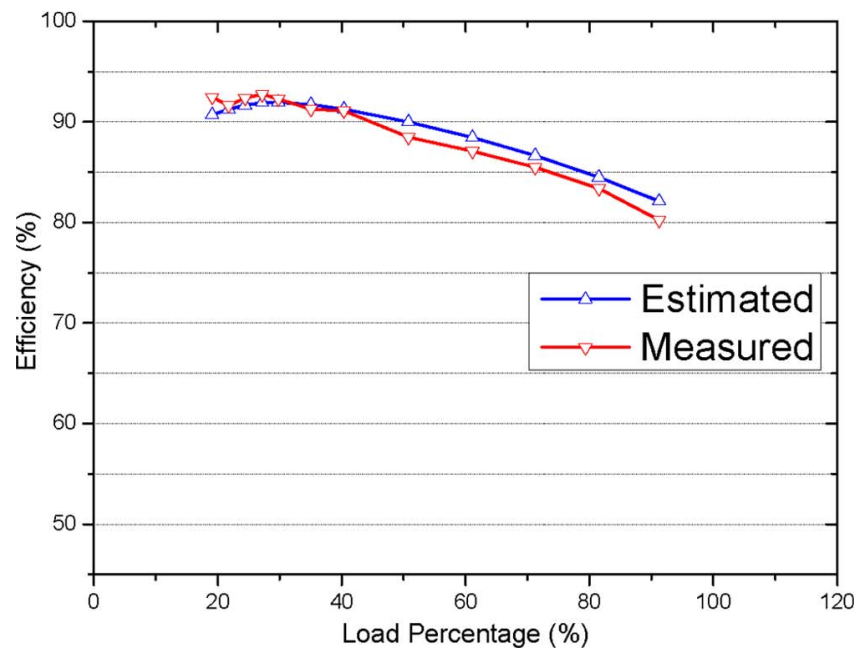

(a)

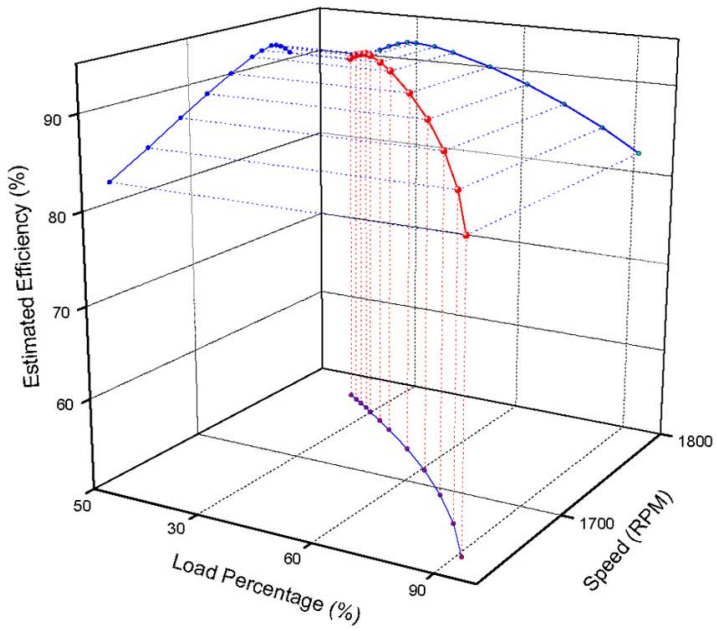

(b)

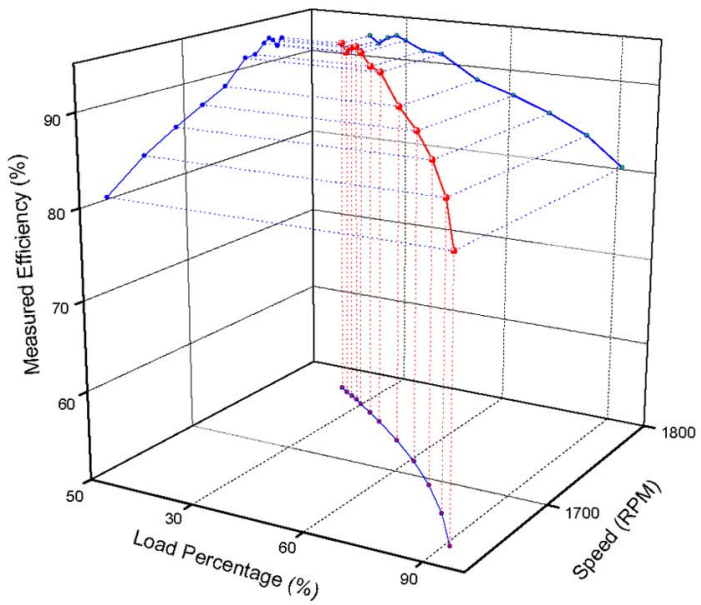

(c)

Fig. 5. Estimated and measured efficiencies of Motor II (GE, 7.5 hp, NEMA-A, TEFC, $1755 \mathrm{r} / \mathrm{min}$ ). (a) Comparison of estimated and measured efficiencies. (b) Estimated efficiencies versus load percentage and speed. (c) Measured efficiencies versus load percentage and speed.

To summarize, the agreement between the estimated and measured efficiencies in the experimental results validates the proposed NAGT method.

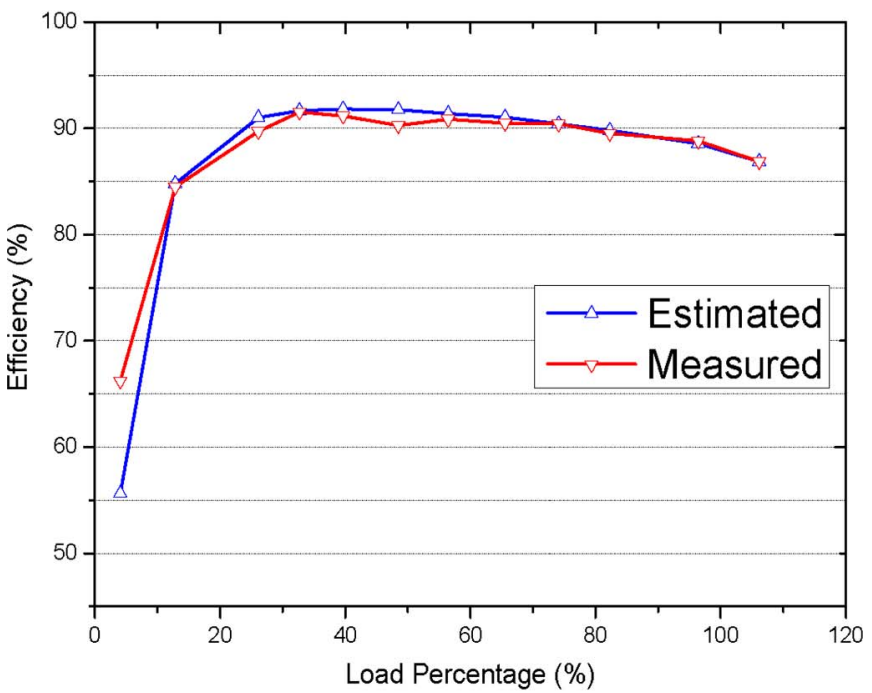

(a)

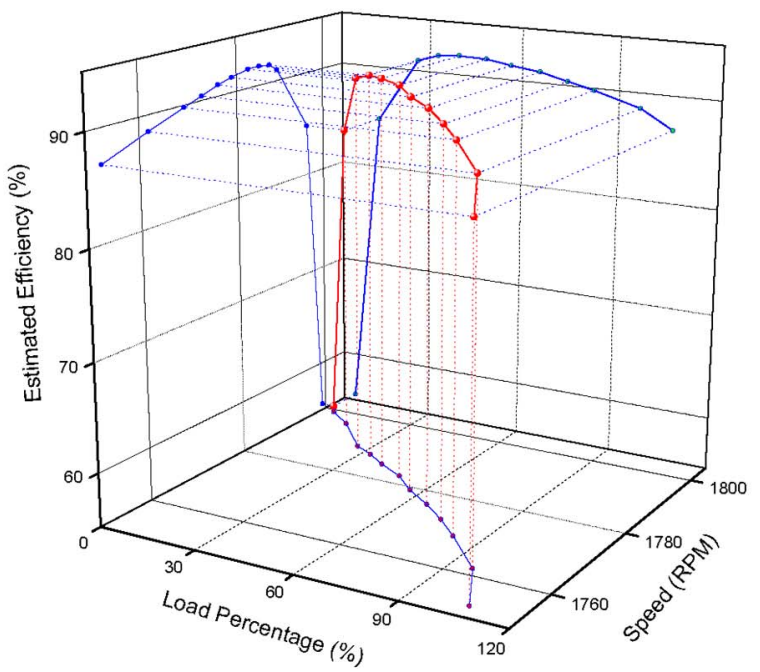

(b)

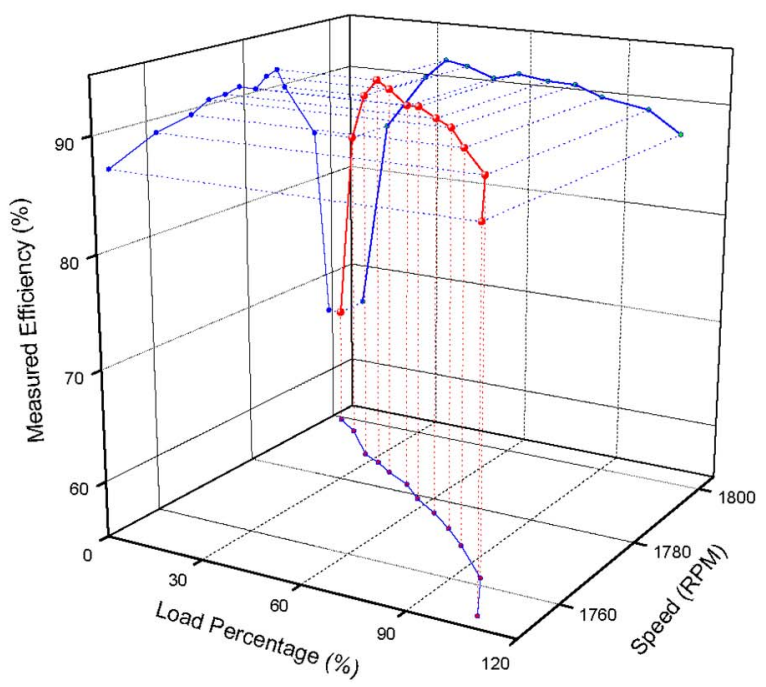

(c)

Fig. 6. Estimated and measured efficiencies of Motor III (Marathon, $10 \mathrm{hp}$, NEMA-B, TEFC, $1750 \mathrm{r} / \mathrm{min}$ ). (a) Comparison of estimated and measured efficiencies. (b) Estimated efficiencies versus load percentage and speed. (c) Measured efficiencies versus load percentage and speed. 


\section{CONCLUSION}

This paper has proposed an online nonintrusive method for in-service motor-efficiency estimation based on AGT using only motor terminal quantities and nameplate information with special considerations of motor condition monitoring requirements. This method has provided a theoretical foundation for low-cost energy evaluation in industrial plants, where motor terminal voltages and currents are readily accessible from the motor control centers without interfering with the motor's normal operations.

This method has been experimentally validated on small induction motors (less than $20 \mathrm{hp}$ ) by testing three induction motors with different physical configurations, such as size, design, and enclosure type. The estimated efficiencies of all three motors agree with the measured efficiencies within $2 \%$ errors during the normal load levels.

A novel and significant contribution of this method is that the efficiency estimation is completely based on motor terminal quantities and motor nameplate information. Costly torque and speed transducers are eliminated.

\section{REFERENCES}

[1] Xenergy, United States Industrial Electric Motor Systems Market Opportunities Assessment, 2002, Washington, DC: Office Energy Efficiency Renewable Energy, U.S. Dept. Energy. Prepared for Oak Ridge Nat. Lab. and DOE's Office of Ind. Technol.

[2] IEEE Standard Test Procedure for Polyphase Induction Motors and Generators, IEEE Standard 112-2004.

[3] B. Lu, T. G. Habetler, and R. G. Harley, "A survey of efficiency-estimation methods for in-service induction motors," IEEE Trans. Ind. Appl., vol. 42, no. 4, pp. 924-933, Jul./Aug. 2006.

[4] E. B. Agamloh, A. K. Wallace, A. von Jouanne, K. J. Anderson, and J. A. Rooks, "Assessment of nonintrusive motor efficiency estimators," IEEE Trans. Ind. Appl., vol. 41, no. 1, pp. 127-133, Jan./Feb. 2005.

[5] J. O. Ojo, V. Ostovic, T. A. Lipo, and J. C. White, "Measurement and computation of starting torque pulsations of salient pole synchronous motors," IEEE Trans. Energy Convers., vol. 5, no. 1, pp. 176-182, Mar. 1990.

[6] G. B. Kliman, R. A. Koegl, W. J. Premerlani, and T. B. Breen, "A demonstration of a sensorless torque meter for AC motors," in Conf. Rec. IEEE IAS Annu. Meeting, Oct. 1996, vol. 1, pp. 633-637.

[7] J. Hsu and B. P. Scoggins, "Field test of motor efficiency and load changes through air-gap torque," IEEE Trans. Ind. Appl., vol. 10, no. 3, pp. 477483, Sep. 1995.

[8] J. Hsu, J. Kueck, M. Olszewski, D. Casada, P. Otaduy, and L. Tolbert, "Comparison of induction motor field efficiency evaluation methods," IEEE Trans. Ind. Appl., vol. 34, no. 1, pp. 117-125, Jan./Feb. 1998.

[9] A. K. Wallace and E. J. Widenbrug, "Motor efficiency determination: From testing laboratory to plant installation," in Proc. IEEE Pulp Paper Ind. Tech. Annu. Conf., Jun. 1999, pp. 190-195.

[10] K. D. Hurst and T. G. Habetler, "Sensorless speed measurement using current harmonic spectral estimation in induction machine drives," IEEE Trans. Power Electron., vol. 11, no. 1, pp. 66-73, Jan. 1996.

[11] S. B. Lee and T. G. Habetler, "An online stator winding resistance estimation technique for temperature monitoring of line-connected induction machines," IEEE Trans. Ind. Appl., vol. 39, no. 3, pp. 685-694, May/Jun. 2003.

[12] P. Zhang, B. Lu, and T. G. Habetler, "A remote and sensorless stator winding resistance estimation method for thermal protection of soft-starterconnected induction machines," IEEE Trans. Ind. Electron., vol. 55, no. 10 , pp. 3611-3618, Oct. 2008.

[13] "DOE Industry Tools," DOE MotorMaster+ Website. [Online]. Available: http://www1.eere.energy.gov/industry/bestpractices/software.html

[14] R. S. Colby and D. L. Flora, "Measured efficiency of high efficiency and standard induction motors," in Conf. Rec. IEEE IAS Annu. Meeting, Oct. 1990 , vol. 1, pp. 18-23.

[15] R. J. Schilling and S. L. Harris, Applied Numerical Methods for Engineers Using MATLAB and C. Pacific Grove, CA: Thomson, 2000

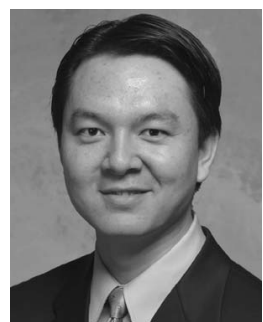

Bin Lu (S'00-M'06) received the B.Eng. degree in automation from Tsinghua University, Beijing, China, in 2001, the M.S. degree in electrical engineering from the University of South Carolina, Columbia, in 2003, and the Ph.D. degree in electrical engineering from the Georgia Institute of Technology, Atlanta, in 2006.

From 2001 to 2003, he was with the VTB group of the University of South Carolina, Columbia, developing a real-time virtual test bed for the hardwarein-the-loop testing of dynamic power systems. In July 2004, he was a Graduate Research Assistant with the power-electronics and motor-diagnostics group of the Georgia Institute of Technology, working on the energy evaluation and condition monitoring of electric machines. In summer of 2006, he was with the General Motors R\&D Center, Warren, MI, as a Graduate Summer Intern. Since October 2006, he has been with the Innovation Center, Eaton Corporation, Milwaukee, WI, where he is currently an Engineering Specialist and Program Manager. He has authored over 30 papers published in refereed journals and international conference proceedings and has five patents pending in these areas. His research interests include electric motor drives and diagnostics, renewable energy and distributed generation, power electronics, modeling and simulation, and application of sensor networks in electric power areas

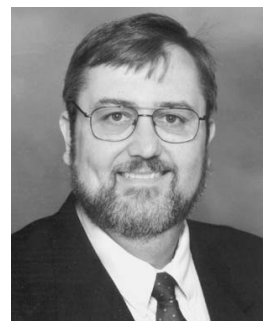

Thomas G. Habetler (S'82-M'83-SM'92-F'02) received the B.S. and M.S. degrees in electrical engineering from Marquette University, Milwaukee, WI, in 1981 and 1984, respectively, and the Ph.D. degree from the University of Wisconsin, Madison, in 1989.

From 1983 to 1985 , he was with the ElectroMotive Division of General Motors as a Project Engineer. Since 1989, he has been with the Georgia Institute of Technology, Atlanta, where he is currently a Professor of electrical engineering with the School of Electrical and Computer Engineering. He has published over 100 papers in the field. He is a regular consultant to industry in the field of condition-based diagnostics for electrical systems. His research interests are in electric machine protection and condition monitoring, and switching converter technology and drives.

Dr. Habetler currently serves as IEEE Division II Director-Elect, is a PastPresident of the IEEE Power Electronics Society, and Past-Chair of the Industrial Power Converter Committee of the IEEE Industry Applications Society. He has received four conference prize paper awards from the Industry Applications Society.

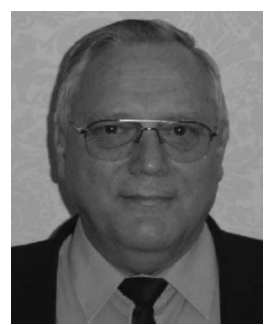

Ronald G. Harley (M'77-SM'86-F'92) received the M.Sc.Eng. degree (cum laude) in electrical engineering from the University of Pretoria, Pretoria, South Africa, in 1965, and the Ph.D. degree from London University, London, U.K., in 1969.

In 1971, he was appointed to the Chair of Electrical Machines and Power Systems at the University of Natal, Durban, South Africa, where he was a Professor of electrical engineering for many years, and also served as the Department Head and Deputy Dean of Engineering. He is currently the Duke Power Company Distinguished Professor with the School of Electrical and Computer Engineering, Georgia Institute of Technology, Atlanta. He has coauthored some 380 papers in refereed journals and international conferences and is the holder of three patents. Ten of the papers attracted prizes from journals and conferences. His research interests include the dynamic behavior and condition monitoring of electric machines, motor drives, power systems and their components, and controlling them by the use of power electronics and intelligent control algorithms.

Dr. Harley is a Fellow of the Institution of Electrical engineers, U.K. $\mathrm{He}$ is also a Fellow of the Royal Society in South Africa and a Founder Member of the Academy of Science in South Africa formed in 1994. During 2000 and 2001, he was one of the IEEE Industry Applications Society's six Distinguished Lecturers. He was the Vice-President of Operations of the IEEE Power Electronics Society (2003 to 2004) and Chair of the Atlanta Chapter of the IEEE Power Engineering Society. He is currently the Chair of the Distinguished Lecturers and Regional Speakers program of the IEEE Industry Applications Society. He received the Cyrill Veinott Award in 2005 from the Power Engineering Society for his outstanding contributions to the field of electromechanical energy conversion. 


\title{
A Remote and Sensorless Stator Winding Resistance Estimation Method for Thermal Protection of Soft-Starter-Connected Induction Machines
}

\author{
Pinjia Zhang, Student Member, IEEE, Bin Lu, Member, IEEE, and Thomas G. Habetler, Fellow, IEEE
}

\begin{abstract}
This paper proposes a remote and sensorless stator winding resistance estimation method for thermal protection of soft-starter-connected induction motors. By changing the gate drive signals of the thyristors in the soft starter, a small adjustable dc bias can be intermittently injected to the motor for the estimation of the stator winding resistance. Based on online and continuous monitoring of the stator winding resistance, the stator winding temperature can be monitored using only motor voltage and current. In addition, the torque pulsation caused by the injected dc bias is analyzed. It can also be controlled under an acceptable level by adjusting the level of the injected dc signal. The influence of cable resistance is also studied, and a compensation method is proposed. The proposed method has been verified by experimental results from two induction motors. The proposed stator resistance estimation method can provide remote, sensorless, and accurate thermal protection for soft-starter-connected induction motors.
\end{abstract}

Index Terms-Induction motor, sensorless, signal injection, soft starter, stator resistance, thermal protection.

\section{INTRODUCTION}

$\mathbf{T}$ HERMAL protection is one of the most important aspects in motor condition monitoring. Thirty percent of motor failures are related to stator winding insulation [1]. It is commonly assumed that the motor's life is reduced by $50 \%$ for every $10{ }^{\circ} \mathrm{C}$ increase above its stator winding temperature limit. Therefore, accurate monitoring of the stator winding temperature is crucial for motor protection purposes. Aside from the direct stator winding temperature measurement, the thermal model-based and the motor parameter-based temperature estimation methods are two major techniques for thermal protection. The thermal model-based methods estimate the stator winding temperature using motor thermal models [2]-[10]. However, due to the thermal parameter variation and the difficulty of thermal parameter identification, the accuracy of these methods cannot be guaranteed. In addition, due to the change of cooling conditions, the thermal parameters are not

Manuscript received January 31, 2008; revised June 2, 2008. Current version published October 1, 2008. This work was supported in part by the Department of Energy and in part by Eaton Corporation.

P. Zhang and T. G. Habetler are with the School of Electrical and Computer Engineering, Georgia Institute of Technology, Atlanta, GA 30332 USA (e-mail: pzhang@ece.gatech.edu; thabetler@ece.gatech.edu).

B. Lu is with the Innovation Center, Eaton Corporation, Milwaukee, WI 53216 USA (e-mail: binlu@ieee.org).

Color versions of one or more of the figures in this paper are available online at http://ieeexplore.ieee.org.

Digital Object Identifier 10.1109/TIE.2008.927241 constant and need to be identified for each motor under each specific cooling condition. On the other hand, the induction motor parameter-based approaches are developed to estimate the average winding temperatures from the winding resistances.

Stator winding resistance $\left(R_{s}\right)$ estimation is broadly used not only in thermal protection but also in motor controls [11]-[15], fault diagnosis [16], [17], efficiency evaluation [18], etc. Over the years, various $R_{s}$ estimation methods have been proposed for different purposes. Generally, they are divided into three major categories: 1) direct measurement; 2) equivalent-circuitbased method; and 3) signal-injection-based method. Direct methods, such as the IEEE Std-118 [19], give the most accurate $R_{s}$ estimates, but it suffers from the following: 1) resistance is only measured at a certain temperature, and the resistance variations due to temperature changes are not considered and 2) the motor has to be disconnected from service to perform the required tests. The equivalent-circuit-based methods use the motor current and voltage to calculate the stator resistance, based on the induction motor equivalent circuit [20]-[28]. They are nonintrusive and can respond to changes in the cooling conditions, but are generally too sensitive to motor parameters variations. In [29], it was proposed to create a dc bias in the stator supply voltage, and the dc component of the voltage and current to calculate the stator resistance was used. Although this approach is claimed to be accurate and robust to the variations in cooling conditions and motor parameters, it suffers from its highly intrusive nature: an extra dc injection circuit needs to be installed in series with one of the motor leads. Additionally, due to the current limits of semiconductor devices, previous signalinjection-based methods cannot be directly applied to motors beyond $100 \mathrm{hp}$.

To provide accurate thermal protection for the soft-starterconnected induction motors, this paper proposes to inject an adjustable dc bias by changing the gate control signals for the solid-state switches in the soft starter. By monitoring the stator winding resistance estimated from dc injection, the stator winding temperature can be estimated. The torque pulsation caused by the injected dc signal is analyzed. It can also be controlled under an acceptable level by adjusting the level of the injected dc signal. In addition, practical considerations are discussed, including the influence of cable resistance and the measurement offsets. Compensation methods are also proposed. The experimental results show that this method can provide remote, sensorless, and accurate thermal protection for the soft-starter-connected induction motors. 


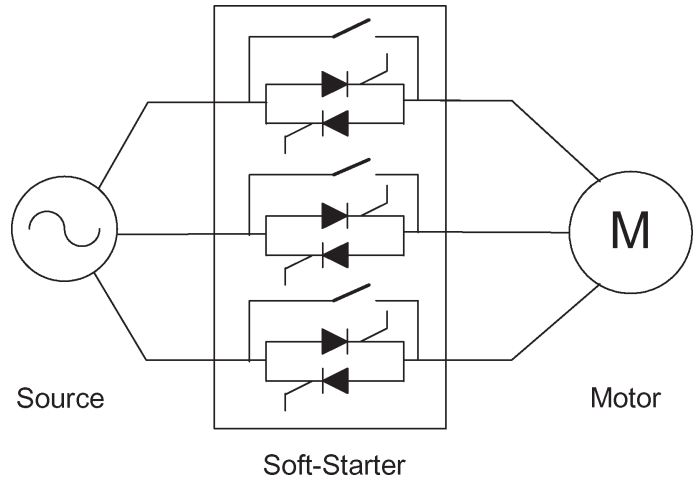

Fig. 1. Basic structure of the soft starter.
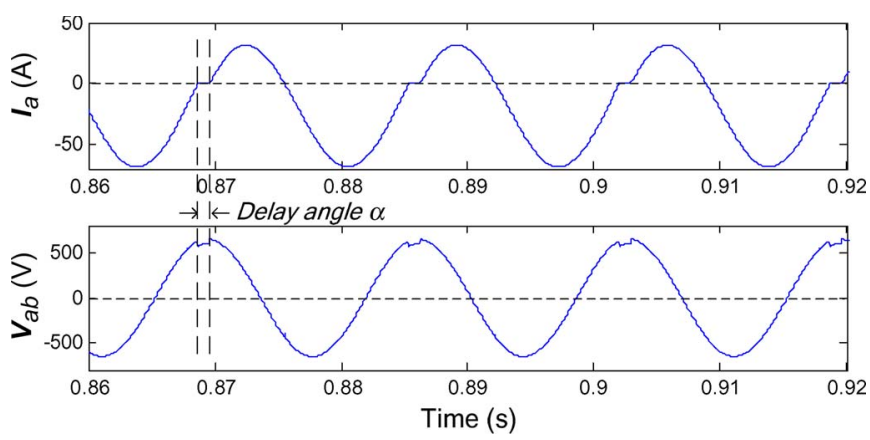

Fig. 2. Motor line voltage and phase current during DIM.

\section{A DC Signal Injection Method Using Soft STARTER FOR $R_{s}$ ESTIMATION}

\section{A. DC Signal Injection Method Using Soft Starter}

The soft starter normally contains multiple antiparallel solidstate switches (e.g., thyristors) to control the current flow and, in turn, the terminal voltages of the motor. The soft starter limits the transient voltages and currents, avoids the inrush currents, and results in a "soft" motor start. After starting, the soft starter enters the "bypass" mode when the contactors are closed to minimize the power dissipation. The basic structure of a soft starter with antiparallel thyristors is shown in Fig. 1.

A new gate drive control mode, namely, the "dc injection mode (DIM)," is proposed in this paper to inject dc components in the motor line voltages and phase currents. During the dc injection period, only one contactor (corresponding to only one phase, e.g., phase $\boldsymbol{a}$ ) in the soft starter is kept open, while the other two contactors still work normally as in the bypass mode. Instead of using the symmetrical operation mode, a short delay is introduced to the gate drive signal of only the forward (backward)-conducting thyristor of phase $\boldsymbol{a}\left(\boldsymbol{V}_{G 1}\right)$ after phase $\boldsymbol{a}$ current's rising (falling) zero crossing. After the DIM period, the phase $\boldsymbol{a}$ contactor is closed so that the soft starter returns to normal bypass operation. Fig. 2 shows the typical waveforms of the motor line voltage $\left(v_{a b}\right)$ and phase current $\left(i_{a}\right)$, while a small delay angle of $\alpha\left(\alpha<30^{\circ}\right)$ is being added.

The dc components in the input voltages and currents do not "pass through" the air gap of the induction motors and, as a result, have no impact on the rotor circuit. Therefore, the equivalent dc model of the induction motor with a soft starter

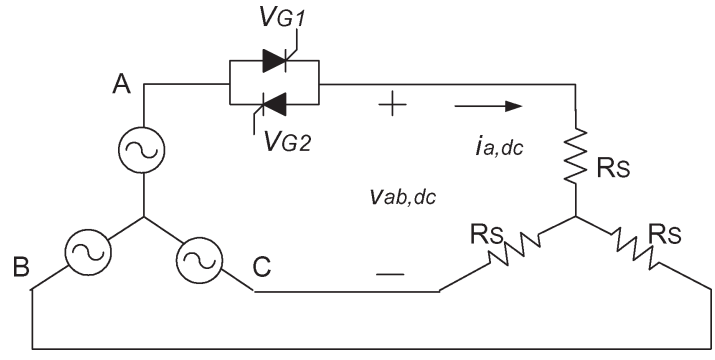

Fig. 3. DC equivalent circuit of motor, source, and soft starter.

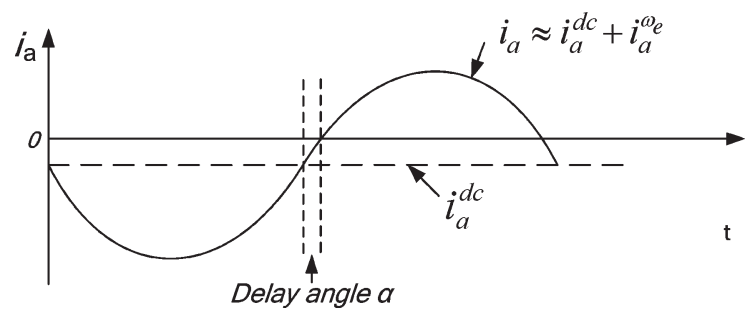

Fig. 4. Approximation of motor phase current during DIM.

can be illustrated as shown in Fig. 3. The stator resistance $R_{s}$ can be estimated from the terminal voltages and currents as

$$
R_{s}=\frac{2 \cdot v_{a b}^{\mathrm{dc}}}{3 \cdot i_{a}^{\mathrm{dc}}}
$$

where $v_{a b}^{\mathrm{dc}}$ and $i_{a}^{\mathrm{dc}}$ are the $\mathrm{dc}$ components of the motor line voltage $v_{a b}$ and phase current $i_{a}$, respectively.

Based on the estimated $R_{s}$ from dc signal injection, the stator winding temperature $T_{s}$ can be monitored. The $R_{s}$ variation is linearly proportional to the $T_{s}$ variation, i.e.,

$$
\hat{T}_{s}=T_{s 0}+\frac{\left(\hat{R}_{s}-R_{s 0}\right)}{\alpha R_{s 0}}
$$

where $T_{s 0}$ and $R_{s 0}$ represent the $T_{s}$ and the $R_{s}$ at room temperature, $\hat{T}_{s}$ and $\hat{R}_{s}$ are the estimated $T_{s}$ and $R_{s}$ from dc injection, and $\alpha$ is the temperature coefficient of resistivity.

\section{B. Analysis of DC Component in Voltage and Current}

As shown in Fig. 2, due to the small delay angle $\alpha\left(\alpha<30^{\circ}\right)$, it can be assumed that $v_{a b}$ only consists of the dc and the line frequency $\left(\omega_{e}\right)$ components, i.e.,

$$
v_{a b} \approx v_{a b}^{\mathrm{dc}}+v_{a b}^{\omega_{e}} .
$$

Similarly, the phase current $i_{a}$ can be approximately denoted as

$$
i_{a} \approx i_{a}^{\mathrm{dc}}+i_{a}^{\omega_{e}}
$$

where $i_{a}^{\mathrm{dc}}$ and $i_{a}^{\omega_{e}}$ is the dc component and the line frequency component of $i_{a}$, respectively, as shown in Fig. 4.

Therefore, the dc component in $i_{a}$ can be approximately derived as

$$
i_{a}^{\mathrm{dc}} \approx-\hat{I}_{a}^{\omega_{e}} \sin (\alpha)
$$


where $\hat{I}_{a}^{\omega_{e}}$ is the peak value of the line frequency component in $i_{a}$.

\section{Evaluation of Torque Pulsation}

Let $\vec{v}_{d q s}, \vec{i}_{d q s}$, and $\vec{\lambda}_{d q s}$ be the stator voltage, stator current, and total flux linkage space vectors in the $d-q$ stationary reference frame. The air-gap torque $T_{\mathrm{ag}}$ can be calculated as the cross product of $\vec{\lambda}_{d q s}$ and $\vec{i}_{d q s}$, i.e.,

$$
T_{\mathrm{ag}}=\frac{3 P}{4}\left|\vec{\lambda}_{d q s} \otimes \vec{i}_{d q s}\right|
$$

where $P$ is the number of poles [30].

The flux linkage vector can be estimated based on the stator voltage and current vectors as

$$
\vec{\lambda}_{d q s}=\int\left(\vec{v}_{d q s}-R_{s} \vec{i}_{d q s}\right) d t \approx \int \vec{v}_{d q s} d t .
$$

The flux and current space vectors can be decomposed into vectors at multiples of the fundamental frequency as

$$
\begin{aligned}
& \vec{\lambda}_{d q s}=\sum_{n=-\infty}^{+\infty} \vec{\lambda}_{d q s}^{n \cdot 60} \\
& \vec{i}_{d q s}=\sum_{n=-\infty}^{+\infty} \vec{i}_{d q s}^{n \cdot 60}, \quad n \in Z
\end{aligned}
$$

where the superscript of each decomposed vector indicates its rotating direction and angular speed in the vector space, assuming that the supply frequency is $60 \mathrm{~Hz}$.

The correlation of each component in the injected signals to the torque pulsation can be evaluated separately based on this decomposition analysis. The frequency of the torque variation caused by flux linkage vector $\vec{\lambda}_{d q s}^{f_{1}}$ and current vector $\vec{i}_{d q s}^{f_{2}}$ is $\left|f_{1}-f_{2}\right|$. Therefore, the air-gap torque in (7) can be extended as

$$
\begin{aligned}
T_{\mathrm{ag}} & =\frac{3 P}{4}\left|\left(\sum_{n=-\infty}^{+\infty} \vec{\lambda}_{d q s}^{n \cdot 60}\right) \otimes\left(\sum_{n=-\infty}^{+\infty} \vec{i}_{d q s}^{n \cdot 60}\right)\right| \\
& =\frac{3 P}{4}\left|\vec{\lambda}_{d q s}^{60} \otimes \vec{i}_{d q s}^{60}\right|+\frac{3 P}{4}\left|\vec{\lambda}_{d q s}^{60} \otimes \vec{i}_{d q s}^{\mathrm{dc}}\right|+\xi_{T} \\
& =T_{1}^{\mathrm{dc}}+T_{2}^{60}+\xi_{T}
\end{aligned}
$$

where $T_{1}^{\mathrm{dc}}$ and $T_{2}^{60}$ represent the $\mathrm{dc}$ and the $60-\mathrm{Hz}$ major components in the air-gap torque, respectively, and $\xi_{T}$ is the remaining high-frequency torque components. Neglecting highorder harmonics in the flux linkage and stator current, the $\mathrm{dc}$ and the $60-\mathrm{Hz}$ components in the air-gap torque can be, respectively, denoted as

$$
\begin{aligned}
& \left|T_{\mathrm{ag}}^{\mathrm{dc}}\right| \approx\left|T_{1}^{\mathrm{dc}}\right| \approx \frac{3 P}{4}\left|\vec{\lambda}_{d q s}^{60} \otimes \vec{i}_{d q s}^{60}\right| \\
& \left|T_{\mathrm{ag}}^{60}\right| \approx\left|T_{2}^{60}\right| \approx \frac{3 P}{4}\left|\vec{\lambda}_{d q s}^{60} \otimes \vec{i}_{d q s}^{\mathrm{dc}}\right| .
\end{aligned}
$$

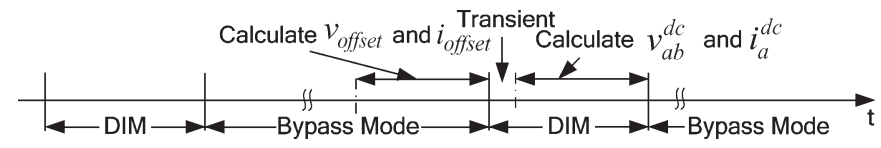

Fig. 5. Measurement offset compensation.

By using (7), (10) can be derived as

$$
\begin{aligned}
\left|T_{\mathrm{ag}}^{\mathrm{dc}}\right| & \approx\left|T_{1}^{\mathrm{dc}}\right| \approx \frac{3 P}{4}\left|\vec{\lambda}_{d q s}^{60} \otimes \vec{i}_{d q s}^{60}\right| \\
& \approx \frac{3 P}{4}\left|\int \vec{v}_{d q s}^{60} d t \otimes \vec{i}_{d q s}^{60}\right|=\frac{3 P}{4}\left|\vec{\lambda}_{d q s}^{60}\right|\left|\vec{i}_{d q s}^{60}\right| \cos (\varphi)
\end{aligned}
$$

where $\cos (\varphi)$ is the power factor.

The dc component in the air-gap torque is induced by the fundamental frequency component in the phase current, as in the bypass mode; whereas the $60-\mathrm{Hz}$ component in the air-gap torque is the torque distortion caused by the injected dc current. It should be noted that the negative sequence current caused by the dc injection is negligible compared to the injected dc component; therefore, the major harmonic in the air-gap torque is the $60-\mathrm{Hz}$ component caused by the injected dc current.

The percentage torque pulsation can be simply derived using (5) and (10) as

$$
\frac{\left|T_{\mathrm{ag}}^{60}\right|}{\left|T_{\mathrm{ag}}^{\mathrm{dc}}\right|} \approx \frac{\left|\vec{\lambda}_{d q s}^{60} \otimes \vec{i}_{d q s}^{\mathrm{dc}}\right|}{\left|\vec{\lambda}_{d q s}^{60} \otimes \vec{i}_{d q s}^{60}\right|}=\frac{\left|\vec{\lambda}_{d q s}^{60}\right|\left|\vec{i}_{d q s}^{\mathrm{dc}}\right|}{\left|\vec{\lambda}_{d q s}^{60}\right|\left|\vec{i}_{d q s}^{60}\right| \cos (\varphi)}=\frac{\sin (\alpha)}{\cos (\varphi)} .
$$

Therefore, the percentage torque pulsation caused by the injected dc signal can be controlled within an acceptable range by controlling the delay angle $\alpha$.

\section{Practical Considerations}

\section{A. DIM Interval and Period}

Since dc injection causes torque pulsation and extra power dissipation in the soft starter and the motor, it is not necessary to inject the dc signal and estimate $R_{s} / T_{s}$ continuously, given a typical motor thermal time constant. The soft starter can be periodically operated in DIM for a minimal time interval that is sufficient to obtain an accurate estimate of $R_{s}$ yet small enough not to cause unacceptable torque pulsation and additional power dissipation. From the experimental results of this work, the soft starter is suggested to operate in DIM for $0.5 \mathrm{~s}$ to obtain an accurate estimate of $R_{s}$.

Given a typical motor thermal time constant, a DIM period of 5-10 min is sufficient for thermal protection purposes, depending on the requirements of practical application. Therefore, the motor performance is only affected by dc injection for $0.5 \mathrm{~s}$ every $5-10 \mathrm{~min}$. In this paper, for validation purposes, the motor is operated in DIM for $0.5 \mathrm{~s}$ every $60 \mathrm{~s}$. 
TABLE I

Stator Winding Resistance Estimation ERror Caused by Cable Resistance

\begin{tabular}{|c|c|c|c|c|c|}
\hline HP & 2 & 5 & 10 & 20 & 50 \\
\hline Typical Rs (Ohm) & $1-2$ & $0.3-1.5$ & $0.15-1$ & $0.1-0.5$ & $0.05-0.2$ \\
\hline Min. Gauge Size (AWG\#) & 12 & 9 & 6 & 3 & 00 \\
\hline Cable Resistance (Ohms/1000 feet) & 1.619 & 0.9077 & 0.4028 & 0.2009 & 0.077 \\
\hline Max. $\Delta$ Rs/Rs caused by cable length of 50 feet $(\%)$ & 0.3 & 0.5 & 0.4 & 0.4 & 0.3 \\
\hline Max. $\Delta$ Rs/Rs caused by cable length of 100 feet $(\%)$ & 0.6 & 1.0 & 0.7 & 0.7 & 0.5 \\
\hline Max. $\Delta$ Rs/Rs caused by cable length of 200 feet (\%) & 1.2 & 2.1 & 1.5 & 1.4 & 1.0 \\
\hline
\end{tabular}

\section{B. Voltage and Current Measurements}

Since $v_{a b}^{\mathrm{dc}}$ and $i_{a}^{\mathrm{dc}}$ are obtained from the means of $v_{a b}$ and $i_{a}$ during DIM, the dc offsets in the voltage and current measurements influence the accuracy of the $R_{s}$ estimate. Therefore, the dc offsets of the measurements must be compensated.

Assuming that the dc component in the supply voltage can be neglected, the offsets can be calculated by the means of $v_{a b}$ and $i_{a}$ for an integer number of cycles in the bypass mode before each DIM, when there is no injected dc signal, as shown in Fig. 5. In this paper, the means of the $v_{a b}$ and $i_{a}$ measurements for 15 cycles in the bypass mode before each DIM are calculated as measurement offsets. After compensation for the measurement offsets, the update rule for $R_{s}$ calculation is modified from (1) to

$$
R_{s}=\frac{2 \cdot\left(v_{a b}^{\mathrm{dc}}-v_{\text {offset }}\right)}{3 \cdot\left(i_{a}^{\mathrm{dc}}-i_{\text {offset }}\right)} .
$$

\section{Cable Resistance}

If the soft starter is installed in the motor control center and the motor terminals are not accessible, the resistance of the cable connecting the induction motor to the soft starter may not be negligible compared to $R_{s}$. The $R_{s}$ estimate by (1) will become

$$
R_{s}=\frac{2 \cdot v_{a b}^{\mathrm{dc}}}{3 \cdot i_{a}^{\mathrm{dc}}}-R_{\text {cable }}
$$

To obtain accurate $R_{s}$ estimates, the cable resistance must be compensated by measurement or estimation of $R_{\text {cable }}$. When measuring $R_{\text {cable }}$ is not possible, given the cable number in the American wire gauge (AWG) standard, $R_{\text {cable }}$ can be estimated based on the resistivity $\rho$ given by the AWG standard, the approximate length $l$ of the cable, and the ambient temperature $T_{A}$ as

$$
\hat{R}_{\text {cable }}=\rho l+\alpha \rho l\left(T_{A}-T_{0}\right)
$$

where $\alpha$ is the temperature coefficient of resistivity and $T_{0}$ is the room temperature, assuming that the cable temperature is the same as the ambient temperature. The error of $R_{s}$ estimate caused by the inaccurate estimate of the cable temperature is given as

$$
\Delta R_{s}=\hat{R}_{\text {cable }}-R_{\text {cable }}=\alpha R_{\text {cable } 0} \Delta T_{\text {cable }}
$$

TABLE II

NAMEPLATE INFORMATION OF EXPERIMENTAL SETUP

\begin{tabular}{|c|c|c|c|}
\hline \multicolumn{5}{|c|}{ Induction Motor 1 } \\
\hline HP & 5 & Brand & Marathon \\
\hline Model. NO. & 184 TTFS6026 & RPM & 1755 \\
\hline Volts & 460 & NEMA & B \\
\hline SF & 1.15 & Nom. Eff. & 90.2 \\
\hline F.L. AMPS & 6.2 & ENCL. & ODP \\
\hline \multicolumn{4}{|c|}{ Induction Motor 2 } \\
\hline HP & 30 & Brand & Lincoln \\
\hline Model. NO. & TV2632 & RPM & 1765 \\
\hline Volts & $230 / 460$ & NEMA & B \\
\hline SF & 1.15 & Nom. Eff. & 88.5 \\
\hline F.L. AMPS & $74 / 37$ & ENCL. & TEFC \\
\hline \multicolumn{4}{|c|}{ Soft-Starter for Experimental Testing } \\
\hline Brand & Eaton & CAT. NO. & IT.S 811 N \\
\hline Volts & $200 / 230 / 460 / 575$ & HP & $10 / 10 / 25 / 30$ \\
\hline Max. AMPS & 37 & Frequency & $47-63$ \\
\hline \multicolumn{4}{|c|}{}
\end{tabular}

where $\Delta R_{s}$ is the error of $R_{s}$ estimate, and $\Delta T_{\text {cable }}$ is the actual cable temperature above the ambient temperature.

Table I shows the typical $R_{s}$ for the induction motor rated from 2 to $50 \mathrm{hp}$. The minimal gauge size of the cable is given, considering the rated current for the motor of each rating and the current carrying ability of the cable listed by the AWG standard. For a cable temperature error $\Delta T_{\text {cable }}$ of $10{ }^{\circ} \mathrm{C}$, the error of $R_{s}$ estimate is calculated, considering the worst case, using (16). It can be seen in Table I that when the cable resistance is estimated using (15), the $R_{s}$ estimation error caused by the cable resistance is within an acceptable range. For example, for a 10-hp induction motor, the $R_{s}$ estimation error caused by a $200-\mathrm{ft}$ cable is within $1.5 \%$. Therefore, the proposed scheme can provide remote and reliable $R_{s}$ estimate even when the motor terminals are not accessible.

\section{EXPERIMENTAL VALIDATION}

\section{A. Experimental Setup}

The proposed thermal monitoring scheme has been validated using a 5-hp open-drip-proof induction motor and a 30-hp totally-enclosed-fan-cooling induction motor, whose nameplate information is shown in Table II. The stator resistances of the two motors are 1.36 and $0.329 \Omega$, respectively, under room temperature. The gate drive control signals of a soft starter are programmed to inject dc signals.

The experimental setup is shown in Figs. 6 and 7. The motor terminal voltages and phase currents are measured and stored 


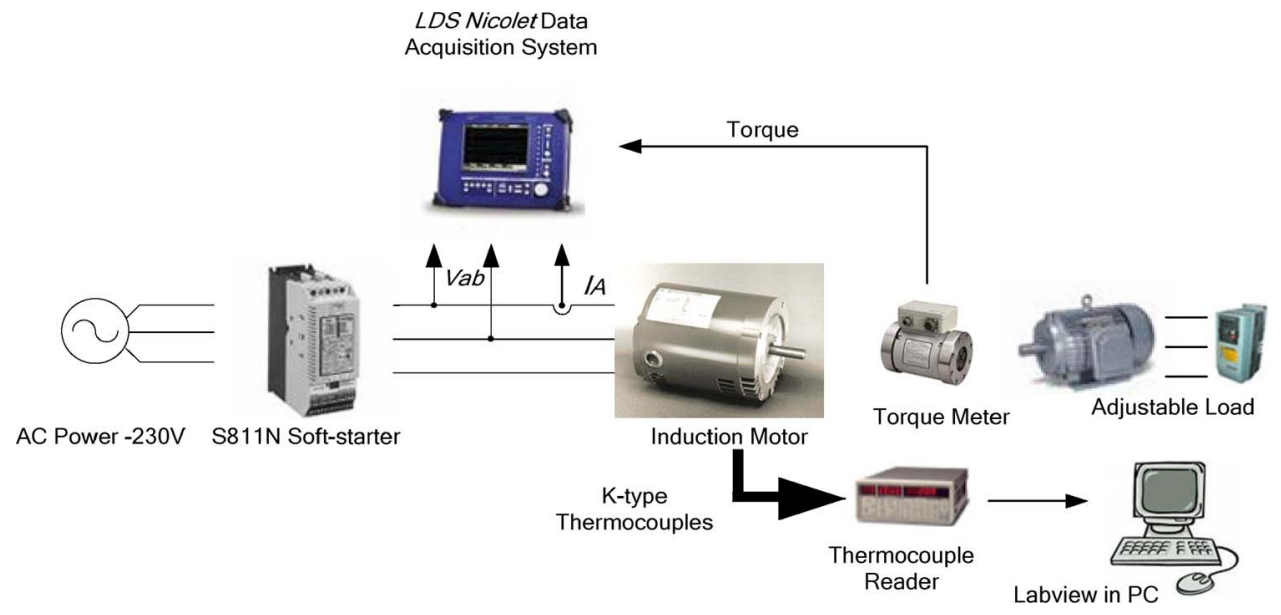

Fig. 6. Overall experimental setup.
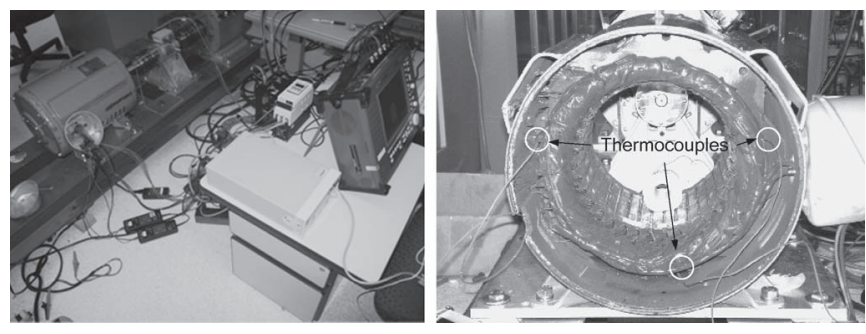

Fig. 7. Experiment setup.

using an LDS Nicolet data-acquisition system, with 16-bit analog-to-digital conversion resolution at a sampling frequency of $100 \mathrm{kHz}$. A 30-hp drive-connected induction generator serves as the adjustable load. A Himmelstein digital torque meter is connected between the induction motor and the load to measure the output torque for torque pulsation analysis. The motor is instrumented with six K-type thermocouples at different locations in the stator winding to record its average winding temperature for validation purposes, as shown in Fig. 7.

\section{B. Torque Pulsation Analysis}

To test the induced torque pulsation by the injected dc signal, motor 1 is operated under $80 \%$ of the rated load with a dcinjection delay angle of $20^{\circ}$. The measured $v_{a b}$ and $i_{a}$ and the output torque are shown in Fig. 8. The fast Fourier transforms (FFTs) of $v_{a b}, i_{a}$, and the output torque are normalized with respect to the $60-\mathrm{Hz}$ components in $v_{a b}, i_{a}$, and the dc component of the output torque, respectively, as shown in Fig. 9. It can been seen in Fig. 9 that the high-frequency components in $i_{a}$ and $v_{a b}$ can be neglected compared to the dc and $60-\mathrm{Hz}$ components, which confirms the assumption in (3) and (4). The major components in the output torque are the dc and $60-\mathrm{Hz}$ components induced by the $60-\mathrm{Hz}$ and dc components in the phase current, respectively.

The percentage torque pulsation of the measured output torque caused by the injected dc signal is $36.8 \%$, whereas the estimated torque pulsation from the delay angle $\alpha$ by (12) gives $\sin \left(20^{\circ}\right) / \cos (\varphi)=43.3 \%$, with a measured power factor
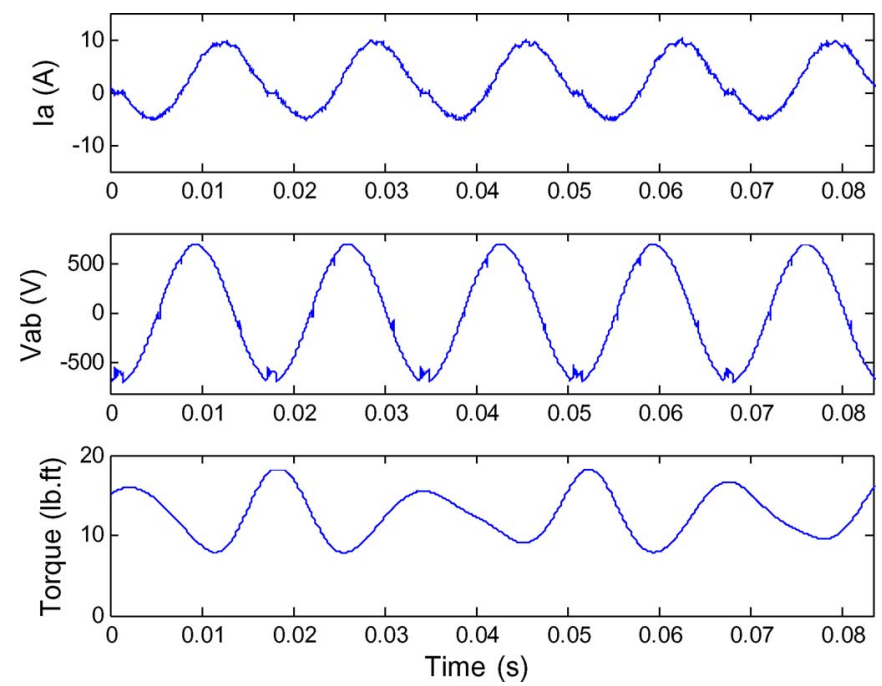

Fig. 8. Measured voltage, current, and output torque of motor 1.
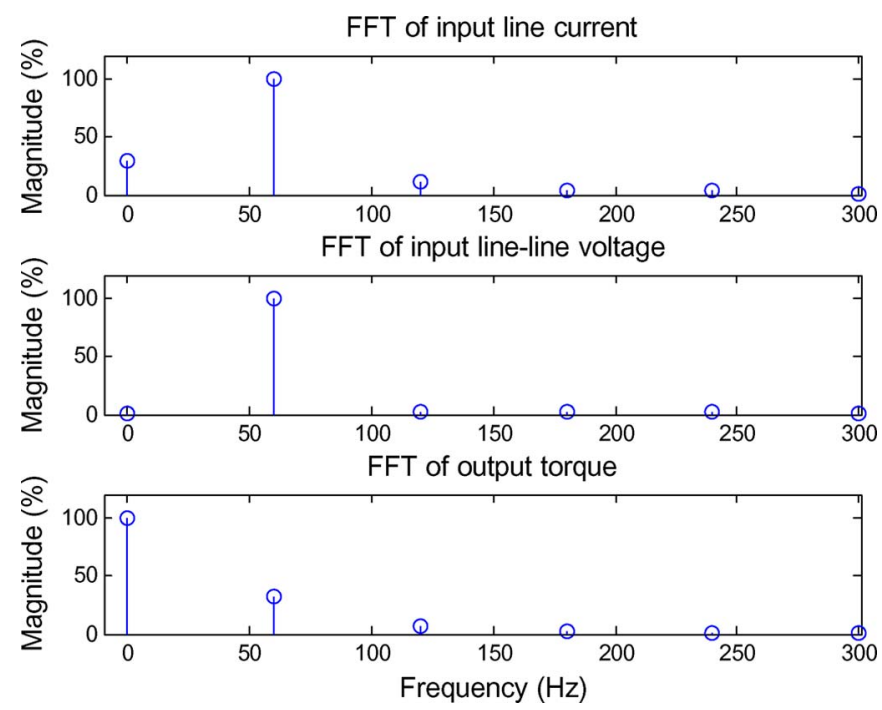

Fig. 9. Normalized FFTs of the measured voltage, current, and output torque.

$\cos (\varphi)=0.79$. This shows that by using (12), the maximum delay angle $\alpha$ can be adjusted online, given an acceptable torque pulsation level. 

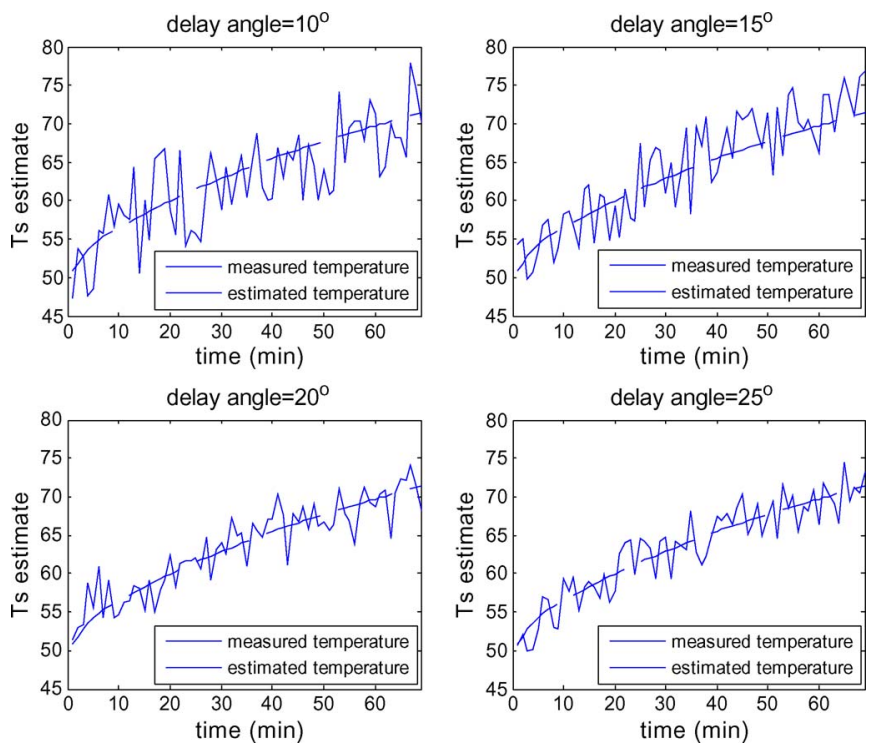

Fig. 10. Stator winding temperature estimation results (motor 1).
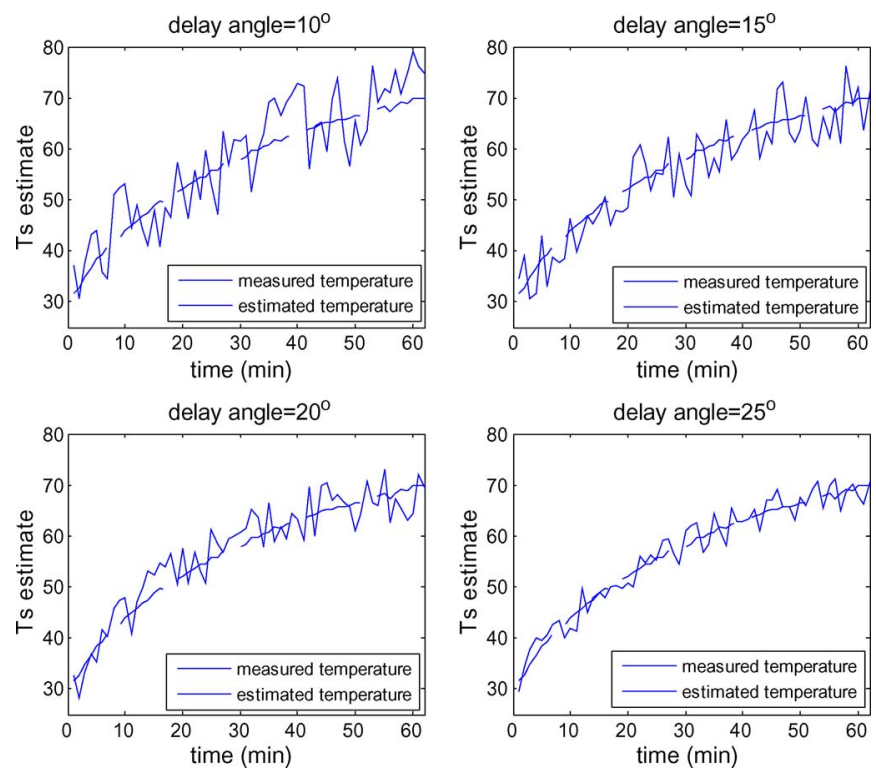

Fig. 11. Stator winding temperature estimation results (motor 2).

It should be noted that torque pulsation is only induced in DIM, in which the motor is only operated for $0.5 \mathrm{~s}$ every $60 \mathrm{~s}$. In the bypass mode, the motor performance is not affected.

\section{Stator Resistance and Temperature Estimation Results}

After determining the maximal delay angle to limit the torque pulsation under an acceptable level, the stator winding temperature $T_{s}$ can be monitored using (2) based on the stator resistance estimation from dc injection. It should be noted that the $T_{s}$ estimation accuracy is dependent on the level of the injected dc signal.

The estimated $T_{s}$ based on dc injection for motors 1 and 2 are shown in Figs. 10, and 11, respectively. The tested motors are operated under full load with different delay angles (i.e., 10, 15, 20 , and $25^{\circ}$ ). The measured temperatures are calculated using the average temperature measured from the preinstalled thermocouples for validation purposes. It can be seen in Figs. 10 and 11 that the accuracy of the $T_{s}$ estimate improves for a larger delay angle. However, from (12), the torque pulsation caused by the delay angle also increases as the delay angle increases. Therefore, determining the delay angle is a tradeoff between stator temperature accuracy and torque pulsation. Fig. 12(a) and (b) shows the mean square error (mse) of the $T_{s}$ estimation under a full-load condition and the percentage torque pulsation as functions of delay angle for motors 1 and 2, respectively.

\section{Influence of Cable Resistance}

To test the influence of the cable resistance, a 48 -ft 12 AWG cable is installed from the supply power to motor 1 . From the AWG standard, the resistivity of the 12 AWG cable is $1.588 \Omega / 1000 \mathrm{ft}$. Using (15), the cable resistance at room temperature is calculated as $0.0762 \Omega$. Motor 1 is operated under a full-load condition with a delay angle of $20^{\circ}$. The $T_{s}$ estimation results with and without cable resistance compensation are compared in Fig. 13. It can be seen in Fig. 13 that the proposed cable resistance compensation method in Section III-C can effectively reduce the $T_{s}$ estimation error caused by the cable resistance, which allows remote thermal monitoring of the motors.

\section{CONCLusion}

This paper has proposed a remote and sensorless stator winding resistance estimation method for thermal protection of soft-starter-connected induction motors. A dc-injection method has been developed to estimate the stator winding resistance by changing the gate drive signals of the thyristors in the soft starter. Based on the estimated stator resistance, the average stator winding temperature can be monitored. The torque pulsation caused by the signal injection is analyzed. It can also be controlled for improving the resistance/temperature estimation accuracy while minimizing the impact of torque pulsation caused by the injected injection. In addition, the accuracy of the proposed method can be greatly improved by considering the dc offsets in the voltage and current measurements and the resistance of the power cable from the soft starter to the motor terminals. A cable resistance compensation method has been proposed and enables this sensorless thermal monitoring and protection scheme to be implemented remotely at the soft starter or other motor control devices. The proposed methods are validated by experimental results from two induction motors with different enclosures. The main advantages of the proposed scheme are summarized as follows.

1) Sensorless: The stator winding temperature can be estimated using only the motor line voltage and phase current measurements (already existing in soft starter) without additional sensors attached on the motor.

2) Remote: By using the proposed cable resistance compensation method, the motor winding resistance/temperature can be accurately determined remotely at the soft starter. As a result, remote motor winding thermal monitoring and protection becomes feasible. 


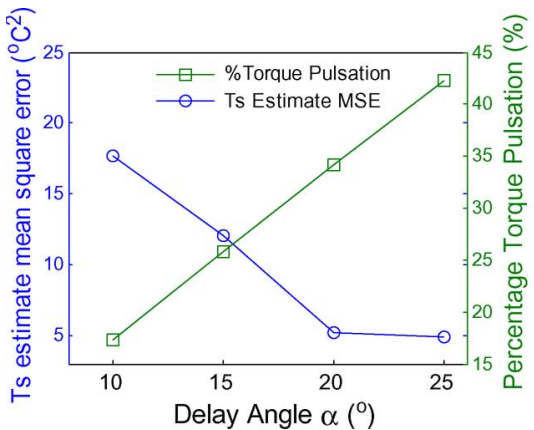

(a)

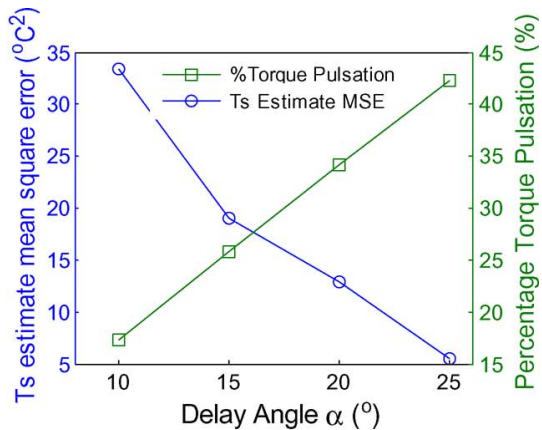

(b)

Fig. 12. MSE of $T_{s}$ estimation and \%torque pulsation as functions of delay angle. (a) Motor 1. (b) Motor 2.

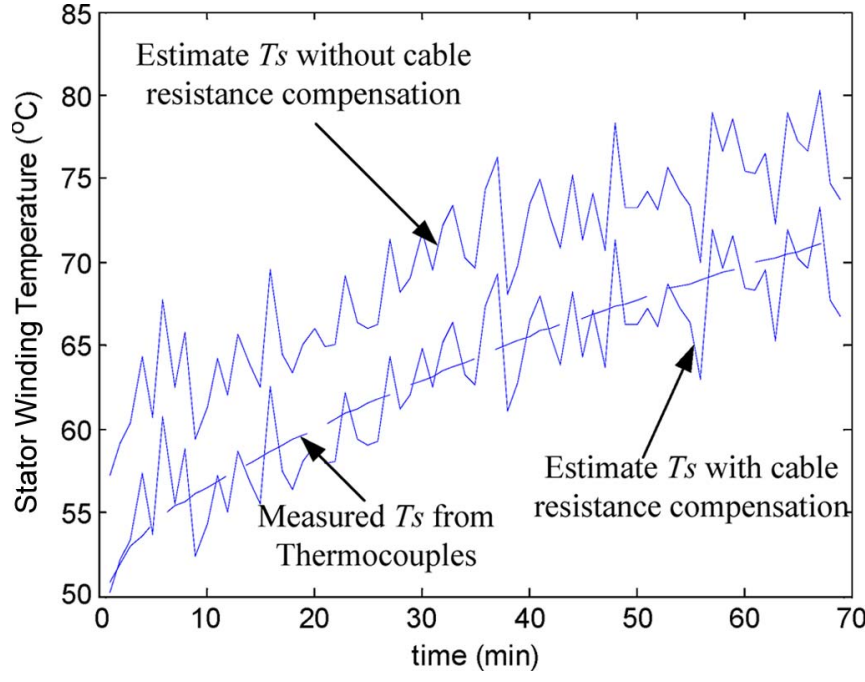

Fig. 13. $T_{s}$ estimation with and without cable resistance compensation.

3) Accurate: From the experimental results, the root mean square error of the stator winding temperature estimation using the proposed methods is within $2.5^{\circ} \mathrm{C}$ at a $25^{\circ}$ delay angle when the winding temperature varies from $30^{\circ} \mathrm{C}$ to $70{ }^{\circ} \mathrm{C}$.

4) Flexible: The torque pulsation caused by the dc injection and the estimation accuracy are found to be both related to the delay angle. The delay angle can be optimally controlled to compromise between the estimation accuracy and the induced torque pulsation during the resistance/temperature estimation.

\section{REFERENCES}

[1] "Report of large motor reliability survey of industrial and commercial installations-Part I," IEEE Trans. Ind. Appl., vol. IA-21, no. 4, pp. 853864, Jul./Aug. 1985

[2] M. S. Abou-El-Ela, A. I. Megahed, and O. P. Malik, "Thermal model based digital relaying algorithm for induction motor protection," in Proc. Can. Conf. Elect. Comput. Eng., 1996, vol. 2, pp. 1016-1019.

[3] S. E. Zocholl, "Motor analysis and thermal protection," IEEE Trans. Power Del., vol. 5, no. 3, pp. 1275-1280, Jul. 1990.

[4] K. D. Hurst and T. G. Habetler, "A thermal monitoring and parameter tuning scheme for induction machines," in Conf. Rec. IEEE IAS Аnпи. Meeting, 1997, vol. 1, pp. 136-142.

[5] M. A. Valenzuela, P. V. Verbakel, and J. A. Rooks, "Thermal evaluation for applying TEFC induction motors on short-time and intermittent duty cycles," IEEE Trans. Ind. Appl., vol. 39, no. 1, pp. 45-52, Jan./Feb. 2003.

[6] J. F. Moreno, F. P. Hidalgo, and M. D. Martinez, "Realisation of tests to determine the parameters of the thermal model of an induction machine,"
Proc. Inst. Elect. Eng.-Electric Power Applications, vol. 148, no. 5, pp. 393-397, Sep. 2001.

[7] H. Nestler and P. Sattler, "On-line-estimation of temperatures in electrical machines by an observer," Elect. Mach. Power Syst., vol. 21, no. 1, pp. 3950, Jan. 1993.

[8] A. Bousbaine, M. McCormick, and W. F. Low, "In-situ determination of thermal coefficients for electrical machines," IEEE Trans. Energy Convers., vol. 10, no. 3, pp. 385-391, Sep. 1995.

[9] P. H. Mellor, D. Roberts, and D. R. Turner, "Lumped parameter thermal model for electrical machines of TEFC design," Proc. Inst Elect. Eng.-Electric Power Applications, vol. 138, no. 5, pp. 205-218, Sep. 1991.

[10] D. Staton, A. Boglietti, and A. Cavagnino, "Solving the more difficult aspects of electric motor thermal analysis in small and medium size industrial induction motors," IEEE Trans. Energy Convers., vol. 20, no. 3, pp. 620-628, Sep. 2005.

[11] J. Catala i Lopez, L. Romeral, A. Arias, and E. Aldabas, "Novel fuzzy adaptive sensorless induction motor drive," IEEE Trans. Ind. Electron., vol. 53, no. 4, pp. 1170-1178, Aug. 2006.

[12] J. Salomaki, M. Hinkkanen, and J. Luomi, "Sensorless control of induction motor drives equipped with inverter output filter," IEEE Trans. Ind. Electron., vol. 53, no. 4, pp. 1188-1197, Jun. 2006.

[13] M. Cirrincione, M. Pucci, G. Cirrincione, and G. A. Capolino, "Sensorless control of induction motors by reduced order observer with MCA EXIN + based adaptive speed estimation," IEEE Trans. Ind. Electron., vol. 54, no. 1, pp. 150-166, Feb. 2007.

[14] Q. Gao, G. Asher, and M. Sumner, "Sensorless position and speed control of induction motors using high-frequency injection and without offline precommissioning," IEEE Trans. Ind. Electron., vol. 54, no. 5, pp. 2474 2481, Oct. 2007.

[15] M. Barut, S. Bogosyan, and M. Gokasan, "Speed-sensorless estimation for induction motors using extended Kalman filters," IEEE Trans. Ind. Electron., vol. 54, no. 1, pp. 272-280, Feb. 2007.

[16] M. S. Ballal, Z. J. Khan, H. M. Suryawanshi, and R. L. Sonolikar, "Adaptive neural fuzzy inference system for the detection of inter-turn insulation and bearing wear faults in induction motor," IEEE Trans. Ind. Electron., vol. 54, no. 1, pp. 250-258, Feb. 2007.

[17] J. H. Jung, J. J. Lee, and B. H. Kwon, "Online diagnosis of induction motors using MCSA," IEEE Trans. Ind. Electron., vol. 53, no. 6, pp. 1842 1852, Dec. 2006.

[18] D. de Almeida Souza, W. C. P. de Aragao Filho, and G. C. D. Sousa, "Adaptive fuzzy controller for efficiency optimization of induction motors," IEEE Trans. Ind. Electron., vol. 54, no. 4, pp. 2157-2164, Aug. 2007.

[19] IEEE Standard Test Code for Resistance Measurement, May 2004. IEEE Standard 118-1978

[20] R. Beguenane and M. E. H. Benbouzid, "Induction motors thermal monitoring by means of rotor resistance identification," IEEE Trans. Energy Convers., vol. 14, no. 3, pp. 566-570, Sep. 1999.

[21] S. B. Lee, T. G. Habetler, R. G. Harley, and D. J. Gritter, "A stator and rotor resistance estimation technique for conductor temperature monitoring," in Conf. Rec. IEEE IAS Annu. Meeting, 2000, vol. 1, pp. 381-387.

[22] B. Karanayil, M. F. Rahman, and C. Grantham, "Investigation of an online rotor resistance identification with a new stator resistance observer for induction motor drive using artificial neural networks," in Proc. IEEE 34th Anпи. PESC, 2003, vol. 4, pp. 1883-1888.

[23] M. Boussak and K. Jarray, "A high-performance sensorless indirect stator flux orientation control of induction motor drive," IEEE Trans. Ind. Electron., vol. 53, no. 1, pp. 41-49, Feb. 2006. 
[24] M. J. Duran, J. L. Duran, F. Perez, and J. Fernandez, "Induction-motor sensorless vector control with online parameter estimation and overcurrent protection," IEEE Trans. Ind. Electron., vol. 53, no. 1, pp. 154-161, Feb. 2006.

[25] B. Karanayil, M. Fazlur Rahman, and C. Grantham, "Online stator and rotor resistance estimation scheme using artificial neural networks for vector controlled speed sensorless induction motor drive," IEEE Trans. Ind. Electron., vol. 54, no. 1, pp. 167-176, Feb. 2007.

[26] M. Hasegawa, "Robust-adaptive-observer design based on $\gamma$-positive real problem for sensorless induction-motor drives," IEEE Trans. Ind. Electron., vol. 53, no. 1, pp. 76-85, Feb. 2006.

[27] M. Rashed, P. F. A. MacConnell, A. F. Stronach, and P. Acarnley, "Sensorless indirect-rotor-field-orientation speed control of a permanent-magnet synchronous motor with stator-resistance estimation," IEEE Trans. Ind. Electron., vol. 54, no. 3, pp. 1664-1675, Jun. 2007.

[28] C. Lascu and G. D. Andreescu, "Sliding-mode observer and improved integrator with DC-offset compensation for flux estimation in sensorlesscontrolled induction motors," IEEE Trans. Ind. Electron., vol. 53, no. 3, pp. 785-794, Jun. 2006.

[29] S. B. Lee and T. G. Habetler, "An online stator winding resistance estimation technique for temperature monitoring of line-connected induction machines," IEEE Trans. Ind. Appl., vol. 39, no. 3, pp. 685-694, May/ Jun. 2003.

[30] J. S. Hsu, H. H. Woodson, and W. F. Weldon, "Possible errors in measurement of air-gap torque pulsations of induction motors," IEEE Trans. Energy Convers., vol. 7, no. 1, pp. 202-208, Mar. 1992.

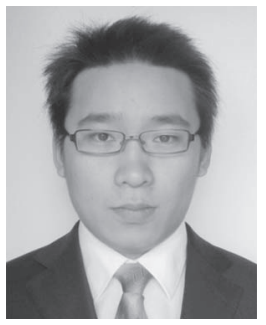

Pinjia Zhang (S'06) received the B.Eng. degree in electrical engineering from Tsinghua University, Beijing, China, in 2006. He is currently working toward the Ph.D. degree in the School of Electrical and Computer Engineering, Georgia Institute of Technology, Atlanta.

Since July 2006, he has been working on condition monitoring of electric machines, in collaboration with Eaton Corporation, as a graduate Research Assistant in the electric power group of the Georgia Institute of Technology. His research interests include electric machine diagnostics, motor drives, power electronics, and the application of artificial intelligence in power system.

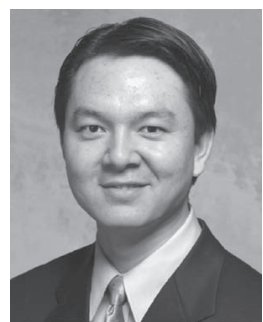

Bin Lu (S'00-M'06) received the B.Eng. degree in automation from Tsinghua University, Beijing, China, in 2001, the M.S. degree in electrical engineering from the University of South Carolina, Columbia, in 2003, and the Ph.D. degree in electrical engineering from the Georgia Institute of Technology, Atlanta, in 2006, respectively.

From 2001 to 2003, he was with the VTB Group, University of South Carolina, Columbia, developing a real-time virtual test bed for the hardware-inthe-loop testing of dynamic power systems. Since July 2004, he has been a graduate Research Assistant in the power electronics and motor diagnostics group of the Georgia Institute of Technology, working on the energy evaluation and condition monitoring of electric machines. In summer 2006, he joined the Manufacturing Research Laboratory, R\&D Center, General Motors, Warren, MI, as a Graduate Summer Intern. Since October 2006, he has been with the Innovation Center, Eaton Corporation, Milwaukee, WI, where he is currently an Engineering Specialist and a Program Manager. He has authored over 30 papers published in refereed journals and international conference proceedings and has five patents pending in these areas. His research interests include electric motor drives and diagnostics, renewable energy, power electronics, modeling and simulation, and application of wireless sensor networks in electric power areas.

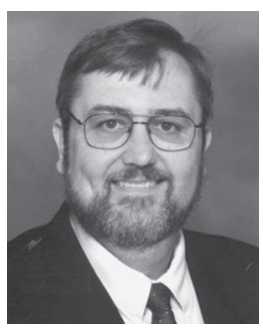

Thomas G. Habetler (S'82-M'83-SM'92-F'02) received the B.S. and M.S. degrees in electrical engineering from Marquette University, Milwaukee, WI, in 1981 and 1984, respectively, and the Ph.D. degree from the University of Wisconsin, Madison, in 1989.

From 1983 to 1985 , he was with the ElectroMotive Division, General Motors, as a Project Engineer. Since 1989, he has been with the Georgia Institute of Technology, Atlanta, where he is currently a Professor of electrical engineering in School of Electrical and Computer Engineering. He has authored over 100 published papers in this field. He is a regular consultant to industry in the field of condition-based diagnostics for electrical systems. His research interests are electric machine protection and condition monitoring, switching converter technology, and drives.

Dr. Habetler has received four conference prize paper awards from the Industry Applications Society. He currently serves as the IEEE Division II Director-Elect, is a former President of the IEEE Power Electronics Society, and is a former Chair of the Industrial Power Converter Committee of the IEEE Industry Applications Society. 


\title{
A Survey of Available Methods for
}

\section{Evaluating In-Service Motor Efficiency}

\author{
EATON WSN Research Report
}

\section{Prepered by}

Bin Lu

Prof. Thomas G. Habetler

Prof. Ronald G. Harley

Oct. 26 $^{\text {th }}, 2004$

SCHOOL OF ELECTRICAL AND COMPUTER ENGINEERING GEORGIA INSTITUTE OF TECHNOLOGY

ATLANTA, GA 30332 


\section{TALBE OF CONTENTS}

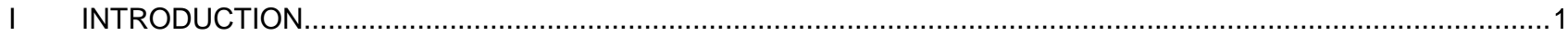

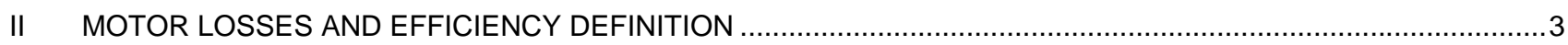

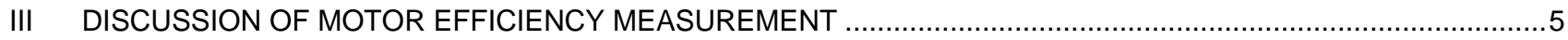

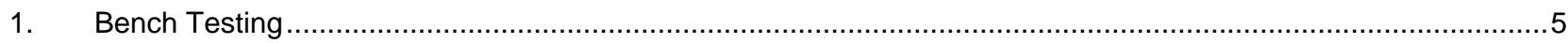

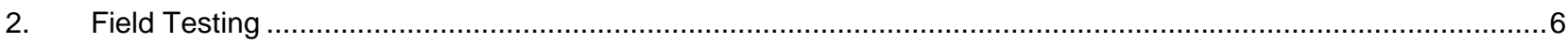

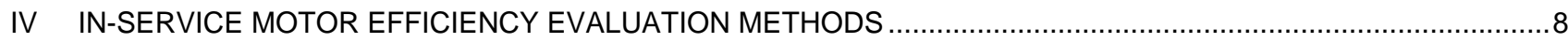

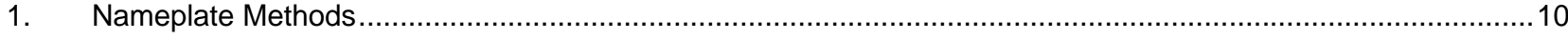

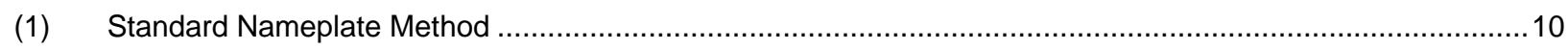

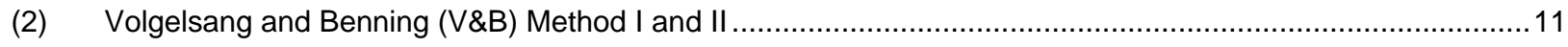

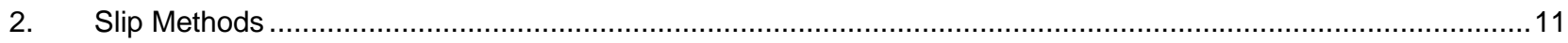

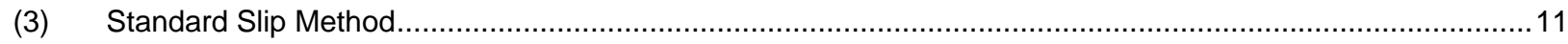

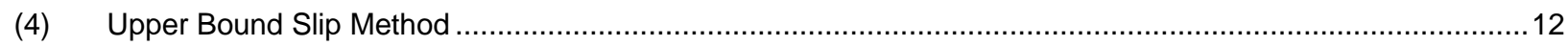

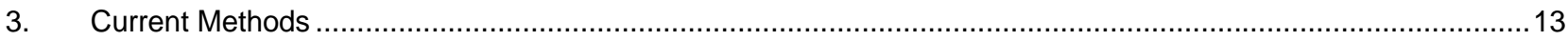

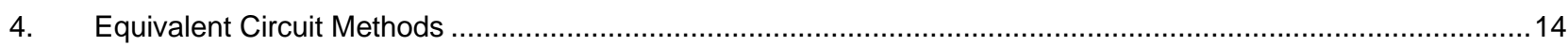

(5) Standard Equivalent Circuit Method (IEEE Std-112 Method F) ...................................................... 15

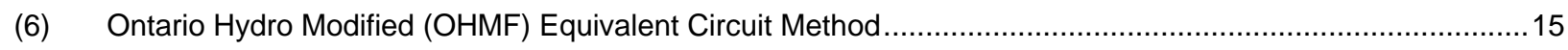

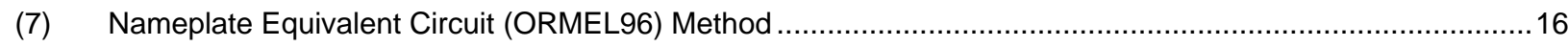

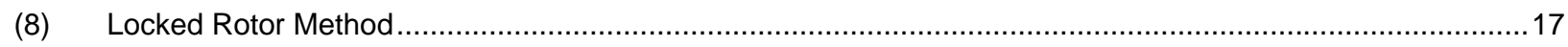

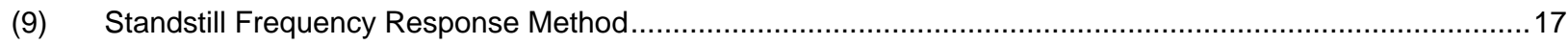

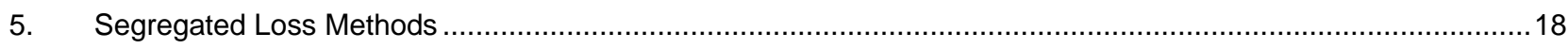

(10) Standard Segregated Loss Method (IEEE Std-112 Method E) …................................................. 18

(11) Ontario Hydro Modified (OHME) Segregated Loss Method................................................................... 19

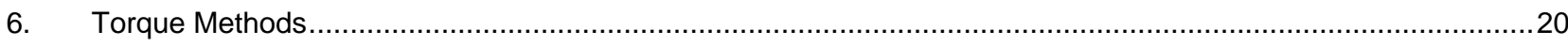

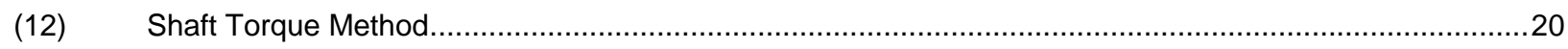

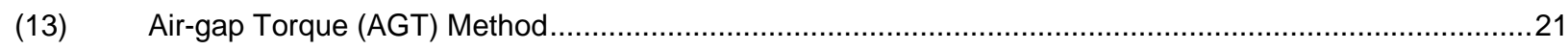

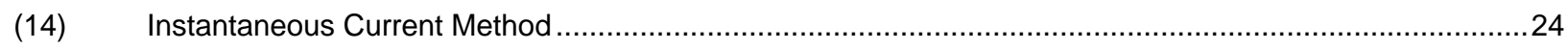

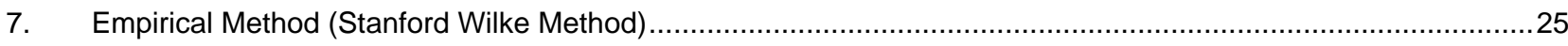

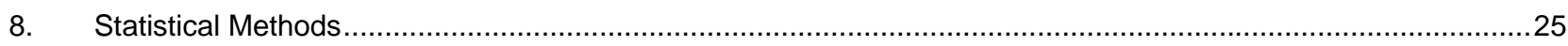

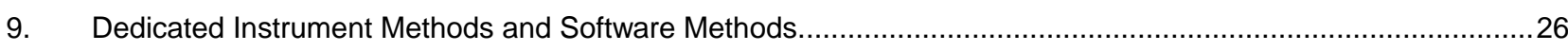

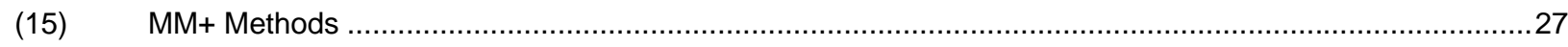

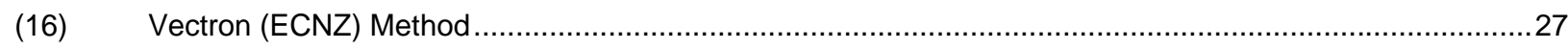

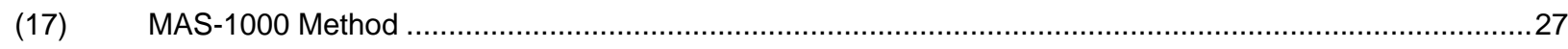

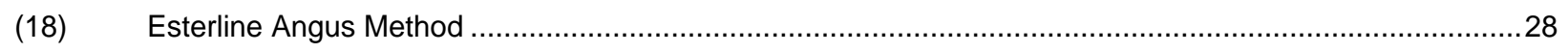

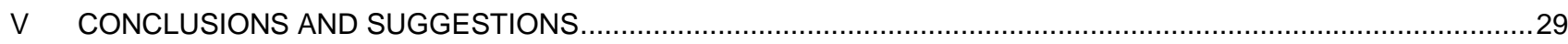

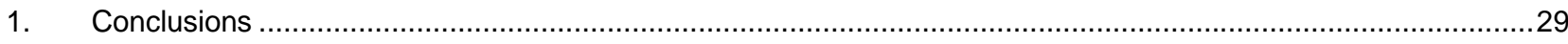

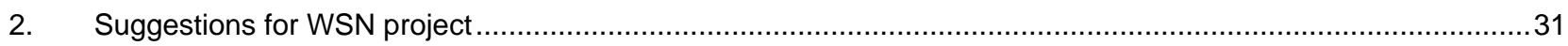

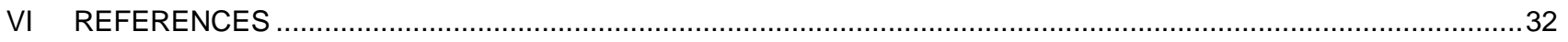




\section{LIST OF TABLES}

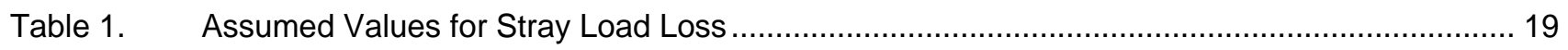

Table 2. Comparison of the Three Suggested Methods ................................................................... 30

Table 3. Comparison of the Testing Results Using Three Suggested Methods.................................... 30

\section{LIST OF FIGURES}

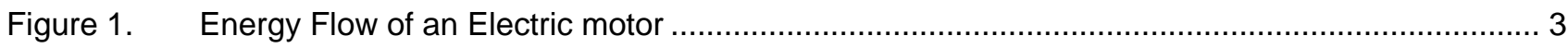

Figure 2. Assumed and Actual Load-versus-Current Curves. ....................................................... 14

Figure 3. Equivalent Circuit of IEEE Std-112 Method F....................................................... 15

Figure 4. Anticipated Efficiency Estimation Errors versus Evaluation Methods................................... 29 


\section{INTRODUCTION}

In the Eaton WSN research project, the focus is on two main objectives, efficiency estimation and condition monitoring. In this report, the various methods of determining the motor efficiency are discussed in details.

Over the years, many motor efficiency estimation methods have been proposed. A series of relatively comprehensive laboratory assessments have been conducted back in 1990's, and have become main sources of this survey.

In March of 1996, a team at the Oak Ridge National Lab (ORNL), under contract to the Bonneville Power Administration (BPA) and Pacific Gas and Electric (PG\&E), reviewed 28 of the already in use or proposed for use efficiency estimation methods and evaluated them according to their intrusiveness and cost of equipment. ${ }^{[1][2][3]}$

Shortly after the first report, the same team selected 6 candidate methods among these 28 methods for more detailed evaluation under the support of the Motor Challenge Program sponsored by Department of Energy (DOE). ${ }^{[4]}$ These 6 methods particularly target on estimating motor efficiency and load under field conditions. These 6 methods were divided into three groups according to their intrusion level and accuracy, ranging from highly intrusive methods to completely non-intrusive methods. The report also suggested one best candidate method out of each group based on the laboratory testing results.

Based on above reviews, in April of 1999 the Washington State University Co-Operative Extension Energy Program (WSUCEEP), under contract with BPA and PG\&E, subcontracted with the Motor System Resource Facility (MSRF) at Oregon State University (OSU) to test the 12 most promising in-service motor efficiency testing methods. ${ }^{[5][6][7]}$ This testing also investigated the intrusion level of these efficiency estimation methods and their achieved level of accuracies. A more detailed test of four non-intrusive efficiency methods was also performed and the results were compared. 
In our previous WSN report “An Investigation on Commercially Available Energy Usage Estimation Products” ${ }^{[28]}$, we investigated the available energy usage estimation products on the market. These products chose different methods in efficiency estimation. However, since the industry expects the energy usage estimation and diagnosis to be done without shutting down the motor, most of these products adopted non-intrusive or low-intrusive methods.

The measurements needed for each method are different, but most of them require the following common data: input line-to-line voltage, input phase current, and winding temperature. Some methods require the nameplate information (rated voltage, current, horsepower, speed, etc), stator resistance, or rotor speed (slip). Among these, the measurements/estimates of the stator resistance and rotor speed have been regarded as the stumbling blocks of various efficiency estimation methods for years.

However, over recent years research in this area has solved (or at least relieved) the difficulty of obtaining the stator resistance and rotor speed. Recent research has produced quite accurate speed estimates through stator current harmonic spectral analysis when the machine is working under normal stable operation. ${ }^{[8][9][10]}$ Also some “in situ” stator resistance measurement methods have been proposed which inject a DC component into the stator voltage and current. The accurate nonintrusive measurement of stator resistance may still need more research, but alternatively it can be measured and recorded at the time of new machine installation and at scheduled maintenance intervals or estimated from nameplate data. Therefore, in the efficiency estimation methods described hereafter, the stator resistance and rotor speed can be regarded as known variables.

In the rest of this survey, over 18 available motor efficiency testing methods are introduced and compared.

Finally, based on the preliminary research, three best in-service motor efficiency estimation methods are suggested for the WSN project ranging from low-intrusive to high-intrusive. 


\section{MOTOR LOSSES AND EFFICIENCY DEFINITION}

Figure 1 shows the energy flow of an electric motor. The total losses of the motor are of our interest, since finding the efficiency is equivalent with finding the total losses.

There are, in general, five components of motor losses, as follows: ${ }^{[11]}$

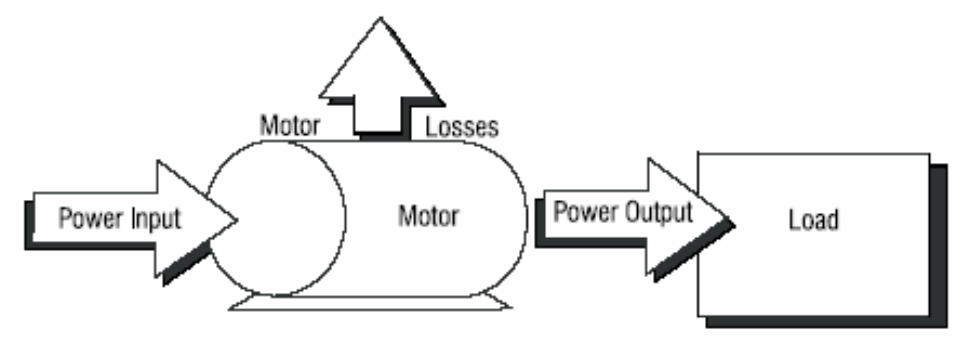

Figure 1. Energy Flow of an Electric motor

Stator resistance losses $\left(\mathrm{W}_{\mathrm{s}}\right.$, ) are the losses in the stator windings equal to $1.5 \mathrm{I}^{2} \mathrm{R}_{\mathrm{s}}$ for a three-phase motor where I is the measured or calculated RMS current per line terminal at specified load; and $\mathrm{R}_{\mathrm{S}}$ is the average dc resistance between any two line terminals corrected to the specified temperature.

Rotor resistance losses $\left(\mathrm{W}_{\mathrm{r}}\right.$ ) are the losses in the rotor windings equal to $3 \mathrm{I}_{2}{ }^{2} \mathrm{R}_{\mathrm{r}}$ for a three-phase motor where $I_{2}$ is the rotor phase current and $R_{r}$ is the rotor dc resistance corrected to the specified temperature.

Core losses ( $\mathrm{W}_{\text {core }}$ ) constitute the hysteresis and eddy current losses in the iron. These losses vary approximately with the square of the input voltage, but for fixed input voltage these remain approximately constant from no load to full load. These losses are commonly measured by running the machine as a motor at rated voltage and frequency without connected load (no-load test). 
Windage and friction losses $\left(\mathrm{W}_{\mathrm{fw}}\right)$ are the mechanical losses due to bearing friction and windage. These losses are also approximately constant from no load to full load. It is also a common practice to use no-load test to measure these losses.

Stray load losses $\left(\mathrm{W}_{\mathrm{LL}}\right)$ are the fundamental and high-frequency losses in the structure of the motor, circulating current losses in the stator winding, and harmonic losses in the rotor conductors under load. These losses are proportional to the square of the rotor current. The stray load losses can be further divided into two parts: stator stray loss $\left(\mathrm{W}_{\mathrm{LLs}}\right)$ and rotor stray loss $\left(\mathrm{W}_{\mathrm{LLr}}\right)$.

The relationship between the input power, output power and the losses are described by the following equation:

$$
W_{\text {Losses }}=P_{\text {input }}-P_{\text {output }}=W_{S}+W_{r}+W_{\text {core }}+W_{f w}+W_{L L s}+W_{L L r}
$$

The efficiency is defined by different ways as the following ${ }^{[3]}$

$$
\begin{aligned}
& \eta=\frac{P_{\text {output }}}{P_{\text {input }}} \times 100 \% \\
& =100 \%-\frac{W_{\text {Losses }}}{P_{\text {input }}} \times 100 \% \\
& =\frac{P_{\text {em }}-W_{f w}}{P_{\text {input }}} \times 100 \% \\
& =\frac{T_{\text {air-gap }} \cdot 2 \pi \frac{r p m}{60}-W_{f w}-W_{L L r}}{P_{\text {input }}} \times 100 \%
\end{aligned}
$$




\section{DISCUSSION OF MOTOR EFFICIENCY MEASUREMENT}

\section{Bench Testing}

In general, bench testing provides the most accurate testing methods of motor efficiency. A load absorption device (dynamometer, pump or generator) is used to absorb the power generated by the motor and a torque measuring device is used to measure the torque on the motor shaft (shaft torque) as it provides power to the load absorber. In addition, a speed measuring device is used to measure the speed of the rotor. The output power is simply calculated by multiplying the measured torque times the speed.

Errors can be introduced through a number of ways:

(1) The accuracy of the output power depends on the accuracy of two measured variables, speed and torque. Speed is relatively precise. While the measurement of torque is prone to error if one strain gauge is installed on the bench and is used to measure a wide range shaft torque.

(2) The input power is calculated by multiplying the measured input voltage waveform and input current waveform. Errors can be introduced by the phase shifts associated with the current transformer and the difference in sample time between the samples of the input currents and voltages.

(3) The power supply at the test bench may also produce some error in to the efficiency determination. For example, a three percent voltage imbalance can result in a negative sequence current flow and subsequent heating which requires nearly a $10 \%$ derating of the motor. ${ }^{[12]}$

While errors are not the main drawback of bench testing method, the most significant problem is the time required to remove the motor form service, transport it to the test facility, perform the testing, reinstall it, including necessary alignment. There are significant expenses in downtime and manpower, which would not be acceptable for most cases. 


\section{Field Testing}

The most significant advantage of the in-service test methods is that the motor does not have to be removed from the service and thus the manufacturing process is not interrupted. Measurements can be taken quickly with clamp-on current transformers (CTs) and voltage probes. Typically, these measurements are taken at the motor control center (MCC). Usually field testing is the only practical method for efficiency testing because neither the removal of the motor nor the operation downtime is permitted by the user.

Another significant advantage of performing in-service testing is that the motor's service condition can be measured and recorded for evaluation. As previously discussed, the voltage imbalance and harmonics distortion can have a severe negative effect on the efficiency determination. The estimation would be more precise if these negative factors are known before evaluation.

The fundamental disadvantage with field testing is that motor output torque (shaft torque) is not measured. Thus motor output power is not measured. Instead, input currents, voltages, power factors, and speed are measured (extracted) in conjunction with nameplate data to estimate power output and efficiency. The various efficiency estimation methods are generally divided into two categories:

(1) Approximate efficiency based only on motor speed;

(2) Approximate efficiency based on a mathematical model, or equivalent circuit, of the motor.

The methods of approximating efficiency in the field without measuring motor torque output and without using an equivalent circuit typically rely on a measurement of motor speed or power, such as the "Stanford Method" and the slip method. These methods are in reality approximations and yield efficiencies that are often more than 15\% from the true value.

More sophisticated methods use techniques for calculating motor torque without actually measuring it and depend on the use of a motor equivalent circuit or some other mathematical model of the motor's electro-mechanical power transfer mechanism. The degree of the accuracy of these methods is directly related to the degree of accuracy of the parameters in the equivalent circuit model. 
Development of a high accuracy equivalent circuit is normally done with information obtained from no load testing and resistance testing of the disconnected motor. Equivalent circuit development is described in length in IEEE Standard 112, IEEE Standard Test Procedure for Polyphase Induction Motors and Generators [5]. In typical industrial applications, however, the user generally will not permit the motor to be disconnected to measure stator resistance, or uncoupled operation of the motor to measure the no load power input. For this reason, an alternate, less accurate, method of developing the equivalent circuit is often used.

An equivalent circuit may be developed by using motor nameplate data and data from Motor Master or standards developed by IEEE. Motor Master is a database of motor performance information for over 10,000 motors available on diskette. It is possible to develop an equivalent circuit from this information because the nameplate usually provides performance information, such as the locked rotor kVA code and NEMA Design type, which can be used in a set of equations to solve for the equivalent circuit constants. Sometimes more information is available from Motor Master than is available on the nameplate. The problem with this approach is that the nameplate and Motor Master data are generic design data and may not be accurate in reflecting such parameters as the actual speed and power factor when the motor is delivering rated horsepower.

The unique aspect of the equivalent circuit method is that the circuit can be solved for any running speed. When the circuit is solved, it will calculate a motor power factor. This power factor can then be compared with the actual measured power factor to assess the accuracy of the equivalent circuit. If the calculated power factor does not match the measured power factor, the equivalent circuit parameter of magnetizing reactance can be adjusted until a match is found. Magnetizing reactance, does, in fact, decrease as a motor ages. As the magnetizing reactance decreases, the efficiency also decreases. Thus the equivalent circuit can be adjusted to match the present condition of the motor if the power factor can be measured. 


\section{IN-SERVICE MOTOR EFFICIENCY EVALUATION METHODS}

There are many methods pertinent to field efficiency evaluation in the literature, and new methods are appearing almost every year. An in-service motor efficiency evaluation can consist of a single basic method or can be built using a combination of different basic methods. This section lists the most promising motor efficiency methods which are categorized according to their theoretical bases and error sources. Some of these methods are not applicable for field in-service testing, but they are also included here for the following reasons:

1) It is the "backbone" method in the certain category.

2) It best illustrates the physical nature of the certain series of methods.

3) Most of the other sophisticated methods are the variations of a single or a combination of these basic methods after some simplification or modification.

Generally, these methods can be categorized as follows [2][14]:

- Nameplate methods

- Slip methods

- Current methods

- Equivalent circuit methods

- Segregated loss methods

- Torque methods

- Empirical methods

- Statistical methods

- Dedicated instrument methods

All methods calculate efficiency according to the definition 


$$
\text { Efficiency }=\frac{(\text { Shaft output power })}{(\text { Electrical input power to drive system })}
$$

The shaft output power is the input power minus the losses. How to assess losses and evaluate output power gives rise to fundamental differences among the various methods. Consequently, the accuracies of methods are different.

The degree of intrusiveness of a field evaluation method is determined by what data are required to be measured in the field and the difficulty of performing the measurements. One or more of the following measurements may be involved: ${ }^{[2]}$

- nameplate reading;

- $\quad$ speed measured by optotachometer (Note: This can be neglected now.);

- currents measured by clamp-on transducer;

- $\quad$ voltages measurement;

- $\quad$ input power measurement;

- $\quad$ stator winding resistance reading (Note: This can be neglected now.);

- $\quad$ winding temperature data;

- no-load data measured with uncoupled shaft;

- $\quad$ shaft torque measurement.

The data may be acquired in the format of RMS meter readings or digitally sampled waveforms.

The cost associated with the labor, material, and downtime for implementing safety requirements for data collections can be used as a gauge to weigh intrusiveness. Planning may also affect intrusiveness. If a decoupled no-load test is required and the motor power supply is available during scheduled downtime, it may be possible to conduct this kind of test without affecting production.

The most promising efficiency evaluation methods are briefly introduced in the rest of this section. 


\section{Nameplate Methods}

The least intrusive field evaluation method is to obtain motor information from the nameplate. There are several versions of the nameplate methods. They are reported in [1][2][4][7]. They all use the nameplate data of the motor for the efficiency estimation.

\section{(1) Standard Nameplate Method}

In this method, it is assumed that the efficiency of the motor is constant and equal to the nameplate value. This works best when the efficiency-load curve is fairly flat, so that the full-load efficiency is applicable for most load conditions. But this is not always true for all kinds of motors. ${ }^{[2]}$ Hence, the nameplate method may be applicable for some motors, but could result in substantial inaccuracies for other motor types.

With this nameplate method, three additional problems may occur. First, the nameplate data may be given according to a method other than IEEE Standard 112 Method B. Second, the motor may have been rewound. Third, the field environment pertinent to the voltage unbalance and harmonics content may be different from that the nameplate data is derived from.

Nameplate efficiencies of a given motor can be evaluated according to different standards. The three most frequently used standards are the National Electrical Manufacturers Association (NEMA) that uses IEEE Standard 112, the Japanese Electrotechnical Committee (JEC), and the International Electrotechnical Commission (TEC). These three standards are not in agreement and may result in a given motor being stamped with rather different efficiencies.

Rewound motors introduce additional uncertainty, since the nameplate data may no longer be valid. Some engineers suggest that, after each rewinding to the same horsepower and same number of poles, a two percentage points reduction of efficiency should be considered. However, a different opinion indicates that the efficiency should not be reduced if the rewinding follows Electrical Apparatus Service Association (EASA) standards. 
Reference [2] reported that under the worst situation, the efficiency can be off ten percentage points from the nameplate efficiency. However, the nameplate method is the most non-intrusive method. And the bottom line is that a nameplate method is better than no field evaluation at all.

\section{(2) Volgelsang and Benning (V\&B) Method I and II}

The Volgelsang and Benning (V\&B) method I and II are variations of the standard nameplate method, and they are used in a dedicated commercially available instrument. ${ }^{[7]}$ V\&B option I requires testing at three conditions: uncoupled (no-load test), normally loaded, and unpowered (off). The original method requires a reflector to be attached to rotating equipment to allow the speed to be recorded. (Note: This speed measurement can be substituted by current signature analysis in nowadays.) In Option II, testing is accomplished without uncoupling, and motor nameplate data are substituted, but the accuracy is assumed to be reduced.

The principles of this instrument are based on theory closely exposed in the patent [13]. The approach of this instrument is based on the identification and segregation of the losses of the induction motor. It calculates an upper bound efficiency by allocating the iron and the friction losses to the stator losses and a lower efficiency boundary by allocating the iron and friction losses to the rotor. The stator resistance has to be measured and entered into the program. Nameplate data, RMS electrical quantities, and measured speed are used to estimate the operating efficiency.

\section{Slip Methods}

There are several versions of slip methods. They are reported in [1][2][4][7][14]. All rely on a measurement of motor speed to find the slip.

\section{(3) Standard Slip Method}

This method presumes that the percentage of load is closely proportional to the percentage of the ratio of measured slip to full-load slip. The obvious error is that the slip ratio represents the percentage of load and the efficiency is not equal to the percentage of load. Alternatively, one can also measure the power into the motor and approximate the power out of the motor by multiplying 
the rated horsepower of the motor by the ratio of the measured slip to rated slip. The operating efficiency of the motor is thus approximated using the following relationship:

$$
\text { Efficiency }=\frac{(\text { Measured Slip } / \text { Rated Slip }) \times \text { Rated Output Power }}{\text { Input Power }}
$$

This method can be enhanced by correcting the rated nameplate speed for voltage variations, especially when the motor-efficiency-versus-load curve is not flat. This is done by taking the square of the ratio of the actual voltage to nameplate voltage and multiplying this with the rated speed. However, the estimation results could still have a relative big error, since the nameplate speed is

allowed to deviate as much as 20 percent from the actual rated speed by NEMA MG1. ${ }^{[12]}$

The no-load speed of induction motors is always close to the synchronous speed. Subsequently, the projection of a light load through the standard slip method is relatively more accurate than the projection of a heavy load.

The main attraction of the standard slip method is its simplicity. However, several authors, e.g. [2], [16] and [18], have observed that the accuracy of the method suffers badly from several causes. Reference [18] provides an excellent discussion of the drawbacks of the slip methods, particularly the standard slip method.

\section{(4) Upper Bound Slip Method}

The upper bound with resistance method is reported in [7]. It first calculates the stator losses by calculating the $I^{2} R_{s}$ losses of the stator, obtained from the stator resistance measurement and the operating current measurement. Next, the rotor copper losses are calculated with the standard slip method out of the air-gap power, which is assumed to be equal to the input power minus stator resistance losses. The resulting efficiency estimation will always be higher than the operating efficiency since it neglects stray, friction, windage, and core losses. 
As in the nameplate method, a large increase in accuracy could be obtained by entering the information obtained on a no-load test. With that information, friction, windage, and core losses can be obtained accurately and load-dependent stray-load losses with a fair accuracy. But the need of noload test for an accurate measurement is obviously the drawback of this method.

\section{Current Methods}

The Current Method is included in [1][2][4][7][14]. The current method is another approach that uses a minimum of field measurements in conjunction with manufacturer's data to estimate motor efficiency at normal operating loads. There are also several alternative current methods. Like the slip methods, the main attraction of the current method is its simplicity.

This method presumes that the percentage of load is closely proportional to the percentage of the ratio of measured current to full-load current. The shaft output power is, thus, approximated using equation (a). $I_{\mathrm{fl}}$ is the nameplate full load current and $\mathrm{I}$ is the measured current. The expression of shaft output power is also defined by equation (b) in some circumstances. As illustrated in Figure 2, the load is normally overestimated using equation (a); while using equation (b), the load evaluation is normally underestimated. The average of the two approaches may give a more accurate shaft output power.

$$
\begin{gathered}
\text { (Shaft Output Power) }=\frac{I}{I_{f l}} \cdot(\text { Rated Output Power) } \\
\text { (Shaft Output Power) }=\frac{\left(I-I_{n l}\right)}{\left(I_{f l}-I_{n l}\right)} \cdot(\text { Rated Output Power) }
\end{gathered}
$$

The simple current method, equation (a), does not require a no-load current value. The refined equation (b) requires a no-load test to obtain the no-load data. Just as with the slip method, to obtain efficiency one will have to either use typical efficiency-versus-load curves or measure input power. 


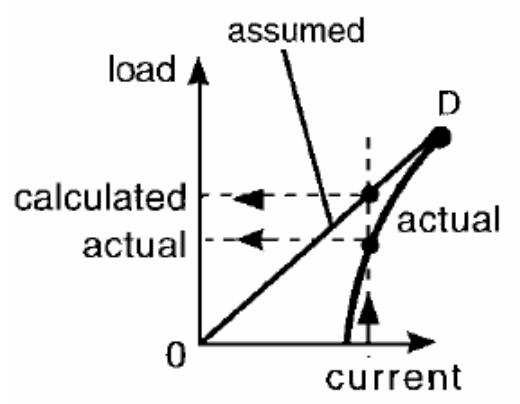

(a) Overestimated

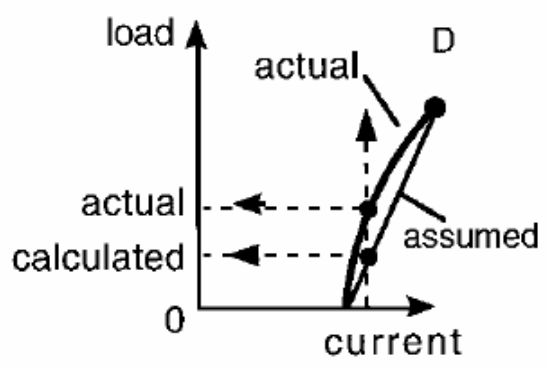

(b) Underestimated

Figure 2. $\quad$ Assumed and Actual Load-versus-Current Curves.

Reference [18] provides an excellent discussion of this problem and provides an improved version of the above equations. They also conclude that even the improved version can have major inaccuracies depending on the shape of the motor performance curve and the load condition.

Reference [2] and [18] summarize the advantages of the current method:

1) The NEMA Standard MG1-12.47 permits only half the tolerance in nameplate, fullload current as it does full-load slip.

2) Motor current measured by a clamp-on probe has a low intrusion level.

The chief disadvantages of the current method:

1) Current, unlike slip, does not vary linearly with load because there is a magnetizing current even when the motor is operating at no load. Therefore, this method also has a significant inherent inaccuracy.

2) The need for no-load test is not accepted in most in-service testing.

\section{Equivalent Circuit Methods}

The performance of an electric motor, at least with regard to efficiency, can be calculated from its equivalent electric circuit. These methods permit one to compute estimates of the efficiency of the motor when it is operating at loads other than those at which measurements were made. 
(5) Standard Equivalent Circuit Method (IEEE Std-112 Method F)

The efficiency estimation method $\mathrm{F}$ in the IEEE Standard 112-1996 is the standard equivalent circuit method. ${ }^{[11]}$ The IEEE Standard112 Method F is not a useful field-test for efficiency. Its additional removed-rotor and reverse rotation tests to directly measure the fundamental frequency and high frequency stray load losses are too invasive and user unfriendly. The basic Method F requires an impedance test and the complete no-load, variable voltage test. It requires volts, watts, amperes, slip, stator winding temperature, or stator winding resistance to be measured at two values of voltage while operating at no load. In one case, measurements are made at rated voltage while operating at no load. In the other case, measurements are made while operating at no load with voltage reduced until slip is equal to that obtained at the normal operating load. Once these measurements are made, an iterative procedure is used to determine the parameters of the equivalent circuit. The iterative procedure requires one to either know the design value of the ratio $\mathrm{X}_{1} / \mathrm{X}_{2}$ or to use the standard NEMA design value. The equivalent circuit of Method F is shown in Figure 3.

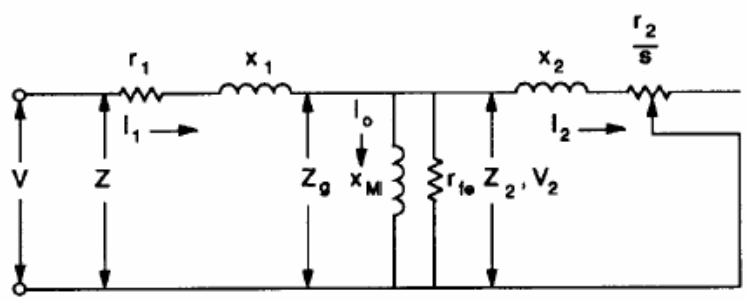

Figure 3. $\quad$ Equivalent Circuit of IEEE Std-112 Method F.

Although this method is expected to be quite accurate, it is still considered to be too intrusive for inservice testing. This method is kept here mainly as the basis of other modified methods.

\section{(6) Ontario Hydro Modified (OHMF) Equivalent Circuit Method}

This method is included in [14][16]. This is a modified version of the IEEE Standard Method F proposed by Ontario Hydro. ${ }^{[16]}$ 
A no-load and a full-load test, both at rated voltage, must be run. In turn, this requires one to disconnect the motor being tested from its load. Line voltage, input power, line current, power factor, and stator resistance at load temperature are measured after operating at no load and at full load. The slip is also measured at full load.

This method eliminates the need for a variable voltage as required by IEEE Standard 112, Method F. ${ }^{[11]}$ The equivalent circuit used by this method is slightly different from that of Method F. In this version of the equivalent circuit, the impedance elements $\left(\mathrm{r}_{\mathrm{fo}}\right.$ and $\mathrm{X}_{\mathrm{M}}$ ) of the magnetizing branch are shown in series while that of Method F is shown in parallel.

\section{(7) Nameplate Equivalent Circuit (ORMEL96) Method}

The least intrusive method to estimate efficiency is based on the use of an equivalent circuit derived from the motor's nameplate data. Once the equivalent circuit of a motor is known, its running efficiency at any load can be determined simply by measurement of the motor speed. The nameplate data provides information about the motor's rated performance, locked rotor current, and design type.

The ORMEL96 method was developed by Oak Ridge National Laboratory (ORNL) in 1996. ${ }^{11}$ [4][7][14] The ORMEL 96 method finds the equivalent circuit from the nameplate data plus the value of the stator resistance. It was found in [7] that the accuracy of the predicted efficiencies increases substantially if the stator resistance is known. However, if the stator resistance is not known, it can also be estimated from motor nameplate data. ${ }^{[14]}$ ORMEL96 also has an advanced mode which allows the user to adjust certain "tunable parameters".

Even with typical nameplate information of older conventional motors and rewound motors, this method has been shown in limited testing to provide an average accuracy of less than 3.5 percentage points. $^{[14]}$ 


\section{(8) Locked Rotor Method}

This method is included in [14][23]. It differs from the previous equivalent circuit. It adds a second rotor loop.

Reference [23] presented a procedure that uses two locked rotor tests to determine the parameters of an equivalent circuit with two rotor loops. An alternative procedure is to use a single locked rotor in conjunction with a load test to determine these parameters. In both cases, a no-load test must also be run. With these parameters in hand, they then develop a method for computing the efficiency of the motor from the equivalent circuit relationships.

The advantages of this two-rotor loop method are these areas:

1) The procedure for determining parameters of equivalent circuit is not iterative like that of IEEE Std-112 Method F.

2) According to the authors, the two-reactance loop equivalent circuit represents doublecage and deep-bar rotor motors better than the single rotor loop equivalent circuits.

This method has two principal disadvantages:

1) It requires a complete no-load test with the motor connected to a variable voltage power source.

2) It requires connecting the motor to a variable frequency source. This is too massive and user unfriendly for a good field test.

\section{(9) Standstill Frequency Response Method}

This method is included in [14] [24]. Like the previous Locked Rotor Method, it also differs from the standard equivalent circuit. It also has two rotor loops.

Reference [24], sponsored by the Electric Power Research Institute (EPRI), investigated the sensibility of determining the electrical equivalent circuit parameters of induction motors by using the standstill frequency response test. The approach investigated by the EPRI study was to measure 
the impedance of a motor, with its rotor stationary, over a frequency range of 0.01 to $500 \mathrm{~Hz}$. The parameters of the equivalent circuit are then derived from these data.

The major advantage of this method over the IEEE Std-112 Method F is that the low-voltage, noload test is not required. The level of applied voltage is much lower than that of the low-voltage, noload test. If a packaged test device with a variable frequency source is developed and made commercially available, then the only drawback would be the no-load test to determine friction and windage.

\section{Segregated Loss Methods}

The segregated loss methods are the most straightforward of the efficiency testing methods because they simply estimate the magnitudes of each motor power loss component. Some of the methods are quite complicated and intrusive, while others rely on empirical factors to estimate the losses.

\section{(10) Standard Segregated Loss Method (IEEE Std-112 Method E)}

The efficiency estimation method E in the IEEE Standard 112-1996 is the standard segregated loss method. ${ }^{[11]}$ Same with IEEE Std-112 Method F, Method E is not a useful method for motor inservice testing, since it requires additional removed-rotor and reverse-rotation tests used to directly measure the fundamental frequency and high-frequency, stray-load losses. Method E is seldom used in the field because it requires a variable load and a variable voltage power supply. Some important properties of method E are listed as follows:

1) Method E specifies a comprehensive no load test.

2) Method E requires test under load at six equally spaced load points with four between 25 percent and 100 percent of full load and two greater than 100 percent and less than 150 percent.

3) Method E specifies an assumed value for stray load losses at rated load.

4) The repeatability of Method E is improved by requiring the adjustment of all resistance and slip measurements to a specified temperature. 
Note that Method E requires variable load tests, so the motor being tested must be connected to a variable load. In most circumstances in the field this would be quite disruptive to normal operation of the system. This again shows the high intrusiveness of Method E. Like Method F, this method is kept here mainly as the basis of other modified segregated loss methods.

\section{(11) Ontario Hydro Modified (OHME) Segregated Loss Method}

This method is included in [1][2][4][7][14]. Ontario Hydro ${ }^{[16]}$ proposed a segregated loss method that simplifies Method E much further. It uses an empirical value of 3.5\% 4.2\% of rated input power to find the no-load losses rather than actually performing no-load testing. ${ }^{[2]}$ As a further refinement, this value could be optimized by testing samples of motors at different horsepower levels. The stray load losses are estimated based on the IEEE 112 standard assumed values as Table 1. ${ }^{[11]}$

Table 1. $\quad$ Assumed Values for Stray Load Loss

\begin{tabular}{|c|c|c|}
\hline \multicolumn{2}{|c|}{ Machine rating } & $\begin{array}{c}\text { Stray-load loss percent of rated } \\
\text { output }\end{array}$ \\
\hline $1-125 \mathrm{hp}$ & $1-90 \mathrm{~kW}$ & $1.8 \%$ \\
\hline $126-500 \mathrm{hp}$ & $91-375 \mathrm{~kW}$ & $1.5 \%$ \\
\hline $501-2499 \mathrm{hp}$ & $376-1850 \mathrm{~kW}$ & $1.2 \%$ \\
\hline $2500 \mathrm{hp}$ and greater & $1851 \mathrm{~kW}$ and greater & $0.9 \%$ \\
\hline
\end{tabular}

This method can be simplified even further by using assumed value 0.8 for rated power factor. The stator resistance at load is estimated based on a simple approximation using the motor current to estimate the temperature rise. The only other measurements required are power into the motor and motor speed. The author of [7] has experimented with OHME Method and found it to provide an accuracy of $\pm 3 \%$ or $\pm 4 \%$. Report [4] claimed that an error of about $1 \%$ was obtained using OHME Method for a 7.5hp motor. 


\section{Torque Methods}

According to the definition in Section II, the efficiency of a motor can be defined as following equations.

$$
\begin{aligned}
& \eta=\frac{P_{\text {output }}}{P_{\text {input }}} \times 100 \% \\
& =\frac{T_{\text {shaft }} \cdot \omega_{r}}{P_{\text {input }}} \times 100 \% \\
& =\frac{T_{\text {air }- \text { gap }} \cdot \omega_{r}-W_{f w}-W_{L L r}}{P_{\text {input }}} \times 100 \%
\end{aligned}
$$

With the input power and the rotor speed measured/estimated, the efficiency can easily be determined if shaft torque is known. This is the basic principle of all the torque methods.

\section{(12) Shaft Torque Method}

This method is discussed in [2]. Regardless of how sophisticated a method is, it remains difficult to assess all the stray-load losses accurately in the field. The most straightforward torque method, Shaft Torque Method, is to measure the shaft torque (and therefore output power) directly from the shaft, without any need to calculate losses. The shaft torque method offers the most accurate field efficiency evaluation method. It is also highly intrusive.

A custom-built torque coupling may be used to replace the existing coupling. Torque signals can be obtained through slip rings. Laser and telemetry technologies may also be used without going through slip rings for signal noise reductions. Many different ways have been proposed in the report ORNL/TM-13165. ${ }^{[4]}$ The accuracy of this method depends on the quality of the torque sensors, the signal noise, and the shaft alignment of the motor and its load. Downtime is required for preparing and replacing the shaft torque coupling. 


\section{(13) Air-gap Torque (AGT) Method}

In 1995, Hsu and Scoggins at the Oak Ridge National Lab developed the Air-gap Torque Method based on well-known air-gap equations. ${ }^{[3]}$ This method has been approved by several experiments ${ }^{[2][3][4][7]}$ to be a very promising motor efficiency estimation method. It is also regarded by us as one of the most potential efficiency estimation methods for WSN project. Therefore, this method is explained in details here.

A. Equations of Power, Air-Gap Torque, and Efficiency

\section{a) Power Equations}

The instantaneous input power of a three-phase induction motor is the summation of products of the instantaneous phase voltages, $\mathrm{v}_{\mathrm{a}}, \mathrm{v}_{\mathrm{b}}, \mathrm{v}_{\mathrm{c}}$, and phase currents, $\mathrm{i}_{\mathrm{a}}, \mathrm{i}_{\mathrm{b}}$, and $\mathrm{i}_{\mathrm{c}}$.

$$
\text { Power }=v_{a} i_{a}+v_{b} i_{b}+v_{c} i_{c}
$$

A portion of this instantaneous power includes the charging and discharging of the energy stored in the windings. Therefore, this instantaneous power cannot represent the instantaneous torque even at a constant speed after subtracting the losses. However, the average value of instantaneous power at full load represents the input power of the motor for the efficiency calculation. The conventional single-frequency power equation using power factor, RMS current and voltage is a special case of the average instantaneous power.

b) Review of Air-Gap Torque Equations

The following voltage equations are for the three-phase armature windings,

$$
v_{a}=\frac{d \psi_{a}}{d t}+r i_{a}, v_{b}=\frac{d \psi_{b}}{d t}+r i_{b}, v_{c}=\frac{d \psi_{c}}{d t}+r i_{c}
$$


Where

$\Psi_{a}, \Psi_{b}, \Psi_{c}=$ flux linkages of windings a, b, and c, respectively

$r=$ the phase resistance

Substituting into (1) to give

$$
\text { Power }=\left[i_{a}\left(\frac{d \psi_{a}}{d t}+r i_{a}\right)+i_{b}\left(\frac{d \psi_{b}}{d t}+r i_{b}\right)+i_{c}\left(\frac{d \psi_{c}}{d t}+r i_{c}\right)\right]
$$

From (2), the flux linkages can also be given as

$$
\begin{aligned}
& \psi_{a}=\int\left(v_{a}-r i_{a}\right) d t \\
& \psi_{b}=\int\left(v_{b}-r i_{b}\right) d t \\
& \psi_{c}=\int\left(v_{c}-r i_{c}\right) d t
\end{aligned}
$$

Subtracting the copper losses and the terms pertinent to the energy stored in the windings, the air gap torque equation can be written as

$$
\text { Torque }[\mathrm{N} \cdot \mathrm{m}]=\frac{P}{2 \cdot \sqrt{3}}\left\{\begin{array}{cc}
\left(i_{\mathrm{A}}-i_{\mathrm{B}}\right) \cdot \int & {\left[v_{\mathrm{CA}}-R\left(i_{\mathrm{C}}-i_{\mathrm{A}}\right)\right] d t} \\
-\left(i_{\mathrm{C}}-i_{\mathrm{A}}\right) \cdot \int & {\left[v_{\mathrm{AB}}-R\left(i_{\mathrm{A}}-i_{\mathrm{B}}\right)\right] d t}
\end{array}\right\}
$$

where $\mathrm{P}$ is the number of poles, $i_{A}, i_{B}$, and $i_{C}$ are the line currents, and $\mathrm{R}$ is half of the line-to-line resistance value.

When using either three leads for $\mathrm{Y}$-connected motors without a neutral connection or three leads for delta-connected motors, (4) can be further simplified by using

$$
i_{B}=-\left(i_{A}+i_{C}\right)
$$


c) Numerical Evaluation of Integrals

The integrals of windings corresponding to lines CA and AB in (6) represent flux linkages. Since the time increment between data points is small, a simple trapezoidal method can be used. Other methods using Simpson's rule or Gauss's rule for numerical evaluation of integrals can also be applied.

$$
\begin{gathered}
\int\left[v_{c a}-R\left(i_{c}-i_{a}\right)\right] d t \\
\int\left[-v_{B A}-R\left(2 \bullet i_{A}-i_{C}\right)\right] d t
\end{gathered}
$$

d) Efficiency Equations

As mentioned earlier, the shaft output power is calculated from the shaft speed (rpm) and shaft torque.

$$
P_{\text {output }}=T_{\text {air-gap }} \cdot \omega_{r}=T_{\text {air-gap }} \cdot 2 \pi \cdot \frac{r p m}{60}
$$

where the shaft torque is the difference of air-gap torque and torque losses corresponding to mechanical loss and stray loss produced by rotor current.

$$
T_{\text {shaft }}=T_{\text {air-gap }}-\frac{W_{f w}}{\left(2 \pi \cdot \frac{r p m}{60}\right)}-\frac{W_{L L r}}{\left(2 \pi \cdot \frac{r p m}{60}\right)}
$$

From (7), (8), and the definition of efficiency, the efficiency, $\eta$, yields

$$
\eta==\frac{T_{\text {air }- \text { gap }} \cdot\left(2 \pi \frac{r p m}{60}\right)-W_{f w}-W_{L L r}}{P_{\text {input }}} \times 100 \%
$$


B. Discussions of the Air-gap Torque Method.

A significant difference between the AGT Method and the IEEE Std-112 Method E is that the AGT Method considers the losses associated with the negative torques produced by the negative sequence voltages and currents, which are the products of unbalanced voltages and harmonics. Because in the real world the power supply is commonly polluted with unbalanced voltages and harmonics, the AGT Method has a unique advantage over the IEEE Std-112 Method E for in-service motor efficiency assessment.

The advantages of the AGT Method:

1) Air-gap torque can be measured while the motor is running.

2) The AGT Method should continue to provide optimum accuracy when the phase powers are unbalanced.

3) This method can be used for non-induction motors such as the adjustable speed, brushless dc motors.

The major disadvantages of the method:

1) The voltage and current waveforms must be acquired with a reasonably high sampling rate so that a high accuracy of the waveform shape may be achieved. A sample rate of $5,000 \mathrm{~Hz}$ was adopted in the testing of reference. ${ }^{[3]}$

2) A measurement of a no-load power into the motor must be made. This could be avoided by combining some other methods like Empirical Methods or Statistical Methods. The non-intrusiveness can be improved at the price of accuracy reducing.

\section{(14) Instantaneous Current Method}

This method is reported in [7]. It is based on an input-output power approach. Although this method is called "Instantaneous Current Method”, it is actually a variation of AGT Method. 
The input power to the motor is measured on the electrical terminals, and the output power is estimated by the multiplication of speed and torque. Speed is obtained by analyzing the instantaneous electrical quantities measured and calculated at the electrical inputs. Air-gap torque is calculated via the dq-axis theory for electrical machinery. Output torque (shaft torque) is estimated by subtracting an estimate of friction, windage, and stray load loss from the calculated air-gap torque.

This method is implemented in a dedicated instrument called Explorer II by Baker Instrument Company and is indeed an intrusive method. In our previous WSN report ${ }^{[28]}$, the Explorer II by Baker Instrument Company was evaluated as the best commercially available instrument for energy usage estimation and condition monitoring in today's market.

\section{Empirical Method (Stanford Wilke Method)}

This method is reported in [1][2]. Empirical Method is also called as "Stanford Wilke Method". It was developed at Stanford University with K. Wilke as one of its developers. The method was developed to be applicable to motors between 10 and 50 horsepower. Some of the empirical assumptions were taken from IEEE Standard 112 while others arose from the author's experience. It is anticipated that the method will yield efficiency estimates with error within $\pm 4 \%$ or better. Reference [2] claimed that this method yields efficiency estimate with error about 6\% for both balanced and unbalanced voltage conditions.

\section{Statistical Methods}

The Statistical Methods are discussed in [1][2]. Empirical equations are set up to use minimal numbers of measured data for input power and efficiency estimations. Usually, application of this method is restricted to the group of motors for which empirical equations were derived.

The statistical results can be quite different for the same variable. A good example is the stray load loss estimation. NEMA MG1 ${ }^{[12]}$ paragraph 20.52 states that, if stray load loss is not measured, the 
value of stray load loss at rated load shall be assumed to be $1.2 \%$ of the rated output for motors rated less than 2500 hp and 0.9\% for motors rated 2500 hp and greater. IEEE Standard 112 Section 5.4.4 ${ }^{\text {[11] }}$ gives different assumed stray load loss values for motors rated less than 2500 hp, as shown in Table 1.

Additionally, some non-U.S. standards use $0.5 \%$ of rated output as the stray load loss at rated load. The statistical approach is commonly used with other basic methods. For instance, the previously discussed OHME Method suggests the combined windage, friction, and core losses to be 3.5\% of the rated input power. The stray load losses are estimated based on the IEEE Standard 112 standard assumed values, as shown in Table 1.

This method can be simplified even further by using assumed values for rated power factor (e.g. 0.8). Approximations can also be made for the temperature rise of the winding, and the winding resistance can even be estimated by measuring the line-to-line resistance taken from the circuit breaker and subtracting the estimated cable resistance. The only other measurements required are power into the motor and motor speed.

\section{Dedicated Instrument Methods and Software Methods}

Many of the motor efficiency methods have been incorporated into commercially available products, including both software and instruments. These methods are listed very briefly in this part.

Obviously, these methods could be categorized into previously discussed methods according to their physical nature. However, due to some commercial reasons, the algorithms behind some of these products have not been clearly reported. On the other hand, some of the previously discussed methods, such as the V\&B Method I II and the ORMEL96 Method have also been implemented into products. They are not listed here because relatively complete knowledge of these methods is available in the literature. 
(15)

The Motor Master Plus (MM+) is a free software tool developed by the Washington State University under the support of the US Department of Energy. This tool features motor inventory management tools, maintenance log tracking, efficiency analysis, savings evaluation, energy accounting, and environmental reporting capabilities. The lasted version, MotorMaster+ 4.0 software, includes a catalog of over 20,000 AC motors. It includes its own efficiency estimation methods as well as some other methods, such as ORMEL96 Method.

There are three MM+ methods used in MM+ software: MM+ Power Method, MM+ Current Method and MM+ Slip Method. These three methods are based on nameplate and normal load operation. They allow efficiency to be computed/estimated at the normal load. Electrical and/or speed readings are required at normal load. No uncoupled or unpowered readings are required. Reference [14] provides testing results using these methods.

\section{(16) Vectron (ECNZ) Method}

This method is reported in [7]. The Vectron Method was developed for the Electricity Corporation of New Zealand (ECNZ). It requires testing at a load $<10 \%$ and a load $>50 \%$, and unpowered (off). It also uses an optical tachometer based on an attached reflective strip. The manufacturer claims that the efficiency at full load conditions can be determined from testing at a load $>50 \%$ but less than full load. The manufacturer also claims that this method can correct for off nominal voltage conditions up to $5 \%$.

\section{(17) MAS-1000 Method}

This method is reported in [7]. The MAS-1000 Method is based upon an instrument MAS-1000 developed by Niagara Instruments. The latest version of this instrument, MAS-1000 LA, has been introduced in our previous WSN report ${ }^{[28]}$. 
A magnetic reluctance speed sensor is used, although a strobe tachometer can be substituted if readings are manually entered, but the former is preferred. The system is based on an Intel 486 processor, and the manufacturer claims that Motor Master Plus (MM+) software developed by DOE could be loaded and used with the tester for additional convenience in motor systems management.

\section{(18) Esterline Angus Method}

The Esterline Angus method is tested by [7]. It is a pure software method dedicated for a certain instrument. It requires only generic equipment and custom software. Tests are required while uncoupled, at normal load, and unpowered. Surface and ambient temperature are required. 


\section{CONCLUSIONS AND SUGGESTIONS}

\section{Conclusions}

The previous section introduced over 18 available motor efficiency evaluation methods. These methods are categorized into the following 9 categories:

1. Nameplate methods

2. Slip methods

3. Current methods

4. Equivalent circuit methods

5. Segregated loss methods

6. Torque methods

7. Empirical methods

8. Statistical methods

9. Dedicated instrument methods

The first 6 categories are of our main interest in WSN project. The intrusiveness and corresponding accuracy of these 6 categories of methods are compared in Figure $4 .{ }^{[2]}$ Clearly, the more intrusive the method is, the more accurate estimation it can obtain.

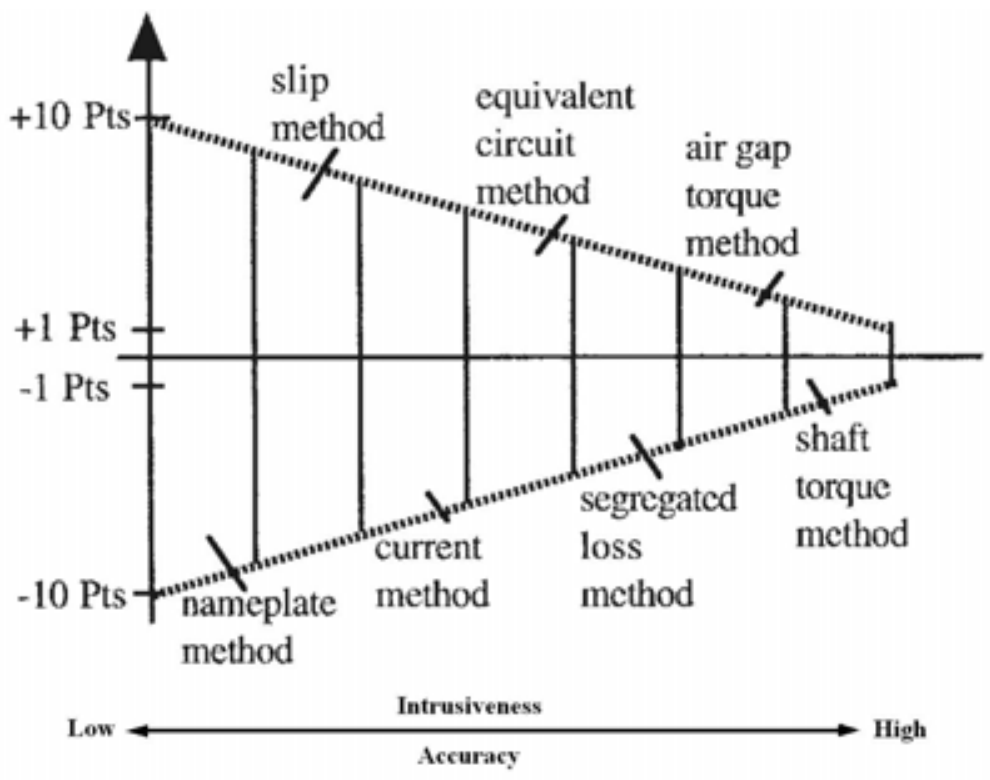

Figure 4. Anticipated Efficiency Estimation Errors versus Evaluation Methods. 
After overall evaluation on the 18 available efficiency estimation methods, we suggest the following three methods as candidates for the WSN research project at different level of intrusion.

1. Low Intrusion Level Method:

2. Medium Intrusion Level Method:

3. High Intrusion Level Method:
ORMEL96 Method (Equivalent circuit method)

OHME Method (Segregated loss method)

Air-gap Torque Method (Torque method)

These three suggested methods are compared in Table 2.

Table 2. Comparison of the Three Suggested Methods

\begin{tabular}{|c|c|c|c|c|c|c|c|c|c|}
\hline $\begin{array}{c}\text { Motor Efficiency } \\
\text { Testing Methods }\end{array}$ & \multicolumn{6}{|c|}{ Tests and Measurements/Estimations Required } & \multicolumn{3}{c|}{ Performance } \\
\cline { 2 - 12 } & $\begin{array}{c}\text { Lo } \\
\text { Lod }\end{array}$ & $\begin{array}{c}\text { Full } \\
\text { Load }\end{array}$ & $\begin{array}{c}\text { Unpower } \\
\text { (Off) }\end{array}$ & $\begin{array}{c}\text { Variable } \\
\text { Voltage }\end{array}$ & Speed & $\begin{array}{c}\text { Nameplate } \\
\text { Info }\end{array}$ & $\begin{array}{c}\text { Stator } \\
\text { Resistance }\end{array}$ & Intrusiveness & Accuracy \\
\hline ORMEL96 Method & No & No & No & No & Yes & Yes & Optional & Low & Low \\
\hline OHME Method & No & No & No & No & Yes & Yes & Yes & Medium & Medium \\
\hline AGT Method & Yes & No & No & No & Yes & No & Yes & High & High \\
\hline
\end{tabular}

Reference [4] has done a complete testing using these three methods with 12 motors ranging in size from 5hp to 100hp. Seven of the motors were older standard efficiency design (two had been rewound), the other five were energy efficient designs. The motors were tested with no load, and at 25\%, 50\%, 75\% and full load. Tests were conducted with two voltage conditions: phase balanced within $0.5 \%$ and unbalanced to $3 \%$. Table 3 shows the testing results using these three methods.

Table 3. Comparison of the Testing Results Using Three Suggested Methods

\begin{tabular}{|c|c|c|c|c|c|}
\hline Efficiency (\%) / Error (\%) & $\mathbf{7 . 5}$ hp 72\% Load & $\mathbf{1 0}$ hp 40\% Load & 5hp 81\% Load & 5hp 31\% Load & Avg Error \\
\hline Direct Measurement & 84.5 & 85.2 & 86.2 & 74.3 & $/$ \\
\hline ORMEL96 Method & $82.2 /-2.72$ & $84.9 /-0.35$ & $81.9 /-4.99$ & $67.0 /-9.83$ & $4.47 \%$ \\
\hline OHME Method & $83.6 /-1.07$ & $85.3 /+0.12$ & $85.8 /-0.46$ & $73.9 /-0.54$ & $\mathbf{0 . 5 3 \%}$ \\
\hline AGT Method & $85.0 /+0.59$ & $85.4 /+0.23$ & $86.8 /+0.69$ & $74.6 /-0.40$ & $\mathbf{0 . 4 8} \%$ \\
\hline
\end{tabular}

The average estimation error obtained for the ORMEL96 Method is around 4.5\%. It is also noted that the accuracy of the method improves as the load increases because the method does not use no 
load data. Study shows that if the motor load is above 50\%, the method accuracy is better than the average and reasonably accurate results are obtained. In cases where the load is less than 50\% it is less important to know the actual efficiency.

The average estimation error for the OHME Method, 0.53\%, is probably not representative of what is obtainable with less sophisticated data acquisition equipment in the field. However, an accuracy of $2 \%$ to $3 \%$ is likely obtainable.

The AGT Method is proved to be very accurate yielding efficiency estimates that were within $0.5 \%$ of the actual values. The authors of [4] believed that the laboratory results are typical of what can be attained in the field. ${ }^{[4]}$

\section{Suggestions for WSN project}

All of these three suggested methods can be used in WSN project. ORMEL96 and OHME Methods don't need future modifications if stator resistance can be correctly estimated. As for AGT Method, since it requires no-load test which is not accepted in WSN project, some modifications are needed. The modifications could be considered from the following approaches:

(1) If the motor been tested has a regular scheduled maintenance, the no-load test could be done during the operation downtime.

(2) If the nameplate information of the motor been tested is available, the no-load data could be estimated from the nameplate data.

(3) If none of the above is available, the no-load losses could be estimated from some empirical values, like what has been done in OHME Method. 


\section{REFERENCES}

[1] J. Kueck, J. Gray, R. Driver, J. Hsu, “Assessment of Available Methods for Evaluating InService Motor Efficiency,” Oak Ridge National Laboratory report, ORNL/TM-13237, 1996.

[2] J. Hsu, J. Kueck, M. Olszewski, D. Casada, P. Otaduy, L. Tolbert, "Comparison of Induction Motor Field Efficiency Evaluation Methods," IEEE Trans. IAS, Vol. 34, No. 1, Jan/Feb 1998.

[3] J. Hsu, B. P. Scoggins, "Field Test of Motor Efficiency and Load Changes through AirGap Torque,” IEEE Trans. Energy Conversion, Vol.10, No. 3, Sep. 1995.

[4] J. D. Kueck, M. Olszewski, D. A. Casada, J. Hsu, P. J. Otaduy, L. M. Tolbert, "Assessment of Methods for Estimating Motor Efficiency and Load Under Field Conditions,” Oak Ridge National Laboratory report, ORNL/ TM-13165, 1996.

[5] Douglass, John G., "Efficacy of Methods for Estimating In-Service Motor Efficiency," Washington State University Co-Operative Extension Energy Program report prepared for the Pacific Gas and Electric Company and the Bonneville Power Administration, June 1997.

[6] Douglass, John G., "In-Service Motor Testing," Washington State University CoOperative Extension Energy Program report\#99-040, Nov. 1999.

[7] A. Wallance, A. Von Jouanne, E. Wiedenbrug, E. Matheson, J. Douglass, “A Laboratory Assessment of In-Service and Non-Intrusive Motor Efficiency Testing Methods," Electric Power Components and Systems, 29: 517-529, 2001.

[8] K.D. Hurst, T.G. Habetler, "Sensorless Speed Measurement Using Current Harmonic Spectral Estimation in Induction Machine Drives," IEEE Trans. Power Electronics, Vol. 11, No. 1, Jan. 1996. 
[9] K.D. Hurst, T.G. Habetler, “A comparison of Spectrum Estimation Techniques for Sensorless Speed Detection in Induction Machines,” IEEE Trans. IAS, Vol. 33, No. 4, July/August 1997.

[10] Patrick L. Jansen, Robert D. Lorenz, “Transducerless Position and Velocity Estimation in Induction and Salient AC Machines,” IEEE Trans. IAS, Vol. 31, No. 2, March/April 1995.

[11] IEEE Standard Test Procedure for Polyphase Induction Motors and Generators, IEEE 1121996.

[12] NEMA - MG 1, 2003.

[13] W. Benning, "Method of Determining the Efficiency of Asynchronous Motors and Apparatus for Carrying Out the Method," United States Patent 5,659,232, 1997.

[14] J. D. Kueck, “Development of a Method for Estimating Motor Efficiency and Analyzing Motor Condition,” IEEE Pulp and Paper Industry Technical Conference, pp.67-72, 1998.

[15] John R. Holmquist, James A. Rooks, Michael E. Richter, "Practical Approach for Determining Motor Efficiency in the Field Using Calculated and Measured Values,” IEEE Trans. IAS, Vol. 40, No. 1, pp.242-248, Jan/Feb 2004.

[16] “In-Plant Electric Motor Loading and Efficiency Techniques,” Ontario Hydro.

[17] R. L. Nailen, “Can Field Tests Prove Motor Efficiency?” IEEE Trans. IAS, Vol. 25, No. 3, May/June 1989.

[18] R. L. Nailen, “Finding True Power Output Isn’t Easy,” Electrical Apparatus, Feb 1994.

[19] A. K. Wallace, E. J. Wiedenbrug, "Motor Efficiency Determination: From Testing Laboratory to Plant Installation,” IEEE Pulp and Paper Conference, 1999.

[20] A. K. Wallace, T. E. Rollman, "High Efficiency Testing Laboratory for Motors, Drives, \& Generators,” PEVD96 Conf. Proc., pp. 220-225, 1996.

[21] A. K. Wallace, J. A. Rooks, and J. R. Holmquist, “Comparison Testing of IEEE Standards 841 Motors,” in Proc. IEEE 2000 Pulp and Paper Industry Tech. Conf., 2001, pp. 10-15. 
[22] A. Nagornyy, A. K. Wallace, A. Von Jouanne, “Stray Load Loss Efficiency Connections,” IEEE Industry Applications Magazine, May/June 2004.

[23] Antonio Dell'Aquila, “A New Test Method for Determination of Induction Motor Efficiency,” Technical Papers from the IEEE Power Engineering Society Winter Meeting, 1984.

[24] “Derivation of Induction Motor Models from Standstill Frequency Response Tests,” GS6250, Electric Power Research Institute, July 1991.

[25] “Determining Electric Motor Load and Efficiency,” Fact Sheet of Motor Challenge, DOE.

[26] “Replacing an Oversized and Under-loaded Electric Motor," Fact Sheet of Motor Challenge, DOE.

[27] Motor Systems Resource Facility, "Non-Intrusive Motor Efficiency Estimators," Northwest Energy Efficiency Alliance Report, NEEA Report \#04-126, March 2004.

[28] Bin Lu, Thomas G. Habetler, Ronald Harley, “An investigation on Commercially Available Energy Usage Estimation Products,” EATON WSN Research Report, Sep. 2004. 


\title{
A Survey of Available Methods for
}

\section{Evaluating In-Service Motor Efficiency}

\author{
UPDATED VERSION
}

Feb 25 ${ }^{\text {th }}, 2005$

EATON WSN Research Report

\author{
Prepared by \\ Bin Lu \\ Prof. Thomas G. Habetler \\ Prof. Ronald G. Harley
SCHOOL OF ELECTRICAL AND COMPUTER ENGINEERING GEORGIA INSTITUTE OF TECHNOLOGY
ATLANTA, GA 30332




\section{TALBE OF CONTENTS}

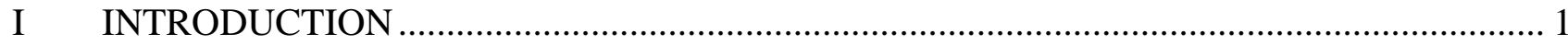

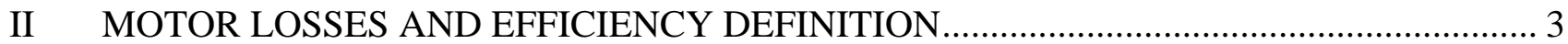

III DISCUSSION OF MOTOR EFFICIENCY MEASUREMENT …………………................... 5

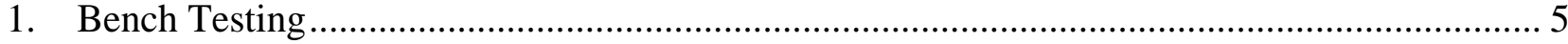

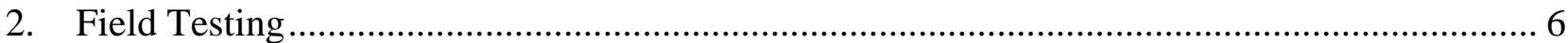

IV IN-SERVICE MOTOR EFFICIENCY EVALUATION METHODS ........................................ 8

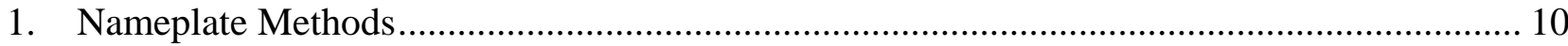

(1) Standard Nameplate Method ...................................................................................... 10

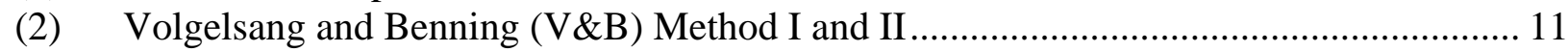

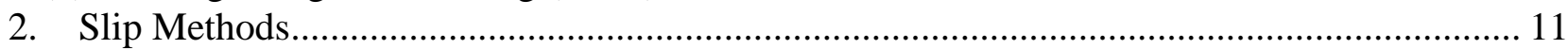

(3) Standard Slip Method ............................................................................................. 11

(4) Upper Bound Slip Method ....................................................................................... 12

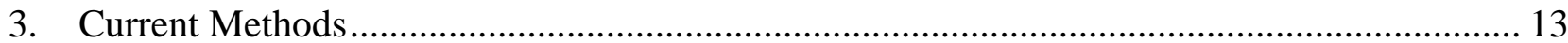

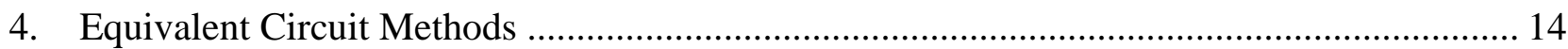

(5) Standard Equivalent Circuit Method (IEEE Std-112 Method F) ........................................ 15

(6) Ontario Hydro Modified (OHMF) Equivalent Circuit Method .......................................... 15

(7) Nameplate Equivalent Circuit (ORMEL96) Method ......................................................... 16

(8) Locked Rotor Method ........................................................................................... 17

(9) Standstill Frequency Response Method .......................................................................... 17

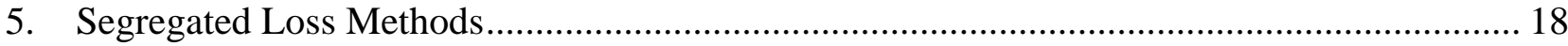

(10) Standard Segregated Loss Method (IEEE Std-112 Method E) ........................................... 18

(11) Ontario Hydro Modified (OHME) Segregated Loss Method ............................................. 19

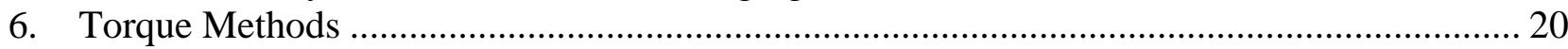

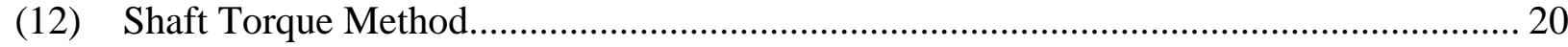

(13) Air-gap Torque (AGT) Method.................................................................................... 21

(14) Instantaneous Current Method ....................................................................................... 24

7. Empirical Method (Stanford Wilke Method) ………….......................................................... 25

8. Statistical Methods ......................................................................................................... 25

9. Dedicated Instrument Methods and Software Methods .......................................................... 26

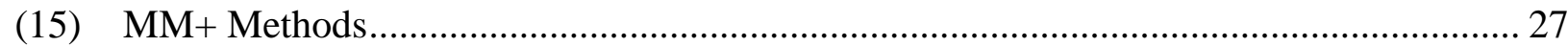

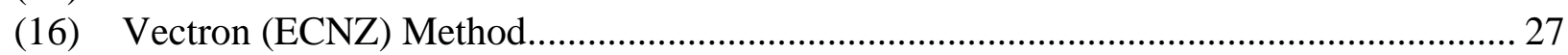

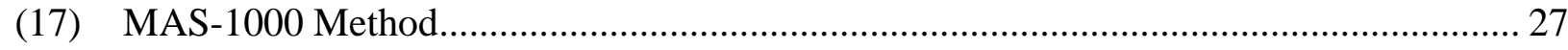

(18) Esterline Angus Method............................................................................................... 28

V PROPOSITION OF A MODIFIED AIR-GAP TORQUE METHOD ........................................... 29

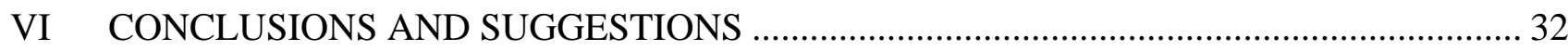

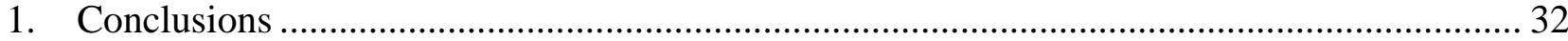

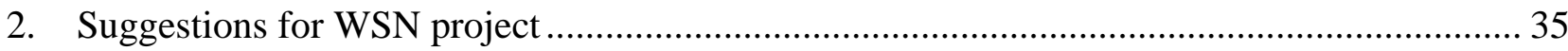

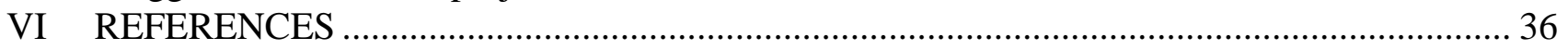




\section{LIST OF TABLES}

Table 1. Assumed Values for Stray Load Loss .................................................................... 19

Table 2. Comparison of the Motor Efficiency Estimation Methods ............................................ 33

Table 3. Comparison of Four Suggested/Proposed Methods ...................................................... 34

Table 4. Comparison of the Testing Results Using Suggested Methods .................................... 34

\section{LIST OF FIGURES}

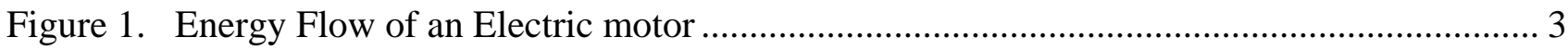

Figure 2. Assumed and Actual Load-versus-Current Curves................................................... 14

Figure 3. Equivalent Circuit of IEEE Std-112 Method F. ..................................................... 15

Figure 4. Anticipated Efficiency Estimation Errors versus Evaluation Methods........................... 32 


\section{INTRODUCTION}

In the Eaton WSN research project, the focus is on two main objectives, efficiency estimation and condition monitoring. In this report, the various methods of determining the motor efficiency are discussed in details.

Over the years, many motor efficiency estimation methods have been proposed. A series of relatively comprehensive laboratory assessments have been conducted back in 1990's, and have become main sources of this survey.

In March of 1996, a team at the Oak Ridge National Lab (ORNL), under contract to the Bonneville Power Administration (BPA) and Pacific Gas and Electric (PG\&E), reviewed 28 of the already in use or proposed for use efficiency estimation methods and evaluated them according to their intrusiveness and cost of equipment. ${ }^{[1][2][3]}$

Shortly after the first report, the same team selected 6 candidate methods among these 28 methods for more detailed evaluation under the support of the Motor Challenge Program sponsored by Department of Energy (DOE). ${ }^{[4]}$ These 6 methods particularly target on estimating motor efficiency and load under field conditions. These 6 methods were divided into three groups according to their intrusion level and accuracy, ranging from highly intrusive methods to completely non-intrusive methods. The report also suggested one best candidate method out of each group based on the laboratory testing results.

Based on above reviews, in April of 1999 the Washington State University Co-Operative Extension Energy Program (WSUCEEP), under contract with BPA and PG\&E, subcontracted with the Motor System Resource Facility (MSRF) at Oregon State University (OSU) to test the 12 most promising in-service motor efficiency testing methods. ${ }^{[5][6][7]}$ This testing also investigated the intrusion level of these efficiency estimation methods and their achieved level of accuracies. A more detailed test of four non-intrusive efficiency methods was also performed and the results were compared. 
In our previous WSN report “An Investigation on Commercially Available Energy Usage Estimation Products" [31], we investigated the available energy usage estimation products on the market. These products chose different methods in efficiency estimation. However, since the industry expects the energy usage estimation and diagnosis to be done without shutting down the motor, most of these products adopted non-intrusive or low-intrusive methods.

The measurements needed for each method are different, but most of them require the following common data: input line-to-line voltage, input phase current, and winding temperature. Some methods require the nameplate information (rated voltage, current, horsepower, speed, etc), stator resistance, or rotor speed (slip). Among these, the measurements/estimates of the stator resistance and rotor speed have been regarded as the stumbling blocks of various efficiency estimation methods for years.

However, over recent years research in this area has solved (or at least relieved) the difficulty of obtaining the stator resistance and rotor speed. Recent research has produced quite accurate speed estimates through stator current harmonic spectral analysis when the machine is working under normal stable operation. ${ }^{[8][9][10]}$ Also some "in situ” stator resistance measurement methods have been proposed which inject a DC component into the stator voltage and current. The accurate nonintrusive measurement of stator resistance may still need more research, but alternatively it can be measured and recorded at the time of new machine installation and at scheduled maintenance intervals or estimated from nameplate data. Therefore, in the efficiency estimation methods described hereafter, the stator resistance and rotor speed can be regarded as known variables.

In the rest of this report, over 18 available motor efficiency testing methods from literature are introduced and compared. After that, a modified air-gap torque (MAGT) method is proposed by Georgia Tech specially for WSN research.

Finally, based on the literature review results, three best in-service motor efficiency estimation methods and the proposed modified AGT method are suggested for the WSN project ranging from non-intrusive to high-intrusive. 


\section{MOTOR LOSSES AND EFFICIENCY DEFINITION}

Figure 1 shows the energy flow of an electric motor. The total losses of the motor are of our interest, since finding the efficiency is equivalent with finding the total losses.

There are, in general, five components of motor losses, as follows: ${ }^{[13]}$

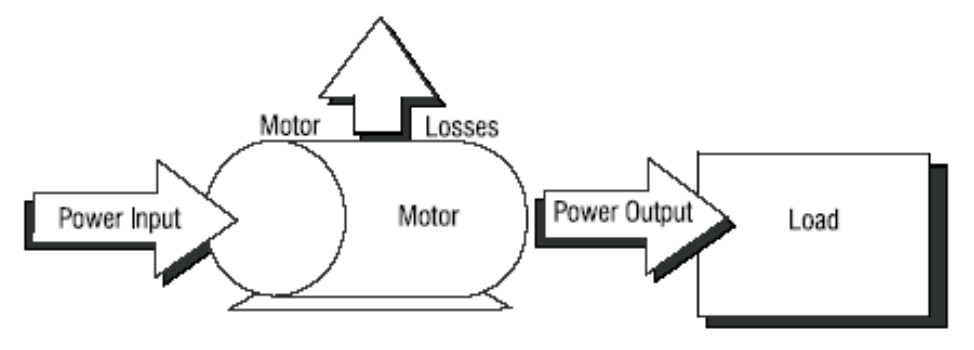

Figure 1. Energy Flow of an Electric motor

Stator resistance losses $\left(\mathrm{W}_{\mathrm{s}}\right.$, ) are the losses in the stator windings equal to $1.5 \mathrm{I}^{2} \mathrm{R}_{\mathrm{s}}$ for a three-phase motor where I is the measured or calculated RMS current per line terminal at specified load; and $\mathrm{R}_{\mathrm{s}}$ is the average dc resistance between any two line terminals corrected to the specified temperature.

Rotor resistance losses $\left(\mathrm{W}_{\mathrm{r}}\right.$ ) are the losses in the rotor windings equal to $3 \mathrm{I}_{2}{ }^{2} \mathrm{R}_{\mathrm{r}}$ for a three-phase motor where $I_{2}$ is the rotor phase current and $R_{r}$ is the rotor dc resistance corrected to the specified temperature.

Core losses ( $\mathrm{W}_{\text {core }}$ ) constitute the hysteresis and eddy current losses in the iron. These losses vary approximately with the square of the input voltage, but for fixed input voltage these remain approximately constant from no load to full load. These losses are commonly measured by running the machine as a motor at rated voltage and frequency without connected load (no-load test). 
Windage and friction losses $\left(\mathrm{W}_{\mathrm{fw}}\right)$ are the mechanical losses due to bearing friction and windage. These losses are also approximately constant from no load to full load. It is also a common practice to use no-load test to measure these losses.

Stray load losses $\left(\mathrm{W}_{\mathrm{LL}}\right)$ are the fundamental and high-frequency losses in the structure of the motor, circulating current losses in the stator winding, and harmonic losses in the rotor conductors under load. These losses are proportional to the square of the rotor current. The stray load losses can be further divided into two parts: stator stray loss $\left(\mathrm{W}_{\mathrm{LLs}}\right)$ and rotor stray loss $\left(\mathrm{W}_{\mathrm{LLr}}\right)$.

The relationship between the input power, output power and the losses are described by the following equation:

$$
W_{\text {Losses }}=P_{\text {input }}-P_{\text {output }}=W_{S}+W_{r}+W_{\text {core }}+W_{f w}+W_{L L s}+W_{L L r}
$$

The efficiency is defined by different ways as the following ${ }^{[3]}$

$$
\begin{aligned}
& \eta=\frac{P_{\text {output }}}{P_{\text {input }}} \times 100 \% \\
& =100 \%-\frac{W_{\text {Losses }}}{P_{\text {input }}} \times 100 \% \\
& =\frac{P_{\text {em }}-W_{f w}}{P_{\text {input }}} \times 100 \% \\
& =\frac{T_{\text {air-gap }} \cdot 2 \pi \frac{r p m}{60}-W_{f w}-W_{L L r}}{P_{\text {input }}} \times 100 \%
\end{aligned}
$$




\section{DISCUSSION OF MOTOR EFFICIENCY MEASUREMENT}

\section{Bench Testing}

In general, bench testing provides the most accurate testing methods of motor efficiency. A load absorption device (dynamometer, pump or generator) is used to absorb the power generated by the motor and a torque measuring device is used to measure the torque on the motor shaft (shaft torque) as it provides power to the load absorber. In addition, a speed measuring device is used to measure the speed of the rotor. The output power is simply calculated by multiplying the measured torque times the speed.

Errors can be introduced through a number of ways:

(1) The accuracy of the output power depends on the accuracy of two measured variables, speed and torque. Speed is relatively precise. While the measurement of torque is prone to error if one strain gauge is installed on the bench and is used to measure a wide range shaft torque.

(2) The input power is calculated by multiplying the measured input voltage waveform and input current waveform. Errors can be introduced by the phase shifts associated with the current transformer and the difference in sample time between the samples of the input currents and voltages.

(3) The power supply at the test bench may also produce some error in to the efficiency determination. For example, a three percent voltage imbalance can result in a negative sequence current flow and subsequent heating which requires nearly a $10 \%$ derating of the motor. ${ }^{[14]}$

While errors are not the main drawback of bench testing method, the most significant problem is the time required to remove the motor form service, transport it to the test facility, perform the testing, reinstall it, including necessary alignment. There are significant expenses in downtime and manpower, which would not be acceptable for most cases. 


\section{Field Testing}

The most significant advantage of the in-service test methods is that the motor does not have to be removed from the service and thus the manufacturing process is not interrupted. Measurements can be taken quickly with clamp-on current transformers (CTs) and voltage probes. Typically, these measurements are taken at the motor control center (MCC). Usually field testing is the only practical method for efficiency testing because neither the removal of the motor nor the operation downtime is permitted by the user.

Another significant advantage of performing in-service testing is that the motor's service condition can be measured and recorded for evaluation. As previously discussed, the voltage imbalance and harmonics distortion can have a severe negative effect on the efficiency determination. The estimation would be more precise if these negative factors are known before evaluation.

The fundamental disadvantage with field testing is that motor output torque (shaft torque) is not measured. Thus motor output power is not measured. Instead, input currents, voltages, power factors, and speed are measured (extracted) in conjunction with nameplate data to estimate power output and efficiency. The various efficiency estimation methods are generally divided into two categories:

(1) Approximate efficiency based only on motor speed;

(2) Approximate efficiency based on a mathematical model, or equivalent circuit, of the motor.

The methods of approximating efficiency in the field without measuring motor torque output and without using an equivalent circuit typically rely on a measurement of motor speed or power, such as the "Stanford Method" and the slip method. These methods are in reality approximations and yield efficiencies that are often more than 15\% from the true value.

More sophisticated methods use techniques for calculating motor torque without actually measuring it and depend on the use of a motor equivalent circuit or some other mathematical model of the motor's electro-mechanical power transfer mechanism. The degree of the accuracy of these methods is directly related to the degree of accuracy of the parameters in the equivalent circuit model. 
Development of a high accuracy equivalent circuit is normally done with information obtained from no load testing and resistance testing of the disconnected motor. Equivalent circuit development is described in length in IEEE Standard 112, IEEE Standard Test Procedure for Polyphase Induction Motors and Generators [5]. In typical industrial applications, however, the user generally will not permit the motor to be disconnected to measure stator resistance, or uncoupled operation of the motor to measure the no load power input. For this reason, an alternate, less accurate, method of developing the equivalent circuit is often used.

An equivalent circuit may be developed by using motor nameplate data and data from Motor Master or standards developed by IEEE. Motor Master is a database of motor performance information for over 10,000 motors available on diskette. It is possible to develop an equivalent circuit from this information because the nameplate usually provides performance information, such as the locked rotor kVA code and NEMA Design type, which can be used in a set of equations to solve for the equivalent circuit constants. Sometimes more information is available from Motor Master than is available on the nameplate. The problem with this approach is that the nameplate and Motor Master data are generic design data and may not be accurate in reflecting such parameters as the actual speed and power factor when the motor is delivering rated horsepower.

The unique aspect of the equivalent circuit method is that the circuit can be solved for any running speed. When the circuit is solved, it will calculate a motor power factor. This power factor can then be compared with the actual measured power factor to assess the accuracy of the equivalent circuit. If the calculated power factor does not match the measured power factor, the equivalent circuit parameter of magnetizing reactance can be adjusted until a match is found. Magnetizing reactance, does, in fact, decrease as a motor ages. As the magnetizing reactance decreases, the efficiency also decreases. Thus the equivalent circuit can be adjusted to match the present condition of the motor if the power factor can be measured. 


\section{IN-SERVICE MOTOR EFFICIENCY EVALUATION METHODS}

There are many methods pertinent to field efficiency evaluation in the literature, and new methods are appearing almost every year. An in-service motor efficiency evaluation can consist of a single basic method or can be built using a combination of different basic methods. This section lists the most promising motor efficiency methods which are categorized according to their theoretical bases and error sources. Some of these methods are not applicable for field in-service testing, but they are also included here for the following reasons:

1) It is the "backbone" method in the certain category.

2) It best illustrates the physical nature of the certain series of methods.

3) Most of the other sophisticated methods are the variations of a single or a combination of these basic methods after some simplification or modification.

Generally, these methods can be categorized as follows [2][16]:

- Nameplate methods

- Slip methods

- Current methods

- Equivalent circuit methods

- Segregated loss methods

- Torque methods

- Empirical methods

- Statistical methods

- Dedicated instrument methods

All methods calculate efficiency according to the definition 


$$
\text { Efficiency }=\frac{(\text { Shaft output power })}{(\text { Electrical input power to drive system })}
$$

The shaft output power is the input power minus the losses. How to assess losses and evaluate output power gives rise to fundamental differences among the various methods. Consequently, the accuracies of methods are different.

The degree of intrusiveness of a field evaluation method is determined by what data are required to be measured in the field and the difficulty of performing the measurements. One or more of the following measurements may be involved: ${ }^{[2]}$

- $\quad$ nameplate reading;

- $\quad$ speed measured by optotachometer (Note: This can be neglected now.);

- currents measured by clamp-on transducer;

- $\quad$ voltages measurement;

- $\quad$ input power measurement;

- $\quad$ stator winding resistance reading (Note: This can be neglected now.);

- $\quad$ winding temperature data;

- no-load data measured with uncoupled shaft;

- $\quad$ shaft torque measurement.

The data may be acquired in the format of RMS meter readings or digitally sampled waveforms.

The cost associated with the labor, material, and downtime for implementing safety requirements for data collections can be used as a gauge to weigh intrusiveness. Planning may also affect intrusiveness. If a decoupled no-load test is required and the motor power supply is available during scheduled downtime, it may be possible to conduct this kind of test without affecting production.

The most promising efficiency evaluation methods are briefly introduced in the rest of this section. 


\section{Nameplate Methods}

The least intrusive field evaluation method is to obtain motor information from the nameplate. There are several versions of the nameplate methods. They are reported in [1][2][4][7]. They all use the nameplate data of the motor for the efficiency estimation.

\section{(1) Standard Nameplate Method}

In this method, it is assumed that the efficiency of the motor is constant and equal to the nameplate value. This works best when the efficiency-load curve is fairly flat, so that the full-load efficiency is applicable for most load conditions. But this is not always true for all kinds of motors. ${ }^{[2]}$ Hence, the nameplate method may be applicable for some motors, but could result in substantial inaccuracies for other motor types.

With this nameplate method, three additional problems may occur. First, the nameplate data may be given according to a method other than IEEE Standard 112 Method B. Second, the motor may have been rewound. Third, the field environment pertinent to the voltage unbalance and harmonics content may be different from that the nameplate data is derived from.

Nameplate efficiencies of a given motor can be evaluated according to different standards. The three most frequently used standards are the National Electrical Manufacturers Association (NEMA) that uses IEEE Standard 112, the Japanese Electrotechnical Committee (JEC), and the International Electrotechnical Commission (TEC). These three standards are not in agreement and may result in a given motor being stamped with rather different efficiencies.

Rewound motors introduce additional uncertainty, since the nameplate data may no longer be valid. Some engineers suggest that, after each rewinding to the same horsepower and same number of poles, a two percentage points reduction of efficiency should be considered. However, a different opinion indicates that the efficiency should not be reduced if the rewinding follows Electrical Apparatus Service Association (EASA) standards. 
Reference [2] reported that under the worst situation, the efficiency can be off ten percentage points from the nameplate efficiency. However, the nameplate method is the most non-intrusive method. And the bottom line is that a nameplate method is better than no field evaluation at all.

\section{(2) Volgelsang and Benning (V\&B) Method I and II}

The Volgelsang and Benning (V\&B) method I and II are variations of the standard nameplate method, and they are used in a dedicated commercially available instrument. ${ }^{[7]} \mathrm{V} \& \mathrm{~B}$ option I requires testing at three conditions: uncoupled (no-load test), normally loaded, and unpowered (off). The original method requires a reflector to be attached to rotating equipment to allow the speed to be recorded. (Note: This speed measurement can be substituted by current signature analysis in nowadays.) In Option II, testing is accomplished without uncoupling, and motor nameplate data are substituted, but the accuracy is assumed to be reduced.

The principles of this instrument are based on theory closely exposed in the patent [15]. The approach of this instrument is based on the identification and segregation of the losses of the induction motor. It calculates an upper bound efficiency by allocating the iron and the friction losses to the stator losses and a lower efficiency boundary by allocating the iron and friction losses to the rotor. The stator resistance has to be measured and entered into the program. Nameplate data, RMS electrical quantities, and measured speed are used to estimate the operating efficiency.

\section{Slip Methods}

There are several versions of slip methods. They are reported in [1][2][4][7][16]. All rely on a measurement of motor speed to find the slip.

\section{(3) Standard Slip Method}

This method presumes that the percentage of load is closely proportional to the percentage of the ratio of measured slip to full-load slip. The obvious error is that the slip ratio represents the percentage of load and the efficiency is not equal to the percentage of load. Alternatively, one can also measure the power into the motor and approximate the power out of the motor by multiplying 
the rated horsepower of the motor by the ratio of the measured slip to rated slip. The operating efficiency of the motor is thus approximated using the following relationship:

$$
\text { Efficiency }=\frac{(\text { Measured Slip } / \text { Rated Slip }) \times \text { Rated Output Power }}{\text { Input Power }}
$$

This method can be enhanced by correcting the rated nameplate speed for voltage variations, especially when the motor-efficiency-versus-load curve is not flat. This is done by taking the square of the ratio of the actual voltage to nameplate voltage and multiplying this with the rated speed. However, the estimation results could still have a relative big error, since the nameplate speed is allowed to deviate as much as 20 percent from the actual rated speed by NEMA MG1. ${ }^{[14]}$

The no-load speed of induction motors is always close to the synchronous speed. Subsequently, the projection of a light load through the standard slip method is relatively more accurate than the projection of a heavy load.

The main attraction of the standard slip method is its simplicity. However, several authors, e.g. [2], [18] and [20], have observed that the accuracy of the method suffers badly from several causes. Reference [20] provides an excellent discussion of the drawbacks of the slip methods, particularly the standard slip method.

\section{(4) Upper Bound Slip Method}

The upper bound with resistance method is reported in [7]. It first calculates the stator losses by calculating the $I^{2} R_{s}$ losses of the stator, obtained from the stator resistance measurement and the operating current measurement. Next, the rotor copper losses are calculated with the standard slip method out of the air-gap power, which is assumed to be equal to the input power minus stator resistance losses. The resulting efficiency estimation will always be higher than the operating efficiency since it neglects stray, friction, windage, and core losses. 
As in the nameplate method, a large increase in accuracy could be obtained by entering the information obtained on a no-load test. With that information, friction, windage, and core losses can be obtained accurately and load-dependent stray-load losses with a fair accuracy. But the need of noload test for an accurate measurement is obviously the drawback of this method.

\section{Current Methods}

The Current Method is included in [1][2][4][7][16]. The current method is another approach that uses a minimum of field measurements in conjunction with manufacturer's data to estimate motor efficiency at normal operating loads. There are also several alternative current methods. Like the slip methods, the main attraction of the current method is its simplicity.

This method presumes that the percentage of load is closely proportional to the percentage of the ratio of measured current to full-load current. The shaft output power is, thus, approximated using equation (a). $I_{\mathrm{fl}}$ is the nameplate full load current and $\mathrm{I}$ is the measured current. The expression of shaft output power is also defined by equation (b) in some circumstances. As illustrated in Figure 2, the load is normally overestimated using equation (a); while using equation (b), the load evaluation is normally underestimated. The average of the two approaches may give a more accurate shaft output power.

$$
\begin{gathered}
\text { (Shaft Output Power) }=\frac{I}{I_{f l}} \cdot(\text { Rated Output Power) } \\
\text { (Shaft Output Power })=\frac{\left(I-I_{n l}\right)}{\left(I_{f l}-I_{n l}\right)} \cdot(\text { Rated Output Power) }
\end{gathered}
$$

The simple current method, equation (a), does not require a no-load current value. The refined equation (b) requires a no-load test to obtain the no-load data. Just as with the slip method, to obtain efficiency one will have to either use typical efficiency-versus-load curves or measure input power. 


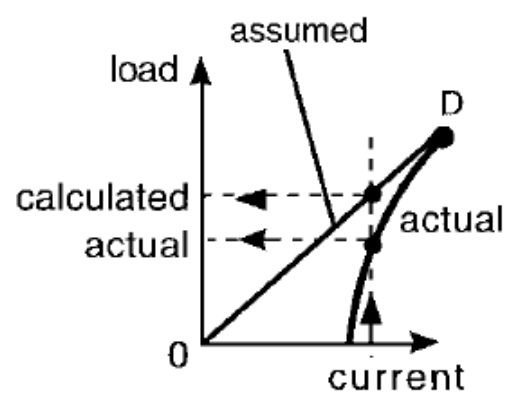

(a) Overestimated

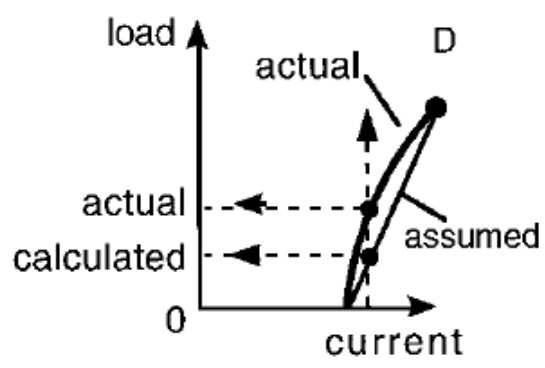

(b) Underestimated

Figure 2. $\quad$ Assumed and Actual Load-versus-Current Curves.

Reference [20] provides an excellent discussion of this problem and provides an improved version of the above equations. They also conclude that even the improved version can have major inaccuracies depending on the shape of the motor performance curve and the load condition.

Reference [2] and [20] summarize the advantages of the current method:

1) The NEMA Standard MG1-12.47 permits only half the tolerance in nameplate, fullload current as it does full-load slip.

2) Motor current measured by a clamp-on probe has a low intrusion level.

The chief disadvantages of the current method:

1) Current, unlike slip, does not vary linearly with load because there is a magnetizing current even when the motor is operating at no load. Therefore, this method also has a significant inherent inaccuracy.

2) The need for no-load test is not accepted in most in-service testing.

\section{Equivalent Circuit Methods}

The performance of an electric motor, at least with regard to efficiency, can be calculated from its equivalent electric circuit. These methods permit one to compute estimates of the efficiency of the motor when it is operating at loads other than those at which measurements were made. 


\section{(5) Standard Equivalent Circuit Method (IEEE Std-112 Method F)}

The efficiency estimation method F in the IEEE Standard 112-1996 is the standard equivalent circuit method. ${ }^{[13]}$ The IEEE Standard112 Method F is not a useful field-test for efficiency. Its additional removed-rotor and reverse rotation tests to directly measure the fundamental frequency and high frequency stray load losses are too invasive and user unfriendly. The basic Method F requires an impedance test and the complete no-load, variable voltage test. It requires volts, watts, amperes, slip, stator winding temperature, or stator winding resistance to be measured at two values of voltage while operating at no load. In one case, measurements are made at rated voltage while operating at no load. In the other case, measurements are made while operating at no load with voltage reduced until slip is equal to that obtained at the normal operating load. Once these measurements are made, an iterative procedure is used to determine the parameters of the equivalent circuit. The iterative procedure requires one to either know the design value of the ratio $\mathrm{X}_{1} / \mathrm{X}_{2}$ or to use the standard NEMA design value. The equivalent circuit of Method F is shown in Figure 3.

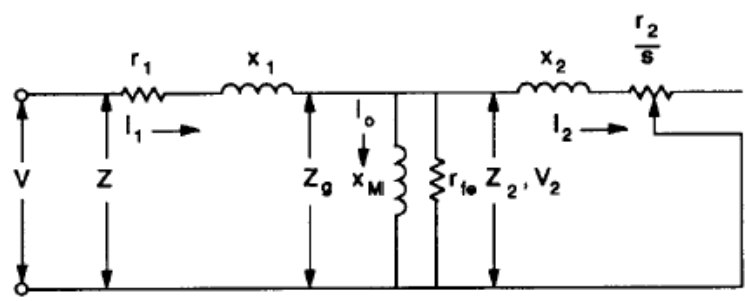

Figure 3. $\quad$ Equivalent Circuit of IEEE Std-112 Method F.

Although this method is expected to be quite accurate, it is still considered to be too intrusive for inservice testing. This method is kept here mainly as the basis of other modified methods.

\section{(6) Ontario Hydro Modified (OHMF) Equivalent Circuit Method}

This method is included in [16][18]. This is a modified version of the IEEE Standard Method F proposed by Ontario Hydro. ${ }^{[18]}$ 
A no-load and a full-load test, both at rated voltage, must be run. In turn, this requires one to disconnect the motor being tested from its load. Line voltage, input power, line current, power factor, and stator resistance at load temperature are measured after operating at no load and at full load. The slip is also measured at full load.

This method eliminates the need for a variable voltage as required by IEEE Standard 112, Method F. ${ }^{[13]}$ The equivalent circuit used by this method is slightly different from that of Method F. In this version of the equivalent circuit, the impedance elements $\left(\mathrm{r}_{\mathrm{f} 0}\right.$ and $\left.\mathrm{X}_{\mathrm{M}}\right)$ of the magnetizing branch are shown in series while that of Method F is shown in parallel.

\section{(7) Nameplate Equivalent Circuit (ORMEL96) Method}

The least intrusive method to estimate efficiency is based on the use of an equivalent circuit derived from the motor's nameplate data. Once the equivalent circuit of a motor is known, its running efficiency at any load can be determined simply by measurement of the motor speed. The nameplate data provides information about the motor's rated performance, locked rotor current, and design type.

The ORMEL96 method was developed by Oak Ridge National Laboratory (ORNL) in 1996. ${ }^{[1]}$ [4][7][16] The ORMEL 96 method finds the equivalent circuit from the nameplate data plus the value of the stator resistance. It was found in [7] that the accuracy of the predicted efficiencies increases substantially if the stator resistance is known. However, if the stator resistance is not known, it can also be estimated from motor nameplate data. ${ }^{[16]}$ ORMEL96 also has an advanced mode which allows the user to adjust certain "tunable parameters".

Even with typical nameplate information of older conventional motors and rewound motors, this method has been shown in limited testing to provide an average accuracy of less than 3.5 percentage points. ${ }^{[16]}$ 


\section{(8) Locked Rotor Method}

This method is included in [16][25]. It differs from the previous equivalent circuit. It adds a second rotor loop.

Reference [25] presented a procedure that uses two locked rotor tests to determine the parameters of an equivalent circuit with two rotor loops. An alternative procedure is to use a single locked rotor in conjunction with a load test to determine these parameters. In both cases, a no-load test must also be run. With these parameters in hand, they then develop a method for computing the efficiency of the motor from the equivalent circuit relationships.

The advantages of this two-rotor loop method are these areas:

1) The procedure for determining parameters of equivalent circuit is not iterative like that of IEEE Std-112 Method F.

2) According to the authors, the two-reactance loop equivalent circuit represents doublecage and deep-bar rotor motors better than the single rotor loop equivalent circuits.

This method has two principal disadvantages:

1) It requires a complete no-load test with the motor connected to a variable voltage power source.

2) It requires connecting the motor to a variable frequency source. This is too massive and user unfriendly for a good field test.

\section{(9) Standstill Frequency Response Method}

This method is included in [16] [26]. Like the previous Locked Rotor Method, it also differs from the standard equivalent circuit. It also has two rotor loops.

Reference [26], sponsored by the Electric Power Research Institute (EPRI), investigated the sensibility of determining the electrical equivalent circuit parameters of induction motors by using the standstill frequency response test. The approach investigated by the EPRI study was to measure 
the impedance of a motor, with its rotor stationary, over a frequency range of 0.01 to $500 \mathrm{~Hz}$. The parameters of the equivalent circuit are then derived from these data.

The major advantage of this method over the IEEE Std-112 Method F is that the low-voltage, noload test is not required. The level of applied voltage is much lower than that of the low-voltage, noload test. If a packaged test device with a variable frequency source is developed and made commercially available, then the only drawback would be the no-load test to determine friction and windage.

\section{Segregated Loss Methods}

The segregated loss methods are the most straightforward of the efficiency testing methods because they simply estimate the magnitudes of each motor power loss component. Some of the methods are quite complicated and intrusive, while others rely on empirical factors to estimate the losses.

\section{(10) Standard Segregated Loss Method (IEEE Std-112 Method E)}

The efficiency estimation method E in the IEEE Standard 112-1996 is the standard segregated loss method. ${ }^{[13]}$ Same with IEEE Std-112 Method F, Method E is not a useful method for motor inservice testing, since it requires additional removed-rotor and reverse-rotation tests used to directly measure the fundamental frequency and high-frequency, stray-load losses. Method E is seldom used in the field because it requires a variable load and a variable voltage power supply. Some important properties of method $\mathrm{E}$ are listed as follows:

1) Method E specifies a comprehensive no load test.

2) Method E requires test under load at six equally spaced load points with four between 25 percent and 100 percent of full load and two greater than 100 percent and less than 150 percent.

3) Method E specifies an assumed value for stray load losses at rated load.

4) The repeatability of Method $E$ is improved by requiring the adjustment of all resistance and slip measurements to a specified temperature. 
Note that Method E requires variable load tests, so the motor being tested must be connected to a variable load. In most circumstances in the field this would be quite disruptive to normal operation of the system. This again shows the high intrusiveness of Method E. Like Method F, this method is kept here mainly as the basis of other modified segregated loss methods.

\section{(11) Ontario Hydro Modified (OHME) Segregated Loss Method}

This method is included in [1][2][4][7][16]. Ontario Hydro ${ }^{[18]}$ proposed a segregated loss method that simplifies Method E much further. It uses an empirical value of 3.5\% 4.2\% of rated input power to find the no-load losses rather than actually performing no-load testing. ${ }^{[2]}$ As a further refinement, this value could be optimized by testing samples of motors at different horsepower levels. The stray load losses are estimated based on the IEEE 112 standard assumed values as Table 1. [13]

Table 1. $\quad$ Assumed Values for Stray Load Loss

\begin{tabular}{|c|c|c|}
\hline \multicolumn{2}{|c|}{ Machine rating } & $\begin{array}{c}\text { Stray-load loss percent of rated } \\
\text { output }\end{array}$ \\
\hline $1-125 \mathrm{hp}$ & $1-90 \mathrm{~kW}$ & $1.8 \%$ \\
\hline $126-500 \mathrm{hp}$ & $91-375 \mathrm{~kW}$ & $1.5 \%$ \\
\hline $501-2499 \mathrm{hp}$ & $376-1850 \mathrm{~kW}$ & $1.2 \%$ \\
\hline $2500 \mathrm{hp}$ and greater & $1851 \mathrm{~kW}$ and greater & $0.9 \%$ \\
\hline
\end{tabular}

This method can be simplified even further by using assumed value 0.8 for rated power factor. The stator resistance at load is estimated based on a simple approximation using the motor current to estimate the temperature rise. The only other measurements required are power into the motor and motor speed. The author of [7] has experimented with OHME Method and found it to provide an accuracy of $\pm 3 \%$ or $\pm 4 \%$. Report [4] claimed that an error of about $1 \%$ was obtained using OHME Method for a 7.5hp motor. 


\section{Torque Methods}

According to the definition in Section II, the efficiency of a motor can be defined as following equations.

$$
\begin{aligned}
& \eta=\frac{P_{\text {output }}}{P_{\text {input }}} \times 100 \% \\
& =\frac{T_{\text {shaft }} \cdot \omega_{r}}{P_{\text {input }}} \times 100 \% \\
& =\frac{T_{\text {air }- \text { gap }} \cdot \omega_{r}-W_{f w}-W_{L L r}}{P_{\text {input }}} \times 100 \%
\end{aligned}
$$

With the input power and the rotor speed measured/estimated, the efficiency can easily be determined if shaft torque is known. This is the basic principle of all the torque methods.

\section{(12) Shaft Torque Method}

This method is discussed in [2]. Regardless of how sophisticated a method is, it remains difficult to assess all the stray-load losses accurately in the field. The most straightforward torque method, Shaft Torque Method, is to measure the shaft torque (and therefore output power) directly from the shaft, without any need to calculate losses. The shaft torque method offers the most accurate field efficiency evaluation method. It is also highly intrusive.

A custom-built torque coupling may be used to replace the existing coupling. Torque signals can be obtained through slip rings. Laser and telemetry technologies may also be used without going through slip rings for signal noise reductions. Many different ways have been proposed in the report ORNL/TM-13165. ${ }^{[4]}$ The accuracy of this method depends on the quality of the torque sensors, the signal noise, and the shaft alignment of the motor and its load. Downtime is required for preparing and replacing the shaft torque coupling. 


\section{(13) Air-gap Torque (AGT) Method}

In 1995, Hsu and Scoggins at the Oak Ridge National Lab developed the Air-gap Torque Method based on well-known air-gap equations. ${ }^{[3]}$ This method has been approved by several experiments ${ }^{[2][3][4][7]}$ to be a very promising motor efficiency estimation method. It is also regarded by us as one of the most potential efficiency estimation methods for WSN project. Therefore, this method is explained in details here.

A. Equations of Power, Air-Gap Torque, and Efficiency

\section{a) Power Equations}

The instantaneous input power of a three-phase induction motor is the summation of products of the instantaneous phase voltages, $\mathrm{v}_{\mathrm{a}}, \mathrm{v}_{\mathrm{b}}, \mathrm{v}_{\mathrm{c}}$, and phase currents, $\mathrm{i}_{\mathrm{a}}, \mathrm{i}_{\mathrm{b}}$, and $\mathrm{i}_{\mathrm{c}}$.

$$
\text { Power }=v_{a} i_{a}+v_{b} i_{b}+v_{c} i_{c}
$$

A portion of this instantaneous power includes the charging and discharging of the energy stored in the windings. Therefore, this instantaneous power cannot represent the instantaneous torque even at a constant speed after subtracting the losses. However, the average value of instantaneous power at full load represents the input power of the motor for the efficiency calculation. The conventional single-frequency power equation using power factor, RMS current and voltage is a special case of the average instantaneous power.

b) Review of Air-Gap Torque Equations

The following voltage equations are for the three-phase armature windings,

$$
v_{a}=\frac{d \psi_{a}}{d t}+r i_{a}, v_{b}=\frac{d \psi_{b}}{d t}+r i_{b}, v_{c}=\frac{d \psi_{c}}{d t}+r i_{c}
$$


Where

$\Psi_{a}, \Psi_{b}, \Psi_{c}=$ flux linkages of windings a, b, and c, respectively

$r=$ the phase resistance

Substituting into (1) to give

$$
\text { Power }=\left[i_{a}\left(\frac{d \psi_{a}}{d t}+r i_{a}\right)+i_{b}\left(\frac{d \psi_{b}}{d t}+r i_{b}\right)+i_{c}\left(\frac{d \psi_{c}}{d t}+r i_{c}\right)\right]
$$

From (2), the flux linkages can also be given as

$$
\begin{aligned}
& \psi_{a}=\int\left(v_{a}-r i_{a}\right) d t \\
& \psi_{b}=\int\left(v_{b}-r i_{b}\right) d t \\
& \psi_{c}=\int\left(v_{c}-r i_{c}\right) d t
\end{aligned}
$$

Subtracting the copper losses and the terms pertinent to the energy stored in the windings, the air gap torque equation can be written as

$T_{\text {air-gap }}=\frac{\text { poles }}{2 \sqrt{3}}\left\{\left(i_{A}-i_{B}\right) \cdot \int\left[v_{C A}-R_{S}\left(i_{C}-i_{A}\right)\right] d t-\left(i_{C}-i_{A}\right) \cdot \int\left[v_{A B}-R_{S}\left(i_{A}-i_{B}\right)\right] d t\right\}$

where $\mathrm{P}$ is the number of poles, $i_{A}, i_{B}$, and $i_{C}$ are the line currents, and $\mathrm{R}$ is half of the line-to-line resistance value.

When using either three leads for Y-connected motors without a neutral connection or three leads for delta-connected motors, (4) can be further simplified by using

$$
i_{B}=-\left(i_{A}+i_{C}\right)
$$


c) Numerical Evaluation of Integrals

The integrals of windings corresponding to lines CA and AB in (6) represent flux linkages. Since the time increment between data points is small, a simple trapezoidal method can be used. Other methods using Simpson's rule or Gauss's rule for numerical evaluation of integrals can also be applied.

$$
\begin{gathered}
\int\left[v_{c a}-R\left(i_{c}-i_{a}\right)\right] d t \\
\int\left[-v_{B A}-R\left(2 \bullet i_{A}-i_{C}\right)\right] d t
\end{gathered}
$$

\section{d) Efficiency Equations}

As mentioned earlier, the shaft output power is calculated from the shaft speed (rpm) and shaft torque.

$$
P_{\text {output }}=T_{\text {air-gap }} \cdot \omega_{r}=T_{\text {air-gap }} \cdot 2 \pi \cdot \frac{r p m}{60}
$$

where the shaft torque is the difference of air-gap torque and torque losses corresponding to mechanical loss and stray loss produced by rotor current.

$$
T_{\text {shaft }}=T_{\text {air-gap }}-\frac{W_{f w}}{\left(2 \pi \cdot \frac{r p m}{60}\right)}-\frac{W_{L L r}}{\left(2 \pi \cdot \frac{r p m}{60}\right)}
$$

From (7), (8), and the definition of efficiency, the efficiency, $\eta$, yields

$$
\eta=\frac{T_{\text {air-gap }} \cdot\left(2 \pi \frac{r p m}{60}\right)-W_{f w}-W_{L L r}}{P_{\text {input }}} \times 100 \%
$$


B. Discussions of the Air-gap Torque Method.

A significant difference between the AGT Method and the IEEE Std-112 Method E is that the AGT Method considers the losses associated with the negative torques produced by the negative sequence voltages and currents, which are the products of unbalanced voltages and harmonics. Because in the real world the power supply is commonly polluted with unbalanced voltages and harmonics, the AGT Method has a unique advantage over the IEEE Std-112 Method E for in-service motor efficiency assessment.

The advantages of the AGT Method:

1) Air-gap torque can be measured while the motor is running.

2) The AGT Method should continue to provide optimum accuracy when the phase powers are unbalanced.

3) This method can be used for non-induction motors such as the adjustable speed, brushless dc motors.

The major disadvantages of the method:

1) The voltage and current waveforms must be acquired with a reasonably high sampling rate so that a high accuracy of the waveform shape may be achieved. A sample rate of $5,000 \mathrm{~Hz}$ was adopted in the testing of reference. ${ }^{[3]}$

2) A measurement of a no-load power into the motor must be made. This could be avoided by combining some other methods like Empirical Methods or Statistical Methods. The non-intrusiveness can be improved at the price of accuracy reducing.

\section{(14) Instantaneous Current Method}

This method is reported in [7]. It is based on an input-output power approach. Although this method is called "Instantaneous Current Method”, it is actually a variation of AGT Method. 
The input power to the motor is measured on the electrical terminals, and the output power is estimated by the multiplication of speed and torque. Speed is obtained by analyzing the instantaneous electrical quantities measured and calculated at the electrical inputs. Air-gap torque is calculated via the dq-axis theory for electrical machinery. Output torque (shaft torque) is estimated by subtracting an estimate of friction, windage, and stray load loss from the calculated air-gap torque.

This method is implemented in a dedicated instrument called Explorer II by Baker Instrument Company and is indeed an intrusive method. In our previous WSN report ${ }^{[31]}$, the Explorer II by Baker Instrument Company was evaluated as the best commercially available instrument for energy usage estimation and condition monitoring in today’s market.

\section{Empirical Method (Stanford Wilke Method)}

This method is reported in [1][2]. Empirical Method is also called as "Stanford Wilke Method". It was developed at Stanford University with K. Wilke as one of its developers. The method was developed to be applicable to motors between 10 and 50 horsepower. Some of the empirical assumptions were taken from IEEE Standard 112 while others arose from the author's experience. It is anticipated that the method will yield efficiency estimates with error within $\pm 4 \%$ or better. Reference [2] claimed that this method yields efficiency estimate with error about 6\% for both balanced and unbalanced voltage conditions.

\section{Statistical Methods}

The Statistical Methods are discussed in [1][2]. Empirical equations are set up to use minimal numbers of measured data for input power and efficiency estimations. Usually, application of this method is restricted to the group of motors for which empirical equations were derived.

The statistical results can be quite different for the same variable. A good example is the stray load loss estimation. NEMA MG1 ${ }^{[14]}$ paragraph 20.52 states that, if stray load loss is not measured, the 
value of stray load loss at rated load shall be assumed to be $1.2 \%$ of the rated output for motors rated less than $2500 \mathrm{hp}$ and 0.9\% for motors rated $2500 \mathrm{hp}$ and greater. IEEE Standard 112 Section 5.4.4 ${ }^{\text {[13] }}$ gives different assumed stray load loss values for motors rated less than 2500 hp, as shown in Table 1.

Additionally, some non-U.S. standards use $0.5 \%$ of rated output as the stray load loss at rated load. The statistical approach is commonly used with other basic methods. For instance, the previously discussed OHME Method suggests the combined windage, friction, and core losses to be 3.5\% of the rated input power. The stray load losses are estimated based on the IEEE Standard 112 standard assumed values, as shown in Table 1.

This method can be simplified even further by using assumed values for rated power factor (e.g. 0.8). Approximations can also be made for the temperature rise of the winding, and the winding resistance can even be estimated by measuring the line-to-line resistance taken from the circuit breaker and subtracting the estimated cable resistance. The only other measurements required are power into the motor and motor speed.

\section{Dedicated Instrument Methods and Software Methods}

Many of the motor efficiency methods have been incorporated into commercially available products, including both software and instruments. These methods are listed very briefly in this part.

Obviously, these methods could be categorized into previously discussed methods according to their physical nature. However, due to some commercial reasons, the algorithms behind some of these products have not been clearly reported. On the other hand, some of the previously discussed methods, such as the V\&B Method I II and the ORMEL96 Method have also been implemented into products. They are not listed here because relatively complete knowledge of these methods is available in the literature. 
(15)

The Motor Master Plus (MM+) is a free software tool developed by the Washington State University under the support of the US Department of Energy. This tool features motor inventory management tools, maintenance log tracking, efficiency analysis, savings evaluation, energy accounting, and environmental reporting capabilities. The lasted version, MotorMaster+ 4.0 software, includes a catalog of over 20,000 AC motors. It includes its own efficiency estimation methods as well as some other methods, such as ORMEL96 Method.

There are three MM+ methods used in MM+ software: MM+ Power Method, MM+ Current Method and MM+ Slip Method. These three methods are based on nameplate and normal load operation. They allow efficiency to be computed/estimated at the normal load. Electrical and/or speed readings are required at normal load. No uncoupled or unpowered readings are required. Reference [16] provides testing results using these methods.

\section{(16) Vectron (ECNZ) Method}

This method is reported in [7]. The Vectron Method was developed for the Electricity Corporation of New Zealand (ECNZ). It requires testing at a load $<10 \%$ and a load $>50 \%$, and unpowered (off). It also uses an optical tachometer based on an attached reflective strip. The manufacturer claims that the efficiency at full load conditions can be determined from testing at a load $>50 \%$ but less than full load. The manufacturer also claims that this method can correct for off nominal voltage conditions up to $5 \%$.

\section{(17) MAS-1000 Method}

This method is reported in [7]. The MAS-1000 Method is based upon an instrument MAS-1000 developed by Niagara Instruments. The latest version of this instrument, MAS-1000 LA, has been introduced in our previous WSN report ${ }^{[31]}$. 
A magnetic reluctance speed sensor is used, although a strobe tachometer can be substituted if readings are manually entered, but the former is preferred. The system is based on an Intel 486 processor, and the manufacturer claims that Motor Master Plus (MM+) software developed by DOE could be loaded and used with the tester for additional convenience in motor systems management.

\section{(18) Esterline Angus Method}

The Esterline Angus method is tested by [7]. It is a pure software method dedicated for a certain instrument. It requires only generic equipment and custom software. Tests are required while uncoupled, at normal load, and unpowered. Surface and ambient temperature are required. 


\section{PROPOSITION OF A MODIFIED AIR-GAP TORQUE METHOD}

In 1995, Hsu and Scoggins ${ }^{[3]}$ developed the Air-gap Torque (AGT) Method based on air-gap equations ${ }^{[30]}$. This method has been approved by several experiments to be a very effective motor efficiency estimation method. ${ }^{[2][3][4][7]}$ The input power to the motor is calculated from input voltages and currents, and the output power is estimated from rotor speed and shaft torque. Air-gap torque is calculated from the integral of currents and voltages subtracting the stator IR drop. Shaft torque is estimated by subtracting the torque losses associated with the mechanical loss $\left(W_{f w}\right)$ and stray load loss $\left(W_{L L r}\right)$ from the calculated air-gap torque.

The AGT Method requires the following data to be measured: line voltages, currents, rotor speed, and stator resistance. In order to estimate $W_{c o r e}, W_{f w}$ and $W_{L L r}$, no-load test must be run. The requirement of speed, stator resistance measurements, and the no-load test are the main drawback of this method, which makes it an "intrusive” method.

In order to make it a "non-intrusive” method and still keep satisfied accuracy, we present a Modified Air-gap Torque (MAGT) Method by making the following modifications on the AGT Method:

1) The rotor shaft speed no longer needs to be measured. It is estimated at very high accuracy by applying input current spectrum estimation techniques. ${ }^{[9][10]}$

2) The stator resistance is estimated online using the input voltages and currents, instead of direct measurement from unpowered testing or rough approximation from the nameplate data. ${ }^{[11][12]}$

3) No-load test can be avoided, since it is prohibited for in-service motor operation, especially for the applications where both energy usage evaluation and condition monitoring are considered. Instead, $W_{\text {core }}, W_{f w}$ and $W_{L L r}$ are obtained by three options at different levels of accuracy/intrusiveness: 
- If the motor being tested is to be newly installed or has a regular scheduled maintenance, the no-load test could be done during the operation downtime.

- If the nameplate information of the motor is available, the no-load losses are estimated from the nameplate data.

- If neither of the above is available, the no-load losses are estimated from empirical values, like in the OHME Method ${ }^{[18]}$, the combined no-load losses are assumed to be $3.5 \%$ of input rated power and stray load loss is estimated from Table 2 of IEEE Standard 112-1996 ${ }^{[13]}$.

The brief procedure of this MAGT Method is as follows:

The air-gap torque is calculated from the cross product of air-gap flux and input current in d-q system, as shown in Equation 1.

$$
T_{\text {air-gap }}=\frac{3}{2} \cdot \frac{\text { poles }}{2}\left|\lambda_{q d s} \times i_{q d s}\right|
$$

Equation 1

If the motor line-to-neutral voltages are not available, or the negative sequence is of interest, the airgap torque can be calculated using Equation 2 instead.

$T_{\text {air }- \text { gap }}=\frac{\text { poles }}{2 \sqrt{3}}\left\{\left(i_{A}-i_{B}\right) \cdot \int\left[v_{C A}-R_{S}\left(i_{C}-i_{A}\right)\right] d t-\left(i_{C}-i_{A}\right) \cdot \int\left[v_{A B}-R_{S}\left(i_{A}-i_{B}\right)\right] d t\right\} \quad$ Equation 2

Where, the d-q air-gap flux is the integral of input voltage subtracting the stator IR drop, as shown in Equation 3.

$$
\lambda_{q d s}=\int\left(v_{q d s}-i_{q d s} \cdot R_{S}\right) d t
$$

Equation 3

Finally, the motor efficiency is obtained as Equation 4.

$$
\eta=\frac{T_{\text {air-gap }} \cdot \omega_{\text {rotor }}-W_{f w}-W_{L L r}}{P_{\text {input }}} \times 100 \% \quad \text { Equation } 4
$$

Where, $R_{S}, \omega_{\text {rotor }}, W_{f w}$, and $W_{L L r}$ are estimated as previously discussed. 
The advantages of this MAGT Method are:

1) Air-gap torque can be measured while the motor is running. (In-service testing)

2) It is a "non-intrusive" motor efficiency estimation method.

3) This method can be used for non-induction motors such as the adjustable speed, brushless dc motors.

The non-intrusiveness is obtained by sacrificing the accuracy, since the estimate of speed, stator resistance, and no-load data produces additional errors. Based on the physical nature, the accuracy is anticipated to be around 2\%, which is better than OHME Method (2-3\%) and worse than AGT Method (0.4-0.5\%). ${ }^{[4]}$ 


\section{CONCLUSIONS AND SUGGESTIONS}

\section{Conclusions}

The previous sections introduced over 18 available motor efficiency evaluation methods. These methods are categorized into the following 9 categories:

1. Nameplate methods

2. Slip methods

3. Current methods

4. Equivalent circuit methods

5. Segregated loss methods

6. Torque methods

7. Empirical methods

8. Statistical methods

9. Dedicated instrument methods

The first 6 categories are of our main interest in WSN project. The intrusiveness and corresponding accuracy of these 6 categories of methods are compared in Figure $4 .{ }^{[2]}$ Clearly, the more intrusive the method is, the more accurate estimation it can obtain.

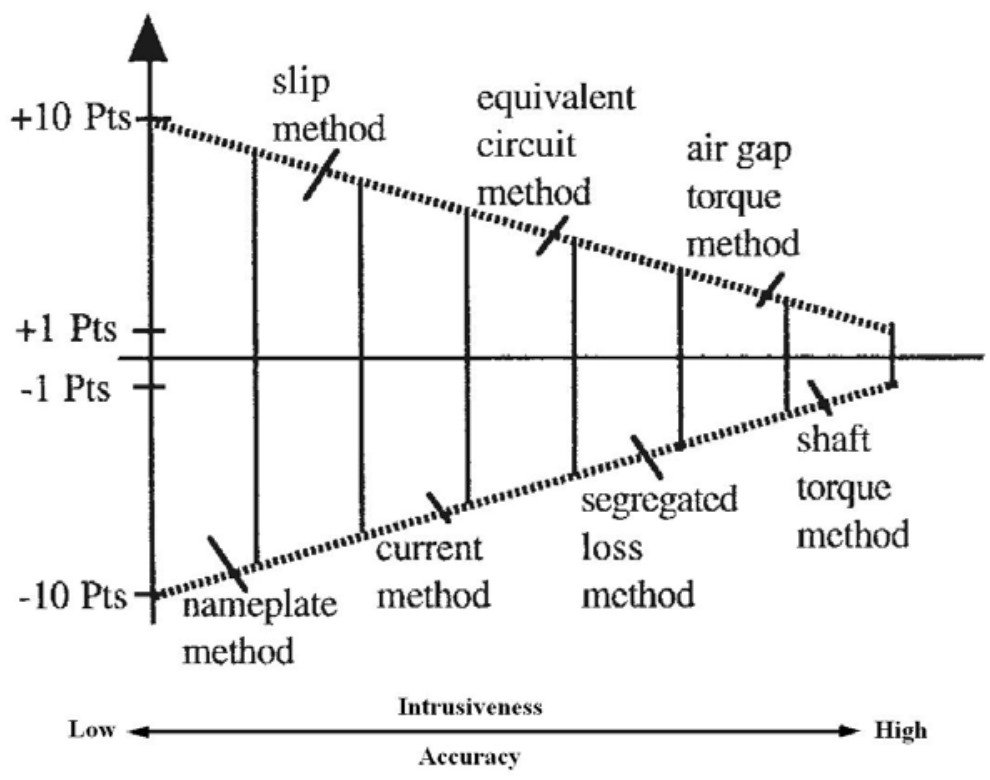

Figure 4. Anticipated Efficiency Estimation Errors versus Evaluation Methods. 
The details of these 6 categories of methods including the proposed MAGT Method are summarized and compared in Table 2.

Table 2. Comparison of the Motor Efficiency Estimation Methods

\begin{tabular}{|c|c|c|c|c|c|c|c|c|c|c|}
\hline \multirow{2}{*}{$\begin{array}{l}\text { Motor Efficiency } \\
\text { Testing Methods }\end{array}$} & \multicolumn{8}{|c|}{ Tests and Measurements/Estimations Required? } & \multicolumn{2}{|c|}{ Performance } \\
\hline & $\begin{array}{l}\text { No } \\
\text { Load }\end{array}$ & $\begin{array}{l}\text { Full } \\
\text { Load }\end{array}$ & $\begin{array}{c}\text { Un- } \\
\text { power }\end{array}$ & $\begin{array}{l}\text { Variable } \\
\text { Volt/Freq }\end{array}$ & Speed & Torque & $\begin{array}{l}\text { Nameplate } \\
\text { Info }\end{array}$ & $\begin{array}{c}\text { Stator } \\
\text { Resistance }\end{array}$ & $\begin{array}{l}\text { Intru- } \\
\text { siveness }\end{array}$ & $\begin{array}{l}\text { Anticipated } \\
\text { Error }\end{array}$ \\
\hline Standard Nameplate Method & No & No & No & No & No & No & Yes & No & Low & $10 \%{ }^{[2]}$ \\
\hline V\&B Method I & Yes & No & Yes & No & Measure & No & No & Measure & High & N/A \\
\hline V\&B Method II & No & No & Yes & No & Measure & No & Yes & Measure & Medium & $2.5 \%{ }^{[7]}$ \\
\hline Standard Slip Method & No & No & Yes & No & Measure & No & Yes & Measure & Low & $7 \%{ }^{[2]}$ \\
\hline Upper Bound Slip Method & Yes & No & Yes & No & Measure & No & Yes & Measure & Medium & $8 \%{ }^{[7]}$ \\
\hline Standard Current Method & Yes & No & No & No & No & No & Yes & No & Medium & $6 \%[2]$ \\
\hline IEEE Std 112-1996 Method F & Yes & No & Yes & Yes & Measure & No & Yes & Measure & High & $5 \%{ }^{[2]}$ \\
\hline ORMEL96 Method & No & No & Optional & No & Measure & No & Yes & Optional & Low & $4 \%{ }^{[4]}$ \\
\hline Locked Rotor Method & Yes & No & No & Yes & Measure & No & Yes & No & High & N/A \\
\hline Standstill Freq. Resp. Method & Yes & No & Yes & Yes & Measure & No & No & Measure & High & N/A \\
\hline IEEE Std 112-1996 Method E & Yes & Yes & Yes & Yes & Measure & No & Yes & Measure & High & $3 \%{ }^{[2]}$ \\
\hline OHME Method & No & No & No & No & Measure & No & Yes & Measure & Medium & $2-3 \%^{[4]}$ \\
\hline Air-gap Torque (AGT) Method & Yes & No & No & No & Measure & Estimate & No & Measure & High & $0.4-0.5 \%^{[4]}$ \\
\hline Shaft Torque Method & No & No & No & No & Measure & Measure & No & No & High & $<0.5 \%{ }^{[2]}$ \\
\hline Modified AGT (MAGT) Method & No & No & No & No & Estimate & No & Optional & Estimate & None & $2 \%$ \\
\hline
\end{tabular}

After overall evaluation on these efficiency estimation methods, we suggest/propose the following four methods as candidates for the WSN research project at different level of intrusion.

1. Non-intrusive method:

2. Low-intrusive method:

3. Medium-intrusive method:

4. High-intrusive method:
MAGT Method (Torque method)

ORMEL96 Method (Equivalent circuit method)

OHME Method (Segregated loss method)

AGT Method (Torque method) 
These four methods are compared in Table 3. The performance errors of the methods in the table are anticipated from their physical natures.

Table 3. Comparison of Four Suggested/Proposed Methods

\begin{tabular}{|c|c|c|c|c|c|c|c|c|c|c|}
\hline \multirow{2}{*}{$\begin{array}{l}\text { Motor Efficiency } \\
\text { Testing Methods }\end{array}$} & \multicolumn{8}{|c|}{ Tests and Measurements/Estimations Required } & \multicolumn{2}{|c|}{ Performance } \\
\hline & \begin{tabular}{|c|} 
No \\
Load \\
\end{tabular} & \begin{tabular}{|c|} 
Full \\
Load \\
\end{tabular} & \begin{tabular}{|c|}
$\begin{array}{c}\text { Un- } \\
\text { power }\end{array}$ \\
\end{tabular} & $\begin{array}{c}\text { Variable } \\
\text { Volt/Freq }\end{array}$ & Speed & Torque & \begin{tabular}{|c|}
$\begin{array}{c}\text { Nameplate } \\
\text { Info }\end{array}$ \\
\end{tabular} & $\begin{array}{c}\text { Stator } \\
\text { Resistance }\end{array}$ & \begin{tabular}{|c|} 
Intru- \\
siveness
\end{tabular} & \begin{tabular}{|c}
$\begin{array}{c}\text { Anticipated } \\
\text { Error }\end{array}$ \\
\end{tabular} \\
\hline Modified AGT (MAGT) Method & No & No & No & No & Estimate & No & Optional & Estimate & None & $2 \%$ \\
\hline ORMEL96 Method & No & No & Optional & No & Measure & No & Yes & Optional & Low & $4 \%{ }^{[4]}$ \\
\hline OHME Method & No & No & No & No & Measure & No & Yes & Measure & Medium & $2-3 \%{ }^{[4]}$ \\
\hline Air-gap Torque (AGT) Method & Yes & No & No & No & Measure & Estimate & No & Measure & High & $0.4-0.5 \%{ }^{[4]}$ \\
\hline
\end{tabular}

Reference [4] has done a complete testing using three out of the four methods (ORMEL96, OHME, AGT) with 12 motors ranging in size from 5hp to 100hp. Seven of the motors were older standard efficiency design (two had been rewound), the other five were energy efficient designs. The motors were tested with no load, and at 25\%, 50\%, 75\% and full load. Tests were conducted with two voltage conditions: phase balanced within $0.5 \%$ and unbalanced to $3 \%$. Table 4 shows the testing results using these three methods.

Table 4. Comparison of the Testing Results Using Suggested Methods

\begin{tabular}{|c|c|c|c|c|c|}
\hline Efficiency (\%) / Error (\%) & $\mathbf{7 . 5}$ hp 72\% Load & $\mathbf{1 0}$ hp 40\% Load & 5hp 81\% Load & 5hp 31\% Load & $\begin{array}{c}\text { Reported } \\
\text { Error in [4] }\end{array}$ \\
\hline Direct Measurement & 84.5 & 85.2 & 86.2 & 74.3 & $/$ \\
\hline ORMEL96 Method & $82.2 /-2.72$ & $84.9 /-0.35$ & $81.9 /-4.99$ & $67.0 /-9.83$ & $4.47 \%$ \\
\hline OHME Method & $83.6 /-1.07$ & $85.3 /+0.12$ & $85.8 /-0.46$ & $73.9 /-0.54$ & $\mathbf{0 . 5 3 \%}$ \\
\hline AGT Method & $85.0 /+0.59$ & $85.4 /+0.23$ & $86.8 /+0.69$ & $74.6 /-0.40$ & $\mathbf{0 . 4 8} \%$ \\
\hline
\end{tabular}

The average estimation error obtained for the ORMEL96 Method is around 4.5\%. It is also noted that the accuracy of the method improves as the load increases because the method does not use no-load test data. Study shows that if the motor load is above $50 \%$, the method accuracy is better than the average and reasonably accurate results are obtained. In cases where the load is less than $50 \%$ it is less important to know the actual efficiency. 
The average estimation error for the OHME Method, 0.53\%, is probably not representative of what is obtainable with less sophisticated data acquisition equipment in the field. However, an accuracy of $2 \%-3 \%$ is likely obtainable.

The AGT Method is proved to be very accurate yielding efficiency estimates that were within $0.5 \%$ of the actual values. The authors of [4] believed that the laboratory results are typical of what can be attained in the field. ${ }^{[4]}$

\section{Suggestions for WSN project}

MAGT, ORMEL96, OHME and AGT Methods are suggested/proposed as candidate methods for WSN project. When these methods are used in WSN research, the rotor speed is estimated from stator current harmonics instead of being measured directly using speed transducers.

ORMEL96 and OHME Methods don’t need future modifications if rotor speed and stator resistance can be correctly estimated.

As for the AGT Method, since it requires no-load test which is not accepted in WSN project, some modifications are required. These modifications actually convert the AGT method into MAGT method. These modifications include:

(1) If the motor been tested has a regular scheduled maintenance, the no-load test could be done during the operation downtime.

(2) If the nameplate information of the motor been tested is available, the no-load data could be estimated from the nameplate data.

(3) If none of the above is available, the no-load losses could be estimated from some empirical values, like what has been done in OHME Method. 


\section{REFERENCES}

[1] J. Kueck, J. Gray, R. Driver, and J. Hsu, “Assessment of available methods for evaluating in-service motor efficiency,” Oak Ridge National Laboratory report, ORNL/TM-13237, 1996.

[2] J. Hsu, J. Kueck, M. Olszewski, D. Casada, P. Otaduy, and L. Tolbert, "Comparison of induction motor field efficiency evaluation methods," IEEE Trans. Industrial Applications, vol. 34, no. 1, Jan./Feb. 1998.

[3] J. Hsu and B. P. Scoggins, "Field test of motor efficiency and load changes through airgap torque,” IEEE Trans. Energy Conversion, vol. 10, no. 3, Sept. 1995.

[4] J. D. Kueck, M. Olszewski, D. A. Casada, J. Hsu, P. J. Otaduy, and L. M. Tolbert, “Assessment of methods for estimating motor efficiency and load under field conditions," Oak Ridge National Laboratory report, ORNL/ TM-13165, 1996.

[5] J. G. Douglass, "Efficacy of methods for estimating in-service motor efficiency," Washington State University Co-Operative Extension Energy Program report prepared for the Pacific Gas and Electric Company and the Bonneville Power Administration, June 1997.

[6] J. G. Douglass, “In-service motor testing," Washington State University Co-Operative Extension Energy Program report\#99-040, Nov. 1999.

[7] A. Wallance, A. Von Jouanne, E. Wiedenbrug, E. Matheson, and J. Douglass, “A laboratory assessment of in-service and non-intrusive motor efficiency testing methods," Electric Power Components and Systems, vol. 29, pp. 517-529, 2001.

[8] K. D. Hurst and T. G. Habetler, "Sensorless speed measurement using current harmonic spectral estimation in induction machine drives,” IEEE Trans. Power Electronics, vol. 11, no. 1, Jan. 1996. 
[9] K. D. Hurst and T. G. Habetler, “A comparison of spectrum estimation techniques for sensorless speed detection in induction machines," IEEE Trans. Industrial Applications, vol. 33, no. 4, July/August 1997.

[10] P. L. Jansen and R. D. Lorenz, "Transducerless position and velocity estimation in induction and salient ac machines,” IEEE Trans. Industrial Applications, vol. 31, no. 2, March/April 1995.

[11] T. G. Habetler, F. Profumo, G. Griva, M. Pastorelli, and A. Bettini, "Stator resistance tuning in a stator-flux field-oriented drive using an instantaneous hybrid flux estimator," IEEE Trans. Power Electronics, vol. 13, no. 1, pp. 125-133, Jan. 1998.

[12] S. B. Lee, "Sensorless stator winding temperature estimation for induction machines," Ph.D. Thesis, Georgia Institute of Technology, May 2001.

[13] “Test Procedure for Polyphase Induction Motors and Generators,” IEEE Standard 1121996, 1996.

[14] NEMA - MG 1, 2003.

[15] W. Benning, "Method of determining the efficiency of asynchronous motors and apparatus for carrying out the method," United States Patent 5,659,232, 1997.

[16] J. D. Kueck, "Development of a method for estimating motor efficiency and analyzing motor condition,” IEEE Pulp and Paper Industry Technical Conference, pp. 67-72, 1998.

[17] J. R. Holmquist, J. A. Rooks, and M. E. Richter, "Practical approach for determining motor efficiency in the field using calculated and measured values," IEEE Trans. Industrial Applications, vol. 40, no. 1, pp. 242-248, Jan./Feb. 2004.

[18] “In-plant electric motor loading and efficiency techniques,” Ontario Hydro.

[19] R. L. Nailen, “Can field tests prove motor efficiency?” IEEE Trans. Industrial Applications, vol. 25, no. 3, May/June 1989.

[20] R. L. Nailen, “Finding true power output isn’t easy,” Electrical Apparatus, Feb. 1994. 
[21] A. K. Wallace and E. J. Wiedenbrug, "Motor efficiency determination: from testing laboratory to plant installation,” IEEE Pulp and Paper Conference, 1999.

[22] A. K. Wallace and T. E. Rollman, "High efficiency testing laboratory for motors, drives, and generators,” in Proc. EVD96 Conf., pp. 220-225, 1996.

[23] A. K. Wallace, J. A. Rooks, and J. R. Holmquist, "Comparison testing of IEEE standards 841 motors,” in Proc. IEEE 2000 Pulp and Paper Industry Tech. Conf., 2001, pp. 10-15.

[24] A. Nagornyy, A. K. Wallace and A. Von Jouanne, "Stray load loss efficiency connections,” IEEE Industry Applications Magazine, May/June 2004.

[25] A. Dell'Aquila, “A new test method for determination of induction motor efficiency,” Technical Papers from the IEEE Power Engineering Society Winter Meeting, 1984.

[26] “Derivation of induction motor models from standstill frequency response tests," Report GS-6250, Electric Power Research Institute, July 1991.

[27] “Determining electric motor load and efficiency,” Fact Sheet of Motor Challenge, DOE.

[28] “Replacing an oversized and under-loaded electric motor," Fact Sheet of Motor Challenge, DOE.

[29] Motor Systems Resource Facility, “Non-intrusive motor efficiency estimators,” Northwest Energy Efficiency Alliance Report, NEEA Report \#04-126, March 2004.

[30] J. O. Ojo, V. Ostovic, T. A. Lipo, and J. C. White, "Measurement and computation of starting torque pulsations of salient pole synchronous motors," IEEE Trans. Energy Conversion, vol. 5, issue 1, pp. 176-182, March 1990.

[31] B. Lu, T. G. Habetler, and R. G. Harley, “An investigation on commercially available energy usage estimation products,” EATON WSN Research Report, Sept. 2004. 


\title{
Incipient Bearing Fault Detection via Motor Stator Current Noise Cancellation using Wiener Filter
}

\author{
Wei Zhou, Member, IEEE, Bin Lu, Member, IEEE, \\ Thomas G. Habetler, Fellow, IEEE, and Ronald G. Harley, Fellow, IEEE
}

\begin{abstract}
Current-based monitoring can offer significant economic savings and implementation advantages over traditional vibration monitoring for bearing fault detection. The key issue in current-based bearing fault detection is to extract bearing fault signature from the motor stator current. Since bearing fault signature in the stator current is typically very subtle, especially when the fault is at an incipient stage, it is difficult to detect the fault signature directly. Therefore, in this paper the bearing fault signature is detected alternatively by estimating and removing non-bearing fault components via a noise cancellation method. In this method all the components of the stator current that are not related to bearing faults are regarded as noise and are estimated by a Wiener filter. Then all these noise components are cancelled out by their estimates in a real time fashion and a fault indicator is established based on the remaining components which are mainly caused by bearing faults. Machine parameters, bearing dimensions, nameplate values, and the stator current spectrum distribution are not required in the method. The results of on-line experiments with a 20-horsepower induction motor under multiple load levels have confirmed the effectiveness of this method.

Index terms - bearings (mechanical), sensorless condition monitoring, fault diagnosis, motor current signature analysis (MCSA), vibration, noise cancellation, Wiener filter
\end{abstract}

\section{INTRODUCTION}

$\mathrm{B}$ EARING faults account for over $40 \%$ of all induction machine faults [1], and their detection is highly desired to prevent damages of industrial processes. Conventional bearing detection techniques are sensor-based techniques. The most common sensors used are vibration sensors such as accelerometers. Since vibration sensors are expensive and their installations are only justified to large electric machines and/or very important processes, there is a need to develop inexpensive, better if non-intrusive, monitoring techniques. Current-based (sensorless) monitoring techniques in fact meet the need, since they usually do not require additional sensors above those already used for metering, controls, and electrical protection [2]-[5]. Conse-

This work was financially supported in part by the US Department of Energy and Eaton Corporation under Grant DE-FC36-04GO14000. The views presented in this article are not necessarily attributable to any of those organizations mentioned in the article. The work presented in this article has been filed as a patent at USPTO on June $3^{\text {rd }}, 2008$.

Wei Zhou is with Southern California Edison, Rosemead, CA 91770 USA. (email: weizhou@ece.gatech.edu).

Bin $\mathrm{Lu}$ is with Eaton Corporation - Innovation Center, 4201 North 27th Street, Milwaukee, WI, 53216, U.S.A. (e-mail: binlu@ieee.org).

Thomas G. Habetler and Ronald G. Harley are with the School of Electrical and Computer Engineering, Georgia Institute of Technology, Atlanta, GA, 30332, U.S.A. (e-mail: \{ thabetler, rharley\} @ece.gatech.edu). quently, current-based monitoring techniques have great economic benefits and potential to be adopted by industry.

The challenge, however, in current-based bearing fault detection is to extract fault signature from the motor stator current. For different types of bearing faults, fault signatures can be in different forms. According to different stages of the fault development process, bearing faults can be categorized as: 1) single-point defects, which typically occur at the very late stage of the bearing life or due to severe system failures, and 2) generalized roughness, which occurs when the bearing starts to degrade while it is still operable [6]. Single-point defects can be created off-line, for example, by drilling holes in the bearing components such as the inner raceway, the outer raceway, the balls, and the cage of a typical rolling element bearing. As a result, the faults may be detected by identifying the corresponding characteristic fault frequencies in the stator current [7]. In fact there has been many research works focusing on the detection of single-point bearing faults [8]-[15]. In contrast, many bearing faults developed through months or years on-line in industrial processes are generalized-roughness bearing faults, especially at an early stage [6]. Such faults can also be generated in a shorter time period, but only by using some special experiment setups (an example can be found in Section IV of this paper). Generalized-roughness faults exhibit degraded bearing surfaces, but not necessarily distinguished defects. As a result, the characteristic fault frequencies do not necessarily exist in the stator current [6], [12], [16].

Most sensorless bearing fault detection techniques such as [7]-[15] in the literature rely on locating and processing the characteristic bearing fault frequencies in stator current and, therefore, may not be suitable for detecting generalized-roughness faults. Though the mean spectral deviation (MSD) method in [17] is designed to detect generalized roughness faults, it has several disadvantages. First, it requires thorough knowledge of stator current spectrum distribution, to design a bank of filters to notch non-bearing fault components. In this method, non-bearing fault components include supply harmonics, load harmonics, eccentricity harmonics, slot harmonics, and broken bar harmonics. Second, it assumes that the components at the notch frequencies do not carry fault information, which may not be true in practice. For example, it has been shown in [16] that contamination in bearings can result in an increase in the magnitude of eccentricity harmonics. Third, machine speed has to be measured or estimated. Some machine parameters are also required in this method. 
In contrast to the existing sensorless techniques, the method proposed in this paper aims to detect the generalized-roughness bearing faults, while it does not require thorough knowledge of stator current spectrum distribution as well as the frequencies of non-bearing fault components. Machine parameters, bearing dimensions, and nameplate values are not required in this method either.

The method proposed in this paper is based on the concept of noise cancellation. First all the components not related to bearing faults are regarded as noise and are estimated by a Wiener filter. Then all these noise components are cancelled out by their estimate in a real-time fashion and a fault indicator is established based on the remaining components which are directly related to the bearing failure.

The concept, implementation and performance of the proposed method are discussed in Section II. A few important practical implementation considerations are discussed in Section III. Experimental results are presented in Section IV and conclusions in Section V.

\section{StATOR CURRENT NoISE CANCELlation}

\section{1 Concept and Model}

The dominant components in the stator current of a typical induction motor are the supply fundamental and its multiple harmonics, the eccentricity harmonics, the slot harmonics, the saturation harmonics, and other components from unknown sources including environmental noise [18]. Since these dominant components exist before and after the presence of a bearing fault, a large body of the information they carry is not related to the bearing fault. In this sense, they are basically noise to the bearing fault detection problem. To discover the fault signature, these noise components are desired to be removed. (Note: the noise here and hereafter refer to the components in the stator current that are not related to bearing faults, unless where stated otherwise.)

Since at a given motor operating point, these dominant noise components (in the form of sinusoidal) essentially do not change, either in magnitude or in frequency, they can be reliably predicted by a predictor using the most recent samples of the stator current. Canceling the real time stator current by the prediction of these noise components yields the remaining components of the stator current that mainly contains bearing fault signatures. The noise cancellation process is modeled as in Fig. 1.

Conceptually, the predictor in the model can be in any form; for example, it can be a neural network, or a digital filter. In this research, Wiener filter is chosen and the system performance is analyzed from the signal processing viewpoint.

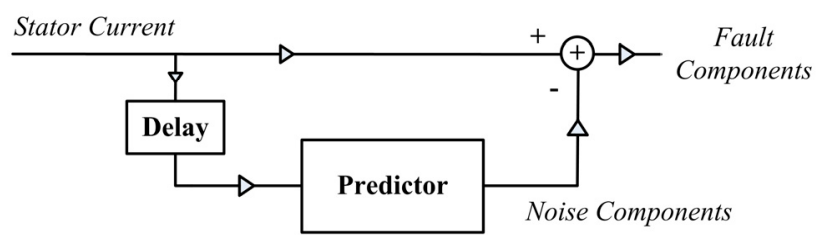

Fig. 1. Noise cancellation model for bearing fault detection.

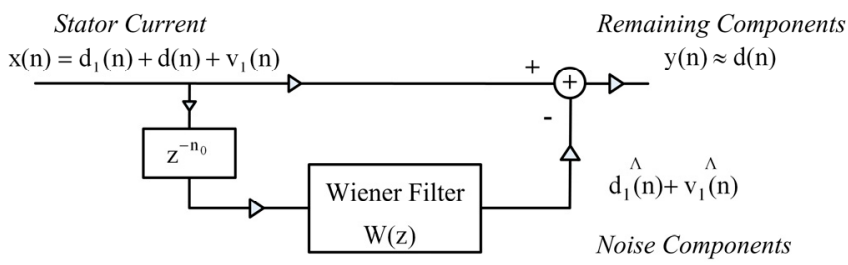

Fig. 2. Noise cancellation model with a Wiener filter as predictor.

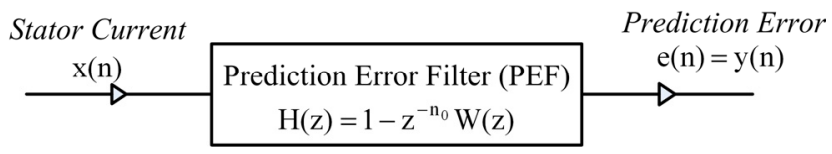

Fig. 3. Interpretation of the noise cancellation method in view of prediction error filtering.

This paper follows the standard definition of a "discrete-time signal," $x(n)$, which arises as result of sampling a continuous-time signal $x(t)$ with an analog-to-digital (A/D) converter. In this work, the continuous-time signal $x(t)$ can be chosen as any one of the three-phase stator currents of a motor. To achieve a better performance with a higher signal-to-noise ratio, it is preferred to use the stator "notch current" with fundamental frequency component filtered out using a hardware notch filter, as in Section IV. The signal $x(t)$ is sampled at a rate of $f_{s}=1 / T_{s}$ samples per second, then the sampled signal $x(n)$ is related to $x(t)$ as

$$
\mathrm{x}(\mathrm{n})=\mathrm{x}\left(\mathrm{nT}_{\mathrm{s}}\right)
$$

When the predictor in Fig. 1 is a Wiener filter, the model can be redrawn in Fig. 2, where

$\mathrm{x}(\mathrm{n})$ is the motor stator current (i.e., original phase current or notch current);

$\mathrm{d}_{1}(\mathrm{n})$ is the noise components;

$\mathrm{d}(\mathrm{n})$ is the bearing fault components;

$\mathrm{v}_{1}(\mathrm{n})$ is the measurement noise;

$y(n)$ is the remaining components in the stator current after noise cancellation;

$\mathrm{d}_{1}(\mathrm{n})$ is the estimated noise components;

$\mathrm{v}_{1}(\mathrm{n})$ is the estimated measurement noise;

$\mathrm{z}^{-\mathrm{n}_{0}}$ is the delay of $\mathrm{n}_{0}$ data samples; and

$\mathrm{W}(\mathrm{z})=\sum_{\mathrm{j}=0}^{\mathrm{p}} \mathrm{w}(\mathrm{j}) \mathrm{z}^{-\mathrm{n}_{0}}$ is a Wiener filter with a order of $\mathrm{p}$. 
From the model, one can see that if the Wiener filter $\mathrm{W}(\mathrm{z})$ has good performance, i.e., if $\hat{d_{1}}(\mathrm{n})+\mathrm{v}_{1}(\mathrm{n}) \approx \mathrm{d}_{1}(\mathrm{n})+\mathrm{v}_{1}(\mathrm{n})$, then the remaining components in the stator current after noise cancellation consist of the fault components $d(n)$ that are induced by the bearing fault. Usually, the measurement noise $\mathrm{v}_{1}(\mathrm{n})$ is negligible, given today's advanced data acquisition technology.

Another interpretation of the noise cancellation method is that the remaining components in the model in Fig. 2 can be viewed as the prediction error of the Wiener filter [19]. As the bearing fault develops and the condition for the prediction Wiener filter gets worse, the prediction error increases. This interpretation is illustrated in an equivalent model shown in Fig. 3.

\section{2 Wiener Filter Design}

The Wiener filter in Fig. 2 should be designed such that it predicts only the noise components. This can be achieved by designing the Wiener filter using the stator current data associated with healthy bearing conditions. By doing so, no bearing fault information is embedded into the coefficients of the filter, since all the components in the stator current at healthy bearing conditions are noise. Therefore, as bearing faults develop, the Wiener filter predicts only the noise components. Correspondingly, due to the degraded predicting ability of the filter in the presence of a bearing fault, the prediction error in Fig. 3 increases.

The Wiener filter design system is shown in Fig. 4, where the stator current $\mathrm{x}(\mathrm{n})$ doesn't contain the fault components $d(n)$ in the system since it is at healthy bearing conditions. In practice, it usually takes long time (typically months or years depending on the applications) for a bearing to start to fail, it is well justified to assume such bearing conditions are accessible. Even for bearings that already exhibit early deterioration, the proposed method would still apply, since the additional bearing fault signatures after the filter is determined can still be detected by the Wiener filter

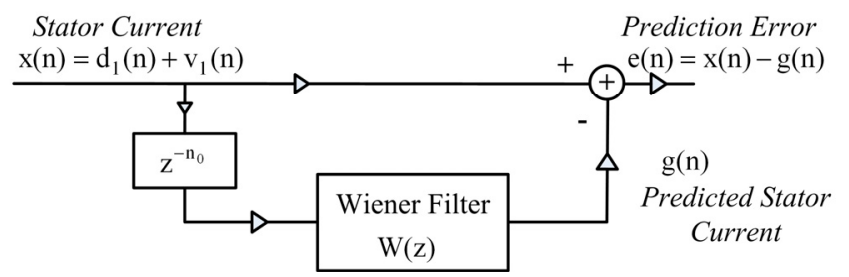

Fig. 4. Weiner filter design system corresponding to healthy bearing conditions.

The objective of the Weiner filter design is to find the filter coefficients, $\mathrm{w}(\mathrm{k}), \mathrm{k}=0,1, \ldots, \mathrm{p}$, that minimize the prediction error $\xi$ in the mean-square sense [19]
$\xi=E\left\{\left.e(n)\right|^{2}\right\}=E\left\{\left|x(n)-\sum_{k=0}^{p} w(k) x\left(n-n_{0}-k\right)\right|^{2}\right\}$

where $\mathrm{E}\{\cdot\}$ stands for expectation.

To minimize $\xi$, it is necessary and sufficient that the first-order derivative of $\xi$ with respect to $\mathrm{w}(\mathrm{k})$ be zero for $\mathrm{k}$ $=0,1, \ldots, \mathrm{p}$,

$$
\frac{\partial \xi}{\partial \mathrm{w}(\mathrm{k})}=\frac{\partial}{\partial \mathrm{w}(\mathrm{k})} \mathrm{E}\left\{\left.\mathrm{e}(\mathrm{n})\right|^{2}\right\}=\mathrm{E}\left\{2 \mathrm{e}(\mathrm{n}) \frac{\partial \mathrm{e}(\mathrm{n})}{\partial \mathrm{w}(\mathrm{k})}\right\}=0 .
$$

Since

$$
e(n)=x(n)-\sum_{j=0}^{p} w(j) x\left(n-n_{0}-j\right)
$$

the first-order derivative of e(n) with respect to $\mathrm{w}(\mathrm{k})$ is

$$
\frac{\partial \mathrm{e}(\mathrm{n})}{\partial \mathrm{w}(\mathrm{k})}=-\mathrm{x}\left(\mathrm{n}-\mathrm{n}_{0}-\mathrm{k}\right) \text {. }
$$

Substituting (5) into (3), it yields

$$
\mathrm{E}\left\{\mathrm{e}(\mathrm{n}) \mathrm{x}\left(\mathrm{n}-\mathrm{n}_{0}-\mathrm{k}\right)\right\}=0, \quad k=0,1, \ldots, p,
$$

or equivalently,

$$
E\left\{\left[x(n)-\sum_{j=0}^{p} w(j) x\left(n-n_{0}-j\right)\right] x\left(n-n_{0}-k\right)\right\}=0,
$$

Assuming signal $\mathrm{x}(\mathrm{n})$ is wide-sense stationary (WSS), then the autocorrelation sequences of $\mathrm{x}(\mathrm{n})$ is

$$
\mathrm{E}\{\mathrm{x}(\mathrm{n}-\mathrm{j}) \mathrm{x}(\mathrm{n}-\mathrm{k})\}=\mathrm{r}_{\mathrm{x}}(k-j),
$$

and (7) can be simplified as

$$
\sum_{\mathrm{j}=0}^{\mathrm{p}} \mathrm{w}(\mathrm{j}) \mathrm{r}_{\mathrm{x}}(\mathrm{k}-\mathrm{j})=\mathrm{r}_{\mathrm{x}}\left(\mathrm{n}_{0}+\mathrm{k}\right), \quad k=0,1, \ldots, p
$$

In a matrix form, (9) can be written as

$$
\left[\begin{array}{cccc}
r_{x}(0) & r_{x}(1) & \cdots & r_{x}(p) \\
r_{x}(1) & r_{x}(0) & \cdots & r_{x}(p-1) \\
\vdots & \vdots & & \vdots \\
r_{x}(p) & r_{x}(p-1) & \cdots & r_{x}(0)
\end{array}\right]\left[\begin{array}{c}
w(0) \\
w(1) \\
\vdots \\
w(p)
\end{array}\right]=\left[\begin{array}{c}
r_{x}\left(n_{0}\right) \\
r_{x}\left(n_{0}+1\right) \\
\vdots \\
r_{x}\left(n_{0}+p\right)
\end{array}\right],
$$

or denoted by

$$
\mathrm{R}_{\mathrm{x}} \underline{\mathrm{w}}=\underline{\mathrm{r}} .
$$

In practice, the autocorrelation sequences are replaced by the ensemble averages to implement the method. The matrix $R_{x}$ is a symmetric Toeplitz matrix, and can be 
solved efficiently by the Levinson-Durbin Recursion algorithm [19].

\section{II.3 System Performance}

The performance of the system is examined in this subsection. Specifically, the effects of the presence of the fault components $d(n)$ on the prediction error are evaluated. First a general equation describing the prediction error will be given, and then analyses based on the equation will be performed for healthy bearing conditions and bearing fault conditions, respectively.

The general equation for the mean square prediction error of the system is defined in (2). Upon expansion, (2) can be rewritten as

$$
\begin{aligned}
\xi= & {\left[r_{x}(0)-\sum_{k=0}^{p} w(k) r_{x}\left(n_{0}+k\right)\right] } \\
& +\sum_{k=0}^{p} w(k)\left[\sum_{j=0}^{p} w(j) r_{x}(k-j)-r_{x}\left(n_{0}+k\right)\right]
\end{aligned} .
$$

For healthy bearing conditions, since $\mathrm{w}(\mathrm{k}), \mathrm{k}=0,1, \ldots, \mathrm{p}$, are solution to (11), the second term on the right hand side (RHS) of (12) is zero. Therefore, the prediction error at healthy bearing conditions is

$$
\xi_{\min }=E\left\{|e(n)|^{2}\right\}=r_{x}(0)-\sum_{k=0}^{p} w(k) r_{x}\left(n_{0}+k\right) .
$$

For healthy bearing conditions, from $x(n)=d_{1}(n)+v_{1}(n)$, the following relations can be derived by assuming that the measurement noise is broadband noise and that it is uncorrelated with any other component in the stator current, i.e.,

$$
\begin{gathered}
\mathrm{r}_{\mathrm{x}}(0)=\mathrm{r}_{\mathrm{d}_{1}}(0)+\mathrm{r}_{\mathrm{v}_{1}}(0), \text { and } \\
\mathrm{r}_{\mathrm{x}}(\mathrm{k})=\mathrm{r}_{\mathrm{d}_{1}}(\mathrm{k}), \quad \mathrm{k} \neq 0 .
\end{gathered}
$$
let

To further investigate the performance of the system,

$$
d_{1}(n)=\sum_{m=1}^{M} A_{m} \sin \left(\omega_{m} n+\varphi_{m}\right)
$$

where $\mathrm{A}_{\mathrm{m}}, \omega_{\mathrm{m}}, \varphi_{\mathrm{m}}, \mathrm{m}=1, \ldots, \mathrm{M}$, are the amplitudes, the frequencies and the angles of $\mathrm{M}$ noise components in the stator current.

Since

$$
r_{d_{1}}(k)=\sum_{m=1}^{M} \frac{A_{m}^{2}}{2} \cos \left(\omega_{m} k\right)
$$

(13) can be rewritten as

$$
\xi_{\min }=\sum_{\mathrm{m}=1}^{\mathrm{M}} \frac{\mathrm{A}_{\mathrm{m}}^{2}}{2}\left\{1-\sum_{\mathrm{k}=0}^{\mathrm{p}} \mathrm{w}(\mathrm{k}) \cos \left[\omega_{\mathrm{m}}\left(\mathrm{n}_{0}+\mathrm{k}\right)\right]\right\}+\mathrm{r}_{\mathrm{v}_{\mathrm{l}}}(0) \text {. }
$$

Similarly, for bearing fault conditions, let

$$
d(n)=\sum_{\mathrm{q}=1}^{\mathrm{Q}} \mathrm{B}_{\mathrm{q}} \sin \left(\omega_{\mathrm{q}} \mathrm{n}+\varphi_{\mathrm{q}}\right)
$$

where $\mathrm{B}_{\mathrm{q}}, \omega_{\mathrm{q}}, \varphi_{\mathrm{q}}, \mathrm{q}=1, \ldots, \mathrm{Q}$, are the amplitudes, the frequencies and the angles of $\mathrm{Q}$ fault components in the stator current injected by the bearing fault. For bearing fault conditions, the mean square prediction error can still be calculated from the general equation (12). The second term on the RHS of (12) now is not zero due to the presence of the fault signal $d(n)$.

Similarly, for bearing fault conditions, from $x(n)=d_{1}(n)+d(n)+v_{1}(n)$, the following can be derived under the assumptions

$$
\begin{aligned}
& r_{x}(0)=r_{d_{1}}(0)+r_{d}(0)+r_{v_{1}}(0)+2 r_{d_{1} d}(0), \text { and } \\
& r_{x}(k)=r_{d_{1}}(k)+r_{d}(k)+2 r_{d_{1} d}(k), \quad k \neq 0 .
\end{aligned}
$$

For $\omega_{\mathrm{q}} \neq \omega_{\mathrm{m}}$, after some mathematic manipulations, the prediction error for bearing fault conditions is obtained as

$$
\begin{aligned}
& \xi=\xi_{\text {min }}+\sum_{\mathrm{q}=1}^{\mathrm{Q}} \frac{\mathrm{B}_{\mathrm{q}}^{2}}{2}\left\{1-\sum_{\mathrm{k}=0}^{\mathrm{p}} \mathrm{w}(\mathrm{k}) \cos \left[\omega_{\mathrm{q}}\left(\mathrm{n}_{0}+\mathrm{k}\right)\right]\right\} \\
& +\sum_{\mathrm{q}=1}^{\mathrm{Q}} \frac{\mathrm{B}_{\mathrm{q}}^{2}}{2}\left\{\sum_{\mathrm{k}=0}^{\mathrm{p}} \mathrm{w}(\mathrm{k})\left[\sum_{\mathrm{j}=0}^{\mathrm{p}} \mathrm{w}(\mathrm{j}) \cos \left(\omega_{\mathrm{q}}(\mathrm{k}-\mathrm{j})-\cos \left(\omega_{\mathrm{q}}\left(\mathrm{n}_{0}+\mathrm{k}\right)\right)\right]\right\}\right.
\end{aligned}
$$

where $\xi_{\min }$ is the prediction error corresponding to healthy bearing conditions.

Several observations can be made based on (20). They are listed as follows.

1) For healthy bearing conditions, all $\mathrm{B}_{\mathrm{q}}$ 's are zero, and (20) is reduced to (17), as expected.

2) The prediction error increases as the bearing fault develop; the degree of the increment is proportional to the power of the fault signal. For this reason, the fault index is chosen to be the root-mean-square (RMS) value of the noise-cancelled stator current.

3) For the generalized-roughness type of bearing faults, the frequencies of the fault signal, $\omega_{q}$ 's, are hard to locate, and may spread out. Also the magnitudes of the fault components, $\mathrm{B}_{\mathrm{q}}$ 's, are small. These two factors make it difficult to detect a specific fault component. However, the method considers the collective effect of 
the fault components after noise cancellation, and thus facilitates bearing fault detection.

4) If $\omega_{\mathrm{q}}=\omega_{\mathrm{m}}$, there will be a lesser increment in the prediction error, since the $3^{\text {rd }}$ term on RHS of (20) will be zero while the $2^{\text {nd }}$ term will be nonzero. This means, even if the fault frequencies overlap the noise components' frequencies (For example, when bearing faults augment the eccentricity of the motor), by (20) the index still increases as the bearing fault develop.

5) When bearing fault signature exhibits broadband behavior in the spectrum of the stator current, i.e., $d(n)$ is a broadband signal, then its effect on the prediction error is similar to that of $\mathrm{v}_{1}(\mathrm{n})$. From (17), it can be seen that the fault index still increases at this situation.

\section{IMPLEMENTATION CONSIDERATIONS}

\section{1 Fault Index and Threshold Selection}

As observed in Section II, the prediction error of the Wiener filter (i.e., the noise-cancelled stator current) increases as the bearing fault develops; the degree of the increment is proportional to the power of the bearing fault signal. Therefore, the fault index is chosen to be the rootmean-square (RMS) value of the noise-cancelled stator current. In practice, a fault index threshold of $15-25 \%$ of RMS value change above the baseline can be used to set the warning for maintenance action. To reduce the false positives caused by possible bad current data (e.g., utility power transients), it is also suggested to use a moving average of multiple RMS values as the fault index to minimize the impact of a single bad data point.

The overall increasing trend in the RMS value of the noise-cancelled stator current indicates an increasing trend of the machine vibration as the bearing deteriorates over time. This is particularly true during the bearing impending failure stage, where generalized roughness dominates the fault signatures. However, it has also been observed that the fault index variation tends to increase as bearing wears further. Therefore, it can be expected that a statistical approach that combines both the average of the RMS values and their variation of the noise-cancelled stator current would have better performance. A method based on statistical process control (SPC) has been investigated by the same authors in [20], where the fault index threshold is automatically determined by a SPC routine based on the noise-cancelled stator current samples.

Generally speaking, the strategy of selecting fault index threshold will have to vary in different applications based on the users' expectations of warning information in terms of timing and severity. It is important to point out that the main objective of such online bearing fault detection system is to give early indication of impending bearing faults to better schedule traditional maintenance activities instead of replacing them.

\section{2 Motor with Various Loading Conditions}

The bearing fault detection methods using Wiener filter have been developed to be applicable to motors that operate under fixed frequency and both constant- and variable-load conditions. This covers the line-connected motors and motors connected to relays, meters, and starters, where the motor current frequency equals to the utility frequency. Fig. 5 and Fig. 6 show the flow charts of the proposed methods under constant- and variable-load conditions, respectively.

The stator current data are sampled periodically, e.g., every 5-15 minutes depending on the application. Each data set contains 10-second of stator current data sampled at 4 $\mathrm{kHz}$ or above. A routine checks the data and ensures the motor operates at steady state and constant frequency. At the first run, the Wiener filter coefficients are determined as in Section II and the RMS value of the noise-cancelled stator current is used as a baseline for future fault detection. The details of the constant-load method and its experimental results are discussed in Section IV.

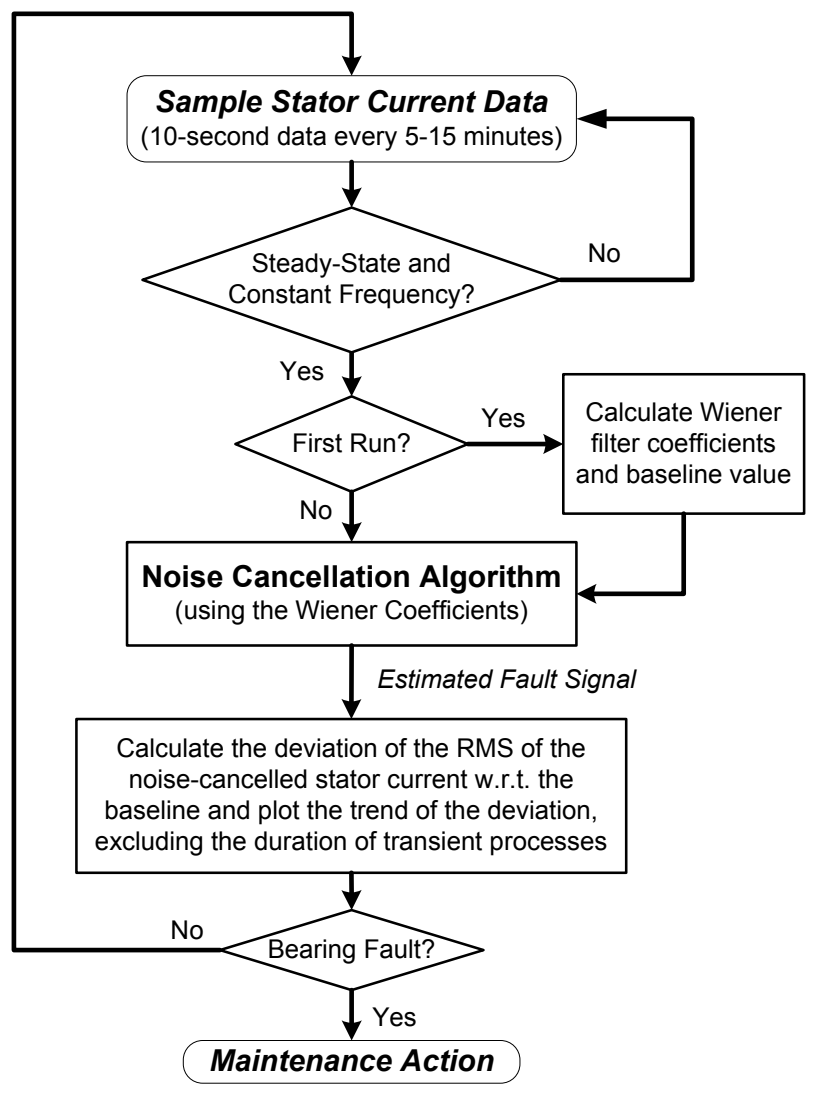

Fig. 5. Flow chart of bearing fault detection scheme under constant load. 


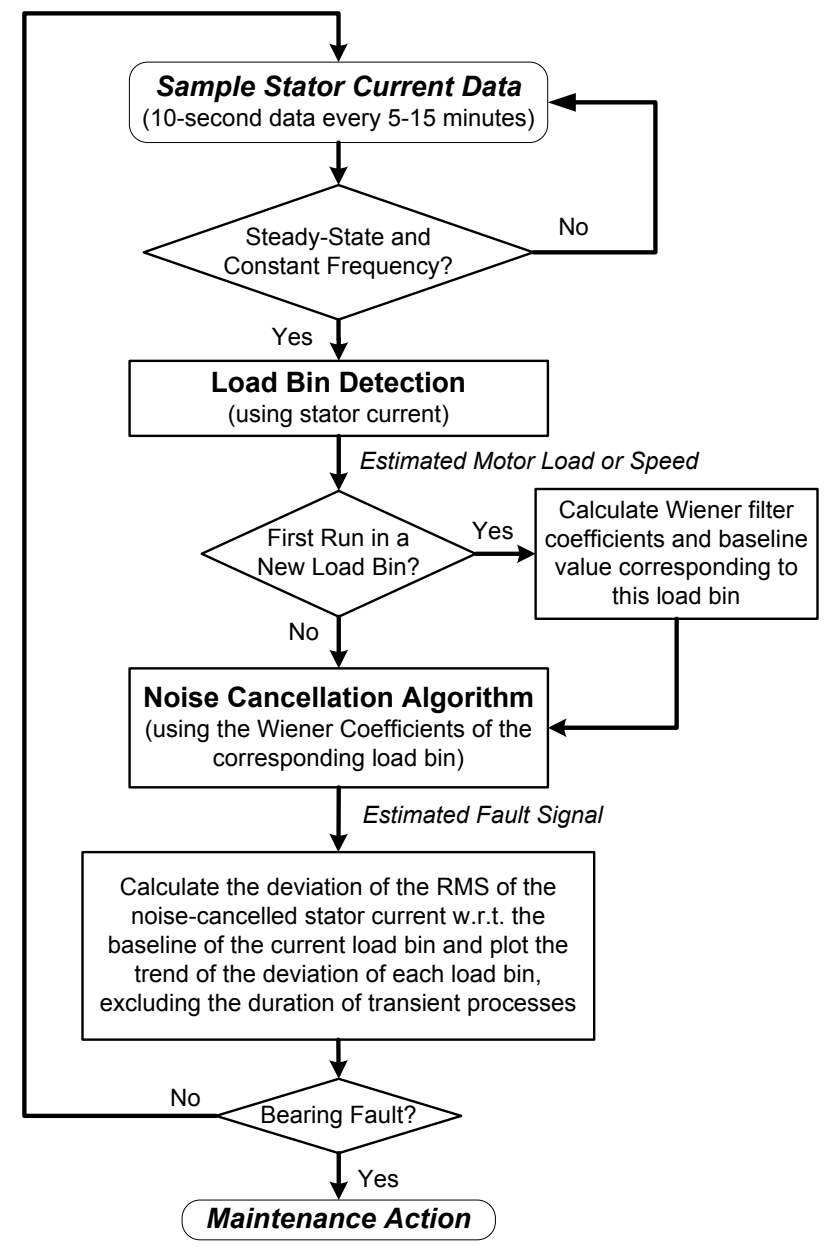

Fig. 6. Flow chart of bearing fault detection scheme under variable load.

The variable-load method inherits most logic from the constant-load method. The main difference in the variableload method is that the entire load range is divided into several load bins (e.g., 10\% for each load bin.) Each load bin has a dedicated Wiener filter with unique coefficients and a baseline value of noised cancelled current. The present load bin at a time can be determined either from the current percentage calculated as a ratio of the stator current and rated motor current, or from motor speed estimated from current harmonics as in [21] (in case the stator notch current is used.) Details of variable-load methods are out of the scope of this paper. They are available in [20].

For inverter-fed motors, the frequency of the stator current may vary when the load and/or speed/torque references change. Therefore, the proposed method cannot be directly applied. More research needs to be done in this area. A promising approach could be to apply adaptive filtering techniques to estimate stator current noises dynamically [19].

\section{EXPERIMENTAL SETUP AND RESUltS}

It has been demonstrated in [22] that the act of disassembling and reassembling a motor could change the spectrum of the stator current. Therefore, sensoreless bearing fault detection techniques should be evaluated by using the data that are acquired as bearing faults are developed online (in situ) to avoid unclearness on the sources (the act vs. a bearing fault) of the spectrum changes.

In this research, the experimental method developed in [22] is improved and then used to generate bearing faults on-line. The experimental setup used in this research is drawn in Fig. 7. This setup uses shaft current [17] which flows through the test bearing to accelerate the aging process of that bearing. To further shorten the time to fail the bearing, the bearing is soaked in a degreasing solution to remove all of the grease and then is repacked with about $5 \%$ volume of the original grease before it is installed in the motor. Prior to this degreasing process, the test bearing is brand new, enabling to track the entire bearing failure process. The experience from the authors with this setup is that bearings start to fail within several days with a proper level of the shaft current. This amount of time compares several months or years in the industry.

With this setup, a 20-horsepower, 230-V, three-phase, four-pole induction motor was used. The test bearings (front-end) are typed SKF 6309 2Z. The motor was fed directly from the mains $(230 \mathrm{~V}, 60 \mathrm{~Hz})$. The ac voltage source of $120 \mathrm{~V}$ (single phase, $60 \mathrm{~Hz}$ ) supplied the shaft current. The rear end bearing, Type $63072 \mathrm{Z}$, is insulated from the stator and the frame. A dynamometer connected to the shaft via an electrically insulated coupling, provided for the adjustable load. The accelerometer was mounted on the stator to measure the vibration $(\mathrm{mV} / \mathrm{G})$. In this setup, different from that used in [22], the shaft current circuit is isolated from both the motor circuit and the measurement circuit, to eliminate interferences between them.

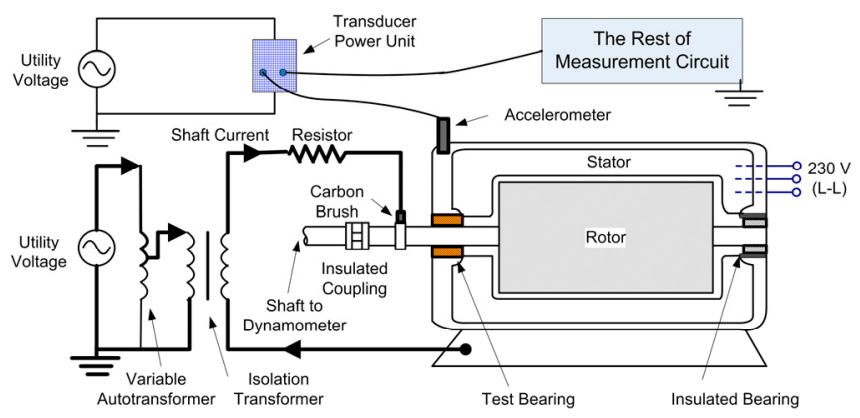

Fig. 7. Experiment setup for generating bearing faults on-line by using the shaft current method.

One phase stator current of the motor was measured and notch filtered at $60 \mathrm{~Hz}$ before it was sampled. The notch filter was employed to best utilize the dynamic range of the data acquisition system. The sampling frequency was $6 \mathrm{kHz}$ 
and 10 -second data was sampled every 15 minutes. The length of the Wiener filter was chose as 100 data samples and its input delay was one data sample (i.e., one cycle of $60 \mathrm{~Hz}$ of the most recent samples was used to predict the next sample). For each new test bearing, the motor ran continuously until an incipient bearing fault occurred. The incipient bearing faults were identified by an increase in the machine vibration and/or the emission of screeching sounds from the bearing.

The results from two experiments performed in this research are plotted in Fig. 8 and Fig. 9, respectively. In these figures, the top plot illustrates the RMS value of the noisecancelled stator current; the middle and the bottom plots illustrate the RMS value of the vibration acceleration and the vibration velocities, respectively. The RMS value of the noise-cancelled stator current was calculated every 15 minutes and was used as the fault index. For the vibration data, different quantities were calculated, including the RMS value of the vibration acceleration from 0 to $1 \mathrm{kHz}$, the RMS values of the vibration velocity from $10 \mathrm{~Hz}$ to $1 \mathrm{kHz}$ (the ISO 10816 metric) and from $1 \mathrm{kHz}$ to $3 \mathrm{kHz}$. The vibration accelerations were measured via the accelerometer as shown in Fig. 5. The vibration velocities were calculated from the vibration accelerations [23].

From Fig. 8, it can be seen that both the noise-cancelled stator current RMS and the vibration RMS increase as the fault develops. Specifically, according to the vibration acceleration from 0 to $1 \mathrm{kHz}$ and the vibration velocity from 1 $\mathrm{kHz}$ to $3 \mathrm{kHz}$, the incipient fault builds up during the time between the $30^{\text {th }}$ hour and the $55^{\text {th }}$ hour. During the same time period, the noise cancellation method responds well, by offering relatively large values compared to the baseline level. From the 60th hour to the end of the experiment, both the noise-cancelled current RMS and the vibration RMS remain at relatively high levels due to the degraded bearing condition. Also from Fig. 8, it can be seen that the ISO 10816 metric reflects the bearing health as expected.

In the top plot in Fig. 9, an abnormal peak at the second data point (i.e., at the end of the first half hour) is probably due to bad data, since all the points around it are still at a low level. Except this point, generally speaking, the noise cancellation curve (the top plot) loosely follows those vibration curves (the middle and the bottom plots). During the time between the $10^{\text {th }}$ hour to the $35^{\text {th }}$ hour, the incipient fault builds up as seen on the $1 \mathrm{k}-3 \mathrm{k} \mathrm{Hz}$ vibration velocity curve. Correspondingly, there is substantial increase in the noise-cancelled current. From the $40^{\text {th }}$ hour to the end of the experiment, the vibration (the acceleration $0-1 \mathrm{kHz}$ and the velocity $10-1 \mathrm{k} \mathrm{Hz}$ ) and the noise-cancelled current become unstable due to the deteriorated bearing condition. The machine emits screeching sounds after 40 hours' running; and the sound level increases until the experiment is stopped during the $50^{\text {th }}$ hour.

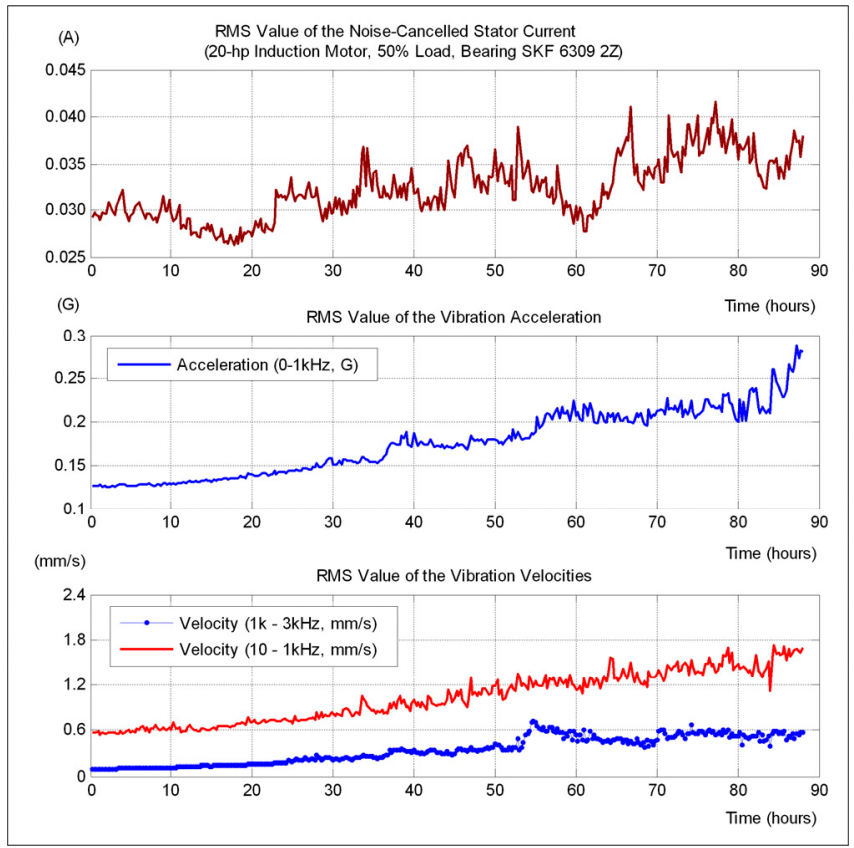

Fig. 8. Results from bearing type-6309 at a 50\% load level. (top) The RMS value of the noise-cancelled stator current increases as the fault develops. (middle and bottom) The RMS value of the vibration increases as the fault develops.

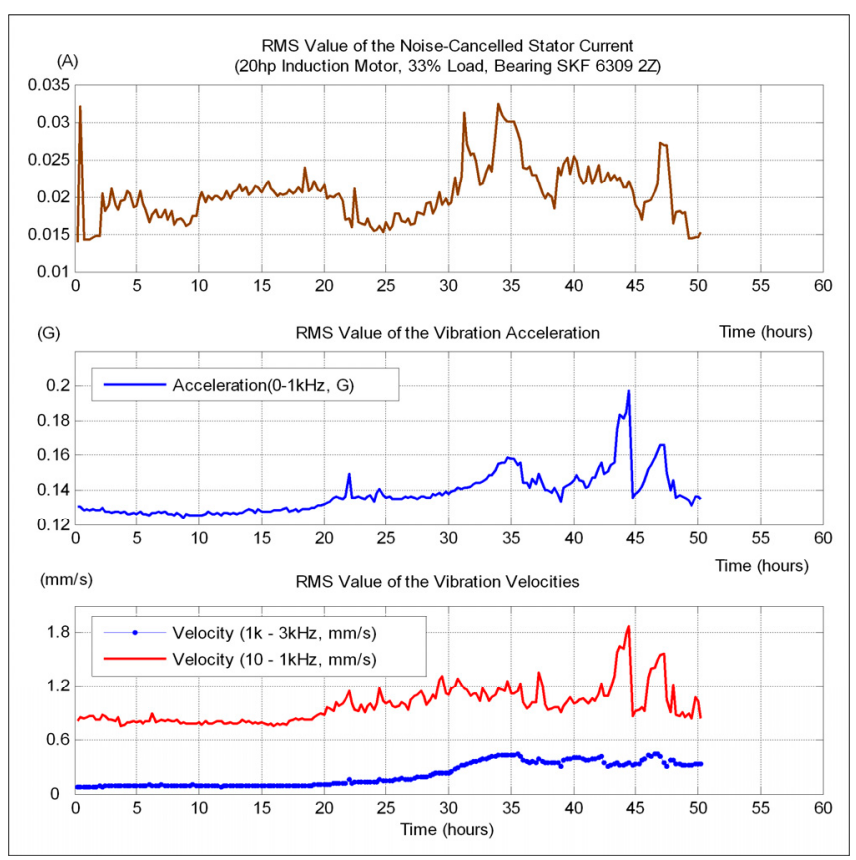

Fig. 9. Results from bearing type-6309 at a 33\% load level. (top) The RMS value of the noise-cancelled stator current indicates the presence of the incipient bearing fault. (middle and bottom) The RMS value of the vibration indicates the presence of the incipient bearing fault. 


\section{CONCLUSIONS}

This paper has presented a new current-based technique to detect incipient bearing faults by applying a noise cancellation method. The method decomposes stator current into the noise components and the fault-related components. The noise components are estimated by a Wiener filter and are cancelled in a real-time fashion. The RMS value of the noise-cancelled stator current is calculated as the fault index. Theoretical analysis has shown the robustness of the method on detection of incipient bearing faults.

Two on-line experiments have been performed. The results of those experiments have confirmed the effectiveness of the proposed method. In those experiments, the RMS value of the noise-cancelled stator current increased significantly as the faults developed. The proposed method is simple and easy to implement. Machine parameters, bearing dimensions, nameplate values, and the stator current spectrum distribution are not required in this method. To implement the method, only one filter is needed. Further more, efficient algorithms (such as the Levinson-Durbin Recursion algorithm) are available to compute the parameters of the filter. To expand the applicability of the proposed method, implementation strategies for both constant- and variable-load conditions are provided. Potential research approach for inverter-fed motors is also briefly addressed.

\section{REFERENCES}

[1] IEEE Motor reliability working group, "Report on large motor reliability survey of industrial and commercial installations," IEEE Trans. Ind. Appl., vol. IA-21, no. 4, pp. 853-872, Jul./Aug. 1985.

[2] K. Kim, A. G. Parlos, and R. M. Bharadwaj, "Sensorless fault diagnosis of induction motors," IEEE Trans. Ind. Electron., vol. 50, no. 5, pp. 1038-1051, May 2003.

[3] T. W. S. Chow and S. Hai, "Induction machine fault diagnostic analysis with wavelet technique," IEEE Trans. Ind. Electron., vol. 51, no. 3, pp. 558-565, Mar. 2004.

[4] J. H. Jung, J. J. Lee, and B. H. Kwon, "Online diagnosis of induction motors using MCSA," IEEE Trans. Ind. Electron., vol. 53, no. 6, pp. 1842-1852, Jun. 2006.

[5] B. Akin, U. Orguner, H. A. Toliyat, and M. Rayner, "Low order PWM inverter harmonics contributions to the inverterfed induction machine fault diagnosis," IEEE Trans. Ind. Electron., vol. 55, no. 2, pp. 610-619, Feb. 2008.

[6] J. R. Stack, T. G. Habetler, and R. G. Harley, "Fault classification and fault signature production for rolling element bearings in electric machines," IEEE Trans. Ind. Appl., vol. 40, no. 3, pp. 735-739, May/Jun. 2004.

[7] R. R. Schoen, T. G. Habetler, F. Kamran, and R. G. Bartfield, "Motor bearing damage detection using stator current monitoring," IEEE Trans. Ind. Appl., vol. 31, no. 6, pp. 12741279, Nov./Dec. 1995.

[8] B. Yazici and G. B. Kliman, "An adaptive statistical timefrequency method for detection of broken bars and bearing faults in motors using stator current," IEEE Trans. Ind. Appl., vol. 35, no. 2, pp. 442-452, Mar./Apr. 1999.

[9] L. Eren and M. J. Devaney, "Bearing damage detection via wavelet packet decomposition of the stator current," IEEE Trans. Instrum. Meas., vol. 53, no. 2, pp. 431-436, Apr. 2004.

[10] M. E. H. Benbouzid, M. Vieira, and C. Theys, "Induction motors' faults detection and localization using stator current advanced signal processing techniques," IEEE Trans. Power Electron., vol. 14, no. 1, pp. 14-22, Jan. 1999.

[11] M. E. H. Benbouzid, "A review of induction motors signature analysis as a medium for faults detection," IEEE Trans. Ind. Electron., vol. 47, no. 5, pp. 984-993, Oct. 2000.

[12] M. Blodt, P. Granjon, B. Raiso, and G. Rostaing, "Models for bearing damage detection in induction motors using stator current monitoring," in Proc. IEEE International Symposium Ind. Electron., vol. 55. no. 4, pp. 1813-1822, Apr. 2008.

[13] M. S. Ballal, Z. J. Khan, H. M. Suryawanshi, and R. L. Sonolikar, "Adaptive neural fuzzy inference system for the detection of inter-turn insulation and bearing wear faults in induction motor," IEEE Trans. Ind. Electron., vol. 54, no. 1, pp. 250-258, Jan. 2007.

[14] J. R. Stack, R. G. Harley, and T. G. Habetler, "An amplitude modulation detector for fault diagnosis in rolling element bearings," IEEE Trans. Ind. Electron., vol. 51, no. 5, pp. 1097-1102, May 2004.

[15] S. Wu and T. W. S. Chow, "Induction machine fault detection using SOM-based RBF neural networks," IEEE Trans. Ind. Electron., vol. 51, no. 1, pp. 183-194, Jan. 2004.

[16] A. M. Knight, and S. P. Bertani, "Mechanical fault detection in a medium-sized induction motor using stator current monitoring," IEEE Trans. Energy Convers., vol. 20, no. 4, pp 753760, Dec. 2005.

[17] J. R. Stack, T. G. Habetler, and R. G. Harley, “ Bearing fault detection via autoregressive stator current modeling," IEEE, Trans., Ind., Appl., vol. 40, no. 3, pp 740-747, May/Jun. 2004.

[18] S. J. Yang, Low-Noise Electrical Motors. New York, NY: Oxford University Press, 1981, pp15-21.

[19] M. H. Hayes, Statistical Digital Signal Processing and Modeling. New York, NY: Wiley, 1996, pp265-269; 165-166; 337-353.

[20] W. Zhou, T. G. Habetler, and R. G. Harley, "Bearing fault detection via stator current noise cancellation and statistical control," IEEE Trans. Ind. Electron., in press, vol. 55, no. 12, pp. $4260-469$, Dec. 2008.

[21] K. D. Hurst and T. G. Habetler, "Sensorless speed measurement using current harmonic spectral estimation in induction machine drives," IEEE Trans. Power Electron., vol. 11, no. 1, pp. 66-73, Jan. 1996.

[22] J. R. Stack, T. G. Habetler, and R. G. Harley, "Experimentally generating faults in rolling element bearings via shaft current," IEEE, Trans., Ind., Appl., vol. 41, no. 1, pp. 25-29, Jan./Feb. 2005.

[23] Mechanical Vibration-Evaluation of Machine Vibration by Measurements on Nonrotating Parts-Part 1: General Guidelines, ISO 10816-1:1995(E), 1995. 


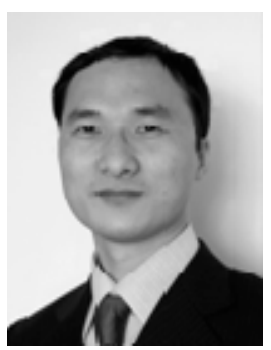

Wei Zhou (M'2006) received his Bachelor degree from Wuhan University, Wuhan, China, 1999, and two Master degrees from Wuhan University, 2002 and Georgia Institute of Technology, 2006 respectively, and his Ph.D. degree from Georgia Institute of Technology, 2007.

From Aug. 2002 to Aug. 2003, he was with Guangdong Electric Power Design Institute, Guangzhou, China, as a bulk power system planner. From Aug. 2006 to Dec. 2006, he was with Electric Power Group, LLC, as a Research Engineer Intern working on large-scale power grid reliability monitoring. From Aug. 2007 to Aug. 2008, he was with Midwest Independent Transmission System Operator (Midwest ISO), Carmel, IN as a market administration engineer under market operation. He is currently with Southern California Edison, Rosemead, CA working on California wholesale electricity market analysis in the department of Market Strategy and Resource Planning. His research interests include energy markets operation and analysis, power system planning and analysis, power system reliability analysis, and electric machine condition monitoring.

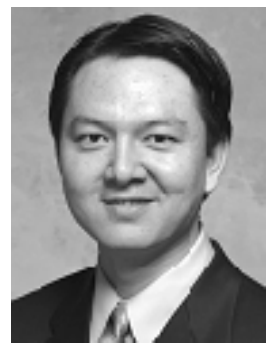

Bin Lu (S'00-M'06) received the B.Eng. degree in automation from Tsinghua University, Beijing, China, in 2001, the M.S. degree in electrical engineering from the University of South Carolina, Columbia, SC, in 2003, and the Ph.D. degree in electrical engineering from the Georgia Institute of Technology, Atlanta, GA, USA in 2006, respectively.

From 2001 to 2003, he was with the VTB Group University of South Carolina, Columbia, where his master thesis work built the foundation of a real-time virtual test bed for the hardware-in-the-loop testing of dynamic power systems. Since July 2004, he has been a graduate Research Assistant in the power electronics and motor diagnostics group of the Georgia Institute of Technology, working on the energy evaluation and condition monitoring of electric machines. In 2006 summer, he worked at the General Motors R\&D Center at Warren, MI, as a graduate summer intern. Since October 2006, he has been with the Innovation Center, Eaton Corporation, Milwaukee, WI, where he is currently an Engineering Specialist and a Program Manager. He has authored over 40 papers published in refereed journals and international conference proceedings and has seven patent applications in these areas. His research interests include electric motor drives and diagnostics, renewable energy, power electronics, modeling and simulation, and application of wireless sensor networks in electric power areas.

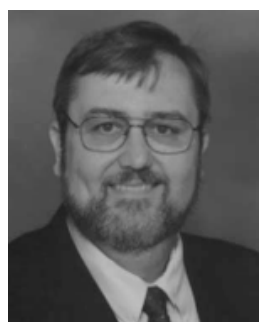

Thomas G. Habetler (S'82-M'83 -SM'92-F'02) received the B.S.E.E. degree in 1981 and the M.S. degree in 1984, both in electrical engineering, from Marquette University, Milwaukee, WI, and the Ph.D. degree from the University of Wisconsin-Madison, in 1989.

From 1983-1985 he was employed by the ElectroMotive Division of General Motors as a Project Engineer. Since 1989 he has been with the Georgia Institute of Technology, Atlanta, USA, where he is currently a Professor of Electrical Engineering. His research interests are in electric machine protection and condition monitoring, switching converter technology and drives. Dr. Habetler has published over 100 papers in the field. He is a regular consultant to industry in the field of condition-based diagnostics for electrical systems.

Dr. Habetler has received four conference prize paper awards from the Industry Applications Society. He currently serves as IEEE Division II Director-Elect, is a Past-President of the IEEE Power Electronics Society, and Past-Chair of the Industrial Power Converter Committee of the IEEE Industry Applications Society. Dr. Habetler is a Fellow of IEEE.

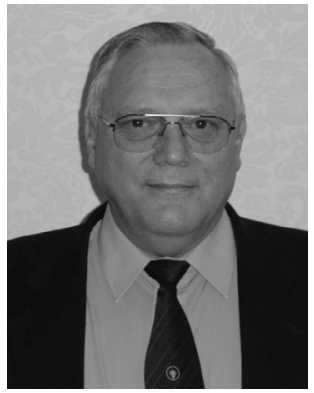

Ronald G. Harley (M'77-SM'86-F'92) received the MScEng degree (cum laude) in electrical engineering from the University of Pretoria, South Africa in 1965, and the Ph.D. degree from London University in 1969.

In 1971 he was appointed to the Chair of Electrical Machines and Power Systems at the University of Natal in Durban, South Africa. At the University of Natal in South Africa he was a professor of Electrical Engineering for many years, including the Department Head and Deputy Dean of Engineering. He is currently the Duke Power Company Distinguished Professor at the Georgia Institute of Technology, Atlanta, USA. His research interests include the dynamic behavior and condition monitoring of electric machines, motor drives, power systems and their components, and controlling them by the use of power electronics and intelligent control algorithms. He has coauthored some 400 papers in refereed journals and international conferences and three patents. Altogether 10 of the papers attracted prizes from journals and conferences.

Dr. Harley is a Fellow of the British IEE, and a Fellow of the IEEE. He is also a Fellow of the Royal Society in South Africa, and a Founder Member of the Academy of Science in South Africa formed in 1994. During 2000 and 2001 he was one of the IEEE Industry Applications Society's six Distinguished Lecturers. He was the Vice-President of Operations of the IEEE Power Electronics Society (2003-2004) and Chair of the Atlanta Chapter of the IEEE Power Engineering Society. He is currently Chair of the Distinguished Lecturers and Regional Speakers program of the IEEE Industry Applications Society. He received the Cyrill Veinott Award in 2005 from the Power Engineering Society for outstanding contributions to the field of electromechanical energy conversion. 


\title{
An Investigation on Commercially Available Energy Usage Estimation Products
}

\author{
EATON WS R Rearch Report \\ Prepared by \\ Bin Lu \\ Prof. Thomas G. Habetler \\ Prof. Ronald G. Harley
}

SCHOOL OF ELECTRICAL AND COMPUTER ENGINEERING
GEORGIA INSTITUTE OF TECHNOLOGY

ATLANTA, GA 30332 


\section{INTRODUCTION}

Currently, many companies have developed products for estimating the energy usage of electric machines; more specifically, induction machines. Available products are both software- and hardware-based.

The algorithms behind these products are primarily based on methods published in the technical literature. In the Eaton WSN research project, the focus is on two main objectives, efficiency estimation and condition monitoring. The products described hereafter overlap this domain, but none of them are based on wireless data collection or communication.

The condition monitoring functions claimed in these products are still in a beginning stage with very limited precision and applicability in industry. The specific functions of these products will be discussed in more detail in the rest of this report.

Over the years, many efficiency estimation methods have been proposed. As an example, Oak Ridge National Lab (ORNL) compared 28 methods on electric machine efficiency estimation methods on 1996, ranging from highly intrusive methods to completely non-intrusive methods. Since industry expects the estimation and diagnosis to be done without shutting down the motor, most of these products adopted a low-intrusive approach. Different products chose different methods in efficiency estimation. The measurements needed for each method are different, but most of them require the following common data: input line-to-line voltage, input phase current, and winding temperature. Some methods require the rated nameplate information (voltage, current, horsepower, speed, etc), stator resistance, or rotor speed (slip).

The measurements/estimates of the stator resistance and rotor speed have been regarded as the stumbling blocks of various efficiency estimation methods for years. However, recent research has produced quite accurate speed estimates through stator current harmonic spectral analysis when the machine is working under normal stable operation. (K.D. Hurst, T.G. Habetler, "A comparison of Spectrum Estimation Techniques for Sensorless Speed Detection in Induction Machines," IEEE Trans. Ind. App. July 1997). Also some "in situ" stator resistance measurement methods have been proposed which inject a DC component into the stator voltage and current. The accurate nonintrusive measurement of stator resistance may still need some more research, but alternatively it can be measured and recorded at the time of new machine installation and then at scheduled maintenance intervals.

Based on the preliminary research, several efficiency estimation methods are suggested for the WSN project. They are discussed in detail in a separate report. 


\section{MOTOR ENERGY MONITORING AND EFFICIENCY ESTIMATION PRODUCTS}

Several currently available commercial products have been investigated and compared. The six most important products on energy usage of electric machines are summarized below. For all six products, an owner's manual with detailed specifications is available on the respective websites.

\section{Explorer Series II (Baker Instrument Co.):}
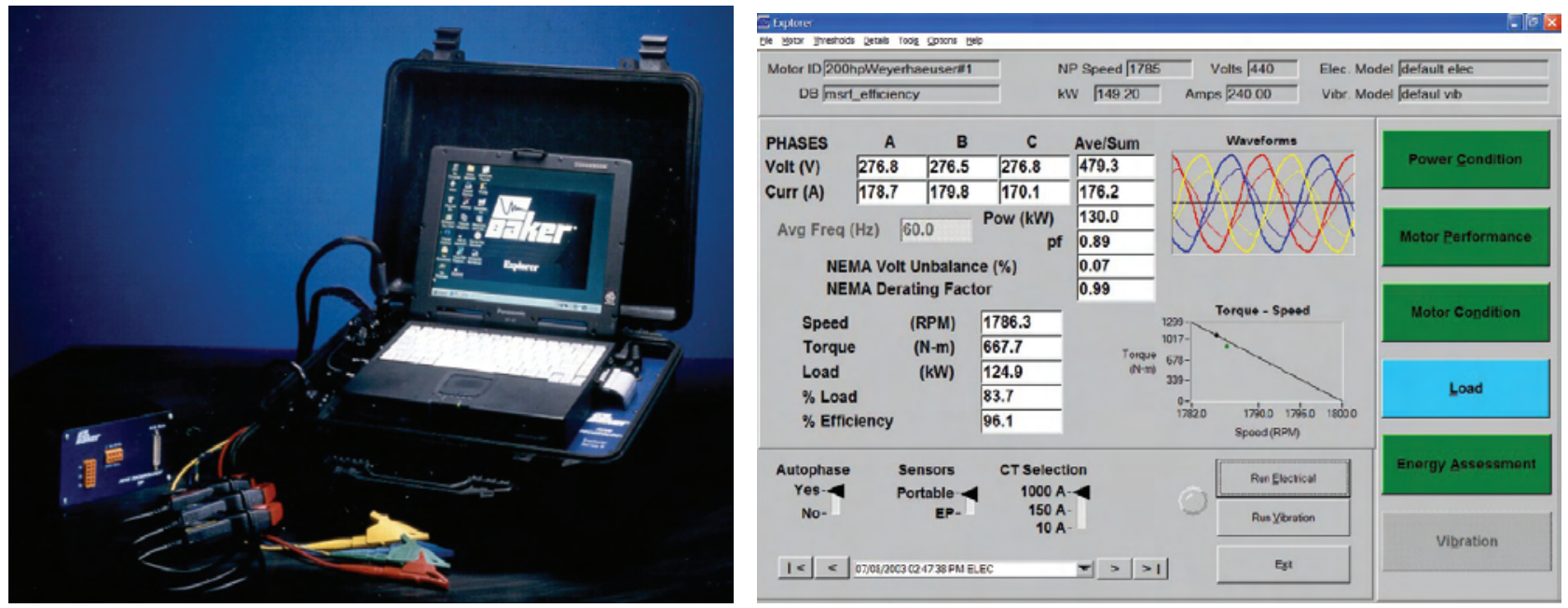

Fig. 1: Explorer Series II (Baker Instrument Co.)

The Explorer Series II is a relatively new product developed by Baker Instrument Co. for online monitoring of power circuit issues, overall motor health, load, and performance of electric machines. It estimates motor speed by current signature analysis and estimate torque from the instantaneous product of current magnitude and voltage magnitude. It performs five major functions: (1) identifying possible power circuit problem, (2) examining overall motor power conditions, (3) monitoring the load condition, (4) observing motor performance, (5) and estimating the energy usage. Among these functions, efficiency estimation is its prime function.

As shown in the Fig. 1, the Explorer II is a truly portable instrument which uses a laptop computer for analysis algorithms, data collection and display. The current probes are by AEMC and a set of three is provided to cover specific ranges with reasonable accuracy.

The Explorer II includes the DOE Motor Master+ software, which calculates the motor condition and energy savings directly given the measured data. Specifically, it performs the following tests: voltage level, voltage balance, distortion, service factor, overcurrent condition, efficiency, rotor bar condition, operating condition, torque ripple, load history, and vibration. 


\section{MCEmax ${ }^{\mathrm{TM}}$ (PdMA Co.):}
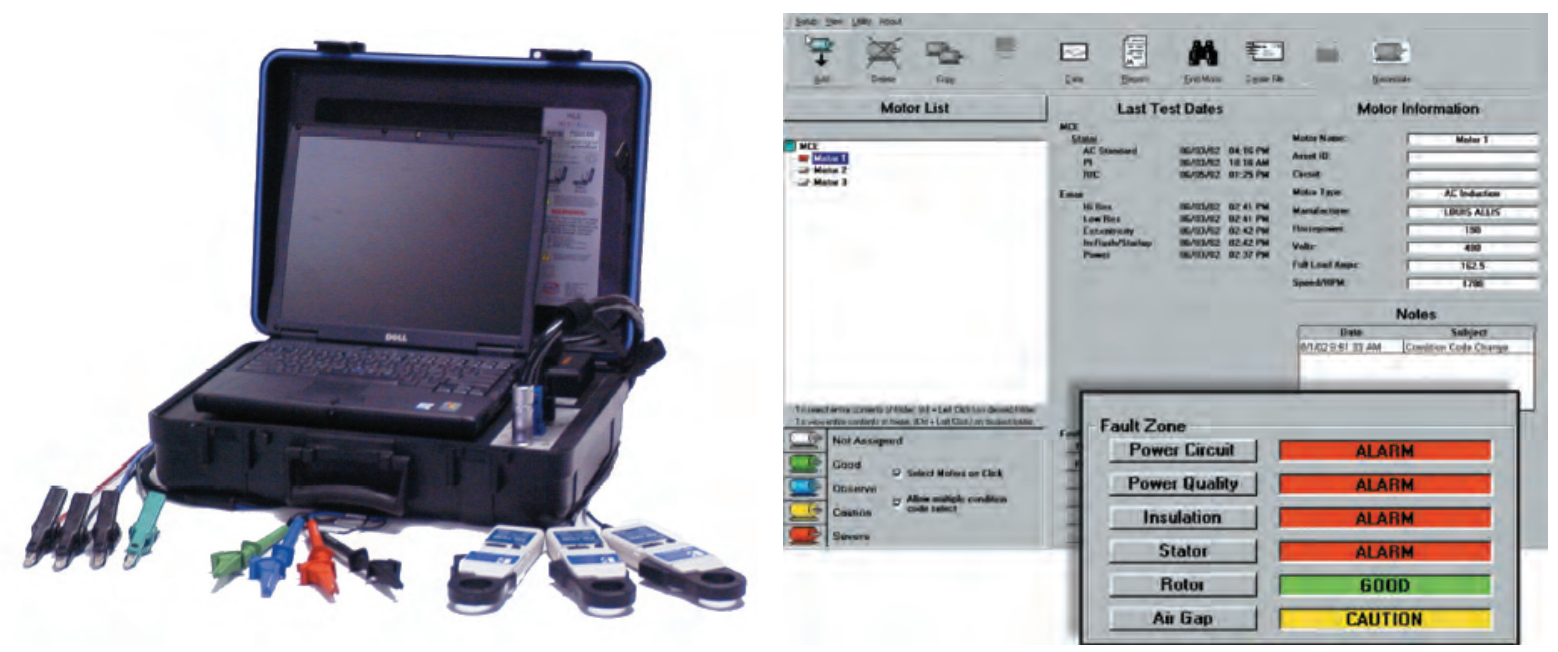

Fig. 2: MCEmax ${ }^{\mathrm{TM}}$ (PdMA Co.)

The MCEmax by PdMA was developed from their original motor problem diagnostic instrument MCE, which uses stator current signature to detect problems such as mechanical and electrical unbalance, bearing degradation, broken rotor bars, etc. Unlike from Explorer II, PdMA MCE series are mainly for condition monitoring instead of efficiency estimation. MCEmax is the most powerful one in this series. It has the functions to complement the diagnostics to providing motor output power and efficiency estimates. The algorithms used by this monitor, however, are not based on detailed signature analysis, but rather on simple fluctuations in measured terminal parameters.

As shown in Fig. 2, the MCEmax uses a laptop computer which contains the necessary analysis software and provides graphic display of the test results. It is a truly portable instrument which operates from a rechargeable battery. The voltage probes shown in Fig. 2 are of good quality and the current clamps are very accurate, battery powered by LEM. The LEM probes are smaller than the equivalent rated AEMC units (used in Explorer II) which could be a problem when connecting to a small MCC cabinet.

The MCEmax tests a wide range of potential faults: stator, rotor, air gap, power quality, power circuit and insulation. The MCEmax is more versatile compared with other products in terms of machine types. It tests AC induction machine, synchronous machine, wound rotor, DC, specialty motors, generators and transformers. 


\section{Motor Efficiency Wizard $\stackrel{\text { TM }}{\text { (Reliance Electric, Rockwell) }}$}
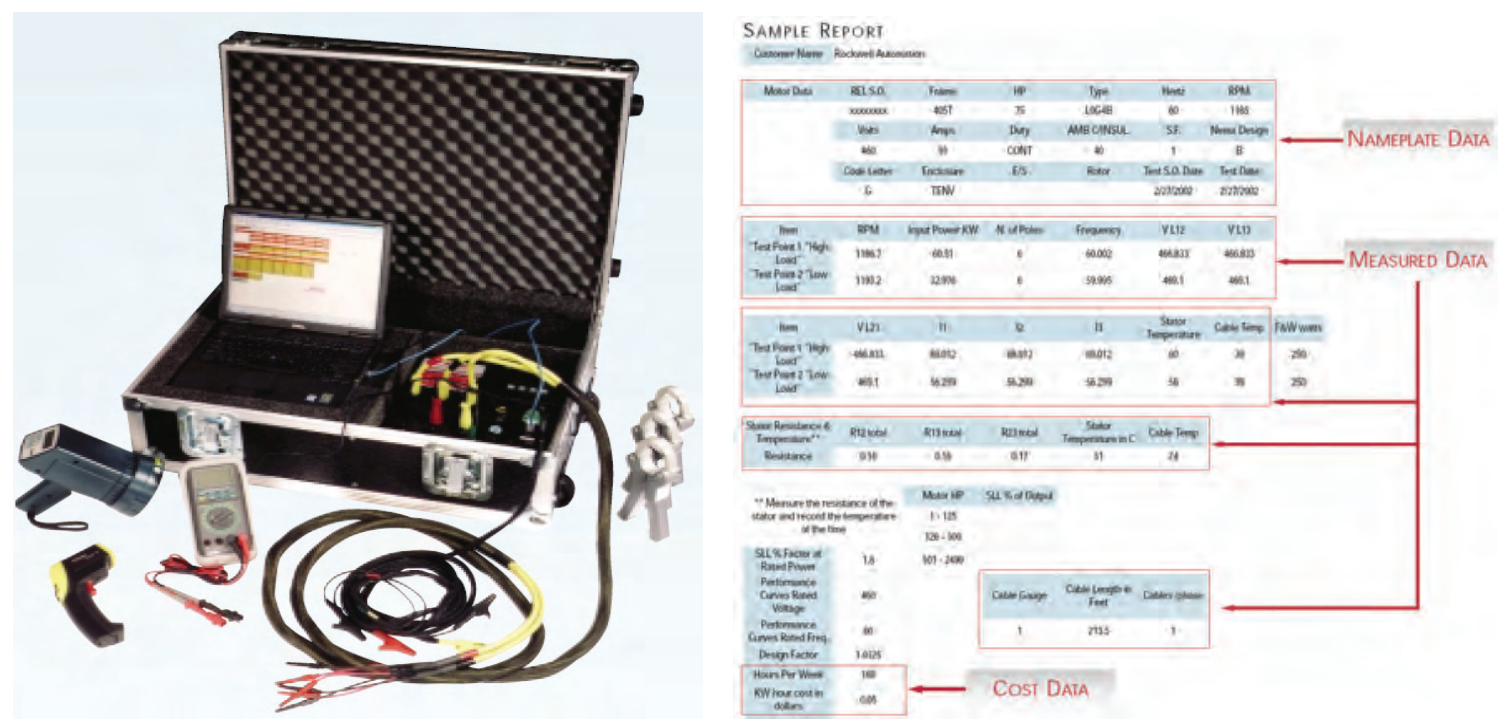

Fig. 3: Motor Efficiency Wizard ${ }^{\mathrm{TM}}$ (Reliance)

The Motor Efficiency Wizard ${ }^{\mathrm{TM}}$ is developed by the Reliance Electric division of Rockwell for squirrel cage induction motor efficiency estimation. Unlike the Explorer II and MCEmax, this product has no condition monitoring capability.

As shown in Fig. 3, the Motor Efficiency Wizard Kit consists of the following items:

- Allen-Bradley ${ }^{\circ}$ Portable Power Monitor

- Three Current Transformers

- Infrared Temperature Instrument

- Digital Strobe RPM Sensor

- Digital Multi Meter, Monitoring Software and Cable

- Set of Alligator Clips

- Dell ${ }^{\circ}$ Lattitude ${ }^{\circ}$ Laptop Computer

- RSPower $32^{\mathrm{mm}}$ Software

- Microsoft Office XP Package

- Reliance Motor Efficiency Wizard Software

- RSLinx" Software

Reliance claims that this product can measure the efficiency of any squirrel cage induction motor inservice with an accuracy of $1 \%$. However, the "in-service” here means without disconnecting the motor, an is therefore "non-intrusive". The estimation process, however, requires an accurate measurement of the motor's stator resistance, and as a result, the motor needs to be stopped and disconnected from the power supply. 


\section{EMPATH ${ }^{\mathrm{TM}} \underline{2000}$ (Framatome Co., France):}

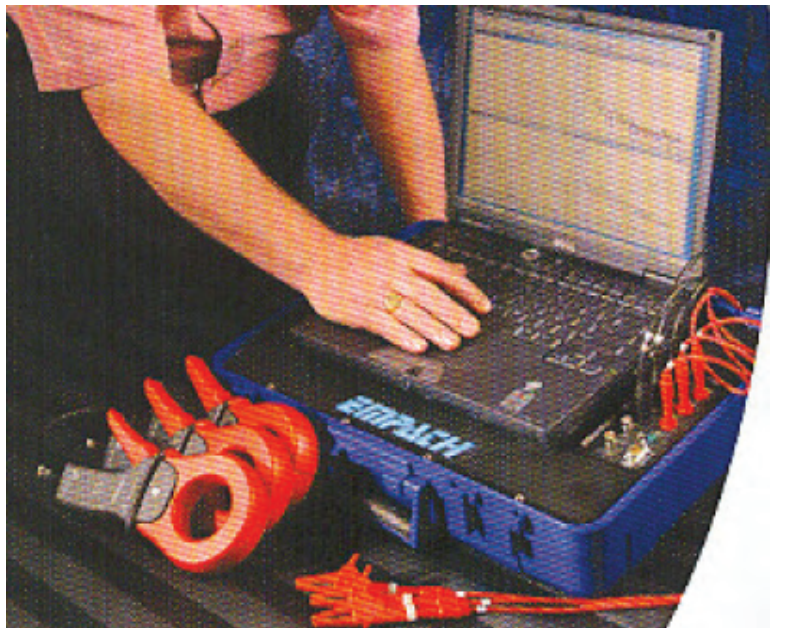

Fig. 4(a): EMPATH ${ }^{\mathrm{TM}} 2000$ (Framatome Co.)

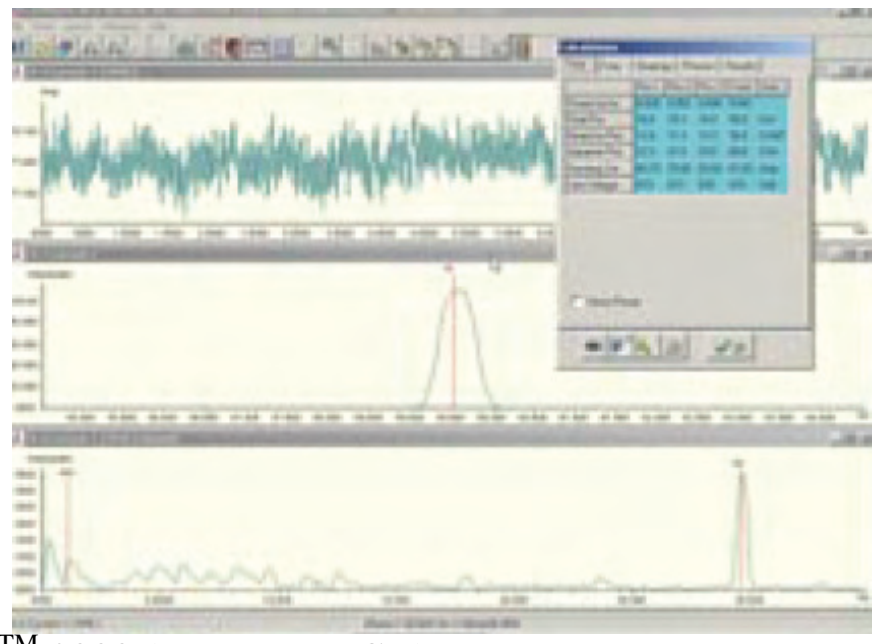

(2000 (Framatome Co.)

Framatome is a French company producing a wide variety of equipment in support of the nuclear industry. The Electric Motor Performance Analysis and Trending Hardware (EMPATH 2000) was developed by Framatome to measure and analyze electric motor stator current and voltage to obtain condition-related information. So far, this system is mainly for condition monitoring. The efficiency estimation algorithm is still under development and has not been incorporated into the system.

As shown in Fig. 4(a), the EMPATH system contains a laptop computer with a 16-channel, 16-bit A/D card, and analysis software to store data and display. The only data measured are three phase voltages and currents. EMPATH demodulates the signals from the $60 \mathrm{~Hz}$ carrier and apply FFT on them to get the final test results.

The estimation results include:

- Rotor bar and stator deterioration and severity level

- Rotor-stator eccentricity characteristics

- Motor speed and slip

- Average running current - an indicator of motor torque

- Power factor

- Torsional vibration and dynamic loading

- Misalignment, mechanical unbalance

- Bearing degradation

- Current/voltage variations

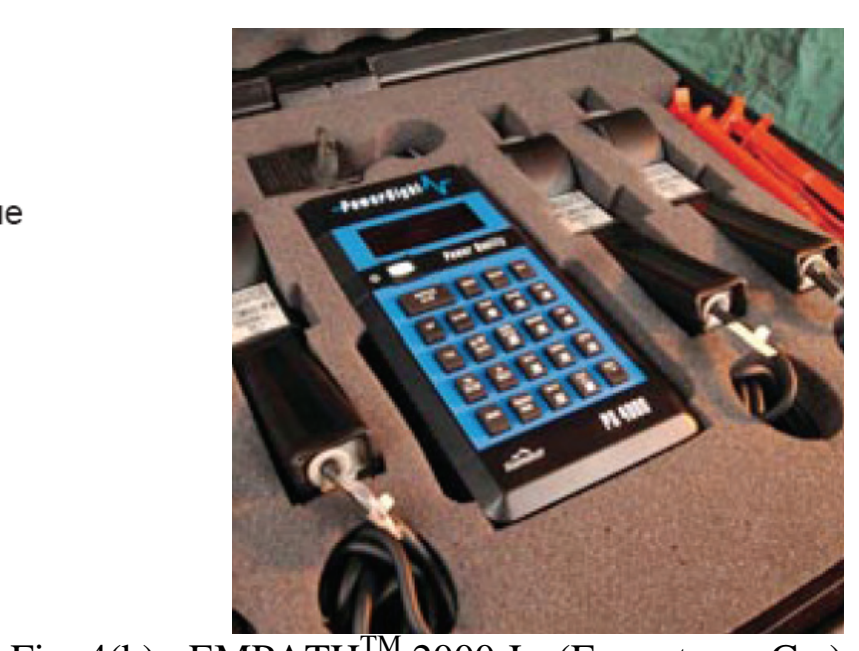

Fig. 4(b): EMPATH ${ }^{\mathrm{TM}} 2000 \mathrm{Jr}$. (Framatome Co.)

Many of the core capabilities of the EMPATH 2000 system now are available in a more compact, more affordable motor analysis system, called EMPATH Jr., as shown in Fig. 4(b). 


\section{MAS-1000 Series (Digital Instruments):}

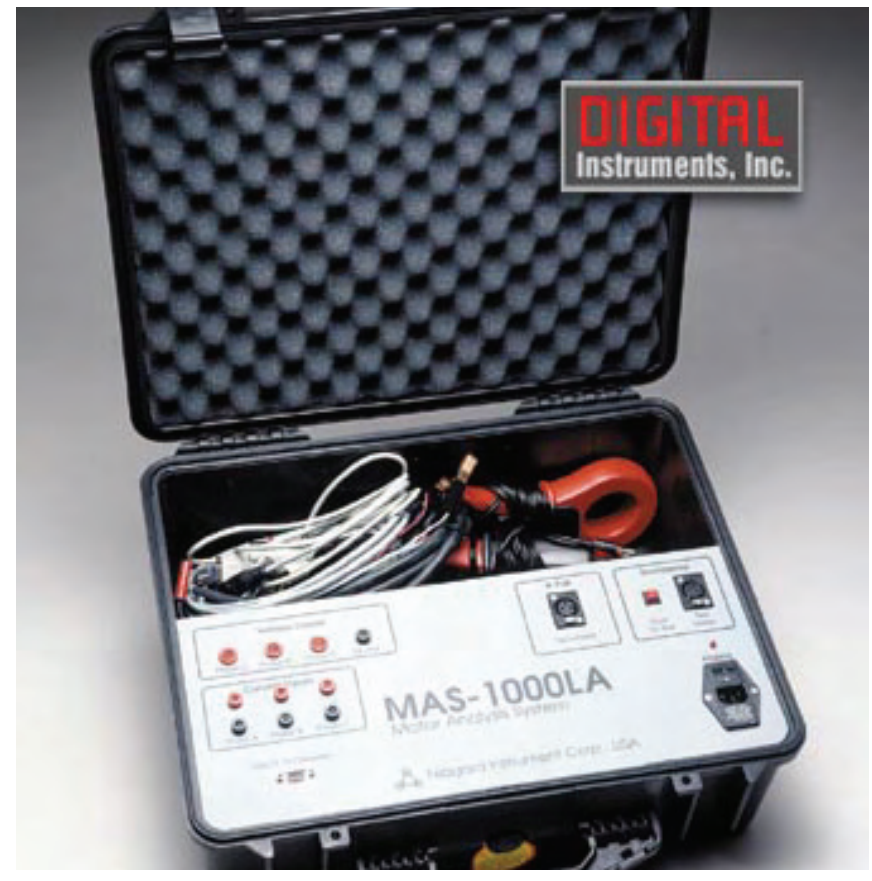

Fig. 5: MAS-1000 LA (Digital Instruments Co.)

The previous version of MAS-1000 LA is the MAS-1000 produced by Niagara Instruments. This series of systems are mainly for efficiency estimation purpose. Previous evaluation of MAS-1000 was found to give reasonable assessment of operational efficiency but it required the attachment of a reflective tape on the motor shaft, and therefore could not be classified as non-intrusive.

Recently the MAS-1000 has been developed to become less obtrusive (and has been purchased by Digital Instruments). The new MAS-1000 LA motor analyzing system uses IEEE Standard 112 Method E (Electric Power Measurement with Loss Segregation) as its estimation method. This requires a No-load test and a test under load. Besides this, stator winding resistance and rotor speed (slip) need to be measured.

The MAS-1000 LA has much less functionality than the systems previously discussed. No evaluation or comparison of this product with other product could be found at this time. 
6. Weyerhaeuser Power Analyzer (Weyerhaeuser Co.):

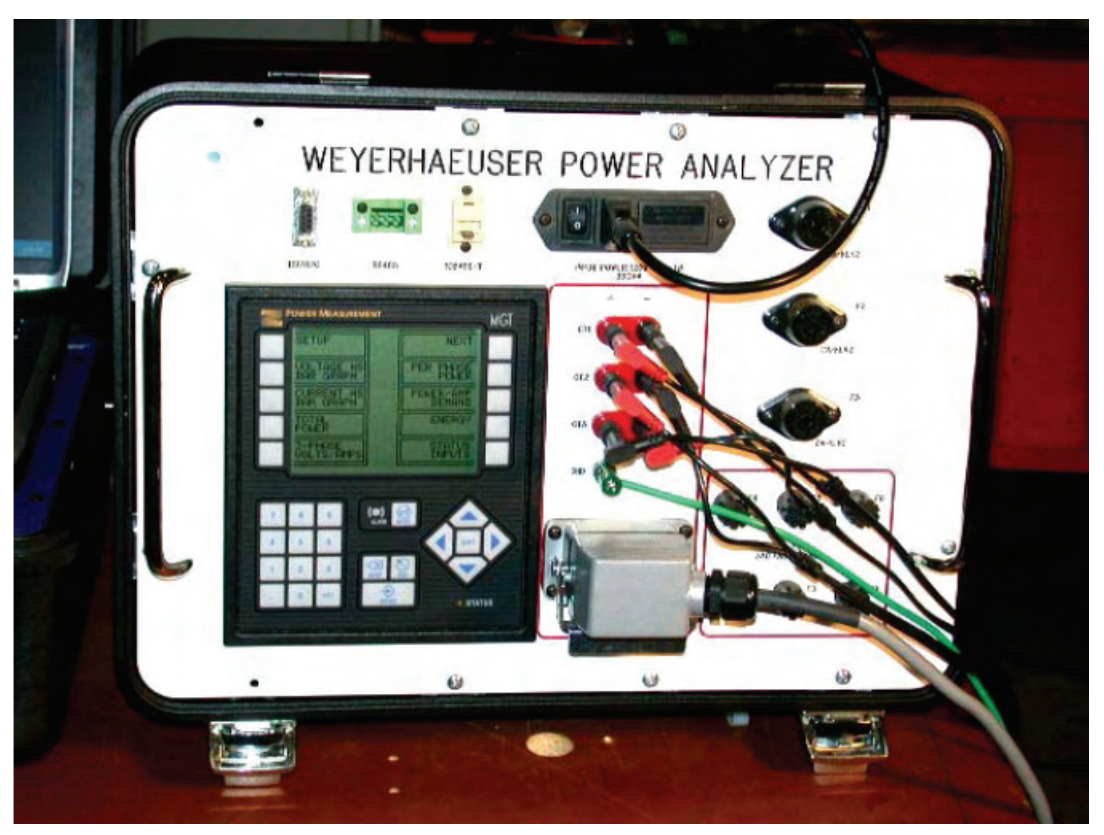

Fig. 6: Weyerhaeuser Power Analyzer (Weyerhaeuser Co.)

The Weyerhaeuser Power Analyzer is an instrument developed by Weyerhaeuser Company for the energy efficiency estimation in their mills. Presently, it is only intended for use in Weyerhaeuser plants. However, the company has reportedly reached an agreement with a possible developer with a view to commercialization.

This instrument is based on a Power Measurements Ltd. (PML) power meter which provides data for a performance approximation based on the Nameplate Equivalent Circuit method and Slip method described in (J. Holmquist, J. Rooks, M. Richter, "Practical Approach for Determining Motor Efficiency in the Field Using Calculated and Measured Values”, Proc IEEE PPIC, June 2003.) The output power estimate is determined from No-load current. If the no-load current is not available, a default value of $30 \%$ of the nameplate rated current is used. Alternatively, for new motors, the noload current can be looked up from the Motor Master Plus documents. 


\section{CONCLUSIONS AND COMMENTS}

The previous section briefly introduced six commercially available products on energy usage analysis. These six products were discussed in a market research report ("Non-Intrusive Motor Efficiency Estimators,” MSRF, NEEA, March 2004) which was proposed in the Motor System Resource Facility (MSRF) at Northwest Energy Efficiency Alliance (NEEA) in March 2004. Among the six instruments:

- Three (Weyerhaeuser, PdMA MCEmax, Baker Explorer) were evaluated on motors with different horsepower ratings (50hp, 100hp, 200hp), over a range of loads (25\% to 120\%), a range of balanced voltage levels ( $90 \%$ to $110 \%)$, and voltage unbalance $(1.25 \%, 2.5 \%)$.

- Two (Framatome EMPATH, MAS-1000 LA) were not evaluated due to the unavailability at testing time.

- One (the Reliance unit) did not meet the definition of "non-intrusion" and therefore was withdrawn.

The testing results led to the following observations:

1. PdMA MCEmax

- Excellent parameter measurement and good speed estimation (but time consuming).

- Portable.

- Nameplate power factor is needed as an input parameter.

- Expensive.

2. Baker Explorer II

- It is a good efficiency and power factor estimator with condition monitoring functions.

- The speed estimation is less accurate than MCEmax due to its faster sampling frequency.

- Portable.

- Relatively inexpensive.

3. Weyerhaeuser

- Still in an early developing stage.

- Large errors in efficiency estimation for motors operating below 50\% of rated load.

4. Overall Comments:

- All instruments got satisfying efficiency estimation at 75\% load or higher.

- All systems are expensive.

- Based on price, ease of use, and efficiency estimation precision, the Explorer II from Baker Instrument is the best at this time. 


\begin{tabular}{|c|c|c|c|c|c|c|c|c|}
\hline Product & $\begin{array}{l}\text { Efficiency } \\
\text { Estimation }\end{array}$ & $\begin{array}{c}\text { Condition } \\
\text { Monitoring }\end{array}$ & $\begin{array}{l}\text { Commercial } \\
\text { Availability }\end{array}$ & Portability & Non-Intrusive & $\begin{array}{l}\text { Relative } \\
\text { Price }\end{array}$ & Other Comments & $\begin{array}{c}\text { Overall } \\
\text { Evaluati } \\
\text { on }\end{array}$ \\
\hline $\begin{array}{c}\text { Explorer II } \\
\text { (Baker Instruments) }\end{array}$ & Good & Yes & Yes & Yes & Yes & Low & $\begin{array}{c}\text { Uses DOE } \\
\text { MotorMaster + }\end{array}$ & 5 \\
\hline $\begin{array}{l}\text { MCEmax } \\
\text { (PdMA) }\end{array}$ & Yes & $\begin{array}{c}\text { Yes } \\
\text { But not good }\end{array}$ & Yes & Yes & Yes & High & $\begin{array}{l}\text { Can deal with IM, } \\
\text { Sync., DC, XFMR }\end{array}$ & 3 \\
\hline $\begin{array}{l}\text { Motor Efficiency Wizard } \\
\text { (Reliance) }\end{array}$ & Yes & No & Yes & Yes & $\begin{array}{c}\text { No } \\
\text { Need no-load test }\end{array}$ & High & $\begin{array}{c}\text { For Squirrel Cage } \\
\text { I.M. only }\end{array}$ & 2 \\
\hline $\begin{array}{c}\text { EAMPATH } \\
\text { (Framatome) }\end{array}$ & $\begin{array}{c}\text { No } \\
\text { Under developing }\end{array}$ & Yes & Yes & Yes & Yes & High & $\begin{array}{c}\text { Has a more compact } \\
\text { version } \\
\text { EMPATH2000 Jr. }\end{array}$ & 4 \\
\hline $\begin{array}{c}\text { MAS-1000 LA } \\
\text { (Digital Instruments) }\end{array}$ & Yes & No & Yes & Yes & $\begin{array}{c}\text { No } \\
\text { Need no-load test }\end{array}$ & Low & $\begin{array}{l}\text { Use IEEE Std-112 } \\
\text { Method E }\end{array}$ & 1 \\
\hline $\begin{array}{l}\text { Power Analyzer } \\
\text { (Weyerhaeuser) }\end{array}$ & Yes & No & No & $\begin{array}{c}\text { Yes } \\
\text { But heavier }\end{array}$ & Yes & N/A & $\begin{array}{l}\text { Need nameplate } \\
\text { information }\end{array}$ & 0 \\
\hline
\end{tabular}




\section{White Paper}

\section{Motor Condition Monitoring Algorithms}

\section{Rotor Fault Detection for Induction Machines \\ Using Wavelet Analysis}

\section{$\underline{\text { Key Features }}$}

$\checkmark$ Non-intrusive

Without interfering with normal operation

$\checkmark$ Online

Continuous rotor health monitoring

$\checkmark$ Inferential

Using current and voltage, no additional sensors needed

$\checkmark$ Low-cost

No additional sensors needed 


\section{Background}

There are around 1.2 billions of electric motors used in the United States, which consume about $57 \%$ of all generated electric power. Over $70 \%$ of the electrical energy used by manufacturing and $90 \%$ in process industries is consumed by motor driven systems [1]. Among these motor systems, squirrel-cage induction motors have a dominant percentage because of the simple and rugged design and easy maintenance.

It is well realized by the industries that degraded energy efficiency of the motor causes increased energy losses and results in more economical losses. However, more energy losses actually come from the unscheduled downtime caused by the unexpected motor failures, which, for some certain industries, can be catastrophic and intolerable. The average downtime cost of different industries is summarized in Fig. 1 [2].

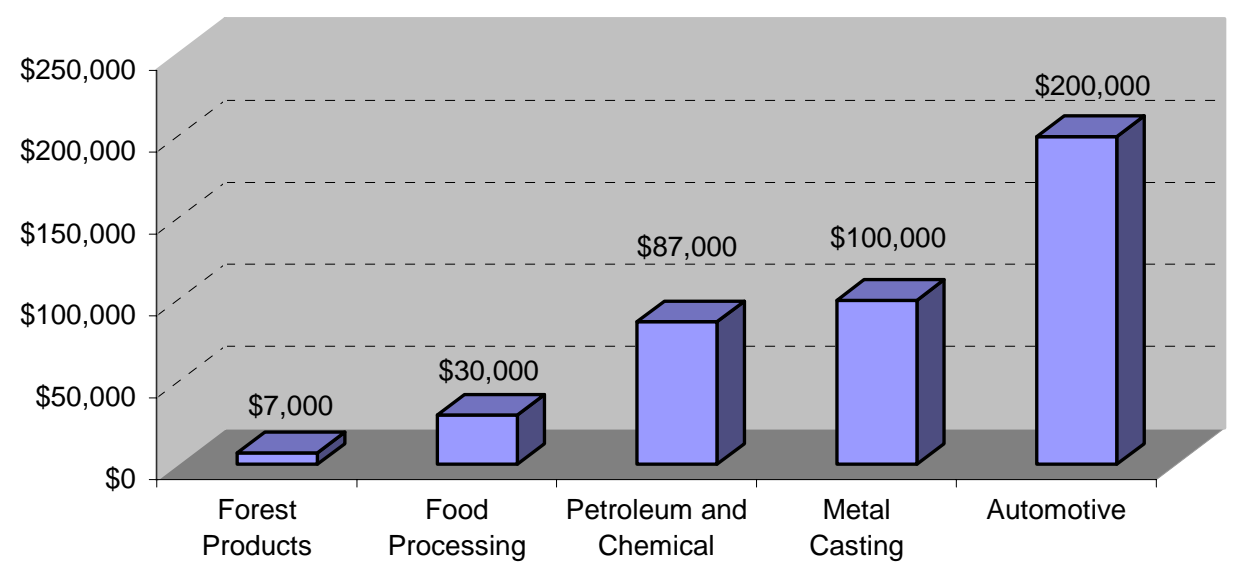

Fig. 1. Average Downtime Cost of Different Industries

It is estimated that about $37 \%$ of the induction motor failures are caused by stator winding faults, $41 \%$ by bearing failures, $10 \%$ by rotor faults, and $12 \%$ by miscellaneous faults. Rotor faults may appear less significant than the bearing faults from these numbers, but it is critical to understand that bearing failures are often resulted from shaft current and increased vibration, which are caused by shaft misalignment, eccentricity, and rotor related faults. Besides, rotor faults may also result in excess heat, decrease efficiency, shorten insulation life, and possibly cause core damage. Therefore, early detection of impending rotor faults and appropriate maintenance can avoid more severe motor failures. Many surveys have shown that broken rotor bar is a serious rotor fault for squirrel-cage induction motors due to arduous duty cycles, especially for large motors.

As a leading industrial manufacturer, Eaton develops a complete portfolio of motor wellness diagnostic algorithms to provide early prediction of various motor and equipment failures, including rotor fault, stator insulation fault, bearing fault, misalignment and eccentricity, etc. This white paper focuses on the rotor fault detection, especially broken rotor bar fault. 


\section{Science Behind Rotor Faults}

\section{Causes}

The following conditions can cause broken rotor bar in induction motor.

$>$ Direct on-line starting with duties, for which the rotor cage was not designed to withstand. This causes high thermal and mechanical stresses.

$>$ Pulsating mechanical loads, such as reciprocating compressors and coal crushers, can cause high mechanical stresses to the rotor cage.

$>$ Imperfections in the manufacturing process of the rotor cage.

\section{Fault Signature}

According to the motor space vector analysis, a 3-phase symmetrical stator winding fed from a symmetrical supply with frequency $f_{1}$ will produce only a resultant forward rotating magnetic field at the synchronous speed in the air-gap. When this magnetic field is applied to the rotor that rotates at frequency $(1-s) f_{1}$, a rotor current at the slip frequency (i.e., $f_{2}=s f_{1}$ ) is induced in the rotor cage, where $s$ is the slip. Any asymmetry of the supply or stator winding impedances will cause a negative sequence component in the stator current, which, in turn, results in a backward rotating magnetic field in the air-gap $\left(-f_{1}\right)$.

The rotor current $\left(f_{2}\right)$ produces a rotating magnetic field with the same number of poles as the stator field but rotating at the slip frequency $f_{2}$ with respect to the rotating rotor. With a symmetrical rotor cage, only a forward rotating field exists $\left(f_{2}\right)$. When rotor asymmetry such as broken rotor bar exists, there will also be a resultant backward rotating field at slip frequency with respect to the forward rotating rotor $\left(-f_{2}\right)$. As a result, this backward rotating field $\left(-f_{2}\right)$ induces an EMF and current in the stator winding at the following frequency,

$$
f_{s b}=f_{1}(1-2 s) .
$$

This is referred to as the lower twice slip frequency sideband due to broken rotor bars. Therefore, there is a cyclic variation of current that causes a torque pulsation at twice slip frequency $\left(2 s f_{1}\right)$ and a corresponding speed oscillation, which is also a function of the drive inertia. 


\section{Conventional Methods}

Conventional techniques of detection of rotor faults, bearing faults, and air-gap eccentricity are based on spectrum analysis of motor voltage, current, and instantaneous input power, e.g., Fast Fourier Transformation (FFT) [3,4]. The accuracy of these techniques depends on the load condition, the signal-to-noise ratio (SNR) of the spectral components being examined, and the ability to maintain a constant speed to allow the operation of the FFT. Some methods use starting current for better SNR, but in practice the starting current is not always available $[5,6]$. The faults that cause rotor asymmetry such as the broken rotor bars can be detected by monitoring the stator current spectral components in Equation (1).

The presence of rotor faults is indicated by an amplitude difference of less than $50 \mathrm{~dB}$ between the fundamental frequency and the left sideband as shown in Fig. 2.

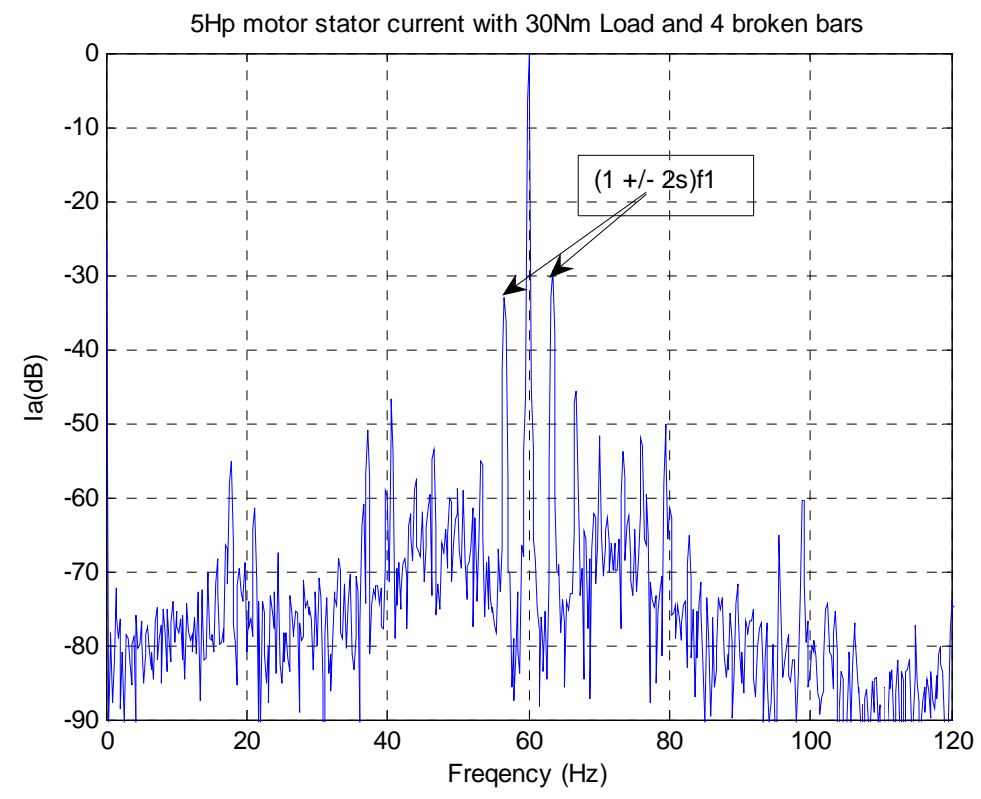

Fig. 2. Stator current spectrum of a $5 \mathrm{hp}$ induction motor with 4 broken rotor bars

Most of the conventional FFT based methods are based on the following assumptions:

$>$ The stator fundamental frequency is constant.

$>$ The load is constant.

$>$ The motor speed is constant and known.

$>$ The motor load is sufficient (i.e., slip is large enough) to separate the sidebands from the fundamental.

While the first assumption holds true for most direct line-connected motors, it does not apply to drive-connected motors. The other assumptions are load specific and therefore not valid in many applications with varying loads, such as compressors, material handling, etc. 


\section{Eaton's Intelligent Solution}

In order to address the challenge of detecting rotor faults under varying load conditions, Eaton has developed a new method using wavelet analysis of per phase input real power over one-cycle (instead of using FFT of motor current). Wavelet allows us to analyze nonstationary waveform and one-cycle average power allows us to detect fault characteristic frequency component $\left(2 s f_{1}\right)$ easily even if with lower per unit slip without removal of fundamental component. An interactive threshold adjusting method helps us to detect rotor faults under varying load conditions.

It can be proven by simple mathematics that for an induction motor with rotor fault, the current and instantaneous input power has the frequency components as shown in Table I.

Table I. Frequency components in the line current and instantaneous input power of an induction motor with rotor faults

\begin{tabular}{|c|c|c|}
\hline & Line Current & Instantaneous Power \\
\hline \multirow{3}{*}{$\begin{array}{c}\text { Frequency } \\
\text { Components }\end{array}$} & $f_{1}$ & $\mathrm{dc}$ \\
\cline { 2 - 3 } & $(1 \pm 2 k s) f_{1}$ & $2 f_{1}$ \\
\cline { 2 - 3 } & & $\left(2 \pm 2 k f_{1}\right.$ \\
\hline
\end{tabular}

Among these frequency components, the rotor fault related components are $2 k s f_{1}$. It needs to be separated out from the other frequency components. The $2 f_{1}$ and $(2 \pm 2 k s) f_{1}$ components are removed by just taking an average of instantaneous power for one fundamental cycle and dc will be removed by just subtracting mean from the signal so finally the remaining signal is having only $2 k s f_{1}$ components.

Then, the resultant waveform is passed through the wavelet analysis. The major advantage of wavelet over FFT or power spectrum analysis is its "multi-resolution" characteristic. Unlike the FFT that requires a certain length of stationary data to guarantee the frequency resolution, wavelet analysis is ideal for transient analysis, where signals have rich harmonics but non-stationary. 


\section{Test Results}

The method has been successfully tested and implemented for different ratings of the motors and the sample results are given here.

\section{HP Induction Motor (3 ph, 460V, 6.8A, 6 pole)}

Fault indexes for different load and different fault severity of a $5 \mathrm{hp}$ induction motor are given in Fig. 3.

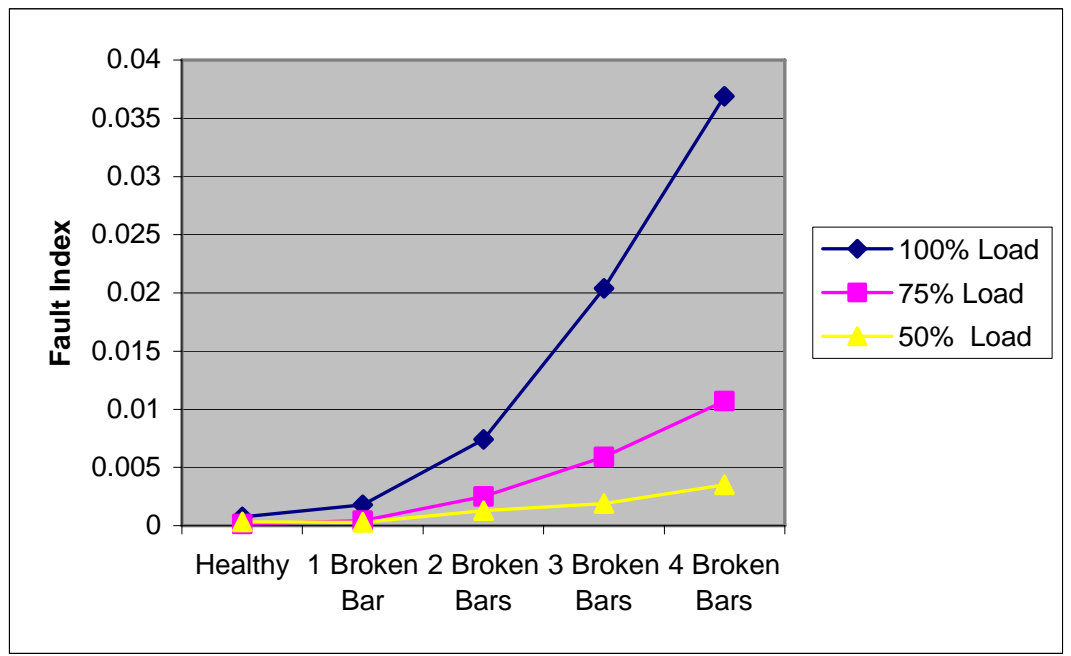

Fig. 3. Fault index Vs. Numbers of broken rotor bars and Load

\section{HP Induction Motor (3 ph, 230V, 5.4A, 2 pole)}

The same fault indexes for a $2 \mathrm{hp}, 100 \%$ loaded motor with different number of broken bars are illustrated in Fig. 4

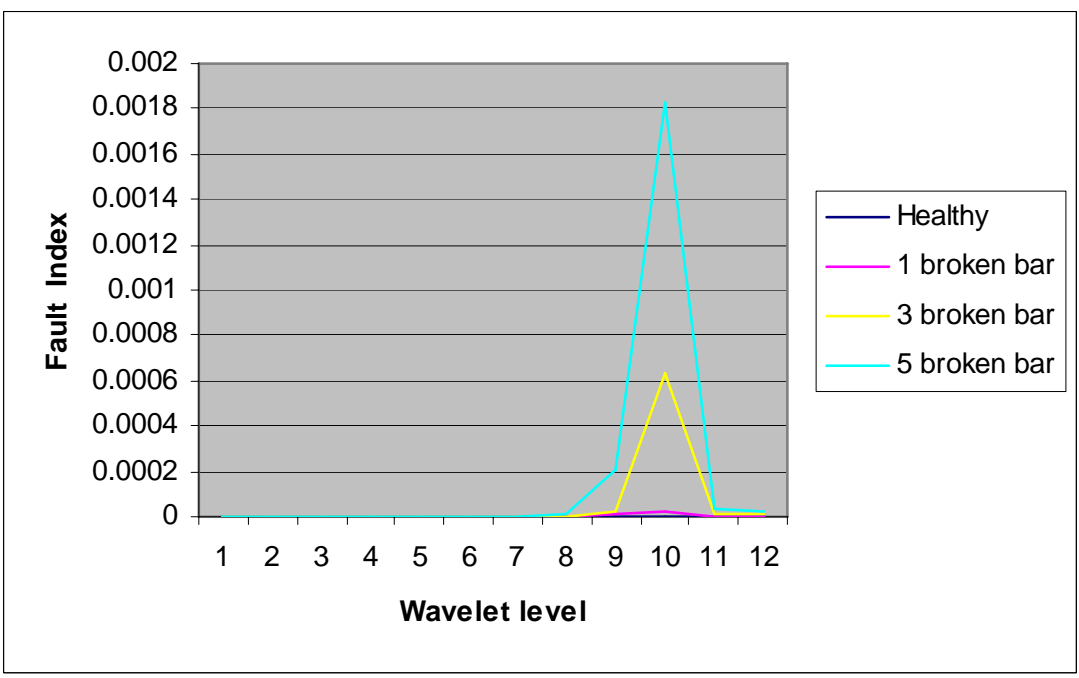

Fig. 4. Fault index Vs. Numbers of broken rotor bars and Wavelet levels 


\section{Deployment, Reference, and Contact}

\section{Deployment Requirements}

$>$ This method requires either single-phase or three-phase line voltages and currents.

$>$ This method requires the nameplate information of the machine.

$>$ This method requires initial baseline computation after deployment.

\section{References}

[1] H. W. Penrose, "Motor circuit analysis for energy, reliability and production cost improvements," ALLTEST Pro, LLC, Old Saybrook, CT.

[2] H. W. Penrose, "Test methods for determining the impact of motor condition on motor efficiency and reliability," ALL-TEST Pro, LLC, Old Saybrook, CT.

[3] G. Didier, E. Temisien, O. Caspa, H. Razik, H. Henao, A. Yazidi, and G.-A. Capolino, "Rotor fault detection using the instantaneous power signature," IEEE International Conference on Industrial Technology, vol. 1, pp. 170-174, Dec. 2004.

[4] Z. Liu, X. Yin, Z. Zhang, and D. Chen, "Online rotor mixed fault diagnosis way based on spectrum analysis of instantaneous power in squirrel cage induction motors," IEEE Trans. Energy Conversion, vol. 19, no. 3, pp. 485-490, Sep. 2004.

[5] H. Douglas, P. Pillay, and A.K. Ziarani, "Broken rotor bar detection in induction machines with transient operating speeds," IEEE Trans. Energy Conversion, vol. 20, no. 1, pp. 135-141, Mar. 2005.

[6] R. Supangat, N. Ertugrul, W.L. Soong, D.A. Gray, C. Hansen, and J. Grieger, "Broken rotor bar fault detection in induction motors using starting current analysis," 2005 European Conference on Power Electronics and Applications, Sep. 2005.

\section{Contact Us}

$\begin{array}{ll}\text { Name: } & \text { Dr. Bin Lu } \\ & \text { Mr. Manish Paghda } \\ \text { Address: } & \text { Eaton Corporation } \\ & \text { Innovation Center } \\ & \text { 4201 N. 27th Street } \\ & \text { Milwaukee, WI 53216 } \\ & \text { USA }\end{array}$

Email: $\quad$ binlu@eaton.com

Telephone: 1-(414)-449-6036

Fax: $\quad$ 1-(414)-449-6131 


\section{Motor Condition Monitoring Algorithms}

\section{Bearing Fault Detection \\ Via Stator Current Noise Cancellation}

Version: 1.0

\section{$\underline{\text { Key Features }}$}

$\checkmark$ Non-intrusive

Without interfering with normal operation

$\checkmark$ Online

Continuous bearing health monitoring

- Inferential

Using current, no additional sensors needed

- Low-cost

No additional sensors needed

- Simple

Independent of motor parameters

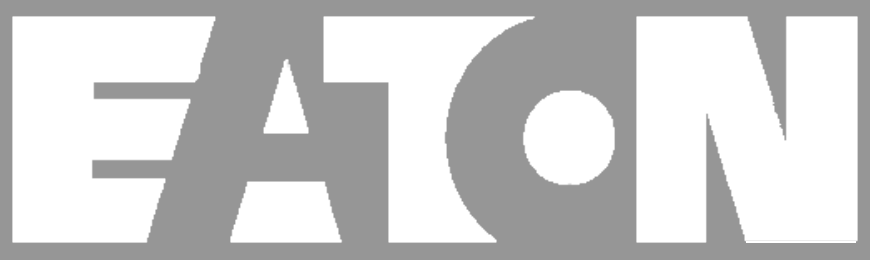

Dr. Bin Lu Eaton Corporation Innovation Center binlu@eaton.com

July 23, 2007 


\section{Background and Technical Challenges}

\section{Bearing Failures}

It is estimated that bearing failures account for approximately $41 \%$ of all machine failures. It is of practical importance to monitor bearing conditions in electrical machines to reduce the damage from bearing failures. Despite many bearing condition monitoring methods (e.g. vibration monitoring, temperature monitoring, chemical monitoring, acoustic emission monitoring, etc), vibration and current monitoring are very popular nowadays. Usually, vibration monitoring is reliable but expensive. In the contrast, current monitoring is economical and non-intrusive. Current monitoring is also suitable for remote monitoring performed in the motor control center.

\section{Why it is difficult to detect bearing faults via current monitoring?}

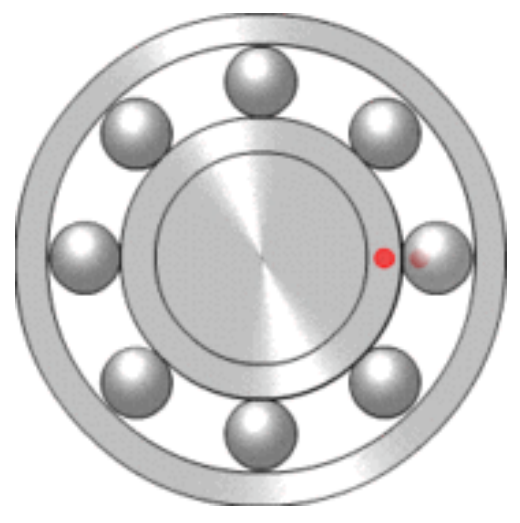

Detecting bearing faults via stator current is a known difficult problem in industry. The difficulty comes from the fact that bearing fault signatures are subtle in the stator current, and the fact that the research is limited in this area. For those in situ developed bearing faults, the fault frequencies are not predictable, which adds another difficulty. However, by some advanced technologies, it is still possible to detect bearing faults via stator current, simply because bearing-related components in machine vibration are, at some level, reflected in the stator current.

Most available current-based techniques rely on the observability of certain frequencies in the stator current. Consequently, they are most effective for detecting single-point defects in bearing surfaces. However, for realistic bearing faults that are developed on-line in industrial settings, especially at an early stage, those frequencies are not observable and may not exist at all. 


\section{Bring New Technology to Traditional Problems}

\section{What is Stator Current Noise Cancellation?}

The Stator Current Noise Cancellation Algorithm is developed for the early detection of in situ bearing faults. It is known that the dominant components in the stator current are not related to bearing faults and subtle bearing fault signatures can be well masked by those dominant components. This algorithm utilizes the advanced noise cancellation technology to separate the bearing fault-related and non-related components in the stator current; it does not rely on localizing specific frequencies. By effectively removing those dominant, bearing fault-unrelated components, the remaining components are more related to bearing faults.

Further analysis can be applied to the remaining components, i.e. the noise-cancelled stator current. Specifically, a warning threshold can be computed such that the measurement frequently falling outside the threshold means a deteriorated bearing condition. Maintenance action can be scheduled based on the processing results. This idea is illustrated by the following figure.

A schematic diagram of the noise cancellation process is illustrated in figure 2 . The SPC processor is described in the next section.

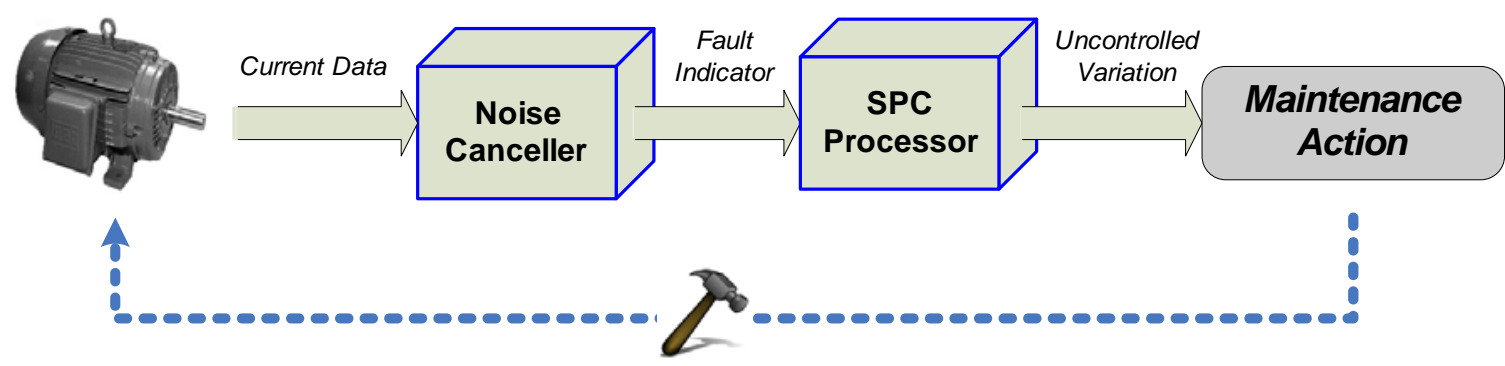

Figure 1: Illustration of current-based bearing condition monitoring by combining SPC and SCNC

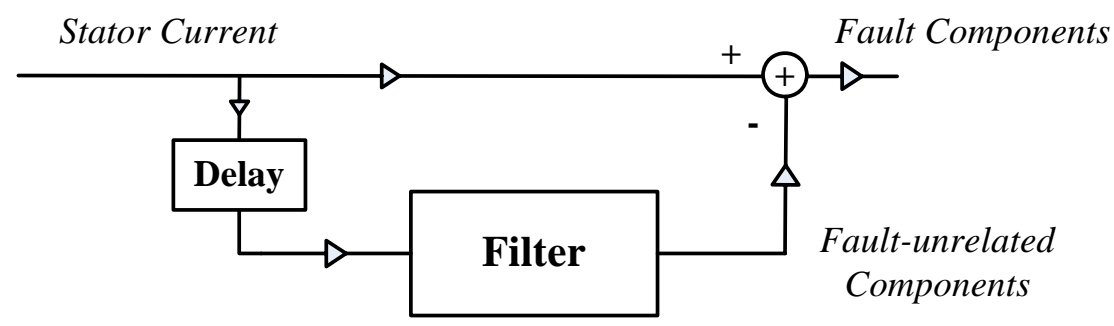

Figure 2: Illustration of the process of the stator current noise cancellation 


\section{Technical Description}

\section{Determination of the warning threshold based on SPC}

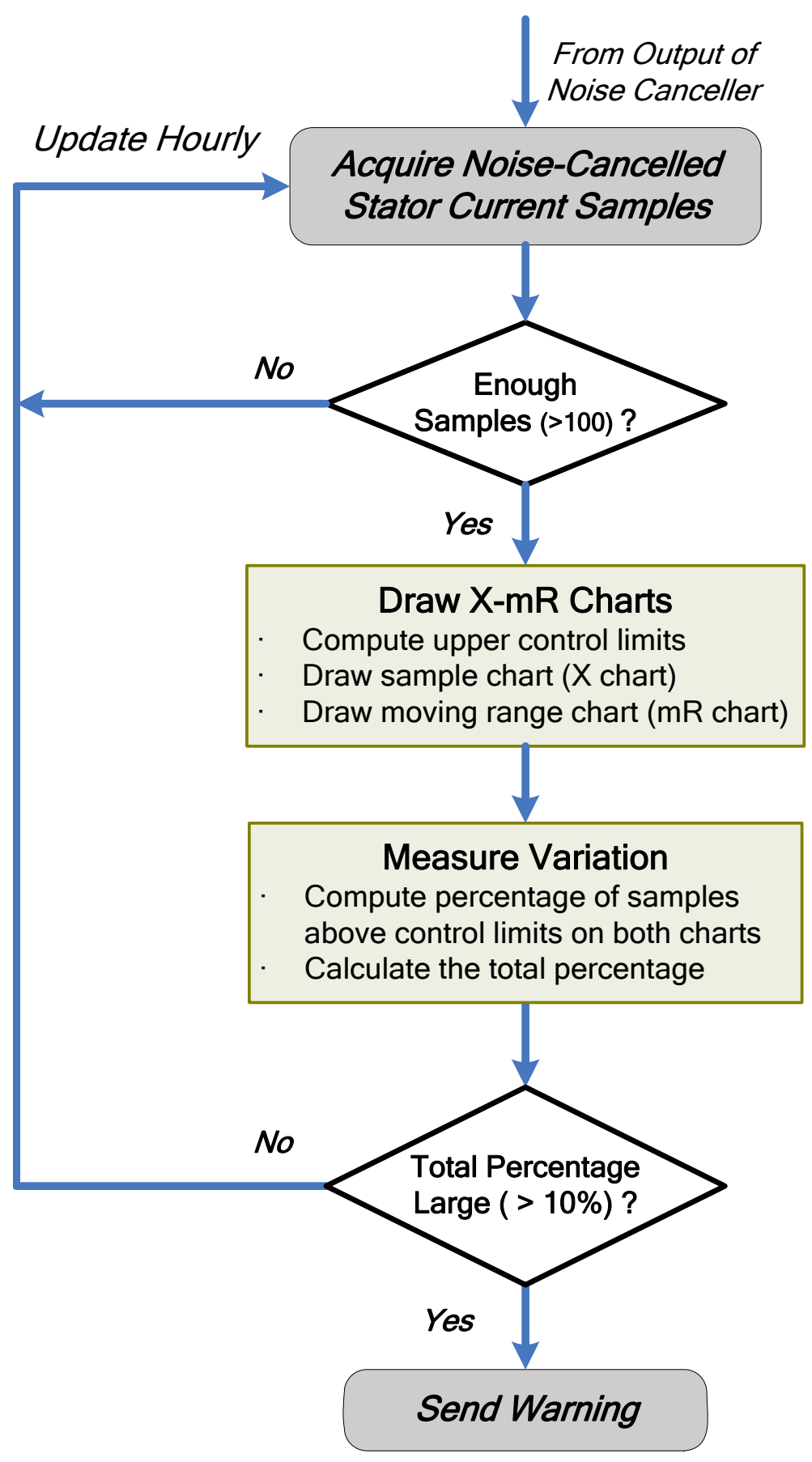

Figure 3: A block diagram of the statistical processor for bearing fault detection
The warning threshold is computed by using available stator current measurements only. Some statistical methods such as the Statistical Process Control serve this purpose.

The RMS value of the noisecancelled stator current can be treated as an output measurement of a complex machine-bearing process. The variation in the measurement can be attributed to random factors and/or assignable causes. Since dominant nonbearing fault components are already removed in the noisecancelled stator current, the uncontrolled variation is most likely caused by bearing faults. Thus, upon identifying uncontrolled variation in the noise-cancelled stator current, a possible bearing fault should be investigated.

A block diagram of the SPC Processor is illustrated in Figure 3. 


\section{Testing Results - Noise Cancellation}

\section{Sample Test Results}

Sample on-line test results are shown in Figure 4. Each point in top plot stands for the average of the RMS of the noise-cancelled stator current in each hour. The middle plot illustrates the difference between adjacent samples of the noise-cancelled stator current along the time. The bottom plot records the RMS of the machine vibration, which indicates that a bearing fault is developing in this test. Please note that a special process is used in the test to expedite the bearing aging process from a few years to less than a week (90 hours in Figure 4).

Figure 4 also shows the overall control limits (warning thresholds) on the top and the mid plots. It can be seen clearly that significant uncontrolled variation takes place on both the charts as the bearing condition degrades.
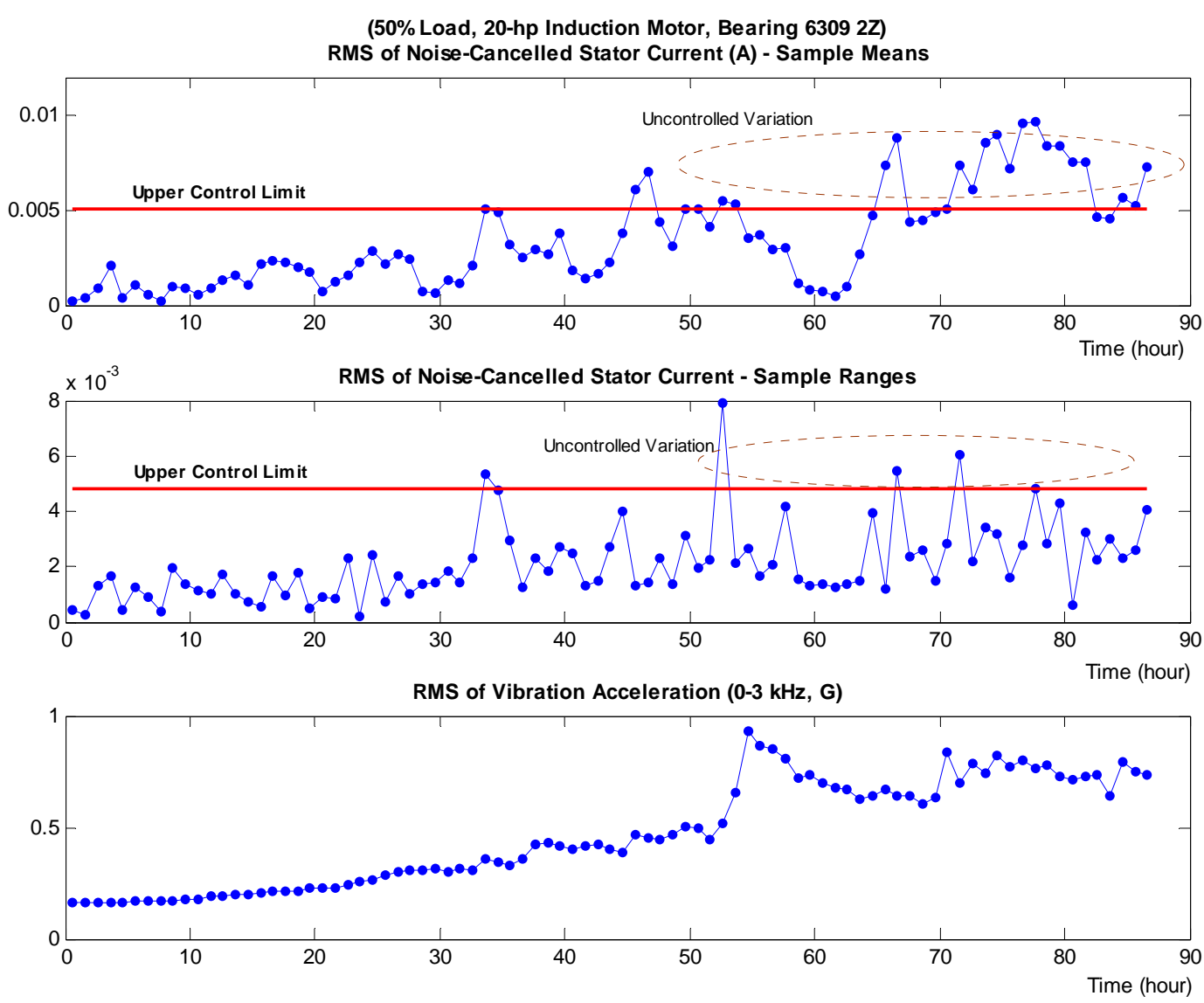

Figure 4: the control charts clearly show uncontrolled variation in the SCNC results as the bearing fault develops 


\section{Testing Results - Bearing Fault Indication}

To quantify the degree of uncontrolled variation in the process along the time, the percentage of the out-of-control $(\mathrm{OOC})$ samples was calculated, following the procedure described in Figure 3. The percentage of the OOC samples on the sample mean chart and the sample range chart, as well as the total percentage, are shown in Figure 5. As seen from the figure, around the 46th hour for this particular test, the total percentage of the OOC samples exceeded $10 \%$ and a warning message was sent. In addition, the total percentage of the out-of-control samples increases significantly along the time, from $3 \%$ to $23 \%$ during the whole experiment. Therefore, the results shown in these figures demonstrated the effectiveness of the stator current noise cancellation algorithm in on-line bearing fault detection.
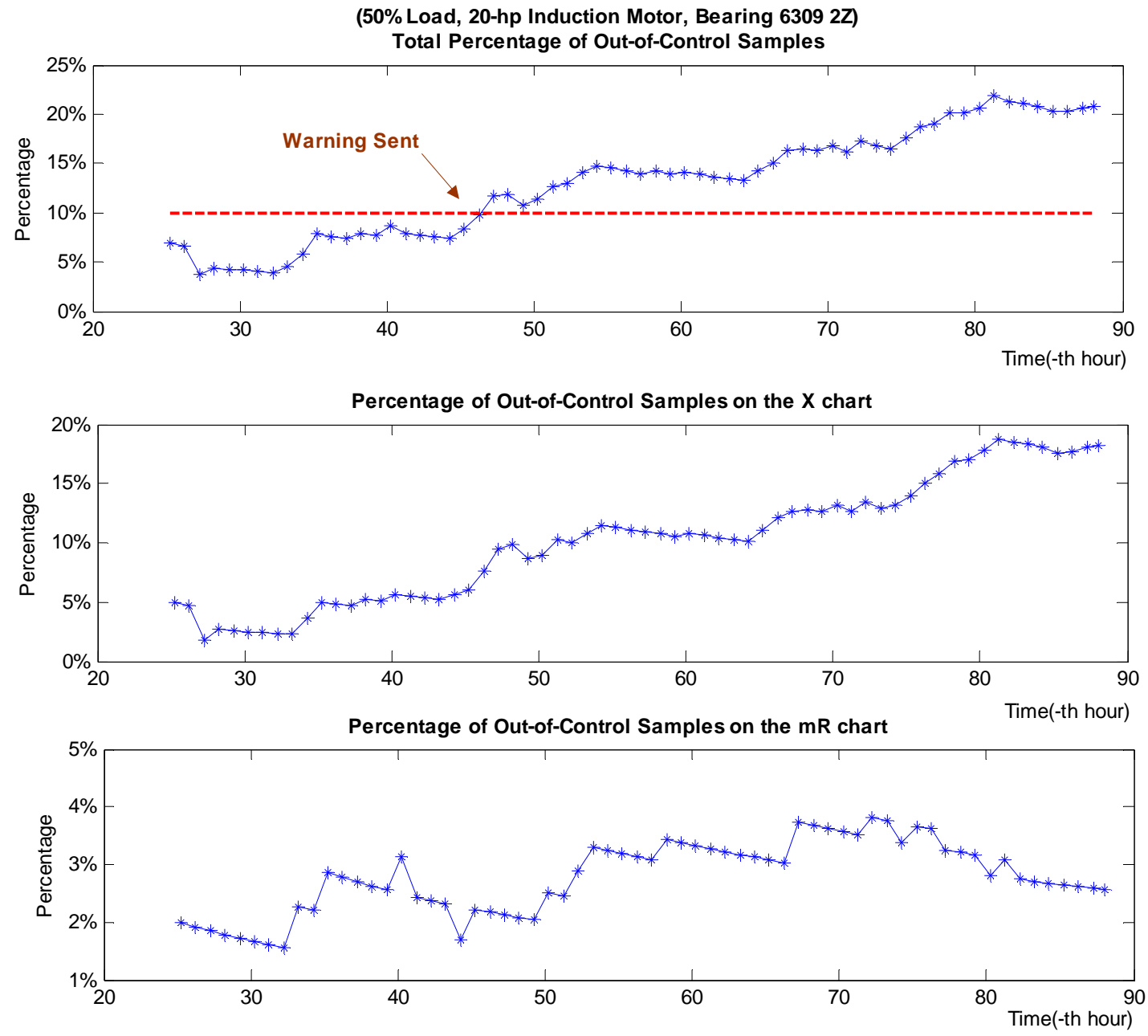

Figure 5: Percentage of the Out-of-Control Samples along the time for constant load experiment 


\section{References and Contact}

\section{References}

[1] T. K. Moon and W. C. Stirling, "Mathematical methods and algorithms for signal processing," Prentice Hall, Upper Saddle River, New Jersey, ISBN 0-201-36186-8, 2000

[2] A. V. Oppenheim and R. W. Schafer, "Discrete-Time Signal Processing," $2^{\text {nd }}$ Edition, Prentice Hall, Upper Saddle River, New Jersey, ISBN: 0-13-754920-2, 1999

[3] D. J. Wheeler and D. S. Chambers, "Understanding statistical process control," $2^{\text {nd }}$ edition, Tennessee: Knoxville, ISBN: 0-945320-13-2, SPC Press, 1992

[4] W. A. Shewhart, "Economic Control of Quality of Manufactured Product," American Society for Quality Press, Milwaukee, Wisconsin, ISBN: 0-87389-076-0, 1980

[5] Mechanical Vibration-Evaluation of Machine Vibration by Measurements on Nonrotating Parts—Part 3: General Guidelines, ISO 10816-3:1998(E), 1998.

[6] P. J. Tavner and J. Pennman, "Condition Monitoring of Electrical Machines," Letchworth, England: Research Studies Press Ltd. 1987.

[7] J. R. Stack, T. G. Habetler, and R. G. Harley, "Fault classification and fault signature production for rolling element bearings in electric machines," IEEE Transactions on Industry Applications, Vol. 40, pp. 735-739, May/June 2004.

\section{Contact Us}

Name: $\quad$ Dr. Bin Lu

Address: $\quad$ Eaton Corporation Innovation Center 4201 N. 27th Street Milwaukee, WI 53216 USA

Email: $\quad$ binlu@eaton.com

Telephone: 1-(414)-449-6036

Fax: $\quad 1-(414)-449-6131$ 


\section{White Paper}

\section{Motor Condition Monitoring Algorithms}

Power Quality Monitoring and Harmonic Analysis

Version: 1.3

\section{$\underline{\text { Key Features }}$}

$\checkmark$ Non-intrusive

Without interfering with normal operation

$\checkmark$ Online

Continuous and online monitoring

$\checkmark$ Inferential

Using only motor currents and voltages

$\checkmark$ Low-cost

No additional sensors needed 


\section{Importance and Problem Classification}

\section{Importance of Good Power Quality}

The quality of electric power has become an important issue for electric utilities and their customers. One of the main problems manufacturing industries is now facing is the distortion in the electrical supply. This power quality problem interrupts the sensitive manufacturing devices and results in very expensive consequences. With the everincreasing use of sophisticated controls and equipment in industrial, commercial, institutional, and governmental facilities, the continuity, reliability, and quality of electrical service has become extremely crucial to many power users. Electrical systems are subject to a wide variety of power quality problems, which can interrupt production processes, affect sensitive equipment, and cause downtime, scrap and capacity losses.

\section{How to Define Power Quality Problem?}

A power quality problem can best be described as, "any variations in the electrical power service, such as voltage dips and fluctuations, momentary interruptions, harmonics and transients, resulting in malfunction or failure of end-use equipment." The following are the major contributors to power quality problems.

\section{Impulsive Transient}

Rapidly occurring voltage sags caused by sudden and large increases in load current are called as "Impulsive Transients," as shown in Fig.1. Impulsive transient is most commonly caused by rapidly varying loads that require a large amount of reactive power such as welders, rock-crushers, sawmills, woodchoppers, metal shredders, and amusement rides. It can cause visible flicker in lights and cause other processes to shut down or malfunction.

\section{Momentary Interruption}

Zero-voltage events for small duration of time called as "Momentary Interruption," as shown in Fig.1. Momentary Interruption can be caused by weather, equipment malfunction, recloser operations, or transmission outages. Interruptions can occur on one or more phases and are typically short duration events. The vast majority of power interruptions are less than 60 seconds.

\section{Voltage Sag and Swell}

The science behind sag and swell can be described, as the sudden, large increase in the current required from a source will cause a larger voltage to be developed across the source impedance. This will result in a reduction in the voltage, as seen by the load, which is called sag. Like a surge, a sudden reduction in the current flow will cause an increase in voltage in inductive/capacitive impedances, which the load may experience is called as swell. These two are the most common power problems encountered. Sags are short-term reductions in voltage, and can cause interruptions to sensitive equipments, such as 


\section{Problem Classification Continues ...}

adjustable-speed drives, relays, and robots. The causes of sags are fuse or breaker operation, motor starting and capacitor switching etc. Sags can occur on multiple phases or on a single phase and can be accompanied by voltage swells on other phases.

\section{Notching}

Line notches are irregularities in the voltage waveforms that appear as notches, as illustrated in Fig.1. They are typically present in the waveform during SCR commutation. The duration of these notches in each ac voltage cycle is typically only a few microseconds, but they can last longer and cause equipment malfunction or resonance with damage or loss to neighboring electrical equipment or the processes they control.

\section{Harmonic Distortions}

Harmonic problems are generally caused by nonlinear loads such as adjustable speed drives, switching power supplies, electronic ballasts for fluorescent lighting, or arcing devices such as welders. Harmonic can cause nearby equipment, such as sensitive computer controls or digital clocks, to malfunction. They can also cause nearby transformers to overheat.
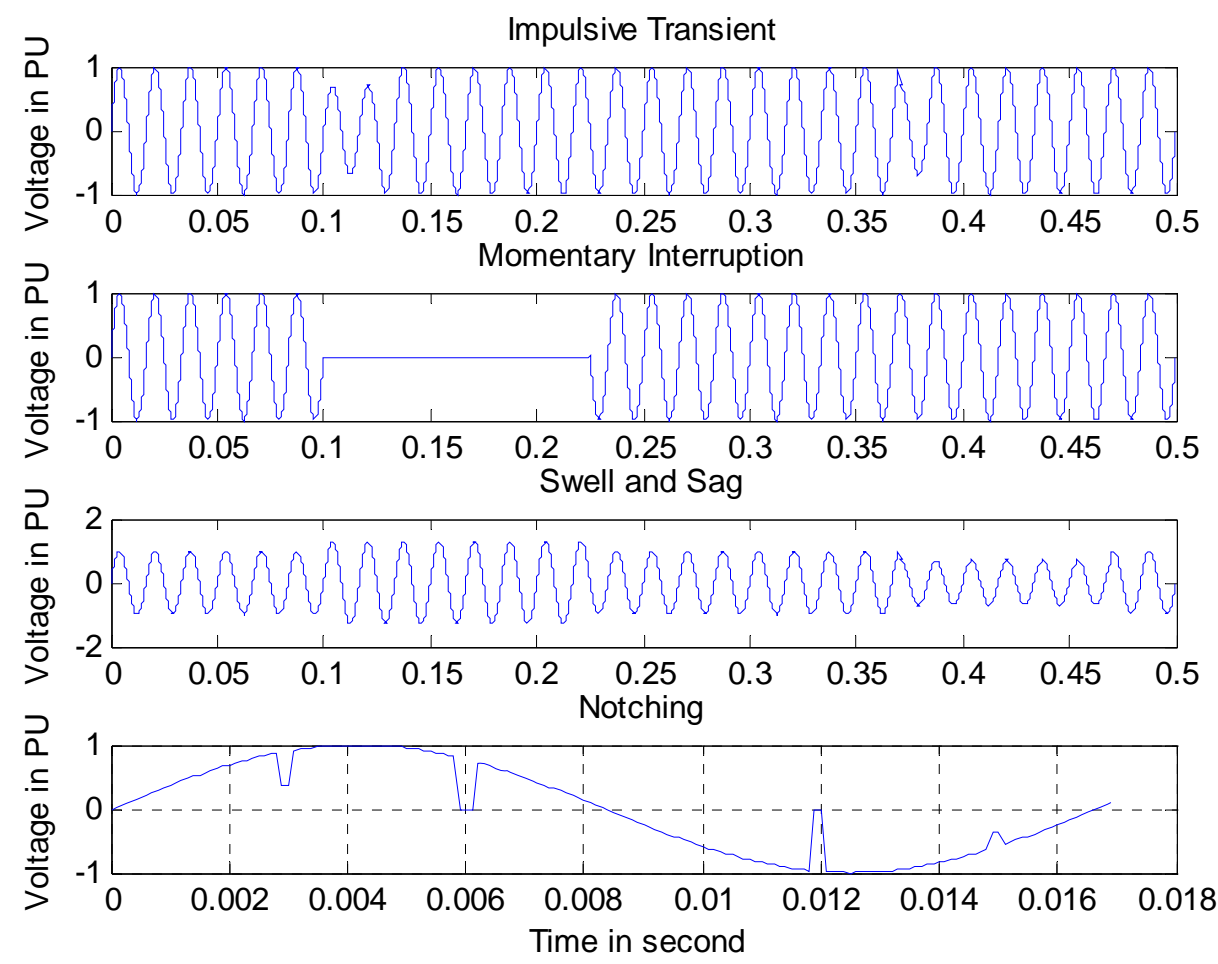

Fig.1. Different types of power quality problems. 


\section{Power Monitoring and Harmonic Analysis}

\section{Eaton's Power Monitoring and Harmonic Analysis Algorithms}

To improve power quality, sources of disturbances must be identified and controlled. Power quality disturbance waveform recognition is often troublesome, because it involves a broad range of disturbance categories. The decision boundaries of disturbance features may overlap. Power quality monitoring and analysis must be able to detect, localize, estimate, and classify various disturbances for the electric power system.

Today, power quality monitoring is an essential service that many utilities perform for their industrial and key commercial customers. As the technology are now available, power quality monitoring is highly effective. The monitoring system can provide information about the quality of the power and the causes of power system disturbances. It can also identify problematic conditions throughout the system before they cause catastrophic failures. Power quality problems are not necessarily limited to the utility power system. Many surveys have shown that the majority of problems are localized within customer facilities.

To provide advanced monitoring and protection functions in our industrial control products, Eaton has developed a comprehensive set of three-phase power monitoring and harmonic analysis algorithms, as summarized in Table I. Besides the numerical results, these algorithms also provide some graphical representations, such as voltage and current spectrum, and voltage swell/sag event curve (ITIC curve).

Table I. Different monitoring parameters

\begin{tabular}{|c|c|c|}
\hline \multicolumn{2}{|c|}{ Eaton Power Monitoring and Harmonic Analysis Algorithms } & \multirow{2}{*}{ Unit } \\
\hline General & Specific & \\
\hline Frequency & System frequency & $\mathrm{Hz}$ \\
\hline Voltage RMS & RMS of all three line to line voltages & Volt \\
\hline Current RMS & RMS of all three phase currents & Amp \\
\hline Voltage Harmonics & 1st, 3rd and 5th order harmonics & Volt \\
\hline Current Harmonics & 1st, 3rd and 5th order harmonics & Amp \\
\hline \multirow{4}{*}{$\begin{array}{c}\text { Positive and Negative Sequence } \\
\text { Components at Fundamental } \\
\text { Frequency }\end{array}$} & RMS of positive-sequence phase voltage & Volt \\
\hline & RMS of negative-sequence phase voltage & Volt \\
\hline & RMS of positive-sequence phase current & Amp \\
\hline & RMS of negative-sequence phase current & Amp \\
\hline Sequence impedances & Positive and negative sequence impedance & Ohm \\
\hline Total Harmonic Distortion (THD) & THD of three-phase voltages and currents & - \\
\hline K-factor & Overall voltage and current $\mathrm{K}$-factors & - \\
\hline Unbalance Factor & Voltage, current, and frequency unbalance factor & - \\
\hline Power Factor & Distortion, displacement, and overall power factors & - \\
\hline Crest Factor & Crest Factor for three-phase voltages and currents & - \\
\hline \multirow{2}{*}{ Derating Factor } & Voltage unbalance derating factor & - \\
\hline & Voltage harmonics derating factor & - \\
\hline
\end{tabular}




\section{Key Features}

The key features of our power monitoring and harmonic analysis algorithms are listed in this section.

\section{RMS Value}

The RMS value internally updated after every sample (Not after every cycle), using concept of circular buffer and Simpson's 1/3 rule.

\section{Harmonics}

Harmonics up to $33^{\text {rd }}$ order are calculated for $60 \mathrm{~Hz}$ fundamental signals with high resolution. Based on these harmonic contents, the total harmonic distortion (THD) and Kfactors are also calculated. Frequency/power spectra of votage and current are also provided, as shown in Fig.2.

\section{Unbalance Factor}

The unbalances (i.e., ratio of positive and negative sequence component) in voltage and current are derived according to the IEEE and NEMA standards [1].

\section{Sequence Impedance}

The sequence impedances (positive and negative) are derived for $1^{\text {st }}, 3^{\text {rd }}$ and $5^{\text {th }}$ Harmonic order using positive and negative sequence voltages and currents.
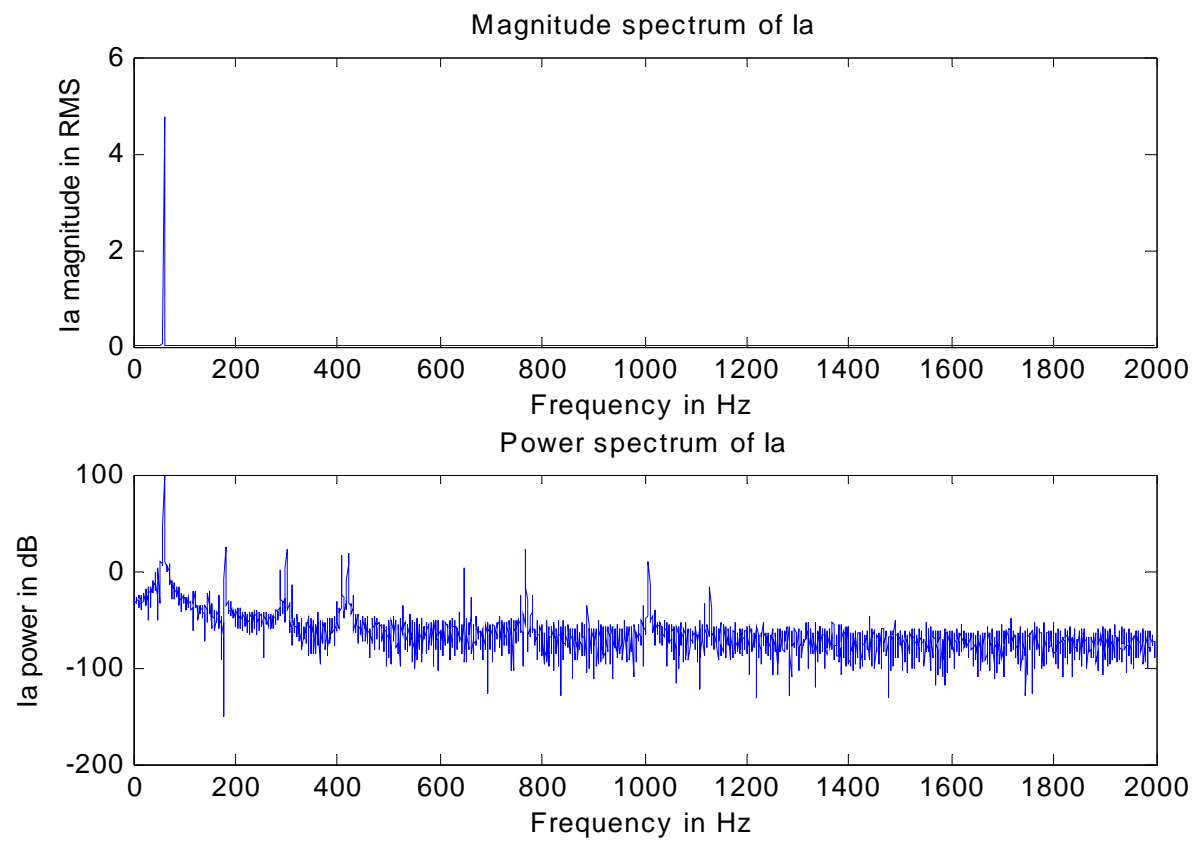

Fig.2. Magnitude and power spectrum of phase-A current 


\section{Key Features Continue...}

\section{Swell and Sag}

Swell and sag in supply voltage are detected according to ITIC (Information Technology Industry Council) curve [2]. ITIC curve defines the limit of change in voltage with time duration, which will not affect the performance of the instrument. The ITIC curve is shown in Fig. 3 with two events violating the curve.

\section{Power Factor}

Due to the use of nonlinear loads, the current and voltage waveforms are not purely sinusoidal. Under such cases, only displacement power factor is not enough for further analysis. Here four types of power factors are calculated and displayed as given in Table I.

\section{Derating Factor}

When unbalances and harmonic contents present in supply, the instrument rating has to be derated. Considering the 3-phase motor application, two derating factors have been derived here using NEMA standard curves.

(1) Voltage unbalance derating factor

(2) Voltage harmonic derating factor

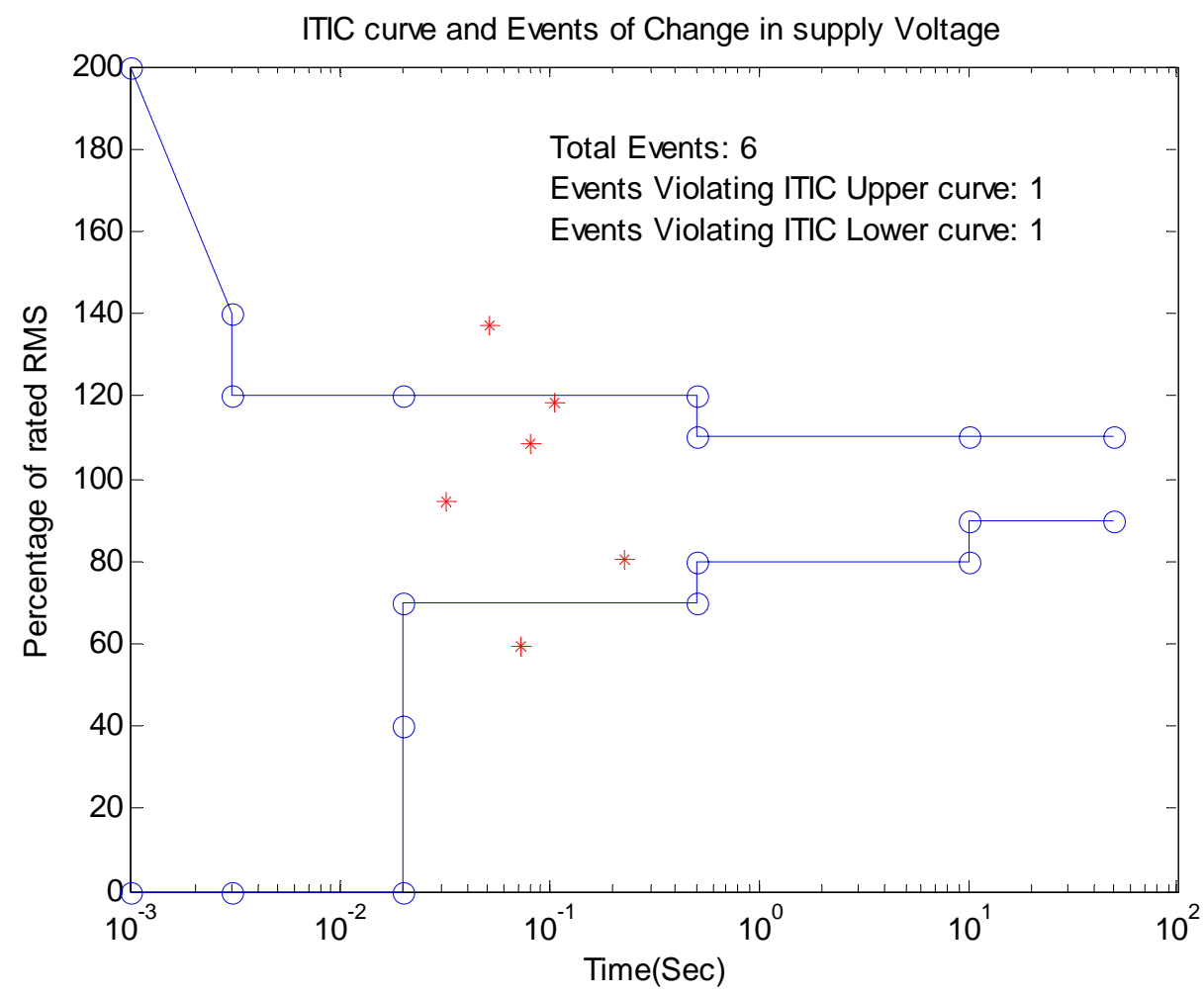

Fig.3. ITIC curve and voltage swell and sag events. 


\section{Reference and Contact}

\section{References}

[1] National Electrical Manufacturers Association (NEMA), "Information guide for general purpose industrial AC small and medium squirrel - cage Induction Motor standard."

[2] ITIC curve published by Technical Committee 3 (TC3) of the Information Technology Industry Council (ITI, formerly known as the Computer \& Business Equipment Manufacturers Association). http://www.itic.org/technical/iticurv.pdf

\section{Contact Us}

Name: $\quad$ Dr. Bin Lu

Address: $\quad$ Eaton Corporation Innovation Center 4201 N. 27th Street Milwaukee, WI 53216 USA

Email: $\quad$ binlu@eaton.com

Telephone: 1-(414)-449-6036

Fax: $\quad 1-(414)-449-6131$ 


\section{Induction Motor Rotor Fault Diagnosis using Wavelet Analysis of One-Cycle Average Power}

\author{
Bin $\mathrm{Lu}$ \\ Innovation Center \\ Eaton Corporation \\ Milwaukee, WI 53216, U.S.A. \\ Email:binlu@ieee.org
}

\author{
Manish Paghda \\ Innovation Center \\ Eaton Technologies Pvt. Ltd. \\ Pune, India \\ Email: ManishPaghda@Eaton.com
}

\begin{abstract}
Conventional techniques of detection of broken rotor bars, bearing faults and air-gap eccentricity were based on frequency domain analysis of voltage, current, and instantaneous input power. The accuracy of these techniques depends on the loading conditions of the machine, the signal-to-noise ratio of the spectral components being examined, and the ability to maintain a constant speed. This paper presents a method for induction motor rotor fault diagnosis using wavelet analysis with higher signal to noise ratio under varying load conditions. It also includes an interactive technique to detect broken rotor bar in varying load conditions. The fault severity is derived by wavelet analysis of single-phase active one-cycle average power. Wavelet allows analyzing non-stationary waveform and one-cycle average power allows detecting fault characteristic frequency component under low load conditions without removing the fundamental component. Finally, the proposed method is verified from the experimental results of two induction motors with different configurations.
\end{abstract}

Keywords - motor diagnosics, wavelet analysis, discrete wavelet transform, interactive threshold method.

\section{INTRODUCTION}

There are around 1.2 billions of electric motors used in the United States, which consume about $57 \%$ of all generated electric power. Over $70 \%$ of the electrical energy used by manufacturing and $90 \%$ in process industries are consumed by motor driven systems [1]. Among these motor systems, squirrel-cage induction motors (SCIM) have a dominant percentage because of their simple design, rugged performance, and easy maintenance.

It is estimated that about $38 \%$ of the induction motor failures are caused by stator winding faults, $40 \%$ by bearing failures, $10 \%$ by rotor faults, and $12 \%$ by miscellaneous faults. Rotor faults may appear less significant than the bearing faults, but it is critical to understand that bearing failures are often resulted from shaft current and increased vibration, which are caused by shaft misalignment, rotor eccentricity, and other rotor related faults. Besides, rotor faults can also result in excess heat, decreased efficiency, reduced insulation life, and iron core damage. Therefore, early detection of impending rotor faults and appropriate maintenance can avoid more severe motor failures. Many industrial surveys have also shown that rotor faults tend to be more common for medium and high voltage motors due to the higher mechanical and thermal stresses [2].
Most conventional methods of detecting rotor faults, bearing faults, and air-gap eccentricity are based on spectrum analysis of motor voltage, current, and instantaneous input power, e.g., Fast Fourier Transform (FFT) [3, 4]. The applicability and accuracy of these methods are greatly affected by the load conditions, signal-to-noise ratio (SNR), and the amount of time domain data available for analysis. Some methods use motor starting current for better SNR, but in practice the starting current is not always available to the diagnostic device $[5,6]$. Most of the FFT based methods are based on the following assumptions:

1. The stator fundamental frequency is constant.

2. The load is constant

3. The motor speed is constant and known.

4. The motor load is sufficient (i.e., slip is large enough) to separate the sidebands from the fundamental.

While the first assumption holds true for most direct lineconnected motors, it does not apply to drive-connected motors. The other assumptions are load specific and therefore not valid in many applications with varying loads, such as compressors.

To address the challenge of rotor fault diagnosis under varying load conditions, the paper describes a new method using wavelet analysis of per phase input power over one cycle. Wavelet allows analyzing non-stationary waveform and onecycle average power allows detecting fault characteristic frequency component under low load conditions without removing the fundamental component. An interactive threshold adjusting method is proposed to detect rotor faults under varying load conditions. Finally, the method has been verified by the experimental results from two induction motors with different number of broken bars at various load conditions.

\section{WAVELET THEORY}

Wavelet transform provides flexibility in describing signals that include regions of different frequency contents. It is important for power quality problems and variable load applications.

The wavelet transform is an expansion of a given waveform into a space defined by a set of orthogonal or orthonormal functions, namely the wavelets. Many different wavelet functions have been proposed. The functions can be continuous (continuous wavelet transform) or discrete (discrete wavelet transform). 


\section{A. Continuous Wavelet Transform}

Unlike Fourier transform, the technique based on wavelets allows to perform, through a multi-resolution analysis (MRA), several overlapped projections of a signal. For a signal $f(t)$, the generating function of the MRA can be expressed as

$$
\varphi_{k}^{j}(t)=2^{-j / 2} \varphi\left(2^{-j} t-k\right),
$$

where, $\varphi$ is the so-called mother wavelet; $j$ indicates the decomposition level, and $k$ is the time shift factor. The wavelet coefficients obtained by applying an orthogonal wavelet are

$$
d_{k}^{j}=\int_{-\infty}^{\infty} f(t) \psi_{k}^{j}(t) d t
$$

where, $\psi_{k}^{j}$ is the wavelet analyzing function. For instance, Debauche, Haar, Morlet, Shannon, etc, could be used.

In short, the mother wavelet is scaled and shifted continuously. At each stage, it is correlated with the original signal and the correlation produces wavelet coefficients.

\section{B. Discrete Wavelet Transform}

In discrete wavelet transform, the mother wavelet is not scaled continuously, but scaled in the power of 2 . Hence, it is easy to implement in digital computers and takes less execution time.

Let $S$ be a discrete-time signal to be decomposed into its approximate and detailed versions using the discrete wavelet analysis. The first level decomposition coefficients are $c A_{1}$ and $c D_{1}$; where, $c A_{1}$ is the approximate version of $S$, and $c D_{1}$ is the detailed representation of $S . c A_{1}$ and $c D_{1}$ are defined as

$$
\begin{aligned}
& c A_{1}=\sum_{k}^{n} L(k-2 n) S(k), \\
& c D_{1}=\sum_{k}^{n} H(k-2 n) S(k),
\end{aligned}
$$

where, $H$ and $L$ are the decomposition filters of $S(n)$ in $c D_{1}$ and $c A_{1}$, respectively.
The next (second) decomposition level is based on cA1 and the coefficients are given by

$$
\begin{aligned}
& c A_{2}(n)=\sum_{k}^{n} L(k-2 n) c A_{1}(k), \\
& c D_{2}(n)=\sum_{k}^{n} H(k-2 n) c A_{1}(k) .
\end{aligned}
$$

Higher-level decompositions can be obtained in a similar fashion. The coefficients $A_{\mathrm{j}}$ and $D_{\mathrm{j}}$ are computed using the tree decomposition algorithm, which allows storing low frequency information of the signal as well as the discontinuities. $H$ and $L$ represent the decomposition filters and $\downarrow 2$ denotes a down sampling by a factor of 2 . Thus, it can be concluded that $c A_{1}$ is the approximate version of the original signal $S$. $L$ behaves as a low-pass filter. If $c D_{1}$ contains only high frequency components of signal $S$, then $H$ behaves as a high-pass filter.

Fig.1 shows the analysis and synthesis of signal $S$ using wavelet decomposition. $H^{\prime}$ ' and $L^{\prime}$ ' are the time-reversed $H$ and $L$ filters, respectively. Once the detail and the approximate coefficients are obtained, the original signal can be reconstructed. In general, this process can be expressed as

$$
S^{\prime}=A_{\mathrm{n}}+D_{\mathrm{n}}+D_{(\mathrm{n}-1)}+D_{(\mathrm{n}-2)}+\ldots \ldots \ldots D_{3}+D_{2}+D_{1} .
$$

Ideally $S^{\prime}$ should be equal to $S$, if the detail and approximate coefficients before reconstruction are not modified. Fig. 2 shows frequency distribution up to 4 levels of decomposition.

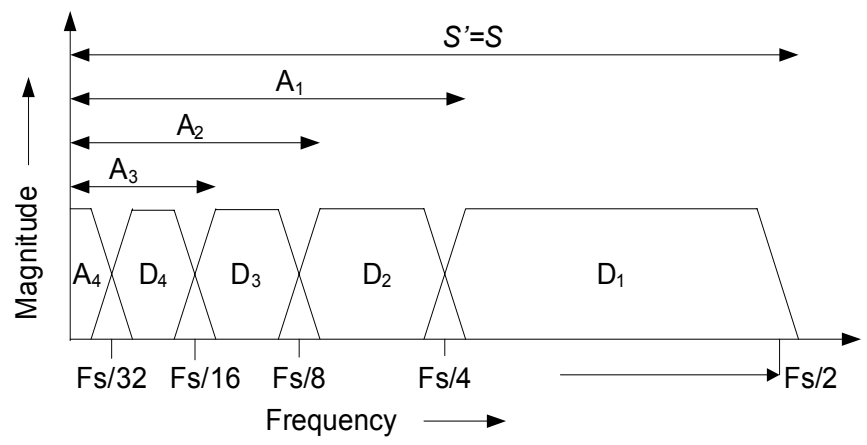

Fig. 2. Frequency distribution in detail and approximate coefficients.

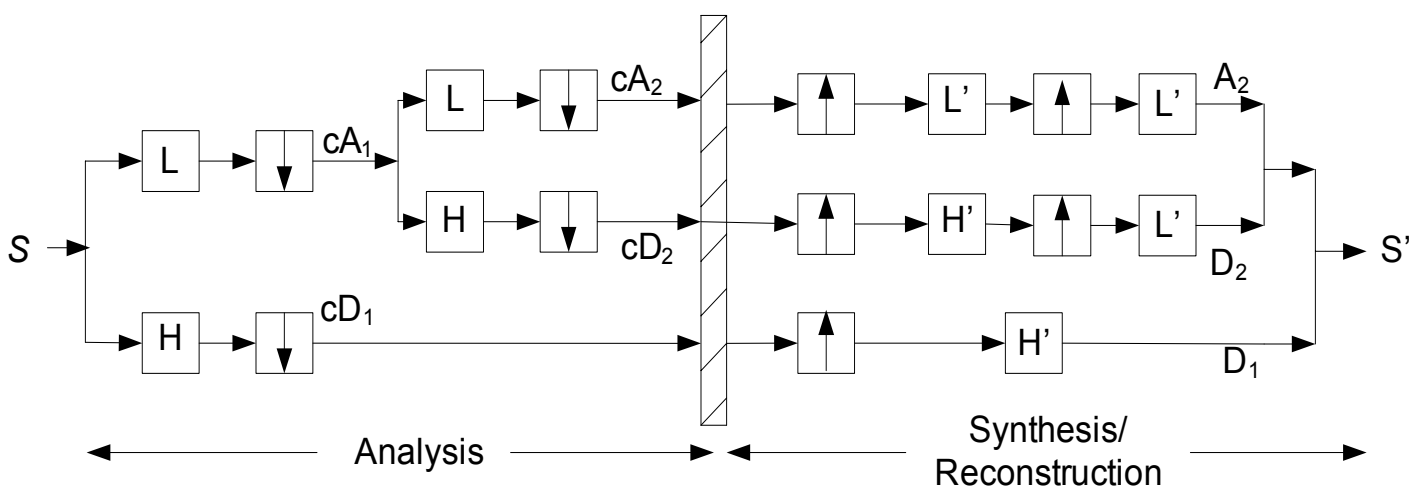

Fig. 1. Wavelet analysis and synthesis of a signal $S$. 


\section{INDUCTION MOTOR ROTOR FAULTS}

In induction motor, the rotor faults, such as the broken rotor bar, can be caused by the following reasons:

1. Direct on-line starting with duties, for which the rotor cage is not designed to withstand, can cause high thermal and mechanical stresses.

2. Pulsating mechanical loads, such as reciprocating compressors and coal crushers, can cause high mechanical stresses to the rotor cage.

3. Imperfections in the manufacturing process of the rotor cage.

According to the space vector analysis, a 3-phase symmetrical stator winding fed from a symmetrical supply with fundamental frequency $f_{1}$ will produce a resultant forward rotating magnetic field at the synchronous frequency in the airgap. When this magnetic field is applied to the rotor that rotates at electrical frequency $(1-s) f_{1}$, a current at slip frequency (i.e., $f_{2}=s f_{1}$ ) is induced in the rotor cage, where $s$ is the slip. Any asymmetry of the supply or stator winding impedances will cause a negative sequence component in the stator current, which, in turn, results in a backward magnetic field in the airgap rotating at synchronous frequency $\left(-f_{1}\right)$.

The rotor current $\left(f_{2}\right)$ produces a rotating magnetic field with the same number of poles as the stator field but rotating at the slip frequency $f_{2}$ with respect to the rotating rotor. With a symmetrical rotor cage, only a forward rotating field exists $\left(f_{2}\right)$. When rotor asymmetry such as broken rotor bar exists, there will also be a resultant backward rotating field at slip frequency $\left(-f_{2}\right)$ with respect to the forward rotating rotor. As a result, this backward rotating field $\left(-f_{2}\right)$ induces an EMF and current in the stator winding at the following frequency,

$$
f_{s b}=f_{1}(1-2 s) \text {. }
$$

This is referred to as the lower twice slip frequency sideband due to broken rotor bars. Therefore, there is a cyclic variation of current that causes a torque pulsation at twice slip frequency ( $\left.2 s f_{1}\right)$ and a corresponding speed oscillation, which is also a function of the drive inertia.

\section{Rotor Fault Signature In InStantaneOUS Motor INPUT POWER}

With $u_{\mathrm{ab}}$ and $i_{\mathrm{a}}$ being the line voltage and phase current, the instantaneous input power of a three-phase motor is given by

$$
p(t)=\sqrt{3} u_{a b} i_{a} .
$$

\section{A. Instantaneous Power of a Healthy Motor}

Neglecting the inherent asymmetry of a normal SCIM, the line voltage and stator current can be written as

$$
\begin{aligned}
& u_{a b}=U_{m} \cos \omega_{1} t \\
& i_{a}=I_{m} \cos \left(\omega_{1} t-\varphi\right)
\end{aligned}
$$

where,

$$
\begin{aligned}
& U_{m}: \text { Peak value of the supply line voltage; } \\
& I_{m}: \text { Peak value of the supply fundamental current; } \\
& \omega_{1}: \text { Supply angular frequency }\left(\omega_{1}=2 \pi f_{1}\right) ; \\
& \varphi: \text { Initial phase angle of the fundamental supply current. }
\end{aligned}
$$

The instantaneous input power at this condition can be expressed as

$$
p(t)=\frac{\sqrt{3}}{2} U_{m} I_{m}\left[\cos \left(2 \omega_{1} t-\varphi\right)+\cos \varphi\right] .
$$

Equation (8) indicates that the spectrum of the current has only the fundamental component at frequency, while, (9) indicates that the spectrum of the instantaneous input power has a component at frequency of $2 f_{1}$ and a dc component, and the latter is the average input power.

\section{B. Instantaneous Power of a Motor with Broken Rotor Bars}

In the case of a faulty rotor, the rotor current deviates from the current distribution of a healthy cage, and the fault characteristic components at frequency of $(1 \pm 2 k s) f_{1}$ appear in the stator current, as

$i_{a}=I_{m} \cos \left(\omega_{1} t-\varphi\right)+\sum_{k=1}^{\infty}\left\{\begin{array}{l}I_{b p k} \cos \left[(1-2 k s) \omega_{1} t-\beta_{b p k}\right] \\ +\left\{I_{b n k} \cos \left[(1+2 k s) \omega_{1} t-\beta_{b n k}\right]\right\}\end{array}\right\}$,

where,

$I_{b p k}$ : Peak value of $(1-2 k s) f_{1}$ component;

$I_{b n k}$ : Peak value of $(1+2 k s) f_{1}$ component;

$\beta_{b p k}$ : Initial phase angle of the $(1-2 k s) f_{1}$ component;

$\beta_{b n k}$ : Initial phase angle of the $(1+2 k s) f_{1}$ component.

Similarly, the instantaneous power can be described as

$$
\begin{aligned}
& p(t)=\frac{\sqrt{3}}{2}\left\{U_{m} I_{m}\left[\cos \left(2 \omega_{1} t-\varphi\right)+\cos \varphi\right]\right. \\
& +\sum_{k=1}^{\infty}\left\{U_{m} I_{b p k}\left[\cos \left((2-2 k s) \omega_{1} t-\varphi_{b p k}\right)+\cos \left(2 k s \omega_{1} t+\varphi_{b p k}\right)\right]\right. \\
& \left.\left.+U_{m} I_{b n k}\left[\cos \left((2+2 k s) \omega_{1} t-\varphi_{b n k}\right)+\cos \left(2 k s \omega_{1} t-\varphi_{b n k}\right)\right]\right\}\right\}
\end{aligned}
$$

It can be seen from (11) that the spectrum of the instantaneous input power contains:

1. A dc component,

2. A component at frequency of $2 f_{1}$,

3. Components at frequencies of $(2 \pm 2 k s) f_{1}$,

4. Additional components at frequencies of $2 k s f_{1}$.

The additional components at $2 k s f_{1}$ are caused by the rotor faults. They can be used as the characteristic signature to detect rotor faults. The frequencies of these characteristic components are distinct from the supply frequency $f_{1}$, enabling the rotor faults to be detected. 


\section{Rotor FaUlt Diagnosis USIng WaVelet ANalysis}

As mentioned above, rotor fault characteristic components exist in both motor current and in instantaneous input power, when rotor fault exists. In this paper, a one-cycle average input power (average of instantaneous input power over one electric cycle) is used to detect the fault signature frequency. The average input power is used in the method because it eliminates the $2 f_{1}$ component and the dc component can be easily filtered out to achieve better SNR.

Different wavelet decomposition levels of average input power give different frequency ranges from $F_{\mathrm{S}} / 2$ to $F_{\mathrm{S}} / 32$ and further down to $0 \mathrm{~Hz}$, as shown in Fig. 2, where $F_{\mathrm{S}}$ is the analog to digital sampling frequency. Using the slip of the motor and stator frequency, the fault signature component $2 k_{s} f_{1}$ can be found. Based on this rotor fault characteristic frequency, the corresponding wavelet level can be determined. Finally, the rotor fault can be detected/predicted by comparing the energy level of that specific wavelet with baseline value or preset threshold. In case of broken bar faults, the number of broken bar could be estimated also. The overall block diagram of the proposed method is illustrated in Fig. 3.

In the experiments, overlapping of frequencies between two successive signal levels has been observed. This can be avoided by properly selecting the mother wavelet. In this paper, Daubechies-40 is used as the mother wavelet [7]. Level D9 to D14 and A14 and their respective frequency ranges are shown in Fig. 4 (with $F_{\mathrm{S}}$ being $4 \mathrm{kHz}$ as an example). In the experiment presented in Section VII, the fault signature $2 \mathrm{ks} f_{1}$ is $3.5031 \mathrm{~Hz}$, which falls in level 10 (D10). Then, the signal energy of the interested D10 level is computed. Finally, the fault index is calculated as the per unit energy of the D10, taking the energy of A14 (dc component) as the base value.

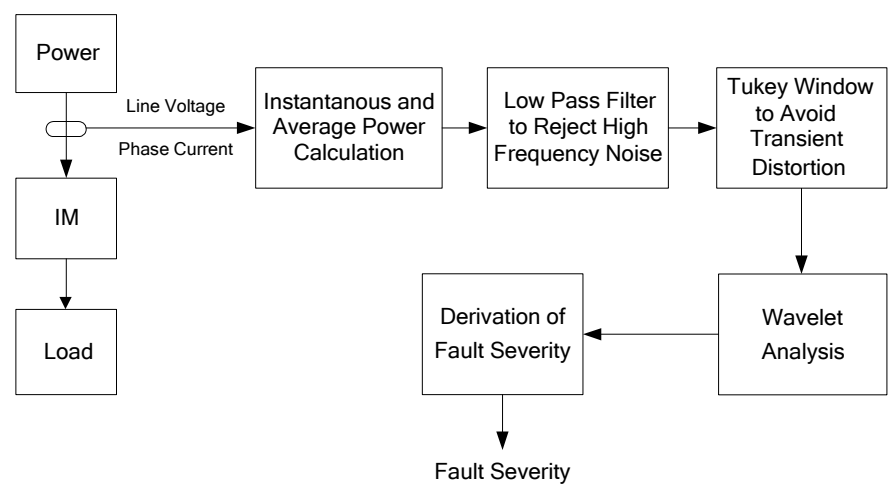

Fig. 3. Overall block diagram of the proposed method.

\section{FAult SEVERITy Determination Scheme}

In practice, by just observing the energy of the interested level, the fault severity cannot be predicted reliably. This is because of the following challenges:

1. It is possible that the frequency of interest $2 s f_{1}$ falls on the borders of a particular level instead of the center of that level.

2. The amplitude of the fault signature component $2 s f_{1}$ is different for different ratings of motor running under the same load.

3. The amplitude of the fault signature component $2 s f_{1}$ is not a constant in variable load conditions for the same motor.
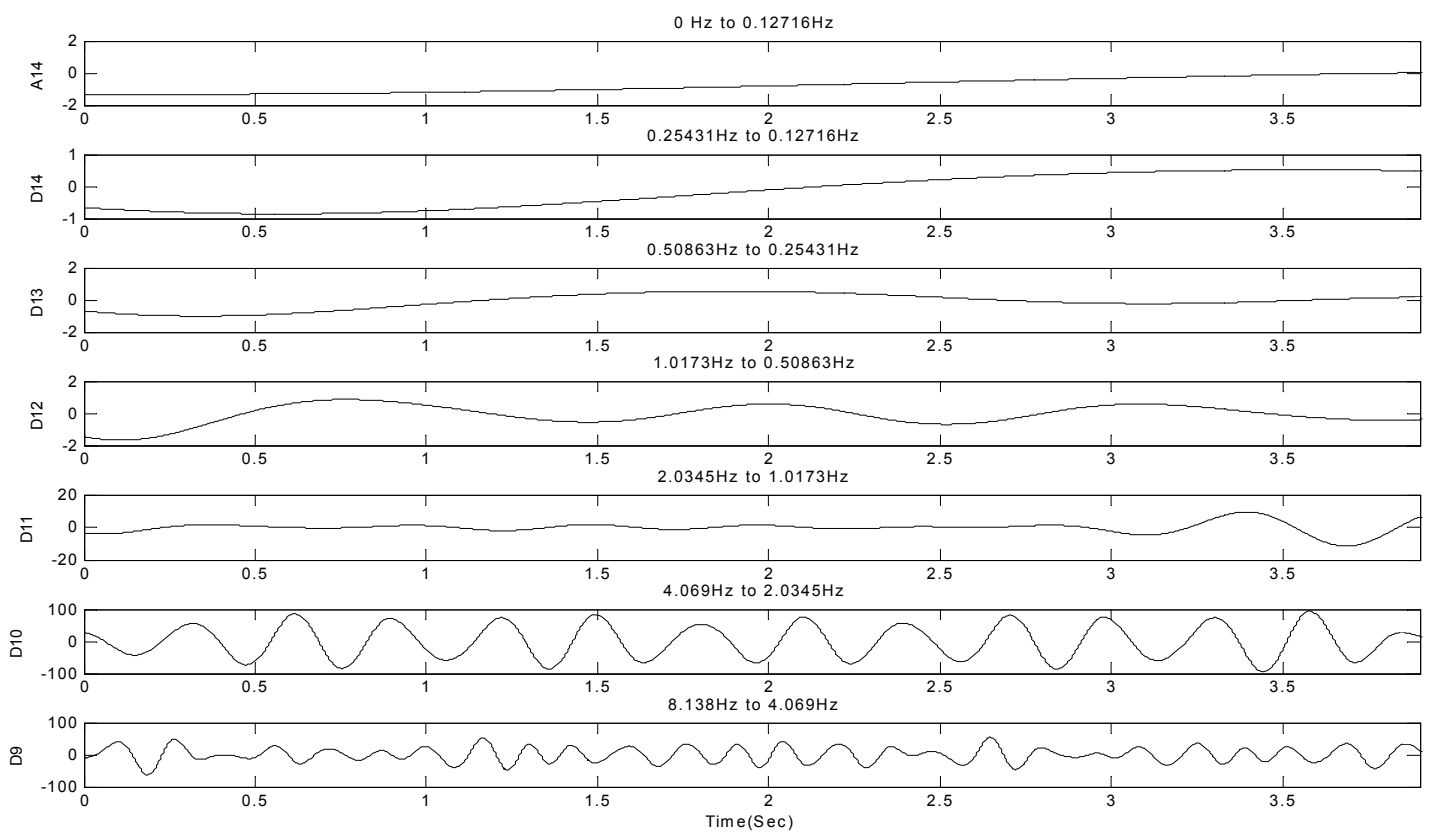

Fig. 4. Various decomposition levels of Wavelet analysis. 
To resolve the first challenge, the interest level, where the fault frequency lies in, is divided into an optimal number of "bins". Then, based on the location of the fault frequency, the energy of one of the following three conditions is used for calculating fault index: (1) current level, (2) current and previous level, (3) current and next level. This method holds good for broken bar detection as the fault characteristic component is at lower frequency (typically $0-5 \mathrm{~Hz}$ ), where the decomposition levels have smaller width.

To resolve the second challenge, a concept of baseline index is implemented in this method. Baseline index is chosen as the fault index (the magnitude of the fault signature component) when the motor is healthy; or, more practically, when the algorithm is first applied to a given motor in the field. Here, the assumption is that when the motor diagnostic device (where the proposed algorithm resides) is first installed in the field, the motor under monitoring does not have existing rotor faults. Due to the fact that rotor fault normally develops slowly; this assumption is well positioned on a general basis. Upon the installation, the average of the first three fault characteristic energy values is taken as the baseline index. After that, at any given moment, the present fault index is taken and the ratio between the present and baseline indices is used to detect the fault and determine the fault severity. Ideally, when the motor is healthy, this ratio should be close to unity. As rotor faults develop, this ratio will increase and its value is used to predict the severity of the fault.

To resolve the third challenge, the entire load range (e.g., from no load to $150 \%$ of the rated load) is divided into small "bins". The span of each bin is configurable and can be preset upon installation based on specific motor applications. In each bin, an independent baseline index is determined when the motor first enters this bin after installation. Eventually, there will be one base index for each possible load bin.

The detailed procedures are provided in the Appendix.

\section{EXPERIMENTAL VALIDATION}

The proposed method has been validated from experimental results. Two induction motors with different configurations have been tested. The key motor parameters are listed in Table I. In the experimental setup, the motors are line-connected to a $230 / 460$ volts mains supply. A dc generator connected to resistor boxes serves as the dynamometer. The voltages and currents are slightly unbalanced, and reflect the actually motor working condition. The motor line voltages and phase currents are sampled for 5 seconds at $4 \mathrm{kHz}$.

These fault indices under various load and motor health conditions are calculate according to the procedure discussed in Section V and VI. The final results are presented in Fig. 5 and Fig. 6. Fig. 5 shows the results of Motor A, where the rotor fault indices increase as the load increases and the severity of fault increases. Fig. 6 shows the results of Motor B, where the fault indices $\left(2 k s f_{1}=3.5031 \mathrm{~Hz}\right)$ in wavelet level D10 (2.0345$4.069 \mathrm{~Hz}$ ) clearly differentiate the increasing severity of rotor faults from healthy rotor to 5 broken rotor bars.

\section{CONCLUSIONS}

The paper has presented an induction motor rotor fault diagnostic method based on discrete wavelet analysis of average input power. It has also proposed an interactive fault severity determination scheme to handle various implementation challenges.

This method tends to address the challenges faced by most of the conventional rotor fault detection methods, including varying load conditions, limited data length, complicated filter design, etc. In this method, wavelet analysis allows analyzing non-stationary waveform and one-cycle average input power allows detecting rotor fault characteristic component under low load conditions without removing fundamental frequency. The proposed method can be applied to motors working under both steady state and varying load conditions. The efficacy of this method has been validated using experimental results from two induction motors with different configurations.

TABLE I. KEY PARAMETERS OF THE MOTORS IN EXPERIMENT

\begin{tabular}{|c|c|c|c|c|c|}
\hline Motor & HP & Volts & Current & Frequency & RPM \\
\hline A & 2 & $230 / 460$ & $5.4 / 2.7$ & 60 & 3450 \\
\hline B & 5 & $230 / 460$ & $13.6 / 6.8$ & 60 & 1165 \\
\hline
\end{tabular}

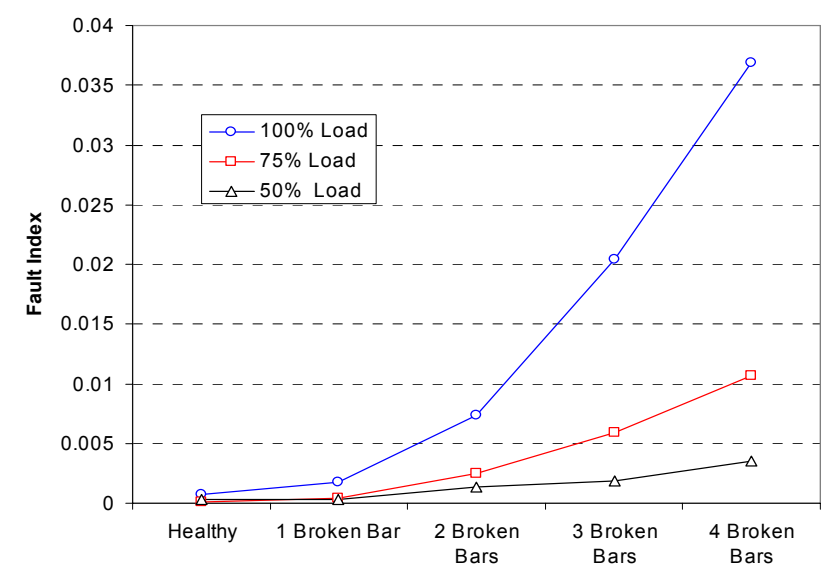

Fig. 5. Fault index vs. numbers of broken rotor bars at various load conditions for motor B.

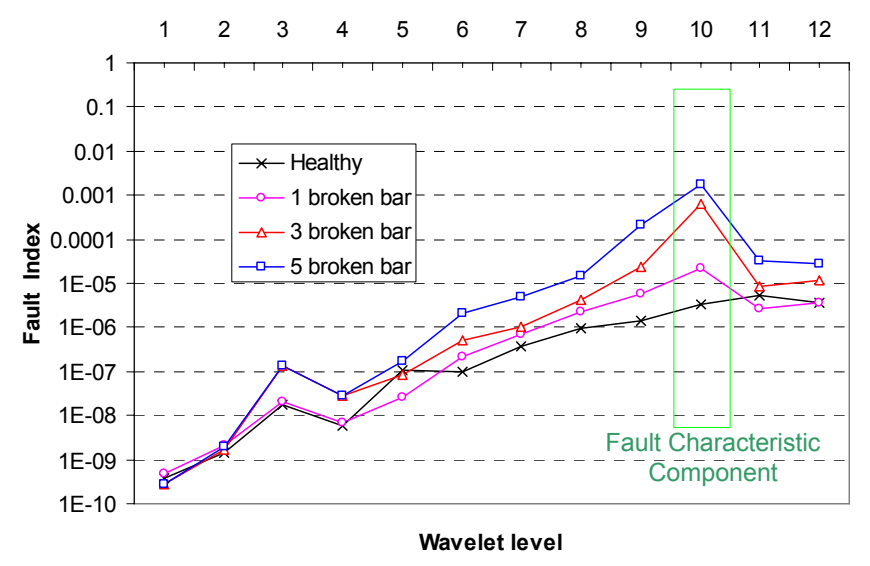

Fig. 6. Energy of wavelet levels for motor A with different number of broken rotor bars. $\left(2 k s f_{1}=3.5031 \mathrm{~Hz}\right.$, Fault related level $\left.=\mathrm{D} 10\right)$ 


\section{ACKNOWLEDGMENT}

This research was supported by a United States Department of Energy (DOE) award (DE-FC36-04GO14000).

\section{REFERENCES}

[1] H. W. Penrose, "Motor circuit analysis for energy, reliability and production cost improvements," ALL-TEST Pro, LLC, Old Saybrook, CT.

[2] IEEE Motor Reliability Working Group, "Report of large motor reliability survey of industrial commercial installations," Part I, IEEE Trans. Ind. Applicat., vol IA-21, pp. 853-872, July/Aug. 1985.

[3] G. Didier, E. Temisien, O. Caspa, H. Razik, H. Henao, A. Yazidi, and G.-A. Capolino, "Rotor fault detection using the instantaneous power signature," IEEE International Conference on Industrial Technology, vol. 1, pp. 170-174, Dec. 2004
[4] Z. Liu, X. Yin, Z. Zhang, and D. Chen, "Online rotor mixed fault diagnosis way based on spectrum analysis of instantaneous power in squirrel cage induction motors," IEEE Trans. Energy Conversion, vol. 19, no. 3, pp. 485-490, Sep. 2004.

[5] H. Douglas, P. Pillay, and A.K. Ziarani, "Broken rotor bar detection in induction machines with transient operating speeds," IEEE Trans. Energy Conversion, vol. 20, no. 1, pp. 135-141, Mar. 2005.

[6] R. Supangat, N. Ertugrul, W.L. Soong, D.A. Gray, C. Hansen, and J. Grieger, "Broken rotor bar fault detection in induction motors using starting current analysis," 2005 European Conference on Power Electronics and Applications, Sep. 2005.

[7] J.A. Antonino-Daviu, M.R. Guasp, J.R. Folch, M.P. Molina Palomares, "Validation of a new method for the diagnosis of rotor bar failures via wavelet transforms in industrial induction machines," IEEE Trans. Ind. Applicat., vol. 42, no. 4, July/August 2006.

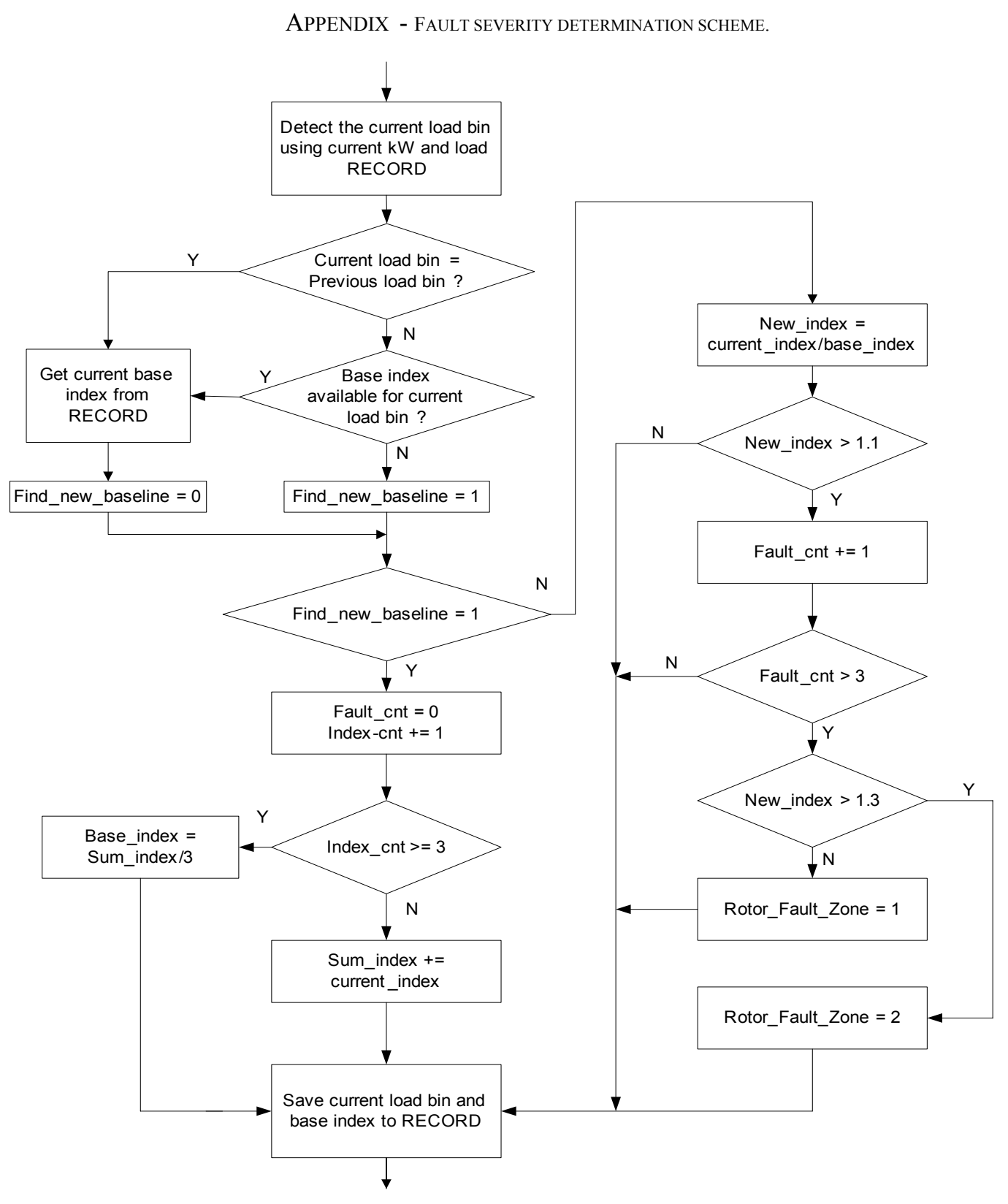




\title{
Solving Induction Motor Equivalent Circuit using Numerical Methods for an In-Service and Nonintrusive Motor Efficiency Estimation Method
}

\author{
Bin Lu, Wei Qiao, Thomas G. Habetler, and Ronald G. Harley \\ School of Electrical and Computer Engineering \\ Georgia Institute of Technology \\ Atlanta, GA 30332, USA \\ \{binlu,weiqiao,thabetler,rharley\}@ece.gatech.edu
}

\begin{abstract}
Motor efficiency evaluation enables the energy savings in industry. However, because of the uninterrupted characteristic of industrial processes, traditional methods defined in IEEE Std-112 cannot be used for these in-service motors. A novel nonintrusive method for in-service motor efficiency estimation based on a modified induction motor equivalent circuit has been developed by the authors. A highly nonlinear and 4-dimensional system of equations needs to be solved to obtain the parameters of the motor equivalent circuit and finally the motor efficiency. This paper continues this topic and presents an in-depth discussion on solving these motor parameters using three numerical methods under various conditions. Newton's method exhibits the best suitability in this application because of its simplicity and fast convergence. In the rare cases where Newton's method does not converge, the particle swam optimization and simulated annealing methods are used. Finally, the proposed motor efficiency estimation method is verified by the experimental results from a 4-pole $7.5 \mathrm{hp}$ TEFC induction motor. The performances of these three numerical methods are evaluated and compared.
\end{abstract}

Keywords - efficiency estimation; in-service testing; induction motors; equivalent circuit; numerical methods; Newton's method; particle swam optimization; simulated annealing method

\section{INTRODUCTION}

Motor efficiency evaluation enables the energy savings in industry. However, because of the uninterrupted characteristic of industrial processes, traditional methods defined in IEEE Std-112 cannot be used. Nonintrusive motor efficiency estimation methods have to be developed for in-service motor testing.
Induction motor equivalent circuit based methods are one of the least intrusive categories of motor efficiency estimation methods. Over the years, many methods have been developed based on induction motor equivalent circuit. The IEEE Std-112 F method is the standard equivalent circuit method [1]. Although this method is expected to be quite accurate, the required no-load, variable voltage, removed-rotor, and reverse rotation tests make it impossible to be used in in-service testing. Later, the standard 112-F method is modified by Ontario Hydro by eliminating the variable voltage test [2]. However, a no load test and a full load test both under rated voltage are still required. In addition, direct stator resistance measurement is also needed. To further reduce the intrusion levels, a modified equivalent circuit based method is developed by Oak Ridge National Lab in [2]. It is a low-intrusion method, however, the parameters of the equivalent circuit are solved from imaginary rated load condition and locked rotor condition, which completely rely on motor nameplate information and may have up to $20 \%$ inaccuracies according to NEMA MG1 [3]. Another interesting method calculates the motor parameters using two different motor operating points [4]. However, it requires rather intrusive measurements of stator resistance and stator winding temperature. Besides, the solution of motor parameters requires the actual value of stator leakage reactance, which is not available for inservice testing.

To overcome the problems in these traditional methods, a novel nonintrusive method for in-service motor efficiency estimation based on a modified induction motor equivalent circuit is proposed by the same authors in [5]. A highly nonlinear and 4-dimenstional system of equations needs to be solved to obtain the parameters of the motor equivalent circuit and finally the motor efficiency. 
This paper continues this topic and presents an in-depth discussion on solving motor parameters using numerical methods under various conditions. Section II briefly reviews the nonintrusive motor efficiency estimation method proposed in [5]. Section III suggests the three numerical methods to solve the motor equivalent circuit parameters: Newton's method, particle swam optimization (PSO), and simulated annealing method. Finally in section $\mathrm{IV}$, the performances of three methods are compared using experimental results from a 4-pole $7.5 \mathrm{hp} \mathrm{TEFC} \mathrm{induction}$ motor.

\section{A Nonintrusive EQUIVALENT CiRCUIT METHOD}

In [5], the same authors propose a nonintrusive method for in-service motor efficiency estimation based on a modified induction motor equivalent circuit using only motor terminal quantities and nameplate information. Only a few cycles of line voltages and currents from two different operating points and motor nameplate information are required to develop the equivalent circuit.

A modified induction motor equivalent circuit is used in this method, as shown in Fig. 1. To simplify the problem, only the positive sequence equivalent circuit is considered here. $V_{1}$ is the stator phase voltage phasor. $I_{1}$ and $I_{2}$ are the stator and rotor phase current phasors, respectively. $R_{l}, R_{2}$, and $R_{C}$ are the stator, rotor, and core resistances, respectively; and $X_{1}, X_{2}$, and $X_{m}$ are the stator leakage, rotor leakage, and magnetizing reactances, respectively.

The rotor stray-load loss, $W_{L L r}$, is defined in [1] as

$$
W_{L L r}=\left(\frac{I_{2}}{I_{2 \_ \text {rated }}}\right)^{2} W_{L L r_{-} \text {rated }}=3\left|\widetilde{I}_{2}\right|^{2} R_{L L}
$$

where, $I_{2}$ is the magnitude of the rotor current, and the subscript "rated" denotes the rated load condition.
Therefore, an equivalent stray-load resistor, $R_{L L}$, can be added in series with the rotor circuit

$$
R_{L L}=\frac{W_{L L r_{r} \text { rated }}}{3 I_{2 \_ \text {rated }}^{2}}=\text { const. }
$$

Since the stray-load loss is primarily determined by the

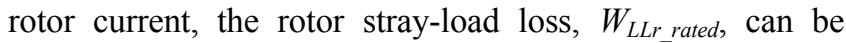
estimated using the assumed stray-load values defined in IEEE Std-112, as in Table I.

TABLE I

ASSUMED VALUES FOR STRAY LOAD LOSS IN IEEE STD-112

\begin{tabular}{|c|c|c|}
\hline \multicolumn{2}{|c|}{ Machine Rating } & $\begin{array}{c}\text { Stray load loss percent of } \\
\text { rated output }\end{array}$ \\
\hline $1-125 \mathrm{hp}$ & $1-90 \mathrm{~kW}$ & $1.8 \%$ \\
\hline $126-500 \mathrm{hp}$ & $91-375 \mathrm{~kW}$ & $1.5 \%$ \\
\hline $501-2499 \mathrm{hp}$ & $376-1850 \mathrm{~kW}$ & $1.2 \%$ \\
\hline $2500 \mathrm{hp}$ and up & $1851 \mathrm{~kW}$ and up & $0.9 \%$ \\
\hline
\end{tabular}

Considering that the stator resistance, $R_{l}$, and slip, $s$, can be estimated sensorlessly from motor voltages and currents as discussed in [5], and the stator and rotor leakage reactances have a specific ratio $\left(k=X_{1} / X_{2}=1.0,0.67\right.$, or 0.43 ) for a certain NEMA design [3], the input impedance can be expressed as a function $F$ in term of only four independent unknown variables: $X_{1}, R_{C}, X_{m}$, and $R_{2}$,

$$
\begin{aligned}
& Z_{\text {in }}=Z_{1}+Z_{a g}=Z_{1}+Z_{m} / / Z_{2} \\
& =Z_{1}+\frac{Z_{m} Z_{2}}{Z_{m}+Z_{2}}=F\left(X_{1}, R_{C}, X_{m}, R_{2}\right)
\end{aligned}
$$

where, $Z_{1}, Z_{2}$, and $Z_{m}$ are the stator, rotor, and magnetizing impedances, respectively. $Z_{\text {in }}$ is the total motor input impedance.

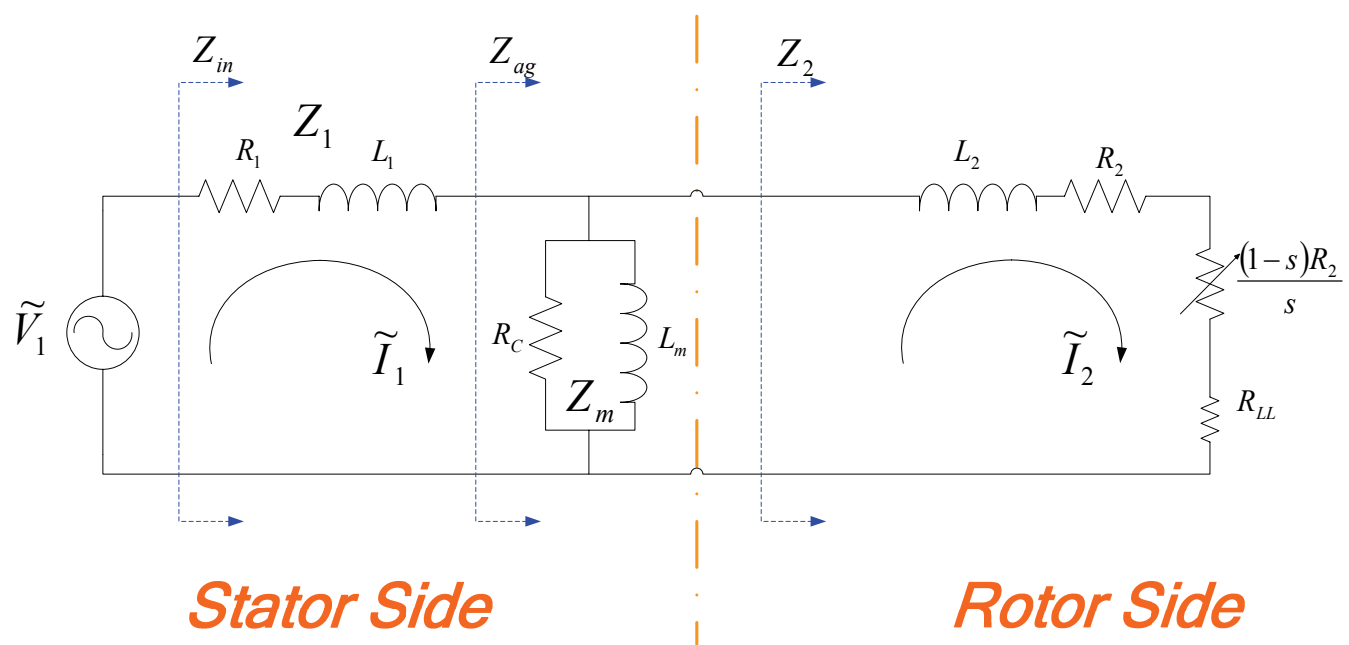

Fig. 1. A modified induction motor positive sequence equivalent circuit with an added equivalent stray-load resistor. 
Splitting the real and imaginary parts in (1), two independent equations can be obtained at each motor load point.

$$
\left\{\begin{array}{l}
\operatorname{real}\left(\frac{\widetilde{V}_{1}}{\widetilde{I}_{1}}\right)=\operatorname{real}\left(Z_{\text {in }}\right)=\operatorname{real}\left[F\left(X_{1}, R_{C}, X_{m}, R_{2}\right)\right] \\
\operatorname{imag}\left(\frac{\widetilde{V}_{1}}{\widetilde{I}_{1}}\right)=\operatorname{imag}\left(Z_{\text {in }}\right)=\operatorname{imag}\left[F\left(X_{1}, R_{C}, X_{m}, R_{2}\right)\right]
\end{array}\right.
$$

Expanding (2), two independent equations can be obtained at each load points as (3).

In order to solve four independent unknowns, a set of four independent equations are developed from two carefully selected load points. The details on solving such a highly nonlinear and multi-dimensional system of equations are given in section III.

After the parameters of the equivalent circuit are solved, the motor efficiency at any load can be simply computed as

$$
\begin{aligned}
& \eta=\frac{P_{\text {output }}}{P_{\text {input }}}=\frac{3\left|\widetilde{I}_{2}\right|^{2} \frac{(1-s)}{s} R_{2}-W_{f w}}{3\left|\widetilde{I}_{1}\right|^{2} \operatorname{real}\left(Z_{\text {in }}\right)} \\
& =\frac{3\left|\widetilde{I}_{1}\left(\frac{Z_{m}}{Z_{m}+Z_{2}}\right)\right|^{2} \frac{(1-s)}{s} R_{2}-W_{f w}}{3\left|\widetilde{I}_{1}\right|^{2} \operatorname{real}\left(Z_{\text {in }}\right)}
\end{aligned}
$$

where, the friction and windage loss, $W_{f w}$, is taken as a constant percentage of the rated motor horse power, e.g., $1.2 \%$ for 4-pole motors below $200 \mathrm{hp}$, as suggested by many statistical motor efficiency estimation methods.

\section{SOlVING MOTOR PARAMETERS USING NUMERICAL METHODS}

In order to solve four independent unknowns

$$
\boldsymbol{x}=\left[X_{1}, R_{c}, X_{m}, R_{2}\right],
$$

a set of four independent nonlinear equations

$$
\boldsymbol{g}(\boldsymbol{x})=\left[g_{1}(\boldsymbol{x}) ; g_{2}(\boldsymbol{x}) ; g_{3}(\boldsymbol{x}) ; g_{4}(\boldsymbol{x})\right]
$$

are developed from two carefully selected load points according to (3).
Too close load points will result in ill-conditioned equations; while too distant conditions will result in additional errors caused by the parameter variations due to temperature change, flux saturation, etc.

Solving such a highly nonlinear and multi-dimensional system of equations is not trivial. Three numerical methods (Newton's method [6], PSO [7], [8], and simulated annealing method [6]) have been studied and implemented. Newton's method is very simple and fast. But its convergence highly depends on the initial guess. When it converges, it finds the solution in only a few iterations. In the rare cases where Newton's method does not converge, the PSO and simulated annealing methods can be used. Both of them target on global optimization and can converge from a general zero initial condition, but their converging speeds are much slower.

\section{A. Newton's Method}

Newton's method is a very fast root finding method based on approximating $\boldsymbol{g}(\boldsymbol{x})$ locally with a two-term Taylor series. It is the most widely used root finding method in engineering applications because of its simplicity and fast quadratic convergence. Since this problem is a 4dimensional nonlinear algebraic system, an extended Newton's vector method is used. It requires the computation of a $4 \times 4$ Jacobian matrix

$$
\boldsymbol{J}(\boldsymbol{x})=\partial \boldsymbol{g}(\boldsymbol{x}) / \partial \boldsymbol{x}
$$

In this problem, fortunately, the explicit expression of the Jacobian matrix $\boldsymbol{J}(\boldsymbol{x})$ can be obtained offline, and the computation of partial differentiations is not needed at each iteration. The procedure of Newton's vector method is available in [6].

Like any non-bracketing method, Newton's method is not guaranteed to converge in all cases. This is the major disadvantage of this method. Its convergence highly depends on the initial point $\boldsymbol{x}_{\boldsymbol{0}}$. If an initial point is chosen to be close to the solution, it converges to the solution very rapidly. Fortunately, in this problem, a reasonable initial point can be always obtained from the following rough estimations.

$$
\left\{\begin{array}{l}
\operatorname{real}\left(\frac{\widetilde{V}_{1}}{\widetilde{I}_{1}}\right)=R_{1}+\frac{R_{C} X_{m}\left(\frac{R_{2}}{s}+R_{L L}\right)\left[R_{C} X_{m}+\frac{R_{C} X_{1}}{k}+\left(\frac{R_{2}}{s}+R_{L L}\right) X_{m}\right]-\frac{R_{C} X_{m} X_{1}}{k}\left[R_{C}\left(\frac{R_{2}}{s}+R_{L L}\right)-\frac{X_{m} X_{1}}{k}\right]}{\left[R_{C}\left(\frac{R_{2}}{s}+R_{L L}\right)-\frac{X_{m} X_{1}}{k}\right]^{2}+\left[R_{C} X_{m}+\frac{R_{C} X_{1}}{k}+\left(\frac{R_{2}}{s}+R_{L L}\right) X_{m}\right]^{2}} \\
\operatorname{imag}\left(\frac{\widetilde{V}_{1}}{\widetilde{I}_{1}}\right)=X_{1}+\frac{\frac{R_{C} X_{m} X_{1}}{k}\left[R_{C} X_{m}+\frac{R_{C} X_{1}}{k}+\left(\frac{R_{2}}{s}+R_{L L}\right) X_{m}\right]+R_{C} X_{m}\left(\frac{R_{2}}{s}+R_{L L}\right)\left[R_{C}\left(\frac{R_{2}}{s}+R_{L L}\right)-\frac{X_{m} X_{1}}{k}\right]}{\left[R_{C}\left(\frac{R_{2}}{s}+R_{L L}\right)-\frac{X_{m} X_{1}}{k}\right]^{2}+\left[R_{C} X_{m}+\frac{R_{C} X_{1}}{k}+\left(\frac{R_{2}}{s}+R_{L L}\right) X_{m}\right]^{2}}
\end{array}\right.
$$


It has been experimentally established that the stator leakage reactance $X_{1}$ and the magnetizing reactance $X_{m}$ obey the relation

$$
X_{1}=\alpha \cdot X_{m}
$$

where, the ratio $\alpha$ is a constant ranging from 0.02 to 0.07 for a specific motor.

The magnitude of no load current $I_{1-N L}$ can be estimated as a certain percent of full load current, e.g., $20 \%$. Then, the magnitude of the no load input impedance $\left|Z_{\text {in } N L}\right|$ can be obtained by

$$
\left|Z_{\text {in_ } N L}\right|=\frac{V_{1 \_N L}}{I_{1 \_N L}}
$$

Considering the core loss resistance $R_{C}$ is very large and its contribution in $\left|Z_{\text {in_NL }}\right|$ is negligible, the no load reactance can be roughly estimated by

$$
X_{1}+X_{m} \approx \sqrt{\left|Z_{\text {in_NLL }}\right|^{2}-R_{1}^{2}}
$$

From (4) to (6), the initial guess of $X_{I}$ is

$$
X_{1}=\frac{\alpha}{\alpha+1} \sqrt{\left(\frac{V_{1 \_N L}}{I_{1 \_N L}}\right)^{2}-R_{1}^{2}}
$$

The initial guess of $R_{C}$ can be set as one order of magnitude larger than $X_{m}$. The rotor resistance $R_{2}$ can be set in the same order of magnitude of $R_{l}$. It has been observed from experiments that for almost all cases, the above initial guess can result in final convergence.

\section{B. Particle Swarm Optimization}

The PSO method is an evolutionary computational algorithm inspired by the paradigm of birds flocking [7]-[8]. It has been successfully used for both continuous nonlinear and discrete binary optimization [8]. The PSO algorithm searches for the optimal solution from a population of moving particles. Each particle represents a potential solution. It has a position represented by a position vector $X_{i}$ and a moving velocity represented by a velocity vector $V_{i}$ in the problem space. Each particle keeps track of its coordinates in the problem space, which are associated with the best position it has achieved so far. This position is called individual best position $X_{i, p b e s t}$. Furthermore, the best position among all the particles obtained so far in the population is kept track of by all particles as $X_{\text {gbest }}$, which is called swarm best position. The PSO algorithm is implemented in the following iterative procedure to search for the optimal solution. (i) Initialize a population of particles with random positions and velocities of $M$ dimensions in the problem space.

(ii) Define a fitness measure function to evaluate the performance of each particle.

(iii) Compare particle's present position $X_{i}$ with particle's $X_{i, p b e s t}$ based on the fitness evaluation. If current position $X_{i}$ is better than $X_{i, p b e s t}$, then set $X_{i, p b e s t}$ equal to the current position $X_{i}$.

(iv) If $X_{i, p b e s t}$ is updated, then compare particle's $X_{i, p b e s t}$ with the swarm best position $X_{\text {gbest }}$ based on the fitness evaluation. If $X_{i, p b e s t}$ is better than $X_{\text {gbest }}$, then set $X_{\text {gbest }}$ equal to the current position $X_{i, p b e s t}$.

(v) At iteration $k$, a new velocity for particle $i$ is updated by

$$
\begin{gathered}
V_{i}(k+1)=w V_{i}(k)+c_{1} \varphi_{1}\left(X_{i, p b e s t}-X_{i}(\mathrm{k})\right)+ \\
c_{2} \varphi_{2}\left(X_{\text {gbest }}-X_{i}(\mathrm{k})\right), \quad i=1,2, \cdots, N
\end{gathered}
$$

(vi) Based on the updated velocity, each particle then changes its position according to the following equation,

$$
X_{i}(k+1)=X_{i}(k)+V_{i}(k+1), \quad i=1,2, \cdots, N
$$

(vii) Repeat steps (iii)-(vi) until a criterion, usually a sufficiently good fitness or a maximum number of iterations is achieved. The final value of $X_{\text {gbest }}$ is regarded as the optimal solution of the problem.

In (6), $c_{1}$ and $c_{2}$ are positive constants representing the weighting of the acceleration terms that guide each particle toward the individual best and the swarm best positions $X_{i, p b e s t}$ and $X_{\text {gbest }}$, respectively; $\varphi_{1}$ and $\varphi_{2}$ are random numbers in the range $[0,1] ; w$ is a positive inertia weight developed to provide better control between exploration and exploitation; $N$ is the number of particles in the swarm.

In this problem, the fitness measure function $f(x)$ for each particle is defined as

$$
f(\boldsymbol{x})=\|\boldsymbol{g}(\boldsymbol{x})\|
$$

where $\|\cdot\|$ represents the Euclidean norm.

In this problem, the values of $c_{1}$ and $c_{2}$ in (6) are chosen as 2; the number of particles $N$ is chosen as 20; the inertia constant $w$ starts with 0.9 and linearly decreases to 0.4 when the iteration number reaches a pre-specified maximum number during the simulation.

\section{Simulated Annealing Method}

Similar to PSO, the simulated annealing method is also a statistical optimization technique. It is based on an analogy with the annealing of metal and searches for a global minimum of the objective function.

The simulated annealing method has two stages to reach the global optimization. The first stage is a random global search based on simulated annealing. As the temperature of metal is gradually lowered from above its melting point, the 
atoms lose thermal mobility and decay to lower energy states. Eventually, the atoms settle into global energy state minimum. The simulated annealing methods simulate this annealing process by gradually lowering an artificial temperature $T$. The energy states of artificial atoms are associated with $\boldsymbol{x}$. Their changes $\Delta \boldsymbol{x}$ are described using a Gaussian distribution with zero mean. The convergence speed of simulated annealing is often accelerated by considering lower and upper bonds on $\boldsymbol{x}$ and $f(\boldsymbol{x})$. A localization parameter, $0<\gamma<1$, is set empirically to adjust the scope of searching local minima.

The second stage is an efficient local search. Many local minimum optimization methods can be utilized, such as the conjugate-gradient method and penalty function method. The detailed procedure of the simulated annealing method is available in [6].

In this problem, the root finding process for the nonlinear 4-dimentional system of equations can be converted to an optimization process using the simulated annealing method as

$$
\begin{array}{lr}
\text { Minimize: } & f(\boldsymbol{x})=\|\boldsymbol{g}(\boldsymbol{x})\|^{2}=\boldsymbol{g}(\boldsymbol{x}) \boldsymbol{g}(\boldsymbol{x}) \\
\text { Subject to: } & \boldsymbol{a}<\boldsymbol{x}<\boldsymbol{b}
\end{array}
$$

A localization parameter of $\gamma=0.3-0.5$ provides best convergence speed. The lower and upper bonds vectors, $\boldsymbol{a}$ and $\boldsymbol{b}$, can be roughly computed from the motor nameplate data.

\section{EXPERIMENTAL RESULTS}

The proposed motor efficiency estimation method has been verified by both computer simulations and motor experiments. The parameters of the motor used in the experiments are listed in Table II.

In the experimental setup, the three-phase induction motor is line-connected to a 230 -volt mains supply. A dc generator connected to resistor boxes serves as the dynamometer. The unbalances in the voltages and currents are negligible $\left(V / V_{+}<1 \%\right.$ and $\left.I / I_{+}<3 \%\right)$. The line voltages and currents are sampled at $2 \mathrm{kHz}$ and collected using a NI LabVIEW data acquisition system. The actual efficiency is directly calculated from the shaft torque, measured by an in-line rotary torque transducer.

The motor equivalent circuit is solved using data from two load conditions: (1) $19.09 \%$ rated load, $1775 \mathrm{rpm}$, and (2) $71.26 \%$ rated load, $1694 \mathrm{rpm}$. The motor parameters are solved using all three methods. As discussed in section III, Newton's method is regarded as the major solver for this application. The experimental results validate its fast convergence. Using the initial guess calculated from motor nameplate data, this algorithm converges in only 6 iterations. The results of Newton's method is summarized in Table III, which shows the iterations number $k$, the current estimate $\boldsymbol{x}$, and the norm of $\boldsymbol{g}(\boldsymbol{x})$ at the current estimate.

Compared with Newton's method, the PSO and simulated annealing methods converge much more slowly. However, these two methods can converge from a general zero initial guess. The same equations are solved using these three methods on a computer with Pentium $43.4 \mathrm{GHz}$ processor and $512 \mathrm{Mb}$ RAM. The iterations and CPU time required by each method to reach final solution are compared in Table IV. Because both the PSO and simulated annealing methods are statistical techniques, their iterations and computation time can vary from time to time. In Table IV, the iterations and CPU time used by the PSO and simulated annealing methods are the average of 10 repeated experiments.

TABLE II. PARAmeters OF THE INDUCTION MOTOR IN EXPERIMENT.

\begin{tabular}{|l|l||l|l|}
\hline Brand & GE & Volts & $230 / 460 \mathrm{~V}$ \\
\hline HP & 7.5 & F.L.AMPS & $18.2 / 9.1 \mathrm{~A}$ \\
\hline CAT. NO. & S231 & RPM & 1755 \\
\hline Design & NEMA-A & Nom. PF & 0.865 \\
\hline Enclosure & TEFC & Nom. Eff. & 0.895 \\
\hline
\end{tabular}

Table III. Newton’s Vector Method Results.

\begin{tabular}{|c|c|c|c|c|c|}
\hline \multirow{2}{*}{$k$} & \multicolumn{4}{|c|}{$\boldsymbol{x}$} & \multirow{2}{*}{$\|\boldsymbol{g}(\boldsymbol{x})\|$} \\
\cline { 2 - 5 } & $X_{1}(\Omega)$ & $R_{C}(\Omega)$ & $X_{m}(\Omega)$ & $R_{2}(\Omega)$ & \\
\hline \hline $\mathbf{0}$ & 0.907794 & 70.949235 & 19.53566 & 0.500000 & 17.457290 \\
\hline $\mathbf{1}$ & 3.41516 & 92.305626 & 55.92357 & 0.534489 & 7.0520789 \\
\hline $\mathbf{2}$ & 1.77525 & 244.494693 & 86.55143 & 0.497332 & 3.0326519 \\
\hline $\mathbf{3}$ & 1.93057 & 1325.90180 & 84.4203 & 0.520816 & 0.6147128 \\
\hline $\mathbf{4}$ & 1.938864 & 824.305199 & 80.9109 & 0.520560 & 0.0258162 \\
\hline $\mathbf{5}$ & 1.938487 & 846.149846 & 80.8698 & 0.520614 & 0.0000644 \\
\hline $\mathbf{6}$ & $\mathbf{1 . 9 3 8 4 8 7}$ & $\mathbf{8 4 6 . 1 1 2 9 2 2}$ & $\mathbf{8 0 . 8 6 9 4 3}$ & $\mathbf{0 . 5 2 0 6 1 4}$ & $\mathbf{0 . 0 0 0 0 0 0 0}$ \\
\hline
\end{tabular}

TABle IV. COMPARISON OF Three Numerical Methods.

\begin{tabular}{|c|c|c|c|}
\hline Methods & Convergence & Iterations & CPU Time \\
\hline Newton's Vector & Local & 6 & $0.185 \mathrm{~s}$ \\
\hline PSO & Global & 2601 & $2.432 \mathrm{~s}$ \\
\hline Simulated Annealing & Global & 3377 & $17.123 \mathrm{~s}$ \\
\hline
\end{tabular}

It can be observed from Table IV that when the Newton's method converges, there is no doubt that it is the best method. In the rare cases where the Newton's method does not converge using the estimated initial guess, both the PSO and the simulated annealing methods can be used. The PSO method is faster, but requires relatively more configuration parameters $\left(c_{1}, c_{2}, w, N\right)$ to be tuned. While, the simulated annealing method is about 10 times slower, but it just needs one localization parameter $(0<\gamma<1)$ to be tuned.

Using the solved motor parameters, the motor efficiency at any load levels can be estimated. Fig. 2 compares the estimated and measured motor efficiencies versus load 
percentage, when the load changes continuously from almost no load to full load conditions. The estimated motor efficiencies show good agreement (within 2-3\% errors) with the measured efficiencies during the normal motor operations (load ranges from $30 \%$ to $90 \%$ of rated load). The errors under very low load conditions (less than $30 \%$ of rated load) are slightly larger (within 10\%), but usually under such low load levels, there is no need to estimate motor efficiencies. The errors are caused by many factors, such as motor parameter variations under different load levels, stator resistance and speed estimation errors, motor nameplate information inaccuracies, etc. The agreement between the estimated and measured efficiencies validates the solved motor parameters and the proposed nonintrusive motor efficiency estimation method.

\section{CONCLUSIONS}

This paper continues the proposal of a novel nonintrusive method for in-service motor efficiency estimation based on a modified induction motor equivalent circuit using only motor terminal quantities and nameplate data. Only a few cycles of line voltages and currents from two different motor operating points and motor nameplate information are required to develop the equivalent circuit. The parameters are obtained by solving a highly nonlinear and 4dimensional system of equations. As the focus of this paper, three numerical methods (Newton's method, PSO, and simulated annealing method) are adopted to solve the motor parameters under various conditions. Newton's method is suggested as the major solver of this application because of its simplicity and fast convergence. In the rare cases where Newton's method does not converge, the PSO and simulated annealing methods can be used using a general zero initial guess. All three suggested numerical methods have been tested on a 4-pole $7.5 \mathrm{hp}$ TEFC induction motor. Experimental results validate that the motor efficiencies estimated using the solved motor parameters agree with the measured efficiencies within $2-3 \%$ errors.

\section{ACKNOWLEDGMENT}

This work is financially supported by U.S. Department of Energy and Eaton Corporation.

\section{REFERENCES}

[1] IEEE Standard Test Procedure for Polyphase Induction Motors and Generators, IEEE Standard 112-2004, Nov. 2004.

[2] J. D. Kueck, M. Olszewski, D. A. Casada, J. Hsu, P. J. Otaduy, and L. M. Tolbert, "Assessment of methods for estimating motor efficiency and load under field conditions," Oak Ridge National Laboratory report, ORNL/ TM-13165, 1996.

[3] NEMA - MG 1 Standard, 2003.

[4] Y. El-Ibiary, "An accurate low-cost method for determining electric motors's efficiency for the purpose of plant energy management," IEEE Trans. Industrial Applications, vol.39, no. 4, July/Aug. 2003, pp. 1205-1210.

[5] B. Lu, T. G. Habetler, and R. G. Harley, "A nonintrusive efficiency estimation method for in-service motor testing using a modified induction motor equivalent circuit," in Proc. of the $37^{\text {th }}$ IEEE Power Electronics Specialist Conference (PESC'06), June 2006.

[6] R. J. Schilling and S. L. Harris, Applied Numerical Methods for Engineers using MATLAB and C. Pacific Grove, CA: Thomson, 2000.

[7] J. Kennedy and R. C. Eberhart, "Particle swarm optimization," in Proc. of IEEE International Conference on Neural Networks, vol. 4, Nov./Dec., 1995, pp.1942-1948.

[8] J. Kennedy and R. C. Eberhart, Swarm Intelligence. San Mateo, CA: Morgan Kaufmann, 2001.

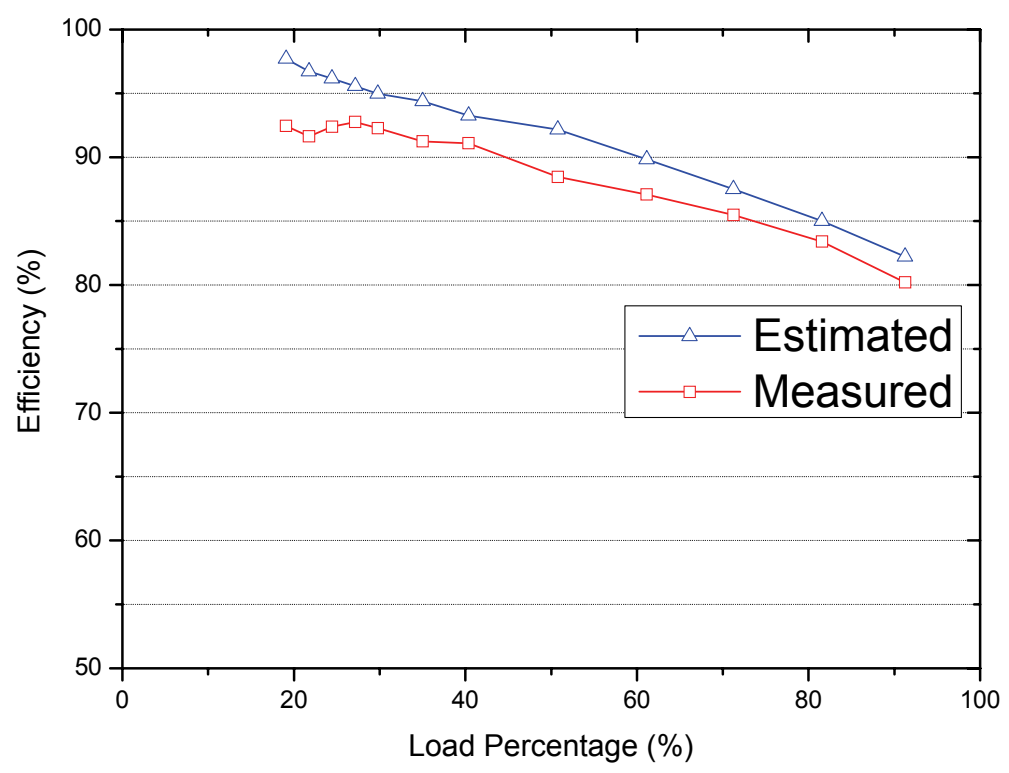

Fig. 2. Efficiency v.s. load curve of the $7.5 \mathrm{hp} \mathrm{GE} \mathrm{TEFC} \mathrm{motor.}$ 


\title{
A Nonintrusive and In-Service Motor-Efficiency Estimation Method Using Air-Gap Torque With Considerations of Condition Monitoring
}

\author{
Bin Lu, Member, IEEE, Thomas G. Habetler, Fellow, IEEE, and Ronald G. Harley, Fellow, IEEE
}

\begin{abstract}
Energy usage evaluation and condition monitoring for electric machines are important in industry for overall energy savings. They are often expected to be implemented in an integrated product because of many common requirements such as data collection. Because of the uninterrupted characteristic of industrial processes, traditional methods defined in IEEE Standard 112 cannot be used for these in-service motors. This paper proposes a truly nonintrusive method for in-service motor-efficiency estimation based on air-gap torque using only motor terminal quantities and nameplate information, with special considerations of motor condition monitoring requirements. Rotor speed and stator resistance, the stumbling blocks of most in-service testing methods, are extracted from motor input currents instead of being measured. The no-load test, which is required for calculating the rotational loss and core loss, is eliminated by using empirical values. Stray-load loss is assumed according to the motor horsepower as suggested in IEEE Standard 112. Finally, the proposed method is validated by testing three induction motors with different configurations. Experimental results show that the proposed method can estimate motor efficiencies with less than $2 \%$ errors under normal load conditions.
\end{abstract}

Index Terms-Air-gap torque (AGT), condition monitoring, efficiency estimation, electric machines, IEEE Standard 112, inservice testing.

\section{NOMENCLATURE}

$V_{s} \quad$ Magnitude of stator phase voltage phasor.

$I_{s} \quad$ Magnitude of stator phase current phasor.

$I_{r} \quad$ Magnitude of rotor phase current phasor.

$v_{a b}, v_{b c}, v_{c a} \quad$ Stator line voltages.

$i_{a}, i_{b}, i_{c} \quad$ Stator phase currents.

$\cos \varphi \quad$ Power factor.

$p \quad$ Number of poles.

$P_{\text {input } \quad \text { Motor input power. }}$

$P_{\text {output }} \quad$ Motor output power or shaft power.

$P_{\text {ag }} \quad$ Air-gap power or electromagnetic power.

Paper IPCSD-08-012, presented at the 2006 Industry Applications Society Annual Meeting, Tampa, FL, October 8-12, and approved for publication in the IEEE TRANSACTIONS ON INDUSTRY APPLICATIONS by the Electric Machines Committee of the IEEE Industry Applications Society. Manuscript submitted for review October 1, 2006 and released for publication February 19, 2008. Current version published November 19, 2008. This work was supported by the United States Department of Energy (DOE) under Grant DE-FC3604GO14000.

B. Lu is with the Innovation Center, Eaton Corporation, Milwaukee, WI 53216 USA (e-mail: binlu@ieee.org).

T. G. Habetler and R. G. Harley are with the School of Electrical and Computer Engineering, Georgia Institute of Technology, Atlanta, GA 30332 USA (e-mail: thabetler@ece.gatech.edu; rharley@ece.gatech.edu).

Color versions of one or more of the figures in this paper are available online at http://ieeexplore.ieee.org.

Digital Object Identifier 10.1109/TIA.2008.2006297

$\begin{array}{ll}P_{m} & \text { Developed mechanical power. } \\ R_{s} & \text { Stator resistance. } \\ R_{r} & \text { Rotor resistance. } \\ s & \text { Motor slip. } \\ T_{\mathrm{ag}} & \text { Air-gap torque or electromagnetic torque. } \\ T_{\mathrm{shaft}} & \text { Shaft torque or output torque. } \\ W_{\mathrm{LLs}} & \text { Stator stray-load loss. } \\ W_{\mathrm{LLr}} & \text { Rotor stray-load loss. } \\ W_{s} & \text { Stator copper loss. } \\ W_{\mathrm{core}} & \text { Core loss. } \\ W_{r} & \text { Rotor copper loss. } \\ W_{\mathrm{fw}} & \text { Friction and windage loss or mechanical loss. } \\ \omega_{\text {syn }} & \text { Synchronous speed. } \\ \omega_{r} & \text { Rotor mechanical speed. } \\ \eta & \text { Motor efficiency. } \\ \theta_{d a} & \text { Angle between } d \text {-axis and stator } a \text {-axis. } \\ v_{d q s} & \text { Stator voltage space vector. } \\ i_{d q s} & \text { Stator current space vector. } \\ \lambda_{d q s} & \text { Total flux linkage space vector. }\end{array}$

\section{INTRODUCTION}

$\mathbf{M}$ OTOR-DRIVEN systems use nearly $70 \%$ of the total electric energy consumed by industry in the United States. In industry, only motors above $500 \mathrm{hp}$ are usually monitored because of their high costs. However, motors below 500 hp make up $99.7 \%$ of the motors in service and consume $71 \%$ of the energy used. On average, these motors operate at no more than $60 \%$ of their rated load because of oversized installations or underloaded conditions, and thus at reduced efficiency which results in wasted energy [1]. Therefore, low-cost methods are needed in monitoring the motor energy usage and health conditions, particularly for medium- and small-size motors.

Energy usage evaluation and condition monitoring for electric machines are important in industry for overall energy savings. They are often expected to be implemented in an integrated product because of many common requirements such as data collections. In industrial plants, the motor terminal voltages and currents are readily available from the motor control centers, since there are already preinstalled potential transformers (PTs) and current transformers (CTs) for protection purpose. The terminal voltage and current measurements bring no additional costs in terms of data collection. Most traditional efficiency evaluation methods also require the rotor speed and shaft torque to be measured to calculate the output power [2]. The speed and torque transducers are very costly, and their installations are highly intrusive. In most cases, it is even 


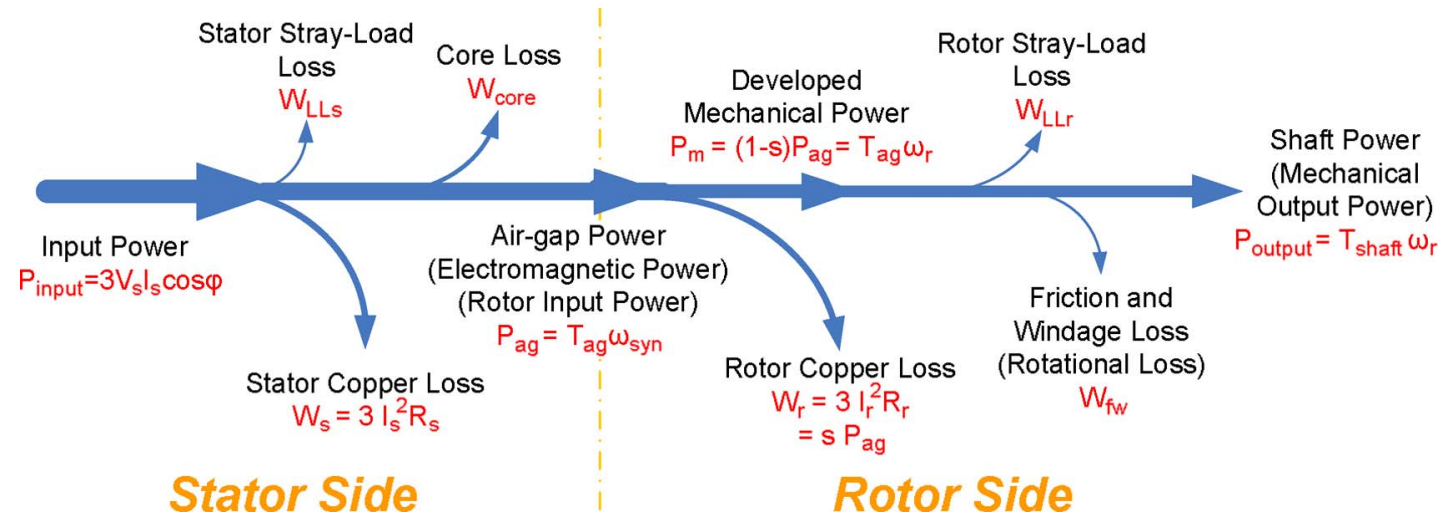

Fig. 1. Power flow and loss definition of induction motors.

not possible to install this equipment because the motors may be buried inside a machine or there is no space to attach such transducers between the motor and the load. Because of the uninterrupted characteristic of continuous industrial processes, traditional methods defined in IEEE Standard 112 cannot be used for these in-service motors. Nonintrusive methods, which only rely on terminal voltages and currents while a motor is running, have brought increasing attention during the recent years for these continuous applications [3], [4].

A possible approach of evaluating efficiency is to use the premeasured motor characteristic efficiencies under representative load conditions during motor development to predict the actual efficiencies in operation. This approach is nonintrusive in nature. However, its usage, in practice, is greatly limited by the following facts: 1) The characteristic efficiencies under representative load conditions are not always available from motor data sheets and 2) the characteristic efficiencies are generic data which could differ greatly from actual efficiencies for a specific motor due to many factors, such as inaccurate nameplate information and different working environments. Therefore, the authors only focus on online methods in this paper.

This paper proposes a nonintrusive air-gap torque (NAGT) method for in-service motor-efficiency estimation using only motor terminal quantities and nameplate information, with special considerations of motor condition monitoring requirements. Rotor speed and stator resistance, the stumbling blocks of most in-service testing methods, are extracted from motor input currents instead of being measured. No-load test, which is required for calculating the rotational loss and core loss, is eliminated by using empirical values. Stray-load loss is assumed according to the motor horsepower as suggested in IEEE Standard 112. As a continuation of the authors' previous work in [3], where a general approach is proposed for nonintrusive efficiency estimation based on a survey study, this paper specifically focuses on the experimental validation of the proposed NAGT method. Three induction motors with different configurations are tested, and the results are presented and analyzed.

\section{ORIGIN AND MOTIVATION}

The key to the electric machine energy usage evaluation is nonintrusive motor-efficiency estimation. The same authors conducted a survey on motor-efficiency estimation methods, specifically considering the advances in sensorless speed estimation and in-service stator resistance estimation techniques during the last decade [3]. Three candidate methods, ORMEL96, OHME, and AGT methods, are suggested in the survey to be improved for nonintrusive and in-service motor testing.

Among these methods, the air-gap torque (AGT) method is regarded as the best in terms of accuracy and ease of implementation. It was developed based on the "AGT" [5] or "sensorless torque" equations [6], developed in 1990s. This method has been verified by several experiments to be an effective motorefficiency estimation method [7]-[9]. The input power to the motor is calculated from input line voltages and phase currents, and the output power is calculated from rotor speed and shaft torque. Air-gap flux is calculated from the integral of currents and voltages subtracting the stator $I R$ drop. Shaft torque is obtained by subtracting the torque losses associated with the friction and windage loss $W_{\mathrm{fw}}$ and rotor stray-load loss $W_{\mathrm{LLr}}$ from the calculated AGT. The power flow and definition of each loss term in an induction motor are shown in Fig. 1.

The AGT method requires the following data to be measured: line voltages, phase currents, rotor speed, and stator resistance. In addition, to measure $W_{\text {core }}$ and $W_{\mathrm{fw}}$, a no-load test must be run. The requirement of speed, stator resistance measurements, and the no-load test are the main drawbacks of this method, which makes it a "high-intrusive" method and prevents its use in in-service motor testing.

To overcome these problems, a "nonintrusive" method based on AGT equations can be developed by making the following improvements to the original AGT method. It still keeps satisfactory accuracy but greatly reduces the intrusion levels.

1) The rotor speed does not come from direct measurement. It is estimated at high accuracy ( 0.005 p.u.) from motor current spectrum analysis extracting slot harmonics from stator currents [10].

2) The stator resistance is estimated using online dc signal injection methods using only the input line voltages and phase currents [11], [12], instead of direct measurement from unpowered testing or rough approximation from the nameplate data.

3) A no-load test should be avoided due to its high intrusiveness. Instead, no-load data are obtained by three options at different levels of accuracy/intrusiveness.

a) If the motor being tested is to be newly installed or has a regular scheduled maintenance, the no-load 
TABLE I

ASSUMEd VALUES FOR STRAY-LOAD LOSS IN IEEE STANDARD 112

\begin{tabular}{|c|c|c|}
\hline \multicolumn{2}{|c|}{ Machine Rating } & $\begin{array}{c}\text { Stray-Load Loss Percent of } \\
\text { Rated Output Power }\end{array}$ \\
\hline $1-125 \mathrm{hp}$ & $1-90 \mathrm{~kW}$ & $1.8 \%$ \\
\hline $126-500 \mathrm{hp}$ & $91-375 \mathrm{~kW}$ & $1.5 \%$ \\
\hline $501-2499 \mathrm{hp}$ & $376-1850 \mathrm{~kW}$ & $1.2 \%$ \\
\hline $2500 \mathrm{hp}$ and up & $1851 \mathrm{~kW}$ and up & $0.9 \%$ \\
\hline
\end{tabular}

test could be done during the scheduled operation downtime to give best accuracy.

b) If the motor under test is available in the motor master database, the no-load data are estimated from the generic design data [13].

c) If neither of the above is available, the no-load data can be estimated from empirical values using only motor nameplate data, similar to the OHME and ORMEL96 methods [8]. More specifically, the combined no-load losses are assumed to be $3.5 \%$ of rated output power, the friction and windage loss is $1.2 \%$ rated output power, and the stray-load loss is estimated from Table I as in IEEE Standard 112 [2].

\section{SENSORLESS Rotor SpeEd Estimation}

Direct measurement of the rotor speed requires a shaftmounted speed encoder or optical tachometer to be installed. This reduces the reliability and increases the cost. During the past decade, numerous sensorless rotor speed estimation schemes have been proposed [3]. A sensorless speed estimation method based on current harmonics has been developed by the same research group in [10]. It is based on the motor magnetic saliency harmonics, which arise from rotor slotting and eccentricity.

The frequency of these rotor and dynamic eccentricity harmonics is related to the rotor speed by

$$
f_{\mathrm{seh}}=f_{1}\left[\left(k R+n_{d}\right) \frac{1-s}{p / 2}+n_{w}\right]
$$

where $f_{\text {seh }}$ is the frequency of rotor-related harmonic components; $f_{1}$ is the supply frequency; $k=0,1,2, \ldots ; R$ is the number of rotor slots; $p$ is the number of poles; $s$ is the motor slip; $n_{d}=0, \pm 1, \pm 2, \ldots$ is the order of rotor eccentricity; and $n_{w}= \pm 1, \pm 3, \ldots$ is the air-gap MMF harmonics order.

The speed estimation process consists of two algorithms: an initialization algorithm and an online speed-detection algorithm. The initialization algorithm employs the eccentricity harmonics to determine the value of $R$ and an optimal set of numbers for $k, n_{d}$, and $n_{w}$, which are usually unknown quantities. After that, the subsequent online speed-detection algorithm estimates the rotor speed by

$$
s=1-\frac{p}{2} \cdot \frac{\frac{f_{\mathrm{seh}}}{f_{1}}-n_{w}}{k R+n_{d}} .
$$

The slip estimate is independent of motor parameters, only requiring the number of poles. The determination of slot harmonic frequency requires the motor to be in steady state so

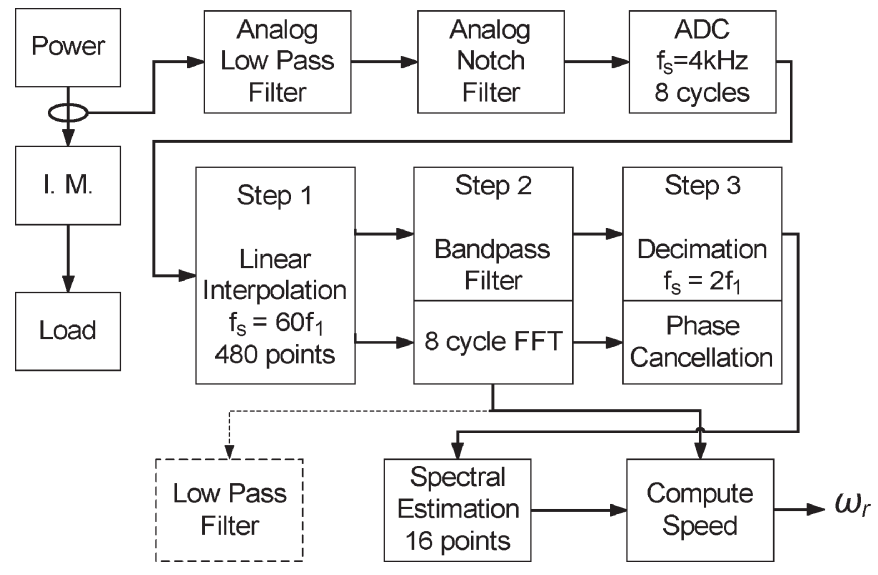

Fig. 2. Speed-detection algorithm using magnetic saliency harmonics.

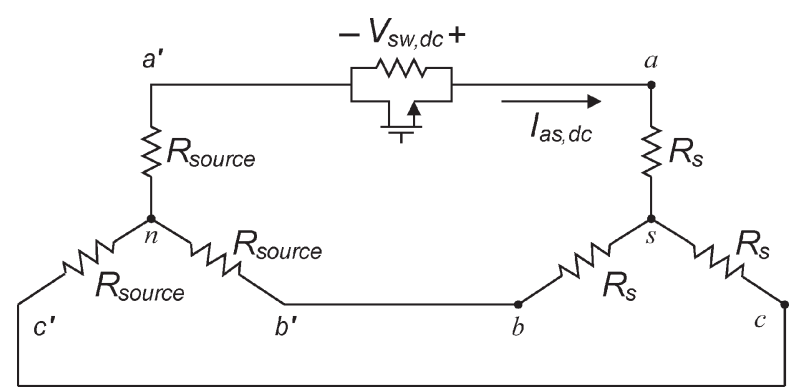

Fig. 3. DC equivalent circuit of source, motor, and dc injection.

that $f_{1}$ and $s$ are nearly constant; thus, this speed-detection method is ideal for online motor-efficiency estimation, where only steady states are concerned. This method provides robust speed estimate down to $1-\mathrm{Hz}$ operation with very high accuracy of within $5 \mathrm{r} / \mathrm{min}$ at high speeds and 0.005 -p.u. slip at low speeds. Fig. 2 shows the overall signal processing algorithm. For simplicity, the details are omitted here and available in [10].

\section{Online Stator Resistance Estimation}

Traditionally, the dc resistance of the stator winding is measured through an unpowered test. The measurement procedure is given in IEEE Standard 118. The advantage of the direct resistance measurement is the high accuracy. However, direct measurement is not acceptable for in-service motor testing because of the following disadvantages.

1) It requires the motor to be disconnected from service to perform an unpowered test. Therefore, the intrusion level is very high.

2) It only records the resistance value at a certain temperature. The actual resistance, however, is a linear function of the winding temperature.

To overcome these problems, many online estimation methods have been developed over the years [3]. Among them, the dc signal injection methods have proven to be very practical, in particular the use of a simple MOSFET-controlled circuit to intermittently inject a controllable dc bias into the motor [11]. The circuit structure is shown in Fig. 3. This method has low power dissipation and torque distortion and is capable of providing accurate stator resistance estimate under motor startup, load variation, and abnormal cooling conditions. 
The installation of the injection circuit can be easily done in the motor control center for mains-fed machines. The stator resistance estimation process can be regarded as nonintrusive after the injection circuit is developed and included. However, the injected dc signal produces unbalances in the stator voltages and currents and causes additional power dissipation and torque distortion. For soft-starter-connected and inverter-fed machines, this simple circuit is not required, since dc signal injection can be easily implemented in a software mode by controlling the excitation signals [12].

\section{NAGT METHOD}

The novelty and importance of this NAGT method come from the fact that only motor terminal quantities and nameplate information are needed for efficiency estimation while the motor is operating in service. The details of this method are given below.

\section{A. Data Measurements: Only Voltages and Currents}

For many induction motors, the neutral points are not accessible from the motor terminals. Therefore, only the line-to-line voltages are available. For a three-phase wye-connected motor, the line voltages and phase currents are assumed to add to zero as in

$$
\begin{aligned}
v_{a b}+v_{b c}+v_{c a} & =0 \\
i_{a}+i_{b}+i_{c} & =0
\end{aligned}
$$

where, $v_{a b}, v_{b c}$, and $v_{c a}$ are the line voltages, and $i_{a}, i_{b}$, and $i_{c}$ are the phase currents.

Let $\theta_{d a}(t)$ be the angle between the $d$-axis and stator $a$-axis at time $t$, and the transformation matrix $T$ is defined as in (4), shown at the bottom of the page.

Assuming that the zero-sequence components in the stator voltages and currents are negligible, the stator voltage and current space vectors, $v_{d q s}$ and $i_{d q s}$, can be derived as in (5), shown at the bottom of the page (time index $t$ omitted from here on for simplicity)

Note that

$$
\begin{aligned}
& v_{a} \cos \theta_{d a}+v_{a} \cos \left(\theta_{d a}-\frac{2 \pi}{3}\right)+v_{a} \cos \left(\theta_{d a}-\frac{4 \pi}{3}\right)=0 \\
& v_{a} \sin \theta_{d a}+v_{a} \sin \left(\theta_{d a}-\frac{2 \pi}{3}\right)+v_{a} \sin \left(\theta_{d a}-\frac{4 \pi}{3}\right)=0
\end{aligned}
$$

From (3)-(6), the stator voltage and current space vectors can be obtained using only two voltage sensors $\left(v_{a b}\right.$ and $v_{c a}$ ) and two current sensors $\left(i_{a}\right.$ and $\left.i_{b}\right)$ as in (7), shown at the bottom of the page.

In industrial plants, the motors are connected to motor control centers, where PTs and CTs are already installed for protection purpose. The proposed efficiency estimation method relies completely on motor terminal voltages and currents, requiring no torque and speed measurements. The entire data collection process can take place at the motor control center with almost no additional cost. Another benefit of this data

$$
T\left(\theta_{d a}(t)\right)=\sqrt{\frac{2}{3}}\left[\begin{array}{ccc}
\cos \theta_{d a}(t) & \cos \left(\theta_{d a}(t)-\frac{2 \pi}{3}\right) & \cos \left(\theta_{d a}(t)-\frac{4 \pi}{3}\right) \\
-\sin \theta_{d a}(t) & -\sin \left(\theta_{d a}(t)-\frac{2 \pi}{3}\right) & -\sin \left(\theta_{d a}(t)-\frac{4 \pi}{3}\right)
\end{array}\right]
$$

$$
\begin{aligned}
v_{d q s}= & {\left[\begin{array}{l}
v_{d s} \\
v_{q s}
\end{array}\right]=T\left(\theta_{d a}\right)\left[\begin{array}{l}
v_{a} \\
v_{b} \\
v_{c}
\end{array}\right] \quad i_{d q s}=\left[\begin{array}{l}
i_{d s} \\
i_{q s}
\end{array}\right]=T\left(\theta_{d a}\right)\left[\begin{array}{c}
i_{a} \\
i_{b} \\
i_{c}
\end{array}\right] } \\
v_{d q s}= & {\left[\begin{array}{l}
\sqrt{\frac{2}{3}}\left[v_{a} \cos \theta_{d a}+v_{b} \cos \left(\theta_{d a}-\frac{2 \pi}{3}\right)+v_{c} \cos \left(\theta_{d a}-\frac{4 \pi}{3}\right)\right] \\
-\sqrt{\frac{2}{3}}\left[v_{a} \sin \theta_{d a}+v_{b} \sin \left(\theta_{d a}-\frac{2 \pi}{3}\right)+v_{c} \sin \left(\theta_{d a}-\frac{4 \pi}{3}\right)\right]
\end{array}\right] } \\
i_{d q s}= & {\left[\begin{array}{l}
\sqrt{\frac{2}{3}}\left[i_{a} \cos \theta_{d a}+i_{b} \cos \left(\theta_{d a}-\frac{2 \pi}{3}\right)+i_{c} \cos \left(\theta_{d a}-\frac{4 \pi}{3}\right)\right] \\
-\sqrt{\frac{2}{3}}\left[i_{a} \sin \theta_{d a}+i_{b} \sin \left(\theta_{d a}-\frac{2 \pi}{3}\right)+i_{c} \sin \left(\theta_{d a}-\frac{4 \pi}{3}\right)\right]
\end{array}\right] }
\end{aligned}
$$

$$
\begin{aligned}
v_{d q s}= & {\left[\begin{array}{l}
\sqrt{\frac{2}{3}} \\
\sqrt{\frac{2}{3}}\left[v_{a b} \cos \left(\theta_{d a} \sin \left(\theta_{d a}-\frac{2 \pi}{3}\right)-v_{c a} \cos \left(\theta_{d a}-\frac{4 \pi}{3}\right)\right]\right. \\
\left.i_{d q s} \sin \left(\theta_{d a}-\frac{4 \pi}{3}\right)\right]
\end{array}\right] } \\
i_{d a} & {\left[\begin{array}{l}
\sqrt{\frac{2}{3}}\left[i_{a} \cos \theta_{d a}+i_{b} \cos \left(\theta_{d a}-\frac{2 \pi}{3}\right)-\left(i_{a}+i_{b}\right) \cos \left(\theta_{d a}-\frac{4 \pi}{3}\right)\right] \\
-\sqrt{\frac{2}{3}}\left[i_{a} \sin \theta_{d a}+i_{b} \sin \left(\theta_{d a}-\frac{2 \pi}{3}\right)-\left(i_{a}+i_{b}\right) \sin \left(\theta_{d a}-\frac{4 \pi}{3}\right)\right]
\end{array}\right] }
\end{aligned}
$$


measurement scheme is that the motor control centers are more easily accessible compared with the motors themselves, which further reduces the deployment cost.

\section{B. AGT Estimation}

The stator voltage equation in the $d-q$ system is

$$
v_{d q s}=R_{s} i_{d q s}+\frac{d \lambda_{d q s}}{d t}+\frac{d \theta_{d a}}{d t}\left[\begin{array}{cc}
0 & -1 \\
1 & 0
\end{array}\right] \lambda_{d q s}
$$

where $\lambda_{d q s}$ is the total flux linkage vector and $R_{s}$ is the average value of the three phase stator resistances.

Choose the stationary reference frame (denoted as the upper script "S"), i.e., $d \theta_{d a} / d t=0$. Thus, the flux linkage is given by the integral of input voltage minus the stator $I R$ drop

$$
\lambda_{d q s}^{\mathrm{S}}=\int\left(v_{d q s}^{\mathrm{S}}-R_{s} i_{d q s}^{\mathrm{S}}\right) d t .
$$

Then, the AGT $T_{\mathrm{ag}}$ can be derived as

$$
\begin{aligned}
T_{\mathrm{ag}}= & \frac{p}{2}\left|\lambda_{d q s}^{\mathrm{S}} \times i_{d q s}^{\mathrm{S}}\right| \\
= & \frac{\sqrt{3} p}{6}\left\{\left(i_{a}-i_{b}\right) \cdot \int\left[v_{c a}+R_{s}\left(2 i_{a}+i_{b}\right)\right] d t\right. \\
& \left.\quad+\left(2 i_{a}+i_{b}\right) \cdot \int\left[v_{a b}-R_{s}\left(i_{a}-i_{b}\right)\right] d t\right\} .
\end{aligned}
$$

The integrals in (10) represent the corresponding flux linkages. Since the sampling frequency of motor voltages and currents is usually large (greater than $2 \mathrm{kHz}$ ), a simple trapezoidal integration method can be used. Other numerical integration methods, e.g., Simpson's rules, can also be applied or better accuracy.

\section{Efficiency Estimation}

The instantaneous input power $P_{\text {input }}$ of a three-phase induction motor can be calculated from instantaneous voltages and currents as

$$
P_{\text {input }}=v_{a} i_{a}+v_{b} i_{b}+v_{c} i_{c}=-v_{c a}\left(i_{a}+i_{b}\right)-v_{a b} i_{b} .
$$

To reduce the ripples caused by the energy stored in the windings, the average value of instantaneous power is used to calculate efficiency.

The shaft output power $P_{\text {output }}$ is the product of the rotor speed $\omega_{r}$ and shaft torque $T_{\text {shaft }}$. The shaft torque is the difference between the AGT and torque losses corresponding to mechanical loss and rotor stray-load loss produced by rotor current and is given by

$$
T_{\text {shaft }}=T_{\mathrm{ag}}-\frac{W_{\mathrm{fw}}}{\omega_{r}}-\frac{W_{\mathrm{LLr}}}{\omega_{r}} .
$$

Since the majority of the stray-load loss is produced by rotor current, the rotor stray-load loss at the rated load is assumed according to Table I.
Finally, the efficiency $\eta$ is

$\eta=\frac{P_{\text {output }}}{P_{\text {input }}}=\frac{T_{\text {shaft }} \cdot \omega_{r}}{P_{\text {input }}}=\frac{T_{\mathrm{ag}} \cdot \omega_{r}-W_{\mathrm{fw}}-W_{\mathrm{LLr}}}{P_{\text {input }}}$.

\section{Discussions}

A significant contribution of this method is that the efficiency estimate is completely based on motor terminal quantities and motor nameplate information, eliminating the use of costly torque and speed transducers. This guarantees the nonintrusive nature of this method.

It can be easily implemented together with motor condition monitoring functions. Almost all condition monitoring algorithms require stator voltages and currents, while some also need stator resistance and rotor speed. When these algorithms are integrated together with the proposed efficiency estimation method, a common application interface can be built using the same collected motor data.

In this method, the air-gap flux (torque) is estimated directly using instantaneous voltage and current data. It does not need phasor computations and, thus, does not limit the analysis within a certain frequency frame. Therefore, the usage of this method can be extended from mains-fed motors to inverter-fed motors with any form of supply, such as space vector PWM, square wave, etc. Moreover, this method also considers the additional losses associated with unbalances and harmonics in the power supply, as well as the stator copper loss and stator stray-load loss.

However, the nonintrusiveness is obtained by sacrificing some accuracy, since the estimates of rotor speed, stator resistance, and no-load loss produce additional errors.

\section{EXPERIMENTAL VALIDATION}

The proposed method has been verified by both computer simulations and real experiments. The computer simulation results are omitted here in order to conserve space.

\section{A. Experimental Setup}

To evaluate the generality of the proposed method, three new induction motors are tested. These motors are intentionally selected with various physical configurations, such as different sizes (7.5 and $10 \mathrm{hp}$ ), designs (NEMA A and B), and enclosures (TEFC and ODP). The motor parameters are listed in Table II.

In the experimental setup, these motors are line connected to a 230-V mains supply. The voltages and currents are slightly unbalanced $\left(I_{-} / I_{+}<10 \%\right)$ and reflect the actual motor working condition. A dc generator feeding resistor loads serves as the dynamometer. Two line voltages $\left(v_{a b}\right.$ and $v_{c a}$ ) and two phase currents $\left(i_{a}\right.$ and $\left.i_{b}\right)$ are sampled using an NI PCI-6250 $\mathrm{M}$-series multifunction data acquisition system with 16-b resolution and 1.25-MS/s single-channel sampling rate and stored in a personal computer using NI LabVIEW. 
TABLE II

PARAMETERS OF THREE INDUCTION MOTORS IN EXPERIMENT

\begin{tabular}{|l|c|c|c|}
\hline & Motor I & Motor II & Motor III \\
\hline Brand & Leeson & GE & Marathon \\
\hline Rated Output Power (hp) & 7.5 & 7.5 & 10 \\
\hline Catalogue NO. & G140417 & S231 & U639 \\
\hline Phase & 3 -phase & 3 -phase & 3 -phase \\
\hline Rated Speed (rpm) & 1760 & 1755 & 1750 \\
\hline Rated Line Voltage (volts) & $230 / 460$ & $230 / 460$ & $230 / 460$ \\
\hline Full Load Current (amps) & $20.0 / 10.0$ & $18.2 / 9.1$ & $25.6 / 12.8$ \\
\hline Nominal Power Factor & 0.72 & 0.865 & 0.86 \\
\hline Nominal Efficiency & 0.885 & 0.895 & 0.895 \\
\hline Stator Resistance (ohms) & 0.326 & 1.044 & 0.223 \\
\hline NEMA Design & B & A & B \\
\hline Enclosure Type & ODP & TEFC & TEFC \\
\hline
\end{tabular}

\section{B. Error Analysis}

To evaluate and validate the accuracy of the estimated efficiencies, the actual motor efficiencies must be measured and their accuracies must be guaranteed. This is a key step in the entire testing process. In the experiment, the actual efficiencies are calculated as the ratio between the mechanical output power and the electrical input power.

The input power is directly calculated from two line-toline terminal voltages and two line currents. The two voltage transducers are LEM LV 25-P with 2500: 1000 conversion ratio, $10-500-\mathrm{V}$ rms voltage range, $\pm 0.8 \%$ accuracy, and $<0.2 \%$ linearity. The two current transducers are LEM LA 55-P with $1: 1000$ conversion ratio, 50-A rms current range, $\pm 0.65 \%$ accuracy, and $<0.15 \%$ linearity. Both of these transducers use Hall effect devices.

The output power is directly calculated from the output shaft torque and the rotor speed. An accurate in-line rotary torque transducer is installed between the motor and the load to measure the shaft torque. The torque transducer is a TQ501$1 \mathrm{~K}$ made by Omega with the following specifications: $10-\mathrm{V}$ dc excitation, $0-1000$-in $\cdot \mathrm{lb}$ torque range, $\pm 0.18 \%$ accuracy, $\pm 0.10 \%$ linearity, $\pm 0.10 \%$ hysteresis, $\pm 0.10 \%$ repeatability, $\pm 1.0 \%$ zero balance, and $6000 \mathrm{r} / \mathrm{min}$ (maximum). The rotor speed is measured by an Extech-461501 noncontact digital tachometer with $\pm 0.10 \%$ accuracy.

The accuracy of the measured efficiencies is highly dependent on the accuracies of the voltage, current, speed, and torque measurements. The accuracy of these measured values depends on the inherent accuracy of the instruments, on the precision of the readings, and the noise present in the measuring system. By using the worst case estimation method [14]

$$
\begin{aligned}
\eta \pm \Delta \eta & =\frac{P_{\text {output }} \pm \Delta P_{\text {output }}}{P_{\text {input }} \pm \Delta P_{\text {input }}} \\
& =\frac{\left(T_{\text {shaft }} \pm \Delta T_{\text {shaft }}\right) \cdot\left(\omega_{r} \pm \Delta \omega_{r}\right)}{\left(V_{s} \pm \Delta V_{s}\right) \cdot\left(I_{s} \pm \Delta I_{s}\right)}
\end{aligned}
$$

TABLE III

EXPERIMENTAL RESULTS OF MOTOR I (LEESON 7.5 hp ODP)

\begin{tabular}{|c|c|c|c|}
\hline $\begin{array}{c}\text { Load } \\
\text { Percentage (\%) }\end{array}$ & $\begin{array}{c}\text { Rotor Speed } \\
(\mathbf{r p m})\end{array}$ & $\begin{array}{c}\text { Estimated } \\
\text { Efficiency } \mathbf{( \% )}\end{array}$ & $\begin{array}{c}\text { Measured } \\
\text { Efficiency }(\%)\end{array}$ \\
\hline 8.54 & 1797.0 & 47.59 & 68.56 \\
\hline 18.54 & 1794.6 & 74.24 & 73.37 \\
\hline 23.50 & 1793.4 & 78.55 & 77.50 \\
\hline 33.14 & 1788.6 & 83.06 & 79.99 \\
\hline 40.23 & 1785.8 & 84.67 & 84.39 \\
\hline 54.37 & 1780.7 & 86.08 & 85.42 \\
\hline 68.22 & 1776.0 & 86.25 & 86.59 \\
\hline 75.07 & 1774.1 & 86.02 & 85.75 \\
\hline 88.75 & 1768.6 & 85.37 & 85.01 \\
\hline 101.51 & 1762.6 & 84.40 & 84.70 \\
\hline 111.66 & 1757.1 & 83.52 & 82.20 \\
\hline 117.53 & 1754.7 & 82.89 & 81.19 \\
\hline
\end{tabular}

the maximum error in the measured efficiencies $\Delta \eta / \eta$ is calculated as

$$
\begin{aligned}
1 \pm \frac{\Delta \eta}{\eta} & =\frac{\left(1 \pm \frac{\Delta T_{\text {shaft }}}{T_{\text {shaft }}}\right) \cdot\left(1 \pm \frac{\Delta \omega_{r}}{\omega_{r}}\right)}{\left(1 \pm \frac{\Delta V_{s}}{V_{s}}\right) \cdot\left(1 \pm \frac{\Delta I_{s}}{I_{s}}\right)} \\
& =\frac{(1 \pm 0.18 \%) \times(1 \pm 0.10 \%)}{(1 \pm 0.80 \%) \times(1 \pm 0.65 \%)} \approx 1 \pm 1.7 \% .
\end{aligned}
$$

Considering that the realistic error is normally much lower than the maximum error $\pm 1.7 \%$, conclusion can be drawn that the measured efficiencies are accurate enough to validate the estimates.

\section{Experiment Results}

The estimated motor efficiencies are calculated using the proposed NAGT method. The line voltages and phase currents are sampled at high frequency $(5 \mathrm{kHz}$ for Motors I and III, $2 \mathrm{kHz}$ for Motor II). Simpson's $1 / 3$ rule is applied for the numerical integration in the air-gap flux (torque) computation for its simplicity and accuracy [15]. The dc offset in the air-gap flux is filtered by a three-cycle $(50 \mathrm{~ms})$ moving average window.

Since the majority of motors in industrial plants operate at about $60 \%$ of their rated load, special attention is paid to $40 \%-90 \%$ load levels in this paper. For extremely low and high load conditions, an accurate motor-efficiency estimate is no longer necessary for making planning decisions. It can be predicted from a typical motor performance curve (efficiency versus load) that under these conditions, the motor has low efficiencies.

In the experiment, all three motors are tested under various load levels, ranging from almost no-load to overload conditions. Since the no-load test could be invasive in a range of circumstances in practice, during the experimental validation, the estimated no-load data are used for efficiency calculation, as suggested in Section II. The total combined no-load losses are estimated to be $3.5 \%$ of rated output power. The friction and windage loss is assumed as $1.2 \%$ rated output power, and the stray-load loss is estimated from Table I. 
TABLE IV

EXPERIMENTAL RESULTS OF MOTOR II (GE 7.5 hp TEFC)

\begin{tabular}{|c|c|c|c|}
\hline $\begin{array}{c}\text { Load } \\
\text { Percentage (\%) }\end{array}$ & $\begin{array}{c}\text { Rotor Speed } \\
\text { (rpm) }\end{array}$ & $\begin{array}{c}\text { Estimated } \\
\text { Efficiency }(\%)\end{array}$ & $\begin{array}{c}\text { Measured } \\
\text { Efficiency }(\%)\end{array}$ \\
\hline 19.09 & 1775.0 & 90.68 & 92.45 \\
\hline 21.77 & 1771.5 & 91.26 & 91.63 \\
\hline 24.45 & 1768.0 & 91.65 & 92.38 \\
\hline 27.17 & 1764.5 & 91.89 & 92.75 \\
\hline 29.77 & 1760.8 & 91.94 & 92.28 \\
\hline 35.03 & 1753.5 & 91.71 & 91.24 \\
\hline 40.37 & 1746.0 & 91.24 & 91.09 \\
\hline 50.78 & 1730.0 & 90.00 & 88.47 \\
\hline 61.14 & 1713.0 & 88.46 & 87.08 \\
\hline 71.26 & 1694.0 & 86.65 & 85.48 \\
\hline 81.56 & 1672.0 & 84.49 & 83.39 \\
\hline 91.27 & 1647.0 & 82.10 & 80.21 \\
\hline
\end{tabular}

TABLE V

EXPERIMENTAL RESUlTS OF MOTOR III (MARATHON $10 \mathrm{hp} \mathrm{TEFC)}$

\begin{tabular}{|c|c|c|c|}
\hline $\begin{array}{c}\text { Load } \\
\text { Percentage (\%) }\end{array}$ & $\begin{array}{c}\text { Rotor Speed } \\
\text { (rpm) }\end{array}$ & $\begin{array}{c}\text { Estimated } \\
\text { Efficiency (\%) }\end{array}$ & $\begin{array}{c}\text { Measured } \\
\text { Efficiency (\%) }\end{array}$ \\
\hline 4.04 & 1799.2 & 55.62 & 66.23 \\
\hline 12.85 & 1796.0 & 84.80 & 84.50 \\
\hline 26.10 & 1789.2 & 91.00 & 89.70 \\
\hline 32.70 & 1787.4 & 91.66 & 91.52 \\
\hline 39.69 & 1785.0 & 91.82 & 91.15 \\
\hline 48.49 & 1782.6 & 91.76 & 90.25 \\
\hline 56.44 & 1779.0 & 91.39 & 90.86 \\
\hline 65.52 & 1776.0 & 91.03 & 90.49 \\
\hline 74.11 & 1772.6 & 90.41 & 90.42 \\
\hline 82.27 & 1768.9 & 89.80 & 89.51 \\
\hline 96.42 & 1761.8 & 88.56 & 88.81 \\
\hline 106.17 & 1752.8 & 86.86 & 86.87 \\
\hline
\end{tabular}

Tables III-V list the experimental results from Motors I-III, respectively, including load percentage, rotor speed, and estimated and measured motor efficiencies. Figs. 4-6 graphically illustrate the estimated and actual measured efficiencies versus the load percentage and rotor speed.

For all three motors, the estimated efficiencies closely agree with the measured efficiencies (within $2 \%$ errors) during the normal motor operations (load ranges from $40 \%$ to $90 \%$ of rated load). Moreover, this method also gives relatively accurate efficiency estimates at very low load (less than 30\% rated load) and high load (greater than $90 \%$ rated load) conditions.

The experimental results also confirm the fact that the generic motor nameplate data could contain a large amount of inaccuracies. The experimental results from Motors I and III agree well with the nameplate data. When the motor operates at the rated load condition, the speed is close to its rated value. The maximum efficiency occurs at normal load levels, which is typically how these motors are designed to operate.

However, the results from Motor II show obvious inconsistencies with the nameplate data. At rated load, the rotor speed drops to around $1640 \mathrm{r} / \mathrm{min}$, which is well below the rated speed of $1755 \mathrm{r} / \mathrm{min}$. Moreover, the maximum efficiency occurs at around 30\% rated load, which is lower than the desired design value. Repetitive tests on two identical GE S231 motors give similar results. It can be concluded that the generic motor template data contain significant inaccuracies for Motor II.

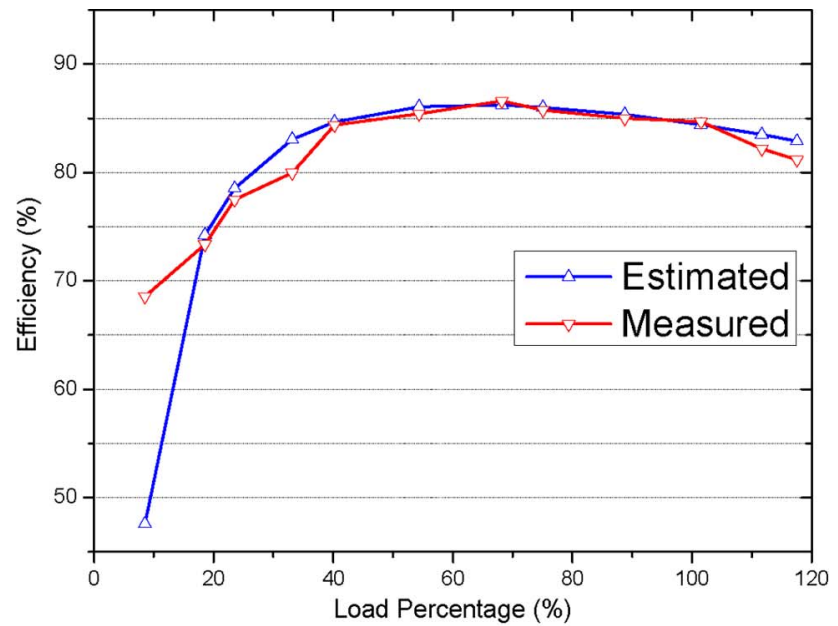

(a)

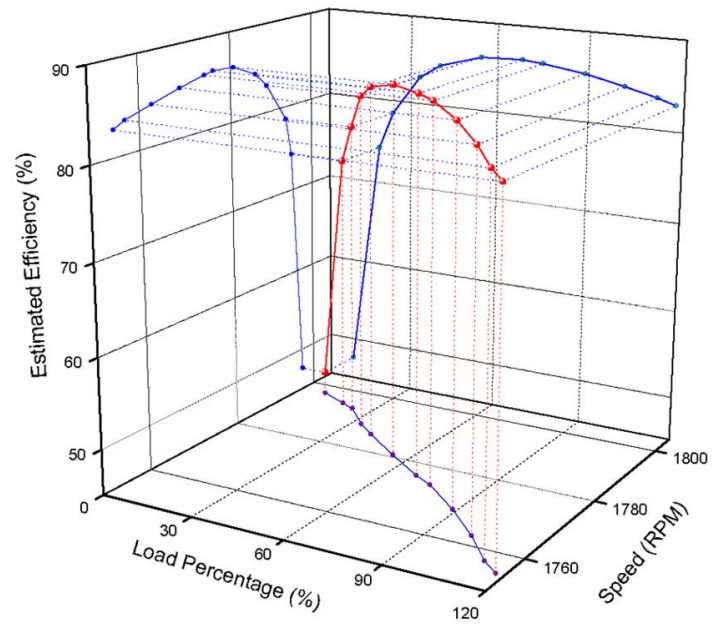

(b)

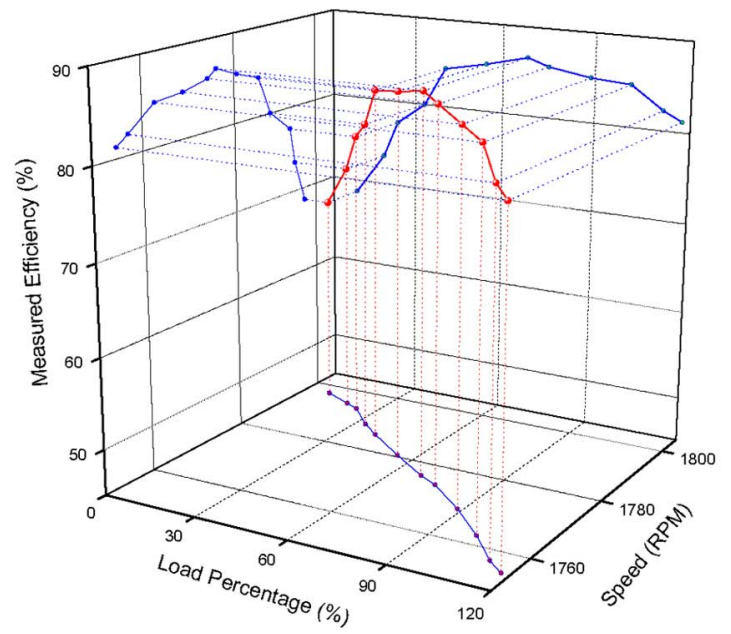

(c)

Fig. 4. Estimated and measured efficiencies of Motor I (Leeson, $7.5 \mathrm{hp}$, NEMA-B, ODP, $1760 \mathrm{r} / \mathrm{min}$ ). (a) Comparison of estimated and measured efficiencies. (b) Estimated efficiencies versus load percentage and speed. (c) Measured efficiencies versus load percentage and speed.

However, since the proposed NAGT method relies mostly on terminal voltages and currents, the inaccuracies in the nameplate data do not generate obvious errors in efficiency estimates, as shown in Fig. 5. 


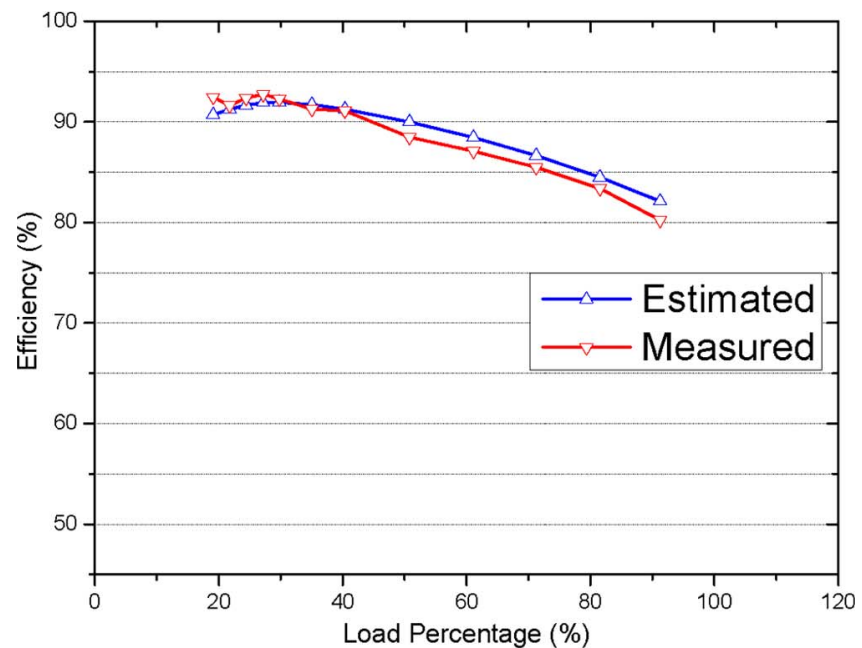

(a)

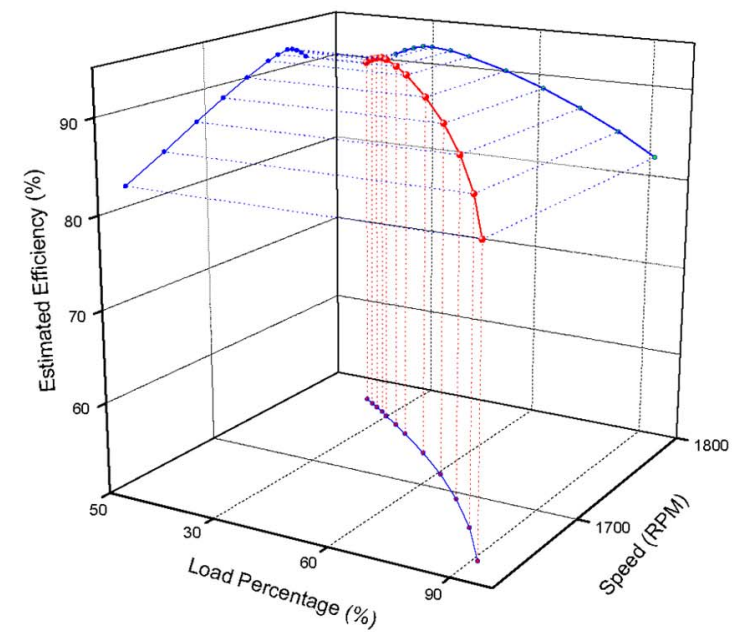

(b)

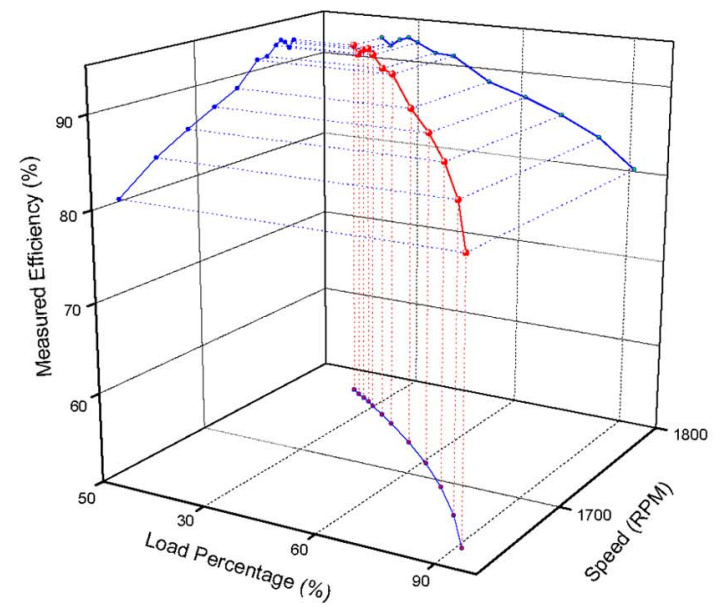

(c)

Fig. 5. Estimated and measured efficiencies of Motor II (GE, 7.5 hp, NEMA-A, TEFC, $1755 \mathrm{r} / \mathrm{min}$ ). (a) Comparison of estimated and measured efficiencies. (b) Estimated efficiencies versus load percentage and speed. (c) Measured efficiencies versus load percentage and speed.

To summarize, the agreement between the estimated and measured efficiencies in the experimental results validates the proposed NAGT method.

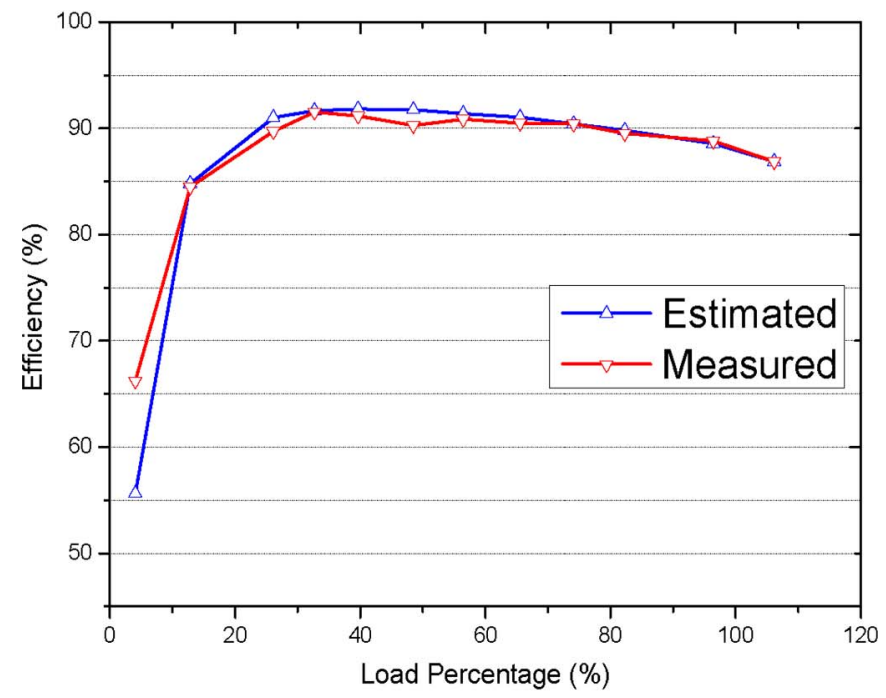

(a)

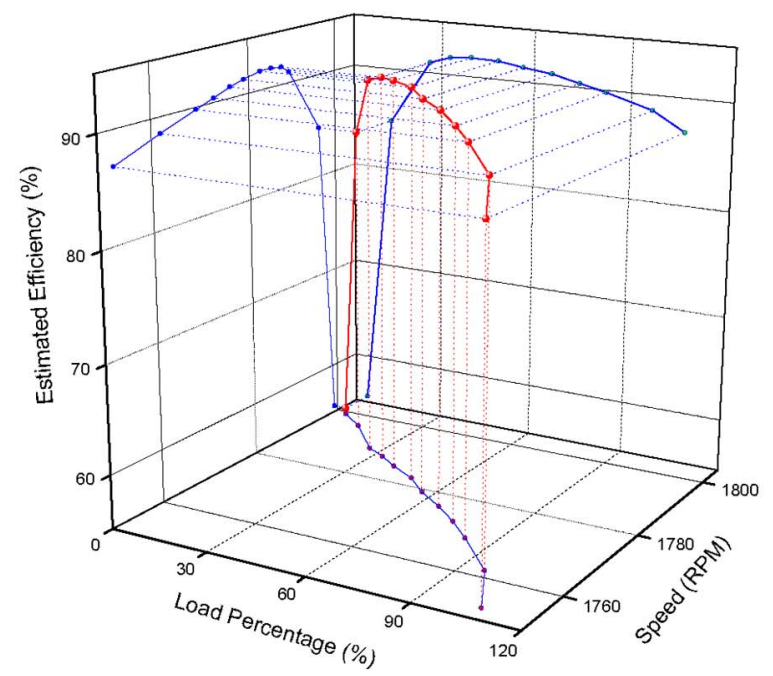

(b)

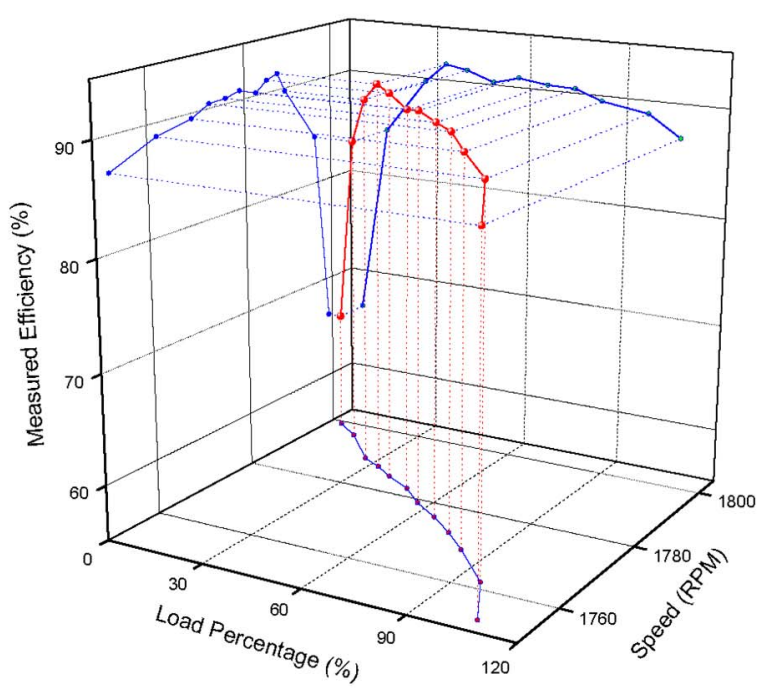

(c)

Fig. 6. Estimated and measured efficiencies of Motor III (Marathon, $10 \mathrm{hp}$, NEMA-B, TEFC, $1750 \mathrm{r} / \mathrm{min}$ ). (a) Comparison of estimated and measured efficiencies. (b) Estimated efficiencies versus load percentage and speed. (c) Measured efficiencies versus load percentage and speed. 


\section{CONCLUSION}

This paper has proposed an online nonintrusive method for in-service motor-efficiency estimation based on AGT using only motor terminal quantities and nameplate information with special considerations of motor condition monitoring requirements. This method has provided a theoretical foundation for low-cost energy evaluation in industrial plants, where motor terminal voltages and currents are readily accessible from the motor control centers without interfering with the motor's normal operations.

This method has been experimentally validated on small induction motors (less than $20 \mathrm{hp}$ ) by testing three induction motors with different physical configurations, such as size, design, and enclosure type. The estimated efficiencies of all three motors agree with the measured efficiencies within $2 \%$ errors during the normal load levels.

A novel and significant contribution of this method is that the efficiency estimation is completely based on motor terminal quantities and motor nameplate information. Costly torque and speed transducers are eliminated.

\section{REFERENCES}

[1] Xenergy, United States Industrial Electric Motor Systems Market Opportunities Assessment, 2002, Washington, DC: Office Energy Efficiency Renewable Energy, U.S. Dept. Energy. Prepared for Oak Ridge Nat. Lab. and DOE's Office of Ind. Technol.

[2] IEEE Standard Test Procedure for Polyphase Induction Motors and Generators, IEEE Standard 112-2004.

[3] B. Lu, T. G. Habetler, and R. G. Harley, "A survey of efficiency-estimation methods for in-service induction motors," IEEE Trans. Ind. Appl., vol. 42, no. 4, pp. 924-933, Jul./Aug. 2006.

[4] E. B. Agamloh, A. K. Wallace, A. von Jouanne, K. J. Anderson, and J. A. Rooks, "Assessment of nonintrusive motor efficiency estimators," IEEE Trans. Ind. Appl., vol. 41, no. 1, pp. 127-133, Jan./Feb. 2005.

[5] J. O. Ojo, V. Ostovic, T. A. Lipo, and J. C. White, "Measurement and computation of starting torque pulsations of salient pole synchronous motors," IEEE Trans. Energy Convers., vol. 5, no. 1, pp. 176-182, Mar. 1990.

[6] G. B. Kliman, R. A. Koegl, W. J. Premerlani, and T. B. Breen, "A demonstration of a sensorless torque meter for AC motors," in Conf. Rec. IEEE IAS Annu. Meeting, Oct. 1996, vol. 1, pp. 633-637.

[7] J. Hsu and B. P. Scoggins, "Field test of motor efficiency and load changes through air-gap torque," IEEE Trans. Ind. Appl., vol. 10, no. 3, pp. 477483, Sep. 1995.

[8] J. Hsu, J. Kueck, M. Olszewski, D. Casada, P. Otaduy, and L. Tolbert, "Comparison of induction motor field efficiency evaluation methods," IEEE Trans. Ind. Appl., vol. 34, no. 1, pp. 117-125, Jan./Feb. 1998.

[9] A. K. Wallace and E. J. Widenbrug, "Motor efficiency determination: From testing laboratory to plant installation," in Proc. IEEE Pulp Paper Ind. Tech. Annu. Conf., Jun. 1999, pp. 190-195.

[10] K. D. Hurst and T. G. Habetler, "Sensorless speed measurement using current harmonic spectral estimation in induction machine drives," IEEE Trans. Power Electron., vol. 11, no. 1, pp. 66-73, Jan. 1996.

[11] S. B. Lee and T. G. Habetler, "An online stator winding resistance estimation technique for temperature monitoring of line-connected induction machines," IEEE Trans. Ind. Appl., vol. 39, no. 3, pp. 685-694, May/Jun. 2003.

[12] P. Zhang, B. Lu, and T. G. Habetler, "A remote and sensorless stator winding resistance estimation method for thermal protection of soft-starterconnected induction machines," IEEE Trans. Ind. Electron., vol. 55, no. 10 , pp. 3611-3618, Oct. 2008.

[13] "DOE Industry Tools," DOE MotorMaster + Website. [Online]. Available: http://www1.eere.energy.gov/industry/bestpractices/software.html

[14] R. S. Colby and D. L. Flora, "Measured efficiency of high efficiency and standard induction motors," in Conf. Rec. IEEE IAS Annu. Meeting, Oct. 1990, vol. 1, pp. 18-23.

[15] R. J. Schilling and S. L. Harris, Applied Numerical Methods for Engineers Using MATLAB and C. Pacific Grove, CA: Thomson, 2000.

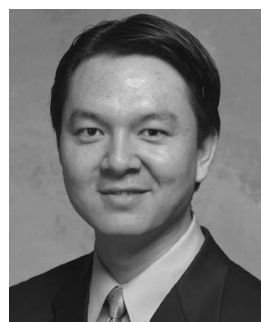

Bin Lu (S'00-M'06) received the B.Eng. degree in automation from Tsinghua University, Beijing, China, in 2001, the M.S. degree in electrical engineering from the University of South Carolina, Columbia, in 2003, and the Ph.D. degree in electrical engineering from the Georgia Institute of Technology, Atlanta, in 2006.

From 2001 to 2003, he was with the VTB group of the University of South Carolina, Columbia, developing a real-time virtual test bed for the hardwarein-the-loop testing of dynamic power systems. In July 2004, he was a Graduate Research Assistant with the power-electronics and motor-diagnostics group of the Georgia Institute of Technology, working on the energy evaluation and condition monitoring of electric machines. In summer of 2006, he was with the General Motors R\&D Center, Warren, MI, as a Graduate Summer Intern. Since October 2006, he has been with the Innovation Center, Eaton Corporation, Milwaukee, WI, where he is currently an Engineering Specialist and Program Manager. He has authored over 30 papers published in refereed journals and international conference proceedings and has five patents pending in these areas. His research interests include electric motor drives and diagnostics, renewable energy and distributed generation, power electronics, modeling and simulation, and application of sensor networks in electric power areas.

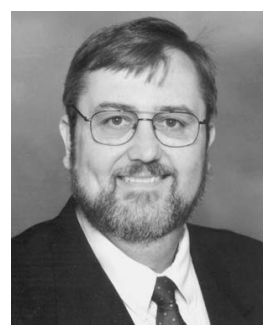

Thomas G. Habetler (S'82-M'83-SM'92-F'02) received the B.S. and M.S. degrees in electrical engineering from Marquette University, Milwaukee, WI, in 1981 and 1984, respectively, and the Ph.D. degree from the University of Wisconsin, Madison, in 1989.

From 1983 to 1985 , he was with the ElectroMotive Division of General Motors as a Project Engineer. Since 1989, he has been with the Georgia Institute of Technology, Atlanta, where he is currently a Professor of electrical engineering with the School of Electrical and Computer Engineering. He has published over 100 papers in the field. He is a regular consultant to industry in the field of condition-based diagnostics for electrical systems. His research interests are in electric machine protection and condition monitoring, and switching converter technology and drives.

Dr. Habetler currently serves as IEEE Division II Director-Elect, is a PastPresident of the IEEE Power Electronics Society, and Past-Chair of the Industrial Power Converter Committee of the IEEE Industry Applications Society. He has received four conference prize paper awards from the Industry Applications Society.

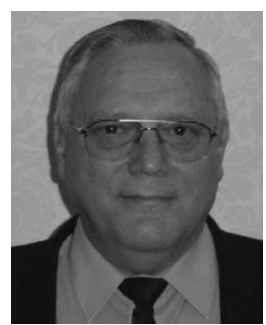

Ronald G. Harley (M'77-SM'86-F'92) received the M.Sc.Eng. degree (cum laude) in electrical engineering from the University of Pretoria, Pretoria, South Africa, in 1965, and the Ph.D. degree from London University, London, U.K., in 1969.

In 1971, he was appointed to the Chair of Electrical Machines and Power Systems at the University of Natal, Durban, South Africa, where he was a Professor of electrical engineering for many years, and also served as the Department Head and Deputy Dean of Engineering. He is currently the Duke Power Company Distinguished Professor with the School of Electrical and Computer Engineering, Georgia Institute of Technology, Atlanta. He has coauthored some 380 papers in refereed journals and international conferences and is the holder of three patents. Ten of the papers attracted prizes from journals and conferences. His research interests include the dynamic behavior and condition monitoring of electric machines, motor drives, power systems and their components, and controlling them by the use of power electronics and intelligent control algorithms.

Dr. Harley is a Fellow of the Institution of Electrical engineers, U.K. $\mathrm{He}$ is also a Fellow of the Royal Society in South Africa and a Founder Member of the Academy of Science in South Africa formed in 1994. During 2000 and 2001, he was one of the IEEE Industry Applications Society's six Distinguished Lecturers. He was the Vice-President of Operations of the IEEE Power Electronics Society (2003 to 2004) and Chair of the Atlanta Chapter of the IEEE Power Engineering Society. He is currently the Chair of the Distinguished Lecturers and Regional Speakers program of the IEEE Industry Applications Society. He received the Cyrill Veinott Award in 2005 from the Power Engineering Society for his outstanding contributions to the field of electromechanical energy conversion. 


\title{
A Nonintrusive Efficiency Estimation Method for In-Service Motor Testing using a Modified Induction Motor Equivalent Circuit
}

\author{
Bin Lu, Thomas G. Habetler, and Ronald G. Harley \\ School of Electrical and Computer Engineering \\ Georgia Institute of Technology \\ Atlanta, GA 30332, USA
}

Phone: 404-894-9829, Fax: 404-894-4641

\{binlu, thabetler, rharley\}@ece.gatech.edu

\begin{abstract}
On average, industrial motors operate at no more than $60 \%$ of their rated load, and thus at reduced efficiency which results in wasted energy. Motor efficiency evaluation enables the energy savings in industry. However, because of the uninterrupted characteristic of industrial processes, traditional methods defined in IEEE Std-112 cannot be used for these in-service motors. This paper proposes a novel nonintrusive method for in-service motor efficiency estimation based on a modified induction motor equivalent circuit using only motor terminal quantities and nameplate information. Rotor speed and stator resistance are extracted from stator voltages and currents. No load test is eliminated by using empirical values instead. Rotor stray-load loss is considered in the equivalent circuit by adding an equivalent resistor in series with the rotor circuit, and its value is derived from the assumed stray-load loss suggested in IEEE Std-112. The parameters of the equivalent circuit are solved using only stator voltage and current phasors and motor nameplate data. Three numerical root finding methods are adopted to guarantee the solution of the parameters under any situations. Finally, the proposed method is verified by testing a $7.5 \mathrm{hp}$ TEFC induction motor. Experimental results are presented and analyzed.
\end{abstract}

Index Terms - Efficiency estimation, in-service testing, IEEE Std-112, induction motors, equivalent circuit, Newton-Raphson method, simulated annealing method, particle swam optimization.

\section{INTRODUCTION}

Motor-driven systems use over $2 / 3$ of the total electric energy consumed by industry in the United States. In industry, motors below $200 \mathrm{hp}$ make up $98 \%$ of the motors in service and consume $85 \%$ of the energy used. On average, these motors operate at no more than $60 \%$ of their rated load because of oversized installations or underloaded conditions, and thus at reduced efficiency which results in wasted energy. Motor efficiency evaluation enables the energy savings in industry. However, because of the uninterrupted characteristic of industrial processes, traditional methods defined in IEEE Std-112 cannot be used. Nonintrusive motor efficiency estimation methods have to be developed for in-service motor testing.

In [1], the same authors present a comprehensive survey on motor efficiency estimation methods and provide suggestions for nonintrusive methods for in-service motor testing. Over twenty methods are summarized into 9 categories according to their physical nature. Among all of these categories, the induction motor equivalent based methods are regarded as one of the least intrusive methods. This is because that the parameters of the equivalent circuit of an induction motor can be extracted from motor input and output variables, such as voltages, currents, speed, etc. And as a result, the efficiency can be computed using the equivalent circuit. A significant advantage of this category of methods is that after the equivalent circuit is developed, the efficiency of any operating point can be estimated.

Over the years, many methods have been developed based on induction motor equivalent circuit. The IEEE Std-112 F method is the standard equivalent circuit method [2]. It requires an impedance test and the complete no-load, variable voltage test. Besides the stray-load loss is measured through an additional removed-rotor and reverse rotation tests. Although this method is expected to be quite accurate, the required tests still make it impossible to be used in in-service testing. The standard 112-F method is modified by Ontario Hydro by eliminating the variable voltage test [3]. However, a no load test and a full load test both under rated voltage are still required. In addition, direct stator resistance measurement is also needed. To further reduce the intrusiveness, a modified equivalent circuit based method is developed by Oak Ridge National Lab in [3]. Stator resistance is estimated using generic relationships based on motor nameplate information. The rotor stay-load loss is considered in the equivalent circuit by adding an extra resistor in the rotor circuit. Only motor voltages, currents, and rotor speed need to be measured for efficiency estimation. It is a very low intrusive method. However, the parameters of the equivalent circuit are solved from imaginary rated load condition and locked rotor condition, which completely rely on motor nameplate information. According to NEMA MG-1, up to $20 \%$ errors are allowed in the motor nameplate information [4]. Therefore, the equivalent circuit developed using this method could have fairly large errors. In [5], another interesting method is proposed, which calculates the motor parameters using two different motor operating points. However, it requires a rather intrusive measurement of stator resistance and stator winding temperature, which are not even possible in many cases. Besides, motor parameters are obtained by solving a set of highly nonlinear and multi-dimensional equations. The detailed methods of solving such equations are not reported. 


\section{A Nonintrusive Equivalent CirCuit Method}

This paper proposes a novel nonintrusive method for in-service motor efficiency estimation based on a modified induction motor equivalent circuit using only motor terminal quantities and nameplate information. Only a few cycles of line voltages and currents from two different motor operating points and motor nameplate information are required to develop the equivalent circuit.

\section{A. Phasor Extraction}

For many wye-connected induction motors, the neutral points are not accessible from the motor terminals. Therefore, only the line-to-line voltages and phase currents are available. The stator line-to-neutral voltages can be obtained through a simple matrix transformation, assuming the zero sequence components are negligible. Then the positive and negative sequence phase voltage and current phasors can be obtained using a digital positive and negative sequence waveform systhesizer. Fig. 1 and Fig. 2 illustrate the positive and negative sequence equivalent circuits of an induction motor with added stray-load resistors, respectively.

For majority of induction motors, the effect of negative sequence in determining the motor equivalent circuit can be neglected, since the negative sequence components in the utility voltage are negligible and the motor stator windings are designed close to perfect symmetry. However, the negative sequence has to be considered when the motor operates under unbalanced conditions, which can be caused by either the utility such as excessive single phase loads or the motor itself such as stator turn faults.

The effect of negative sequence can be considered by applying the same methodology using negative sequence equivalent circuit. For simplicity, only positive sequence voltage and current are considered for the rest of this paper.

\section{B. Rotor Stray-Load Loss Resistor}

The rotor stray-load loss is defined in [2] as

$$
W_{L L r}=\left(\frac{I_{2}}{I_{2 \_ \text {rated }}}\right)^{2} W_{L L r_{-} \text {rated }}=3\left|\tilde{I}_{2}\right|^{2} R_{L L}
$$

Therefore, an equivalent stray-load resistor, $R_{L L}$, can be added in series with the rotor circuit, as shown in Fig. 1 and Fig. 2.

$$
R_{L L}=\frac{W_{L L r_{r} \text { rated }}}{3 I_{2 \_ \text {rated }}^{2}}=\text { const. }
$$

Since the stray-load loss is primarily determined by the rotor current, the rotor stray-load loss, $W_{L L r_{\text {r rated }}}$, can be estimated using the assumed stray-load values defined in IEEE Std-112.

\section{Sensorless Rotor Speed Estimation}

Direct measurement of the rotor speed requires a shaft-mounted speed encoder or optical tachometer to be installed. This reduces the reliability and increases the cost. During the past decade, numerous sensorless rotor speed estimation schemes have been proposed. A sensorless speed estimation method based on stator current harmonics has been developed in the same research group in [6]. It requires only the number of poles from the machine parameters. This method provides robust and parameter-independent speed estimate down to $1 \mathrm{~Hz}$ operation with very high accuracy of within $5 \mathrm{rpm}$ at high speeds and 0.005 p.u. slip at low speeds. Fig. 3 shows the detailed procedure of this method.

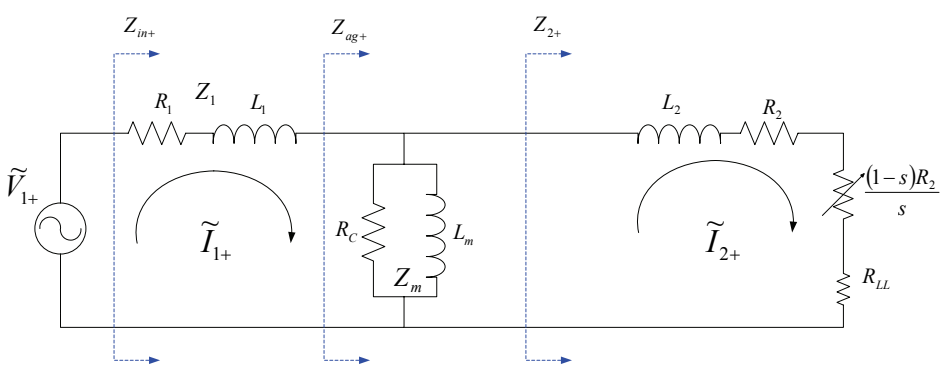

Fig. 1. A modified induction motor positive sequence equivalent circuit with an added equivalent stray-load resistor.

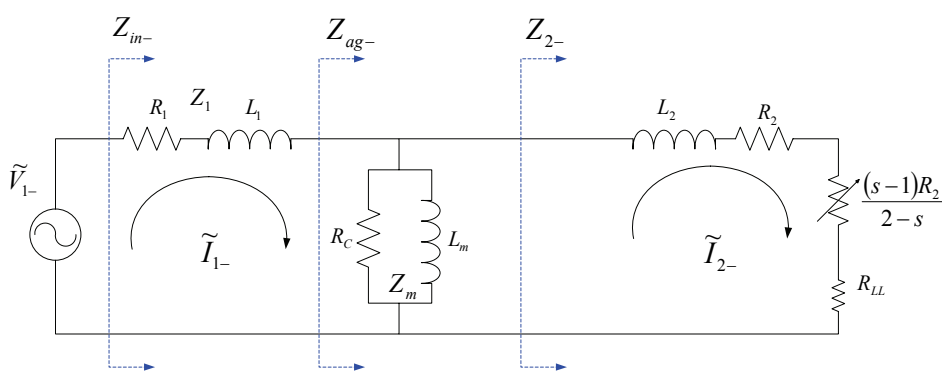

Fig. 2. A modified induction motor negative sequence equivalent circuit with an added equivalent stray-load resistor.

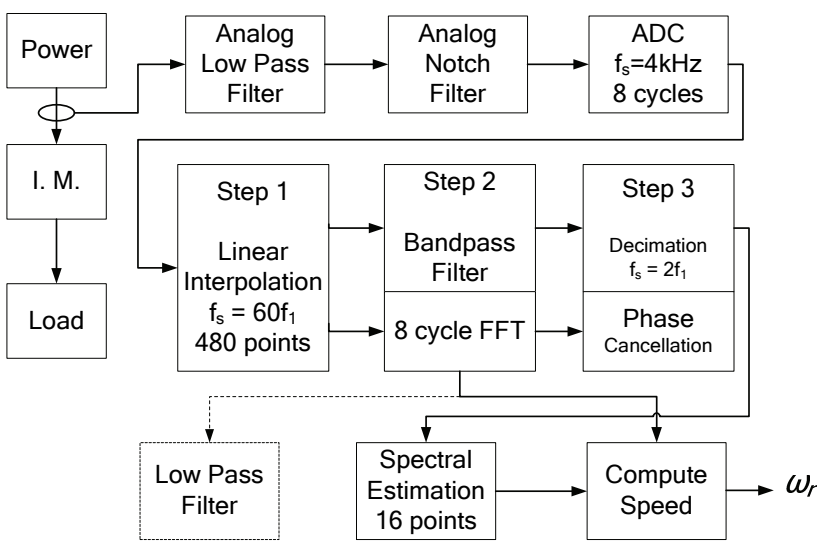

Fig. 3. Speed detection algorithm using rotor slot harmonics. 


\section{Online Stator Resistance Estimation}

Traditionally, the de resistance of the stator winding is measured through an unpowered test. The obvious advantage is its high accuracy. However, direct measurement is not acceptable for in-service motor testing because it requires the motor to be disconnected from service to perform an unpowered test and it records the resistance only at a certain temperature.

To overcome these problems, many online estimation methods have been developed over the years. Among them, the dc signal injection methods have been proved to be very practical. Specially, a simple MOSFET controlled circuit is developed to intermittently inject a controllable dc bias into the motor in [7]. The circuit structure is shown in Fig. 4. This method has very low power dissipation and torque distortion, and is capable of providing accurate stator resistance estimate under motor startup, load variation, and abnormal cooling conditions.

The installation of the injection circuit can be easily done in the motor control center for mains fed machines. The stator resistance estimation process can be regarded as non-intrusive, after the injection circuit is developed and included. However, the injected dc signal produces unbanlances in the stator voltages and currents, and causes additional power dissipation and torque distortion. For drive connected machines, even this simple circuit is not required, since dc signal injection can be easily implemented in a software mode by controlling the PWM signals.

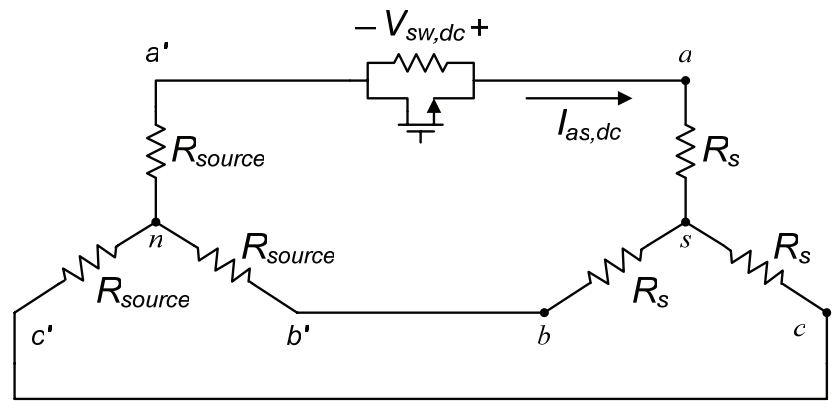

Fig. 4. DC equivalent circuit of source, motor, and dc injection.

\section{E. Input Impedance Functions}

In the modified induction motor positive sequence equivalent circuit in Fig. 1, the following impedance terms are defined (the subscript ' + ' is dropped for simplicity),

$$
\begin{gathered}
Z_{1}=R_{1}+j \omega L_{1} \\
Z_{2}=\frac{R_{2}}{s}+R_{L L}+j \omega L_{2} \\
Z_{m}=R_{C} / / j \omega L_{m}=\frac{j \omega R_{C} L_{m}}{R_{C}+j \omega L_{m}}
\end{gathered}
$$

Considering that the stator resistance, $R_{l}$, and slip, $s$, are known from motor voltages and currents as previously discussed, and stator and rotor leakage inductances have a specific ratio $(1.0,0.67$, or 0.43$)$ for a certain NEMA design [4], the input impedance can be expressed as a function of only four independent unknown parameters: $L_{l}, R_{C}, L_{m}$, and $R_{2}$.

$$
\begin{aligned}
& Z_{\text {in }}=Z_{1}+Z_{a g}=Z_{1}+Z_{m} / / Z_{2} \\
& =Z_{1}+\frac{Z_{m} Z_{2}}{Z_{m}+Z_{2}}=f\left(L_{1}, R_{C}, L_{m}, R_{2}\right)
\end{aligned}
$$

Splitting the real and imaginary parts, two independent equations can be obtained from each motor operating point.

$$
\left\{\begin{array}{c}
\operatorname{real}\left(Z_{\text {in }}\right)=\operatorname{real}\left[f\left(L_{1}, R_{C}, L_{m}, R_{2}\right)\right]=\operatorname{real}\left(\frac{\widetilde{V}_{1}}{\widetilde{I}_{1}}\right) \\
\operatorname{imag}\left(Z_{i n}\right)=\operatorname{imag}\left[f\left(L_{1}, R_{C}, L_{m}, R_{2}\right)\right]=\operatorname{imag}\left(\frac{\widetilde{V}_{1}}{\widetilde{I}_{1}}\right)
\end{array}\right.
$$

\section{F. Solving Motor Parameters using Numerical Methods}

Expanding (2), two independent equations can be obtained at each load points as (3). In order to solve four independent unknowns, a set of four independent equations are developed from two carefully selected load conditions. Too close conditions will result in ill-conditioned equations, while too spread conditions will result in additional errors caused by the parameter variations due to temperature change, flux saturation, etc. Solving such a highly nonlinear and multi-dimensional equations system is not trivial. Three numerical root finding methods, Newton-Raphson method [9], simulated annealing method [9], and particle swarm optimization (PSO) method [10], [11], have been studied and implemented. Newton method converges in a few steps, but a reasonable initial condition is required. Simulated annealing method and particle swarm optimization both target on global optimization. They can converge from a general zero initial condition, but the converging speeds are much slower.

The details of solving motor parameters using these three methods are discussed by the same authors in [12].

$$
\left\{\begin{array}{l}
\operatorname{real}\left(\frac{\widetilde{V}_{1}}{\widetilde{I}_{1}}\right)=R_{1}+\frac{R_{C} X_{m}\left(\frac{R_{2}}{s}+R_{L L}\right)\left[R_{C} X_{m}+\frac{R_{C} X_{1}}{k}+\left(\frac{R_{2}}{s}+R_{L L}\right) X_{m}\right]-\frac{R_{C} X_{m} X_{1}}{k}\left[R_{C}\left(\frac{R_{2}}{s}+R_{L L}\right)-\frac{X_{m} X_{1}}{k}\right]}{\left[R_{C}\left(\frac{R_{2}}{s}+R_{L L}\right)-\frac{X_{m} X_{1}}{k}\right]^{2}+\left[R_{C} X_{m}+\frac{R_{C} X_{1}}{k}+\left(\frac{R_{2}}{s}+R_{L L}\right) X_{m}\right]^{2}} \\
\operatorname{imag}\left(\frac{\widetilde{V}_{1}}{\widetilde{I}_{1}}\right)=X_{1}+\frac{\frac{R_{C} X_{m} X_{1}}{k}\left[R_{C} X_{m}+\frac{R_{C} X_{1}}{k}+\left(\frac{R_{2}}{s}+R_{L L}\right) X_{m}\right]+R_{C} X_{m}\left(\frac{R_{2}}{s}+R_{L L}\right)\left[R_{C}\left(\frac{R_{2}}{s}+R_{L L}\right)-\frac{X_{m} X_{1}}{k}\right]}{\left[R_{C}\left(\frac{R_{2}}{s}+R_{L L}\right)-\frac{X_{m} X_{1}}{k}\right]^{2}+\left[R_{C} X_{m}+\frac{R_{C} X_{1}}{k}+\left(\frac{R_{2}}{s}+R_{L L}\right) X_{m}\right]^{2}}
\end{array}\right.
$$




\section{G. Efficiency Estimation}

After the parameters of the equivalent circuit are solved, the motor efficiency at any load can be simply computed as

$$
\begin{gathered}
\eta=\frac{P_{\text {ouput }}}{P_{\text {input }}}=\frac{3\left|\widetilde{I}_{2}\right|^{2} \frac{(1-s)}{s} R_{2}-W_{f w}}{3\left|\tilde{I}_{1}\right|^{2} \operatorname{real}\left(Z_{\text {in }}\right)} \\
=\frac{3\left|\widetilde{I}_{1}\left(\frac{Z_{m}}{Z_{m}+Z_{2}}\right)\right|^{2} \frac{(1-s)}{s} R_{2}-W_{f w}}{3\left|\widetilde{I}_{1}\right|^{2} \operatorname{real}\left(Z_{\text {in }}\right)}
\end{gathered}
$$

Where, the friction and windage loss, $W_{f w}$, is taken as a constant percentage of the rated horse power, e.g., $1.2 \%$ for 4-pole motors below $200 \mathrm{hp}$, as suggested by many statistical methods in [1].

\section{EXPERIMENTAL RESULTS}

The proposed motor efficiency estimation method has been verified by both computer simulations and motor experiments. The parameters of the motor used in experiment are listed in Table I.

In the experimental setup, a three-phase induction motor is line-connected to a 230 -volt mains supply. A dc generator connected to resistor boxes serves as the dynamometer. The actual efficiency is directly calculated from the shaft torque, measured by an in-line rotary torque transducer.

The unbalances in the voltages and currents are negligible $\left(V_{I_{-}} / V_{l^{+}}<1 \%\right.$ and $\left.I_{I_{-}} / I_{I^{+}}<3 \%\right)$. The line voltages and currents are sampled at $2 \mathrm{kHz}$ and collected using a NI LabVIEW data acquisition system.

The motor equivalent circuit is solved using data from two load conditions: (1) $19.09 \%$ rated load, $1775 \mathrm{rpm}$, and (2) $71.26 \%$ rated load, $1694 \mathrm{rpm}$. The motor parameters are solved using all three methods. As discussed in [12], Newton's method is regarded as the major solver for this application. The experimental results validate its fast convergence. Using the initial guess calculated from motor nameplate data as in [12], this algorithm converges in only 6 iterations. The results of Newton's method is summarized in Table II, which shows the iterations number $k$, the current parameter estimate, and the error at the current estimate.

Compared with Newton's method, the PSO and simulated annealing methods converge much more slowly. However, these two methods can converge from a general zero initial guess. The same equations are solved using these three methods on a computer with Pentium-IV 3.4 $\mathrm{GHz}$ processor and $512 \mathrm{Mb}$ RAM. The iterations and CPU time required by each method to reach final solution are compared in Table III. Because both the PSO and simulated annealing methods are statistical techniques, their iterations and computation time can vary from time to time. In Table III, the iterations and CPU time used by the PSO and simulated annealing methods are the average of 10 repeated experiments.

It can be observed from Table III that when the Newton's method converges, there is no doubt that it is the best method. In the rare cases where the Newton's method does not converge using the estimated initial guess, both the PSO and the simulated annealing methods can be used. The PSO method is faster, but requires relatively more configuration parameters to be tuned. While, the simulated annealing method is about 10 times slower, but it just needs one localization parameter to be tuned.

Using the solved motor parameters, the motor efficiency at any load levels can be estimated. Fig. 5 to Fig. 7 compare the estimated and measured motor efficiencies versus motor speed and load percentage, when the load changes continuously from almost no load to full load conditions. The estimated motor efficiencies show good agreement (within 2-3\% errors) with the measured efficiencies during the normal motor operations (load ranges from $30 \%$ to $90 \%$ of rated load). The errors under very low load conditions (less than $30 \%$ of rated load) are slightly larger (within $10 \%$ ), but usually under such low load levels, there is no need to estimate motor efficiencies. The errors are caused by many factors, such as motor parameter variations under different load levels, stator resistance and speed estimation errors, motor nameplate information inaccuracies, etc.

The agreement between the estimated and measured efficiencies validates the solved motor parameters and the proposed nonintrusive motor efficiency estimation method.

TABle I. PARAmeters of the InduCtion Motor In EXPERIMENT.

\begin{tabular}{|c|c||c|c|}
\hline Brand & GE & Volts & $230 / 460 \mathrm{~V}$ \\
\hline HP & 7.5 & F.L.AMPS & $18.2 / 9.1 \mathrm{~A}$ \\
\hline CAT. NO. & S231 & RPM & 1755 \\
\hline Design & NEMA-A & Nom. PF & 0.865 \\
\hline Enclosure & TEFC & Nom. Eff. & 0.895 \\
\hline
\end{tabular}

Table II. Newton's Vector Method Results.

\begin{tabular}{|c|c|c|c|c|c|}
\hline $\boldsymbol{k}$ & $\boldsymbol{X}_{\boldsymbol{I}} \mathbf{( \Omega )}$ & $\boldsymbol{R}_{\boldsymbol{C}}(\mathbf{\Omega})$ & $\boldsymbol{X}_{\boldsymbol{m}}(\mathbf{\Omega})$ & $\boldsymbol{R}_{\mathbf{2}}(\mathbf{\Omega})$ & Error \\
\hline \hline $\mathbf{0}$ & 0.907794 & 70.949235 & 19.53566 & 0.500000 & 17.457290 \\
\hline $\mathbf{1}$ & 3.41516 & 92.305626 & 55.92357 & 0.534489 & 7.0520789 \\
\hline $\mathbf{2}$ & 1.77525 & 244.494693 & 86.55143 & 0.497332 & 3.0326519 \\
\hline $\mathbf{3}$ & 1.93057 & 1325.90180 & 84.4203 & 0.520816 & 0.6147128 \\
\hline $\mathbf{4}$ & 1.938864 & 824.305199 & 80.9109 & 0.520560 & 0.0258162 \\
\hline $\mathbf{5}$ & 1.938487 & 846.149846 & 80.8698 & 0.520614 & 0.0000644 \\
\hline $\mathbf{6}$ & 1.938487 & 846.112922 & 80.86943 & 0.520614 & 0.0000000 \\
\hline
\end{tabular}

TABle III. Comparison of Three Numerical Methods.

\begin{tabular}{|c|c|c|c|}
\hline Methods & Convergence & Iterations & CPU Time \\
\hline Newton's Vector & Local & 6 & $0.185 \mathrm{~s}$ \\
\hline PSO & Global & 2601 & $2.432 \mathrm{~s}$ \\
\hline Simulated Annealing & Global & 3377 & $17.123 \mathrm{~s}$ \\
\hline
\end{tabular}




\section{CONCLUSIONS}

This paper proposes a novel nonintrusive method for in-service motor efficiency estimation based on a modified induction motor equivalent circuit using only motor terminal quantities and nameplate information. Rotor speed and stator resistance are extracted from stator voltages and currents. No load test is eliminated by using empirical values instead. Stay-load loss is considered in the equivalent circuit by adding an equivalent resistor in the rotor circuit, whose value is derived from the assumed stray-load loss suggested in IEEE Std-112. Only a few cycles of line voltages and currents from two different motor operating points and motor nameplate information are required to develop the equivalent circuit. The parameters are obtained by solving a set of highly nonlinear and multi-dimensional equations. Three numerical root finding methods are adopted to guarantee the solution of the parameters under any situations. The proposed method has been experimentally validated by testing a $7.5 \mathrm{hp} \mathrm{TEFC}$ induction motor. The estimated efficiency agrees with the measured efficiency within $2-3 \%$ errors during the normal motor operations. A significant contribution of this method is that the efficiency estimate is completely based on motor terminal quantities and motor nameplate information.

\section{ACKNOWLEDGMENT}

This work is financially supported by U.S. Department of Energy and Eaton Corporation.

\section{REFERENCES}

[1] B. Lu, T. G. Habetler, and R. G. Harley, "A survey of efficiency estimation methods of in-service induction motors with considerations of condition monitoring," in Proc. 2005 International Electric Machine and Drive Conference (IEMDC'05), May 2005, pp.1365-1372.
[2] IEEE Standard Test Procedure for Polyphase Induction Motors and Generators, IEEE Std. 112-2004, Nov. 2004.

[3] J. D. Kueck, M. Olszewski, D. A. Casada, J. Hsu, P. J. Otaduy, and L. M. Tolbert, "Assessment of methods for estimating motor efficiency and load under field conditions," Oak Ridge National Laboratory report, ORNL/ TM-13165, 1996.

[4] NEMA - MG 1 Standard, 2003.

[5] Y. El-Ibiary, "An accurate low-cost method for determining electric motors's efficiency for the purpose of plant energy management," IEEE Trans. Industrial Applications, vol.39, no. 4, July/Aug. 2003, pp. 1205-1210.

[6] K. D. Hurst and T. G. Habetler, "Sensorless speed measurement using current harmonic spectral estimation in induction machine drives," IEEE Trans. Power Electronics, vol. 11, no. 1, Jan. 1996, pp. 66-73.

[7] S. B. Lee and T. G. Habetler, "An online stator winding resistance estimation technique for temperature monitoring of line-connected induction machines," IEEE Trans. Industrial Applications, vol.39, no. 3, May/June 2003, pp. 685-694.

[8] IEEE Standard Test Procedure for Polyphase Induction Motors and Generators, IEEE Std. 112-1996, Sept. 1996.

[9] R. J. Schilling and S. L. Harris, Applied Numerical Methods for Engineers using MATLAB and C, Pacific Grove, CA: Thomson, 2000.

[10] J. Kennedy and R. C. Eberhart, "Particle swarm optimization," in Proc. IEEE International Conference on Neural Networks, Piscataway, NJ, USA, Nov. 27-Dec. 1, 1995, vol. 4, pp. 1942-1948.

[11] M. Clerc and J. Kennedy, "The particle swarm - explosion, stability, and convergence in a multidimensional complex space," IEEE Trans. Evolutionary Computation, vol. 6, no. 1, Feb. 2002, pp. 58-73.

[12] B. Lu, W. Qiao, T. G. Habetler, and R. G. Harley, "Solving induction motor equivalent circuit using numerical methods for an in-service and nonintrusive motor efficiency estimation method," in Proc. $20065^{\text {th }}$ International Power Electronics and Motion Control Conference (IPEMC'06), Aug. 2006.

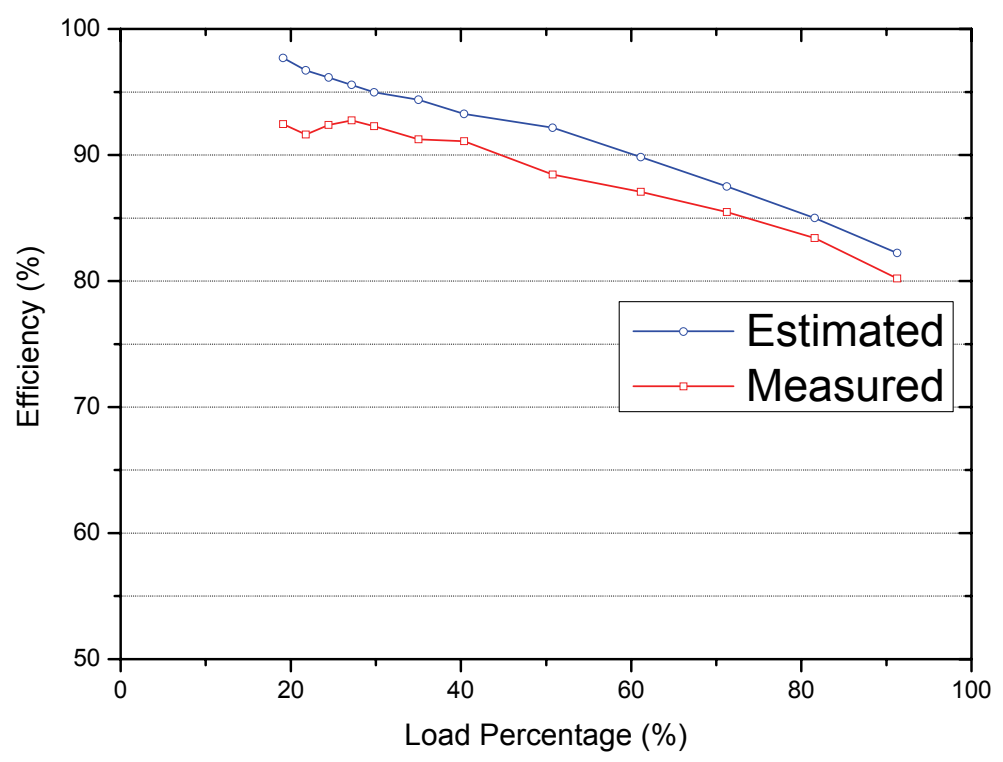

Fig. 5. Estimated and measured efficiencies versus load percentage curve. 


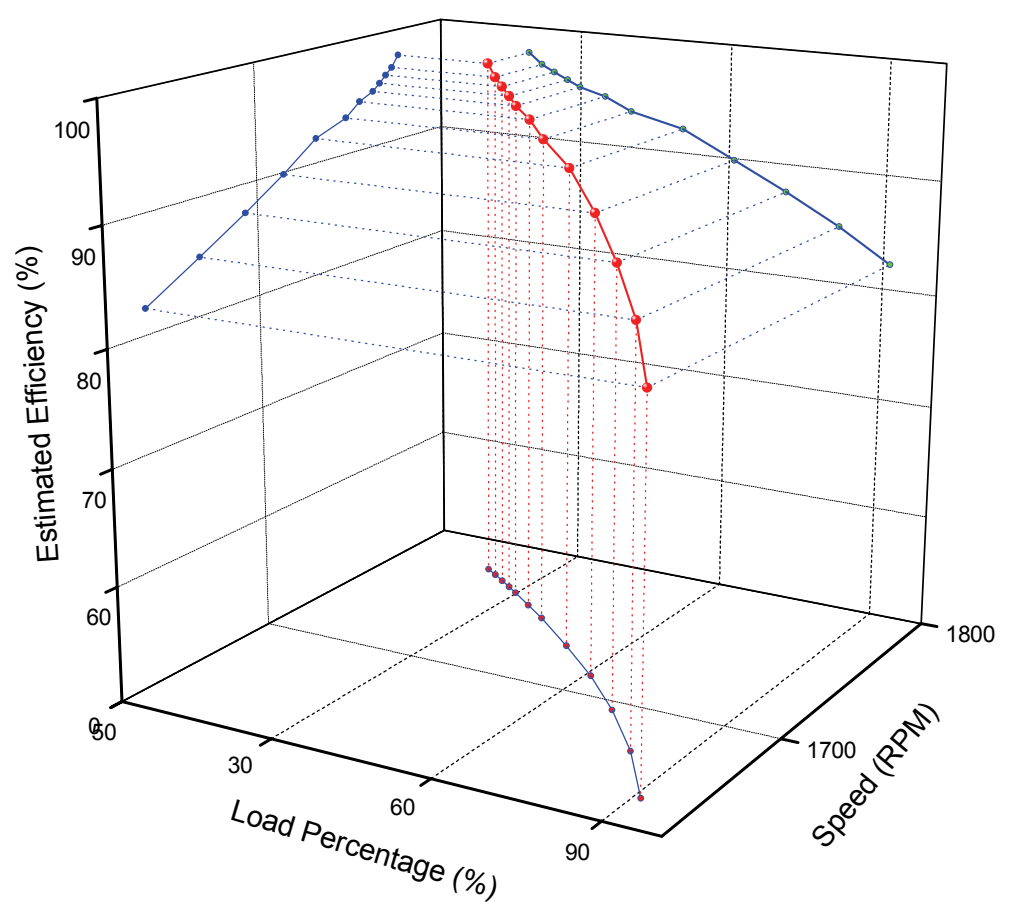

Fig. 6. Estimated efficiency - speed - load curve of the $7.5 \mathrm{hp} \mathrm{GE} \mathrm{TEFC} \mathrm{motor.}$

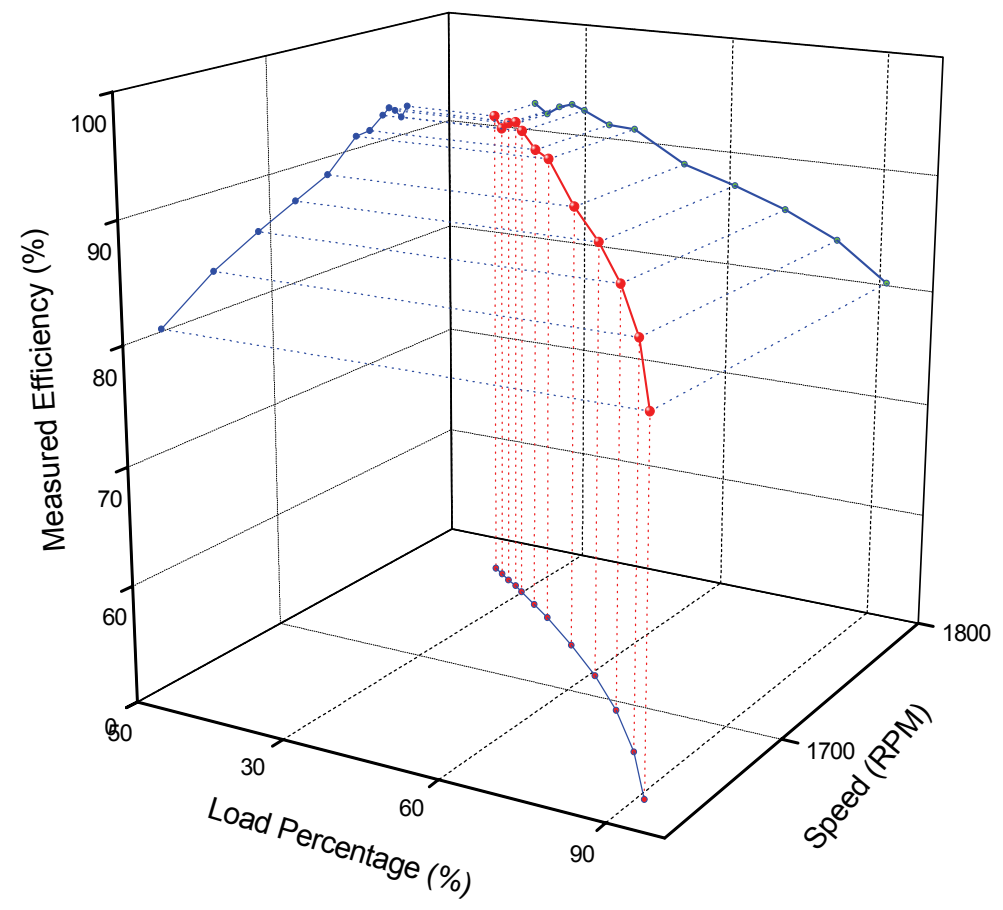

Fig. 7. Measured efficiency - speed - load curve of the 7.5 hp GE TEFC motor. 


\title{
Discussions of Non-intrusive Motor Efficiency Estimation using Wireless Sensor Networks for Medium Size Motors
}

\author{
Bin $\mathrm{Lu}$
}

October 24, 2005 


\section{Review of the MAGT Method}

In the modified air-gap torque (MAGT) method, the motor efficiency, $\eta$, is estimated as

$$
\eta=\frac{P_{\text {output }}}{P_{\text {input }}}=\frac{T_{\text {shaft }} \cdot \omega_{r}}{P_{\text {input }}}=\frac{T_{\text {air-gap }} \cdot \omega_{r}-W_{f w}-W_{L L r}}{P_{\text {input }}}
$$

where, $P_{\text {output }}$ is the output power, $P_{\text {input }}$ is the input power, $\omega_{r}$ is the rotor speed, $T_{\text {shaft }}$ is the shaft torque, $W_{f w}$ is the friction and windage loss, and $W_{L L r}$ is the rotor stray load loss.

In (1), rotor speed is estimated from stator current spectrum; the losses $\left(W_{f w}\right.$ and $\left.W_{L L r}\right)$ are estimated from motor nameplate data; and air-gap torque and input power are both determined by motor input voltages and currents. Therefore, the efficiency estimate in MAGT method is completely based on motor terminal quantities and motor nameplate information. This guarantees the non-intrusive nature of this method, which enables the motor efficiency estimation in a wireless sensor networks (WSN) architecture.

The advantages of this method are:

(1) It is a non-intrusive motor efficiency estimation method, and is suitable for in-service motor testing without interfering with the motor's normal operation.

(2) Only motor terminal voltages and currents are required. The installation of costly speed and torque transducers are eliminated.

(3) Air-gap torque can be estimated while motor is running. Online continuous estimation is possible.

(4) Stator copper loss, core loss, and stator stray load losses have been considered in the air-gap torque calculation.

(5) Losses associated with unbalances in power supply are considered.

(6) It can be used for not only induction motors, but also adjustable-speed motors, and brushless dc motors.

The limitations of this MAGT method are:

(1) It can be applied only for three phase motors, not for single phase motors.

(2) The non-intrusiveness is obtained by sacrificing the accuracy, since the estimate of speed, stator resistance, and losses produces additional errors. 


\section{Discussions on Medium Size Motors}

In industry, small size induction motors (below $200 \mathrm{hp}$ ) make up $98 \%$ of the motors in service and consume $85 \%$ of the energy used. Clearly, improving the efficiencies of these motors improves overall energy savings in industry. This is the original motivation of applying non-intrusive motor energy evaluation using the WSN architecture into industrial plants. However, because small size motors are relatively inexpensive, backups are usually easily available. Besides this, the energy consumed by a small motor is sometimes negligible compared with medium and large size motors. Therefore, the energy evaluation and condition monitoring of small size motors often do not draw enough attention in industry, unless a plant is primarily made up of small motors.

While medium size motors are much more important. They usually work as the very critical components in a plant. They are expensive and normally not available directly from market, but require customized orders. Due to these reasons, these motors do not have backups in most cases. In some special plants that involve high power machinery, such as coal mines and paper mills, the majority of the consumed energy is from medium size motors. In these cases, energy evaluation of medium size motor becomes necessary.

Generally, the proposed MAGT method is applicable for all induction motors regarding their sizes, as long as they are three phase motors. However, because of the structural differences between medium and small size motors, several practical considerations on efficiency estimation, data collection, and wireless communication have to be revisited to extend the non-intrusive energy evaluation scheme in the WSN architecture to medium size motors. The following section lists the preliminary discussions of these practical considerations.

\section{1) High Nominal Efficiency}

Because of the less per unit stator resistance, less stray load losses, and other reasons related to their structures, medium size motors have higher nominal efficiencies than small size motors. For some designs, the nominal efficiency could be higher than $95 \%$. This determines that the accurate efficiency estimate during high load range will be more difficult for medium size motors. This is because that with a certain absolute estimation error, the higher the actual efficiency is, the higher the relative estimation error is. For 
instance, for a small size motor with $85 \%$ efficiency, an efficiency estimate with $\pm 2 \%$ error $(83 \%-87 \%)$ could be regarded as highly accurate. However, for a medium size motor with $96 \%$ efficiency, the same $\pm 2 \%$ error (94\%-98\%) would be relatively inaccurate.

Fortunately, the research of non-intrusive motor efficiency estimation using WSN mainly focuses on motors operating under relatively low load levels, typically $30 \%-70 \%$ rated load in industrial plants, which result in reduced motor efficiencies.

\section{2) Loss Estimation}

A significant difference between the MAGT method and traditional intrusive methods is that the no load losses are not obtained from no load test, but from loss estimation. Since the stator copper loss, core loss, and stator stray load loss are already considered in the air-gap torque calculation, only two loss items are left to be determined: rotor stray load loss, $W_{L L r}$, and friction and windage loss, $W_{f w}$.

The rotor stray load loss is estimated from the empirical values listed in the IEEE Standard-112, as shown in Table 1. Depending on the rated power of a motor, an empirical percentage of rated output power is used to estimate the stray load loss. For instance, for small size motors less than $125 \mathrm{hp}$, stray load loss is assumed to be $1.8 \%$ of rated output power; while for medium size motors over $500 \mathrm{hp}$, stray load loss is smaller and $1.2 \%$ is used.

TABLE 1. ASSUMED VALUES FOR STRAY LOAD LOSS IN IEEE STD-112.

\begin{tabular}{|c|c|c|}
\hline \multicolumn{2}{|c|}{ Machine Rating } & $\begin{array}{c}\text { Stray load loss percent of } \\
\text { rated output power }\end{array}$ \\
\hline $1-125 \mathrm{hp}$ & $1-90 \mathrm{~kW}$ & $1.8 \%$ \\
\hline $126-500 \mathrm{hp}$ & $91-375 \mathrm{~kW}$ & $1.5 \%$ \\
\hline $501-2499 \mathrm{hp}$ & $376-1850 \mathrm{~kW}$ & $1.2 \%$ \\
\hline $2500 \mathrm{hp}$ and up & $1851 \mathrm{~kW}$ and up & $0.9 \%$ \\
\hline
\end{tabular}

The same thing happens for the friction and windage loss. For 4 pole motors less than $200 \mathrm{hp}$, an empirical value of $1.2 \%$ percentage of rated output power is used in MAGT method to estimate friction and windage loss. But when this method is applied for medium size motors, different values may be used to get more accurate efficiency 
estimates. The estimation of friction and windage loss has not been systematically studied and documented in IEEE Std-112. More research should be done in this area. A similar loss estimate has been found in the ORMEL96 method by Oak Ridge National Lab.

\section{3) Stator Resistance Estimation}

The MAGT method proposes different ways to deal with stator resistance, $R_{S}$, at different degrees of accuracy/complexity.

The easiest way is to just use the resistance under normal temperature from the motor manufacturer datasheet and assume it is a constant during the motor operations. Obviously, this rough assumption does not reflect the reality and brings additional errors, especially when motor operates at high load levels.

A more accurate but complex method is to install an additional hardware device between the power supply and the motor terminals to inject a controllable dc bias into the motor. Then, the actual stator resistance can be found from the dc components of the input voltages and currents. This resistance estimation process can be regarded as nonintrusive, after the injection circuit is developed and included.

The dc signal injection method has been proved to be effective and efficient for small size motors. However, when medium size motors are involved, this process may become rather complicated due to the high voltages and large currents in the motor stator winding. Besides, the magnitude of the injected dc voltage needs to be scaled up to get reasonable results. Therefore, the dc signal injection hardware should be redesigned to accommodate these concerns.

\section{4) Accuracy and Cost of Data Collection}

In the proposed non-intrusive energy evaluation system with the WSN architecture, the accuracy of the efficiency estimate is not only determined by the MAGT method itself, but also closely related to the accuracy of sampled motor data, such as the terminal voltages and currents.

At this time, this proposed scheme has been successfully implemented on small size motors $(230 / 460$ volts and less than $10 \mathrm{hp})$. In the demonstration system, highly accurate voltage and current transducers are used to scale the original signals down to the range of 
0-5 volts. More specifically, the voltage transducers are LEM LV 25-P with 10-500 volts rms voltage range, $\pm 0.8 \%$ accuracy, and $<0.2 \%$ linearity; the current transducers are LEM LA 55-P with 50 amps rms current range, $\pm 0.65 \%$ accuracy, and $<0.15 \%$ linearity. Both of these transducers are highly accurate using the Hall effect.

When the same system is applied on medium size motors, both the motor terminal voltages and currents are much larger. The transducers mentioned above are out of consideration. Special VTs and CTs have to be selected for accurate measurement of the motor terminal data. The accuracy and cost of these devices should be carefully considered.

In industrial plant, usually the motor stator voltages and currents are already measured at the $\mathrm{MCC}$ for protection purposes. In that case, no additional data collection devices are required for commissioning this energy evaluation system, but still the accuracy of the measured voltages and currents needs to be carefully verified.

\section{5) A/D Conversion Resolution}

This part follows the discussion in the previous section on the accuracy of data collection.

In the implementation of the proposed system, an analog to digital conversion (ADC) unit is required to sample the scaled $0-5$ volts signals at certain resolution and frequency, which come out of the voltage and currents transducers. Finally, these sampled data are transmitted over the WSN to the Central Supervisory Station, where these data are rescaled to their original values by a computer program for efficiency estimation.

Currently for small size motors, the demonstration system uses a 12-bit, $2 \mathrm{kHz}$ ADC unit to sample the scaled voltages and currents. When this system is extended to medium size motors, the 12-bit resolution may not be enough considering both voltages and currents are many times larger. Higher resolution ADC unit may be used, but it will dramatically increase the cost of the overall system. More research needs to be done to clarify this issue. 


\section{Summary}

To summarize, the proposed MAGT method and non-intrusive energy usage evaluation system using WSN could be applied for three phase medium size induction motors, because of their same physical mechanisms with small size induction motors.

However, because of some structural differences between medium and small size motors, several practical considerations on efficiency estimation, data collection, and wireless communication should be revisited. These considerations are discussed in this report. 


\title{
Wireless Sensor Network for Advanced Energy Management Solutions
}

\section{Appendix B- External Publications}

\author{
DOE Award Number: DE-FC36-04G014000 \\ Reporting Period: January 2004 - June 2009 \\ Principal Investigator: Peter J. Theisen \\ (414) 449-6924 \\ PeterJTheisen@eaton.com \\ Bin Lu, Ph.D. \\ (414) 449-6036 \\ BinLu@eaton.com \\ Charles J. Luebke \\ (414) 449-7264 \\ CharlesJLuebke@eaton.com
}

Recipient: Eaton Corporation Innovation Center 4201 N. $27^{\text {th }}$ Street

Milwaukee, WI 53216 


\section{DOE1 Final Report}

\section{Appendix B:}

\section{A. Publications}

1. B. Lu, T. G. Habetler, and R. G. Harley, "A survey of efficiency-estimation methods of in-service induction motors,” IEEE Transactions on Industry Applications, vol. 42, no. 4, pp. 924-933, Jul./Aug. 2006. Also previously presented in Proc. 2005 International Electric Machine and Drive Conference (IEMDC’05), May 2005, pp. 1365-1372.

2. S. R. Das, K. Srinivasan, and L. R. Pereira, “An Efficient Graph Theoretic Framework For Industrial Wireless Sensor Networks,” in Proc. of the 3rd International Conference on Networked Sensing Systems (INSS 2006), Jun. 2006.

3. B. Lu, T. G. Habetler, R. G. Harley, J. A. Gutiérrez, and D. B. Durocher, "Energy evaluation goes wireless: Applying wireless sensor network in industrial plant energy evaluation and planning systems,” IEEE Industry Applications Magazine, vol. 13, no. 2, pp. 17-23, Mar./Apr. 2007. Also previously presented in Proc. the 52nd Annual IEEE Pulp and Paper Industry Technical Conference (PPIC’06), Jun. 2006, pp. 1-7.

4. B. Lu, W. Cao, and T. G. Habetler, "Error analysis of motor-efficiency estimation and measurement," in Proc the. 38th IEEE Power Electronics Specialist Conference (PESC’07), Jun. 2007, pp. 612-617.

5. W. Zhou, T. G. Habetler, R. G. Harley, "Bearing Condition Monitoring Methods for Electric Machines: A General Review,” in Proc. the 6th IEEE International Symposium on Diagnostics for Electric Machines, Power Electronics and Drives (SDEMPED’07), Sep. 2007, pp. 3-6.

6. W. Zhou, T. G. Habetler, and R. G. Harley, "Stator Current-Based Bearing Fault Detection Techniques: A General Review,” in Proc. the 6th IEEE International Symposium on Diagnostics for Electric Machines, Power Electronics and Drives (SDEMPED’07), Sep. 2007, pp. 7-10.

7. W. Zhou, T. G. Habetler, R. G. Harley, and B. Lu, "Incipient bearing fault detection via stator current noise cancellation using Wiener filter,” in Proc. the 6th IEEE International Symposium on Diagnostics for Electric Machines, Power Electronics and Drives (SDEMPED’07), Sep. 2007, pp. 11-16.

8. B. Lu, W. Cao, I. French, K. J. Bradley, and T. G. Habetler, "Non-intrusive efficiency determination of in-service induction machines using genetic algorithm and air-gap torque methods,” in Proc. the 42nd IEEE Industrial Applications Society Annual Meeting (IAS’07), Sep. 2007, pp. 1186-1192.

9. H. Cao, T. Yan, S. Das, L. Pereira, and B. Lewis, "Using AADL to Analyze and Design Embedded Systems,” Embedded system design magazine (CMP), Aug., 2007. 
10. P. Zhang, B. Lu, and T. G. Habetler, "A remote and sensorless stator winding resistance estimation method for thermal protection of soft-starter-connected induction machines," IEEE Transactions on Industrial Electronics, vol. 55, no. 10, pp. 3611-3618, Oct. 2008. Also previously presented in Proc. the 6th IEEE International Symposium on Diagnostics for Electric Machines, Power Electronics and Drives (SDEMPED’07), Sep. 2007. pp. 197-202.

11. S. Grubic, J. M. Aller, B. Lu, and T. G. Habetler, "A survey on testing and monitoring methods for stator insulation systems of low voltage induction machines focusing on turn insulation problems," IEEE Transactions on Industrial Electronics, vol.55, no. 12, pp.4127-4136, Dec. 2008. Also previously presented in Proc. the 2008 International Conference on Condition Monitoring and Diagnosis (CMD’08), Apr. 2008, pp. 196-203.

12. Ghosh, L. R. Pereira, T. Yan, and H. Cao, "Modeling Wireless Sensor Network Architectures using AADL,” in Proc. of 4th European Congress on Embedded Real Time Software (ERTS' 08), Jan. 2008.

13. B. Lu and M. D. Paghda, "Induction motor rotor fault detection via wavelet analysis of one-cycle average power," in Proc. the 23rd Annual IEEE Applied Power Electronics Conference (APEC'08), Feb. 2008, pp. 1113-1118.

14. P. Zhang, B. Lu, and T. G. Habetler, "Practical implementation of a remote and sensorless stator resistance-based thermal protection method,” in Proc. the 2008 International Conference on Condition Monitoring and Diagnosis (CMD’08), Apr. 2008, pp. 159-162.

15. B. Lu, D. B. Durocher, and P. Stemper, "Online and nonintrusive continuous motor energy and condition monitoring in process industries,” in Proc. the 54th Annual IEEE Pulp and Paper Industry Technical Conference (PPIC’08), Jul. 2008, pp. 18-26.

16. T. Yan, S. R. Das, L. R. Pereira, "SODA: Scalable On-Demand Aggregation for wireless sensor networks,” in Proc. of 2008 IEEE Wireless Hive Networks Conference, Aug. 2008, pp.1-6,

17. P. Zhang, Y. Du, T. G. Habetler, and B. Lu, "A remote and sensorless thermal protection scheme for soft-starter-connected induction motors,” in Proc. the 43rd IEEE Industrial Applications Society Annual Meeting (IAS’08), Oct. 2008, pp. 1-7.

18. B. Lu, T. G. Habetler, and R. G. Harley, "A nonintrusive and in-service motor efficiency-estimation method using air-gap torque with considerations of condition monitoring," IEEE Transactions on Industry Applications, vol. 44, no. 6, pp. 1666-1674, Nov./Dec. 2008. Also previously presented in Proc. the 41st IEEE Industrial Applications Society Annual Meeting (IAS’06), vol. 3, Oct. 2006, pp. 1533-1540.

19. B. Lu and S. Sharma, "A literature review of IGBT fault diagnostic and protection methods for power inverters,"” in Proc. the $43^{\text {rd }}$ IEEE Industrial Applications Society Annual Meeting (IAS’08), Oct. 2008, pp. 1-8. 


\title{
Wireless Sensor Network for Advanced Energy Management Solutions
}

\section{Appendix C- Patent Applications}

\author{
DOE Award Number: DE-FC36-04G014000 \\ Reporting Period: January 2004 - June 2009 \\ Principal Investigator: Peter J. Theisen \\ (414) 449-6924 \\ PeterJTheisen@eaton.com \\ Bin Lu, Ph.D. \\ (414) 449-6036 \\ BinLu@eaton.com \\ Charles J. Luebke \\ (414) 449-7264 \\ CharlesJLuebke@eaton.com
}

Recipient: Eaton Corporation Innovation Center 4201 N. $27^{\text {th }}$ Street

Milwaukee, WI 53216 


\section{Patents Applications}

1. “Ad-Hoc Network and Method Employing Globally Optimized Routes For Packets,” U.S. patent applied, application number: 11/347077. (Publication No. 2007-0177511) From 05-mEDP-026.

2. "Wireless Communication Network and Wireless Control or Monitoring Device Employing An XML Schema," U.S. patent applied, application number: 12/126530. From 05-mEDP-548.

3. "Motor Bearing Fault Detection via Stator Current Noise Cancellation,” U.S. patent applied, application number: 12/132,056. From 07-mITM-164.

4. “System and Method for Motor Fault Detection Using Stator Current Noise Cancellation,” U.S. patent applied, application number: 12/350287. From 07-mITM-164 CIP.

5. "System and Method for Determining Stator Winding Resistance in an AC Motor Using Motor Drives," U.S. patent applied, application number: 12/363,413. From 07-mITM-204

6. “System and Method to Determine Electric Motor Efficiency Nonintrusively," U.S. patent applied, application number: 12/132,151. From 07-mITM-205.

7. "System and Method for Monitoring and Controlling Stator Winding Temperature In a De-Energized AC Motor,” U.S. patent applied, application number: 12/341,044. From 08-mITM-200.

8. “System and Method for Motor Parameter Estimation,” U.S. patent applied, application number: 12/351,582. From 08-MTC-040. 